\title{
Electron-Deficient Alkynes as Dipolarophile in Pd-Catalyzed Enantioselective (3+2) Cycloaddition Reaction with Vinyl Cyclopropanes
}

Wen-Ping Ding, ${ }^{\mathrm{a}, \mathrm{b}}$ Gao-Peng Zhang, ${ }^{\mathrm{a}}$ Yang-Jie Jiang, ${ }^{\mathrm{a}} \mathrm{Juan} \mathrm{Du},{ }^{\mathrm{a}} \mathrm{Xiu}-$ Yan Liu, ${ }^{\mathrm{a}}$ Di Chen, ${ }^{\mathrm{a}}$ ChangHua Ding, ${ }^{* a, c}$ Qing-Hai Deng, ${ }^{* b}$ Xue-Long Hou*a,d

${ }^{a}$ State Key Laboratory of Organometallic Chemistry, Center for Excellence in Molecular Synthesis, Shanghai Institute of Organic Chemistry (SIOC), Chinese Academy of Sciences (CAS), 345 Lingling Road, Shanghai 200032, China.

${ }^{b}$ The Education Ministry Key Lab of Resource Chemistry and Shanghai Key Laboratory of Rare Earth Functional Materials, Shanghai Normal University, Shanghai 200234, China.

${ }^{c}$ Department of Chemistry, Innovative Drug Research Center, Shanghai University, Shanghai 200444, China.

${ }^{d}$ Shanghai-Hong Kong Joint Laboratory in Chemical Synthesis, SIOC, CAS, China.

dingchanghua@ shu.edu.cn; qing-haideng@shnu.edu.cn; xlhou@sioc.ac.cn

\section{Content}

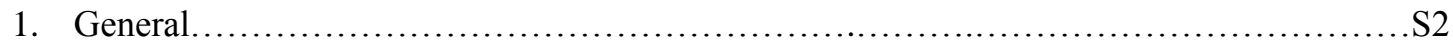

2. Table S1. The impact of reaction conditions on Pd-catalyzed $(3+2)$ cycloaddition reaction of vinyl cyclopropane 1a with alkynyl $\alpha$-ketoester $\mathbf{2 a}$.

3. NMR Data of the Electron-Deficient Alkynes for Figure 2 ............................... 4

4. General experimental procedures for alkynyl $\alpha$-ketoesters $\mathbf{2}$ and the

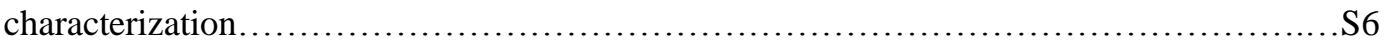

5. General experimental procedure for Scheme 2 and the characterization of

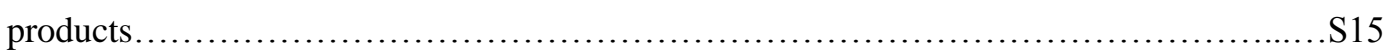

6. General experimental procedure for 1,2-dione 5 and the characterization................S30

7. General experimental procedure for Scheme 3 and the characterization of

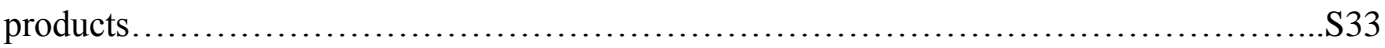

8. Palladium-catalyzed (3+2) cycloaddition of vinylcyclopropane 1a and alkyne 2a on $8 \mathrm{mmol}$

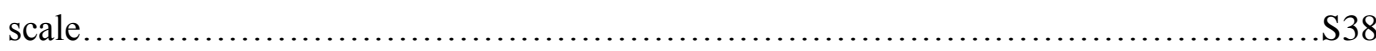

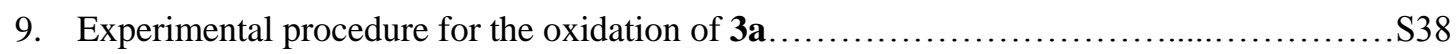

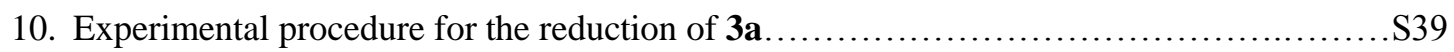

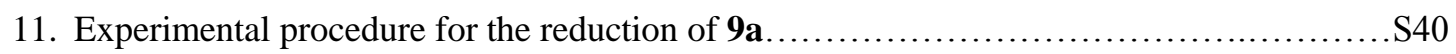

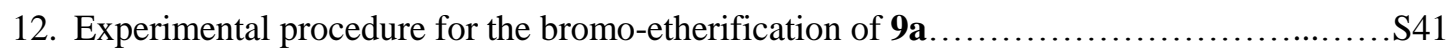

13. X-ray Crystallographic Data of 3a, 9a, and 11a......................................S42

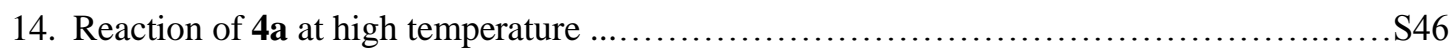

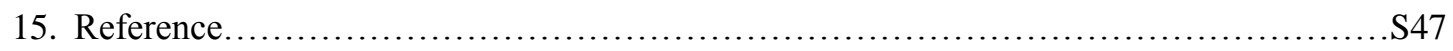

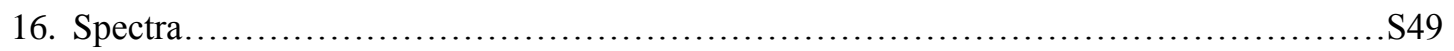




\section{General}

The reactions were carried out in a flame-dried glassware under a dried argon atmosphere. All solvents were purified and dried by using standard methods prior to use. Commercially available reagents were used without further purification. ${ }^{1} \mathrm{H}$ NMR spectra were recorded on a NMR instrument operated at $400 \mathrm{MHz}$. Chemical shifts are reported in ppm from tetramethylsilane with the solvent resonance as the internal standard $\left(\mathrm{CDCl}_{3}: \delta 7.26 \mathrm{ppm}\right)$. Data are reported as follows: chemical shift, multiplicity ( $\mathrm{s}=$ singlet, $\mathrm{d}=$ doublet, $\mathrm{t}=$ triplet, $\mathrm{q}=$ quartet, $\mathrm{br}=$ broad, $\mathrm{m}=$ multiplet or unresolved $)$, coupling constants $(\mathrm{Hz})$, and integration. ${ }^{13} \mathrm{C}$ NMR spectra were recorded on a NMR instrument operated at $100 \mathrm{MHz}$ with complete proton decoupling. ${ }^{19} \mathrm{~F}$ NMR spectra were recorded on a NMR instrument operated at $376 \mathrm{MHz}$. Chemical shifts are reported in ppm from tetramethylsilane with the solvent resonance as the internal standard $\left(\mathrm{CDCl}_{3}: \delta 77.1 \mathrm{ppm}\right)$. Infrared spectra were recorded from thin films of pure samples. Mass and HRMS spectra were measured in EI or ESI mode and the mass analyzer type used for the HRMS was TOF. Thin layer chromatography was performed on pre-coated glass-back plates and visualized with UV light at $254 \mathrm{~nm}$. The melting point were determined by WRS-2 melting point instrument. Flash column chromatography was performed on silica gel. Enantiomer ratios were determined by chiral HPLC analysis in comparison with authentic racemic materials. The signal with the retention time being 3 and 5 min in HPLC chromatograms was the traces of $\mathrm{CH}_{2} \mathrm{Cl}_{2}$ (HPLC grade) that was used to dissolve the sample during the HPLC analysis. The HPLC spectra of $\mathrm{CH}_{2} \mathrm{Cl}_{2}$ were given on the pages S254 and S255. Methyl 3-phenylpropiolate and dimethyl but2-ynedioate were purchased from Tokyo Chemical Industry Co., Ltd. The following compounds were prepared according to the procedures described in literatures: vinylcyclopropanes, ${ }^{1}$ 4-(p-tolyl)but-3-yn-2-one, ${ }^{2}$ iso-propyl 2-chloro-2-oxoacetate, ${ }^{3}$ methyl 2-(methoxy(methyl)amino)-2-oxoacetate, ${ }^{4}$ methyl 2-oxo-4-( $p$-tolyl)but-3ynoate $\mathbf{2 a},{ }^{3}$ ethyl 2-oxo-4-( $p$-tolyl)but-3-ynoate $\mathbf{2 v},{ }^{3}$ iso-propyl 2-oxo-4-( $p$-tolyl)but-3ynoate $2 \mathbf{w},{ }^{3}$ methyl 2-oxooctadec-3-ynoate $2 \mathbf{r},{ }^{4}$ 1-phenyl-4-( $p$-tolyl)but-3-yne-1,2dione 5a, ${ }^{5}$ methyl 2-oxo-4-phenylbut-3-ynoate $\mathbf{2 b},{ }^{3}$ methyl 4-(3-chlorophenyl)-2oxobut-3-ynoate $\mathbf{2} \mathbf{i},{ }^{3}$ 2-(hex-1-en-1-yl)cyclopropane-1,1-dicarbonitrile $\mathbf{1 b}{ }^{6}$ 


\section{Table S1. The impact of reaction conditions on Pd-catalyzed $(3+2)$ cycloaddition}

reaction of vinyl cyclopropane 1a with alkynyl $\alpha$-ketoester $2 a^{a}$

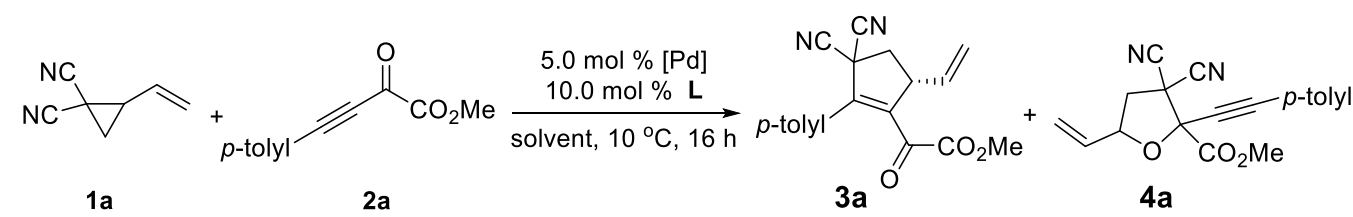

\begin{tabular}{|c|c|c|c|c|c|c|c|}
\hline L11 & L12 & & 13 & L14 & L15 & & \\
\hline entry & $\mathrm{Pd}$ & $\mathbf{L}$ & additive & solvent & $3 \mathbf{a} / 4 \mathbf{a}^{b}$ & $\begin{array}{l}\text { yield } \\
(\%)^{c}\end{array}$ & $\begin{array}{c}\text { ee } \\
(\%)^{d}\end{array}$ \\
\hline $1^{e}$ & $\operatorname{Pd}(\mathrm{dba})_{2}$ & L11 & - & THF & $36 / 64$ & 29 & 29 \\
\hline $2^{e}$ & $\operatorname{Pd}(\mathrm{dba})_{2}$ & L12 & - & THF & $70 / 30$ & 59 & 72 \\
\hline $3^{e}$ & $\operatorname{Pd}(\mathrm{dba})_{2}$ & L13 & - & THF & $84 / 16$ & 84 & 82 \\
\hline $4^{e}$ & $\mathrm{Pd}(\mathrm{dba})_{2}$ & L14 & - & THF & $88 / 12$ & 83 & 85 \\
\hline $5^{e}$ & $\operatorname{Pd}(\mathrm{dba})_{2}$ & L15 & - & THF & $90 / 10$ & 32 & 65 \\
\hline 6 & $\operatorname{Pd}(d b a)_{2}$ & L14 & - & THF & $71 / 29$ & 70 & 88 \\
\hline 7 & $\mathrm{Pd}(\mathrm{dba})_{2}$ & L5 & ${ }_{n} \mathrm{Bu}_{4} \mathrm{NBr}$ & THF & $92 / 8$ & 85 & 52 \\
\hline 8 & $\mathrm{Pd}(\mathrm{dba})_{2}$ & L5 & ${ }_{n} \mathrm{Bu}_{4} \mathrm{NCl}$ & THF & $93 / 7$ & 57 & 16 \\
\hline 9 & $\operatorname{Pd}(\mathrm{dba})_{2}$ & L5 & $\mathrm{KBr}$ & THF & $68 / 32$ & 68 & 88 \\
\hline 10 & $\operatorname{Pd}(\mathrm{dba})_{2}$ & L5 & $\mathrm{LiCl}$ & THF & $76 / 24$ & $65 \%$ & $42 \%$ \\
\hline 11 & $\operatorname{Pd}(\mathrm{dba})_{2}$ & L5 & $\mathrm{LiBr}$ & THF & $98 / 2$ & 83 & 60 \\
\hline 12 & $\mathrm{Pd}(\mathrm{dba})_{2}$ & L5 & $\mathrm{MgBr}_{2}$ & THF & - & trace & - \\
\hline 13 & $\mathrm{Pd}(\mathrm{dba})_{2}$ & L5 & $\mathrm{AgBr}$ & THF & $65 / 35$ & 62 & 88 \\
\hline 14 & $\mathrm{Pd}(\mathrm{dba})_{2}$ & L5 & $3 \AA \mathrm{MS}$ & THF & $64 / 36$ & 61 & 86 \\
\hline 15 & $\mathrm{Pd}(\mathrm{dba})_{2}$ & L5 & - & $\begin{array}{c}\text { DCM/toluene } \\
(1 / 1)\end{array}$ & $75 / 25$ & 63 & 84 \\
\hline
\end{tabular}




\begin{tabular}{|c|c|c|c|c|c|c|c|}
\hline 16 & $\mathrm{Pd}(\mathrm{dba})_{2}$ & L5 & - & $\begin{array}{c}\text { DCM/THF } \\
(1 / 1)\end{array}$ & $90 / 10$ & 82 & 84 \\
\hline 17 & $\mathrm{Pd}(\mathrm{dba})_{2}$ & L5 & - & $\begin{array}{c}\text { DMSO/DME } \\
(1 / 1)\end{array}$ & $98 / 2$ & 86 & 86 \\
\hline 18 & $\operatorname{Pd}(d b a)_{2}$ & L5 & - & $\begin{array}{c}\mathrm{DMSO} / \mathrm{THF} \\
(1 / 1)\end{array}$ & $98 / 2$ & 90 & 66 \\
\hline 19 & $\operatorname{Pd}(\mathrm{dba})_{2}$ & L5 & - & $\begin{array}{c}\mathrm{DCM} / \mathrm{DME} \\
(1 / 1)\end{array}$ & $95 / 5$ & 73 & 88 \\
\hline 20 & $\operatorname{Pd}(\mathrm{dba})_{2}$ & L5 & - & $\begin{array}{c}\text { DCM/DME } \\
(0.2 / 1.8)\end{array}$ & $52 / 48$ & 46 & 88 \\
\hline 21 & $\mathrm{Pd}(\mathrm{dba})_{2}$ & L5 & - & $\begin{array}{c}\text { DCM/DME } \\
(0.5 / 1.5)\end{array}$ & $60 / 40$ & 55 & 89 \\
\hline 22 & $\operatorname{Pd}(\mathrm{dba})_{2}$ & L5 & - & $\begin{array}{c}\text { DCM/DME } \\
(0.8 / 1.2)\end{array}$ & $75 / 25$ & 66 & 89 \\
\hline 23 & $\operatorname{Pd}(d b a)_{2}$ & L5 & - & $\begin{array}{c}\text { DCM/DME } \\
(1.2 / 0.8)\end{array}$ & $94 / 6$ & 80 & 89 \\
\hline 24 & $\mathrm{Pd}(\mathrm{dba})_{2}$ & L5 & - & $\begin{array}{c}\text { DCM/DME } \\
(1.8 / 0.2)\end{array}$ & $96 / 4$ & 83 & 86 \\
\hline 25 & $\mathrm{Pd}_{2}(\mathrm{dba})_{3} \cdot \mathrm{CHCl}_{3}$ & L5 & - & $\begin{array}{c}\mathrm{DCM} / \mathrm{DME} \\
(1.2 / 0.8)\end{array}$ & $91 / 9$ & 85 & 81 \\
\hline 26 & $\operatorname{PdCp}\left(\eta^{3}-\mathrm{C}_{3} \mathrm{H}_{5}\right)$ & L5 & - & $\begin{array}{c}\mathrm{DCM} / \mathrm{DME} \\
(1.2 / 0.8)\end{array}$ & $96 / 4$ & 86 & 86 \\
\hline 27 & $\begin{array}{c}{\left[\mathrm{Pd}\left(\eta^{3}-\right.\right.} \\
\left.\left.\mathrm{C}_{3} \mathrm{H}_{5}\right) \mathrm{Cl}\right]_{2}\end{array}$ & L5 & - & $\begin{array}{c}\text { DCM/DME } \\
(1.2 / 0.8)\end{array}$ & $70 / 30$ & 44 & 81 \\
\hline
\end{tabular}

${ }^{a}$ Conditions: molar ratio of 1a:2a:Pd(dba) $: \mathbf{L}=250: 100: 5: 10 .{ }^{b}$ Determined by ${ }^{1} \mathrm{H} \mathrm{NMR}$ spectroscopy. ${ }^{c}$ Yield of 3a determined by ${ }^{1} \mathrm{H}$ NMR yield using mesitylene as the internal standard. ${ }^{d}$ ee of $\mathbf{3 a}$ determined by chiral HPLC. ${ }^{e}$ Reaction temperature: $25{ }^{\circ} \mathrm{C}$.

3. NMR Data of the Electron-Deficient Alkynes for Figure 2 
4-(p-tolyl)but-3-yn-2-one ${ }^{2}$

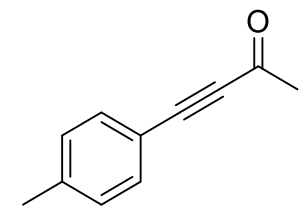

${ }^{1} \mathrm{H}$ NMR (400 MHz, $\left.\mathrm{CDCl}_{3}\right) \delta 7.47(\mathrm{~d}, J=8.0 \mathrm{~Hz}, 2 \mathrm{H}), 7.19(\mathrm{~d}, J=7.9 \mathrm{~Hz}, 2 \mathrm{H}), 2.44$ (s, 3H), 2.39 (s, 3H). ${ }^{13} \mathrm{C}$ NMR (101 MHz, $\left.\mathrm{CDCl}_{3}\right) \delta 184.7,141.5,133.1,129.4,116.7$, 91.0, 88.1, 32.7, 21.7.

methyl 3-phenylpropiolate ${ }^{7}$<smiles>COC(=O)C#Cc1ccccc1</smiles>

${ }^{1} \mathrm{H}$ NMR $\left(400 \mathrm{MHz}, \mathrm{CDCl}_{3}\right) \delta$ 7.58-7.56 (m, 2H), 7.46-7.42 (m, 1H), 7.38-7.34 (m, 2H), $3.83(\mathrm{~s}, 3 \mathrm{H}) .{ }^{13} \mathrm{C}$ NMR $\left(101 \mathrm{MHz}, \mathrm{CDCl}_{3}\right) \delta 154.4,133.0,130.7,128.6,119.5,86.5$, $80.3,52.8$.

dimethyl but-2-ynedioate ${ }^{8}$

$\mathrm{MeO}_{2} \mathrm{C}=\mathrm{CO}_{2} \mathrm{Me}$

${ }^{1} \mathrm{H}$ NMR $\left(400 \mathrm{MHz}, \mathrm{CDCl}_{3}\right) \delta 4.05(\mathrm{~s}, 6 \mathrm{H}) .{ }^{13} \mathrm{C} \mathrm{NMR}\left(101 \mathrm{MHz}, \mathrm{CDCl}_{3}\right) \delta$ 152.1, 74.6, 53.4 .

methyl 2-oxo-4-(p-tolyl)but-3-ynoate $(\mathbf{2 a})^{3}$

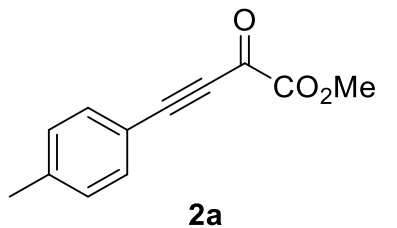

${ }^{1} \mathrm{H}$ NMR (400 MHz, $\left.\mathrm{CDCl}_{3}\right) \delta 7.57(\mathrm{~d}, J=8.0 \mathrm{~Hz}, 2 \mathrm{H}), 7.23(\mathrm{~d}, J=8.0 \mathrm{~Hz}, 2 \mathrm{H}), 3.96$ (s, 3H), 2.41 (s, 3H). ${ }^{13} \mathrm{C}$ NMR (101 MHz, $\left.\mathrm{CDCl}_{3}\right) \delta 169.0,159.7,143.0,133.9,129.6$, 115.9, 99.2, 87.3, 53.6, 21.9. 


\section{General experimental procedures for alkynyl $\alpha$-ketoesters 2 and the characterization}

\section{General method $\mathrm{I}^{3}$}

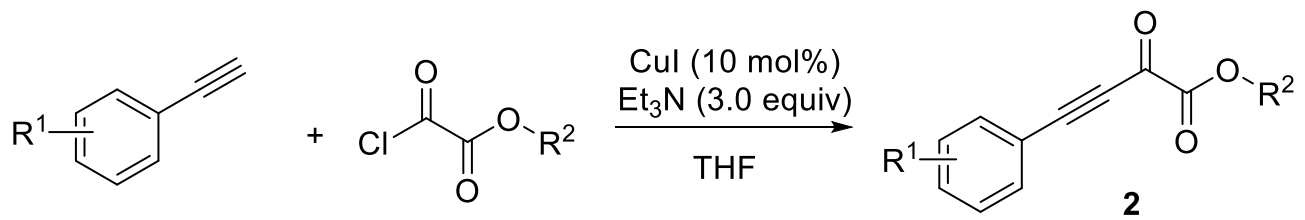

A suspension of $\mathrm{CuI}(328 \mathrm{mg}, 1.7 \mathrm{mmol})$ in THF $(40 \mathrm{~mL})$ was treated with $\mathrm{Et}_{3} \mathrm{~N}$ (7.2 $\mathrm{mL}, 51.7 \mathrm{mmol}$ ) and stirred until a colorless solution formed. The corresponding aryl alkyne (17.2 mmol) and corresponding methyl oxalyl chloride (3.2 mL, $34.4 \mathrm{mmol})$ were added subsequently. The resulting reaction mixture was stirred at room temperature for $18 \mathrm{~h}$. The reaction mixture was quenched with saturated aqueous $\mathrm{NaHCO}_{3}$. The aqueous layer was extracted with ethyl acetate $(20 \mathrm{~mL}$ x 3). The combined organic layers were washed with brine, dried over $\mathrm{Na}_{2} \mathrm{SO}_{4}$ and concentrated under reduced pressure. The resulting residue was purified by flash chromatography on silical gel with petroleum ether and ethyl acetate as eluent to give product.

\section{General method $\mathrm{II}^{4}$}

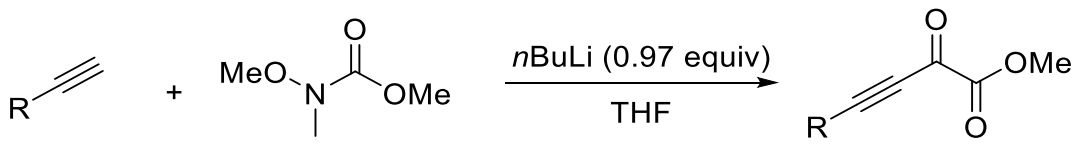

To a solution of alkyne $(8.7 \mathrm{mmol})$ in THF $(15 \mathrm{~mL})$ was added $n$-BuLi $(1.5 \mathrm{M}$ in hexane, $5.1 \mathrm{~mL}, 8.2 \mathrm{mmol})$ at $-30{ }^{\circ} \mathrm{C}$. The mixture was stirred for $3 \mathrm{~h}$ at room temperature and was added dropwise to a solution of methyl methoxymethyl carbamate $(1.0 \mathrm{~g}, 6.80 \mathrm{mmol})$ in $\mathrm{THF}(10 \mathrm{~mL})$ at $-78{ }^{\circ} \mathrm{C}$. The temperature was slowly raised to room temperature and the mixture was stirred for overnight. The solution was filtered on a silica pad and the solvents were removed under reduced pressure. Then the resulting residue was purified by flash chromatography on silica gel with petroleum ether and ethyl acetate as eluent to give product. 
Methyl 4-(4-methoxyphenyl)-2-oxobut-3-ynoate (2c)<smiles>COc1ccc(C#CC(=O)OCc2ccccc2)cc1</smiles>

The compound 2c was obtained according to General method I starting from 1ethynyl-4-methoxybenzene $(1.1 \mathrm{~g}, 8.6 \mathrm{mmol})$. The crude product was purified by flash chromatography on silical gel (petroleum ether / diethyl ether $=10 / 1$ ) to give a light yellow solid (167 mg, yield: $10 \%)$ mp: $77.5-78.2{ }^{\circ} \mathrm{C} .{ }^{1} \mathrm{H}$ NMR $\left(400 \mathrm{MHz}, \mathrm{CDCl}_{3}\right) \delta$ $7.61(\mathrm{~d}, J=8.6 \mathrm{~Hz}, 2 \mathrm{H}), 6.90(\mathrm{~d}, J=8.7 \mathrm{~Hz}, 2 \mathrm{H}), 3.93(\mathrm{~s}, 3 \mathrm{H}), 3.84(\mathrm{~s}, 3 \mathrm{H}) .{ }^{13} \mathrm{C} \mathrm{NMR}$ $\left(101 \mathrm{MHz}, \mathrm{CDCl}_{3}\right) \delta 168.8,162.7,159.9,136.1,114.6,110.6,100.0,87.8,55.5,53.6$. IR (film): 2349, 2323, 2187, 1739, 1726, 1664, 1596, 1081, 790, 731, 682, 671, 664, 495. GCMS (EI) m/z (rel): $218\left(\mathrm{M}^{+}\right), 159$ (100), 144, 131, 116, 88, 77, 62, 51. HRMS (EI) Calcd. For $\mathrm{C}_{12} \mathrm{H}_{10} \mathrm{O}_{4}\left(\mathrm{M}^{+}\right)$: 218.0579; found: 218.0580 .

Methyl 4-(4-fluorophenyl)-2-oxobut-3-ynoate (2d $)^{9}$<smiles>COC(=O)C(=O)C#Cc1ccc(F)cc1</smiles>

The compound 2d was obtained according to General method II starting from 1ethynyl-4-fluorobenzene $(1.0 \mathrm{~g}, 8.7 \mathrm{mmol})$. The crude product was purified by flash chromatography on silical gel (petroleum ether / diethyl ether $=10 / 1)$ to give a yellow solid (447 mg, yield: 25\%). ${ }^{1} \mathrm{H}$ NMR (400 MHz, $\left.\mathrm{CDCl}_{3}\right) \delta$ 7.67-7.63 (m, 2H), 7.12$7.07(\mathrm{~m}, 2 \mathrm{H}), 3.93(\mathrm{~s}, 3 \mathrm{H}) .{ }^{19} \mathrm{~F}$ NMR $\left(376 \mathrm{MHz}, \mathrm{CDCl}_{3}\right) \delta-103.88 .{ }^{13} \mathrm{C} \mathrm{NMR}(101 \mathrm{MHz}$, $\left.\mathrm{CDCl}_{3}\right) \delta 168.9,165.9(\mathrm{~d}, J=257 \mathrm{~Hz}), 159.5,136.3(\mathrm{~d}, J=9 \mathrm{H}), 116.5(\mathrm{~d}, J=22 \mathrm{~Hz})$, $115.1(\mathrm{~d}, J=3 \mathrm{~Hz}), 97.0,87.1,53.7$.

Methyl 4-(4-chlorophenyl)-2-oxobut-3-ynoate (2e) 
<smiles>CC(=O)C#Cc1ccc(Cl)cc1</smiles>

The compound $\mathbf{2 e}$ was obtained according to General method II starting from 1-chloro4-ethynylbenzene (1.9 g, $14 \mathrm{mmol})$. The crude product was purified by flash chromatography on silical gel (petroleum ether / diethyl ether $=10 / 1)$ to give a yellow solid (494 mg, yield: $16 \%$ ). mp: 117.6-118.3 ${ }^{\circ} \mathrm{C} .{ }^{1} \mathrm{H}$ NMR (400 MHz, $\left.\mathrm{CDCl}_{3}\right) \delta 7.58$ $(\mathrm{d}, J=8.3 \mathrm{~Hz}, 2 \mathrm{H}), 7.39(\mathrm{~d}, J=8.3 \mathrm{~Hz}, 2 \mathrm{H}), 3.94(\mathrm{~s}, 3 \mathrm{H}) .{ }^{13} \mathrm{C} \mathrm{NMR}\left(101 \mathrm{MHz}, \mathrm{CDCl}_{3}\right)$ $\delta 168.9,159.5,138.4,134.9,129.3,117.4,96.5,87.7,53.7$. IR (film): 2349, 2344, 2203, 2176, 1737, 1670, 1586, 1258, 1079, 1011, 828, 779, 671, 664, 535. GCMS (EI) $\mathrm{m} / \mathrm{z}$ (rel): $222\left(\mathrm{M}^{+}\right)$, 194, $163(100), 135,99,74,50 . \mathrm{HRMS}$ (EI) Calcd. For $\mathrm{C}_{11} \mathrm{H}_{7} \mathrm{ClO}_{3}\left(\mathrm{M}^{+}\right)$: 222.0084; found: 222.0094 .

Methyl 4-(4-iodophenyl)-2-oxobut-3-ynoate (2f)<smiles>COC(=O)C(=O)C#Cc1ccc(I)cc1</smiles>

The compound $2 \mathbf{f}$ was obtained according to General method I starting from 1ethynyl-4-iodobenzene $(1.0 \mathrm{~g}, 4.4 \mathrm{mmol})$. The crude product was purified by flash chromatography on silical gel (petroleum ether / diethyl ether $=10 / 1$ ) to give a yellow solid (447 mg, yield: 33\%). mp: 121.5-121.6 ${ }^{\circ} \mathrm{C} .{ }^{1} \mathrm{H}$ NMR (400 MHz, $\left.\mathrm{CDCl}_{3}\right) \delta 7.77$ $(\mathrm{d}, J=8.2 \mathrm{~Hz}, 2 \mathrm{H}), 7.35(\mathrm{~d}, J=8.2 \mathrm{~Hz}, 2 \mathrm{H}), 3.94(\mathrm{~s}, 3 \mathrm{H}) .{ }^{13} \mathrm{C} \mathrm{NMR}\left(101 \mathrm{MHz}, \mathrm{CDCl}_{3}\right)$ $\delta 168.9,159.5,138.1,134.8,118.4,99.3,96.7,87.9,53.7$. IR (film): 2345, 2340, 2307, 2199, 1750, 1680, 1302, 1251, 813, 770, 741, 533, 511, 496. GCMS (EI) m/z (rel): 314 $\left(\mathrm{M}^{+}\right), 255$ (100), 128, 100, 74, 50; HRMS (EI) Calcd. For $\mathrm{C}_{11} \mathrm{H}_{7} \mathrm{IO}_{3}\left(\mathrm{M}^{+}\right)$: 313.9440; found: 313.9443 .

Methyl 4-([1,1'-biphenyl]-4-yl)-2-oxobut-3-ynoate (2g) 


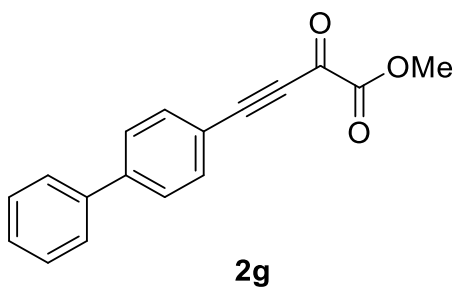

The compound $2 \mathrm{~g}$ was obtained according to General method I starting from 4ethynyl-1,1'-biphenyl (3.1 g, $17.2 \mathrm{mmol})$. The crude product was purified by flash chromatography on silical gel (petroleum ether / diethyl ether $=10 / 1$ ) to give a yellow solid (2.1 g, yield: 47\%). mp: 94.0-94.2 ${ }^{\circ} \mathrm{C} .{ }^{1} \mathrm{H}$ NMR (400 MHz, $\left.\mathrm{CDCl}_{3}\right) \delta$ 7.74-7.72 (m, 2H), 7.65-7.58 (m, 4H), 7.48-7.37 (m, 3H), 3.96 (s, 3H). ${ }^{13} \mathrm{C}$ NMR (101 MHz, $\left.\mathrm{CDCl}_{3}\right) \delta 169.0,159.7,144.7,139.5,134.4,129.0,128.4,127.4,127.2,117.6,98.4$, 87.9, 53.7. IR (film): 2194, 2174, 1741, 1661, 1598, 1255, 1083, 845, 786, 758, 713, 692, 561, 513. GCMS (EI) m/z (rel): $264\left(\mathrm{M}^{+}\right), 205$ (100), 176, 151, 102, 88, 75, 51; HRMS (EI) Calcd. For $\mathrm{C}_{17} \mathrm{H}_{12} \mathrm{O}_{3}\left(\mathrm{M}^{+}\right)$: 264.0786; found: 264.0780.

Methyl 2-oxo-4-(m-tolyl)but-3-ynoate (2h)<smiles>COC(=O)C(=O)C#Cc1cccc(C)c1</smiles>

The compound $\mathbf{2 h}$ was obtained according to General method I starting from 1ethynyl-3-methylbenzene $(2.17 \mathrm{~mL}, 17.2 \mathrm{mmol})$. The crude product was purified by flash chromatography on silical gel (petroleum ether / diethyl ether $=10 / 1$ ) to give a yellow solid (2.2 g, yield: $64 \%$ ). mp: 53.7-53.9 ${ }^{\circ} \mathrm{C} .{ }^{1} \mathrm{H} \mathrm{NMR}\left(400 \mathrm{MHz}, \mathrm{CDCl}_{3}\right) \delta 7.47-$ 7.44 (m, 2H), 7.35-7.25 (m, 2H), 3.94 (s, 3H), 2.35 (s, 3H). $\left.{ }^{13} \mathrm{C} \mathrm{NMR} \mathrm{(101} \mathrm{MHz,} \mathrm{CDCl}_{3}\right)$ $\delta 169.0,159.6,138.7,134.2,132.9,131.0,128.7,118.7,98.6,86.9,53.6,21.1$. IR (film): 2188, 1740, 1726, 1666, 1595, 1312, 1272, 1189, 1095, 1081, 789, 731, 681, 651, 494. GCMS (EI) m/z (rel): $202\left(\mathrm{M}^{+}\right), 143$ (100), 115, 89, 74, 63, 51, 39; HRMS (EI) Calcd. For $\mathrm{C}_{12} \mathrm{H}_{10} \mathrm{O}_{3}\left(\mathrm{M}^{+}\right)$: 202.0630; found: 202.0634 . 
Methyl 4-(3-bromophenyl)-2-oxobut-3-ynoate (2j)<smiles>COC(=O)C(=O)C#Cc1cccc(Br)c1</smiles>

The compound $\mathbf{2} \mathbf{j}$ was obtained according to General method I starting from 1-bromo3-ethynylbenzene $(3.1 \mathrm{~g}, 17.2 \mathrm{mmol})$. The crude product was purified by flash chromatography on silical gel (petroleum ether / diethyl ether $=10 / 1$ ) to give a yellow solid (584 mg, yield: $13 \%$ ). mp: 69.5-71.3 ${ }^{\circ} \mathrm{C} .{ }^{1} \mathrm{H}$ NMR (400 MHz, $\left.\mathrm{CDCl}_{3}\right) \delta$ 7.78-7.76 (m, 1H), 7.63-7.55 (m, 2H), 7.29-7.24 (m, 1H), 3.94 (s, 3H). ${ }^{13} \mathrm{C}$ NMR (101 MHz, $\left.\mathrm{CDCl}_{3}\right) \delta 168.8,159.3,136.1,134.9,132.2,130.2,122.5,120.9,95.5,87.4,53.7 . \mathrm{IR}$ (film): 2183, 1726, 1664, 1270, 1245, 1089, 790, 675, 645, 493. GCMS (EI) $m / z$ (rel): $266\left(\mathrm{M}^{+}\right), 238,207$ (100), 128, 100, 74, 59, 50; HRMS (EI) Calcd. For $\mathrm{C}_{11} \mathrm{H}_{7} \mathrm{BrO}_{3}\left(\mathrm{M}^{+}\right)$: 265.9579; found: 265.9571 .

Methyl 4-(2-fluorophenyl)-2-oxobut-3-ynoate (2k)<smiles>COC(=O)C(=O)C#Cc1ccccc1F</smiles>

2k

The compound $\mathbf{2 k}$ was obtained according to General method I starting from 1ethynyl-2-fluorobenzene $(1.0 \mathrm{~mL}, 8.6 \mathrm{mmol})$. The crude product was purified by flash chromatography on silical gel (petroleum ether / diethyl ether $=10 / 1$ ) to give a yellow solid (1.0 g, yield: 78\%). mp: 79.8-80.0 ${ }^{\circ} \mathrm{C} .{ }^{1} \mathrm{H}$ NMR (400 MHz, $\left.\mathrm{CDCl}_{3}\right) \delta$ 7.65-7.57 $(\mathrm{m}, 1 \mathrm{H}), 7.52-7.46(\mathrm{~m}, 1 \mathrm{H}), 7.22-7.09(\mathrm{~m}, 2 \mathrm{H}), 3.94(\mathrm{~s}, 3 \mathrm{H}) .{ }^{19} \mathrm{~F}$ NMR $(376 \mathrm{MHz}$, $\left.\mathrm{CDCl}_{3}\right) \delta-105.56 .{ }^{13} \mathrm{C} \mathrm{NMR}\left(101 \mathrm{MHz}, \mathrm{CDCl}_{3}\right) \delta 168.8,165.3(\mathrm{~d}, J=261 \mathrm{~Hz}), 159.3$, 135.1, $134.0(\mathrm{~d}, J=8 \mathrm{~Hz}), 124.5$ (d, $J=4 \mathrm{~Hz}), 116.0(\mathrm{~d}, J=2 \mathrm{~Hz}), 108.1(\mathrm{~d}, J=15 \mathrm{~Hz})$, 91.1, 53.7. IR (film): 2194, 1745, 1732, 1668, 1606, 1258, 1113, 1078, 944, 817, 757. 
GCMS (EI) m/z (rel): $206\left(\mathrm{M}^{+}\right)$, 178, 147 (100), 119, 99, 93, 74, 69, 51. HRMS (EI) Calcd. For $\mathrm{C}_{11} \mathrm{H}_{7} \mathrm{FO}_{3}\left(\mathrm{M}^{+}\right)$: 206.0379; found: 206.0376 .

Methyl 4-(2-chlorophenyl)-2-oxobut-3-ynoate (2I)<smiles>COC(=O)C(=O)C#Cc1ccccc1Cl</smiles>

The compound $\mathbf{2 l}$ was obtained according to General method I starting from 1-chloro2-ethynylbenzene $(2.1 \mathrm{~mL}, 17.2 \mathrm{mmol})$. The crude product was purified by flash chromatography on silical gel (petroleum ether / diethyl ether $=10 / 1$ ) to give a yellow solid (3.3 g, yield: 63\%). mp: 93.0-94.2 ${ }^{\circ} \mathrm{C} .{ }^{1} \mathrm{H}$ NMR (400 MHz, $\left.\mathrm{CDCl}_{3}\right) \delta$ 7.67-7.60 $(\mathrm{m}, 1 \mathrm{H}), 7.48-7.38(\mathrm{~m}, 2 \mathrm{H}), 7.35-7.25(\mathrm{~m}, 1 \mathrm{H}), 3.93(\mathrm{~s}, 3 \mathrm{H}) .{ }^{13} \mathrm{C}$ NMR $(101 \mathrm{MHz}$, $\left.\mathrm{CDCl}_{3}\right) \delta 168.9,159.3,138.3,135.4,132.8,129.8,126.8,119.3,93.8,90.7,53.7 . \mathrm{IR}$ (film): 2202, 2178, 1746, 1668, 1584, 1470, 1432, 1269, 1242, 1094, 1084, 1053, 783, 757, 545, 491. GCMS (EI) m/z (rel): $222\left(\mathrm{M}^{+}\right)$, 194, 163 (100), 135, 99, 74, 50, 39; HRMS (EI) Calcd. For $\mathrm{C}_{11} \mathrm{H}_{7} \mathrm{ClO}_{3}\left(\mathrm{M}^{+}\right)$: 222.0084; found: 222.0080.

Methyl 4-(2-bromophenyl)-2-oxobut-3-ynoate (2m)<smiles>COC(=O)C(=O)C#Cc1ccccc1Br</smiles>

The compound $\mathbf{2 m}$ was obtained according to General method I starting from 1bromo-2-ethynylbenzene $(2.1 \mathrm{~mL}, 17.2 \mathrm{mmol})$. The crude product was purified by flash chromatography on silical gel (petroleum ether / diethyl ether $=10 / 1$ ) to give a yellow solid (4.1 g, yield: 88\%). mp: 89.8-90.5 ${ }^{\circ} \mathrm{C} .{ }^{1} \mathrm{H}$ NMR (400 MHz, $\left.\mathrm{CDCl}_{3}\right) \delta$ 7.70-7.61 (m, 2H), 7.39-7.31 (m, 2H), $3.95(\mathrm{~s}, 3 \mathrm{H}) .{ }^{13} \mathrm{C} \mathrm{NMR}\left(101 \mathrm{MHz}, \mathrm{CDCl}_{3}\right) \delta 168.9,159.3$, 135.6, 133.0, 132.8, 127.5, 127.4, 121.6, 95.4, 90.0, 53.7. IR (film): 2199, 2174, 2152, 
$1745,1667,1580,1557,1265,1241,1162,1125,1089,1042,1027,943,779,757,448$. GCMS (EI) m/z (rel): $266\left(\mathrm{M}^{+}\right), 253,238,207$ (100), 128, 100, 74, 59, 50, 39; HRMS (EI) Calcd. For $\mathrm{C}_{11} \mathrm{H}_{7} \mathrm{BrO}_{3}\left(\mathrm{M}^{+}\right)$: 265.9579; found: 265.9573 .

Methyl 4-(2-cyanophenyl)-2-oxobut-3-ynoate (2n)<smiles>COC(=O)C(=O)C#Cc1ccccc1C#N</smiles>

The compound 2n was obtained according to General method I starting from 2ethynylbenzonitrile $(1.0 \mathrm{~g}, 7.8 \mathrm{mmol})$. The crude product was purified by flash chromatography on silical gel (petroleum ether / diethyl ether $=10 / 1$ ) to give a yellow solid (643 mg, yield: 38\%). mp: 108.6-109.4 ${ }^{\circ} \mathrm{C} .{ }^{1} \mathrm{H} \mathrm{NMR}\left(400 \mathrm{MHz}, \mathrm{CDCl}_{3}\right) \delta$ 7.77$7.42(\mathrm{~m}, 2 \mathrm{H}), 7.69-7.58(\mathrm{~m}, 2 \mathrm{H}), 3.97(\mathrm{~s}, 3 \mathrm{H}) .{ }^{13} \mathrm{C} \mathrm{NMR}\left(101 \mathrm{MHz}, \mathrm{CDCl}_{3}\right) \delta$ 168.7, $158.8,134.5,133.3,132.7,131.7,122.6,117.0,116.2,91.4,90.5,53.9$. IR (film): 2360 , 2349, 2330, 2323, 1744, 1681, 1655, 1108, 1073, 792, 778, 528, 499. GCMS (EI) m/z (rel): $213\left(\mathrm{M}^{+}\right)$, 185, 154 (100), 126, 99, 75, 59, 51, 39. HRMS (EI) Calcd. For $\mathrm{C}_{12} \mathrm{H}_{7} \mathrm{NO}_{3}\left(\mathrm{M}^{+}\right):$213.0426; found: 213.0416 .

Methyl 4-(naphthalen-2-yl)-2-oxobut-3-ynoate (2o)<smiles>COC(=O)C(=O)C#Cc1ccc2ccccc2c1</smiles>

20

The compound 20 was obtained according to General method I starting from 2ethynylnaphthalene $(913 \mathrm{mg}, 6 \mathrm{mmol})$. The crude product was purified by flash chromatography on silical gel (petroleum ether / diethyl ether $=10 / 1$ ) to give a yellow solid (180 mg, yield: $13 \%$ ). mp: 109.0-110.1 ${ }^{\circ} \mathrm{C} .{ }^{1} \mathrm{H}$ NMR (400 MHz, $\left.\mathrm{CDCl}_{3}\right) \delta 8.25$ (s, 1H), 7.86-7.83 (m, 3H), 7.64-7.52 (m, 3H), 3.97 (s, 3H). $\left.{ }^{13} \mathrm{C} \mathrm{NMR} \mathrm{(101} \mathrm{MHz,} \mathrm{CDCl}_{3}\right)$ $\delta 169.0,159.7,135.8,134.4,132.5,128.6(2 \mathrm{C}), 128.5,128.4,127.9,127.2,116.1,98.8$, 
87.5, 53.7. IR (film): 2198, 2179, 2151, 1743, 1668, 1293, 1273, 1254, 1226, 1071, 873, 830, 818, 791, 749, 484. GCMS (EI) $m / z$ (rel): $238\left(\mathrm{M}^{+}\right), 179$ (100), 150, 125, 99, 75, 51; HRMS (EI) Calcd. For $\mathrm{C}_{15} \mathrm{H}_{10} \mathrm{O}_{3}\left(\mathrm{M}^{+}\right)$: 238.0630; found: 238.0633 .

Methyl 2-oxo-4-(thiophen-2-yl)but-3-ynoate (2p)<smiles>COC(=O)C(=O)C#Cc1cccs1</smiles>

The compound $\mathbf{2 p}$ was obtained according to General method I starting from 2ethynylthiophene (466 mg, $4.3 \mathrm{mmol}$ ). The crude product was purified by flash chromatography on silical gel (petroleum ether / diethyl ether $=10 / 1$ ) to give a yellow solid (206 mg, yield: 25\%). mp: 86.2-86.4 ${ }^{\circ} \mathrm{C} .{ }^{1} \mathrm{H} \mathrm{NMR}\left(400 \mathrm{MHz}, \mathrm{CDCl}_{3}\right) \delta$ 7.63-7.60 (m, 2H), 7.14-7.08 (m, 1H), 3.94 (s, 3H). $\left.{ }^{13} \mathrm{C} \mathrm{NMR} \mathrm{(101} \mathrm{MHz,} \mathrm{CDCl}_{3}\right) \delta 168.4,159.5$, 138.8, 133.9, 128.1, 118.8, 92.6, 92.4, 53.7. IR (film): 3091, 2171, 1739, 1725, 1654, 1435, 1405, 1245, 1197, 1145, 1133, 1095, 1066, 1036, 716. GCMS (EI) m/z (rel): 194 $\left(\mathrm{M}^{+}\right)$, 166, 135 (100), 107, 81, 77, 63, 45; HRMS (EI) Calcd. For $\mathrm{C}_{9} \mathrm{H}_{6} \mathrm{O}_{3} \mathrm{~S}\left(\mathrm{M}^{+}\right)$: 194.0038; found: 194.0043 .

Methyl 4-cyclohexyl-2-oxobut-3-ynoate (2q)<smiles>COC(=O)C(=O)C#CC1CCCCC1</smiles>

The compound $\mathbf{2 q}$ was obtained according to General method II starting from ethynylcyclohexane $(920 \mathrm{mg}, 8.5 \mathrm{mmol})$. The crude product was purified by flash chromatography on silical gel (petroleum ether / diethyl ether $=10 / 1$ ) to give a yellow oil (835 mg, yield: 51\%). ${ }^{1} \mathrm{H}$ NMR (400 MHz, $\left.\mathrm{CDCl}_{3}\right) \delta 3.88(\mathrm{~s}, 3 \mathrm{H}), 2.70-2.59(\mathrm{~m}$, $1 \mathrm{H}), 1.82-1.86(\mathrm{~m}, 2 \mathrm{H}), 1.67-1.71(\mathrm{~m}, 2 \mathrm{H}), 1.62-1.45(\mathrm{~m}, 3 \mathrm{H}), 1.32-1.35(\mathrm{~m}, 3 \mathrm{H}) .{ }^{13} \mathrm{C}$ NMR (101 MHz, $\left.\mathrm{CDCl}_{3}\right) \delta 169.3,159.7,106.1,79.7,53.5,31.1,29.4,25.5,24.4 . \mathrm{IR}$ (film): 2932, 2856, 2203, 1743, 1679, 1449, 1298, 1273, 1234, 1193, 1157, 1131, 1104 , 
1028. GCMS (EI) m/z (rel): $194\left(\mathrm{M}^{+}\right), 135$ (100), 117, 105, 91, 79, 67, 55, 53, 41. HRMS (EI) Calcd. For $\mathrm{C}_{11} \mathrm{H}_{14} \mathrm{O}_{3}\left(\mathrm{M}^{+}\right)$: 194.0943; found: 194.0951 .

Methyl 2-oxooct-3-ynoate (2s)

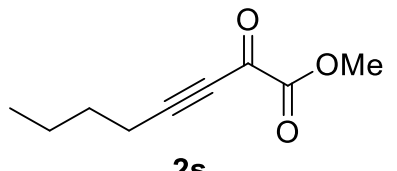

$2 s$

The compound $\mathbf{2 s}$ was obtained according to General method II starting from hex-1yne $(1.9 \mathrm{~mL}, 17 \mathrm{mmol})$. The crude product was purified by flash chromatography on silical gel (petroleum ether / diethyl ether $=10 / 1)$ to give a yellow oil $(581 \mathrm{mg}$, yield: 20\%). ${ }^{1} \mathrm{H}$ NMR (400 MHz, $\left.\mathrm{CDCl}_{3}\right) \delta 3.85(\mathrm{~s}, 3 \mathrm{H}), 2.43(\mathrm{t}, J=7.0 \mathrm{~Hz}, 2 \mathrm{H}), 1.63-1.50$ $(\mathrm{m}, 2 \mathrm{H}), 1.36-1.41(\mathrm{~m}, 2 \mathrm{H}), 0.88(\mathrm{t}, J=7.3 \mathrm{~Hz}, 3 \mathrm{H}) .{ }^{13} \mathrm{C} \mathrm{NMR}\left(101 \mathrm{MHz}, \mathrm{CDCl}_{3}\right) \delta$ 169.1, 159.6, 102.7, 79.6, 53.5, 29.3, 21.9, 19.1, 13.4. IR (film): 2959, 2935, 2874, 2322 2211, 1743, 1680, 1456, 1437, 1381, 1303, 1272, 1194, 1156, 1106, 1051, 1003, 927, 904, 856, 789. GCMS (EI) m/z (rel): $168\left(\mathrm{M}^{+}\right)$, 126, 109 (100), 79, 66, 53, 41, 39. HRMS (EI) Calcd. For $\mathrm{C}_{9} \mathrm{H}_{12} \mathrm{O}_{3}\left(\mathrm{M}^{+}\right)$: 168.0786; found: 168.0784.

Methyl 5-cyclohexyl-2-oxopent-3-ynoate (2t)<smiles>COC(=O)C(=O)C#CCC1CCCCC1</smiles>

$2 \mathrm{t}$

The compound $\mathbf{2 t}$ was obtained according to General method II starting from prop-2yn-1-ylcyclohexane $(1.2 \mathrm{~mL}, 8.5 \mathrm{mmol})$. The crude product was purified by flash chromatography on silical gel (petroleum ether / diethyl ether $=10 / 1$ ) to give a yellow oil (765 mg, yield: 43\%). ${ }^{1} \mathrm{H}$ NMR (400 MHz, $\left.\mathrm{CDCl}_{3}\right) \delta 3.86(\mathrm{~s}, 3 \mathrm{H}), 2.33(\mathrm{~d}, J=6.6$ $\mathrm{Hz}, 2 \mathrm{H}), 1.96-1.45$ (m, 6H), 1.42-0.88 (m, 5H). ${ }^{13} \mathrm{C}$ NMR (101 MHz, $\left.\mathrm{CDCl}_{3}\right) \delta 169.1$, 159.6, 102.0, 80.6, 53.5, 36.6, 32.6, 27.1, 25.9. IR (film): 2924, 2852, 2210, 1743, 1679, 1449, 1308, 1267, 1194, 1155, 995, 902, 796. GCMS (EI) m/z (rel):208 (M+), 150, 149 (100), 126, 105, 93, 83, 67, 55, 53, 41. HRMS (EI) Calcd. For $\mathrm{C}_{12} \mathrm{H}_{16} \mathrm{O}_{3}\left(\mathrm{M}^{+}\right)$: 208.1099; found: 208.1102 . 
Methyl 2-oxo-4-(triethylsilyl)but-3-ynoate (2u)<smiles>CCCCCCCCCC(=O)OC(C)=O</smiles>

The compound $\mathbf{2 u}$ was obtained according to General method II starting from triethyl(ethynyl)silane $(3.6 \mathrm{~mL}, 20 \mathrm{mmol})$. The crude product was purified by flash chromatography on silical gel (petroleum ether / diethyl ether $=10 / 1$ ) to give a yellow oil (1.8 g, yield: 39\%). ${ }^{1} \mathrm{H}$ NMR (400 MHz, $\left.\mathrm{CDCl}_{3}\right) \delta 3.90(\mathrm{~s}, 3 \mathrm{H}), 1.01(\mathrm{t}, J=7.9 \mathrm{~Hz}$, 9H), 0.74-0.66 (m, 6H). ${ }^{13} \mathrm{C} \mathrm{NMR}\left(101 \mathrm{MHz}, \mathrm{CDCl}_{3}\right) \delta 168.6,159.3,105.8,101.4,53.6$, 7.2, 3.6. IR (film): 2958, 2914, 2878, 1761, 1745, 1683, 1095, 1005, 952, 831, 728, 688, 665. GCMS (EI) m/z (rel):197 [(M-C $\left.\left.2 \mathrm{H}_{5}\right)^{+}\right], 169,167$ (100), 141, 117, 111, 89, 83, 59, 53; HRMS (EI) Calcd. For $\mathrm{C}_{9} \mathrm{H}_{13} \mathrm{O}_{3} \mathrm{Si}\left[\left(\mathrm{M}-\mathrm{C}_{2} \mathrm{H}_{5}\right)^{+}\right]$: 197.0634; found: 197.0639.

\section{General experimental procedure for Scheme 2 and the characterization of} products

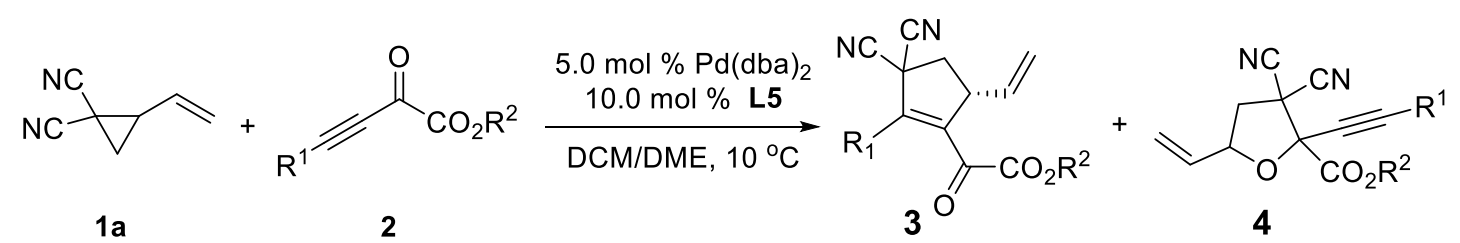

To a flame dried sealing tube were added Pd(dba) $2(5.75 \mathrm{mg}, 0.01 \mathrm{mmol}),(R)-$ SEGPHOS (L5) (12.2 mg, $0.02 \mathrm{mmol}$ ), freshly distilled anhydrous dichloromethane $(1.2 \mathrm{~mL})$ and anhydrous 1,2-dimethoxyethane $(0.8 \mathrm{~mL})$. The resulting mixture was allowed to stir for 30 mins. The $\alpha, \beta$-alkynyl $\alpha$-keto ester $2(0.2 \mathrm{mmol})$ and vinyl cyclopropane 1a $(59.1 \mathrm{mg}, 0.5 \mathrm{mmol})$ were added subsequently. The resulting reaction mixture was stirred at $10{ }^{\circ} \mathrm{C}$ for $16 \mathrm{~h}$. After the volatiles were removed under reduced pressure, the ratio of compounds 3 and 4 was determined by ${ }^{1} \mathrm{H}$ NMR of crude products. Then the resulting residue was purified by preparative TLC with petroleum ether and ethyl acetate as eluent to give product $\mathbf{3}$. 
Methyl-( $R$ )-2-(3,3-dicyano-2-(p-tolyl)-5-vinylcyclopent-1-en-1-yl)-2-oxoacetate (3a)<smiles>C=C[C@H]1CC(C#N)(C(C)=O)C(c2ccc(C)cc2)=C1C(=O)OC</smiles>

Yellow solid, $51.9 \mathrm{mg}$, yield: 81\%, ee: 89\%. HPLC (Chiralpak ID, $4.6 \mathrm{~mm} \times 250 \mathrm{~mm}$, Hexane: $i$-Propanol $=98 / 2,0.5 \mathrm{~mL} / \mathrm{min}, 214 \mathrm{~nm}): \mathrm{t}_{\mathrm{minor}}=46.29 \mathrm{~min}, \mathrm{t}_{\mathrm{major}}=41.1 \mathrm{~min}$; $[\alpha]_{\mathrm{D}}^{30}=-82.6\left(c\right.$ 2.55, $\left.\mathrm{CHCl}_{3}\right)$; mp: 70.9-72.3 ${ }^{\circ} \mathrm{C} .{ }^{1} \mathrm{H}$ NMR $\left(400 \mathrm{MHz}, \mathrm{CDCl}_{3}\right) \delta 7.35-$ $7.23(\mathrm{~m}, 4 \mathrm{H}), 5.89$ (ddd, $J=17.8,10.1,7.9 \mathrm{~Hz}, 1 \mathrm{H}), 5.27(\mathrm{dd}, J=28.7,14.0 \mathrm{~Hz}, 2 \mathrm{H})$, 4.22-4.14 (m, 1H), 3.35 (s, 3H), 3.11 (dd, $J=13.7,8.3 \mathrm{~Hz}, 1 \mathrm{H}), 2.75$ (dd, $J=13.7,4.7$ $\mathrm{Hz}, 1 \mathrm{H}), 2.39$ (s, 3H). ${ }^{13} \mathrm{C} \mathrm{NMR}\left(101 \mathrm{MHz}, \mathrm{CDCl}_{3}\right) \delta 184.2,161.4,146.0,142.6,141.8$, 134.3, 129.8, 128.7, 126.6, 119.2, 114.0, 113.6, 52.7, 48.7, 43.8, 42.4, 21.5. IR (film): 1738, 1687, 1316, 1200, 1176, 923, 819, 763, 727, 685, 507, 440. MS (ES-API) $\mathrm{m} / z$ : $343.1(\mathrm{M}+\mathrm{Na})^{+} ;$HRMS (DART) $m / z$ Calcd for $\mathrm{C}_{19} \mathrm{H}_{17} \mathrm{~N}_{2} \mathrm{O}_{3}(\mathrm{M}+\mathrm{H})^{+}: 321.1234$, found 321.1234 .

Methyl (R)-2-(3,3-dicyano-2-phenyl-5-vinylcyclopent-1-en-1-yl)-2-oxoacetate (3b)<smiles>C=C[C@H]1CC(C#N)(C#N)C(c2ccccc2)=C1C(=O)C(C)=O</smiles>

Yellow solid, $46.9 \mathrm{mg}$, yield: 77\%, ee: 67\%. HPLC (Chiralpak IG, $4.6 \mathrm{~mm} \times 250 \mathrm{~mm}$, Hexane: $i$-Propanol $=98 / 2,0.7 \mathrm{~mL} / \mathrm{min}, 214 \mathrm{~nm}): \mathrm{t}_{\mathrm{minor}}=51.62 \mathrm{~min}, \mathrm{t}_{\mathrm{major}}=45.94 \mathrm{~min}$; $[\alpha]_{\mathrm{D}}^{25}=-53.2\left(c\right.$ 2.34, $\left.\mathrm{CHCl}_{3}\right)$; mp: 109.1-109.8 ${ }^{\circ} \mathrm{C} .{ }^{1} \mathrm{H}$ NMR $\left(400 \mathrm{MHz}, \mathrm{CDCl}_{3}\right) \delta$ 7.54-7.39 (m, 5H), 5.89 ( ddd, $J=17.610 .0,7.6 \mathrm{~Hz}, 1 \mathrm{H}), 5.27(\mathrm{dd}, J=29.1,13.6 \mathrm{~Hz}$, 2H), 4.23-4.14 (m, 1H), 3.32 (s, 3H), 3.11 (dd, $J=13.7,8.3 \mathrm{~Hz}, 1 \mathrm{H}), 2.75$ (dd, $J=13.7$, $4.8 \mathrm{~Hz}, 1 \mathrm{H}) .{ }^{13} \mathrm{C} \mathrm{NMR}\left(101 \mathrm{MHz}, \mathrm{CDCl}_{3}\right) \delta 184.0,161.4,145.7,143.3,134.2,131.2$, 129.5, 129.1, 128.8, 119.3, 113.9, 113.5, 52.7, 48.7, 43.9, 42.4. IR (film): 1731, 1682, 1443, 1276, 1256, 1208, 1186, 1025, 996, 956, 921, 746, 703, 689. MS (DART) $m / z$ : 
$307.1(\mathrm{M}+\mathrm{H})^{+}$. HRMS (DART) $m / z$ Calcd for $\mathrm{C}_{18} \mathrm{H}_{15} \mathrm{~N}_{2} \mathrm{O}_{3}(\mathrm{M}+\mathrm{H})^{+}: 307.1077$, found 307.1079.

Methyl-(R)-2-(3,3-dicyano-2-(4-methoxyphenyl)-5-vinylcyclopent-1-en-1-yl)-2-

oxoacetate (3c)<smiles>C=C[C@H]1CC(C#N)(C#N)C(c2ccc(OC)cc2)=C1C(=O)OC</smiles>

Yellow solid, $20.8 \mathrm{mg}$, yield: 62\%, ee: 85\%. HPLC (Chiralpak IB, $4.6 \mathrm{~mm} \times 250 \mathrm{~mm}$, Hexane: $i$-Propanol $=90 / 10,0.7 \mathrm{~mL} / \mathrm{min}, 254 \mathrm{~nm}$ ): $\mathrm{t}_{\mathrm{minor}}=14.65 \mathrm{~min}, \mathrm{t}_{\mathrm{major}}=15.75 \mathrm{~min}$; $[\alpha]_{\mathrm{D}}{ }^{30}=-113.2\left(c\right.$ 1.00, $\left.\mathrm{CHCl}_{3}\right) . \mathrm{mp}: 89.3-89.7{ }^{\circ} \mathrm{C} .{ }^{1} \mathrm{H}$ NMR $\left(400 \mathrm{MHz}, \mathrm{CDCl}_{3}\right) \delta 7.36$ $(\mathrm{d}, J=8.6 \mathrm{~Hz}, 2 \mathrm{H}), 6.95(\mathrm{~d}, J=8.6 \mathrm{~Hz}, 2 \mathrm{H}), 5.88(\mathrm{ddd}, J=17.6,10.1,7.8 \mathrm{~Hz}, 1 \mathrm{H})$, $5.28(\mathrm{~d}, J=17.0 \mathrm{~Hz}, 1 \mathrm{H}), 5.21(\mathrm{~d}, J=10.2 \mathrm{~Hz}, 1 \mathrm{H}), 4.19-4.11(\mathrm{~m}, 1 \mathrm{H}), 3.82(\mathrm{~s}, 3 \mathrm{H})$, $3.38(\mathrm{~s}, 3 \mathrm{H}), 3.08(\mathrm{dd}, J=13.6,8.3 \mathrm{~Hz}, 1 \mathrm{H}), 2.72(\mathrm{dd}, J=13.7,4.7 \mathrm{~Hz}, 1 \mathrm{H}) .{ }^{13} \mathrm{C} \mathrm{NMR}$ $\left(101 \mathrm{MHz}, \mathrm{CDCl}_{3}\right) \delta 184.2,161.9,161.5,145.9,142.0,134.4,130.4,121.8,119.1$, 114.6, 114.1, 113.7, 55.5, 52.8, 48.6, 43.7, 42.3. IR (film): 1744, 1692, 1657, 1603, $1512,1472,1463,1444,1331,1258,1212,1181,989,965,942,920,840,474 . \mathrm{MS}$ (DART) $m / z: 337.1(\mathrm{M}+\mathrm{H})^{+}$; HRMS (DART) $m / z$ Calcd for $\mathrm{C}_{19} \mathrm{H}_{17} \mathrm{~N}_{2} \mathrm{O}_{4}(\mathrm{M}+\mathrm{H})^{+}$: 337.1183, found 337.1185.

Methyl-(R)-2-(3,3-dicyano-2-(4-fluorophenyl)-5-vinylcyclopent-1-en-1-yl)-2oxoacetate (3d)<smiles>C=CC1CC(C#N)(C#N)C(c2ccc(F)cc2)=C1C(=O)C(C)=O</smiles>

Yellow solid, $54.5 \mathrm{mg}$, yield: 84\%, ee: 73\%. HPLC (Chiralpak IB, $4.6 \mathrm{~mm} \times 250 \mathrm{~mm}$, Hexane: $i$-Propanol $=90 / 10,0.7 \mathrm{~mL} / \mathrm{min}, 254 \mathrm{~nm}$ ): $\mathrm{t}_{\mathrm{minor}}=11.85 \mathrm{~min}, \mathrm{t}_{\mathrm{major}}=12.54 \mathrm{~min}$; $[\alpha]_{\mathrm{D}}{ }^{24}=-70.5\left(c\right.$ 2.73, $\left.\mathrm{CHCl}_{3}\right)$; mp: 110.9-111.5 ${ }^{\circ} \mathrm{C} .{ }^{1} \mathrm{H}$ NMR $\left(400 \mathrm{MHz}, \mathrm{CDCl}_{3}\right) \delta$ 7.46-7.39 (m, 2H), $7.16(\mathrm{t}, J=8.4 \mathrm{~Hz}, 2 \mathrm{H}), 5.86(\mathrm{ddd}, J=15.6,10.0,8.4 \mathrm{~Hz}, 1 \mathrm{H}), 5.30$ 
$(\mathrm{d}, J=17.0 \mathrm{~Hz}, 1 \mathrm{H}), 5.24(\mathrm{~d}, J=10.2 \mathrm{~Hz}, 1 \mathrm{H}), 4.24-4.15(\mathrm{~m}, 1 \mathrm{H}), 3.43(\mathrm{~s}, 3 \mathrm{H}), 3.11$ $(\mathrm{dd}, J=13.7,8.2 \mathrm{~Hz}, 1 \mathrm{H}), 2.73(\mathrm{dd}, J=13.7,5.1 \mathrm{~Hz}, 1 \mathrm{H}) .{ }^{19} \mathrm{~F} \mathrm{NMR}\left(376 \mathrm{MHz}, \mathrm{CDCl}_{3}\right)$ $\delta$-107.29. ${ }^{13} \mathrm{C}$ NMR $\left(101 \mathrm{MHz}, \mathrm{CDCl}_{3}\right) \delta 183.8,165.5(\mathrm{~d}, J=254 \mathrm{~Hz}), 161.3,144.3$, $143.8,134.1,131.1(\mathrm{~d}, J=9 \mathrm{~Hz}), 125.5$ (d, $J=3 \mathrm{~Hz}), 119.5,116.6,116.4,113.7,52.9$, 48.8, 44.0, 42.3. IR (film): 2349, 1735, 1681, 1627, 1599, 1313, 1290, 1278, 1246, 1229, 1199, 1177, 842, 688, 524, 420. MS (DART) $m / z: 325.1(\mathrm{M}+\mathrm{H})^{+} ;$HRMS (DART) $\mathrm{m} / \mathrm{z}$ Calcd for $\mathrm{C}_{18} \mathrm{H}_{14} \mathrm{FN}_{2} \mathrm{O}_{3}(\mathrm{M}+\mathrm{H})^{+}:$: 325.0983, found 325.0985.

Methyl-(R)-2-(2-(4-chlorophenyl)-3,3-dicyano-5-vinylcyclopent-1-en-1-yl)-2oxoacetate (3e)<smiles>C=CC1CC(C#N)(C(C)=O)C(c2ccc(Cl)cc2)=C1C(=O)OC</smiles>

Yellow solid, $50.8 \mathrm{mg}$, yield: 75\%, ee: 83\%. HPLC (Chiralpak IC, $4.6 \mathrm{~mm} \times 250 \mathrm{~mm}$, Hexane: $i$-Propanol $=90 / 10,0.7 \mathrm{~mL} / \mathrm{min}, 214 \mathrm{~nm}$ ): $\mathrm{t}_{\text {minor }}=12.55 \mathrm{~min}, \mathrm{t}_{\text {major }}=16.84 \mathrm{~min}$; $[\alpha]_{\mathrm{D}}^{24}=-83.8\left(c\right.$ 2.54, $\left.\mathrm{CHCl}_{3}\right) ; \mathrm{mp}: 129.8-130.3{ }^{\circ} \mathrm{C} .{ }^{1} \mathrm{H}$ NMR $\left(400 \mathrm{MHz}, \mathrm{CDCl}_{3}\right) \delta$ 7.49-7.44 (m, 2H), 7.40-7.36 (m, 2H), 5.88 (ddd, $J=17.1,10.2,7.9 \mathrm{~Hz}, 1 \mathrm{H}), 5.37-5.23$ $(\mathrm{m}, 2 \mathrm{H}), 4.27-4.20(\mathrm{~m}, 1 \mathrm{H}), 3.46(\mathrm{~s}, 3 \mathrm{H}), 3.14(\mathrm{dd}, J=13.7,8.2 \mathrm{~Hz}, 1 \mathrm{H}), 2.76(\mathrm{dd}, J=$ 13.7, $5.2 \mathrm{~Hz}, 1 \mathrm{H}) .{ }^{13} \mathrm{C} \mathrm{NMR}\left(101 \mathrm{MHz}, \mathrm{CDCl}_{3}\right) \delta 183.7,161.2,144.1,144.0,137.7$, 134.0, 130.2, 129.5, 127.8, 119.7, 113.7, 113.3, 53.0, 48.9, 43.8, 42.4. IR (film): 1736, 1681, 1489, 1288, 1176, 1092, 986, 946, 936, 839, 821, 508, 431. MS (DART) $m / z$ : $341.1(\mathrm{M}+\mathrm{H})^{+}$; HRMS (DART) $m / z$ Calcd for $\mathrm{C}_{18} \mathrm{H}_{14} \mathrm{ClN}_{2} \mathrm{O}_{3}(\mathrm{M}+\mathrm{H})^{+}$: 341.0687, found 341.0689 .

Methyl-(R)-2-(3,3-dicyano-2-(4-iodophenyl)-5-vinylcyclopent-1-en-1-yl)-2oxoacetate (3f) 


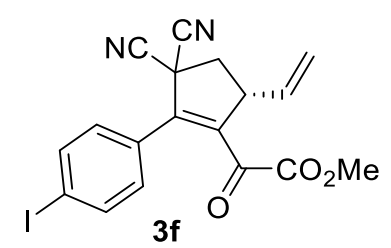

Yellow solid, $44.5 \mathrm{mg}$, yield: 52\%, ee: 58\%. HPLC (Chiralpak IB, $4.6 \mathrm{~mm} \times 250 \mathrm{~mm}$, Hexane: $i$-Propanol = 90/10, $0.7 \mathrm{~mL} / \mathrm{min}, 254 \mathrm{~nm}$ ): $\mathrm{t}_{\text {minor }}=12.74 \mathrm{~min}, \mathrm{t}_{\text {major }}=14.20 \mathrm{~min}$; $[\alpha]_{\mathrm{D}}^{30}=-53.1\left(c\right.$ 1.72, $\left.\mathrm{CHCl}_{3}\right) ; \mathrm{mp}: 95.9-96.1^{\circ} \mathrm{C} .{ }^{1} \mathrm{H}$ NMR $\left(400 \mathrm{MHz}, \mathrm{CDCl}_{3}\right) \delta 7.81$ $(\mathrm{d}, J=8.1 \mathrm{~Hz}, 2 \mathrm{H}), 7.15(\mathrm{~d}, J=8.2 \mathrm{~Hz}, 2 \mathrm{H}), 5.86(\mathrm{ddd}, J=17.6,10.0,8.0 \mathrm{~Hz}, 1 \mathrm{H}), 5.30$ $(\mathrm{d}, J=17.0 \mathrm{~Hz}, 1 \mathrm{H}), 5.24(\mathrm{~d}, J=10.2 \mathrm{~Hz}, 1 \mathrm{H}), 4.23-4.16(\mathrm{~m}, 1 \mathrm{H}), 3.44(\mathrm{~s}, 3 \mathrm{H}), 3.11$ $(\mathrm{dd}, J=13.7,8.2 \mathrm{~Hz}, 1 \mathrm{H}), 2.73(\mathrm{dd}, J=13.7,5.1 \mathrm{~Hz}, 1 \mathrm{H}) .{ }^{13} \mathrm{C} \mathrm{NMR}\left(101 \mathrm{MHz}, \mathrm{CDCl}_{3}\right)$ $\delta 183.6,161.2,144.2,144.0,138.3,134.0,130.2,128.8,119.6,113.7,113.3,97.9,53.0$, 48.9, 43.7, 42.4. IR (film): 2366, 2360, 2349, 1738, 1677, 1281, 1226, 1190, 1119, 1063 , 1050, 1035, 1002, 986, 499, 433. MS (DART) $m / z: 433.0(\mathrm{M}+\mathrm{H})^{+} ;$HRMS (DART) $m / z$ Calcd for $\mathrm{C}_{18} \mathrm{H}_{14} \mathrm{IN}_{2} \mathrm{O}_{3}(\mathrm{M}+\mathrm{H})^{+}:$433.0044, found 433.0046.

Methyl-(R)-2-(2-([1,1'-biphenyl]-4-yl)-3,3-dicyano-5-vinylcyclopent-1-en-1-yl)-2oxoacetate $(\mathbf{3 g})$<smiles>C=CC1CC2(C#N)CC1C(C(=O)OC(C)=O)=C2c1ccc(-c2ccccc2)cc1</smiles>

Yellow solid, $27.2 \mathrm{mg}$, yield: 71\%, ee: 69\%. HPLC (Chiralpak ID, $4.6 \mathrm{~mm} \times 250 \mathrm{~mm}$, Hexane: $i$-Propanol = 90/10, $0.7 \mathrm{~mL} / \mathrm{min}, 214 \mathrm{~nm}$ ): $\mathrm{t}_{\text {minor }}=12.40 \mathrm{~min}, \mathrm{t}_{\mathrm{major}}=10.75 \mathrm{~min}$; $[\alpha]_{\mathrm{D}}{ }^{31}=-59.9\left(c 1.09, \mathrm{CHCl}_{3}\right) ; \mathrm{mp}: 113.1-114.2^{\circ} \mathrm{C} .{ }^{1} \mathrm{H}$ NMR $\left(400 \mathrm{MHz}, \mathrm{CDCl}_{3}\right) \delta 7.69$ $(\mathrm{d}, J=8.3 \mathrm{~Hz}, 2 \mathrm{H}), 7.63-7.57(\mathrm{~m}, 2 \mathrm{H}), 7.55-7.44(\mathrm{~m}, 4 \mathrm{H}), 7.42-7.37$ (m, 1H), 5.92 (ddd, $J=17.6,10.0,8.0 \mathrm{~Hz}, 1 \mathrm{H}), 5.33(\mathrm{~d}, J=17.0 \mathrm{~Hz}, 1 \mathrm{H}), 5.26(\mathrm{~d}, J=10.2 \mathrm{~Hz}, 1 \mathrm{H}), 4.26-$ $4.18(\mathrm{~m}, 1 \mathrm{H}), 3.36(\mathrm{~s}, 3 \mathrm{H}), 3.14(\mathrm{dd}, J=13.7,8.3 \mathrm{~Hz}, 1 \mathrm{H}), 2.78(\mathrm{dd}, J=13.7,4.8 \mathrm{~Hz}$, 1H). ${ }^{13} \mathrm{C} \mathrm{NMR}\left(101 \mathrm{MHz}, \mathrm{CDCl}_{3}\right) \delta 184.0,161.5,145.5,144.0,143.2,139.3,134.2$, 129.3, 129.1, 128.3 (2C), 127.7, $127.1119 .3,114.0,113.6,52.8,48.8,43.8,42.4$. IR (film): 2363, 2354, 2346, 1742, 1678, 1637, 1277, 1201, 1177, 765, 738, 698. MS (DART) $m / z: 383.1(\mathrm{M}+\mathrm{H})^{+}$; HRMS (DART) $m / z$ Calcd for $\mathrm{C}_{24} \mathrm{H}_{19} \mathrm{~N}_{2} \mathrm{O}_{3}(\mathrm{M}+\mathrm{H})^{+}$: 
383.1390, found 383.1390.

Methyl-(R)-2-(3,3-dicyano-2-(m-tolyl)-5-vinylcyclopent-1-en-1-yl)-2-oxoacetate (3h)<smiles>C=CC1CC2(C#N)CC1C(C(=O)C(=O)OC)=C2c1cccc(C)c1</smiles>

Yellow solid, $37.9 \mathrm{mg}$, yield: 59\%, ee: 76\%. HPLC (Chiralpak IB, $4.6 \mathrm{~mm} \times 250 \mathrm{~mm}$, Hexane: $i$-Propanol $=99 / 1,0.7 \mathrm{~mL} / \mathrm{min}, 214 \mathrm{~nm}$ ): $\mathrm{t}_{\text {minor }}=23.98 \mathrm{~min}$, $\mathrm{t}_{\text {major }}=25.55 \mathrm{~min}$; $[\alpha]_{\mathrm{D}}^{31}=-104.3\left(c 1.60, \mathrm{CHCl}_{3}\right)$; mp: 68.5-69.1 ${ }^{\circ} \mathrm{C} .{ }^{1} \mathrm{H} \mathrm{NMR}\left(400 \mathrm{MHz}, \mathrm{CDCl}_{3}\right) \delta$ 7.42$7.29(\mathrm{~m}, 2 \mathrm{H}), 7.28-7.23(\mathrm{~m}, 1 \mathrm{H}), 7.20(\mathrm{~s}, 1 \mathrm{H}), 5.91(\mathrm{ddd}, J=17.2,10.0,8.0 \mathrm{~Hz}, 1 \mathrm{H})$, $5.32(\mathrm{~d}, J=17.0 \mathrm{~Hz}, 1 \mathrm{H}), 5.25(\mathrm{~d}, J=10.2 \mathrm{~Hz}, 1 \mathrm{H}), 4.23-4.15(\mathrm{~m}, 1 \mathrm{H}), 3.34(\mathrm{~s}, 3 \mathrm{H})$, $3.12(\mathrm{dd}, J=13.6,8.3 \mathrm{~Hz}, 1 \mathrm{H}), 2.77(\mathrm{dd}, J=13.7,4.5 \mathrm{~Hz}, 1 \mathrm{H}), 2.39$ (s, 3H). ${ }^{13} \mathrm{C}$ NMR $\left(101 \mathrm{MHz}, \mathrm{CDCl}_{3}\right) \delta 184.1,161.5,146.1,143.0,139.1,134.3,132.0,129.5,129.1$, 125.7, 119.2, 114.0, 113.6, 52.6, 48.7, 43.8, 42.5, 21.3. IR (film): 1671, 1526, 1344, 1198, 855, 730, 718, 504. MS (DART) $m / z: 321.1(\mathrm{M}+\mathrm{H})^{+}$; HRMS (DART) $m / z$ Calcd for $\mathrm{C}_{19} \mathrm{H}_{17} \mathrm{~N}_{2} \mathrm{O}_{3}(\mathrm{M}+\mathrm{H})^{+}:$321.1234, found 321.1236.

Methyl-(R)-2-(2-(3-chlorophenyl)-3,3-dicyano-5-vinylcyclopent-1-en-1-yl)-2oxoacetate (3i)<smiles>C=CC1CC(C#N)(C(C)=O)C(c2cccc(Cl)c2)=C1C(=O)C(C)=O</smiles>

Yellow solid, $46.4 \mathrm{mg}$, yield: 68\%, ee: 73\%. HPLC (Chiralpak IB, $4.6 \mathrm{~mm} \times 250 \mathrm{~mm}$, Hexane: $i$-Propanol $=90 / 10,0.7 \mathrm{~mL} / \mathrm{min}, 214 \mathrm{~nm}): \mathrm{t}_{\mathrm{minor}}=12.00 \mathrm{~min}, \mathrm{t}_{\mathrm{major}}=12.59 \mathrm{~min}$; $[\alpha]_{\mathrm{D}}^{24}=-51.5\left(c 2.00, \mathrm{CHCl}_{3}\right) ; \mathrm{mp}: 78.9-79.4{ }^{\circ} \mathrm{C} .{ }^{1} \mathrm{H}$ NMR $\left(400 \mathrm{MHz}, \mathrm{CDCl}_{3}\right) \delta$ 7.56$7.32(\mathrm{~m}, 4 \mathrm{H}), 5.96-5.81(\mathrm{~m}, 1 \mathrm{H}), 5.35-5.26(\mathrm{~m}, 2 \mathrm{H}), 4.24(\mathrm{dd}, J=12.8,7.6 \mathrm{~Hz}, 1 \mathrm{H})$, $3.48(\mathrm{~s}, 3 \mathrm{H}), 3.14(\mathrm{dd}, J=13.6,8.2 \mathrm{~Hz}, 1 \mathrm{H}), 2.77(\mathrm{dd}, J=13.7,5.0 \mathrm{~Hz}, 1 \mathrm{H}) .{ }^{13} \mathrm{C}$ NMR $\left(101 \mathrm{MHz}, \mathrm{CDCl}_{3}\right) \delta 183.5,161.2,144.5,143.6,135.2,133.9,131.3,131.0,130.5$, 
128.9, 126.9, 119.7, 113.6, 113.2, 53.0, 48.9, 43.8, 42.4. IR (film): 2962, 1258, 1088, 1009, 793, 766, 738, 697, 668. MS (DART) $m / z: 341.1(\mathrm{M}+\mathrm{H})^{+} ;$HRMS (DART) $\mathrm{m} / \mathrm{z}$ Calcd for $\mathrm{C}_{18} \mathrm{H}_{14} \mathrm{ClN}_{2} \mathrm{O}_{3}(\mathrm{M}+\mathrm{H})^{+}: 341.0687$, found 341.0690 .

Methyl-(R)-2-(2-(3-bromophenyl)-3,3-dicyano-5-vinylcyclopent-1-en-1-yl)-2oxoacetate (3j)<smiles>C=C[C@H]1CC(C#N)(C#N)C(c2cccc(Br)c2)=C1C(=O)C(C)=O</smiles>

Yellow solid, $50.7 \mathrm{mg}$, yield: $66 \%$, ee: 74\%. HPLC (Chiralpak IB, $4.6 \mathrm{~mm} \times 250 \mathrm{~mm}$, Hexane: $i$-Propanol $=90 / 10,0.7 \mathrm{~mL} / \mathrm{min}, 254 \mathrm{~nm}$ ): $\mathrm{t}_{\text {minor }}=12.23 \mathrm{~min}, \mathrm{t}_{\text {major }}=13.03 \mathrm{~min}$; $[\alpha]_{\mathrm{D}}^{25}=-54.2\left(c\right.$ 2.53, $\left.\mathrm{CHCl}_{3}\right) ; \mathrm{mp}: 79.0-79.1{ }^{\circ} \mathrm{C} .{ }^{1} \mathrm{H} \mathrm{NMR}\left(400 \mathrm{MHz}, \mathrm{CDCl}_{3}\right) \delta 7.67$ (d, $J=7.8 \mathrm{~Hz}, 1 \mathrm{H}), 7.54$ (s, 1H), 7.44-7.34 (m, 2H), 5.88 (ddd, $J=17.6,10.0,8.0 \mathrm{~Hz}$, 1H), $5.33(\mathrm{~d}, J=17.1 \mathrm{~Hz}, 1 \mathrm{H}), 5.27(\mathrm{~d}, J=10.2 \mathrm{~Hz}, 1 \mathrm{H}), 4.27-4.19(\mathrm{~m}, 1 \mathrm{H}), 3.48$ (s, $3 \mathrm{H}), 3.14(\mathrm{dd}, J=13.7,8.2 \mathrm{~Hz}, 1 \mathrm{H}), 2.76(\mathrm{dd}, J=13.7,5.1 \mathrm{~Hz}, 1 \mathrm{H}) .{ }^{13} \mathrm{C}$ NMR $(101$ $\left.\mathrm{MHz}, \mathrm{CDCl}_{3}\right) \delta 183.5,161.3,144.4,143.5,134.2,133.9,131.8,131.2,130.7,127.2$, 123.1, 119.7, 113.6, 113.2, 53.0, 48.8, 43.8, 42.4. IR (film): 2349, 2323, 1743, 1678, 1200, 1177, 1077, 1053, 1007, 993, 951, 739, 724, 699. MS (DART) m/z: $385.0(\mathrm{M}+$ $\mathrm{H})^{+}$; HRMS (DART) $m / z$ Calcd for $\mathrm{C}_{18} \mathrm{H}_{14} \mathrm{BrN}_{2} \mathrm{O}_{3}(\mathrm{M}+\mathrm{H})^{+}: 385.0182$, found 385.0185 .

Methyl-(R)-2-(3,3-dicyano-2-(2-fluorophenyl)-5-vinylcyclopent-1-en-1-yl)-2-

oxoacetate $(\mathbf{3 k})$<smiles>C=C[C@H]1CC(C#N)(C#N)C(c2ccccc2F)=C1C(=O)OC(C)=O</smiles>

Yellow solid, $51.9 \mathrm{mg}$, yield: 80\%, ee: 77\%. HPLC (Chiralpak IC, $4.6 \mathrm{~mm} \times 250 \mathrm{~mm}$, Hexane: $i$-Propanol $=90 / 10,0.7 \mathrm{~mL} / \mathrm{min}, 214 \mathrm{~nm}): \mathrm{t}_{\text {minor }}=13.58 \mathrm{~min}, \mathrm{t}_{\mathrm{major}}=16.99 \mathrm{~min}$; $[\alpha]_{\mathrm{D}}^{25}=-64.5\left(c 2.58, \mathrm{CHCl}_{3}\right)$; mp: 88.0-88.5 ${ }^{\circ} \mathrm{C} .{ }^{1} \mathrm{H}$ NMR $\left(400 \mathrm{MHz}, \mathrm{CDCl}_{3}\right) \delta 7.58-$ 
$7.41(\mathrm{~m}, 2 \mathrm{H}), 7.33-7.25(\mathrm{~m}, 1 \mathrm{H}), 7.21(\mathrm{t}, J=9.1 \mathrm{~Hz}, 1 \mathrm{H}), 5.92(\mathrm{ddd}, J=17.6,10.0,8.0$ $\mathrm{Hz}, 1 \mathrm{H}), 5.35-5.26(\mathrm{~m}, 2 \mathrm{H}), 4.33-4.25(\mathrm{~m}, 1 \mathrm{H}), 3.47$ (s, 3H), $3.16(\mathrm{dd}, J=13.6,8.2 \mathrm{~Hz}$, $1 \mathrm{H}), 2.78(\mathrm{dd}, J=13.6,4.9 \mathrm{~Hz}, 1 \mathrm{H}) .{ }^{19} \mathrm{~F} \mathrm{NMR}\left(376 \mathrm{MHz}, \mathrm{CDCl}_{3}\right) \delta-110.47 .{ }^{13} \mathrm{C} \mathrm{NMR}$ $(101 \mathrm{MHz},) \delta 183.0,161.3,160.9(\mathrm{~d}, J=252 \mathrm{~Hz}), 145.8,139.2,134.1,133.2(\mathrm{~d}, J=8$ Hz), 130.5, 124.8, 119.6, 117.2 (d, $J=15 \mathrm{~Hz}), 116.5$ (d, $J=22 \mathrm{~Hz}), 113.6,113.2,52.9$, 48.7, 44.0, 42.5. IR (film): 1744, 1700, 1487, 1448, 1432, 1417, 1305, 1290, 1276, 1260 , 958, 943, 759. MS (DART) $m / z: 325.1(\mathrm{M}+\mathrm{H})^{+}$; HRMS (DART) $\mathrm{m} / z$ Calcd for $\mathrm{C}_{18} \mathrm{H}_{14} \mathrm{FN}_{2} \mathrm{O}_{3}(\mathrm{M}+\mathrm{H})^{+}: 325.0983$, found 325.0985 .

Methyl-(R)-2-(2-(2-chlorophenyl)-3,3-dicyano-5-vinylcyclopent-1-en-1-yl)-2oxoacetate (31)<smiles>C=CC1CC(C#N)(C#N)C(c2ccccc2Cl)=C1C(=O)C(C)=O</smiles>

Yellow solid, $56.3 \mathrm{mg}$, yield: 83\%, ee: 77\%. HPLC (Chiralpak ID, $4.6 \mathrm{~mm} \times 250 \mathrm{~mm}$, Hexane: $i$-Propanol $=90 / 10,0.7 \mathrm{~mL} / \mathrm{min}, 214 \mathrm{~nm}$ ): $\mathrm{t}_{\text {minor }}=14.11 \mathrm{~min}, \mathrm{t}_{\mathrm{major}}=7.99 \mathrm{~min}$; $[\alpha]_{\mathrm{D}}{ }^{24}=-46.4\left(c\right.$ 2.82, $\left.\mathrm{CHCl}_{3}\right)$; mp: 100.8-101.3 ${ }^{\circ} \mathrm{C} .{ }^{1} \mathrm{H}$ NMR $\left(400 \mathrm{MHz}, \mathrm{CDCl}_{3}\right) \delta$ 7.55-7.37 (m, 4H), 5.91 (ddd, $J=17.2,9.6,8.0 \mathrm{~Hz}, 1 \mathrm{H}), 5.35$ (d, $J=17.1 \mathrm{~Hz}, 1 \mathrm{H}), 5.28$ (d, $J=10.2 \mathrm{~Hz}, 1 \mathrm{H}), 4.38-4.27(\mathrm{~m}, 1 \mathrm{H}), 3.43(\mathrm{~s}, 3 \mathrm{H}), 3.17(\mathrm{dd}, J=13.6,8.1 \mathrm{~Hz}, 1 \mathrm{H})$, $2.76(\mathrm{dd}, J=13.7,5.4 \mathrm{~Hz}, 1 \mathrm{H}) .{ }^{13} \mathrm{C} \mathrm{NMR}\left(101 \mathrm{MHz}, \mathrm{CDCl}_{3}\right) \delta 182.8,161.6,146.0$, 134.2, 134.1, 132.0, 130.6, 130.2, 128.0, 127.2, 119.6, 113.3, 112.9, 52.8, 48.2, 44.4, 42.4. IR (film): 1731, 1699, 1632, 1327, 1290, 1264, 1249, 1193, 1185, 1149, 1074, 1064, 1039, 1001, 940, 819, 755, 741, 612, 571, 445. MS (DART) $m / z: 341.1(\mathrm{M}+\mathrm{H})^{+}$; HRMS (DART) $m / z$ Calcd for $\mathrm{C}_{18} \mathrm{H}_{14} \mathrm{ClN}_{2} \mathrm{O}_{3}(\mathrm{M}+\mathrm{H})^{+}:$341.0687, found 341.0690.

Methyl-(R)-2-(2-(2-bromophenyl)-3,3-dicyano-5-vinylcyclopent-1-en-1-yl)-2oxoacetate $(\mathbf{3 m})$ 


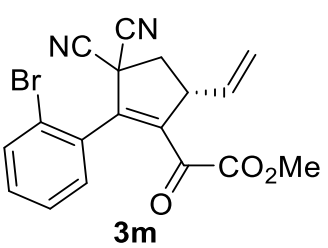

Yellow solid, $60.1 \mathrm{mg}$, yield: 78\%, ee: 78\%. HPLC (Chiralpak ID, $4.6 \mathrm{~mm} \times 250 \mathrm{~mm}$, Hexane: $i$-Propanol $=95 / 5,0.7 \mathrm{~mL} / \mathrm{min}, 214 \mathrm{~nm}$ ): $\mathrm{t}_{\text {minor }}=22.85 \mathrm{~min}, \mathrm{t}_{\text {major }}=11.79 \mathrm{~min}$; $[\alpha]_{\mathrm{D}}^{25}=-37.9\left(c\right.$ 3.05, $\left.\mathrm{CHCl}_{3}\right)$; mp: 94.4-94.9 ${ }^{\circ} \mathrm{C} .{ }^{1} \mathrm{H}$ NMR $\left(400 \mathrm{MHz}, \mathrm{CDCl}_{3}\right) \delta 7.75-$ $7.61(\mathrm{~m}, 1 \mathrm{H}), 7.59-7.30(\mathrm{~m}, 3 \mathrm{H}), 6.19-5.64(\mathrm{~m}, 1 \mathrm{H}), 5.50-5.14(\mathrm{~m}, 2 \mathrm{H}), 4.44-4.18(\mathrm{~m}$, 1H), $3.42(\mathrm{~s}, 3 \mathrm{H}), 3.15(\mathrm{dd}, J=13.6,8.1 \mathrm{~Hz}, 1 \mathrm{H}), 2.81-2.67(\mathrm{~m}, 1 \mathrm{H}) .{ }^{13} \mathrm{C}$ NMR $(101$ $\left.\mathrm{MHz}, \mathrm{CDCl}_{3}\right) \delta 182.8,161.7,134.7,134.0,133.4,132.0,130.4,130.1,127.7,124.1$, 119.5, 113.7, 112.7, 52.8, 48.2, 44.4, 42.6, 42.4. IR (film): 2344, 2323, 1746, 1730, 1700, 1248, 1192, 1184, 1063, 1025, 1002, 940, 819, 696, 679, 670, 664, 645, 613, 573. MS (DART) $m / z: 385.0(\mathrm{M}+\mathrm{H})^{+} ;$HRMS (DART) $m / z$ Calcd for $\mathrm{C}_{18} \mathrm{H}_{14} \mathrm{BrN}_{2} \mathrm{O}_{3}(\mathrm{M}+$ $\mathrm{H})^{+}:$385.0182, found 385.0185 .

Methyl-(R)-2-(3,3-dicyano-2-(2-cyanophenyl)-5-vinylcyclopent-1-en-1-yl)-2oxoacetate (3n)<smiles>C=CC1CN(C#N)C(C#N)(C#N)C1c1ccccc1C#N</smiles>

Yellow solid, $21 \mathrm{mg}$, yield: 32\%, ee: 52\%. HPLC (Chiralpak IB, $4.6 \mathrm{~mm} \times 250 \mathrm{~mm}$, Hexane: $i$-Propanol $=90 / 10,0.7 \mathrm{~mL} / \mathrm{min}, 214 \mathrm{~nm}$ ): $\mathrm{t}_{\mathrm{minor}}=30.12 \mathrm{~min}, \mathrm{t}_{\mathrm{major}}=26.92 \mathrm{~min}$; $[\alpha]_{\mathrm{D}}^{31}=-26.8\left(c\right.$ 1.00, $\left.\mathrm{CHCl}_{3}\right) ; \mathrm{mp}: 119.2-119.3{ }^{\circ} \mathrm{C} .{ }^{1} \mathrm{H}$ NMR $\left(400 \mathrm{MHz}, \mathrm{CDCl}_{3}\right) \delta$ 7.82-7.70 (m, 2H), 7.66-7.59 (m, 2H), $5.90(\mathrm{ddd}, J=17.6,10.0,8.0 \mathrm{~Hz}, 1 \mathrm{H}), 5.39-5.28$ $(\mathrm{m}, 2 \mathrm{H}), 4.50-4.39(\mathrm{~m}, 1 \mathrm{H}), 3.57(\mathrm{~s}, 3 \mathrm{H}), 3.22(\mathrm{dd}, J=13.7,8.0 \mathrm{~Hz}, 1 \mathrm{H}), 2.75(\mathrm{dd}, J=$ 13.7, $5.7 \mathrm{~Hz}, 1 \mathrm{H}) .{ }^{13} \mathrm{C} \mathrm{NMR}\left(101 \mathrm{MHz}, \mathrm{CDCl}_{3}\right) \delta 181.9,161.1,146.9,134.1,133.3$, 133.1, 132.8, 130.9, 129.1, 120.3, 116.4, 114.1, 112.7, 110.0, 53.0, 48.7, 44.4, 42.6. IR (film): 1733, 1697, 1630, 1327, 1290, 1251, 1197, 1185, 1068, 1049, 992, 949, 819, 765, 748, 593, 552. MS (DART) $\mathrm{m} / z: 332.1(\mathrm{M}+\mathrm{H})^{+}$; HRMS (DART) $\mathrm{m} / z$ Calcd for $\mathrm{C}_{19} \mathrm{H}_{14} \mathrm{~N}_{3} \mathrm{O}_{3}(\mathrm{M}+\mathrm{H})^{+}:$332.1030, found 332.1032. 
Methyl-(R)-2-(3,3-dicyano-2-(naphthalen-2-yl)-5-vinylcyclopent-1-en-1-yl)-2oxoacetate (3o)<smiles>C=CC1CC(C#N)(C#N)C(c2ccc3ccccc3c2)=C1C(=O)C(=O)OC</smiles>

Yellow solid, $36.2 \mathrm{mg}$, yield: 51\%, ee: 69\%. HPLC (Chiralpak ID, $4.6 \mathrm{~mm} \times 250 \mathrm{~mm}$, Hexane: $i$-Propanol $=90 / 10,0.7 \mathrm{~mL} / \mathrm{min}, 214 \mathrm{~nm}$ ): $\mathrm{t}_{\text {minor }}=13.52 \mathrm{~min}, \mathrm{t}_{\text {major }}=10.29 \mathrm{~min}$; $[\alpha]_{\mathrm{D}}^{31}=-56.7\left(c\right.$ 1.00, $\left.\mathrm{CHCl}_{3}\right) ; \mathrm{mp}: 110.3-111.5{ }^{\circ} \mathrm{C} .{ }^{1} \mathrm{H}$ NMR $\left(400 \mathrm{MHz}, \mathrm{CDCl}_{3}\right) \delta$ 7.97-7.81 (m, 4H), 7.62-7.53 (m, 3H), 5.94 (ddd, $J=17.6,10.0,7.6 \mathrm{~Hz}, 1 \mathrm{H}), 5.35$ (d, $J$ $=17.0 \mathrm{~Hz}, 1 \mathrm{H}), 5.26(\mathrm{~d}, J=10.2 \mathrm{~Hz}, 1 \mathrm{H}), 4.27-4.20(\mathrm{~m}, 1 \mathrm{H}), 3.16(\mathrm{dd}, J=13.7,8.3$ $\mathrm{Hz}, 1 \mathrm{H}), 3.05(\mathrm{~s}, 3 \mathrm{H}), 2.81(\mathrm{dd}, J=13.7,4.6 \mathrm{~Hz}, 1 \mathrm{H}) .{ }^{13} \mathrm{C} \mathrm{NMR}\left(101 \mathrm{MHz}, \mathrm{CDCl}_{3}\right) \delta$ 184.1, 161.5, 146.1, 143.5, 134.2, 134.0, 132.4, 130.1, 129.3, 128.5, 128.2, 127.9, 127.5, 127.0, 124.2, 119.3, 114.1, 113.6, 52.6, 48.7, 43.7, 42.6. IR (film): 2349, 2344, 1751, $1692,1328,1318,1273,1233,1210,996,966,939,822,790,774,759,477,422$. MS (DART) $m / z: 357.1(\mathrm{M}+\mathrm{H})^{+}$; HRMS (DART) $m / z$ Calcd for $\mathrm{C}_{22} \mathrm{H}_{17} \mathrm{~N}_{2} \mathrm{O}_{3}(\mathrm{M}+\mathrm{H})^{+}$: 357.1234, found 357.1237.

Methyl-(R)-2-(3,3-dicyano-2-(thiophen-2-yl)-5-vinylcyclopent-1-en-1-yl)-2oxoacetate (3p)<smiles>C=CC1CC2(C#N)CC1C(C(=O)C(=O)OC)=C2c1cccs1</smiles>

Yellow viscous oil, $22.9 \mathrm{mg}$, yield: 73\%, ee: 77\%. HPLC (Chiralpak IB, $4.6 \mathrm{~mm} \times 250$ $\mathrm{mm}$, Hexane: $i$-Propanol $=90 / 10,0.7 \mathrm{~mL} / \mathrm{min}, 214 \mathrm{~nm}$ ): $\mathrm{t}_{\mathrm{minor}}=15.24 \mathrm{~min}, \mathrm{t}_{\mathrm{major}}=16.16$ $\min ;[\alpha]_{\mathrm{D}}{ }^{25}=-79.1\left(c 0.65, \mathrm{CHCl}_{3}\right) ;{ }^{1} \mathrm{H} \mathrm{NMR}\left(400 \mathrm{MHz}, \mathrm{CDCl}_{3}\right) \delta 7.57(\mathrm{~d}, J=5.0 \mathrm{~Hz}$, $1 \mathrm{H}), 7.43(\mathrm{~d}, J=3.7 \mathrm{~Hz}, 1 \mathrm{H}), 7.16-7.10(\mathrm{~m}, 1 \mathrm{H}), 5.79(\mathrm{ddd}, J=18.0,10.0,8.8 \mathrm{~Hz}$, 1H), 5.31-5.20 (m, 2H), 4.28-4.19 (m, 1H), $3.61(\mathrm{~s}, 3 \mathrm{H}), 3.14(\mathrm{dd}, J=13.7,7.9 \mathrm{~Hz}, 1 \mathrm{H})$, $2.68(\mathrm{dd}, J=13.7,6.3 \mathrm{~Hz}, 1 \mathrm{H}) .{ }^{13} \mathrm{C} \mathrm{NMR}\left(101 \mathrm{MHz}, \mathrm{CDCl}_{3}\right) \delta 183.7,160.7,142.3$, 
137.5, 134.3, 131.9, 131.1, 130.4, 128.0, 119.9, 113.9, 113.6, 53.1, 49.5, 43.4, 42.6. IR (film): 1737, 1686, 1422, 1261, 1196, 1175, 1049, 935, 849, 718. MS (DART) $\mathrm{m} / z$ : $313.1(\mathrm{M}+\mathrm{H})^{+}$; HRMS (DART) $\mathrm{m} / z$ Calcd for $\mathrm{C}_{16} \mathrm{H}_{13} \mathrm{~N}_{2} \mathrm{O}_{3} \mathrm{~S}(\mathrm{M}+\mathrm{H})^{+}$: 313.0641, found 313.0643.

Methyl-(R)-2-(3,3-dicyano-2-(triethylsilyl)-5-vinylcyclopent-1-en-1-yl)-2-oxoacetate $(3 \mathbf{q})$

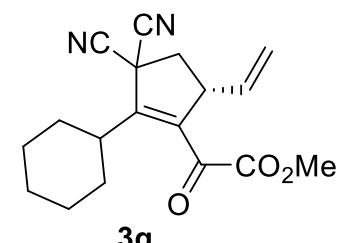

$3 q$

Yellow solid, $55.3 \mathrm{mg}$, yield: 89\%, ee: 86\%. HPLC (Chiralpak IC, $4.6 \mathrm{~mm} \times 250 \mathrm{~mm}$, Hexane: $i$-Propanol $=90 / 10,0.7 \mathrm{~mL} / \mathrm{min}, 214 \mathrm{~nm}): \mathrm{t}_{\text {minor }}=7.67 \mathrm{~min}, \mathrm{t}_{\mathrm{major}}=8.17 \mathrm{~min}$; $[\alpha] \mathrm{D}^{31}=-63.9\left(\right.$ c $\left.2.51, \mathrm{CHCl}_{3}\right) ; \mathrm{mp}: 71.9-73.6{ }^{\circ} \mathrm{C} .{ }^{1} \mathrm{H}$ NMR $\left(400 \mathrm{MHz}, \mathrm{CDCl}_{3}\right) \delta 5.62$ (ddd, $J=16.7,10.3,8.8 \mathrm{~Hz}, 1 \mathrm{H}), 5.20-5.12(\mathrm{~m}, 2 \mathrm{H}), 4.20-4.16(\mathrm{~m}, 1 \mathrm{H}), 3.83(\mathrm{~s}, 3 \mathrm{H})$, $2.96(\mathrm{dd}, J=13.3,7.2 \mathrm{~Hz}, 1 \mathrm{H}), 2.83-2.73(\mathrm{~m}, 1 \mathrm{H}), 2.35(\mathrm{dd}, J=13.3,8.1 \mathrm{~Hz}, 1 \mathrm{H})$, 1.95-1.78 (m, 4H), 1.76-1.60 (m, 3H), 1.38-1.16 (m, 3H). $\left.{ }^{13} \mathrm{C} \mathrm{NMR} \mathrm{(101} \mathrm{MHz,} \mathrm{CDCl}_{3}\right)$ $\delta 184.4,161.3,152.7,141.4,135.1,120.1,114.4,113.9,53.1,48.4,43.8,39.939 .7$, 31.3, 30.5, 26.1, 26.0, 25.2. IR (film): 2934, 2889, 2853, 1742, 1694, 1607, 1418, 1320, 1232, 1213, 1062, 1024, 931. MS (DART) $\mathrm{m} / \mathrm{z}: 313.2(\mathrm{M}+\mathrm{H})^{+} ;$HRMS (DART) $\mathrm{m} / \mathrm{z}$ Calcd for $\mathrm{C}_{18} \mathrm{H}_{21} \mathrm{~N}_{2} \mathrm{O}_{3}(\mathrm{M}+\mathrm{H})^{+}:$313.1547, found 313.1549.

Methyl-(R)-2-(3,3-dicyano-2-tetradecyl-5-vinylcyclopent-1-en-1-yl)-2-oxoacetate (3r)<smiles>C=CC1=C(C(=O)C(C)=O)[C@@H](C=C)CC1(C#N)C#N</smiles>

Yellow solid, $44.3 \mathrm{mg}$, yield: 52\%, ee: 79\%. HPLC (Chiralpak IC, $4.6 \mathrm{~mm} \times 250 \mathrm{~mm}$, Hexane: $i$-Propanol $=98 / 2,0.7 \mathrm{~mL} / \mathrm{min}, 214 \mathrm{~nm}$ ): $\mathrm{t}_{\text {minor }}=10.64 \mathrm{~min}, \mathrm{t}_{\mathrm{major}}=11.37 \mathrm{~min}$; $[\alpha]_{\mathrm{D}}^{32}=-22.3\left(c 2.21, \mathrm{CHCl}_{3}\right)$; mp: $31.9-32.8{ }^{\circ} \mathrm{C} .{ }^{1} \mathrm{H}$ NMR $\left(400 \mathrm{MHz}, \mathrm{CDCl}_{3}\right) \delta 5.76-$ $5.64(\mathrm{~m}, 1 \mathrm{H}), 5.25-5.13(\mathrm{~m}, 2 \mathrm{H}), 4.26-4.17(\mathrm{~m}, 1 \mathrm{H}), 3.86(\mathrm{~s}, 3 \mathrm{H}), 2.98(\mathrm{dd}, J=13.4$, 
$7.6 \mathrm{~Hz}, 1 \mathrm{H}), 2.76-2.63(\mathrm{~m}, 1 \mathrm{H}), 2.63-2.52(\mathrm{~m}, 1 \mathrm{H}), 2.43$ (dd, $J=13.4,6.7 \mathrm{~Hz}, 1 \mathrm{H})$, 1.79-1.60 (m, 2H), 1.25 (s, 22H), 0.94-0.79 (m, $J=6.3 \mathrm{~Hz}, 3 \mathrm{H}) .{ }^{13} \mathrm{C}$ NMR (101 MHz, $\left.\mathrm{CDCl}_{3}\right) \delta 183.7,161.7,150.1,141.1,135.3,119.8,113.8,113.6,53.1,48.4,42.8,42.3$, 31.9, 29.7(3C), 29.6(2C), 29.5, 29.4, 29.3, 29.0, 28.7, 28.4, 22.7, 14.1. IR (film): 2954, 2917, 2851, 1753, 1735, 1693, 1614, 1469, 1442, 1233, 1050, 995, 964, 939, 747, 719, 687. MS (DART) $m / z: 427.3(\mathrm{M}+\mathrm{H})^{+}$; HRMS (DART) $m / z$ Calcd for $\mathrm{C}_{26} \mathrm{H}_{39} \mathrm{~N}_{2} \mathrm{O}_{3}(\mathrm{M}$ $+\mathrm{H})^{+}:$427.2955, found 427.2958 .

Methyl-(R)-2-(3,3-dicyano-2-(triethylsilyl)-5-vinylcyclopent-1-en-1-yl)-2-oxoacetate $(3 \mathbf{s})$<smiles>C=CC1CC(C#N)(C#N)C(CCCC)=C1C(=O)C(C)=O</smiles>

Yellow solid, $32.5 \mathrm{mg}$, yield: 57\%, ee: $83 \%$. HPLC (Chiralpak IC, $4.6 \mathrm{~mm} \times 250 \mathrm{~mm}$, Hexane: $i$-Propanol $=90 / 10,0.7 \mathrm{~mL} / \mathrm{min}, 214 \mathrm{~nm}): \mathrm{t}_{\mathrm{minor}}=8.57 \mathrm{~min}, \mathrm{t}_{\mathrm{major}}=9.09 \mathrm{~min}$; $[\alpha] \mathrm{D}^{30}=-42.4\left(c\right.$ 1.62, $\left.\mathrm{CHCl}_{3}\right) ;{ }^{1} \mathrm{H} \mathrm{NMR}\left(400 \mathrm{MHz}, \mathrm{CDCl}_{3}\right) \delta 5.68(\mathrm{ddd}, J=17.0,10.0$, 8.6 Hz, 1H), 5.20-5.12 (m, 2H), 4.12-4.15 (m, 1H), $3.83(\mathrm{~s}, 3 \mathrm{H}), 2.96$ (dd, $J=13.5,7.6$ $\mathrm{Hz}, 1 \mathrm{H}), 2.72-2.62(\mathrm{~m}, 1 \mathrm{H}), 2.61-2.51(\mathrm{~m}, 1 \mathrm{H}), 2.40$ (dd, $J=13.5,6.7 \mathrm{~Hz}, 1 \mathrm{H}), 1.76-$ $1.56(\mathrm{~m}, 2 \mathrm{H}), 1.47-1.31(\mathrm{~m}, 2 \mathrm{H}), 0.92(\mathrm{t}, J=7.3 \mathrm{~Hz}, 3 \mathrm{H}) .{ }^{13} \mathrm{C} \mathrm{NMR}\left(101 \mathrm{MHz}, \mathrm{CDCl}_{3}\right)$ $\delta 183.7,161.7,150.0,141.1,135.2,119.8,113.8,113.6,53.1,48.4,42.7,42.3,30.3$, 28.4, 22.8, 13.5. IR (film): 2961, 2935, 2874, 2349, 2344, 2323, 1738, 1696, 1262, 1234 , 1205, 1143, 1045, 799. MS (DART) $m / z: 287.1(\mathrm{M}+\mathrm{H})^{+}$; HRMS (DART) $m / z$ Calcd for $\mathrm{C}_{16} \mathrm{H}_{19} \mathrm{~N}_{2} \mathrm{O}_{3}(\mathrm{M}+\mathrm{H})^{+}:$287.1390, found 287.1392.

Methyl-(R)-2-(3,3-dicyano-2-(triethylsilyl)-5-vinylcyclopent-1-en-1-yl)-2-oxoacetate (3t)<smiles>C=C[C@H]1CC(C#N)(C#N)C(CC2CCCCC2)=C1C(=O)C(C)=O</smiles> 
Yellow solid, $50.0 \mathrm{mg}$, yield: 77\%, ee: $82 \%$. HPLC (Chiralpak ID, $4.6 \mathrm{~mm} \times 250 \mathrm{~mm}$, Hexane: $i$-Propanol $=98 / 2,0.7 \mathrm{~mL} / \mathrm{min}, 254 \mathrm{~nm}$ ): $\mathrm{t}_{\text {minor }}=8.49 \mathrm{~min}, \mathrm{t}_{\text {major }}=7.84 \mathrm{~min}$; $[\alpha] \mathrm{D}^{25}=-32.7\left(c\right.$ 2.50, $\left.\mathrm{CHCl}_{3}\right)$; mp: 58.9-59.6 ${ }^{\circ} \mathrm{C} .{ }^{1} \mathrm{H} \mathrm{NMR}\left(400 \mathrm{MHz}, \mathrm{CDCl}_{3}\right) \delta 5.75-$ $5.62(\mathrm{~m}, 1 \mathrm{H}), 5.26-5.14(\mathrm{~m}, 2 \mathrm{H}), 4.23(\mathrm{dd}, J=15.8,7.7 \mathrm{~Hz}, 1 \mathrm{H}), 3.87(\mathrm{~s}, 3 \mathrm{H}), 2.98(\mathrm{dd}$, $J=13.0,7.3 \mathrm{~Hz}, 1 \mathrm{H}), 2.71-2.60(\mathrm{~m}, 1 \mathrm{H}), 2.58-5.47(\mathrm{~m}, 1 \mathrm{H}), 2.41(\mathrm{dd}, J=13.2,7.3 \mathrm{~Hz}$, 1H), 1.90-1.58 (m, 5H), 1.37-0.95 (m, 5H), 0.93-0.74 (m, 1H). ${ }^{13} \mathrm{C}$ NMR (101 MHz, $\left.\mathrm{CDCl}_{3}\right) \delta 184.2,161.4,147.2,143.2,135.1,120.0,113.7,113.6,53.2,48.7,43.2,42.2$, 36.4, 35.7, 33.6, 32.7, 26.0 (2C), 25.9. IR (film): 2948, 2922, 2855, 1727, 1670, 1627, 1452, 1441, 1234, 1202, 1074, 1058, 1039, 1000, 948, 735, 629. MS (DART) $m / z$ : $327.2(\mathrm{M}+\mathrm{H})^{+}$; HRMS (DART) $m / z$ Calcd for $\mathrm{C}_{19} \mathrm{H}_{23} \mathrm{~N}_{2} \mathrm{O}_{3}(\mathrm{M}+\mathrm{H})^{+}: 327.1703$, found 327.1705 .

Methyl-(R)-2-(3,3-dicyano-2-(triethylsilyl)-5-vinylcyclopent-1-en-1-yl)-2-oxoacetate $(3 \mathbf{u})$<smiles>C=C[C@H]1CC(C#N)(C#N)C(CC)=C1C(=O)C(=O)OC</smiles>

Yellow solid, $34.4 \mathrm{mg}$, yield: 50\%, ee: $83 \%$. HPLC (Chiralpak IB, $4.6 \mathrm{~mm} \times 250 \mathrm{~mm}$, Hexane: $i$-Propanol $=98 / 2,0.7 \mathrm{~mL} / \mathrm{min}, 254 \mathrm{~nm}): \mathrm{t}_{\mathrm{minor}}=8.22 \mathrm{~min}, \mathrm{t}_{\mathrm{major}}=8.81 \mathrm{~min}$; $[\alpha]_{\mathrm{D}}^{24}=-56.8\left(c\right.$ 1.72, $\left.\mathrm{CHCl}_{3}\right)$; mp: $61.9-63.2{ }^{\circ} \mathrm{C} .{ }^{1} \mathrm{H} \mathrm{NMR}\left(400 \mathrm{MHz}, \mathrm{CDCl}_{3}\right) \delta 5.67-$ $5.56(\mathrm{~m}, 1 \mathrm{H}), 5.25-5.16(\mathrm{~m}, 2 \mathrm{H}), 4.36-4.26(\mathrm{~m}, 1 \mathrm{H}), 3.87(\mathrm{~s}, 3 \mathrm{H}), 3.06(\mathrm{dd}, J=13.4$, $7.3 \mathrm{~Hz}, 1 \mathrm{H}), 2.38(\mathrm{dd}, J=13.4,9.0 \mathrm{~Hz}, 1 \mathrm{H}), 1.01-0.94(\mathrm{~m}, 9 \mathrm{H}), 0.91-0.80(\mathrm{~m}, 6 \mathrm{H}) .{ }^{13} \mathrm{C}$ NMR $\left(101 \mathrm{MHz}, \mathrm{CDCl}_{3}\right) \delta 185.5,160.6,159.0,147.5,134.7,120.7,115.2,114.3,53.3$, 51.6, 44.8, 43.1, 7.1, 2.7. IR (film): 2958, 2911, 2874, 1735, 1697, 1199, 1154, 1001, 978, 933, 737, 725, 701, 679. MS (DART) $m / z: 345.2(\mathrm{M}+\mathrm{H})^{+}$; HRMS (DART) $\mathrm{m} / \mathrm{z}$ Calcd for $\mathrm{C}_{18} \mathrm{H}_{25} \mathrm{~N}_{2} \mathrm{O}_{3} \mathrm{Si}(\mathrm{M}+\mathrm{H})^{+}$: 345.1629, found 345.1631.

Ethyl (R)-2-(3,3-dicyano-2-(p-tolyl)-5-vinylcyclopent-1-en-1-yl)-2-oxoacetate (3v) 


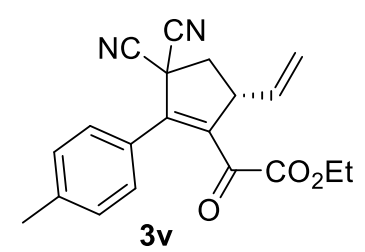

Yellow solid, $54.1 \mathrm{mg}$, yield: $81 \%$, ee: $83 \%$. HPLC (Chiralpak IC, $4.6 \mathrm{~mm} \times 250 \mathrm{~mm}$, Hexane: $i$-Propanol = 90/10, $0.7 \mathrm{~mL} / \mathrm{min}, 214 \mathrm{~nm}$ ): $\mathrm{t}_{\text {minor }}=13.75 \mathrm{~min}, \mathrm{t}_{\text {major }}=21.15 \mathrm{~min}$; $[\alpha]_{\mathrm{D}}^{30}=-75.1\left(\right.$ c 2.70, $\left.\mathrm{CHCl}_{3}\right) ; \mathrm{mp}: 115.2-116.7^{\circ} \mathrm{C} .{ }^{1} \mathrm{H} \mathrm{NMR}\left(400 \mathrm{MHz}, \mathrm{CDCl}_{3}\right) \delta 7.33$ $(\mathrm{d}, J=8.0 \mathrm{~Hz}, 2 \mathrm{H}), 7.26(\mathrm{~d}, J=7.9 \mathrm{~Hz}, 2 \mathrm{H}), 5.90(\mathrm{ddd}, J=17.610 .0,8.4 \mathrm{~Hz}, 1 \mathrm{H})$, $5.31(\mathrm{~d}, J=17.0 \mathrm{~Hz}, 1 \mathrm{H}), 5.23(\mathrm{~d}, J=10.2 \mathrm{~Hz}, 1 \mathrm{H}), 4.22-4.14(\mathrm{~m}, 1 \mathrm{H}), 3.86-3.72(\mathrm{~m}$, 2H), $3.11(\mathrm{dd}, J=13.6,8.3 \mathrm{~Hz}, 1 \mathrm{H}), 2.75(\mathrm{dd}, J=13.6,4.8 \mathrm{~Hz}, 1 \mathrm{H}), 2.39$ (s, 3H), 1.04 $(\mathrm{t}, J=7.1 \mathrm{~Hz}, 3 \mathrm{H}) .{ }^{13} \mathrm{C} \mathrm{NMR}\left(101 \mathrm{MHz}, \mathrm{CDCl}_{3}\right) \delta 184.6,161.0,145.4,142.8,141.8$, 134.4, 129.8, 128.7, 126.8, 119.1, 114.1, 113.7, 62.6, 48.9, 43.7, 42.5, 21.4, 13.5. IR (film): 1726, 1676, 1636, 1281, 1186, 1051, 1018, 910, 819, 729, 714, 699, 504. MS (DART) $m / z: 335.1(\mathrm{M}+\mathrm{H})^{+}$; HRMS (DART) $m / z$ Calcd for $\mathrm{C}_{20} \mathrm{H}_{19} \mathrm{~N}_{2} \mathrm{O}_{3}(\mathrm{M}+\mathrm{H})^{+}$: 335.1390 , found 335.1392 .

Isopropyl (R)-2-(3,3-dicyano-2-(p-tolyl)-5-vinylcyclopent-1-en-1-yl)-2-oxoacetate $(3 \mathbf{w})$<smiles>C=CC1CC(C#N)(C#N)C(c2ccc(C)cc2)=C1C(=O)OCC</smiles>

Yellow solid, $23.6 \mathrm{mg}$, yield: 67\%, ee: $86 \%$. HPLC (Chiralpak IC, $4.6 \mathrm{~mm} \times 250 \mathrm{~mm}$, Hexane: $i$-Propanol $=90 / 10,0.7 \mathrm{~mL} / \mathrm{min}, 214 \mathrm{~nm}): \mathrm{t}_{\operatorname{minor}}=11.47 \mathrm{~min}, \mathrm{t}_{\text {major }}=16.10 \mathrm{~min}$; $[\alpha]_{\mathrm{D}}^{25}=-70.2\left(c\right.$ 1.83, $\left.\mathrm{CHCl}_{3}\right)$; mp: 94.8-96.0 ${ }^{\circ} \mathrm{C} .{ }^{1} \mathrm{H}$ NMR $\left(400 \mathrm{MHz}, \mathrm{CDCl}_{3}\right) \delta$ 7.32$7.29(\mathrm{~m}, 2 \mathrm{H}), 7.27-7.22(\mathrm{~m}, 2 \mathrm{H}), 5.89$ (ddd, $J=19.2,10.4,8.0 \mathrm{~Hz}, 1 \mathrm{H}), 5.30(\mathrm{~d}, J=$ $17.0 \mathrm{~Hz}, 1 \mathrm{H}), 5.22(\mathrm{~d}, J=10.1 \mathrm{~Hz}, 1 \mathrm{H}), 4.68-4.55(\mathrm{~m}, 1 \mathrm{H}), 4.20-4.13(\mathrm{~m}, 1 \mathrm{H}), 3.11(\mathrm{dd}$, $J=13.6,8.2 \mathrm{~Hz}, 1 \mathrm{H}), 2.73(\mathrm{dd}, J=13.6,4.9 \mathrm{~Hz}, 1 \mathrm{H}), 2.37$ (s, 3H), $0.99(\mathrm{~d}, J=6.3 \mathrm{~Hz}$, $6 \mathrm{H}) .{ }^{13} \mathrm{C} \mathrm{NMR}\left(101 \mathrm{MHz}, \mathrm{CDCl}_{3}\right) \delta 185.0,160.3,144.4,142.8,141.8,134.3,129.9$, 128.7, 126.9, 119.2, 114.2, 113.7, 71.2, 49.2, 43.4, 42.6, 21.4, 21.0. IR (film): 1726, 
1672, 1644, 1278, 1201, 1185, 1098, 1053, 928, 778, 508. MS (DART) m/z: 349.2 (M

$+\mathrm{H})^{+}$; HRMS (DART) $m / z$ Calcd for $\mathrm{C}_{21} \mathrm{H}_{21} \mathrm{~N}_{2} \mathrm{O}_{3}(\mathrm{M}+\mathrm{H})^{+}:$349.1547, found 349.1549.

Methyl-(R,E)-2-(3,3-dicyano-5-(hex-1-en-1-yl)-2-(p-tolyl)cyclopent-1-en-1-yl)-2oxoacetate $(\mathbf{3 x})$

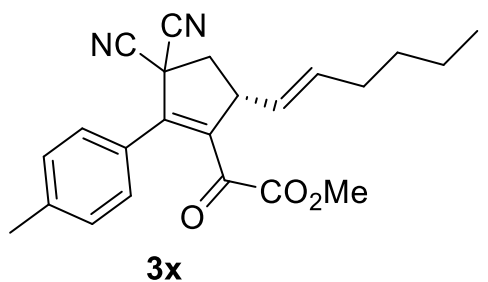

Yellow viscous oil, $41.8 \mathrm{mg}$, yield: 56\%, ee: $30 \%$. HPLC (Chiralpak IC, $4.6 \mathrm{~mm} \times 250$ $\mathrm{mm}$, Hexane: $i$-Propanol $=90 / 10,0.7 \mathrm{~mL} / \mathrm{min}, 214 \mathrm{~nm}): \mathrm{t}_{\mathrm{minor}}=9.82 \mathrm{~min}, \mathrm{t}_{\mathrm{major}}=14.89$ $\min ;[\alpha]_{\mathrm{D}}{ }^{26}=-35.3\left(c 1.3, \mathrm{CHCl}_{3}\right) ;{ }^{1} \mathrm{H}$ NMR $\left(400 \mathrm{MHz}, \mathrm{CDCl}_{3}\right) \delta 7.30(\mathrm{~d}, J=8.1 \mathrm{~Hz}$, 2H), $7.24(\mathrm{~d}, J=8.0 \mathrm{~Hz}, 2 \mathrm{H}), 5.74-5.64(\mathrm{~m}, 1 \mathrm{H}), 5.44(\mathrm{dd}, J=15.3,8.1 \mathrm{~Hz}, 1 \mathrm{H}), 4.17$ - $4.08(\mathrm{~m}, 1 \mathrm{H}), 3.36(\mathrm{~s}, 3 \mathrm{H}), 3.07$ (dd, $J=13.6,8.1 \mathrm{~Hz}, 1 \mathrm{H}), 2.67(\mathrm{dd}, J=13.6,4.9 \mathrm{~Hz}$, 1H), $2.38(\mathrm{~s}, 3 \mathrm{H}), 2.03-1.96(\mathrm{~m}, 2 \mathrm{H}), 1.33-1.23(\mathrm{~m}, 4 \mathrm{H}), 0.85(\mathrm{t}, J=7.1 \mathrm{~Hz}, 3 \mathrm{H}) .{ }^{13} \mathrm{C}$ NMR $\left(101 \mathrm{MHz}, \mathrm{CDCl}_{3}\right) \delta 184.3,161.4,145.1,143.4,141.6,136.0,129.7,128.7,126.8$, 125.8, 114.2, 113.8, 52.7, 48.2, 43.7, 43.1, 32.0, 31.0, 22.0, 21.4, 13.8. IR (film): 3032, 2955, 2926, 2857, 1739, 1683, 1509, 1437, 1276, 1200, 1174, 1048, 1020, 969, 816, 762, 725, 504. MS (DART) $m / z: 377.2(\mathrm{M}+\mathrm{H})^{+}$; HRMS (DART) $\mathrm{m} / z$ Calcd for $\mathrm{C}_{23} \mathrm{H}_{25} \mathrm{~N}_{2} \mathrm{O}_{3}(\mathrm{M}+\mathrm{H})^{+}:$377.1860, found 377.1857.

Methyl 3,3-dicyano-2-(p-tolylethynyl)-5-vinyltetrahydrofuran-2-carboxylate (4a)<smiles>C=CC1CC(C#N)(C#N)C(C#N)(C(=O)OC)O1</smiles>

Yellow viscous oil; ${ }^{1} \mathrm{H}$ NMR (400 MHz, $\left.\mathrm{CDCl}_{3}\right)$ major isomer: $\delta 7.42(\mathrm{~d}, J=7.9 \mathrm{~Hz}$, 2H), $7.17(\mathrm{~d}, J=7.8 \mathrm{~Hz}, 2 \mathrm{H}), 6.00(\mathrm{ddd}, J=17.3,10.2,7.4 \mathrm{~Hz}, 1 \mathrm{H}), 5.47$ (d, $J=17.1$ $\mathrm{Hz}, 1 \mathrm{H}), 5.36(\mathrm{~d}, J=10.3 \mathrm{~Hz}, 1 \mathrm{H}), 5.07-4.99(\mathrm{~m}, 1 \mathrm{H}), 4.01$ (s, 3H), 3.17 (dd, $J=13.4$, $8.4 \mathrm{~Hz}, 1 \mathrm{H}), 2.74(\mathrm{dd}, J=13.5,5.9 \mathrm{~Hz}, 1 \mathrm{H}), 2.37(\mathrm{~s}, 3 \mathrm{H})$. minor isomer: $\delta 7.40(\mathrm{~d}, J=$ 
$7.8 \mathrm{~Hz}, 2 \mathrm{H}), 7.16(\mathrm{~d}, J=7.8 \mathrm{~Hz}, 2 \mathrm{H}), 6.00(\mathrm{ddd}, J=17.3,10.0,7.5 \mathrm{~Hz}, 1 \mathrm{H}), 5.46(\mathrm{~d}, J$ $=17.0 \mathrm{~Hz}, 1 \mathrm{H}), 5.34(\mathrm{~d}, J=10.3 \mathrm{~Hz}, 1 \mathrm{H}), 5.07-4.97(\mathrm{~m}, 1 \mathrm{H}), 4.00(\mathrm{~s}, 3 \mathrm{H}), 3.10(\mathrm{dd}, J$ $=13.1,6.4 \mathrm{~Hz}, 1 \mathrm{H}), 2.87(\mathrm{dd}, J=13.0,9.3 \mathrm{~Hz}, 1 \mathrm{H}), 2.37$ (s, 3H). ${ }^{13} \mathrm{C} \mathrm{NMR}(101 \mathrm{MHz}$, $\mathrm{CDCl}_{3}$ ) major isomer: $\delta 164.3,140.7,134.2,132.1,129.3,120.1,116.8,113.0,112.5$, 92.5, 84.4, 79.6, 78.5, 54.4, 43.8, 42.5, 29.7, 21.6. minor isomer: $\delta$ 164.6, 140.6, 134.8, 132.0, 129.3, 112.0, 116.9, 112.4, 112.1, 92.1, 84.1, 81.2, 79.9, 54.4, 44.7, 42.7, 29.7, 21.6. IR (film): 2226, 1772, 1747, 1510, 1436, 1282, 1258, 1093, 1035, 986, 939, 817 , 793, 533. Major isomer: MS (EI) (for dr) m/z (rel): $320\left(\mathrm{M}^{+}, 9\right), 305$ (12), 261 (15), 242 (11), 218 (11), 175 (40), 143 (100), 57 (16), 55 (9); HRMS (EI) m/z Calcd for $\mathrm{C}_{19} \mathrm{H}_{16} \mathrm{~N}_{2} \mathrm{O}_{3} \mathrm{M}^{+}$: 320.1161, found 320.1171. Minor isomer: MS (EI) (for $\mathrm{dr}$ ) m/z (rel): $320\left(\mathrm{M}^{+}, 1\right), 261$ (12), 242 (37), 227 (21), 175 (29), 144 (14), 143 (100), 115 (11), 91

(9); HRMS (EI) m/z Calcd for $\mathrm{C}_{19} \mathrm{H}_{16} \mathrm{~N}_{2} \mathrm{O}_{3} \mathrm{M}^{+}$: 320.1161, found 320.1155.

\section{General experimental procedure for 1,2-dione 5 and the characterization ${ }^{5}$}

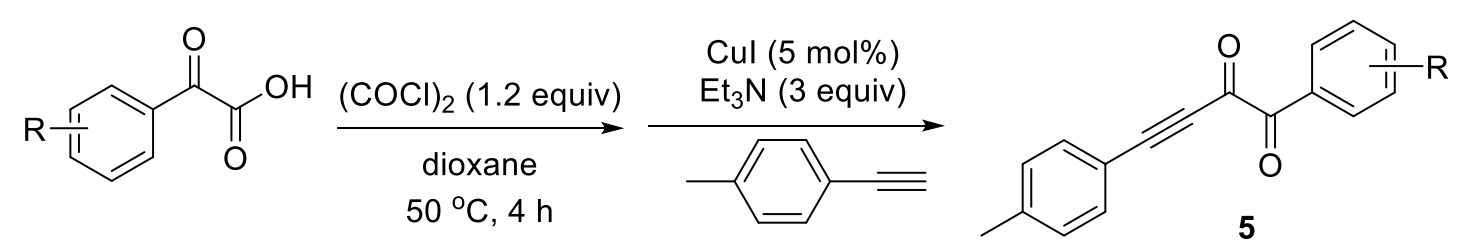

To a flame dried Schlenk flask were added aryl formic acid (20 mmol), 1,4dioxane $(100 \mathrm{~mL})$, and oxalyl chloride $(2.03 \mathrm{~mL}, 24 \mathrm{mmol})$. The resulting mixture was allowed to stir for 4 hours at $50{ }^{\circ} \mathrm{C}$. Then the mixture was cooled to room temperature. CuI (190 mg, 1 mmol, 5 mol\%), 1-ethynyl-4-methylbenzene (2.54 mL, 20.0 mmol, 1 equiv), and dry triethylamine ( $8.4 \mathrm{~mL}, 60 \mathrm{mmol}, 3$ equiv) were successively added to the mixture, and the resulting reaction mixture was stirred at room temperature for overnight. After complete conversion, water $(50 \mathrm{~mL})$ was added and the mixture was extracted with $\mathrm{CH}_{2} \mathrm{Cl}_{2}(3 \times 15 \mathrm{~mL})$. Drying of the organic phase with anhydrous $\mathrm{Na}_{2} \mathrm{SO}_{4}$, and the volatiles were removed under reduced pressure. Then the resulting residue was purified by flash chromatography with petroleum ether and ethyl acetate as 
eluent to give product $\mathbf{5}$.

1,4-di-p-tolylbut-3-yne-1,2-dione (5b)

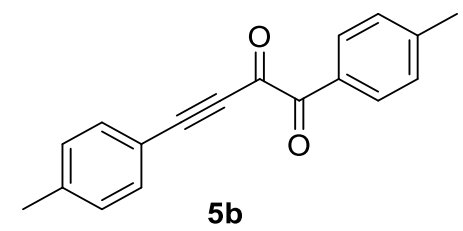

The compound $\mathbf{5 b}$ was obtained according to General method starting from 2-oxo-2( $p$-tolyl)acetic acid $(820 \mathrm{mg}, 5 \mathrm{mmol})$. The crude product was purified by flash chromatography on silical gel (petroleum ether/diethyl ether $=10 / 1$ ) to give a yellow solid (423 mg, yield: 32\%). mp: 53.6-54.7 ${ }^{\circ} \mathrm{C} .{ }^{1} \mathrm{H} \mathrm{NMR}\left(400 \mathrm{MHz}, \mathrm{CDCl}_{3}\right) \delta 7.96(\mathrm{~d}$, $J=8.2 \mathrm{~Hz}, 2 \mathrm{H}), 7.52(\mathrm{~d}, J=8.1 \mathrm{~Hz}, 2 \mathrm{H}), 7.30(\mathrm{~d}, J=8.1 \mathrm{~Hz}, 2 \mathrm{H}), 7.19(\mathrm{~d}, J=8.4 \mathrm{~Hz}$, 2H), 2.43 (s, 3H), 2.38 (s, 3H). ${ }^{13} \mathrm{C}$ NMR (101 MHz, $\left.\mathrm{CDCl}_{3}\right) \delta 188.4,178.9,146.2$, 142.6, 133.7, 130.6, 129.6, 129.5, 129.1, 116.1, 99.8, 87.2, 21.9, 21.8. IR (film): 2182, 1671, 1639, 1602, 1257, 1212, 1180, 1107, 928, 837, 817, 739, 708, 612, 536, 477. GCMS (EI) $m / z$ (rel): $262\left(\mathrm{M}^{+}\right), 234,206,143,119$ (100), 91, 65, 51, 39. HRMS (EI) Calcd. For $\mathrm{C}_{18} \mathrm{H}_{14} \mathrm{O}_{2}\left(\mathrm{M}^{+}\right)$: 262.0994; found: 262.0995 .

1-(4-fluorophenyl)-4-(p-tolyl)but-3-yne-1,2-dione (5c)

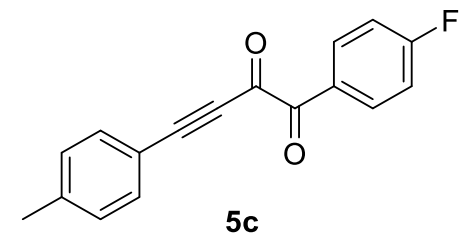

The compound $\mathbf{5 c}$ was obtained according to General method III starting from 2-(4fluorophenyl)-2-oxoacetic acid (948 $\mathrm{mg}, 5.6 \mathrm{mmol})$. The crude product was purified by flash chromatography on silical gel (petroleum ether/diethyl ether $=10 / 1$ ) to give a yellow solid (843 mg, yield: 56\%). mp: 85.7-85.8 ${ }^{\circ} \mathrm{C} .{ }^{1} \mathrm{H}$ NMR (400 $\left.\mathrm{MHz}, \mathrm{CDCl}_{3}\right) \delta$ 8.17-8.07 (m, 2H), $7.53(\mathrm{~d}, J=8.1 \mathrm{~Hz}, 2 \mathrm{H}), 7.23-7.13(\mathrm{~m}, 4 \mathrm{H}), 2.38(\mathrm{~s}, 3 \mathrm{H}) .{ }^{19} \mathrm{~F}$ NMR $\left(376 \mathrm{MHz} \mathrm{CDCl}_{3}\right) \delta-101.29 .{ }^{13} \mathrm{C} \mathrm{NMR}\left(101 \mathrm{MHz}, \mathrm{CDCl}_{3}\right) \delta 186.7,178.0,168.0(\mathrm{~d}, J$ $=261 \mathrm{~Hz}), 142.8,133.8,133.4(\mathrm{~d}, J=9 \mathrm{~Hz}), 129.6,128.2(\mathrm{~d}, J=3 \mathrm{~Hz}), 116.4(\mathrm{~d}, J=$ $22 \mathrm{~Hz}), 116.0,100.2,87.1,21.9$. IR (film): 2189, 1672, 1650, 1593, 1502, 1410, 1255 , 
1231, 1222, 1163, 1103, 931, 811, 776, 741, 708, 667, 612, 515. GCMS (EI) $m / z$ (rel): $266\left(\mathrm{M}^{+}\right), 238,210,143$ (100), 123, 95, 75, 63, 51, 39; HRMS (EI) Calcd. For $\mathrm{C}_{17} \mathrm{H}_{11} \mathrm{FO}_{2}\left(\mathrm{M}^{+}\right)$: 266.0743; found: 266.0736.

1-(4-bromophenyl)-4-(p-tolyl)but-3-yne-1,2-dione (5d)<smiles>CCCCCCCCCC#Cc1ccc(C)cc1</smiles>

The compound 5d was obtained according to General method III starting from 2-(4bromophenyl)-2-oxoacetic acid (916 $\mathrm{mg}, 4 \mathrm{mmol})$. The crude product was purified by flash chromatography on silical gel (petroleum ether/diethyl ether $=10 / 1$ ) to give a yellow solid (541 mg, yield: $42 \%$ ). mp: 98.5-98.8 ${ }^{\circ} \mathrm{C} .{ }^{1} \mathrm{H}$ NMR (400 $\left.\mathrm{MHz}, \mathrm{CDCl}_{3}\right) \delta$ $7.94(\mathrm{~d}, J=8.7 \mathrm{~Hz}, 2 \mathrm{H}), 7.65(\mathrm{~d}, J=8.8 \mathrm{~Hz}, 2 \mathrm{H}), 7.54(\mathrm{~d}, J=8.1 \mathrm{~Hz}, 2 \mathrm{H}), 7.20(\mathrm{~d}, J=$ $7.9 \mathrm{~Hz}, 2 \mathrm{H}), 2.39$ (s, 3H). ${ }^{13} \mathrm{C}$ NMR $\left(101 \mathrm{MHz}, \mathrm{CDCl}_{3}\right) \delta 187.2,177.7,142.8,133.8$, 132.3, 131.9, 130.5, 129.6, 115.9, 100.5, 87.1, 21.9. IR (film): 2176, 1678, 1639, 1601, 1581, 1106, 1067, 926, 844, 815, 753, 722, 705, 685, 572, 472. GCMS (EI) $m / z$ (rel): $326\left(\mathrm{M}^{+}\right), 298,272,183,155,143$ (100), 115, 89, 75, 63, 50. HRMS (EI) Calcd. For $\mathrm{C}_{17} \mathrm{H}_{11} \mathrm{BrO}_{2}\left(\mathrm{M}^{+}\right)$: 325.9942; found: 325.9941 .

1-(4-nitrophenyl)-4-(p-tolyl)but-3-yne-1,2-dione (5e)

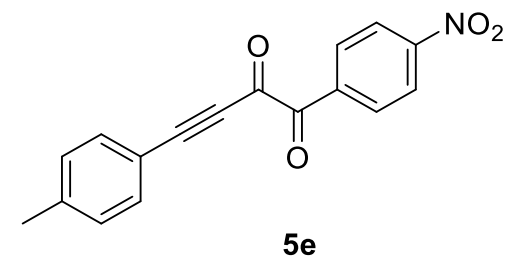

The compound 5e was obtained according to General method III starting from 2-(4nitrophenyl)-2-oxoacetic acid ( $824 \mathrm{mg}, 4.2 \mathrm{mmol}$ ). The crude product was purified by flash chromatography on silical gel (petroleum ether/diethyl ether $=10 / 1$ ) to give a yellow solid (367 mg, yield: 30\%). mp: 147.2-147.4 ${ }^{\circ} \mathrm{C} .{ }^{1} \mathrm{H}$ NMR (400 MHz, $\left.\mathrm{CDCl}_{3}\right) \delta$ 8.35-8.32 (m, 2H), 8.27-8.25 (m, 2H), $7.56(\mathrm{~d}, J=8.1 \mathrm{~Hz}, 2 \mathrm{H}), 7.22(\mathrm{~d}, J=8.1 \mathrm{~Hz}, 2 \mathrm{H})$, $2.40(\mathrm{~s}, 3 \mathrm{H}) .{ }^{13} \mathrm{C} \mathrm{NMR}\left(101 \mathrm{MHz}, \mathrm{CDCl}_{3}\right) \delta 186.1,176.3,143.2,136.5,133.9,131.6$, 
129.7, 123.8, 115.7, 101.5, 87.0, 21.9. IR (film): 2188, 2151, 1747, 1670, 1652, 1471, 1432, 1270, 1242, 1134, 1098, 1053, 1034, 783, 756, 490. MS (DART) m/z: 294.1 (M $+\mathrm{H})^{+}$; HRMS (DART) $m / z$ Calcd for $\mathrm{C}_{17} \mathrm{H}_{12} \mathrm{NO}_{4}(\mathrm{M}+\mathrm{H})^{+}:$294.0761, found 294.0760.

1-(2-bromophenyl)-4-(p-tolyl)but-3-yne-1,2-dione (5f)

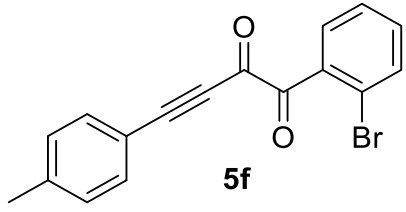

The compound $\mathbf{5 f}$ was obtained according to General method III starting from 2-(2bromophenyl)-2-oxoacetic acid (1.8 g, $8 \mathrm{mmol})$. The crude product was purified by flash chromatography on silical gel (petroleum ether/diethyl ether $=10 / 1$ ) to give a yellow solid (1.0 g, yield: 39\%). mp: 64.8-64.9 ${ }^{\circ} \mathrm{C} .{ }^{1} \mathrm{H}$ NMR $\left(400 \mathrm{MHz}, \mathrm{CDCl}_{3}\right) \delta$ 7.68$7.61(\mathrm{~m}, 2 \mathrm{H}), 7.57(\mathrm{~d}, J=8.0 \mathrm{~Hz}, 2 \mathrm{H}), 7.50-7.40(\mathrm{~m}, 2 \mathrm{H}), 7.23(\mathrm{~d}, J=8.0 \mathrm{~Hz}, 2 \mathrm{H}), 2.41$ (s, 3H). ${ }^{13} \mathrm{C} \mathrm{NMR}\left(101 \mathrm{MHz}, \mathrm{CDCl}_{3}\right) \delta 189.9,175.7,142.7,135.7,133.8,133.7,133.4$, 131.7, 129.6, 127.6, 121.2, 116.1, 100.4, 86.7, 21.9. IR (film): 2922, 2238, 2176, 1914, $1675,1641,1600,1584,1266,1229,1111,1045,1028,955,926,837,815,740,718$, 695, 674, 638, 571, 538. MS (EI) m/z (rel): 326 ( $\left.\mathrm{M}^{+}, 0.1\right), 298$ (4), 270 (8), 183 (18), 155 (9), 143 (100), 115 (8), 89 (11), 75 (10). HRMS (EI) $m / z$ Calcd for $\mathrm{C}_{17} \mathrm{H}_{11} \mathrm{BrO}_{2} \mathrm{M}^{+}$: 325.9942 , found 325.9933 .

7 General experimental procedure for Scheme 3 and the characterization of products

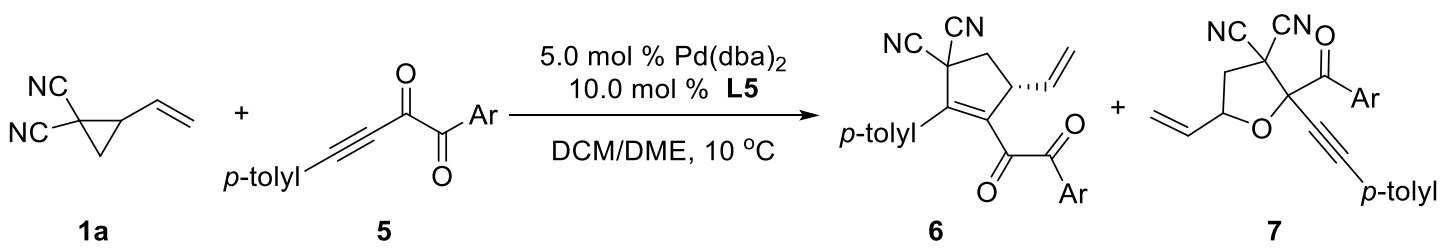

To a flame dried sealing tube were added $\mathrm{Pd}(\mathrm{dba})_{2}(5.75 \mathrm{mg}, 0.01 \mathrm{mmol}),(R)$ SEGPHOS (L5) (12.2 mg, $0.02 \mathrm{mmol})$, freshly distilled anhydrous dichloromethane $(1.2 \mathrm{~mL})$ and anhydrous 1,2-dimethoxyethane $(0.8 \mathrm{~mL})$. The resulting mixture was 
allowed to stir for 30 mins. The 3-yne-1,2-dione 5 (0.2 $\mathrm{mmol})$ and vinyl cyclopropane 1a $(59.1 \mathrm{mg}, 0.5 \mathrm{mmol})$ were added subsequently. The resulting reaction mixture was stirred at $10{ }^{\circ} \mathrm{C}$ for $16 \mathrm{~h}$. After the volatiles were removed under reduced pressure, the ratio of two compounds 6 and 7 was determined by crude ${ }^{1} \mathrm{H}$ NMR. Then the resulting residue was purified by preparative TLC with petroleum ether and ethyl acetate as eluent to give products $\mathbf{6}$.

(R)-3-(2-oxo-2-phenylacetyl)-2-(p-tolyl)-4-vinylcyclopent-2-ene-1,1-dicarbonitrile

$(6 \mathbf{a})$<smiles>C=C[C@H]1CC(C#N)(C#N)C(c2ccc(C)cc2)=C1C(=O)C(=O)c1ccccc1</smiles>

Yellow solid, $54.2 \mathrm{mg}$, yield: 74\%, ee: $85 \%$. HPLC (Chiralpak IC, $4.6 \mathrm{~mm} \times 250 \mathrm{~mm}$, Hexane: $i$-Propanol $=90 / 10,0.7 \mathrm{~mL} / \mathrm{min}, 214 \mathrm{~nm}$ ): $\mathrm{t}_{\text {minor }}=13.14 \mathrm{~min}, \mathrm{t}_{\text {major }}=14.67 \mathrm{~min}$; $[\alpha]_{\mathrm{D}}^{30}=-33.4\left(c 2.32, \mathrm{CHCl}_{3}\right)$; mp: 87.1-88.6 ${ }^{\circ} \mathrm{C} .{ }^{1} \mathrm{H}$ NMR $\left(400 \mathrm{MHz}, \mathrm{CDCl}_{3}\right) \delta 7.61-$ $7.50(\mathrm{~m}, 3 \mathrm{H}), 7.36-7.29(\mathrm{~m}, 2 \mathrm{H}), 7.11(\mathrm{~d}, J=7.6 \mathrm{~Hz}, 2 \mathrm{H}), 6.88(\mathrm{~d}, J=7.5 \mathrm{~Hz}, 2 \mathrm{H})$, 6.06-5.92 (m, 1H), 5.39 (d, $J=17.0 \mathrm{~Hz}, 1 \mathrm{H}), 5.26(\mathrm{~d}, J=10.1 \mathrm{~Hz}, 1 \mathrm{H}), 4.30-4.18(\mathrm{~m}$, $1 \mathrm{H}), 3.14(\mathrm{dd}, J=13.6,8.3 \mathrm{~Hz}, 1 \mathrm{H}), 2.78(\mathrm{dd}, J=13.7,4.6 \mathrm{~Hz}, 1 \mathrm{H}), 2.17(\mathrm{~s}, 3 \mathrm{H}) .{ }^{13} \mathrm{C}$ NMR $\left(101 \mathrm{MHz}, \mathrm{CDCl}_{3}\right) \delta 191.2,190.7,146.1,144.9,141.1,134.6,134.5,131.9,129.5$, 129.3 (2C), 128.3, 126.1, 119.1, 113.9, 113.6, 48.4, 44.4, 42.3, 21.2. IR (film): 1667, 1198, 1179, 937, 924, 817, 758, 730, 704, 684, 667, 615, 501. MS (DART) $m / z: 367.1$ $(\mathrm{M}+\mathrm{H})^{+} ;$HRMS (DART) $m / z$ Calcd for $\mathrm{C}_{24} \mathrm{H}_{19} \mathrm{~N}_{2} \mathrm{O}_{2}(\mathrm{M}+\mathrm{H})^{+}:$367.1441, found 367.1444 .

(R)-3-(2-oxo-2-( $p$-tolyl)acetyl)-2-(p-tolyl)-4-vinylcyclopent-2-ene-1,1-dicarbonitrile $(6 \mathbf{b})$ 


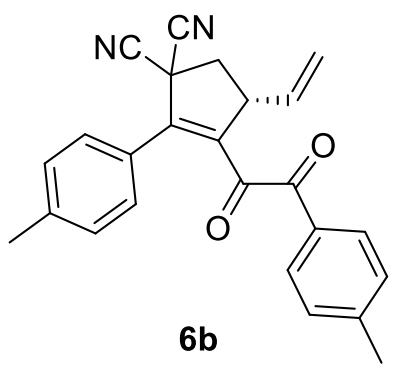

Yellow solid, $66.7 \mathrm{mg}$, yield: $88 \%$, ee: $81 \%$. HPLC (Chiralpak IB, $4.6 \mathrm{~mm} \times 250 \mathrm{~mm}$, Hexane: $i$-Propanol $=99 / 1,0.7 \mathrm{~mL} / \mathrm{min}, 214 \mathrm{~nm}$ ): $\mathrm{t}_{\mathrm{minor}}=28.07 \mathrm{~min}, \mathrm{t}_{\mathrm{major}}=35.37 \mathrm{~min}$; $[\alpha]_{\mathrm{D}}^{32}=-53.0\left(c\right.$ 2.70, $\left.\mathrm{CHCl}_{3}\right) ; \mathrm{mp}: 102.2-103.9^{\circ} \mathrm{C} .{ }^{1} \mathrm{H} \mathrm{NMR}\left(400 \mathrm{MHz}, \mathrm{CDCl}_{3}\right) \delta 7.47$ $(\mathrm{d}, J=8.2 \mathrm{~Hz}, 2 \mathrm{H}), 7.15-7.08(\mathrm{~m}, 4 \mathrm{H}), 6.89(\mathrm{~d}, J=8.1 \mathrm{~Hz}, 2 \mathrm{H}), 5.96(\mathrm{ddd}, J=17.5$, $10.1,7.8 \mathrm{~Hz}, 1 \mathrm{H}), 5.36(\mathrm{~d}, J=17.1 \mathrm{~Hz}, 1 \mathrm{H}), 5.24(\mathrm{~d}, J=10.4 \mathrm{~Hz}, 1 \mathrm{H}), 4.26-4.18(\mathrm{~m}$, 1H), $3.12(\mathrm{dd}, J=13.7,8.2 \mathrm{~Hz}, 1 \mathrm{H}), 2.76(\mathrm{dd}, J=13.7,5.0 \mathrm{~Hz}, 1 \mathrm{H}), 2.38(\mathrm{~s}, 3 \mathrm{H}), 2.18$ (s, 3H). ${ }^{13} \mathrm{C} \mathrm{NMR}\left(101 \mathrm{MHz}, \mathrm{CDCl}_{3}\right) \delta 191.3,190.2$, 145.8, 145.7, 144.9, 141.0, 134.7, $129.7129 .5,129.3,129.2,129.1,126.2,119.1,113.9,113.7,48.5,44.4,42.3,21.9,21.2$. IR (film): 1671, 1604, 1262, 1202, 1176, 923, 752, 736, 726, 604, 507, 483. MS (DART) $m / z: 381.2(\mathrm{M}+\mathrm{H})^{+} ;$HRMS (DART) $m / z$ Calcd for $\mathrm{C}_{25} \mathrm{H}_{21} \mathrm{~N}_{2} \mathrm{O}_{2}(\mathrm{M}+\mathrm{H})^{+}:$381.1598, found 381.1600 .

(R)-3-(2-(4-fluorophenyl)-2-oxoacetyl)-2-(p-tolyl)-4-vinylcyclopent-2-ene-1,1dicarbonitrile (6c)<smiles>C=CC1CC(C#N)(C#N)C(c2ccc(C)cc2)=C1C(=O)C(=O)c1ccc(F)cc1</smiles>

Yellow solid, $51.7 \mathrm{mg}$, yield: $67 \%$, ee: $83 \%$. HPLC (Chiralpak IB, $4.6 \mathrm{~mm} \times 250 \mathrm{~mm}$, Hexane: $i$-Propanol $=90 / 10,0.7 \mathrm{~mL} / \mathrm{min}, 214 \mathrm{~nm}$ ): $\mathrm{t}_{\text {minor }}=9.14 \mathrm{~min}, \mathrm{t}_{\text {major }}=10.89 \mathrm{~min}$; $[\alpha]_{\mathrm{D}}^{30}=-32.4\left(c\right.$ 1.96, $\left.\mathrm{CHCl}_{3}\right) ; \mathrm{mp}: 121.6-121.9{ }^{\circ} \mathrm{C} .{ }^{1} \mathrm{H}$ NMR $\left(400 \mathrm{MHz}, \mathrm{CDCl}_{3}\right) \delta$ 7.67-7.62 (m, 2H), $7.12(\mathrm{~d}, J=8.0 \mathrm{~Hz}, 2 \mathrm{H}), 7.06-7.00(\mathrm{~m}, 2 \mathrm{H}), 6.93(\mathrm{~d}, J=7.9 \mathrm{~Hz}, 2 \mathrm{H})$, $5.97(\mathrm{ddd}, J=17.6,10.0,7.6 \mathrm{~Hz}, 1 \mathrm{H}), 5.39(\mathrm{~d}, J=17.1 \mathrm{~Hz}, 1 \mathrm{H}), 5.27(\mathrm{~d}, J=10.2 \mathrm{~Hz}$ 1H), 4.28-4.21 (m, 1H), $3.16(\mathrm{dd}, J=13.7,8.2 \mathrm{~Hz}, 1 \mathrm{H}), 2.79(\mathrm{dd}, J=13.7,5.1 \mathrm{~Hz}, 1 \mathrm{H})$, 
$2.21(\mathrm{~s}, 3 \mathrm{H}) .{ }^{19} \mathrm{~F}$ NMR $\left(376 \mathrm{MHz}, \mathrm{CDCl}_{3}\right) \delta-101.39 .{ }^{13} \mathrm{C} \mathrm{NMR}\left(101 \mathrm{MHz}, \mathrm{CDCl}_{3}\right) \delta$ 190.9, 188.9, 167.8 (d, $J=259 \mathrm{~Hz}), 146.0,144.9,141.3,134.6,132.4(\mathrm{~d}, J=10 \mathrm{~Hz})$, 129.4, 129.2, 128.4 (d, $J=3$ Hz), 126.1, 119.2, 115.8, 115.6, 113.8, 48.5, 44.3, 42.4, 21.2. IR (film): 1669, 1595, 1506, 1238, 1197, 1152, 929, 877, 853, 809, 743, 602, 504. MS (DART) $m / z: 385.1(\mathrm{M}+\mathrm{H})^{+}$; HRMS (DART) $m / z$ Calcd for $\mathrm{C}_{24} \mathrm{H}_{18} \mathrm{FN}_{2} \mathrm{O}_{2}(\mathrm{M}+$ $\mathrm{H})^{+}: 385.1347$, found 385.1349 .

(R)-3-(2-(4-bromophenyl)-2-oxoacetyl)-2-( $p$-tolyl)-4-vinylcyclopent-2-ene-1,1dicarbonitrile $(\mathbf{6 d})$<smiles>C=C[C@H]1CC(C#N)(C#N)C(c2ccc(C)cc2)=C1C(=O)C(=O)c1ccc(Br)cc1</smiles>

Yellow solid, $66.8 \mathrm{mg}$, yield: 75\%, ee: 70\%. HPLC (Chiralpak IB, $4.6 \mathrm{~mm} \times 250 \mathrm{~mm}$, Hexane: $i$-Propanol = 90/10, $0.7 \mathrm{~mL} / \mathrm{min}, 214 \mathrm{~nm}$ ): $\mathrm{t}_{\text {minor }}=9.40 \mathrm{~min}, \mathrm{t}_{\text {major }}=11.96 \mathrm{~min}$; $[\alpha]_{\mathrm{D}}^{31}=-49.7\left(c\right.$ 2.64, $\left.\mathrm{CHCl}_{3}\right) ; \mathrm{mp}: 103.8-104.5^{\circ} \mathrm{C} .{ }^{1} \mathrm{H} \mathrm{NMR}\left(400 \mathrm{MHz}, \mathrm{CDCl}_{3}\right) \delta 7.51$ $(\mathrm{d}, J=8.5 \mathrm{~Hz}, 2 \mathrm{H}), 7.46(\mathrm{~d}, J=8.6 \mathrm{~Hz}, 2 \mathrm{H}), 7.12(\mathrm{~d}, J=7.9 \mathrm{~Hz}, 2 \mathrm{H}), 6.94(\mathrm{~d}, J=7.8$ $\mathrm{Hz}, 2 \mathrm{H}), 5.97$ (ddd, $J=17.6,10.0,8.0 \mathrm{~Hz}, 1 \mathrm{H}), 5.38(\mathrm{~d}, J=17.0 \mathrm{~Hz}, 1 \mathrm{H}), 5.27$ (d, $J=$ $10.2 \mathrm{~Hz}, 1 \mathrm{H}), 4.27-4.19(\mathrm{~m}, 1 \mathrm{H}), 3.16(\mathrm{dd}, J=13.7,8.2 \mathrm{~Hz}, 1 \mathrm{H}), 2.78$ (dd, $J=13.7,5.1$ $\mathrm{Hz}, 1 \mathrm{H}), 2.22(\mathrm{~s}, 3 \mathrm{H}) .{ }^{13} \mathrm{C} \mathrm{NMR}\left(101 \mathrm{MHz}, \mathrm{CDCl}_{3}\right) \delta 190.8,189.5,146.1,144.9$ 141.4, 134.5, 131.8, 130.8, 130.6, 130.1, 129.4, 129.2, 126.1, 119.3, 113.8, 113.6, 48.5, 44.3, 42.4, 21.2. IR (film): 2948, 2923, 2855, 1727, 1700 1452, 1441, 1268, 1234, 1039, 1000, 948, 803. MS (DART) $m / z: 445.1(\mathrm{M}+\mathrm{H})^{+}$; HRMS (DART) $\mathrm{m} / \mathrm{z}$ Calcd for $\mathrm{C}_{24} \mathrm{H}_{18} \mathrm{BrN}_{2} \mathrm{O}_{2}(\mathrm{M}+\mathrm{H})^{+}:$445.0546, found 445.0549.

(R)-3-(2-(4-nitrophenyl)-2-oxoacetyl)-2-(p-tolyl)-4-vinylcyclopent-2-ene-1,1dicarbonitrile $(\mathbf{6 e})$ 


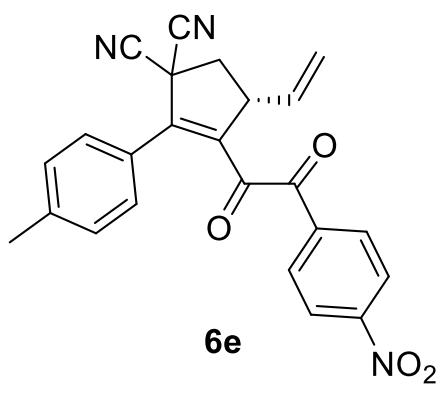

Yellow solid, $37.1 \mathrm{mg}$, yield: 45\%, ee: 78\%. HPLC (Chiralpak IB, $4.6 \mathrm{~mm} \times 250 \mathrm{~mm}$, Hexane: $i$-Propanol $=90 / 10,0.7 \mathrm{~mL} / \mathrm{min}, 214 \mathrm{~nm}$ ): tminor $=22.09 \mathrm{~min}$, tmajor $=29.67$ $\min ;[\alpha]_{\mathrm{D}}{ }^{31}=-54.8\left(c\right.$ 1.00, $\left.\mathrm{CHCl}_{3}\right)$; mp: $120.9-121.3{ }^{\circ} \mathrm{C} .{ }^{1} \mathrm{H}$ NMR $\left(400 \mathrm{MHz}, \mathrm{CDCl}_{3}\right)$ $\delta 8.20(\mathrm{~d}, J=8.7 \mathrm{~Hz}, 2 \mathrm{H}), 7.79(\mathrm{~d}, J=8.7 \mathrm{~Hz}, 2 \mathrm{H}), 7.14(\mathrm{~d}, J=8.0 \mathrm{~Hz}, 2 \mathrm{H}), 6.94(\mathrm{~d}, J$ $=7.8 \mathrm{~Hz}, 2 \mathrm{H}), 5.97(\mathrm{ddd}, J=17.6,10.0,8.0 \mathrm{~Hz}, 1 \mathrm{H}), 5.39(\mathrm{~d}, J=17.0 \mathrm{~Hz}, 1 \mathrm{H}), 5.29$ $(\mathrm{d}, J=10.2 \mathrm{~Hz}, 1 \mathrm{H}), 4.30-4.22(\mathrm{~m}, 1 \mathrm{H}), 3.19(\mathrm{dd}, J=13.8,8.2 \mathrm{~Hz}, 1 \mathrm{H}), 2.81(\mathrm{dd}, J=$ 13.8, 5.2 Hz, 1H), 2.20 (s, 3H). ${ }^{13} \mathrm{C}$ NMR (101 MHz, $\left.\mathrm{CDCl}_{3}\right) \delta 190.2,188.4,150.8$, $146.8,144.5,141.8,136.2,134.4,130.5,129.6,129.2,126.2,123.4,119.5,113.6,113.4$, 48.5, 44.2, 42.5, 21.2. IR (film): 1670, 1526, 1410, 1344, 1198, 937, 926, 815, 730, 718, 504. MS (DART) m/z: $412.1(\mathrm{M}+\mathrm{H})^{+}$; HRMS (DART) $m / z$ Calcd for $\mathrm{C}_{24} \mathrm{H}_{18} \mathrm{~N}_{3} \mathrm{O}_{4}(\mathrm{M}$ $+\mathrm{H})^{+}:$412.1292, found 412.1295 .

(R)-3-(2-(2-bromophenyl)-2-oxoacetyl)-2-(p-tolyl)-4-vinylcyclopent-2-ene-1,1dicarbonitrile (6f)<smiles>C=C[C@H]1CC(C#N)(C#N)C(c2ccc(C)cc2)=C1C(=O)C(=O)c1ccccc1Br</smiles>

Yellow solid, $74.1 \mathrm{mg}$, yield: 83\%, ee: 66\%. HPLC (Chiralpak IB, $4.6 \mathrm{~mm} \times 250 \mathrm{~mm}$, Hexane: $i$-Propanol $=95 / 5,1.5 \mathrm{~mL} / \mathrm{min}, 215 \mathrm{~nm}): \mathrm{t}_{\text {minor }}=7.73 \mathrm{~min}, \mathrm{t}_{\mathrm{major}}=8.64 \mathrm{~min}$; $\left.[\alpha]_{\mathrm{D}}^{31}=-2.6(c) 3.7, \mathrm{CHCl}_{3}\right)$; mp: 81.9-83.2 ${ }^{\circ} \mathrm{C} .{ }^{1} \mathrm{H} \mathrm{NMR}\left(400 \mathrm{MHz}, \mathrm{CDCl}_{3}\right) \delta 7.55(\mathrm{~d}$, $J=7.8 \mathrm{~Hz}, 1 \mathrm{H}), 7.46-7.41(\mathrm{~m}, 1 \mathrm{H}), 7.38-7.21(\mathrm{~m}, 4 \mathrm{H}), 7.04(\mathrm{~d}, J=7.9 \mathrm{~Hz}, 2 \mathrm{H}), 6.01$ (ddd, $J=17.2,10.0,8.0 \mathrm{~Hz}, 1 \mathrm{H}), 5.38(\mathrm{~d}, J=17.0 \mathrm{~Hz}, 1 \mathrm{H}), 5.27(\mathrm{~d}, J=10.2 \mathrm{~Hz}, 1 \mathrm{H})$, 
4.33-4.25 (m, 1H), $3.13(\mathrm{dd}, J=13.7,8.1 \mathrm{~Hz}, 1 \mathrm{H}), 2.78(\mathrm{dd}, J=13.7,5.0 \mathrm{~Hz}, 1 \mathrm{H}), 2.26$ (s, 3H). ${ }^{13} \mathrm{C}$ NMR $\left(101 \mathrm{MHz}, \mathrm{CDCl}_{3}\right) \delta 190.2,189.0,145.7,143.7,141.1,134.8$, 134.4(2C), 132.8, 132.7, 129.5, 129.0, 127.2, 126.3, 122.5, 119.2, 113.8, 113.7, 48.9, 44.8, 42.2, 21.3. IR (film): 1679, 1657, 1584, 1346, 1276, 929, 803, 760, 745, 728, 628, 511, 486. GCMS (EI) m/z (rel): $444\left(\mathrm{M}^{+}\right), 261,234,219,183$ (100), 155, 128, 115, 76; HRMS (EI) Calcd. For $\mathrm{C}_{24} \mathrm{H}_{17} \mathrm{BrN}_{2} \mathrm{O}_{2}\left(\mathrm{M}^{+}\right)$: 444.0473; found: 444.0466.

\section{Palladium-catalyzed $(3+2)$ cycloaddition of vinylcyclopropane 1a and alkyne 2a on $8 \mathrm{mmol}$ scale}

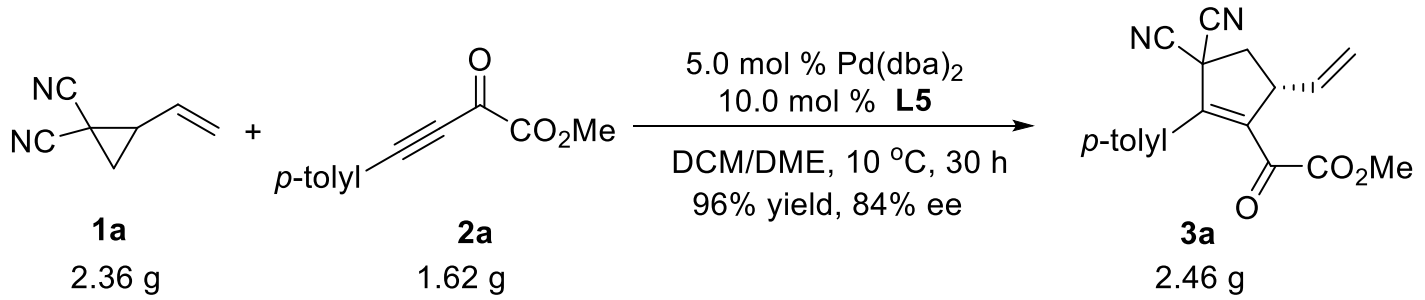

To a flame dried schlenk flask were added $\mathrm{Pd}(\mathrm{dba})_{2}(230 \mathrm{mg}, 0.4 \mathrm{mmol}),(R)-$ SEGPHOS (L5) (488 mg, $0.8 \mathrm{mmol}$ ), freshly distilled anhydrous dichloromethae (48.0 $\mathrm{mL})$ and anhydrous 1,2-dimethoxyethane $(32.0 \mathrm{~mL})$. The resulting mixture was allowed to stir for 30 mins. Alkynyl $\alpha$-keto ester $2 \mathbf{a}(1.62 \mathrm{~g}, 8.0 \mathrm{mmol})$ and vinyl cyclopropane 1a (2.36 g, $0.5 \mathrm{mmol})$ were added subsequently. The resulting reaction mixture was stirred at $10{ }^{\circ} \mathrm{C}$ for $30 \mathrm{~h}$. After the volatiles were removed under reduced pressure, the ratio of compounds $\mathbf{3 a}$ and $\mathbf{4 a}$ was determined by crude ${ }^{1} \mathrm{H}$ NMR. Then the resulting residue was purified by flash chromatography on silica gel with petroleum ether and ethyl acetate as eluent to give product 3a (2.46 g, yield: $96 \%, \mathbf{3 a} / \mathbf{4 a}=100 / 0$, ee: $84 \%$ ).

\section{Experimental procedure for the oxidation of $3 \mathbf{a}^{10}$}

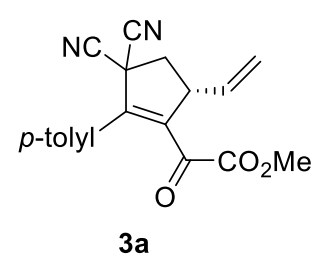

$34 \%$ retrieved

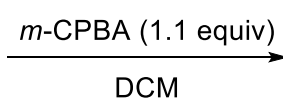

DCM

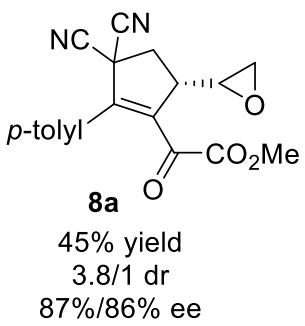


To a solution of $\mathbf{3 a}(160.0 \mathrm{mg}, 0.5 \mathrm{mmol})$ in $\mathrm{CH}_{2} \mathrm{Cl}_{2}(5 \mathrm{~mL})$ was added $m$-CPBA (95 $\mathrm{mg}, 0.55 \mathrm{mmol})$ at ambient temperature and the mixture was stirred overnight in an oil bath of $50{ }^{\circ} \mathrm{C}$. The reaction mixture was quenched with saturated aqueous $\mathrm{Na}_{2} \mathrm{~S}_{2} \mathrm{O}_{3}$ and extracted with $\mathrm{CH}_{2} \mathrm{Cl}_{2}$. The organic layer was washed by saturated aqueous $\mathrm{NaHCO}_{3}\left(20 \mathrm{~mL}\right.$ x 3) and dried over anhydrous $\mathrm{Na}_{2} \mathrm{SO}_{4}$, filtered, and concentrated under vacuo. Then the resulting residue was purified by flash chromatography on silica gel with petroleum ether and ethyl acetate as eluent to give epoxide 8a. White solid, $75.1 \mathrm{mg}$, yield: 45\%, dr: 3.8/1, ee: 87\%/86\%. HPLC: major isomer: (Chiralpak AD-H, $4.6 \mathrm{~mm} \times 250 \mathrm{~mm}$, Hexane: $i$-Propanol $=90 / 10,0.7 \mathrm{~mL} / \mathrm{min}, 254 \mathrm{~nm}): \mathrm{t}_{\text {minor }}=19.62$ min, $\mathrm{t}_{\text {major }}=17.68 \mathrm{~min}$; minor isomer: $\left(\right.$ Chiralpak ID, $4.6 \mathrm{~mm} \times 250 \mathrm{~mm}$, Hexane: $i_{-}$ Propanol $=90 / 10,0.7 \mathrm{~mL} / \mathrm{min}, 214 \mathrm{~nm}): \mathrm{t}_{\text {minor }}=53.14 \mathrm{~min}, \mathrm{t}_{\text {major }}=43.79 \mathrm{~min} ;[\alpha]_{\mathrm{D}}$ major ${ }^{20}=-123.6\left(c\right.$ 2.5, $\left.\mathrm{CHCl}_{3}\right) ;[\alpha]_{\mathrm{D} \text { minor }}{ }^{21}=-49.3\left(c\right.$ 1.2, $\left.\mathrm{CHCl}_{3}\right) ; \mathrm{mp}: 88.5-89.7{ }^{\circ} \mathrm{C} .{ }^{1} \mathrm{H}$ NMR (400 MHz, $\mathrm{CDCl}_{3}$ ) major isomer: $\delta 7.34$ - $7.22(\mathrm{~m}, 4 \mathrm{H}), 3.80$ - $3.73(\mathrm{~m}, 1 \mathrm{H}), 3.38$ (s, 3H), 3.26 - $3.18(\mathrm{~m}, 1 \mathrm{H}), 3.11-3.01(\mathrm{~m}, 1 \mathrm{H}), 2.91-2.81(\mathrm{~m}, J=13.5,3.9 \mathrm{~Hz}, 2 \mathrm{H})$, 2.77 - $2.69(\mathrm{~m}, 1 \mathrm{H}), 2.39(\mathrm{~s}, 3 \mathrm{H})$; minor isomer: $\delta 7.34$ - $7.21(\mathrm{~m}, 4 \mathrm{H}), 3.79$ - $3.65(\mathrm{~m}$, 1H), $3.39(\mathrm{~s}, 3 \mathrm{H}), 3.34$ - $3.22(\mathrm{~m}, 1 \mathrm{H}), 3.14-2.98(\mathrm{~m}, 1 \mathrm{H}), 2.91$ - $2.74(\mathrm{~m}, 2 \mathrm{H}), 2.68$ $2.58(\mathrm{~m}, 1 \mathrm{H}), 2.39(\mathrm{~s}, 3 \mathrm{H}) .{ }^{13} \mathrm{C} \mathrm{NMR}\left(101 \mathrm{MHz}, \mathrm{CDCl}_{3}\right)$ major isomer: $\delta 183.7,161.0$, 147.6, 142.0, 139.9, 129.9, 128.6, 126.5, 114.0, 113.5, 52.8, 51.8, 47.7, 46.0, 43.9, 38.9, 21.5; minor isomer: $\delta 184.3,161.3,146.8,141.9,140.9,129.8,128.7,126.3,113.9$, 113.7, 52.9, 51.9, 47.0, 46.3, 44.2, 38.5, 21.5. IR (film): 1747, 1698, 1342, 1311, 1242 , 1199, 1044, 866, 842, 790, 769, 497, 457. MS (EI) (for dr) $m / z$ (rel): $336\left(\mathrm{M}^{+}, 8\right), 278$ (20), 277 (84), 247 (36), 236 (18), 235 (100), 220 (41), 192 (15), 180 (17); HRMS (EI) $m / z$ Calcd for $\mathrm{C}_{19} \mathrm{H}_{16} \mathrm{~N}_{2} \mathrm{O}_{4} \mathrm{M}^{+}: 336.1110$, found 336.1119 .

\section{Experimental procedure for the reduction of $3 a^{11}$}
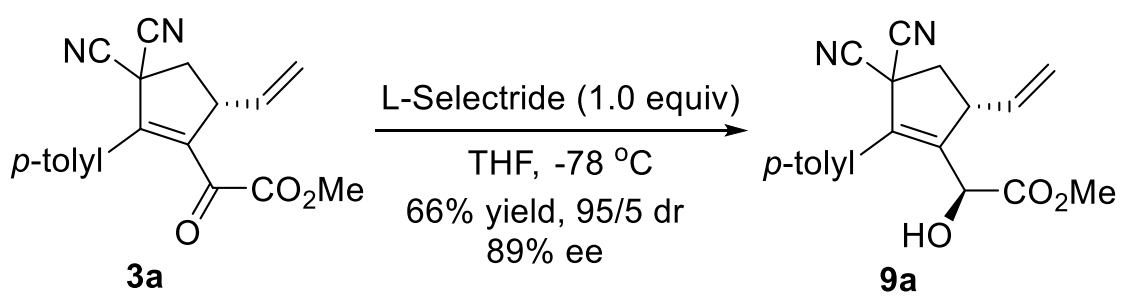

3a (80.0 mg, $0.25 \mathrm{mmol})$ was dissolved in THF $(2.5 \mathrm{~mL})$ and cooled to $-78^{\circ} \mathrm{C}$. L- 
Selectride (1.0 M in THF, $0.3 \mathrm{~mL}, 0.25 \mathrm{mmol}$ ) was added and the solution was stirred for $3 \mathrm{~h}$. Water $(5 \mathrm{~mL})$ was added and the layers were separated. The aqueous layer was extracted with DCM (3 x $5 \mathrm{~mL})$. The combined organic layers were washed with saturated brine $(5 \mathrm{~mL})$, dried over $\mathrm{Na}_{2} \mathrm{SO}_{4}$, filtered, and concentrated under vacuo. Then the resulting residue was purified by preparative TLC with petroleum ether and ethyl acetate as eluent to give product 9a. White solid, $52.7 \mathrm{mg}$, yield: $66 \%$, dr: $95 / 5$, ee: $89 \%$. HPLC: (Chiralpak ID, $4.6 \mathrm{~mm} \times 250 \mathrm{~mm}$, Hexane: $i$-Propanol = 90/10, $0.7 \mathrm{~mL} / \mathrm{min}, 214$ $\mathrm{nm}): \mathrm{t}_{\mathrm{minor}}=33.22 \mathrm{~min}, \mathrm{t}_{\mathrm{major}}=16.34 \mathrm{~min} ;[\alpha]_{\mathrm{D}}{ }^{26}=68.5$ (c 2.1, $\left.\mathrm{CHCl}_{3}\right) ; \mathrm{mp}: 147.9$ $148.5{ }^{\circ} \mathrm{C} .{ }^{1} \mathrm{H}$ NMR $\left(400 \mathrm{MHz}, \mathrm{CDCl}_{3}\right) \delta 7.39(\mathrm{~d}, J=8.0 \mathrm{~Hz}, 2 \mathrm{H}), 7.27(\mathrm{~d}, J=7.7 \mathrm{~Hz}$, 2H), $5.65(\mathrm{dt}, J=17.1,9.6 \mathrm{~Hz}, 1 \mathrm{H}), 5.26(\mathrm{~d}, J=16.9 \mathrm{~Hz}, 1 \mathrm{H}), 5.16(\mathrm{~d}, J=10.0 \mathrm{~Hz}$, $1 \mathrm{H}), 4.75(\mathrm{~d}, J=1.9 \mathrm{~Hz}, 1 \mathrm{H}), 4.01-3.92(\mathrm{~m}, 1 \mathrm{H}), 3.77(\mathrm{~s}, 3 \mathrm{H}), 3.24(\mathrm{~d}, J=2.9 \mathrm{~Hz}, 1 \mathrm{H})$, $3.04(\mathrm{dd}, J=13.6,7.9 \mathrm{~Hz}, 1 \mathrm{H}), 2.62(\mathrm{dd}, J=13.6,5.8 \mathrm{~Hz}, 1 \mathrm{H}), 2.39(\mathrm{~s}, 3 \mathrm{H}) .{ }^{13} \mathrm{C} \mathrm{NMR}$ $\left(101 \mathrm{MHz}, \mathrm{CHCl}_{3}\right) \delta 172.6,144.8,140.0,136.1,134.9,129.7,128.8,127.4,119.6$, 115.1, 114.7, 66.9, 53.2, 48.2, 43.3, 42.8, 21.4. IR (film): 2953, 2922, 1738, 1442, 1261 , 1233, 1086, 946, 935, 819, 711, 547, 503. MS (DART) $m / z: 340.2\left(\mathrm{M}+\mathrm{NH}_{4}\right)^{+} ; \mathrm{HRMS}$ (DART) $m / z$ Calcd for $\mathrm{C}_{19} \mathrm{H}_{22} \mathrm{~N}_{3} \mathrm{O}_{3}\left(\mathrm{M}+\mathrm{NH}_{4}\right)^{+}:$340.1656, found 340.1653.

\section{Experimental procedure for the reduction of $9 \mathrm{a}^{12}$}<smiles>C=C[C@H]1CC2(C#N)C[C@@H]1C([C@H](O)C(=O)OC)=C2C(=O)O[Na]</smiles>

9a

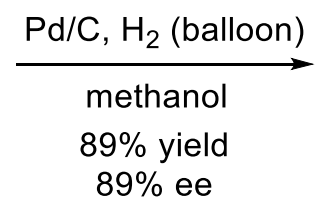

$89 \%$ ee

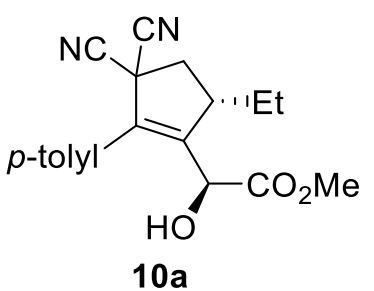

$10 a$

A solution of the $9 \mathrm{a}(20.0 \mathrm{mg}, 0.062 \mathrm{mmol})$ in $1.0 \mathrm{~mL}$ of methanol was added $1.0 \mathrm{mg}$ of $10 \%$ palladium on carbon. The resulting mixture was allowed to stir under $\mathrm{H}_{2}$ balloon at room temperature for 24 hours. The catalyst was filtered off. The combined organic layers were concentrated under vacuo. Then the resulting residue was purified by preparative TLC with petroleum ether and ethyl acetate as eluent to give product 10a. White solid, $17.9 \mathrm{mg}$, yield: $89 \%$, ee: $89 \%$. HPLC: (Chiralpak ID, $4.6 \mathrm{~mm} \times 250$ $\mathrm{mm}$, Hexane: $i$-Propanol $=90 / 10,0.7 \mathrm{~mL} / \mathrm{min}, 214 \mathrm{~nm}$ ): $\mathrm{t}_{\text {minor }}=22.50 \mathrm{~min}, \mathrm{t}_{\text {major }}=15.35$ 
$\min ;[\alpha]_{\mathrm{D}}{ }^{26}=179.4\left(\mathrm{c} 0.89, \mathrm{CHCl}_{3}\right)$; mp: $155.1-156.1{ }^{\circ} \mathrm{C} .{ }^{1} \mathrm{H}$ NMR $\left(400 \mathrm{MHz}, \mathrm{CDCl}_{3}\right)$ $\delta 7.37(\mathrm{~d}, J=7.5 \mathrm{~Hz}, 2 \mathrm{H}), 7.26(\mathrm{~d}, J=7.2 \mathrm{~Hz}, 2 \mathrm{H}), 4.78(\mathrm{~d}, J=2.0 \mathrm{~Hz}, 1 \mathrm{H}), 3.83$ (s, 3H), 3.36-3.26 (m, 1H), $3.17(\mathrm{~d}, J=2.0 \mathrm{~Hz}, 1 \mathrm{H}), 3.01(\mathrm{dd}, J=13.2,7.8 \mathrm{~Hz}, 1 \mathrm{H}), 2.49-$ $2.33(\mathrm{~m}, 4 \mathrm{H}), 1.82-1.68(\mathrm{~m}, 1 \mathrm{H}), 1.28-1.14(\mathrm{~m}, 1 \mathrm{H}), 0.96(\mathrm{t}, J=7.2 \mathrm{~Hz}, 3 \mathrm{H}) .{ }^{13} \mathrm{C} \mathrm{NMR}$ $\left(101 \mathrm{MHz}, \mathrm{CDCl}_{3}\right) \delta 173.4,146.3,139.8,135.3,129.7,128.7,127.8,115.2,114.9,67.2$, 53.5, 45.7, 43.1, 41.8, 24.6, 21.4, 11.7. IR (film): 3514, 2924, 2853, 1736, 1261, 1221 , 1093, 791, 710, 500. MS (EI) (for dr) m/z (rel): $324\left(\mathrm{M}^{+}, 7\right), 279$ (48), 265 (97), 235 (25), 220 (33), 219 (29), 210 (100), 180 (23), 115 (23). HRMS (EI) m/z Calcd for $\mathrm{C}_{19} \mathrm{H}_{20} \mathrm{~N}_{2} \mathrm{O}_{3} \mathrm{M}^{+}:$324.1474, found 324.1479.

\section{Experimental procedure for the bromo-etherification of $9 a^{13}$}

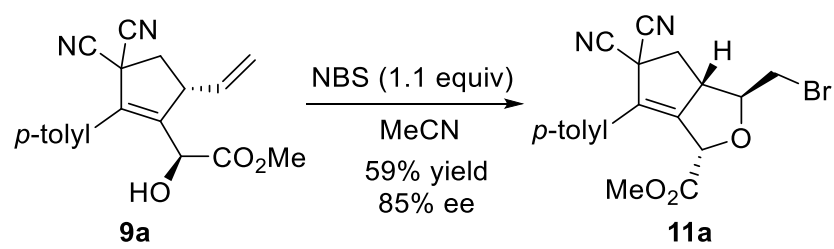

To a solution of $9 \mathbf{a}(32.2 \mathrm{mg}, 0.1 \mathrm{mmol}, 89 \%$ ee $)$ in $\mathrm{MeCN}(1.0 \mathrm{~mL})$ was added NBS (19.6 g, $0.11 \mathrm{mmol})$. The mixture was stirred at room temperature for $4.0 \mathrm{~h}$. The mixture was poured into $10 \%$ aqueous $\mathrm{Na}_{2} \mathrm{~S}_{2} \mathrm{O}_{3}$ solution $(5 \mathrm{~mL})$ and extracted with AcOEt $(2 \mathrm{x}$ $5.0 \mathrm{~mL}$ ). The combined AcOEt layers were washed with saturated aqueous $\mathrm{KHCO}_{3}$ solution $(5 \mathrm{ml})$ and brine $(5 \mathrm{ml})$, dried, filtered and concentrated. The residue was subjected to flash chromatography on silica gel to give product 11a. White solid, 23.6 mg, yield: 59\%, ee: 85\%. HPLC: (Chiralpak ID, $4.6 \mathrm{~mm} \times 250 \mathrm{~mm}$, Hexane:i-Propanol $=90 / 10,0.7 \mathrm{~mL} / \mathrm{min}, 214 \mathrm{~nm}): \mathrm{t}_{\text {minor }}=25.96 \mathrm{~min}, \mathrm{t}_{\text {major }}=38.03 \mathrm{~min} ;[\alpha]_{\mathrm{D}}{ }^{27}=-2.65(\mathrm{c}$ 1.05, $\mathrm{CHCl}_{3}$ ); mp: $118.6-119.4{ }^{\circ} \mathrm{C} .{ }^{1} \mathrm{H} \mathrm{NMR}\left(400 \mathrm{MHz}, \mathrm{CDCl}_{3}\right) \delta 7.28(\mathrm{~d}, J=8.0 \mathrm{~Hz}$, 2H), $7.24(\mathrm{~d}, J=7.9 \mathrm{~Hz}, 2 \mathrm{H}), 5.08(\mathrm{~s}, 1 \mathrm{H}), 4.26(\mathrm{td}, J=8.8,4.0 \mathrm{~Hz}, 1 \mathrm{H}), 3.69-3.60(\mathrm{~m}$, 2H), 3.46-3.38 (m, 1H), 3.24 (s, 3H), $3.15(\mathrm{dd}, J=13.1,6.5 \mathrm{~Hz}, 1 \mathrm{H}), 2.71$ (dd, $J=12.9$, $9.4 \mathrm{~Hz}, 1 \mathrm{H}), 2.39$ (s, 3H). ${ }^{13} \mathrm{C}$ NMR (101 MHz, $\left.\mathrm{CDCl}_{3}\right) \delta 168.6,151.3,140.4,129.6$, 129.5, 128.7, 125.9, 114.1, 113.8, 81.4, 74.2, 55.8, 52.1, 49.4, 42.1, 31.6, 21.4. IR (film): 3203, 1747, 1433, 1206, 1182, 1042, 1026, 820, 736, 662, 504. MS (DART) m/z: 401.0 $(\mathrm{M}+\mathrm{H})^{+}$; HRMS (DART) $m / z$ Calcd for $\mathrm{C}_{19} \mathrm{H}_{18} \mathrm{~N}_{2} \mathrm{O}_{3} \mathrm{Br}(\mathrm{M}+\mathrm{H})^{+}: 401.0495$, found 
401.0492.

13. X-ray Crystallographic Data of 3a, 9a, and 11a
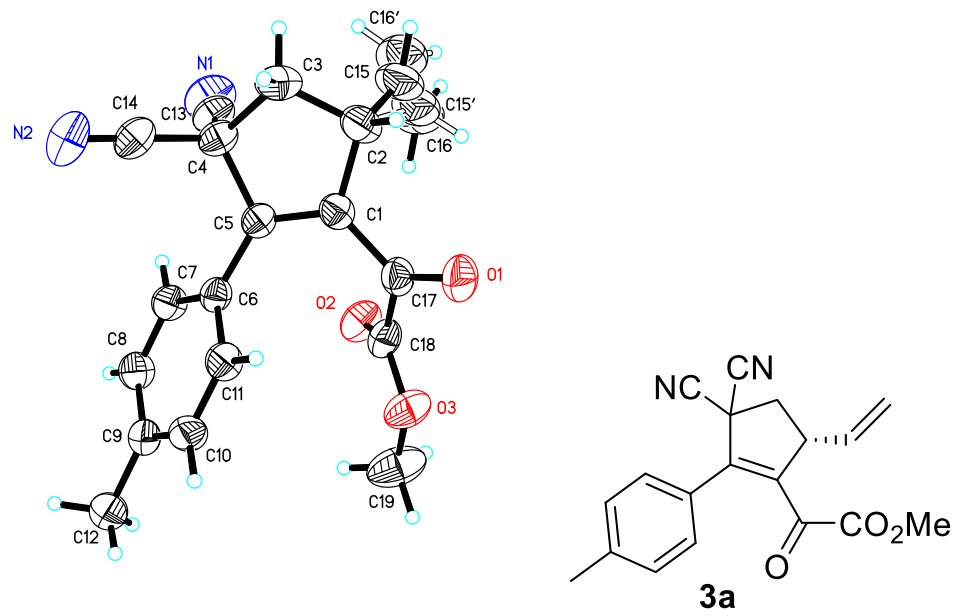

Crystal data and structure refinement for cu_d8v19174_0m.

Identification code

cu_d8v19174_0m

Empirical formula

C19 H16 N2 O3

Formula weight

320.34

Temperature

293(2) K

Wavelength

$1.54178 \AA$

Crystal system

Monoclinic

Space group

P 21

Unit cell dimensions

$$
\begin{array}{ll}
\mathrm{a}=6.1102(3) \AA & \mathrm{a}=90^{\circ} . \\
\mathrm{b}=12.3387(6) \AA & \mathrm{b}=96.016(2)^{\circ} . \\
\mathrm{c}=11.6255(6) \AA & \mathrm{g}=90^{\circ} .
\end{array}
$$

Volume

871.64(8) $\AA^{3}$

Z

2

Density (calculated)

$1.221 \mathrm{Mg} / \mathrm{m}^{3}$

Absorption coefficient

$0.683 \mathrm{~mm}^{-1}$

$\mathrm{F}(000)$

336

Crystal size

$0.190 \times 0.150 \times 0.130 \mathrm{~mm}^{3}$ 
Theta range for data collection

Index ranges

Reflections collected

Independent reflections

Completeness to theta $=67.679^{\circ}$

Absorption correction

Max. and min. transmission

Refinement method

Data / restraints / parameters

Goodness-of-fit on $\mathrm{F}^{2}$

Final R indices [I $>2 \operatorname{sigma}(\mathrm{I})]$

$\mathrm{R}$ indices (all data)

Absolute structure parameter

Extinction coefficient

Largest diff. peak and hole
10.030 to $67.481^{\circ}$.

$-7<=\mathrm{h}<=7,-14<=\mathrm{k}<=14,-12<=\mathrm{l}<=13$

11242

$3017[\mathrm{R}(\mathrm{int})=0.0334]$

$96.3 \%$

Semi-empirical from equivalents

0.7533 and 0.5680

Full-matrix least-squares on $\mathrm{F}^{2}$

$3017 / 41 / 238$

1.080

$\mathrm{R} 1=0.0342, \mathrm{wR} 2=0.0911$

$\mathrm{R} 1=0.0351, \mathrm{wR} 2=0.0924$

$0.04(8)$

$0.037(17)$

0.114 and -0.093 e. $\AA^{-3}$

CCDC 1907291(3a) contains the supplementary crystallographic data for this paper.

The data can be obtained free of charge from The Cambridge Crystallographic Data Centre via www.ccdc.cam.ac.uk/data_request/cif.

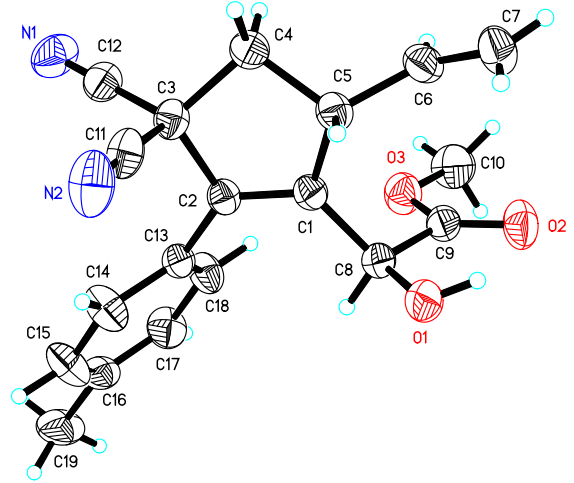

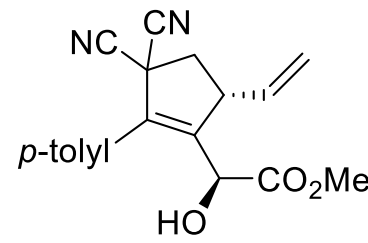

$9 a$ 
Crystal data and structure refinement for cu_dd8v19518_0m.

Identification code cu_dd8v19518_0m

Empirical formula $\quad \mathrm{C} 19 \mathrm{H} 18 \mathrm{~N} 2 \mathrm{O} 3$

Formula weight 322.35

Temperature 293(2) K

Wavelength $\quad 1.54178 \AA$

Crystal system Orthorhombic

Space group P212121

Unit cell dimensions $\mathrm{a}=7.7416(2) \AA \mathrm{a}=90^{\circ}$.

$$
\begin{aligned}
& \mathrm{b}=10.2291(2) \AA \mathrm{b}=90^{\circ} . \\
& \mathrm{c}=22.0387(4) \AA \mathrm{g}=90^{\circ} .
\end{aligned}
$$

Volume 1745.24(6) $\mathrm{A} 3$

Z 4

Density (calculated) $1.227 \mathrm{Mg} / \mathrm{m} 3$

Absorption coefficient $\quad 0.683 \mathrm{~mm}-1$

$\mathrm{F}(000) \quad 680$

Crystal size $0.200 \times 0.150 \times 0.110 \mathrm{~mm} 3$

Theta range for data collection $\quad 4.012$ to $65.499^{\circ}$.

Index ranges $-8<=\mathrm{h}<=7,-11<=\mathrm{k}<=12,-26<=\mathrm{l}<=23$

Reflections collected 10176

Independent reflections $2894[\mathrm{R}(\mathrm{int})=0.0917]$

Completeness to theta $=67.679^{\circ} \quad 91.2 \%$

Absorption correction Semi-empirical from equivalents

Max. and min. transmission $\quad 0.7533$ and 0.4317

Refinement method Full-matrix least-squares on F2

Data / restraints / parameters 2894 / 0 / 229

Goodness-of-fit on F2 1.029

Final R indices $[\mathrm{I}>2 \operatorname{sigma}(\mathrm{I})] \mathrm{R} 1=0.0452, \mathrm{wR} 2=0.1207$

$\mathrm{R}$ indices (all data) $\mathrm{R} 1=0.0476, \mathrm{wR} 2=0.1243$

Absolute structure parameter -0.11(18) 
Extinction coefficient $0.014(5)$

Largest diff. peak and hole $\quad 0.144$ and -0.133 e. $\AA-3$

CCDC 1919661 (9a) contain the supplementary crystallographic data for this paper.

These data can be obtained free of charge from The Cambridge Crystallographic Data

Centre via www.ccdc.cam.ac.uk/data_request/cif.
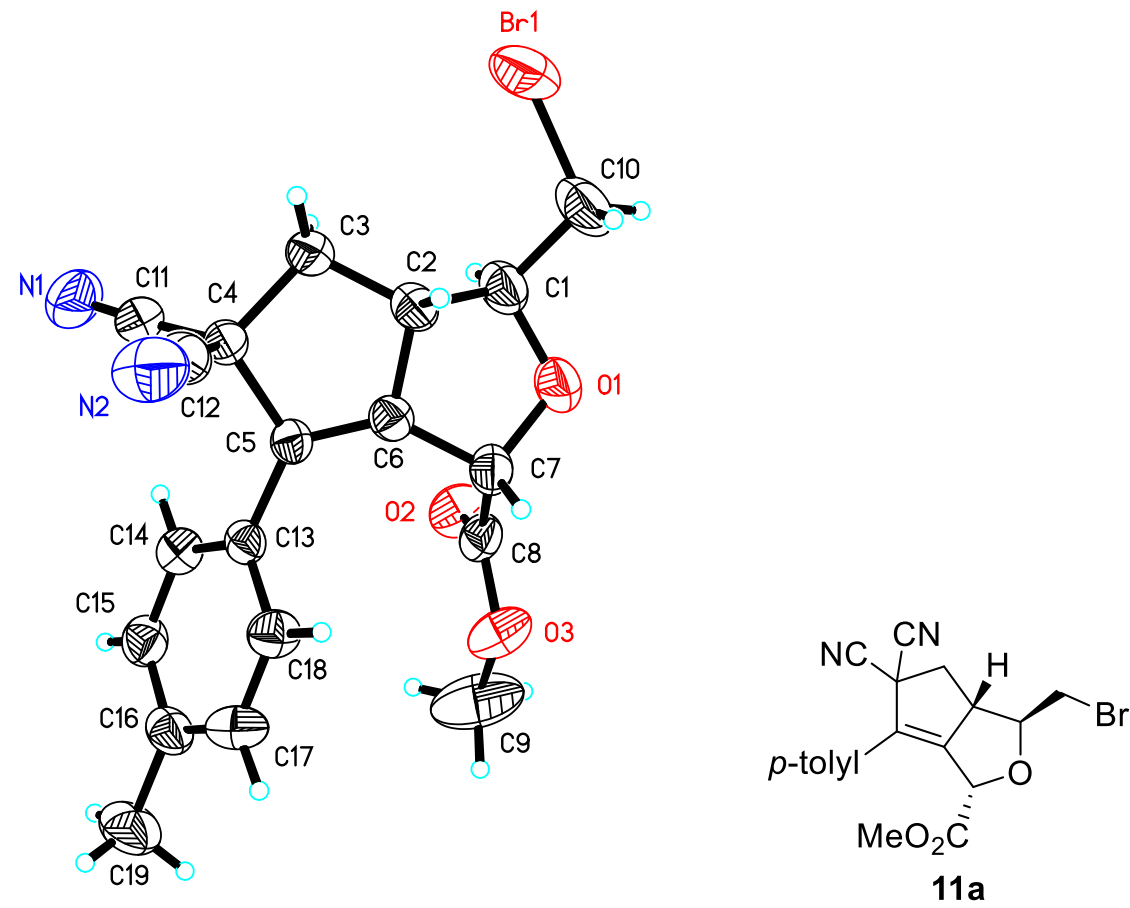

Crystal data and structure refinement for mo_d8v19549_0m.

Identification code mo_d8v19549_0m

Empirical formula $\quad \mathrm{C} 19 \mathrm{H} 17 \mathrm{Br}$ N2 O3

Formula weight 401.25

Temperature 293(2) K

Wavelength $\quad 0.71073 \AA$

Crystal system Monoclinic

Space group $\quad \mathrm{P} 21$

Unit cell dimensions $a=9.6440(4) \AA a=90^{\circ}$.

$$
\begin{aligned}
& \mathrm{b}=9.6225(5) \AA \mathrm{b}=105.0680(10)^{\circ} . \\
& \mathrm{c}=10.4722(5) \AA \mathrm{g}=90^{\circ} .
\end{aligned}
$$

Volume 938.40(8) ^3 
Z 2

Density (calculated) $1.420 \mathrm{Mg} / \mathrm{m} 3$

Absorption coefficient $2.209 \mathrm{~mm}-1$

$\mathrm{F}(000) \quad 408$

Crystal size $0.180 \times 0.150 \times 0.110 \mathrm{~mm}^{3}$

Theta range for data collection $\quad 2.187$ to $25.496^{\circ}$.

Index ranges $-11<=\mathrm{h}<=11,-11<=\mathrm{k}<=11,-12<=\mathrm{l}<=10$

Reflections collected 8963

Independent reflections $3439[\mathrm{R}$ (int) $=0.0295]$

Completeness to theta $=25.242^{\circ} \quad 99.1 \%$

Absorption correction Semi-empirical from equivalents

Max. and min. transmission $\quad 0.7456$ and 0.4021

Refinement method Full-matrix least-squares on F2

Data / restraints / parameters 3439 / 1 / 228

Goodness-of-fit on F2 1.038

Final R indices $[\mathrm{I}>2 \operatorname{sigma}(\mathrm{I})] \mathrm{R} 1=0.0607, \mathrm{wR} 2=0.1735$

$\mathrm{R}$ indices (all data) $\mathrm{R} 1=0.0860, \mathrm{wR} 2=0.1965$

Absolute structure parameter $0.054(11)$

Extinction coefficientn/a

Largest diff. peak and hole 0.455 and -0.451 e. $\AA-3$

CCDC 1922152 (11a) contain the supplementary crystallographic data for this paper.

These data can be obtained free of charge from The Cambridge Crystallographic Data

Centre via www.ccdc.cam.ac.uk/data_request/cif.

\section{Reaction of 4a at high temperature}
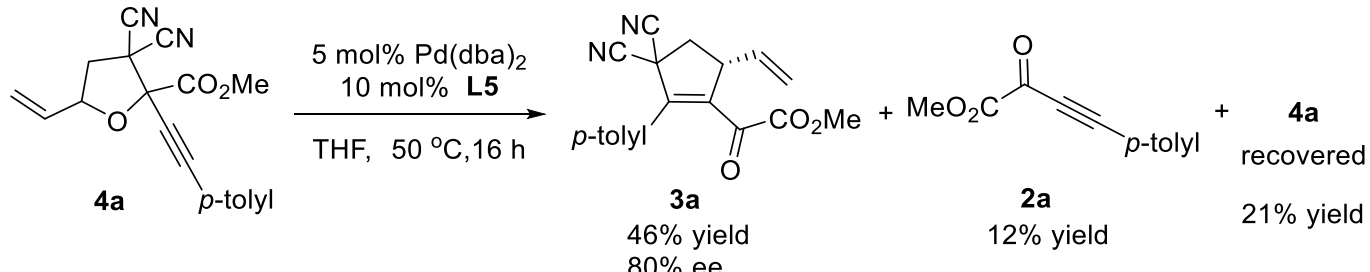
To a flame dried sealing tube were added Pd(dba) 2 (2.43 mg, $0.0042 \mathrm{mmol}),(R)$ SEGPHOS (L5) (5.13 mg, $0.0084 \mathrm{mmol})$, freshly distilled anhydrous THF (2 mL). The resulting mixture was allowed to stir for 30 mins. The methyl 3,3-dicyano-2-( $p$ tolylethynyl)-5-vinyltetrahydrofuran-2-carboxylate $4 \mathbf{a}(27.1 \mathrm{mg}, 0.085 \mathrm{mmol})$ was added. The resulting reaction mixture was stirred at $50{ }^{\circ} \mathrm{C}$ for $16 \mathrm{~h}$. After the volatiles were removed under reduced pressure, the NMR yield of $\mathbf{2 a}(12 \%), \mathbf{3 a}(46 \%)$, and $4 \mathbf{a}$ (21\%) was determined by ${ }^{1} \mathrm{H}$ NMR yield using mesitylene as the internal standard respectively. The sample of 3a for chiral HPLC analysis was obtained by preparative TLC with petroleum ether and ethyl acetate as eluent. The ee value of 3a was determined by chiral HPLC.

\section{Reference}

(1) Dieskau, A.; Holzwarth, M.; Plietker, B. Palladium/N Heterocyclic Carbene (NHC)-Catalyzed Asymmetric (3+2) Cycloaddition Reaction of Vinyl Epoxides with Allenic Amides. J. Am. Chem. Soc. 2012, 134, 5048-5051.

(2) Levashov, A. S.; Buryi, D. M.; Goncharova, O. V.; Konshin, V. V.; Dotsenkoab, V. V.; Andreev, A. A. Tetraalkynylstannanes in The Stille cross Coupling Reaction: a New Effective Approach to Arylalkynes. New J. Chem. 2017, 41, 2910-2918.

(3) Kang, Q. Doctoral thesis of Shanghai Institute of Organic Chemistry, Chinese Academy of Sciences [D] Shanghai: Shanghai Institute of Organic Chemistry, 2008.

(4) Prévost, S.; Ayad, T.; Phansavath, P.; Ratovelomanana-Vidal, V. Total Synthesis of Symbioramide: a Flexible Approach for the Efficient Preparation of Structural Isomers. Adv. Synth. Catal. 2011, 353, 3213-3226.

(5) Kong, X.-W.; Zhang, G.-X.; Yang, S.; Liu, X.-Z.; Fang, X.-Q. N-Heterocyclic Carbene-Catalyzed Umpolung of Alkynyl 1,2-Diketones. Adv. Synth. Catal. 2017, $359,2729-2734$.

(6) Parsley, D.; Plietker, B. Insertion of Imines into Vinylcyclopropanes Catalyzed by Nucleophilic Iron Complexes: A Formal [3+2]-Cycloaddition Strategy for the Synthesis of Substituted Pyrrolidine Derivatives. Synlett. 2014, 25, 2316-2318. 
(7) Shibuya, M.; Sato, T.; Tomizawa, M.; Iwabuchi, Y. Oxoammonium Salt/ $\mathrm{NaClO}_{2}$ : an Expedient, Catalytic System for One-potoxidation of Primary Alcohols to Carboxylic Acids with Broad Substrate Applicability. Chem. Commun. 2009, $1739-1741$.

(8) Huang, Z.-Z.; Sheng, S.-R.; Liu, X.-L.; Lin, S.-Y.; Huang, R.-S. A Convenient Oxidation of 5-Substituted Pyrazol-3(2H)-ones into Methyl 2-Alkynoates Using Poly[4-(diacetoxyiodo)styrene]. J. Chin. Chem. Soc. 2006, 53, 991-994.

(9) Huang, W.-Y.; Gurubrahamam, R.; Chen, K. An Unprecedented Organocascade Synthesis of Functionalized Bicyclic Nitrones from 2-Aminomalonate Derived Nucleophiles and 1-Nitro-1,3-Enynes via Allenes Formation and Subsequent Rearrangement. Adv. Synth. Catal. 2019, 361, 170-175.

(10)Wang, W.-Y.; Wu, J.-Y.; Liu. Q.-R.; Liu, X.-Y.; Ding, C.-H.; Hou, X.-L. Palladium/N Heterocyclic Carbene (NHC)-Catalyzed Asymmetric (3+2) Cycloaddition Reaction of Vinyl Epoxides with Allenic Amides. Org. Lett. 2018, 20, 4773-4776.

(11)Condakes, M. L.; Hung, K; Harwood, S. J.; Maimone, T. J. Total Syntheses of (-)Majucin and (-)-Jiadifenoxolane A, Complex Majucin-Type Illicium Sesquiterpenes. J. Am. Chem. Soc. 2017, 139, 17783-17786.

(12)Cornelius, L. A. M.; Bone, R. G.A.; Hastings, R. H.; Deardorff, M. A.; Scharlach, R. A.; Hauptmann, B. E.; Stankovic, C. S.; Pinnick, H. W. Synthesis of 2Acetylbicyclo[2.2.1]hept-2-eneu. J.Org. Chem. 1993, 58, 3188-3190.

(13)Takeuchi, Y.; Azuma, K.; Takakura, K.; Abe, H.; Kim, H. S.; Wataya, Y.; Harayama , T. Asymmetric synthesis of (1)-febrifugine and (1)-isofebrifugine using yeast reduction. Tetrahedron. 2001, 57, 1213-1218. 
罢蟔器器
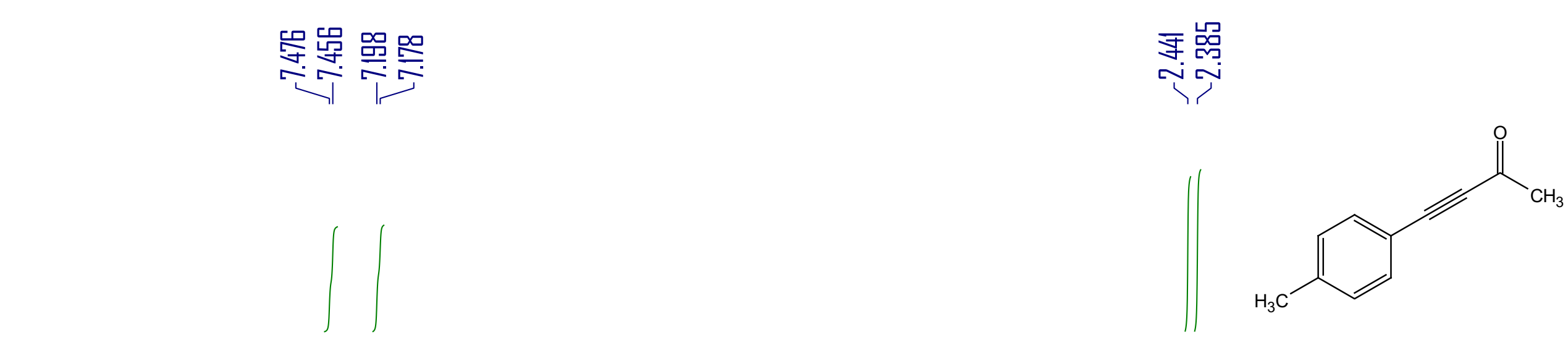

-跑
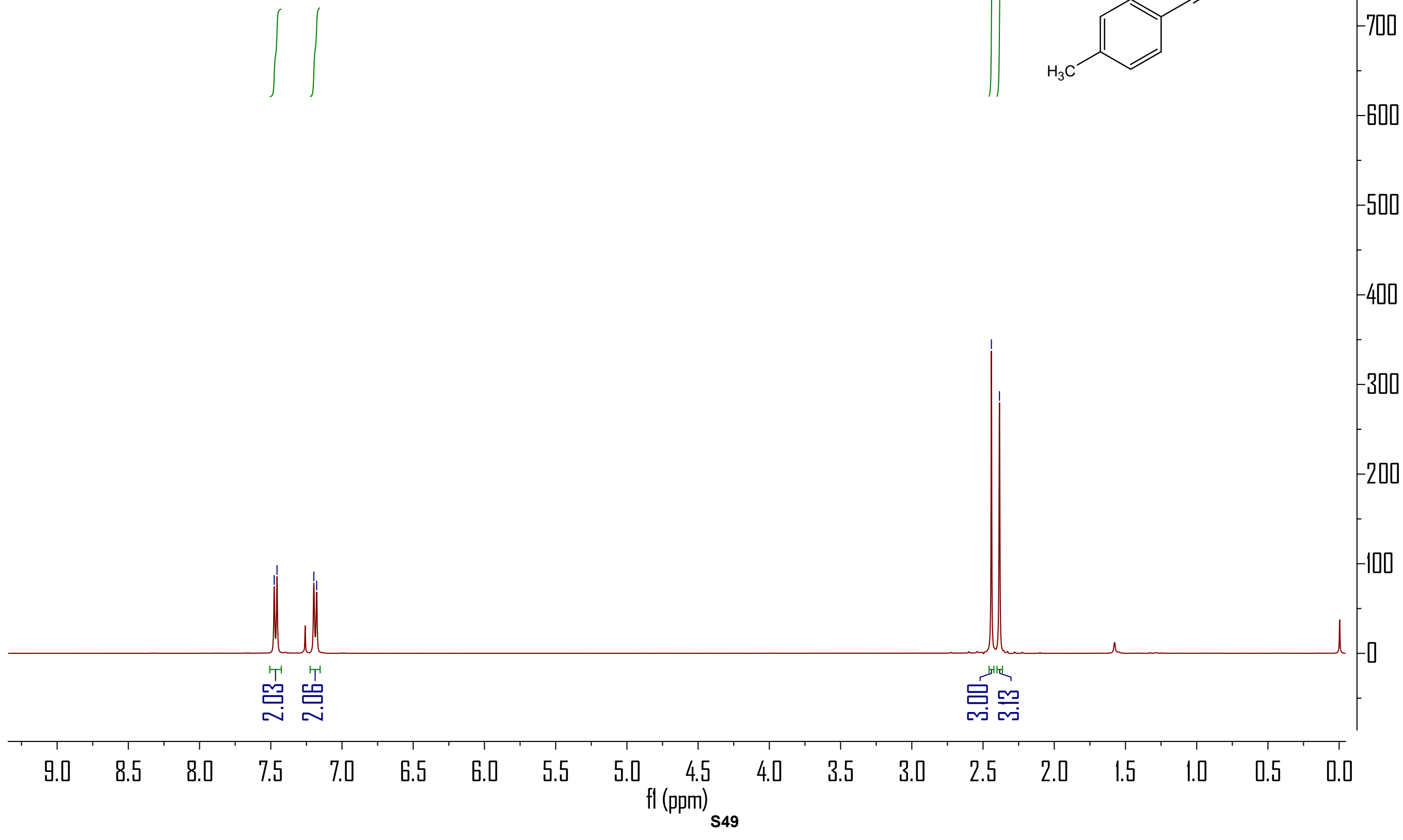


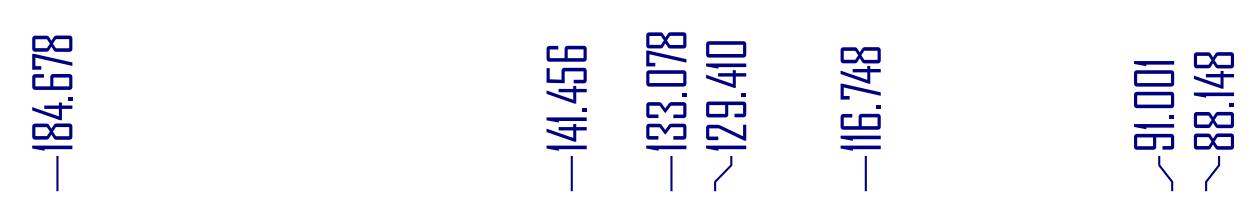

总 吾

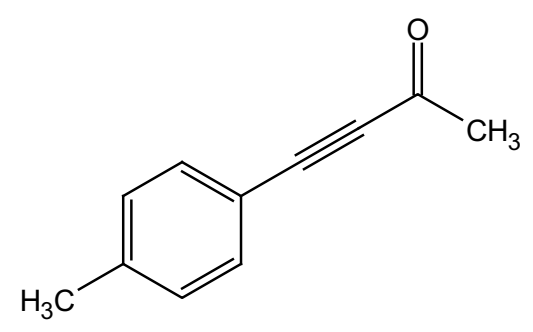



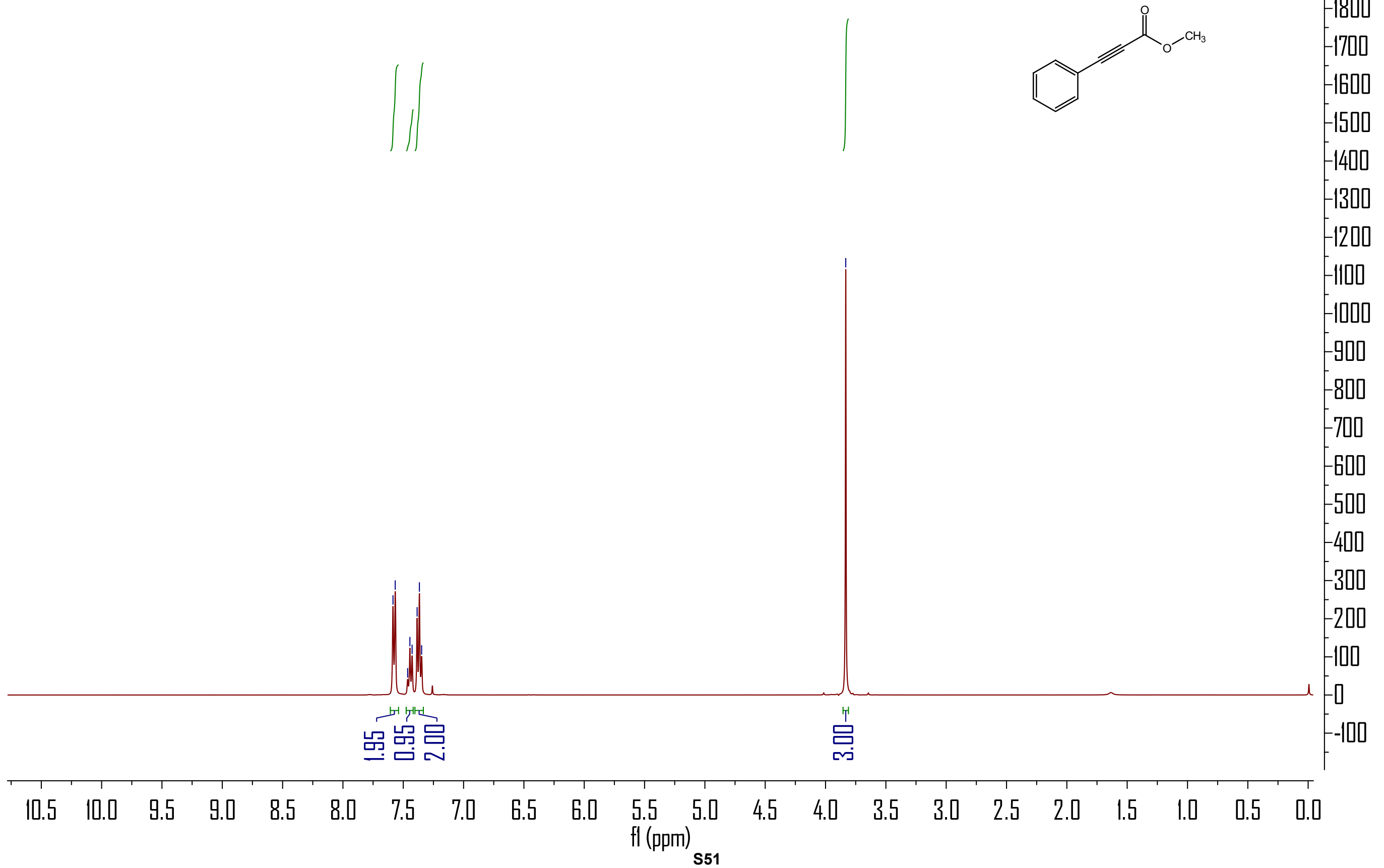


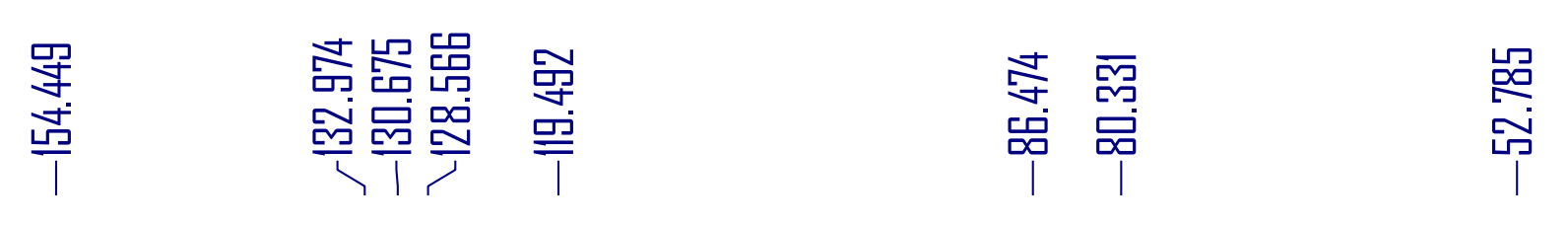
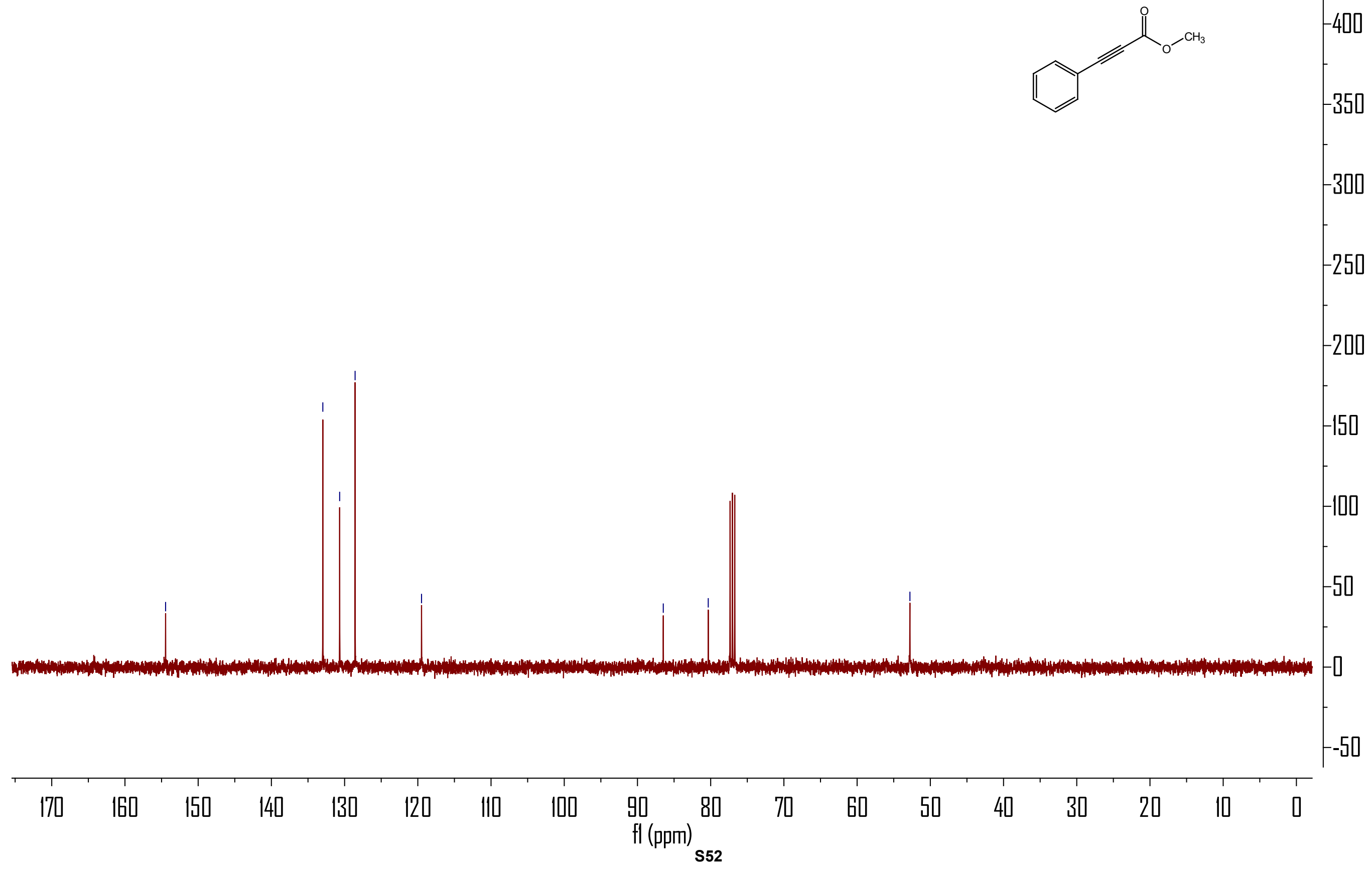
$\mathrm{MeO}_{2} \mathrm{C}=\mathrm{CO}_{2} \mathrm{Me}$ 


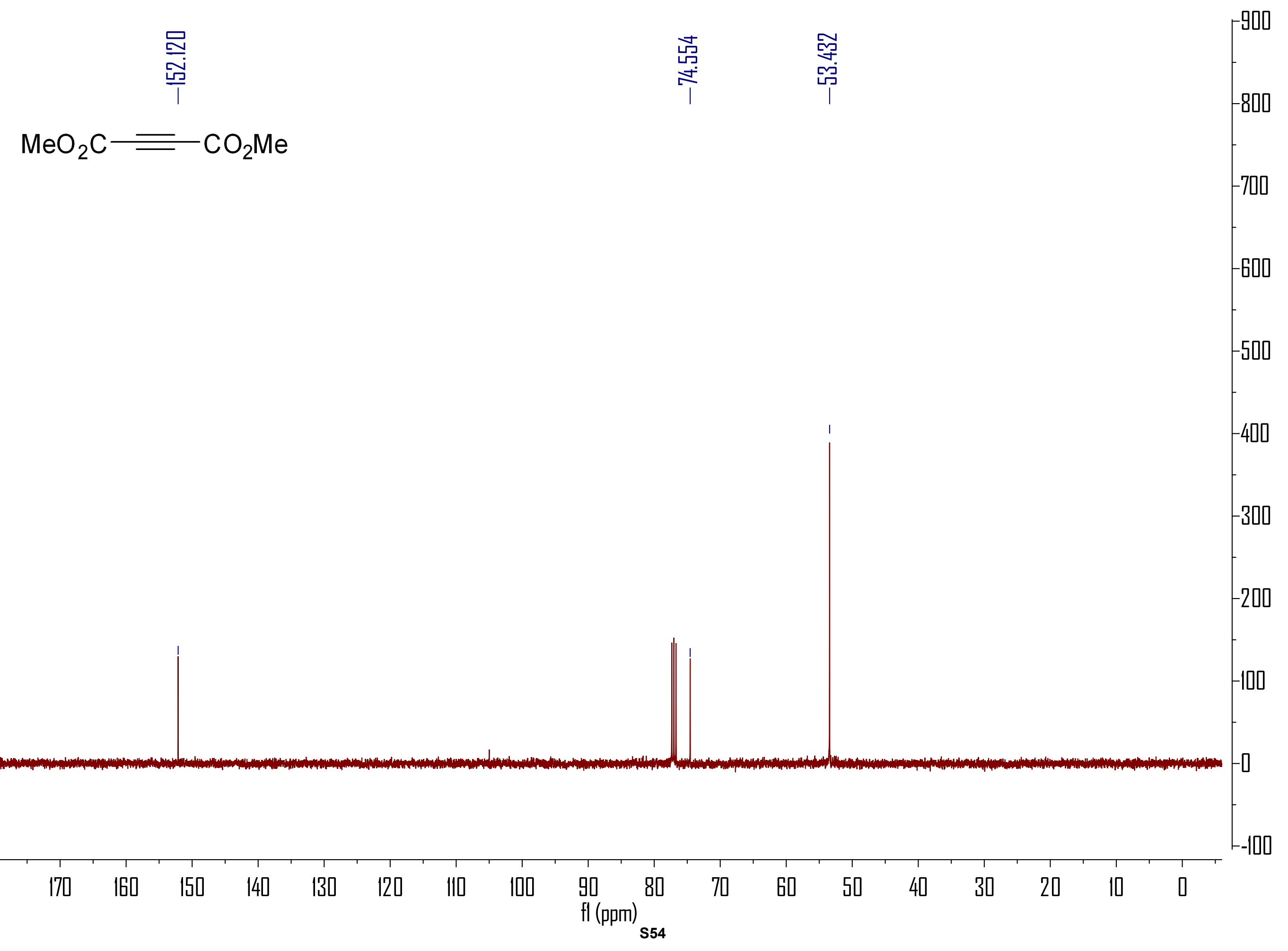


$2 a$
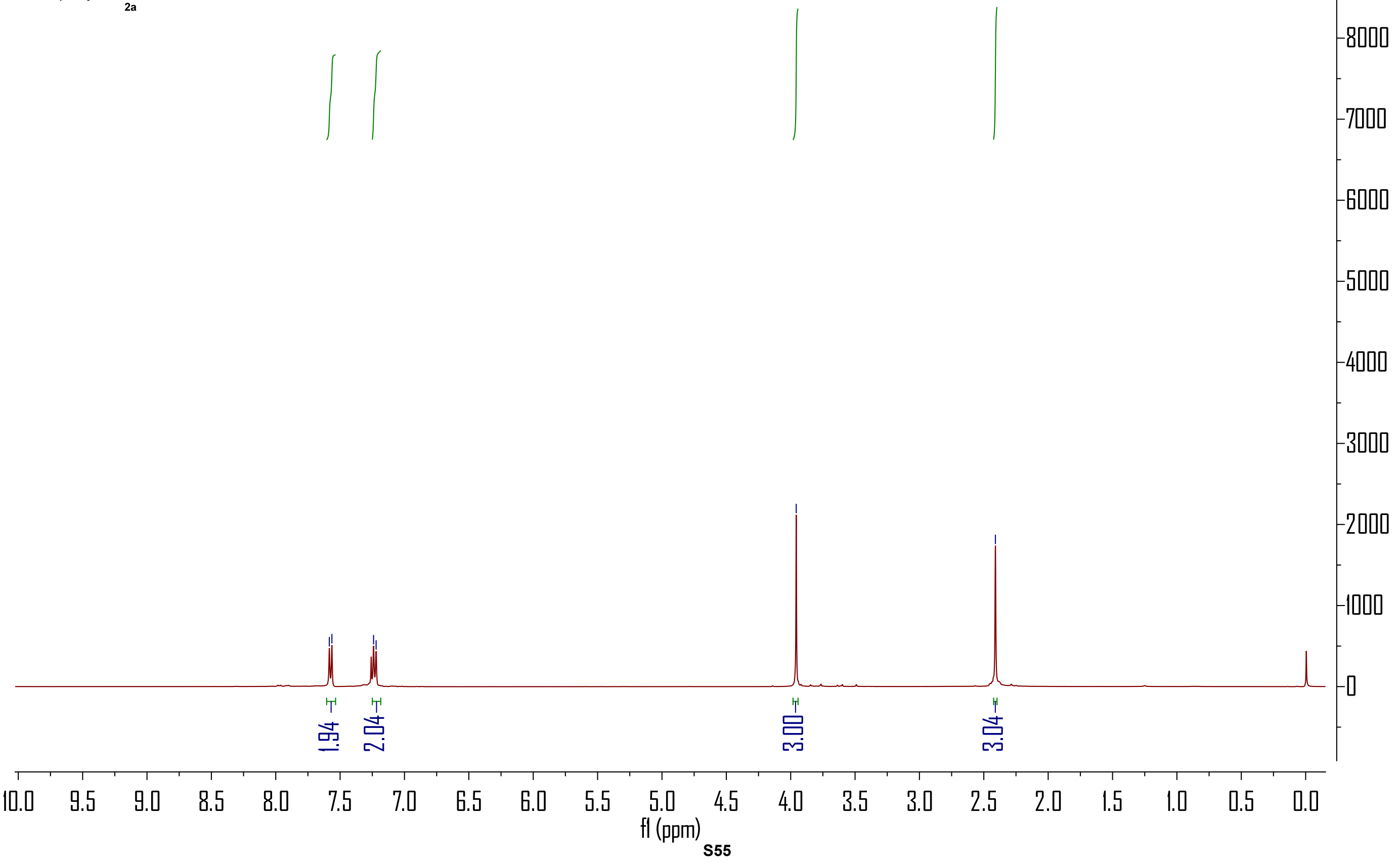


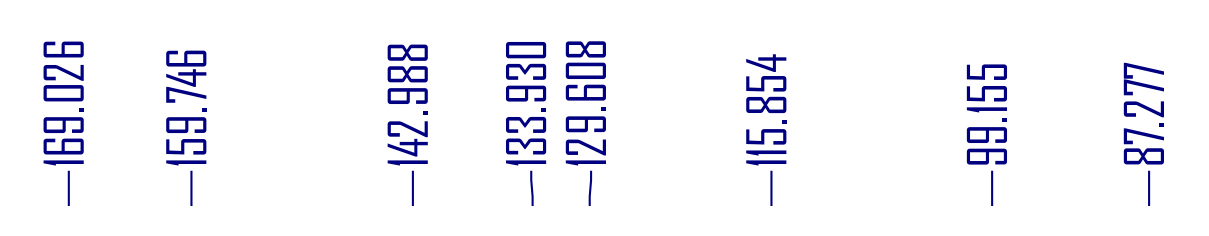

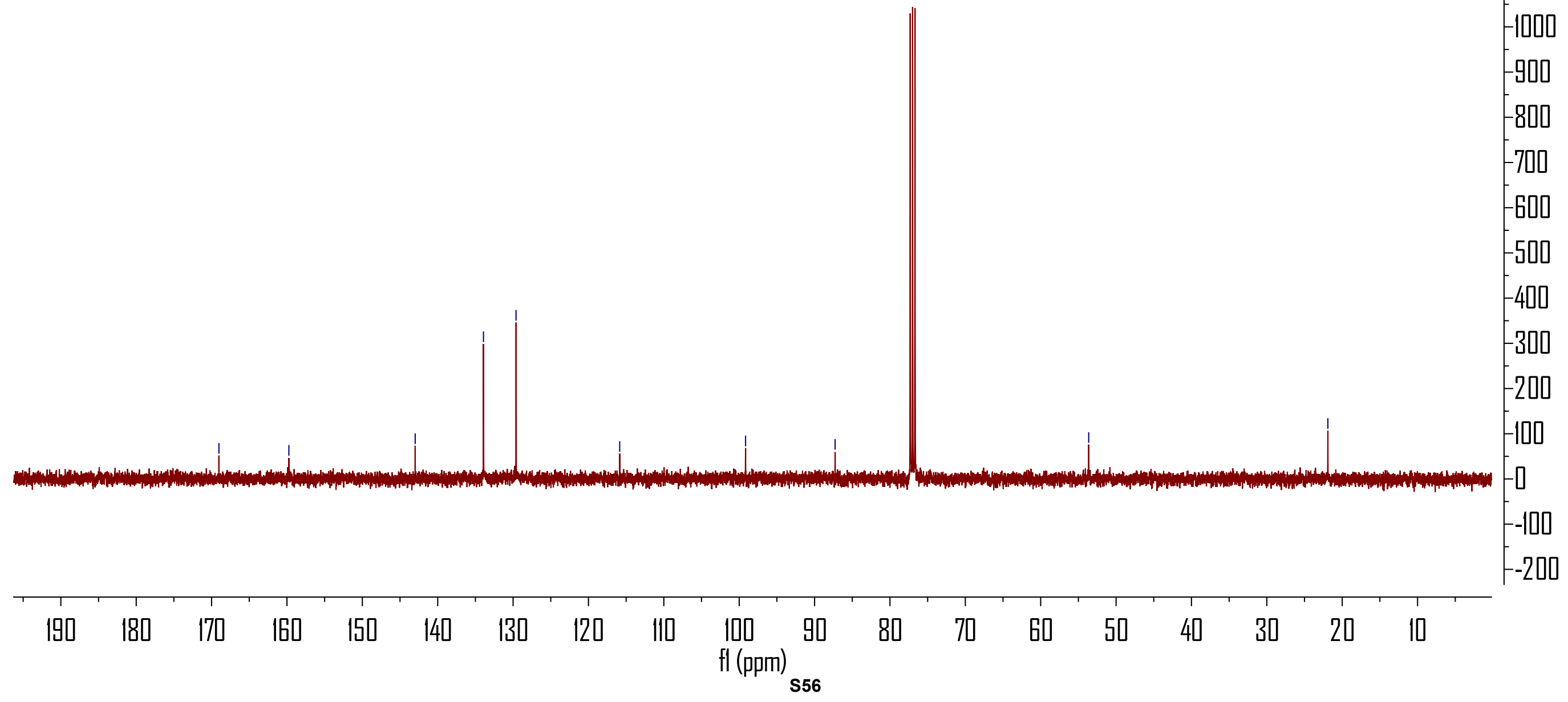




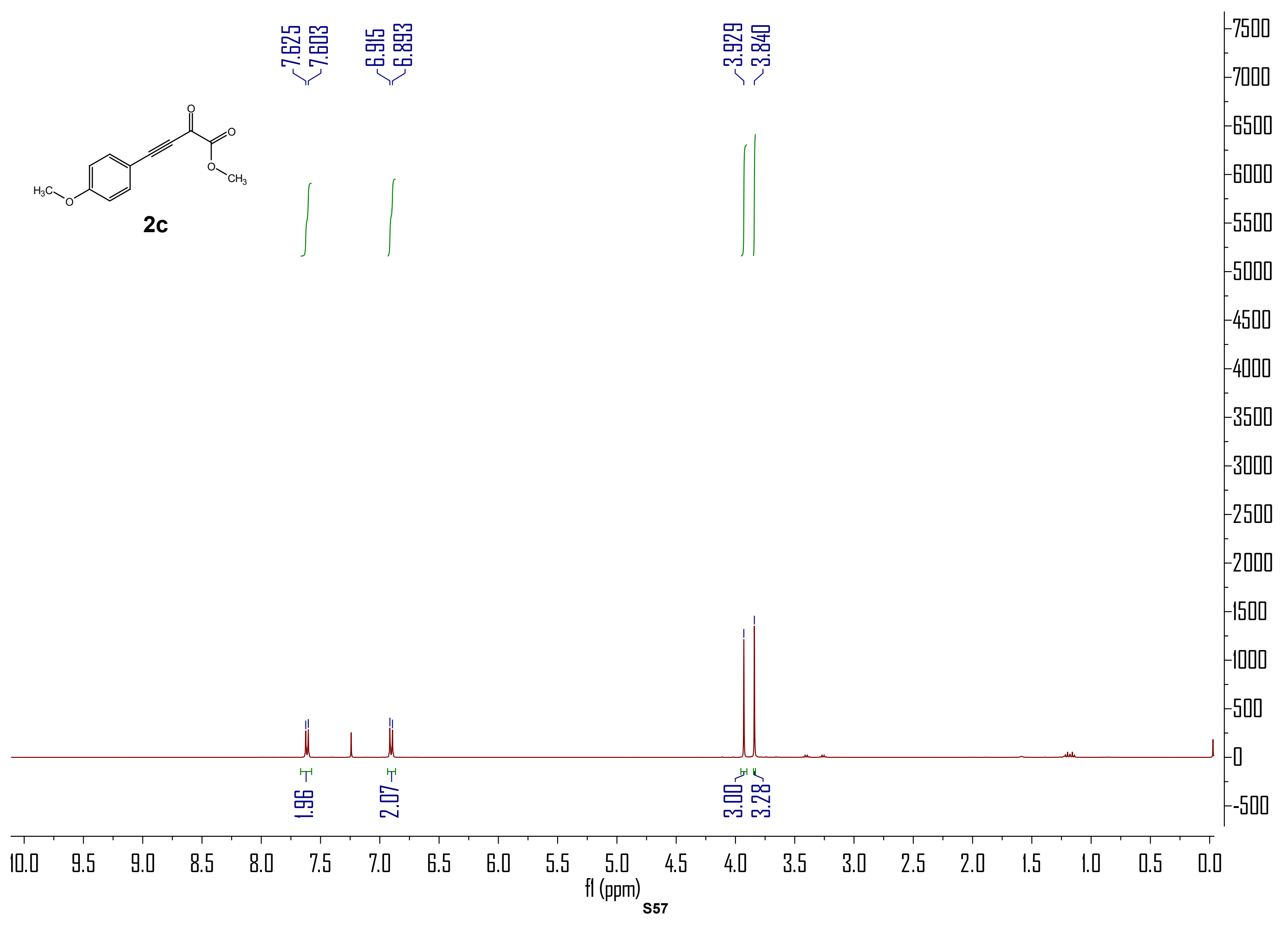




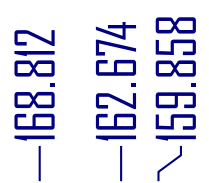

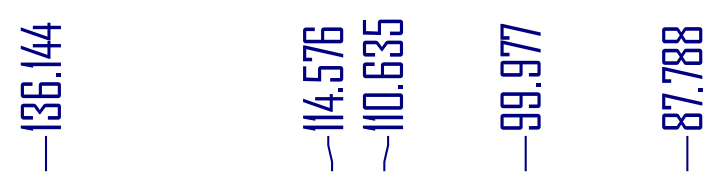
吕莒
品留

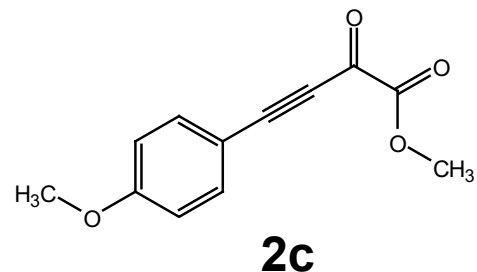

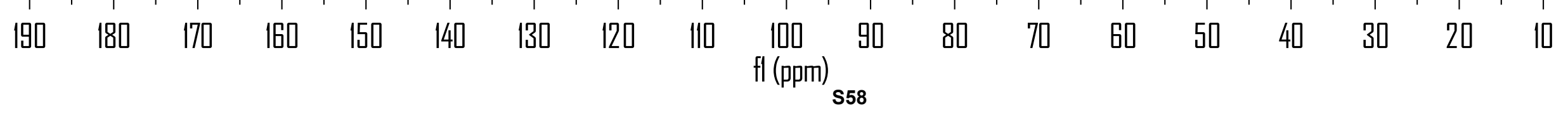




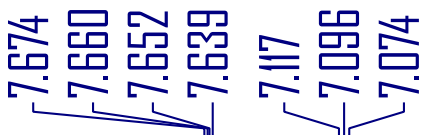
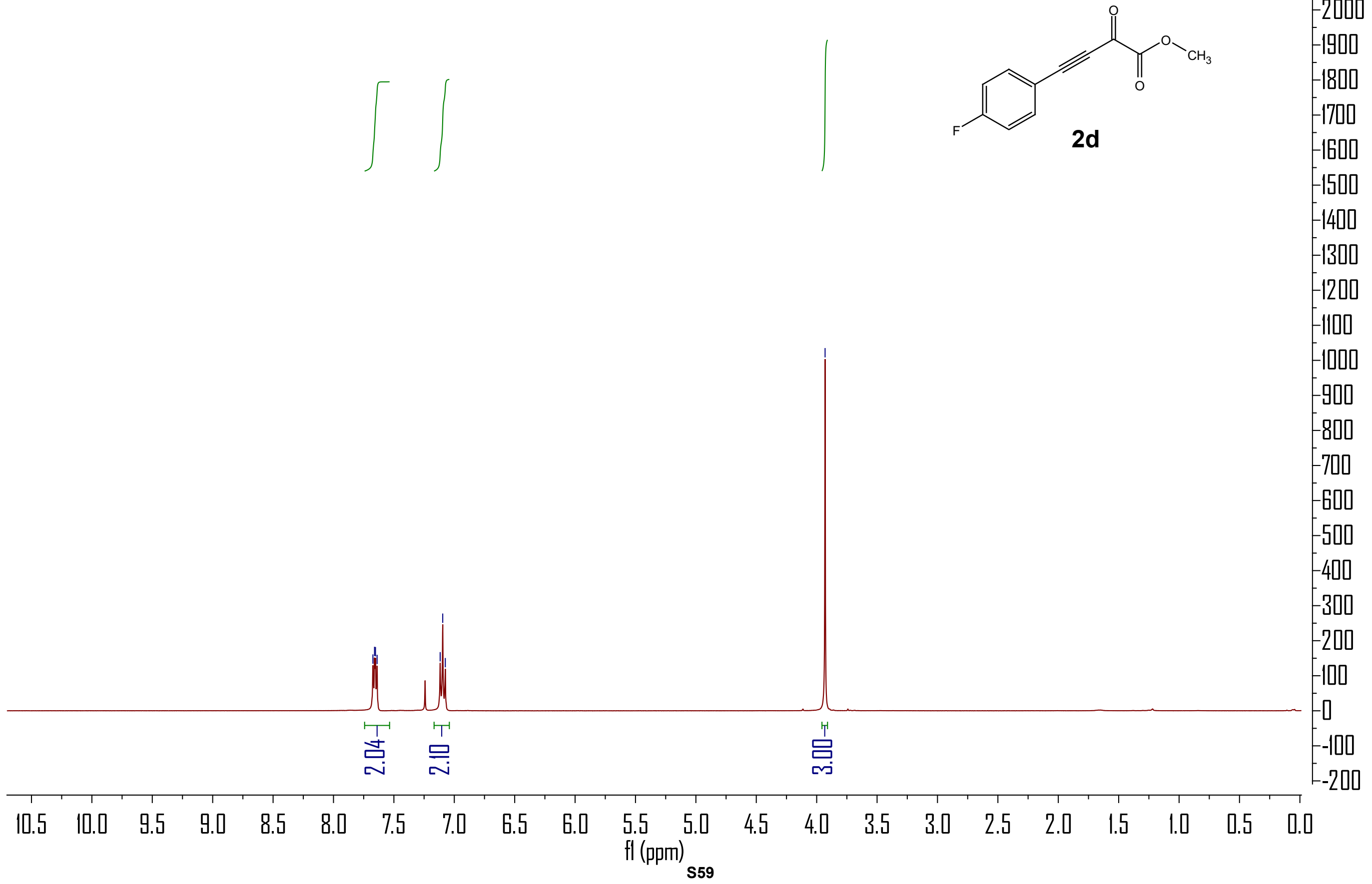
品
学
|

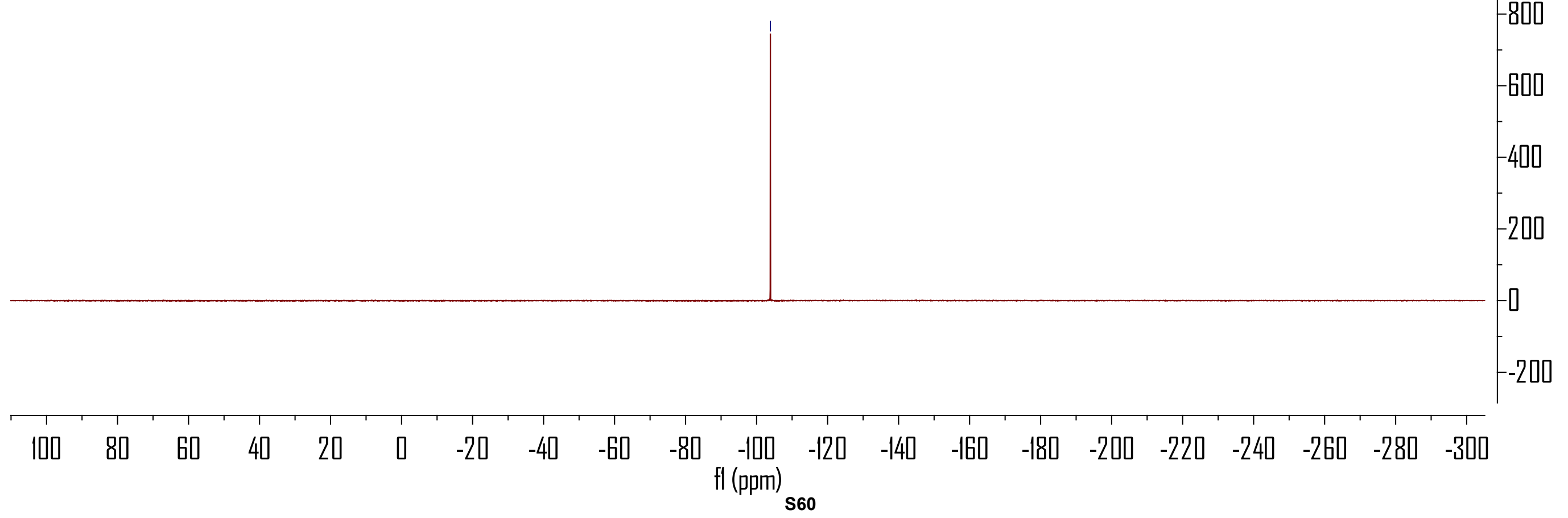




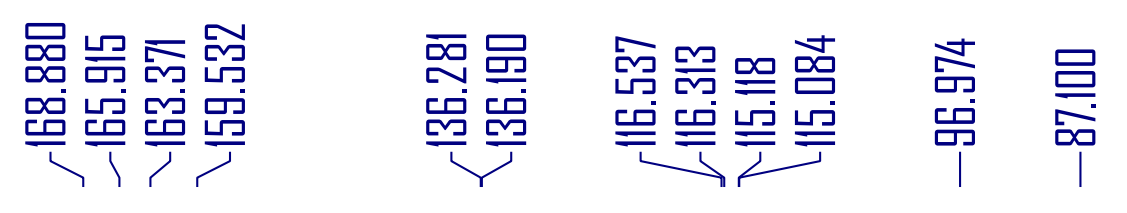
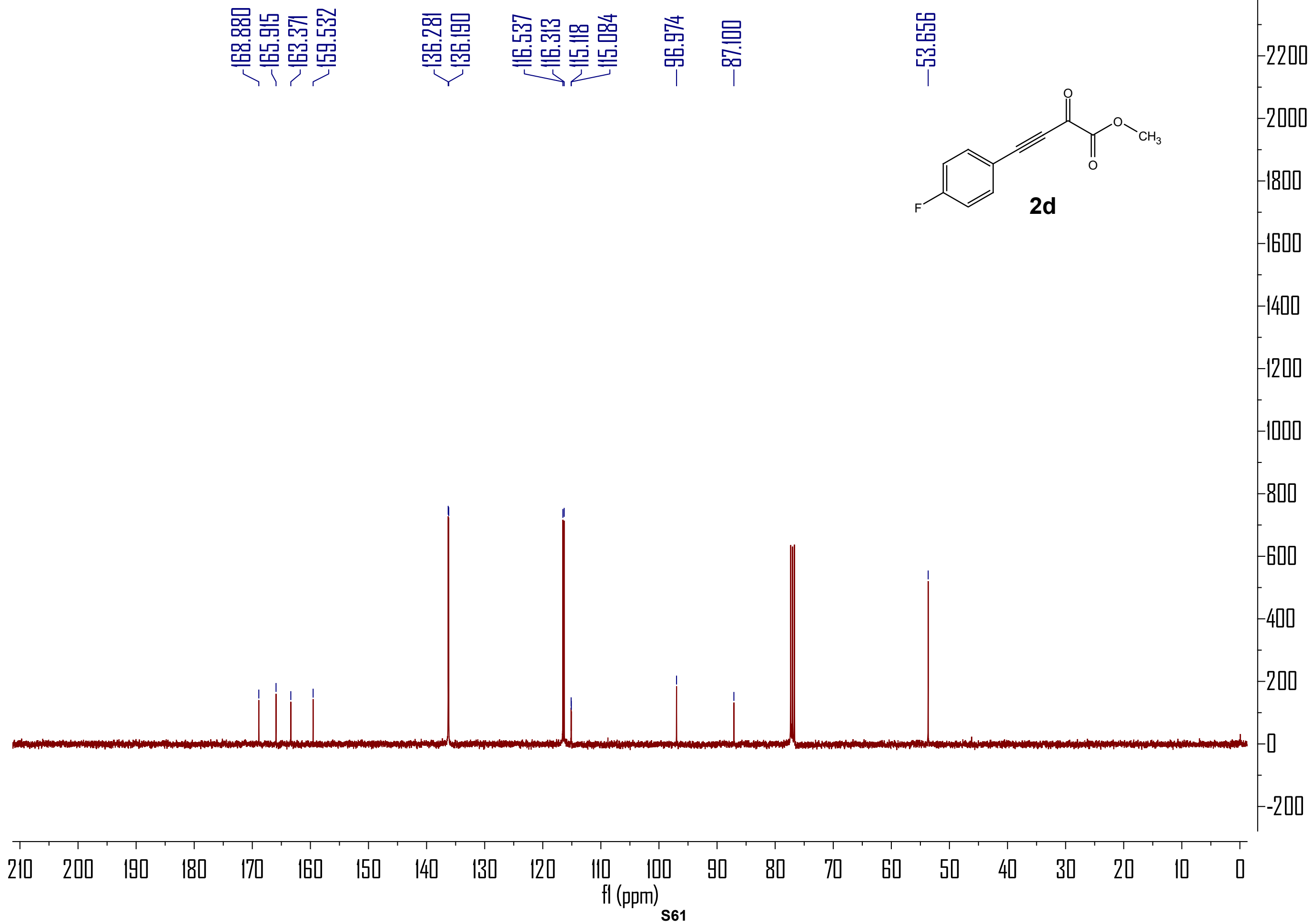


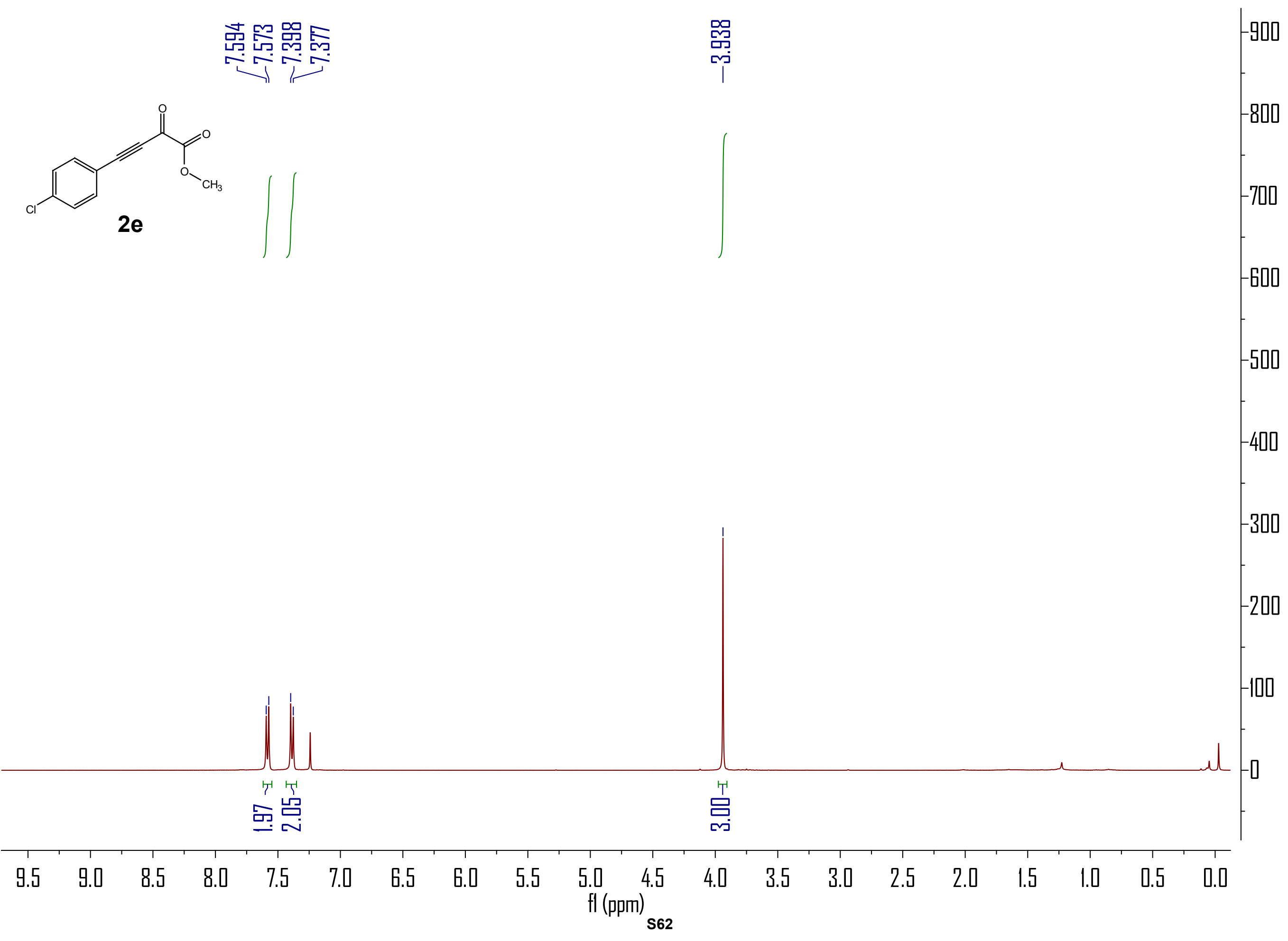




\section{塄䇾}

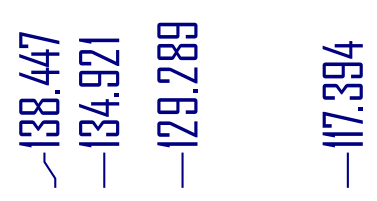

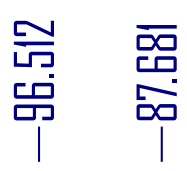

豆

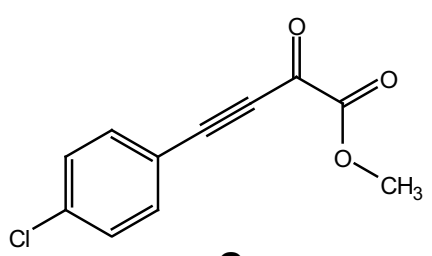

$2 e$

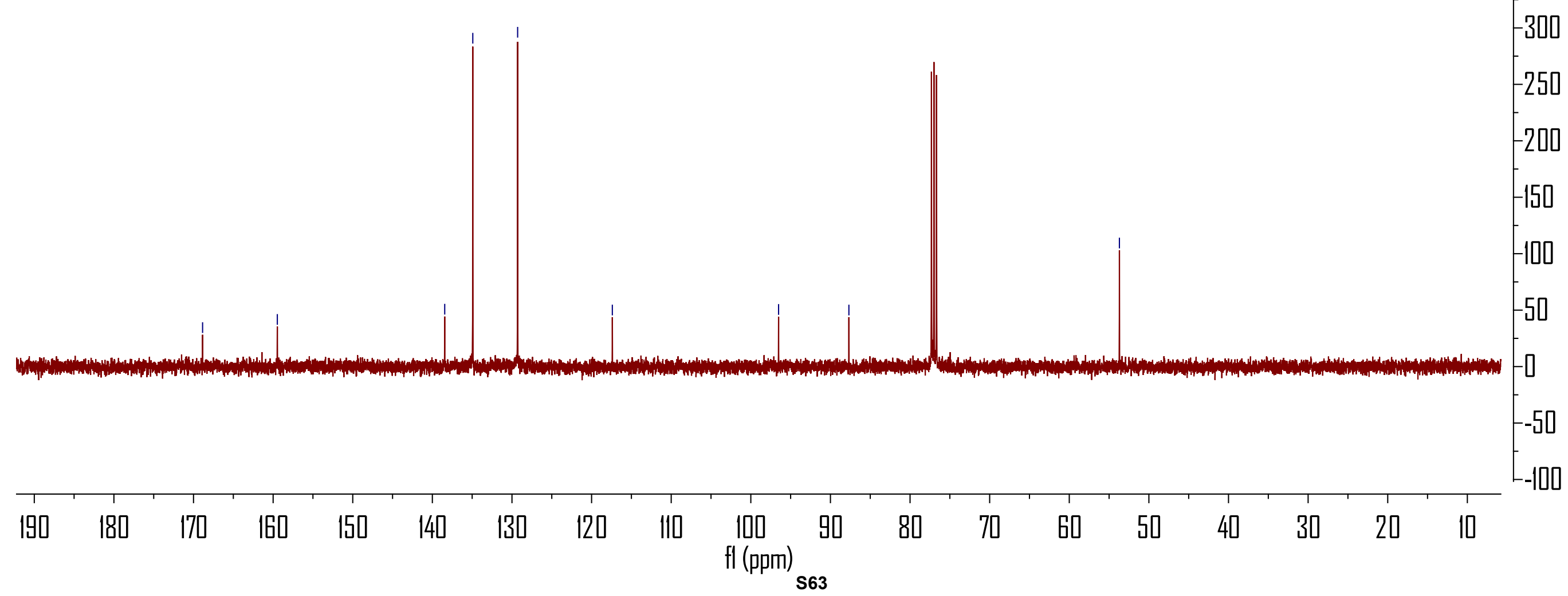


器第嫣

零
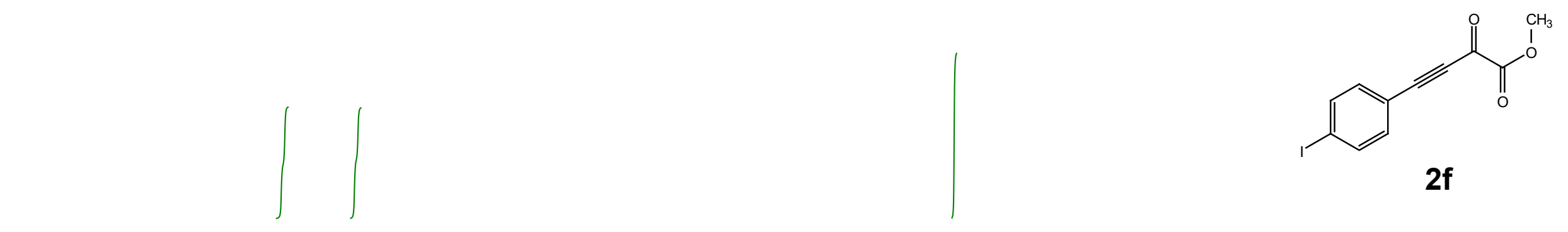

$-260$

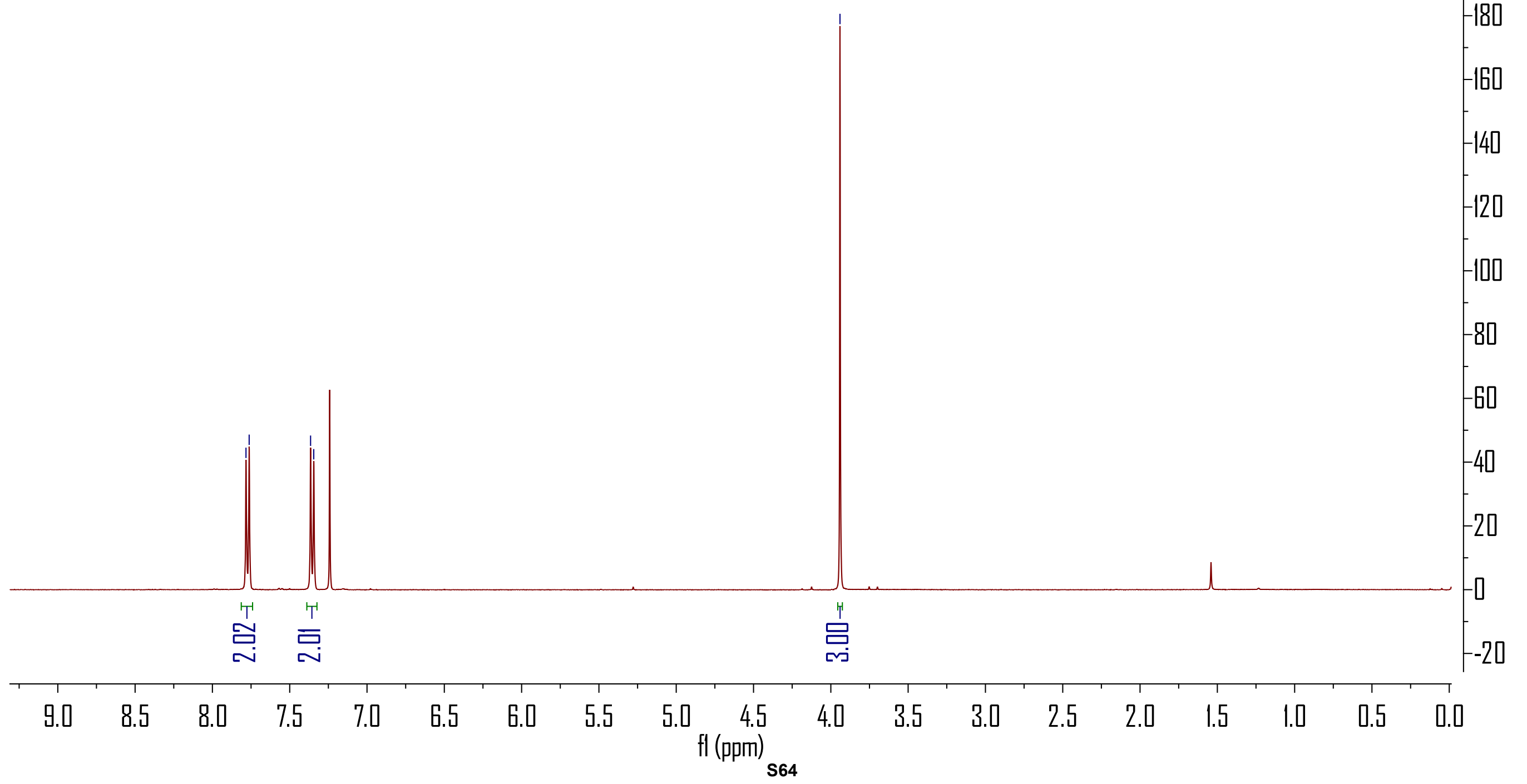




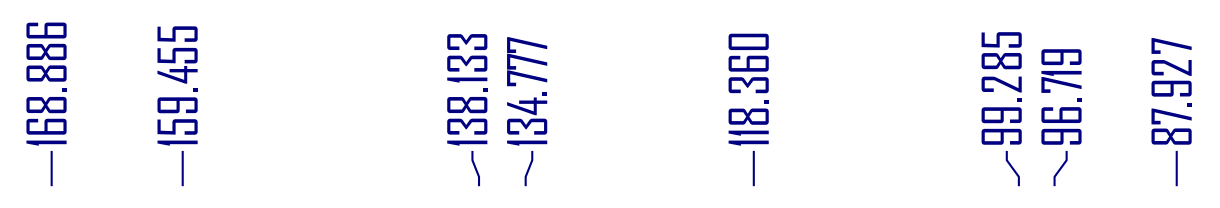

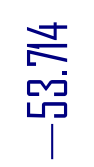

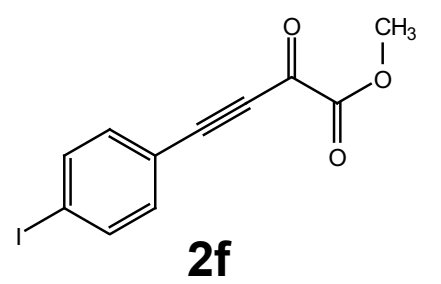

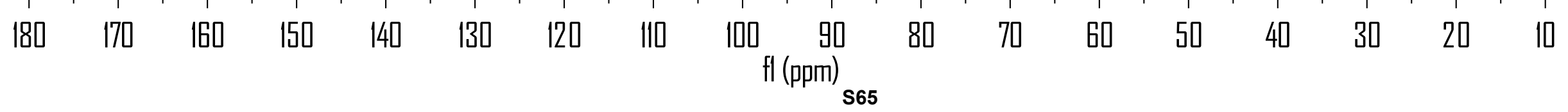




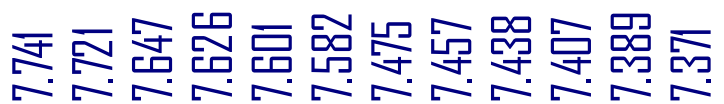

吕
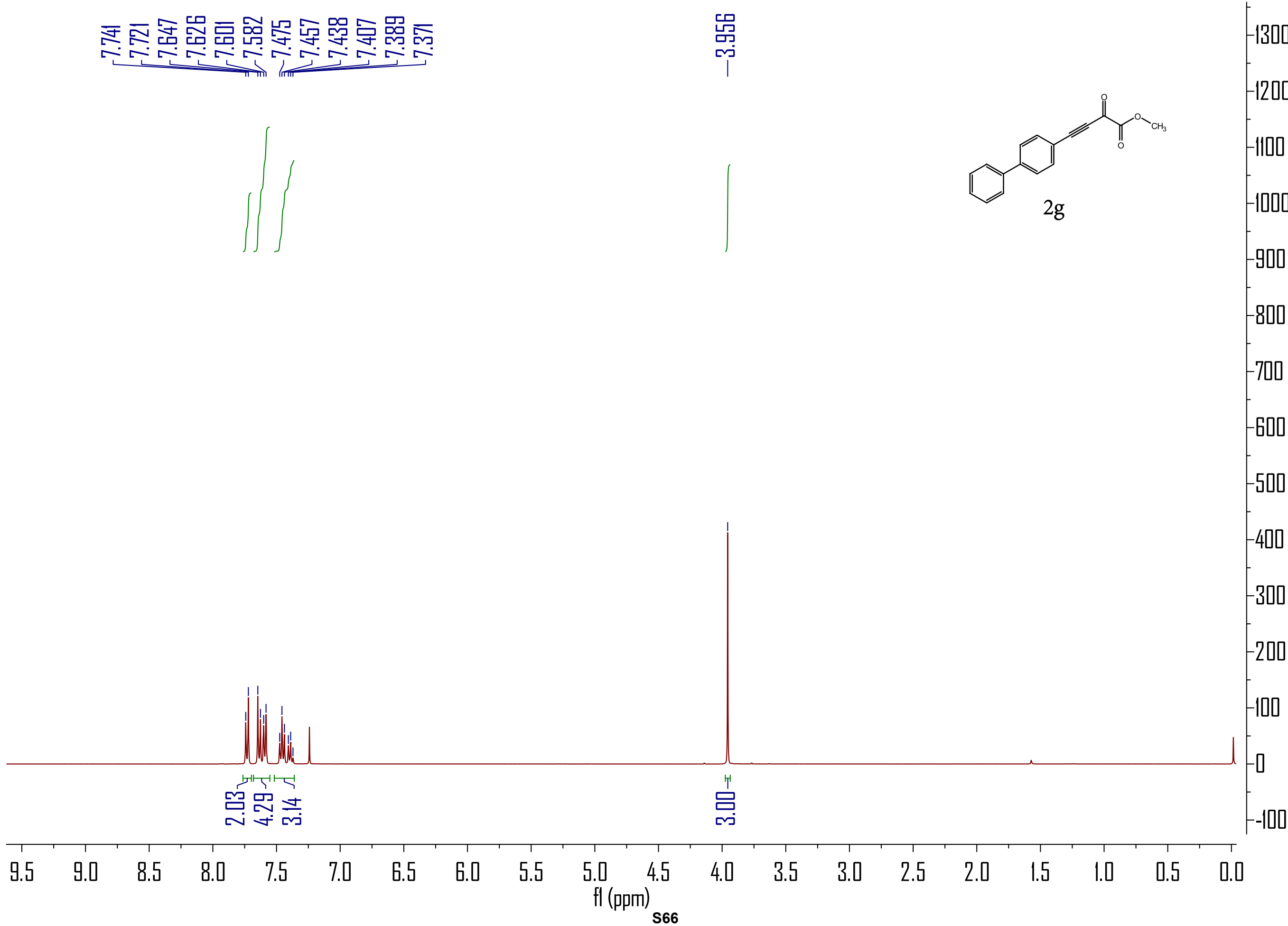


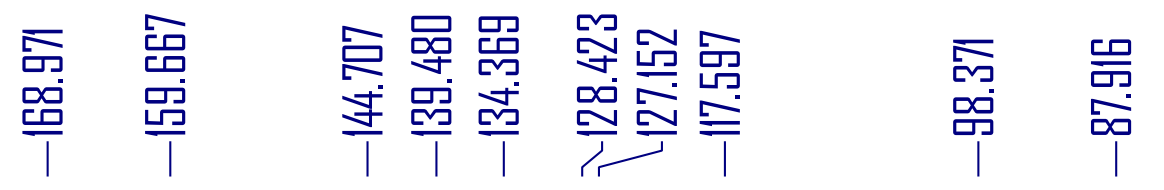

可

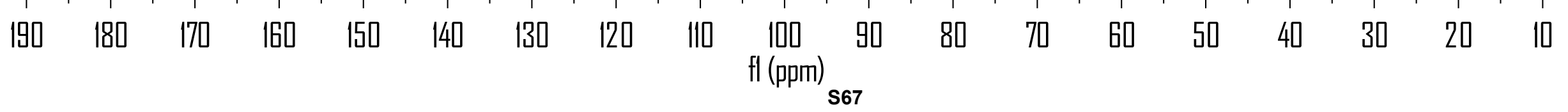



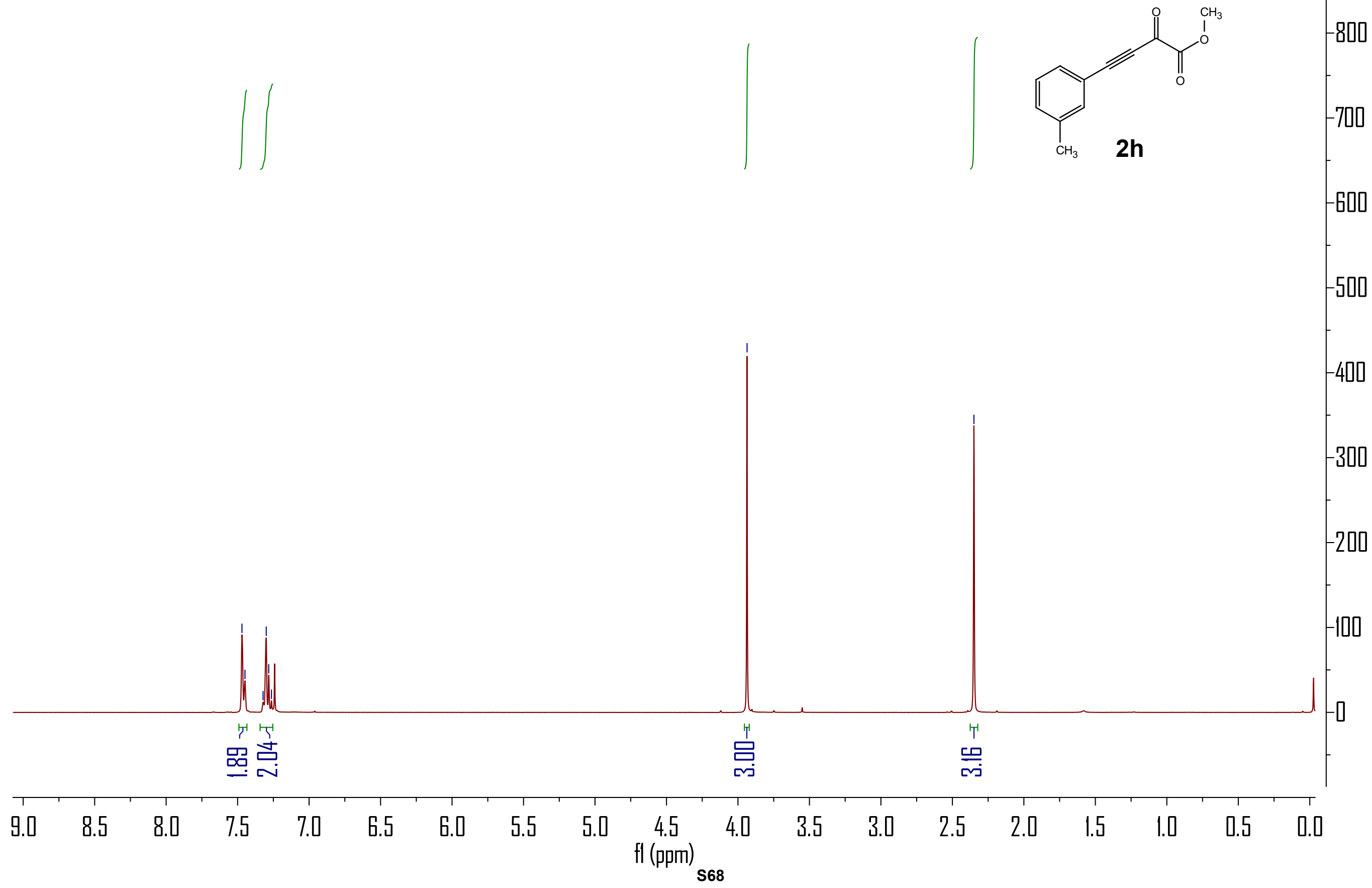


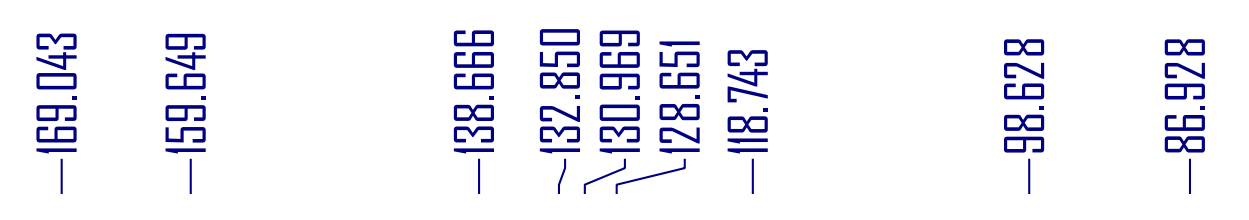

品

骂

$-800$

$-750$

$-700$

$-650$

$2 \mathrm{~h}$

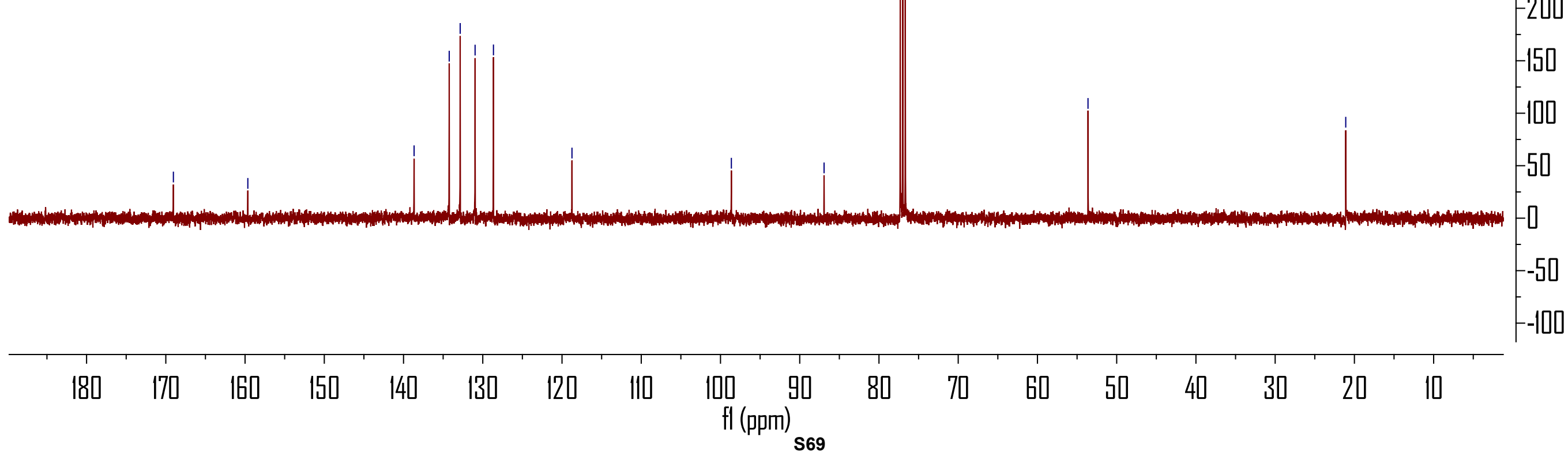




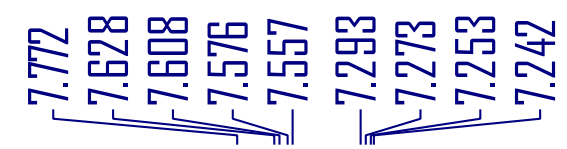

骂
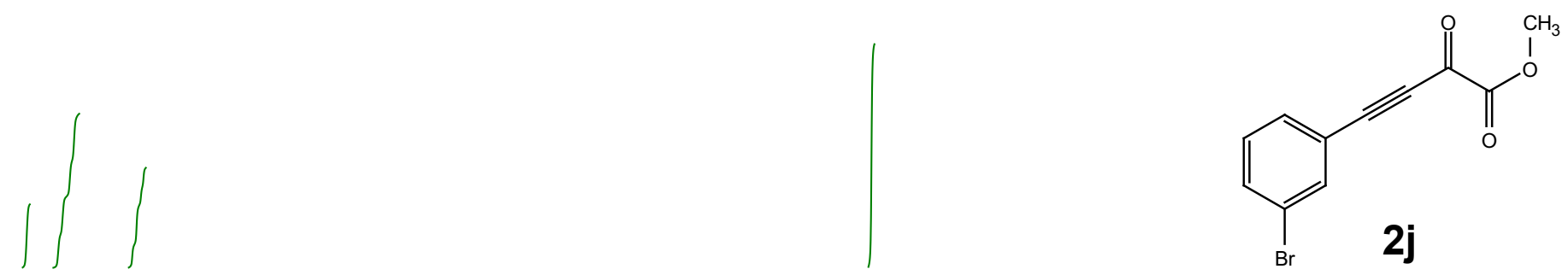

$-1700$

$-1600$

$-1500$

$-1400$

$-1300$

-1200

$-1100$

$-1000$

$-900$

800

700

600

500

400

300

$-200$

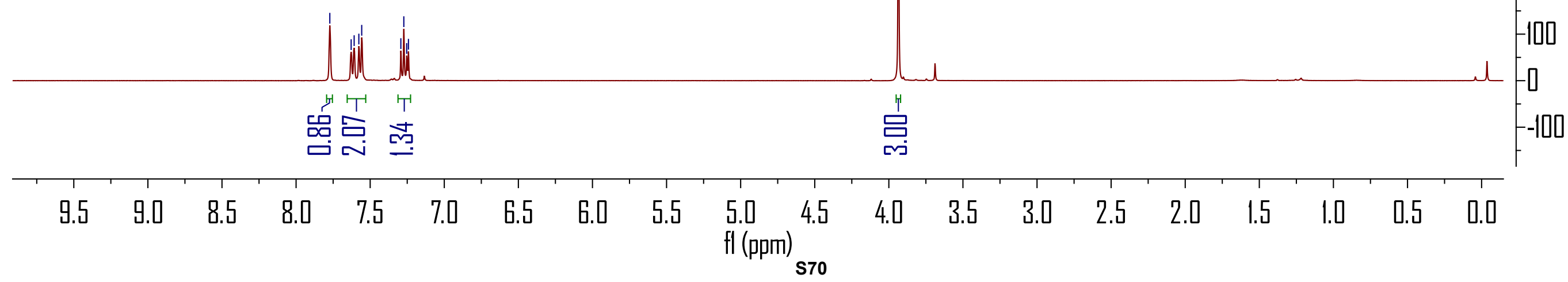




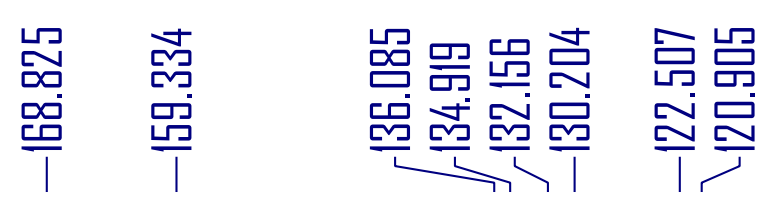

苫品

$\underset{\mathrm{F}}{\stackrel{9}{\mathrm{y}}}$
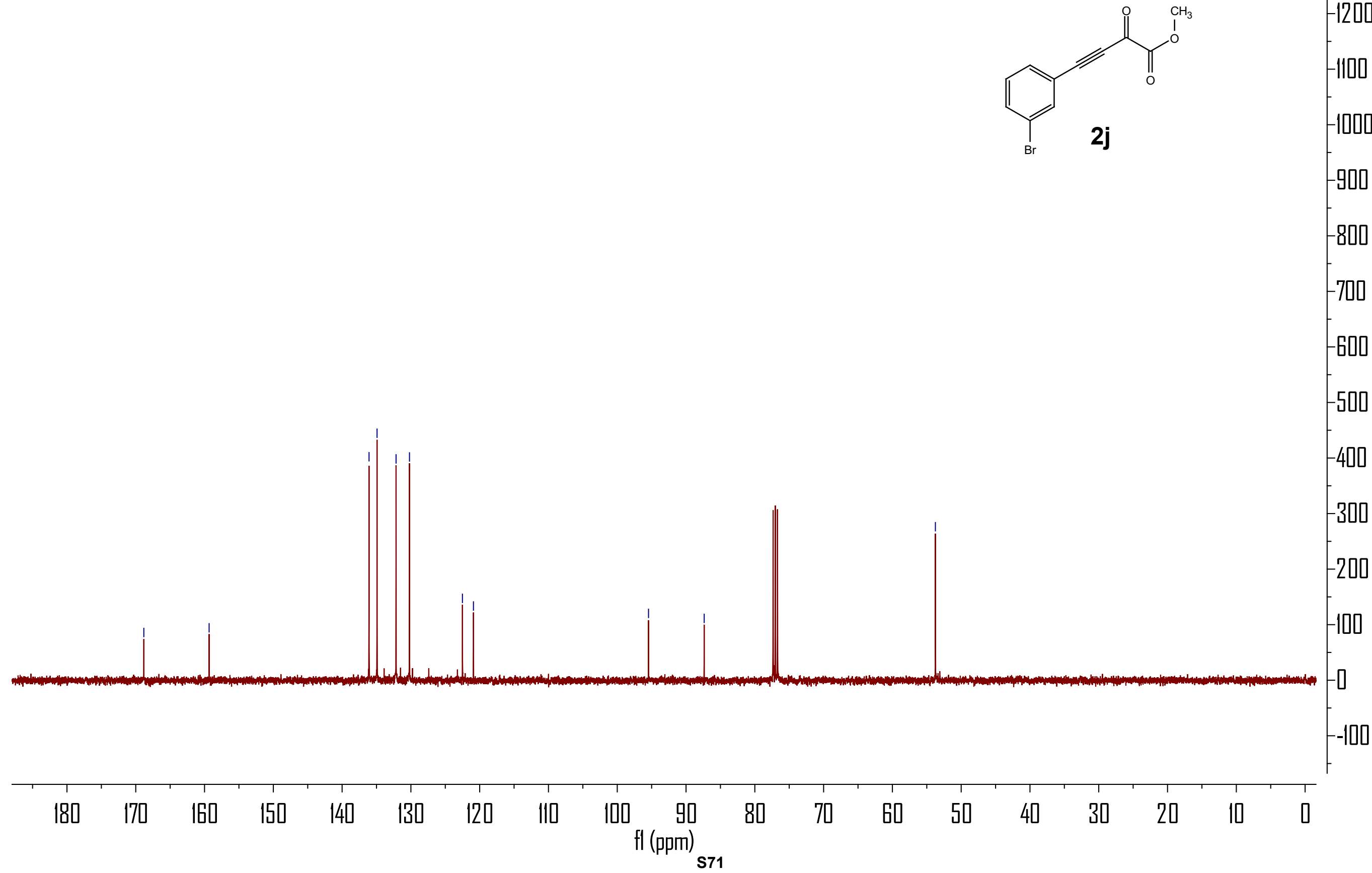

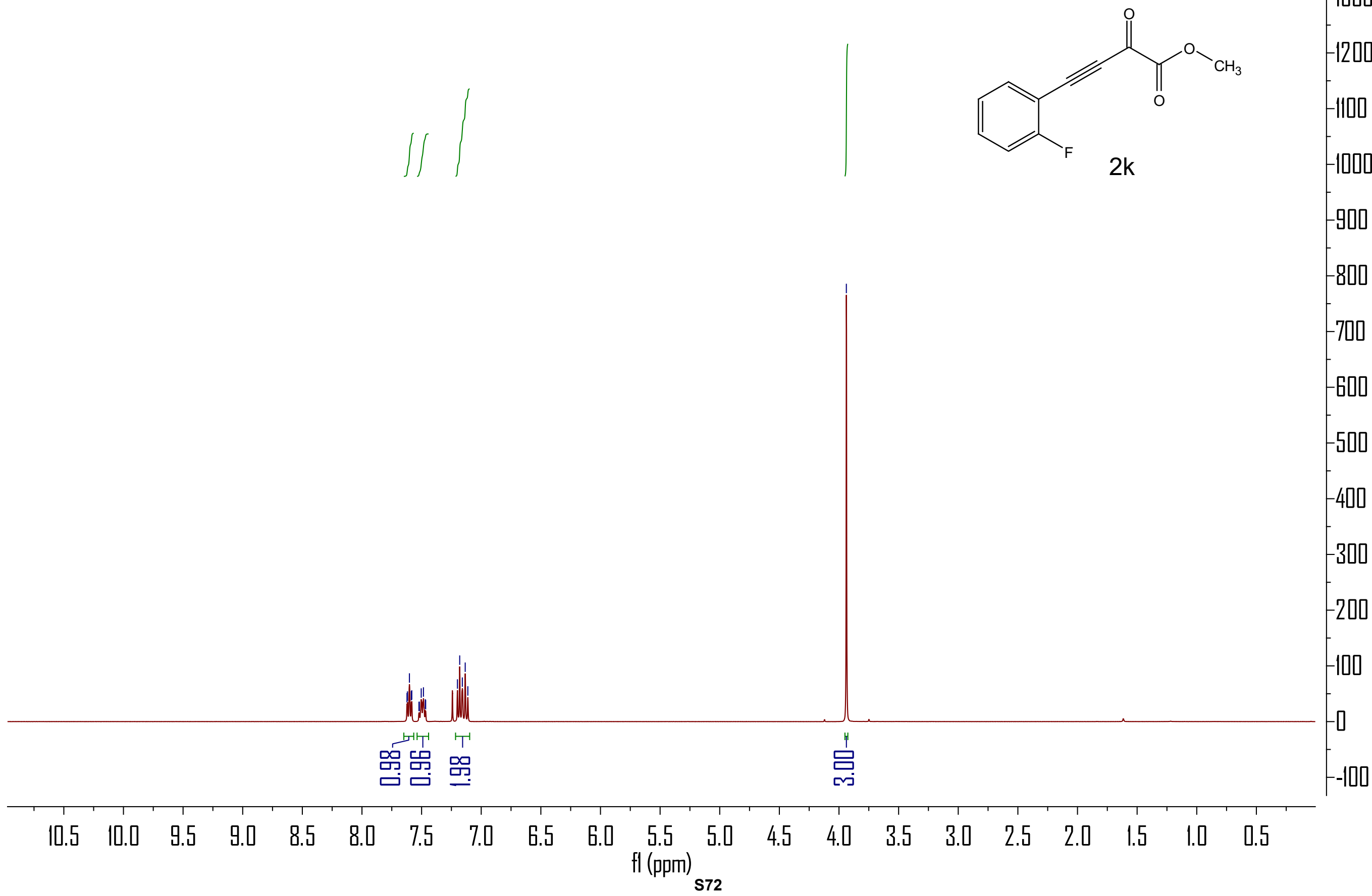
品

$-2300$

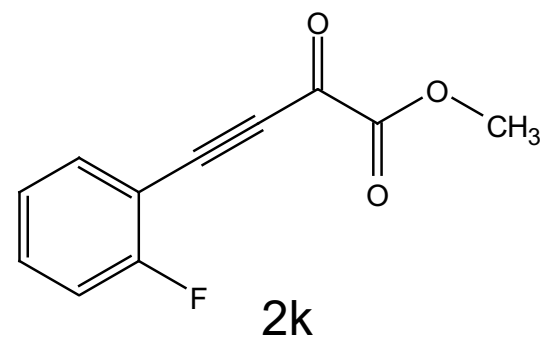

2200

$-2100$

$-2000$

$-1900$

$-1800$

$-1700$

$-1600$

$-1500$

$-1400$

$-1300$

$-1200$

$-1100$

1000

$-900$

$-800$

700

600

500

$-400$

300

$-200$

$-100$

$\begin{array}{llllllllllllllllllll}0 & -10 & -20 & -30 & -40 & -50 & -60 & -70 & -80 & -90 & -100 & -110 & -120 & -130 & -140 & -150 & -160 & -170 & -180 & -190\end{array}$ fl (ppm) 


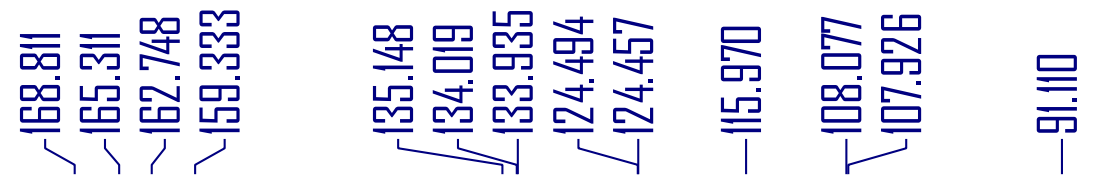

営

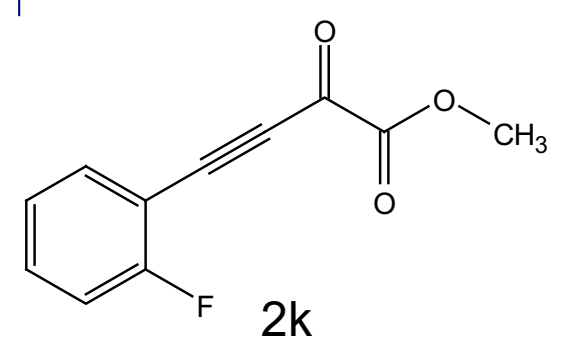

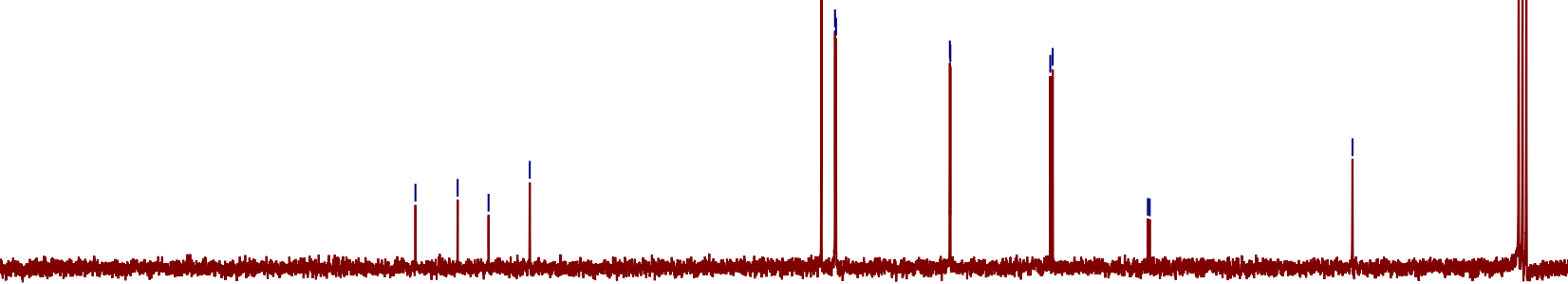

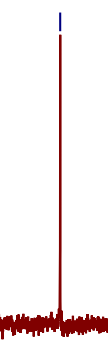



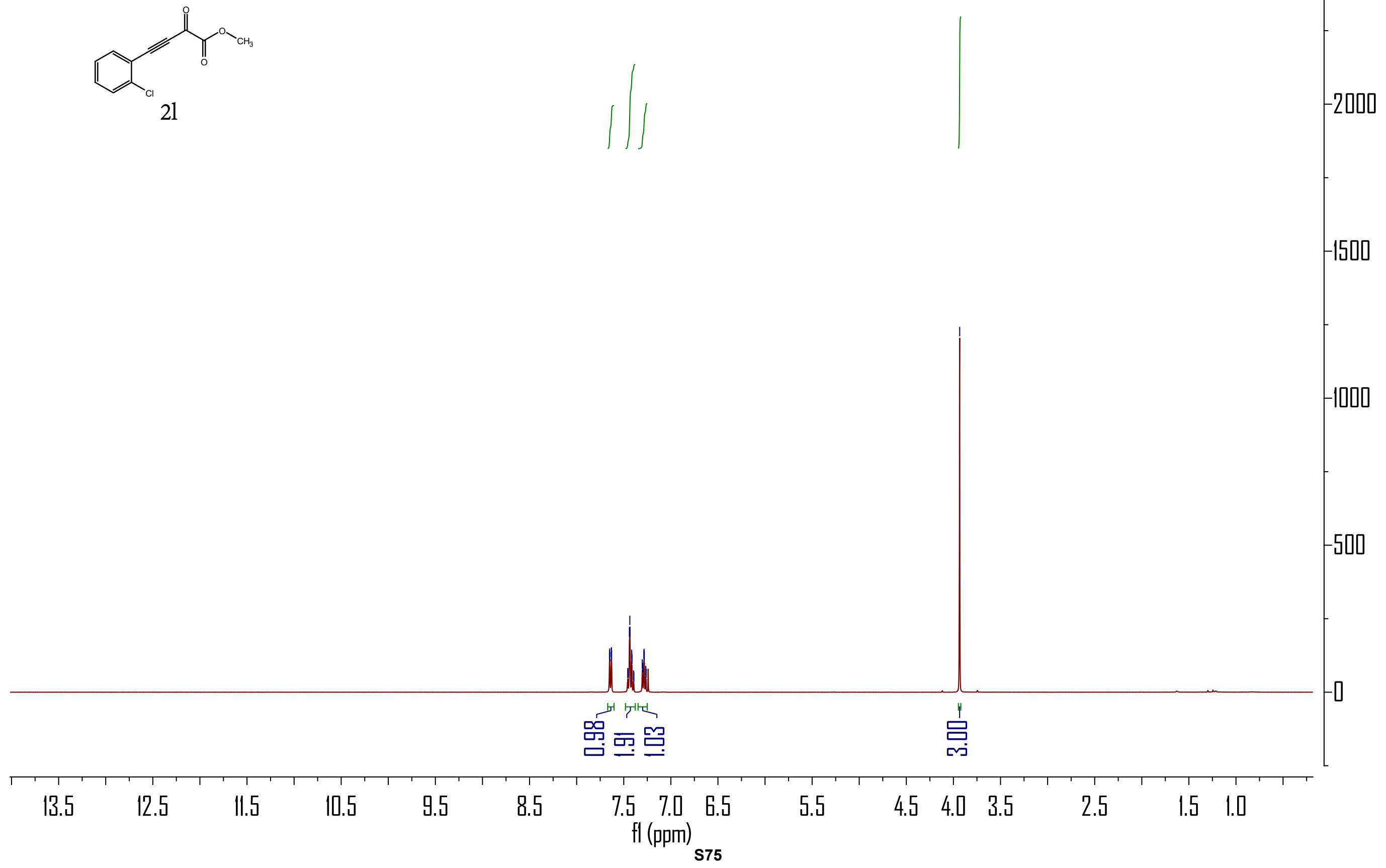


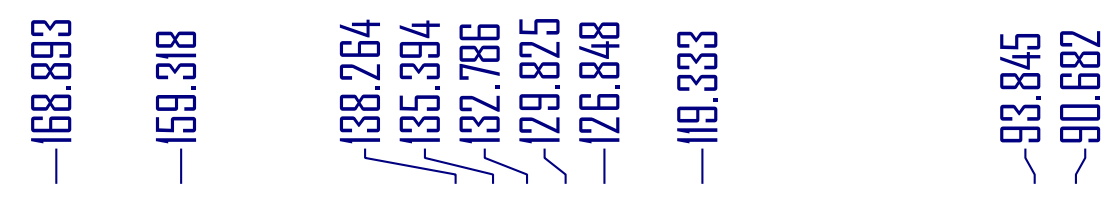

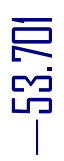

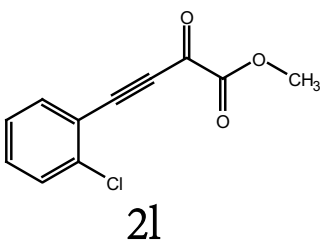

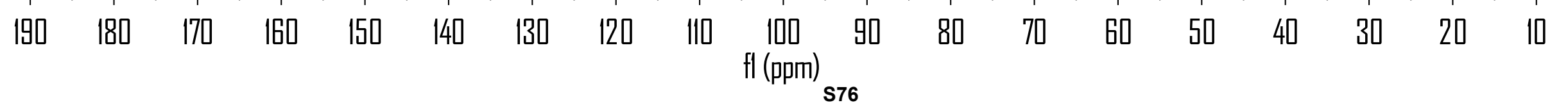



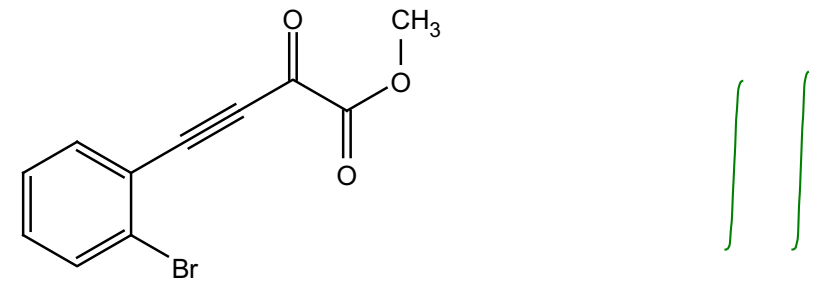

$-1900$

$-1800$

$-1700$

1600

$-1500$

$2 m$

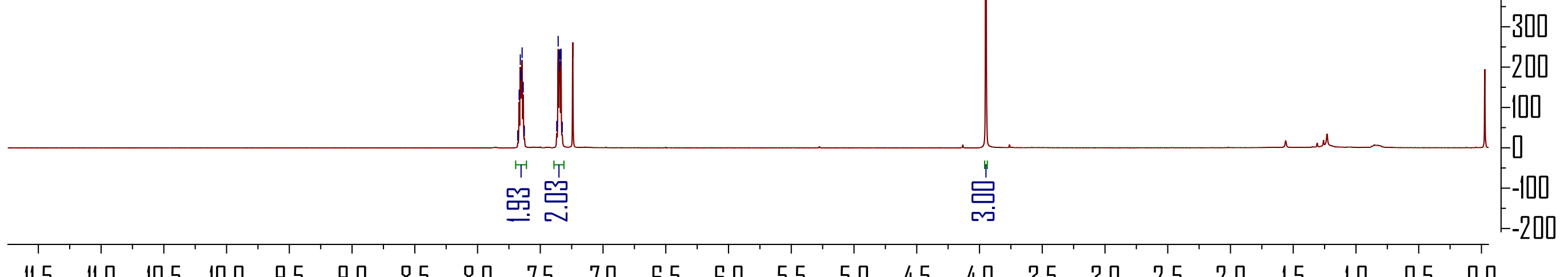

$\begin{array}{llllllllllllllllllllllll}11.5 & 11.0 & 10.5 & 10.0 & 9.5 & 9.0 & 8.5 & 8.0 & 7.5 & 7.0 & 6.5 & 6.0 & 5.5 & 5.0 & 4.5 & 4.0 & 3.5 & 3.0 & 2.5 & 2.0 & 1.5 & 1.0 & 0.5 & 0.0\end{array}$ fl (ppm) 


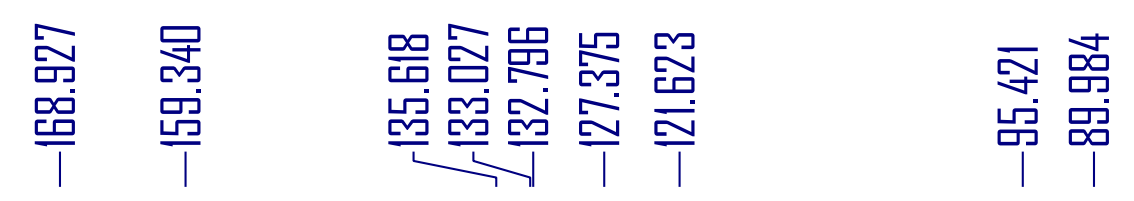

品
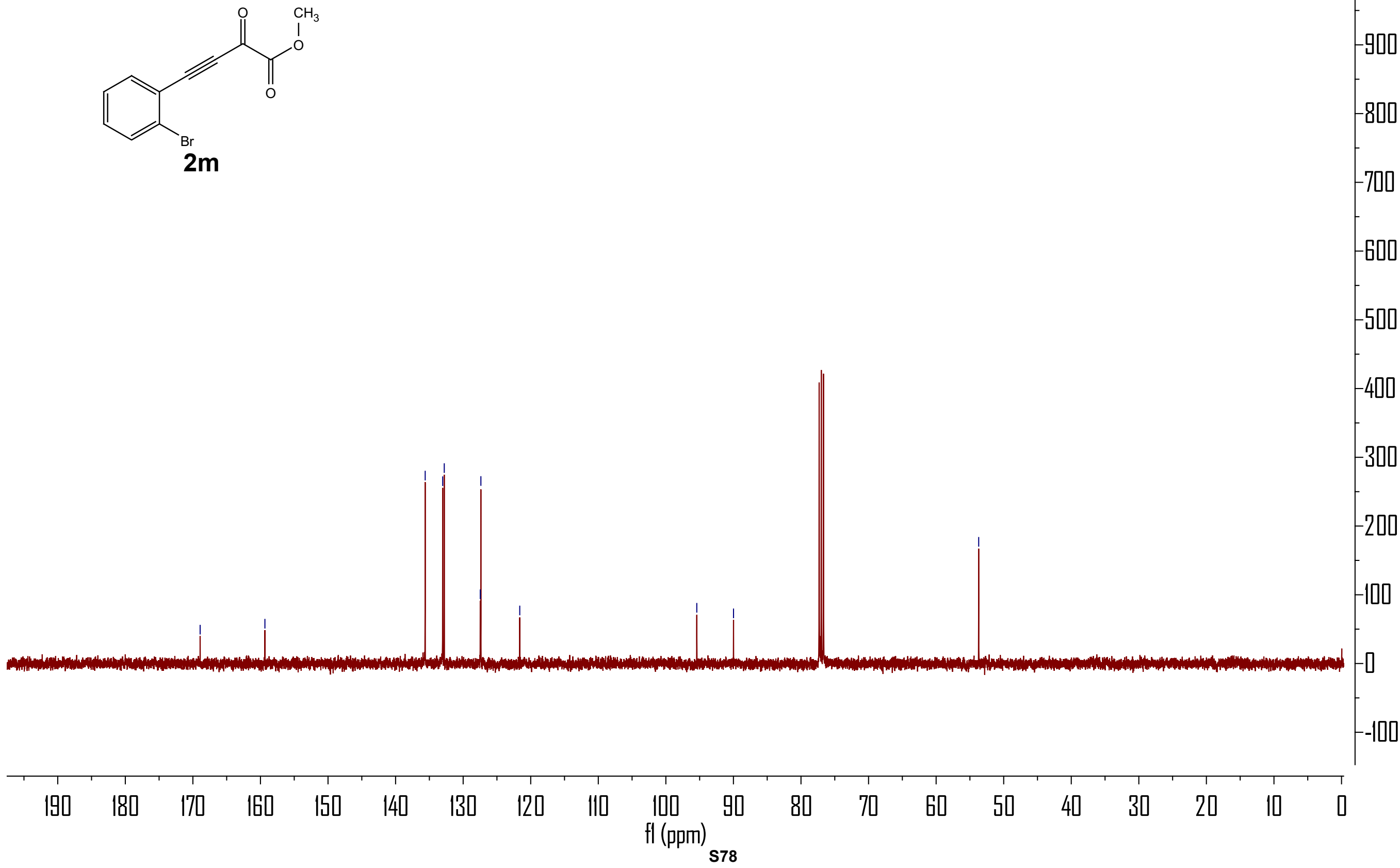


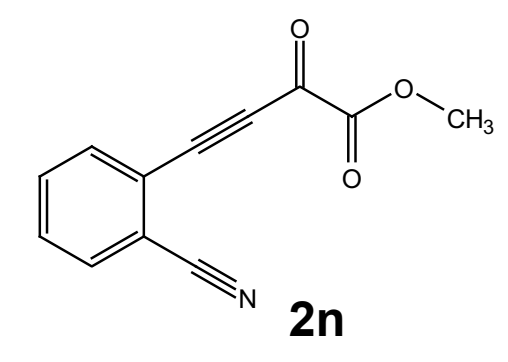




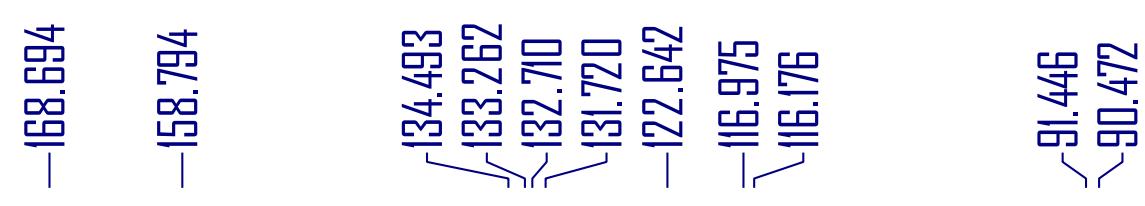

茄
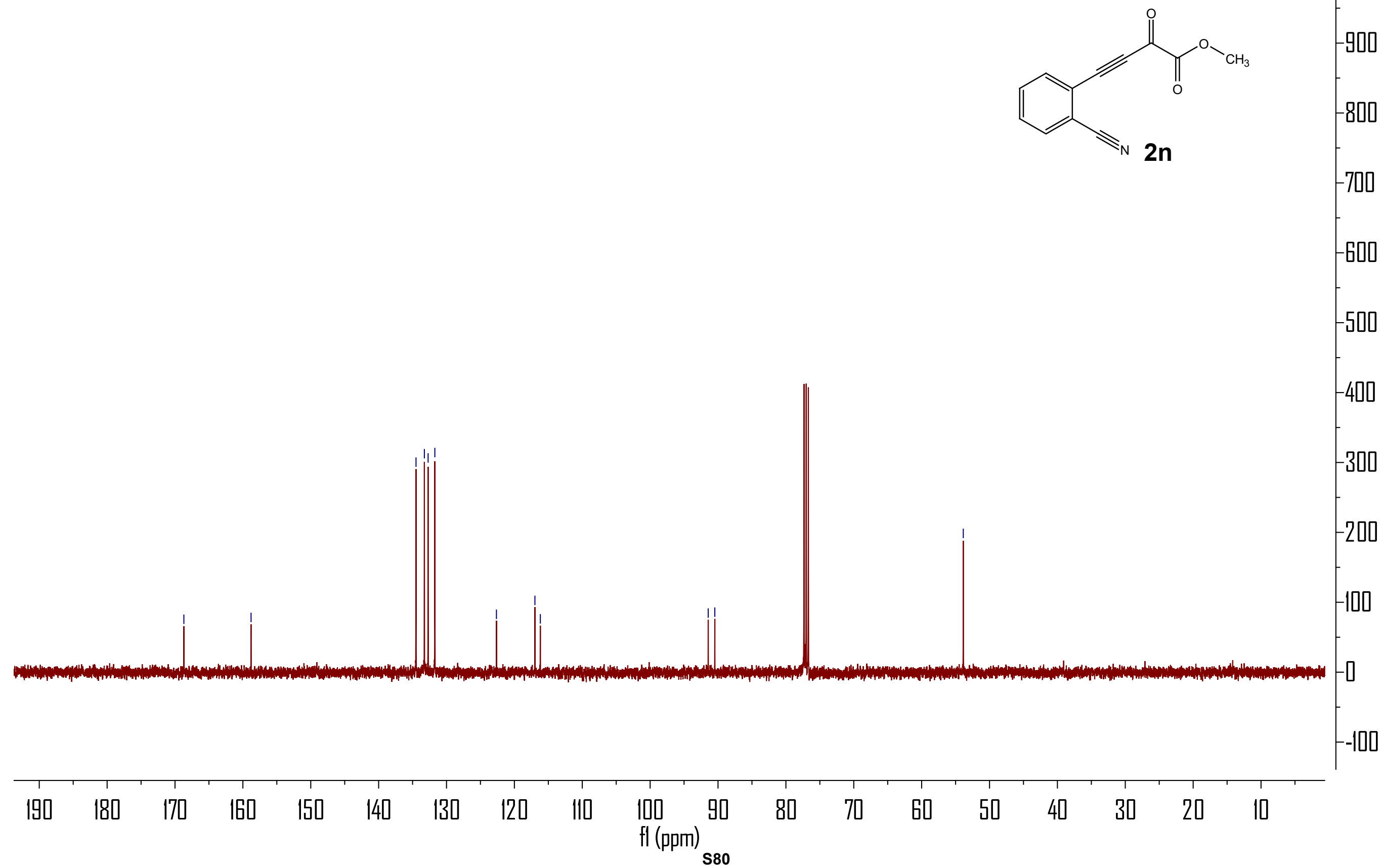


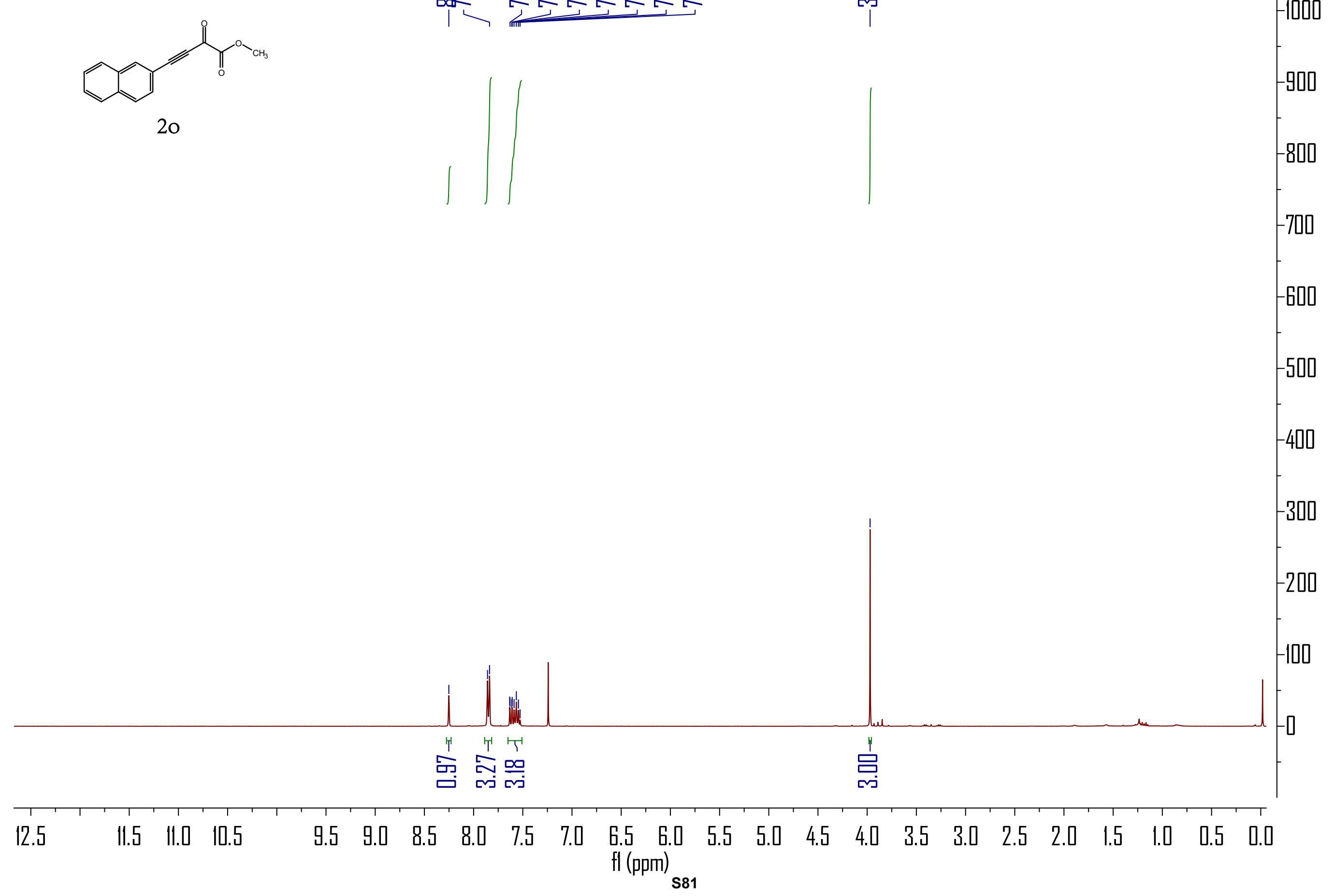




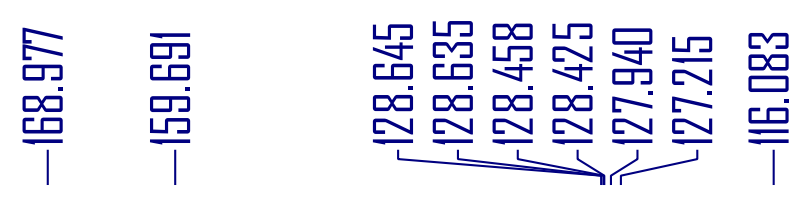

品哭

品

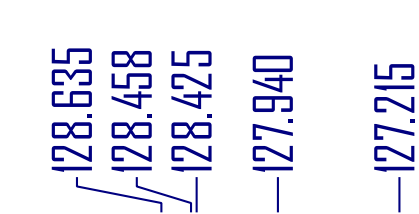

$-2000$

$-1500$

20

$-1600$

$-1500$

$-1400$

$-1300$

$-1200$

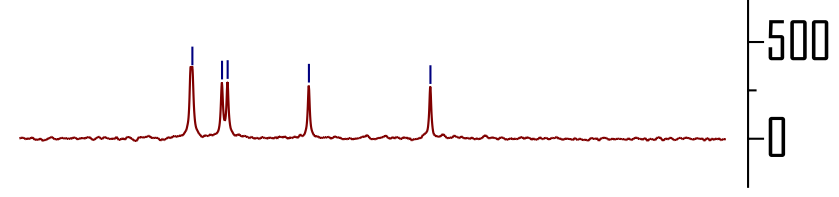

$129 \quad 128 \quad 127 \quad 126$ $\mathrm{fl}(\mathrm{ppm})$ 


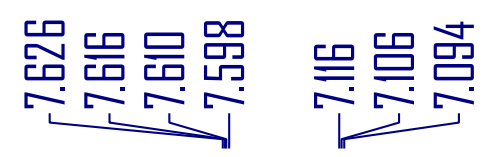

㩊
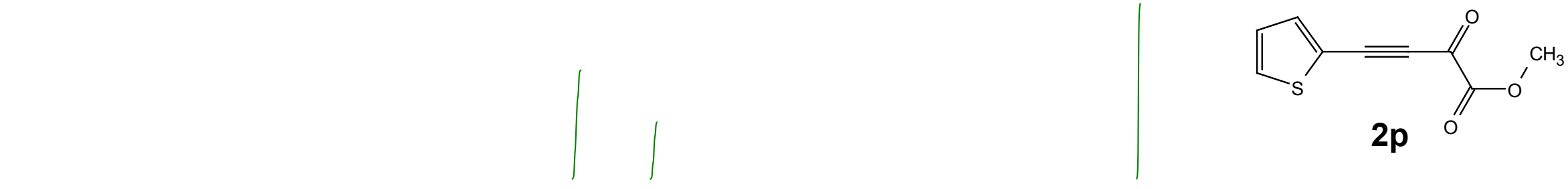


\begin{tabular}{|c|c|c|}
\hline 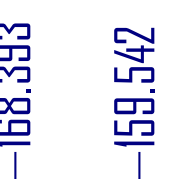 & 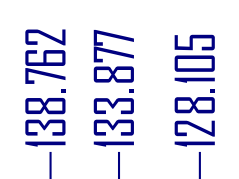 & $\begin{array}{l}\text { 몽 } \\
\text { 哭 } \\
=\end{array}$ \\
\hline
\end{tabular}

㗊
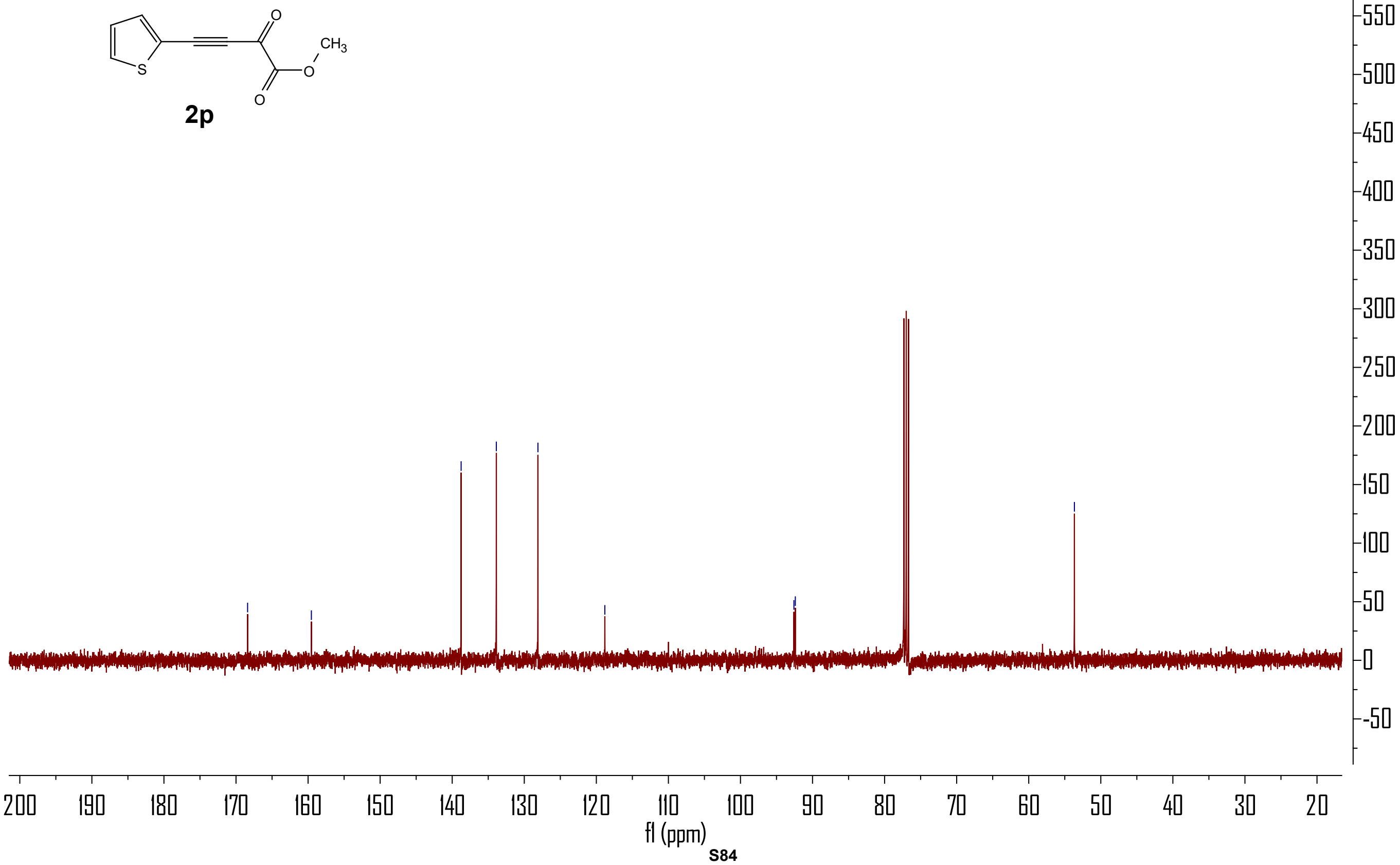


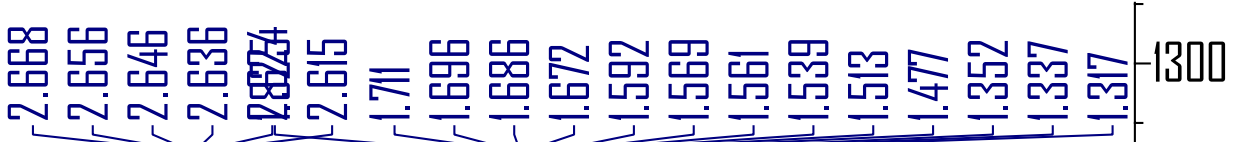

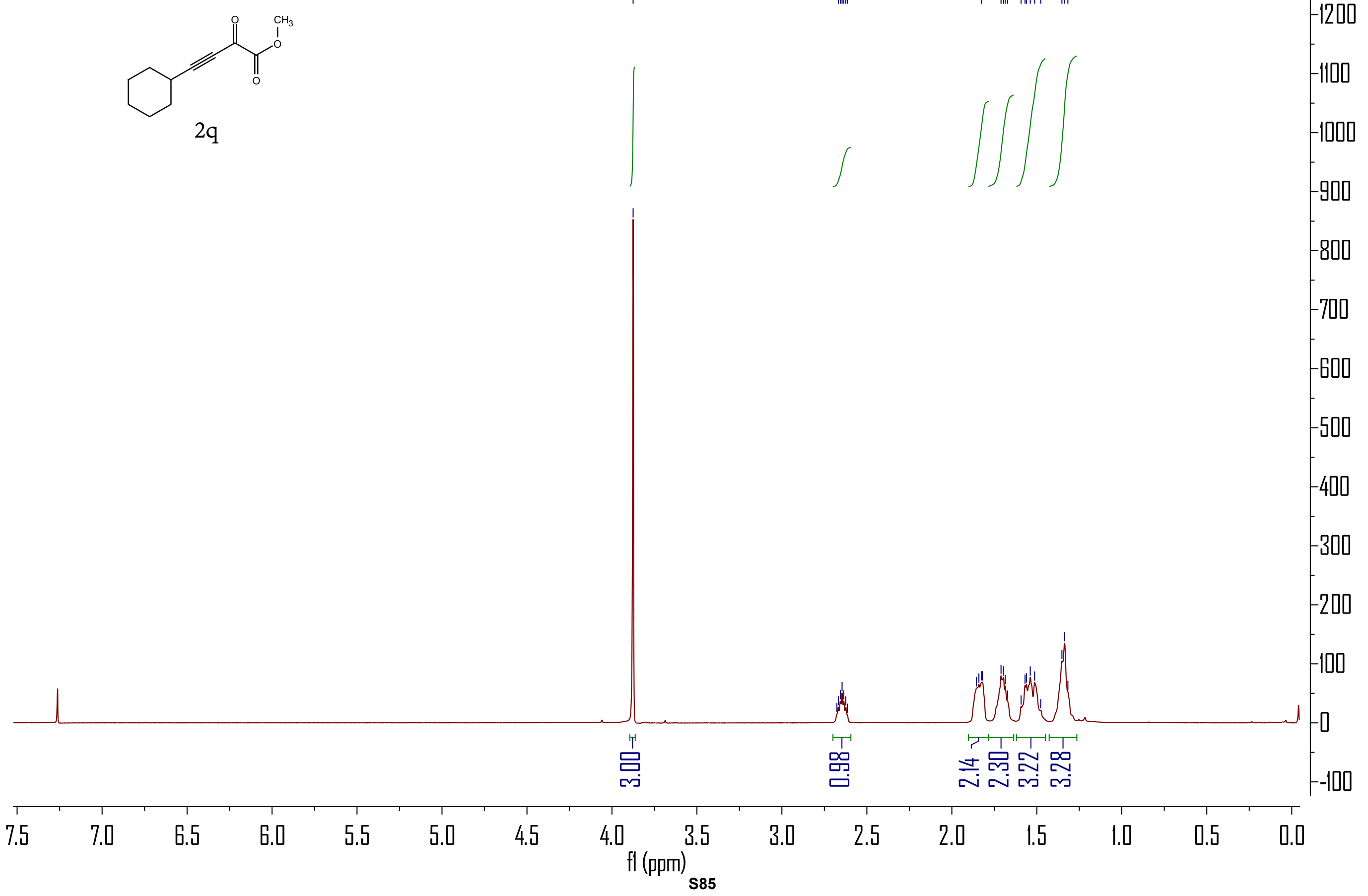


品 嵒
品 品

骂

点

突

品骂品哭罢
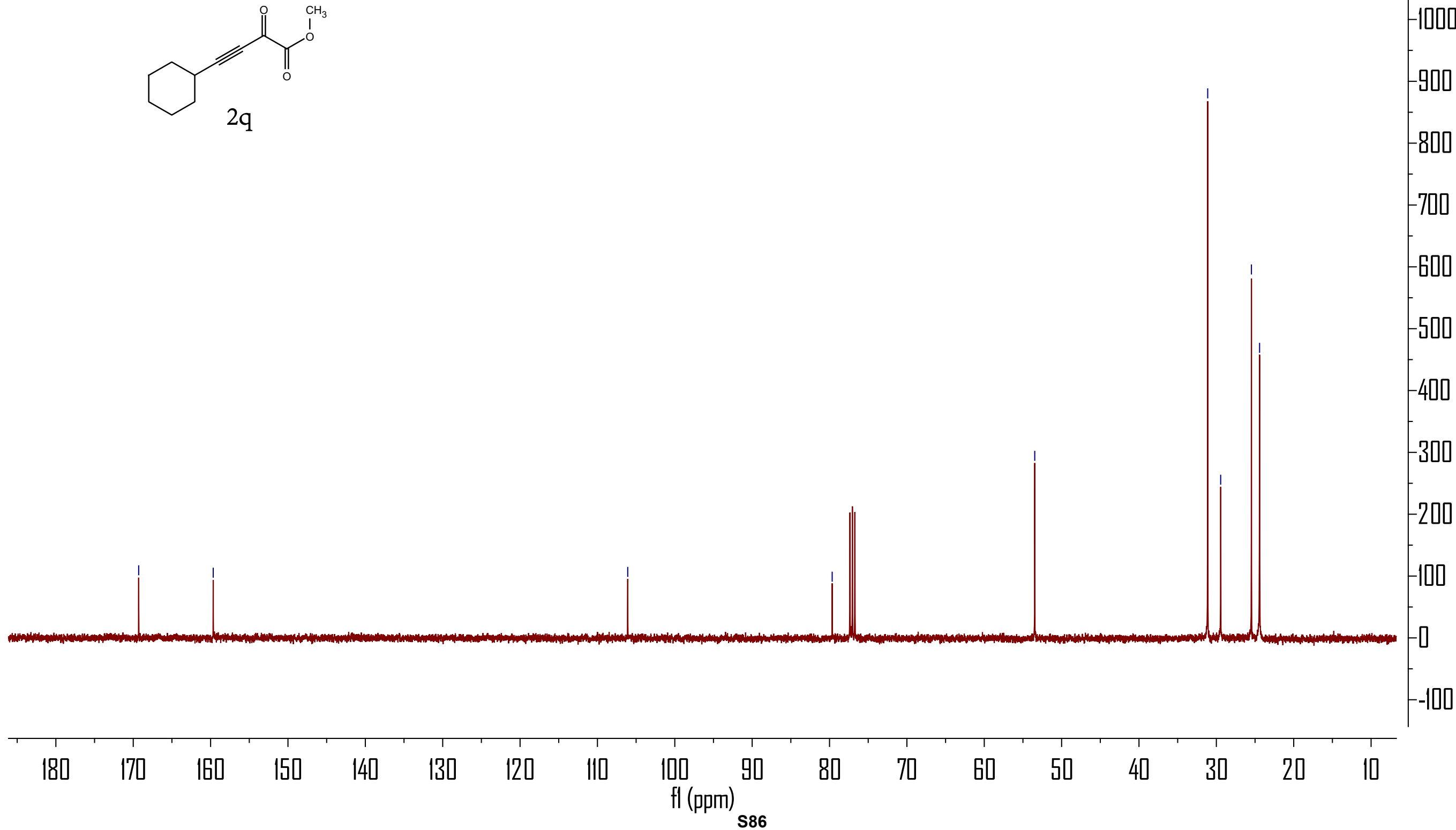


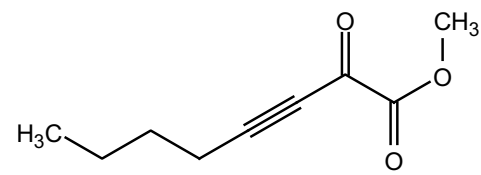

$2 s$
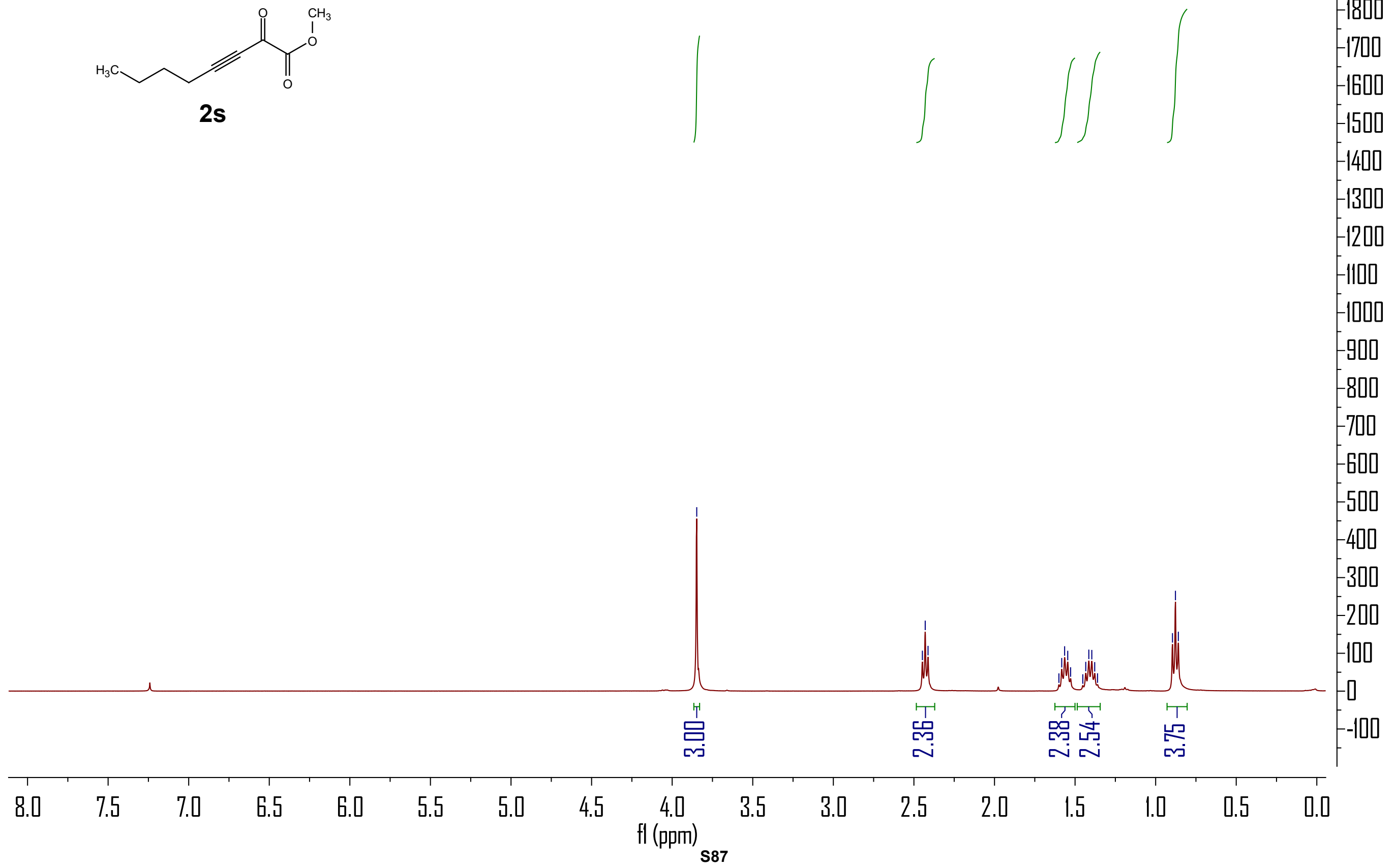
总
品 品

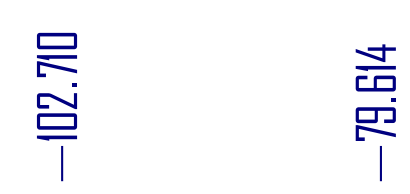

品

品 品 N

-1800
-1700
-1600
-1500
-1400
-1300
-1200
-1100
-1000
-900
-800
-700
-600
-500
-400
-300
-200
-100
-0
-100
-200

180

160

$150 \quad 14$

$130 \quad 120 \quad 110$

I0I (ppm)

的8 

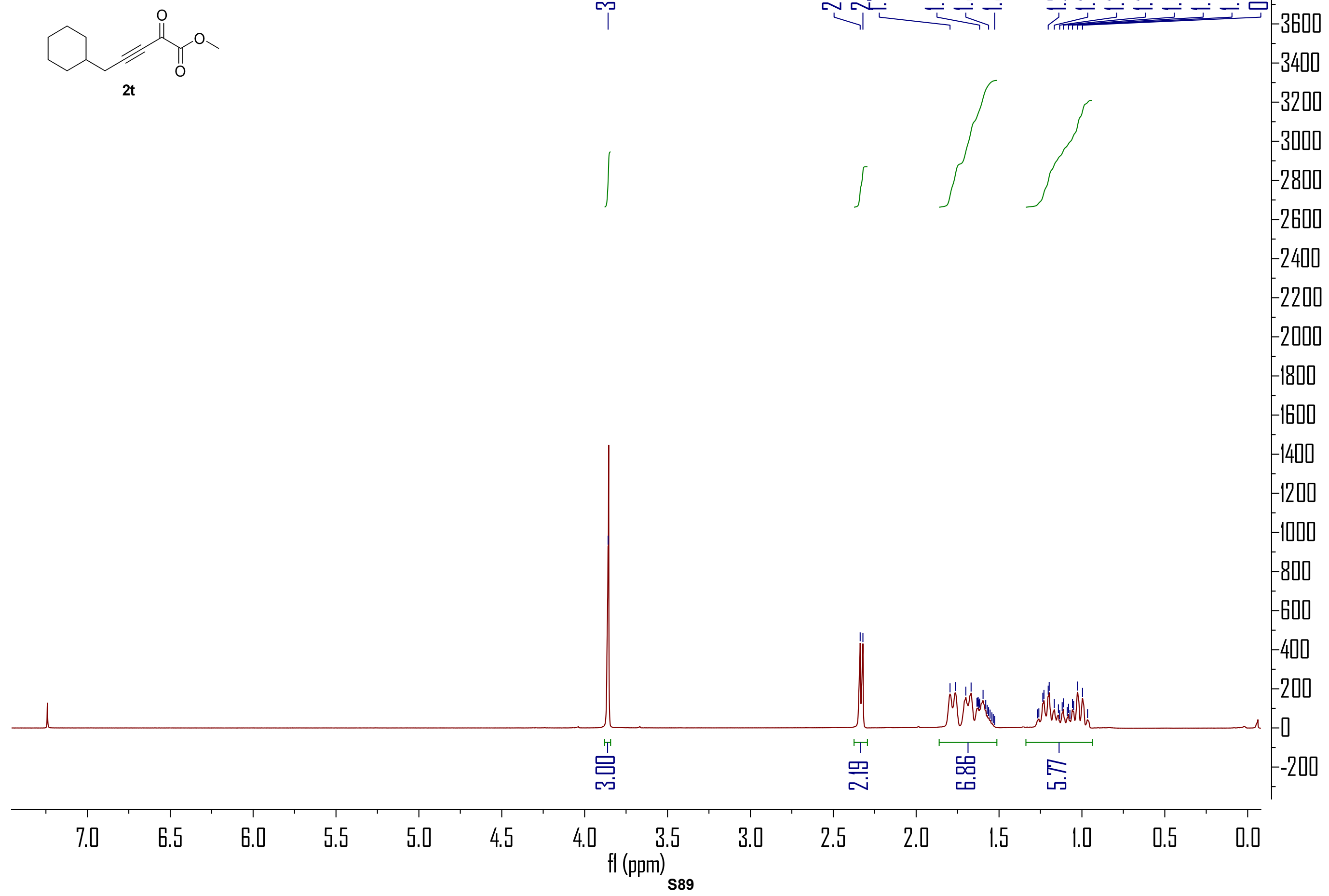
品
品 品
点

$\begin{array}{ll}\text { 名 } & \text { 㟧 } \\ \text { 号 } & \text { 品 }\end{array}$

器嵒嵒品虽

$-2600$

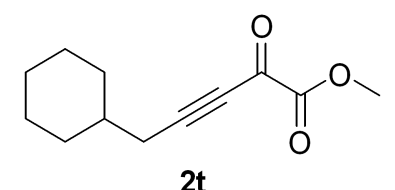

呼 嵒品哭虽

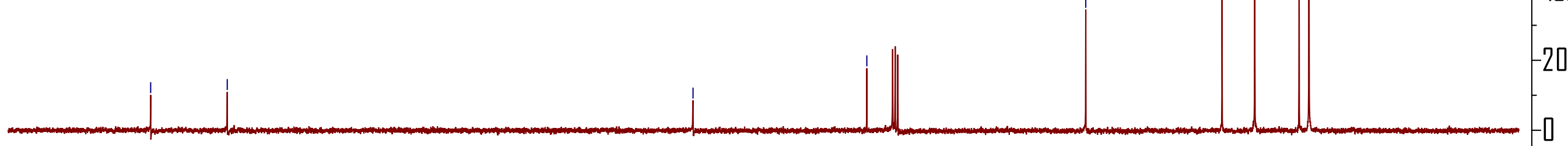

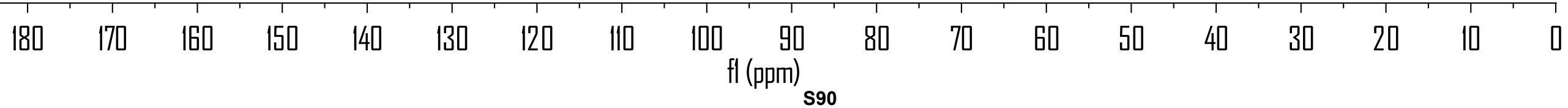




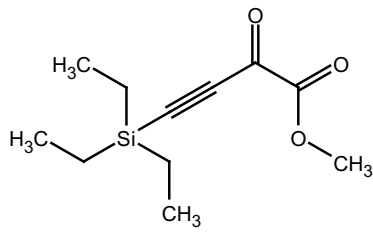

$2 u$

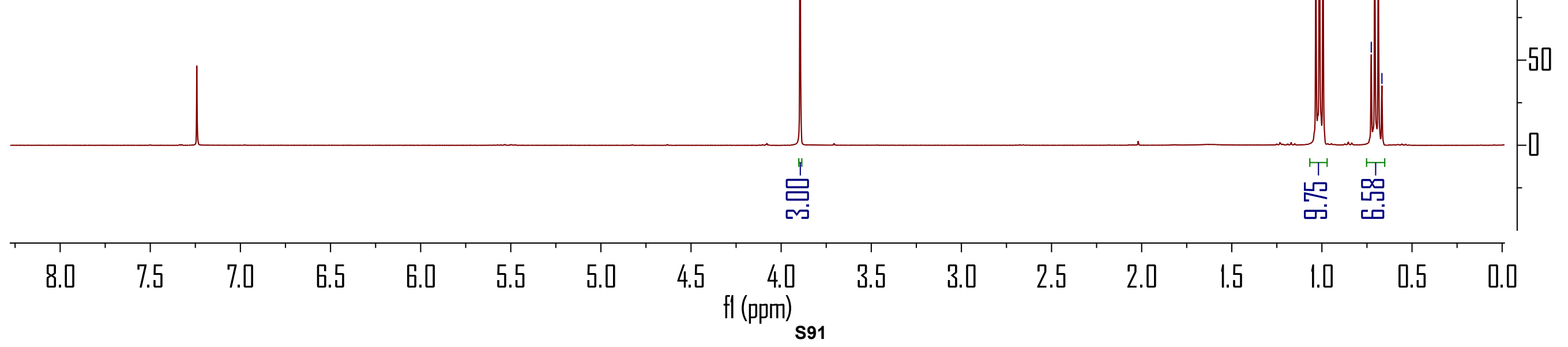




\section{罿翼}

甾顠

몸

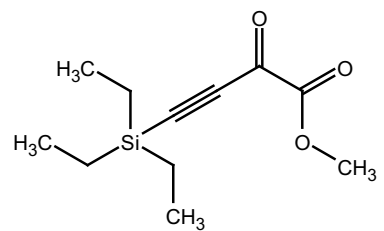

$2 u$

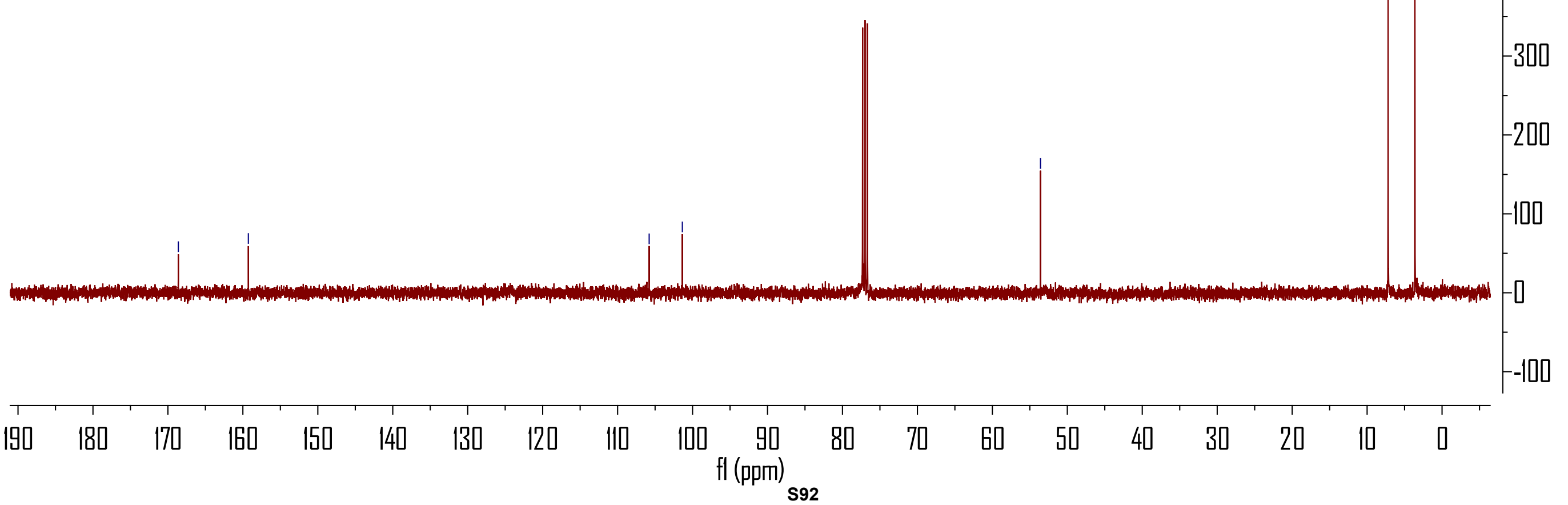




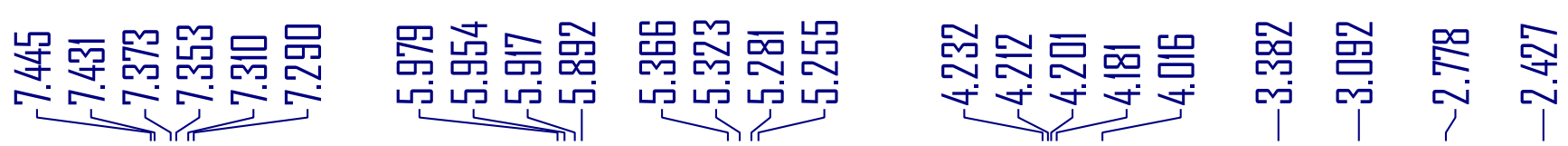

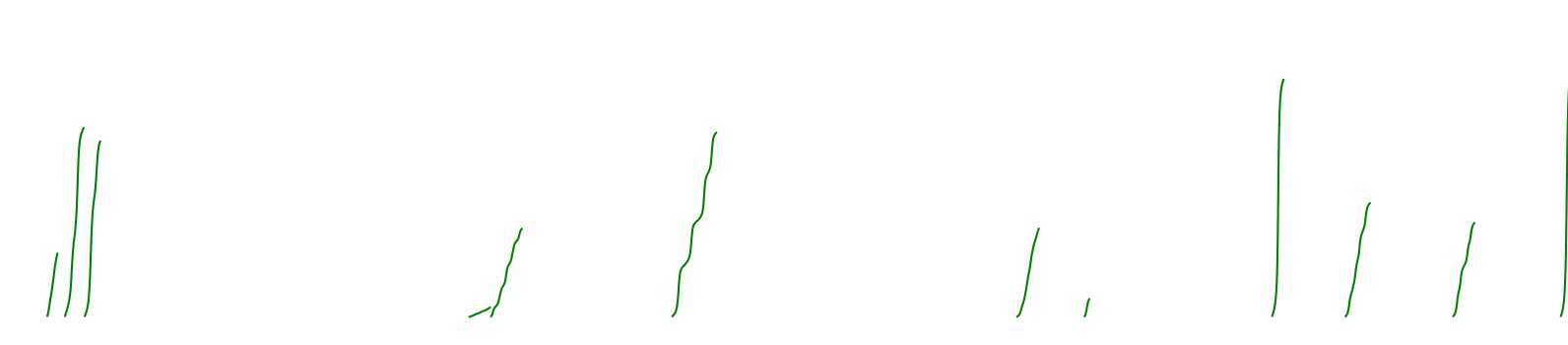

crude product
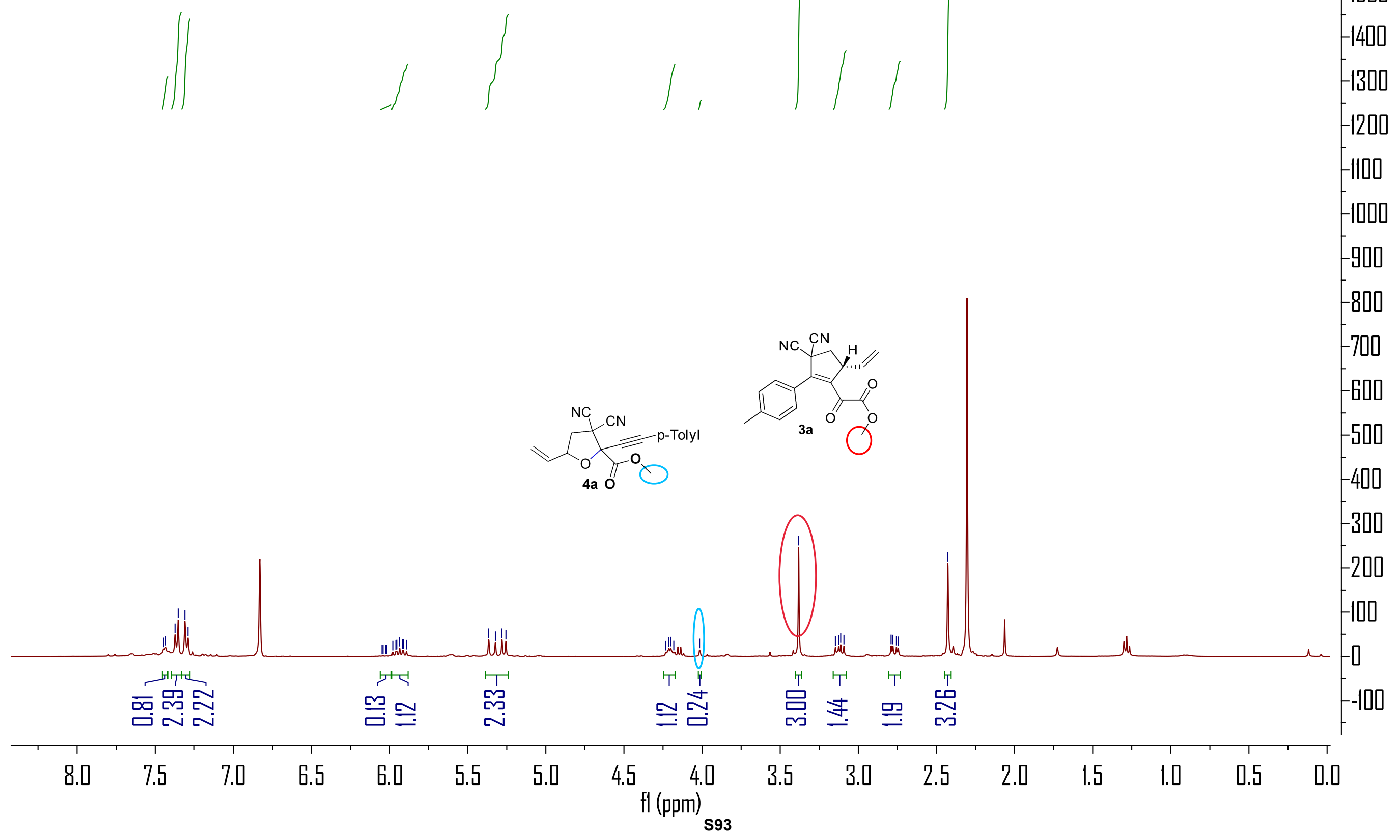


\section{==== Shimadzu LCsolution Analysis Report ====}

G:IDWP-6-31-3-RAC.Icd

$\begin{array}{ll}\text { Acquired by } & \text { : Admin } \\ \text { Sample Name } & \text { : DWP-6-31-3-RAC } \\ \text { Sample ID } & : \text { ID,98/2,0.5,214 } \\ \text { Vail \# } & : \text { 2 uL } \\ \text { Injection Volume } & : \text { DWP-6-31-3-RAC.Icd } \\ \text { Data File Name } & : \text { 123.Icm } \\ \text { Method File Name } & : \\ \text { Batch File Name } & : \text { Default.lcr } \\ \text { Report File Name } & : 2018-6-21 \text { 10:39:32 } \\ \text { Data Acquired } & : 2019-1-820: 13: 58 \\ \text { Data Processed } & \end{array}$

Sample Name

: DWP-6-31-3-RAC

DWP-6-31-3-RAC.Icd

2019-1-8 20:13:58

\section{$<$ Chromatogram $>$}

$\mathrm{mV}$

G:IDWP-6-31-3-RAC.Icd

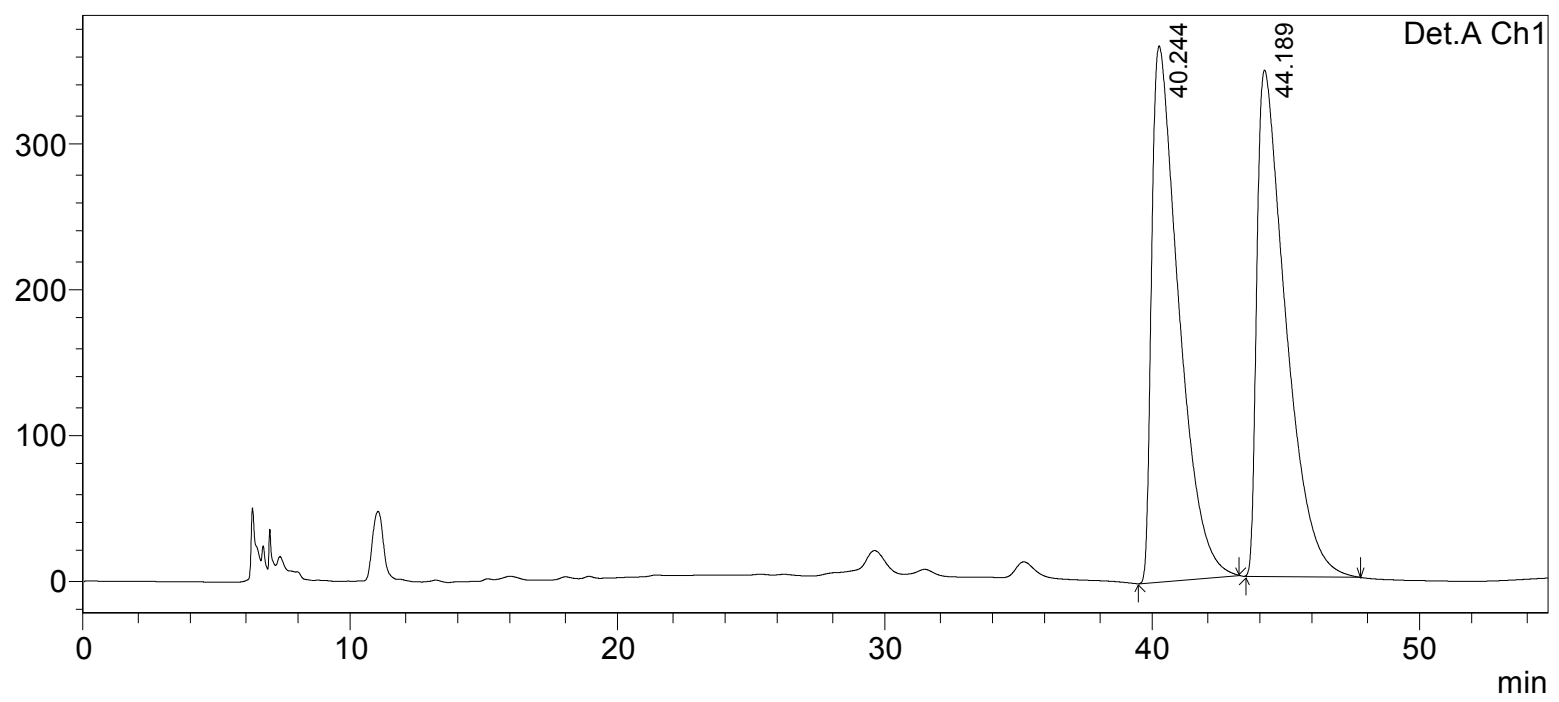

1 Det.A Ch1/214nm

PeakTable

Detector A Ch1 214nm

\begin{tabular}{|r|r|r|r|r|r|}
\hline \multicolumn{1}{|c|}{ Peak\# } & Ret. Time & Area & Height & \multicolumn{1}{|c|}{ Area \% } & \multicolumn{1}{|c|}{ Height \% } \\
\hline 1 & 40.244 & 26072677 & 368687 & 49.924 & 51.434 \\
\hline 2 & 44.189 & 26152504 & 348133 & 50.076 & 48.566 \\
\hline Total & & 52225181 & 716820 & 100.000 & 100.000 \\
\hline
\end{tabular}




\section{==== Shimadzu LCsolution Analysis Report ====}

$\begin{array}{ll}\text { Acquired by } & : \text { Admin } \\ \text { Sample Name } & : \text { dwp-8-101 } \\ \text { Sample ID } & : \text { ID,98/2,0.5,214 } \\ \text { Vail \# } & : \text { uL } \\ \text { Injection Volume } & : \text { dwp-8-101.Icd } \\ \text { Data File Name } & : \text { 123.Icm } \\ \text { Method File Name } & : \\ \text { Batch File Name } & : \text { Default.lcr } \\ \text { Report File Name } & : 2018-8-28 \text { 13:58:16 } \\ \text { Data Acquired } & : 2018-8-2814: 51: 07 \\ \text { Data Processed } & \end{array}$

G:Idwp-8-101.Icd

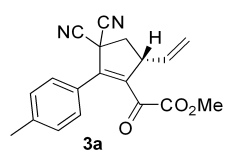

<Chromatogram>

$\mathrm{mV}$

G:Idwp-8-101.Icd

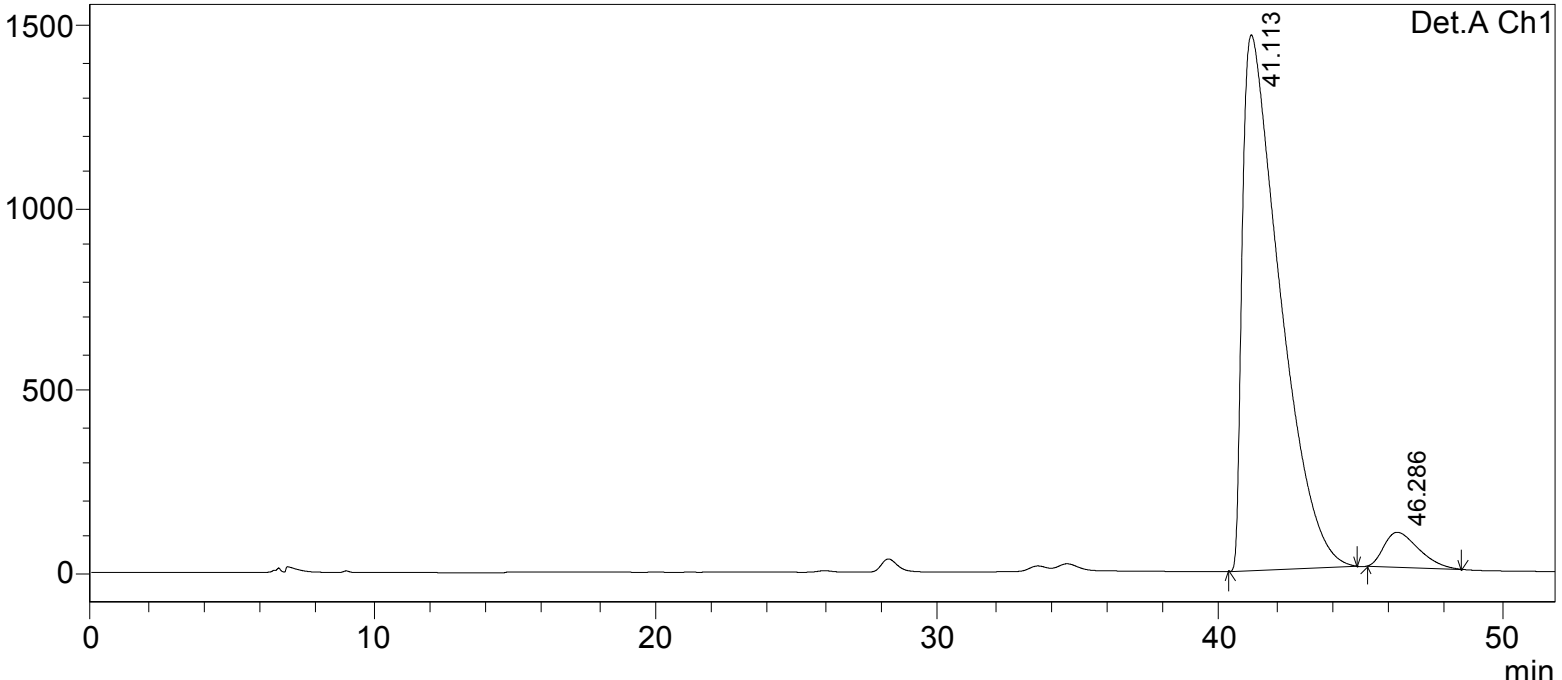

1 Det.A Ch1/214nm

PeakTable

Detector A Ch1 214nm

\begin{tabular}{|r|r|r|r|r|r|}
\hline \multicolumn{1}{|c|}{ Peak\# } & \multicolumn{1}{|c|}{ Ret. Time } & \multicolumn{1}{c|}{ Area } & \multicolumn{1}{c|}{ Height } & \multicolumn{1}{c|}{ Area \% } & \multicolumn{1}{c|}{ Height \% } \\
\hline 1 & 41.113 & 136131934 & 1470302 & 94.284 & 93.869 \\
\hline 2 & 46.286 & 8252956 & 96033 & 5.716 & 6.131 \\
\hline Total & & 144384890 & 1566335 & 100.000 & 100.000 \\
\hline
\end{tabular}


<smiles>C=C[C@@H]1CC(C#N)(C#N)C(c2ccc(C)cc2)=C1C(=O)C(C)=O</smiles>

ecrystallization

\section{==== Shimadzu LCsolution Analysis Report ====}

G:ICHONGIDWP-8-101-RAC.Icd

Acquired by

: Admin

Sample Name

: DWP-11-48-RAC

Sample ID

ID,90/10,0.7,214

Vail \#

Injection Volume

Data File Name

: $1 \mathrm{uL}$

: DWP-8-101-RAC.Icd

Method File Name

Batch File Name

Report File Name

Data Acquired

$: 1 . \mathrm{Icm}$

: Default.Icr

2019-5-27 16:18:01

: 2019-5-27 16:40:35

\section{$<$ Chromatogram $>$}

$\mathrm{mV}$

G:ICHONGIDWP-8-101-RAC.Icd

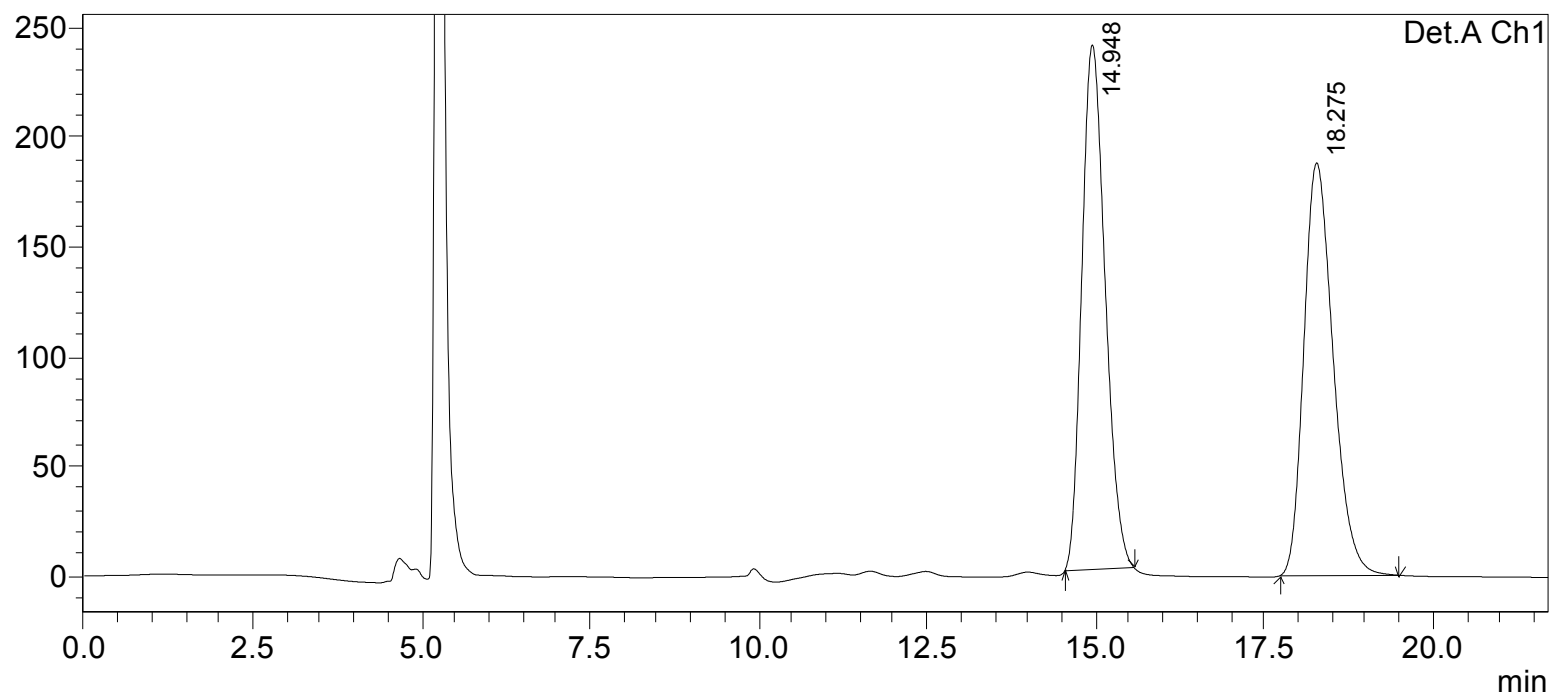

1 Det.A Ch1/214nm

PeakTable

Detector A Ch1 214nm

\begin{tabular}{|r|r|r|r|r|r|}
\hline Peak\# & Ret. Time & \multicolumn{1}{|c|}{ Area } & \multicolumn{1}{c|}{ Height } & \multicolumn{1}{c|}{ Area \% } & \multicolumn{1}{c|}{ Height \% } \\
\hline 1 & 14.948 & 5762693 & 239290 & 50.345 & 55.979 \\
\hline 2 & 18.275 & 5683755 & 188174 & 49.655 & 44.021 \\
\hline Total & & 11446447 & 427463 & 100.000 & 100.000 \\
\hline
\end{tabular}


$\overbrace{3 a}^{\mathrm{CO}_{2} \mathrm{Me}}$

recrystallization

\section{==== Shimadzu LCsolution Analysis Report ====}

$\begin{array}{ll}\text { Acquired by } & \text { : Admin G:ICHONG } \\ \text { Sample Name } & \text { : DWP-8-101-CHONG } \\ \text { Sample ID } & : \text { ID,90/10,0.7,214 } \\ \text { Vail \# } & : \text { 1 uL } \\ \text { Injection Volume } & : \text { DWP-8-101-CHONG.Icd } \\ \text { Data File Name } & : \text { 1.Icm } \\ \text { Method File Name } & : \\ \text { Batch File Name } & \text { : Default.lcr } \\ \text { Report File Name } & : \text { 2019-6-3 15:21:35 } \\ \text { Data Acquired } & : \text { 2019-6-3 15:48:56 } \\ \text { Data Processed } & \end{array}$

<Chromatogram>

G:ICHONGIDWP-8-101-CHONG.Icd

$\mathrm{mV}$

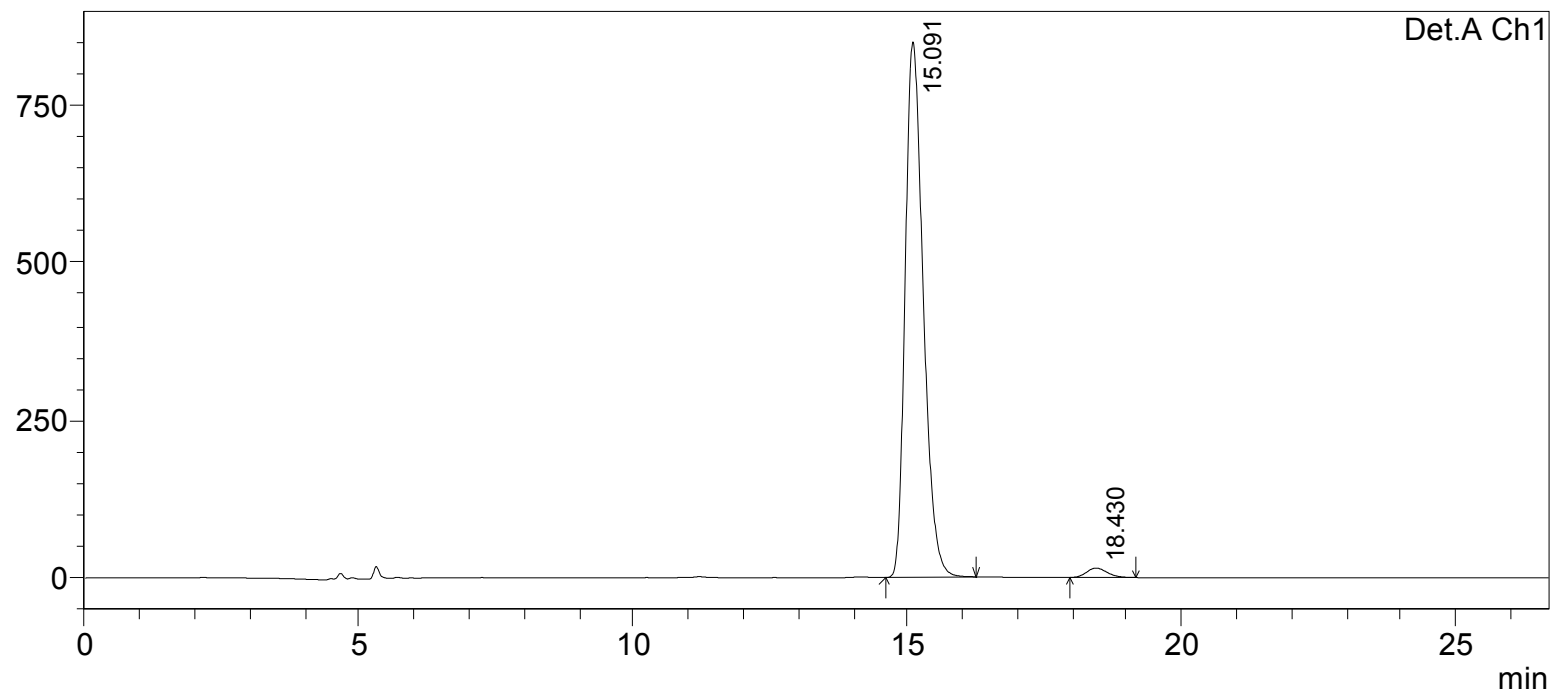

1 Det.A Ch1/214nm

PeakTable

Detector A Ch1 214nm

\begin{tabular}{|r|r|r|r|r|r|}
\hline \multicolumn{1}{|c|}{ Peak\# } & Ret. Time & \multicolumn{1}{|c|}{ Area } & \multicolumn{1}{|c|}{ Height } & \multicolumn{1}{c|}{ Area \% } & \multicolumn{1}{c|}{ Height \% } \\
\hline 1 & 15.091 & 19384410 & 850292 & 98.014 & 98.265 \\
\hline 2 & 18.430 & 392707 & 15015 & 1.986 & 1.735 \\
\hline Total & & 19777117 & 865308 & 100.000 & 100.000 \\
\hline
\end{tabular}




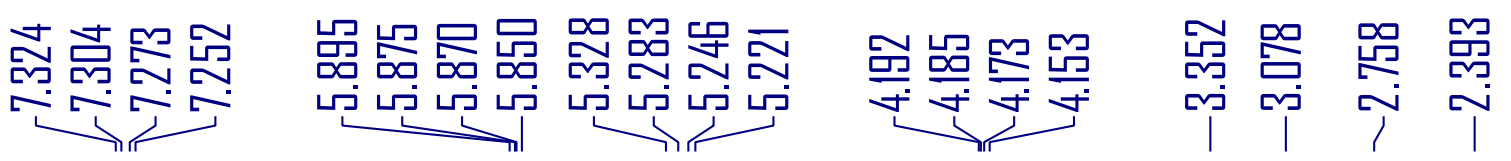

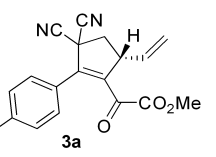
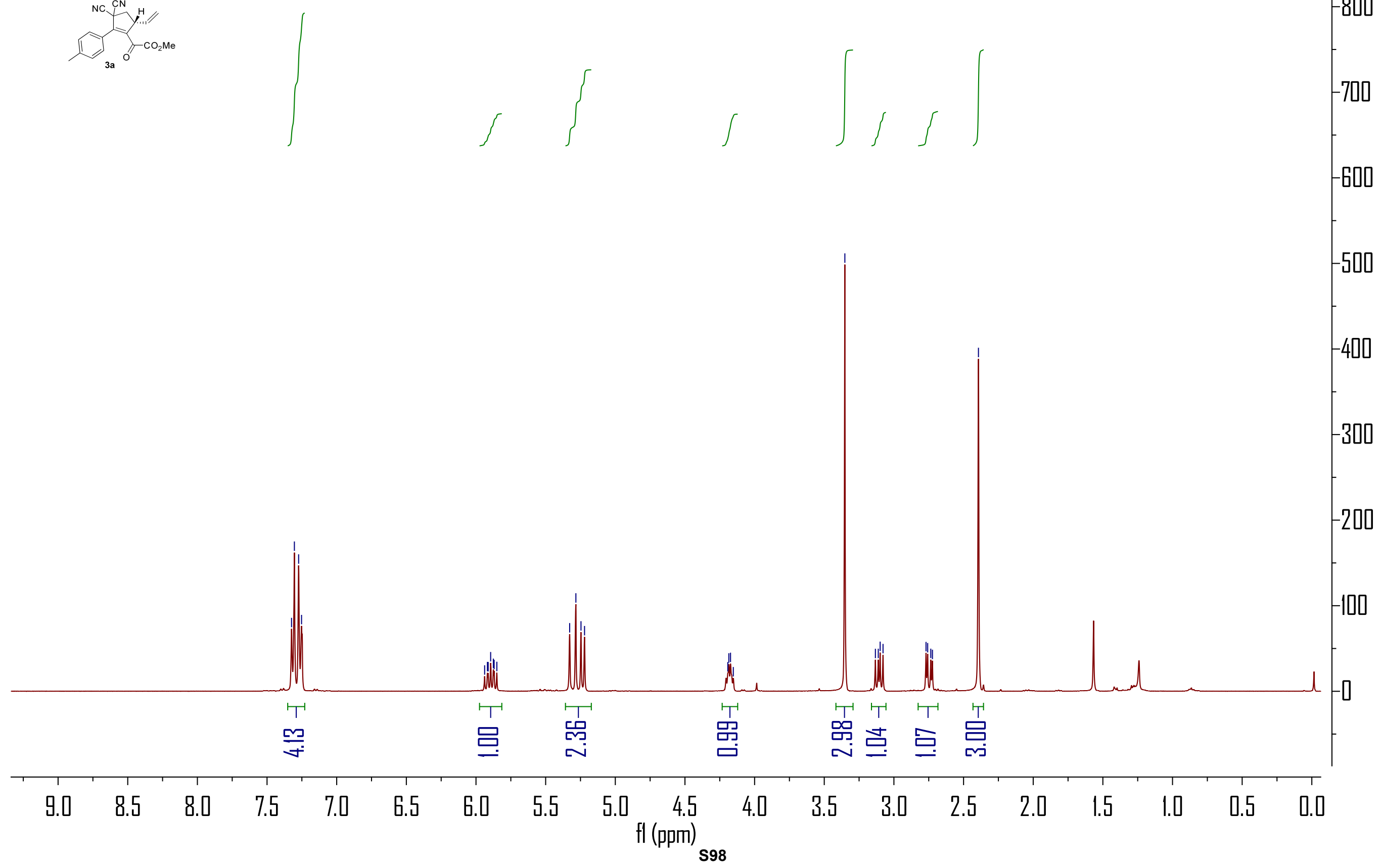


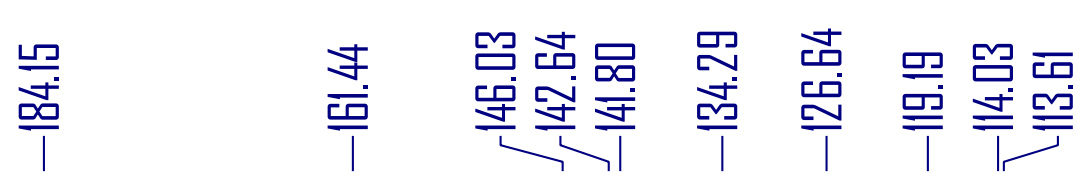

品品吉骂骂

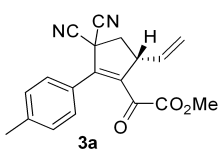

$\stackrel{\text { 邑 }}{=}$

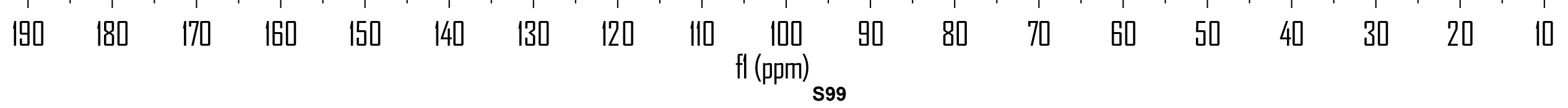




\section{DWP-10-69+- IG 9822140.7}

\begin{tabular}{|llll|}
\hline Sample Name: & DWP-10-69+- IG 982 214 0.7 & Injection Volume: & $\mathbf{3 . 0}$ \\
Vial Number: & RE1 & Channel: & UV_VIS_2 \\
Sample Type: & unknown & Wavelength: & $\mathbf{2 1 4 . 0}$ \\
Control Program: & test-dad4 & Bandwidth: & $\mathbf{4}$ \\
Quantif. Method: & $\mathbf{2 0 1 7 0 6 0 8}$ & Dilution Factor: & $\mathbf{1 . 0 0 0 0}$ \\
Recording Time: & $\mathbf{2 0 1 9 - 1 - 1 9 ~ 2 1 : 2 7}$ & Sample Weight: & $\mathbf{1 . 0 0 0 0}$ \\
Run Time (min): & $\mathbf{6 5 . 0 0}$ & Sample Amount: & $\mathbf{1 . 0 0 0 0}$ \\
\hline
\end{tabular}

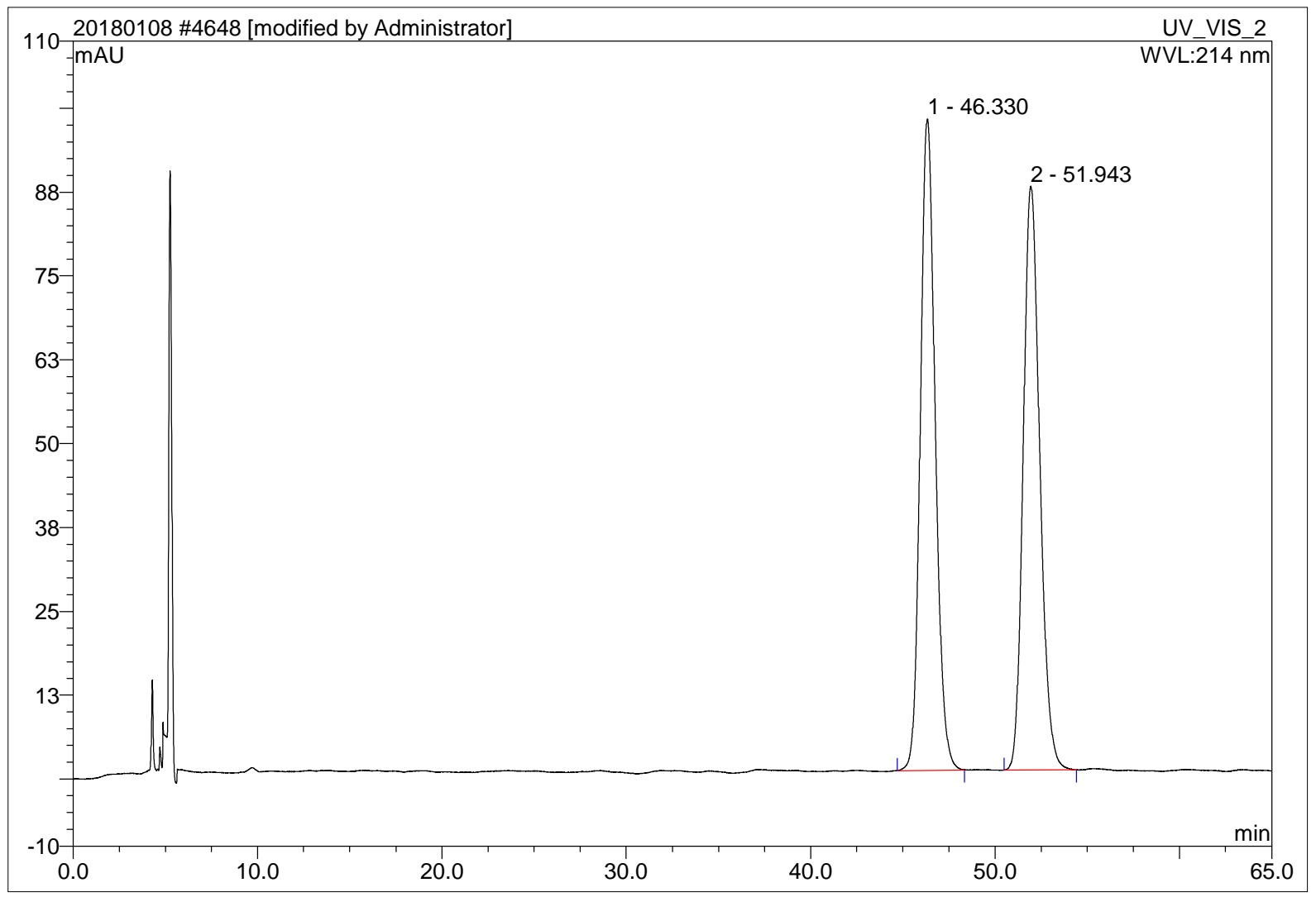

\begin{tabular}{|r|ccrrrrr|}
\hline No. & $\begin{array}{c}\text { Ret.Time } \\
\text { min }\end{array}$ & Peak Name & $\begin{array}{c}\text { Height } \\
\text { mAU }\end{array}$ & $\begin{array}{c}\text { Area } \\
\text { mAU*min }\end{array}$ & $\begin{array}{r}\text { Rel.Area } \\
\%\end{array}$ & Amount & Type \\
\hline 1 & 46.33 & n.a. & 97.159 & 91.111 & 50.17 & n.a. & BMB $^{*}$ \\
2 & 51.94 & n.a. & 87.085 & 90.509 & 49.83 & n.a. & BMB $^{*}$ \\
\hline Total: & & & 184.244 & 181.620 & 100.00 & 0.000 & \\
\hline
\end{tabular}




\section{DWP-10-70 IG 9822140.7}

\begin{tabular}{|llll|}
\hline Sample Name: & DWP-10-70 IG 982 214 0.7 & Injection Volume: & $\mathbf{3 . 0}$ \\
Vial Number: & RC1 & Channel: & UV_VIS_2 \\
Sample Type: & unknown & Wavelength: & $\mathbf{2 1 4 . 0}$ \\
Control Program: & test-dad4 & Bandwidth: & $\mathbf{4}$ \\
Quantif. Method: & $\mathbf{2 0 1 7 0 6 0 8}$ & Dilution Factor: & $\mathbf{1 . 0 0 0 0}$ \\
Recording Time: & $\mathbf{2 0 1 9 - 1 - 1 9 ~ 2 3 : 4 0}$ & Sample Weight: & $\mathbf{1 . 0 0 0 0}$ \\
Run Time (min): & $\mathbf{6 5 . 0 0}$ & Sample Amount: & $\mathbf{1 . 0 0 0 0}$ \\
\hline
\end{tabular}

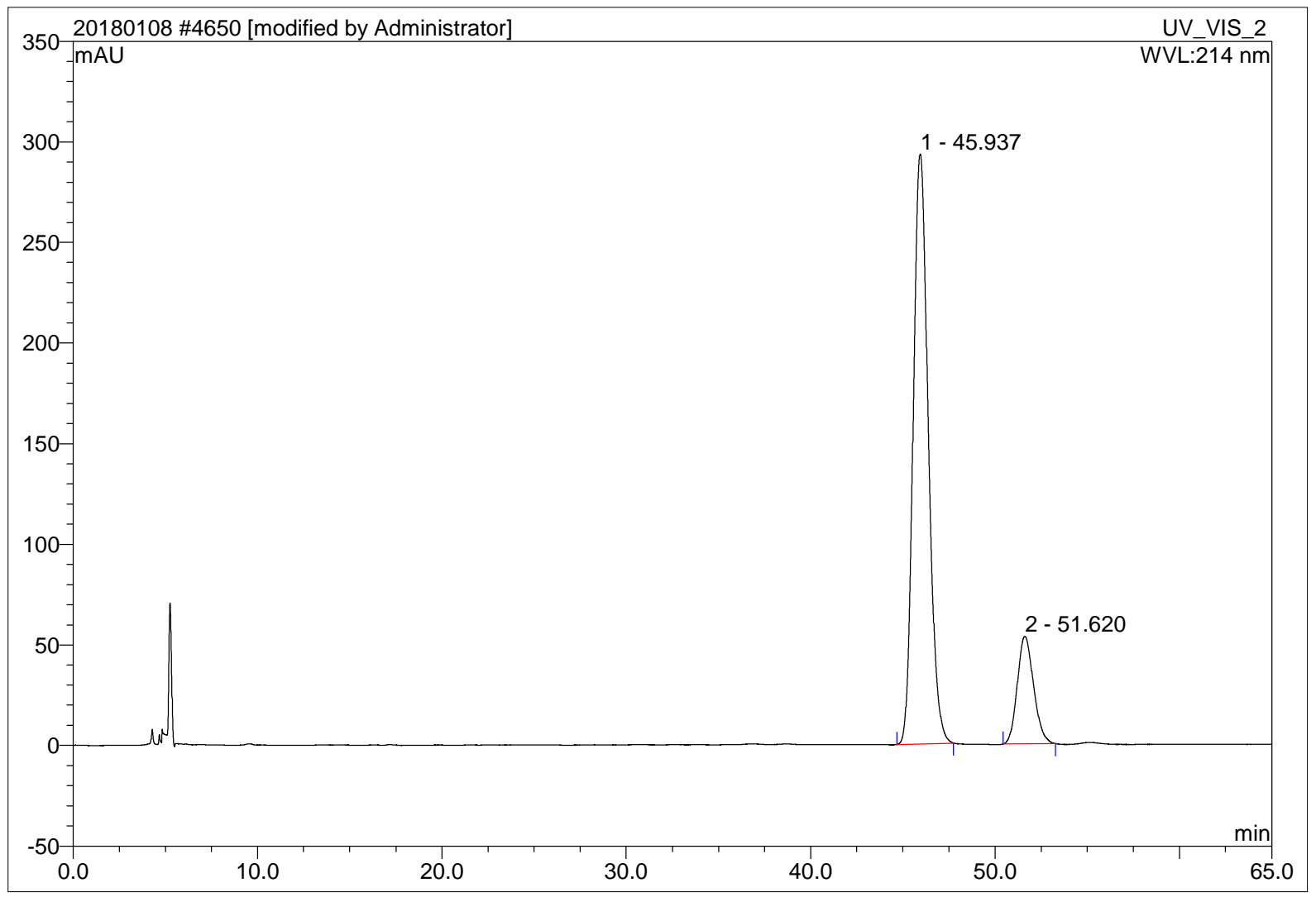

\begin{tabular}{|r|ccrrrrr|}
\hline No. & $\begin{array}{c}\text { Ret.Time } \\
\text { min }\end{array}$ & Peak Name & $\begin{array}{c}\text { Height } \\
\text { mAU }\end{array}$ & $\begin{array}{c}\text { Area } \\
\text { mAU*min }\end{array}$ & $\begin{array}{r}\text { Rel.Area } \\
\%\end{array}$ & Amount & Type \\
\hline 1 & 45.94 & n.a. & 293.332 & 276.190 & 83.43 & n.a. & BMB \\
2 & 51.62 & n.a. & 53.511 & 54.869 & 16.57 & n.a. & BMB \\
\hline Total: & & & 346.843 & 331.058 & 100.00 & 0.000 & \\
\hline
\end{tabular}




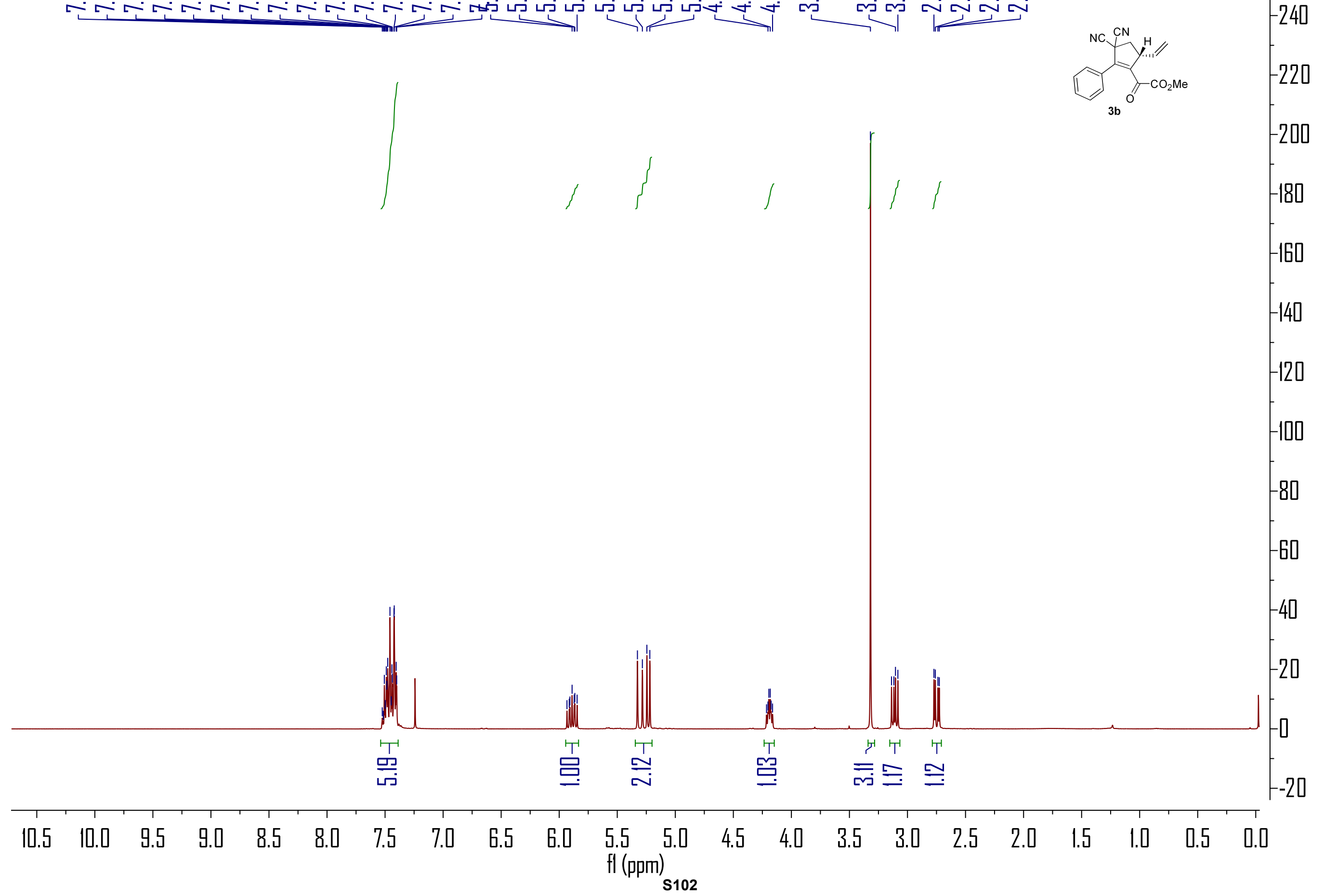




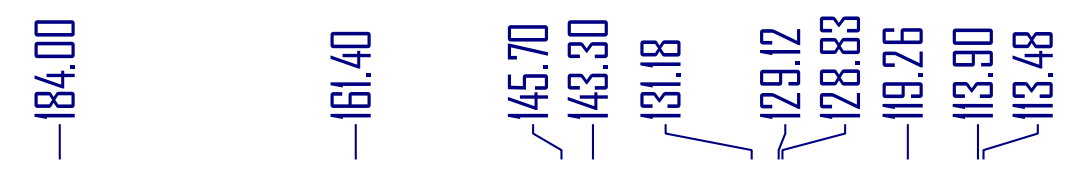

든도

获沵

므 尔듬

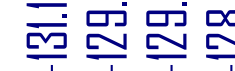

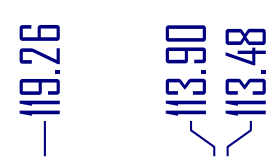

$-1000$

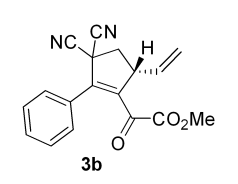

$130 \quad 125 \quad 120 \quad 115$

fl (ppm)

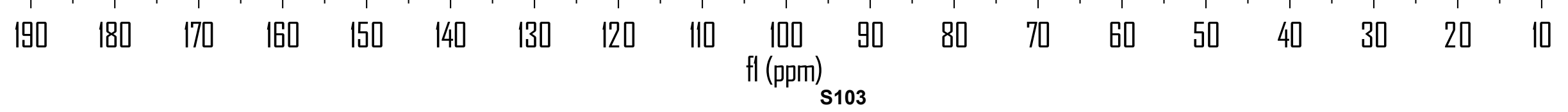




\begin{tabular}{|llll|}
\hline 4481 DWP-10-6+- IB 912540.7 & & \\
& & & \\
\hline Sample Name: & DWP-10-6+- IB 912540.7 & Injection Volume: & $\mathbf{5 . 0}$ \\
Vial Number: & GE4 & Channel: & EXT254NM \\
Sample Type: & unknown & Wavelength: & $\mathbf{2 5 4}$ \\
Control Program: & test-dad6 & Bandwidth: & $\mathbf{0}$ \\
Quantif. Method: & $\mathbf{2 0 1 7 0 6 0 8}$ & Dilution Factor: & $\mathbf{1 . 0 0 0 0}$ \\
Recording Time: & $\mathbf{2 0 1 9 - 1 - 1 1 ~ 1 : 3 4}$ & Sample Weight: & $\mathbf{1 . 0 0 0 0}$ \\
Run Time (min): & $\mathbf{3 0 . 0 0}$ & Sample Amount: & $\mathbf{1 . 0 0 0 0}$ \\
\hline
\end{tabular}

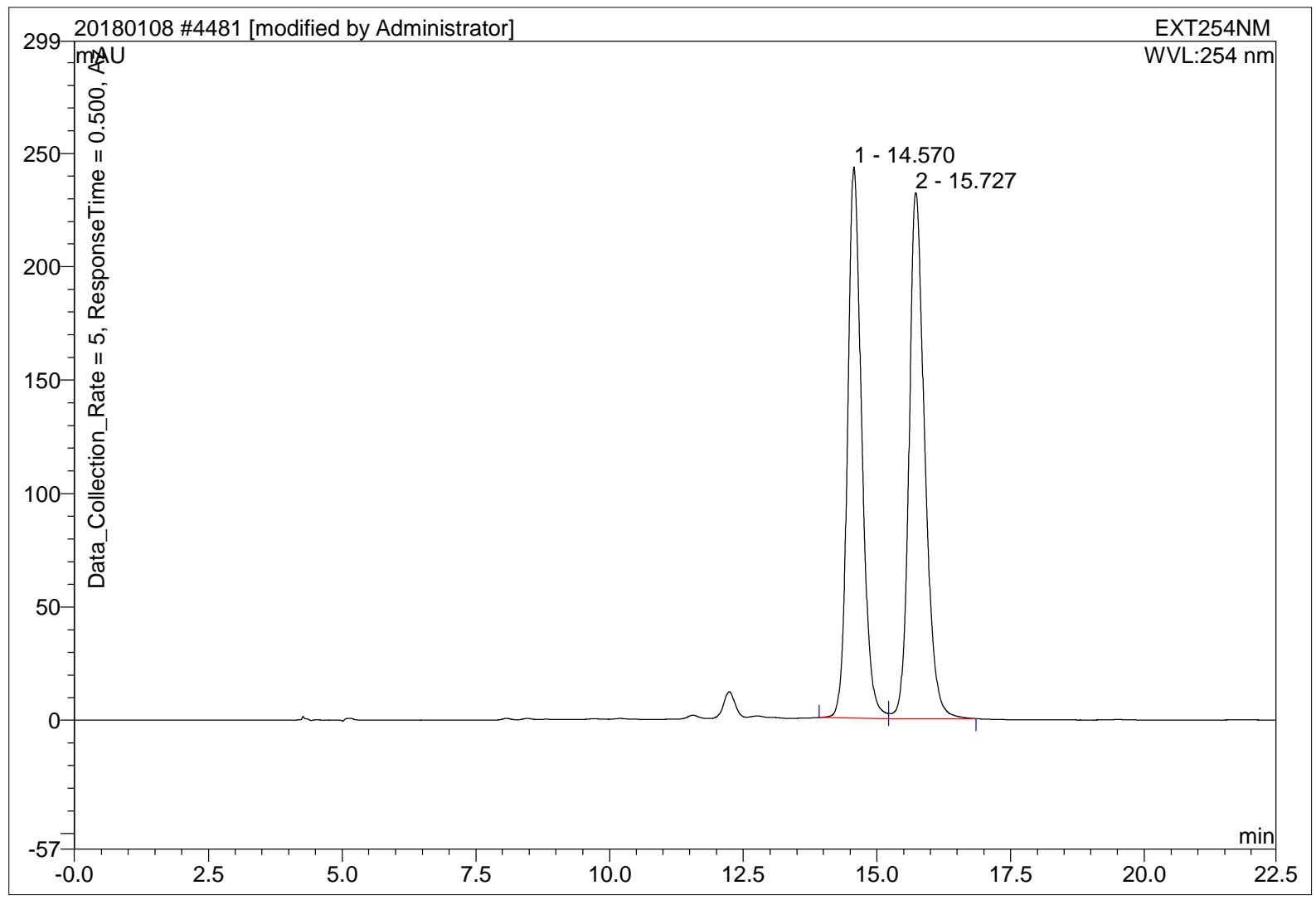

\begin{tabular}{|r|ccrrrrr|}
\hline No. & $\begin{array}{c}\text { Ret.Time } \\
\text { min }\end{array}$ & Peak Name & $\begin{array}{c}\text { Height } \\
\text { mAU }\end{array}$ & $\begin{array}{c}\text { Area } \\
\text { mAU*min }\end{array}$ & $\begin{array}{r}\text { Rel.Area } \\
\%\end{array}$ & Amount & Type \\
\hline 1 & 14.57 & n.a. & 243.030 & 74.843 & 48.53 & n.a. & BM $^{*}$ \\
2 & 15.73 & n.a. & 232.068 & 79.375 & 51.47 & n.a. & MB $^{*}$ \\
\hline Total: & & & 475.099 & 154.217 & 100.00 & 0.000 & \\
\hline
\end{tabular}




\begin{tabular}{|llll|}
\hline 4482 DWP-10-58 IB 912540.7 & & \\
& & & \\
\hline Sample Name: & DWP-10-58 IB 912540.7 & Injection Volume: & $\mathbf{5 . 0}$ \\
Vial Number: & GD4 & Channel: & EXT254NM \\
Sample Type: & unknown & Wavelength: & $\mathbf{2 5 4}$ \\
Control Program: & test-dad6 & Bandwidth: & $\mathbf{0}$ \\
Quantif. Method: & $\mathbf{2 0 1 7 0 6 0 8}$ & Dilution Factor: & $\mathbf{1 . 0 0 0 0}$ \\
Recording Time: & $\mathbf{2 0 1 9 - 1 - 1 1 ~ 2 : 0 5}$ & Sample Weight: & $\mathbf{1 . 0 0 0 0}$ \\
Run Time (min): & $\mathbf{3 0 . 0 0}$ & Sample Amount: & $\mathbf{1 . 0 0 0 0}$ \\
\hline
\end{tabular}

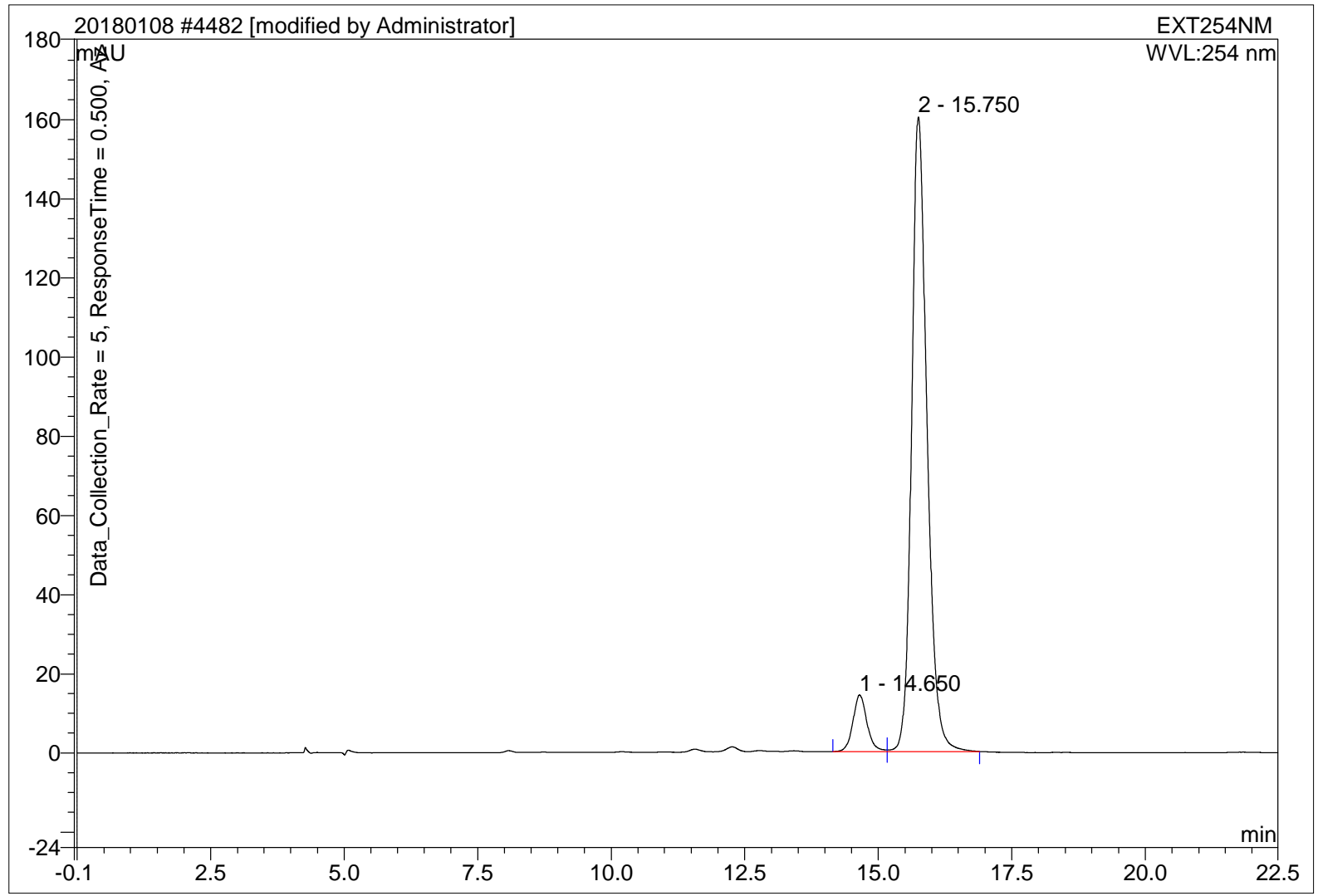

\begin{tabular}{|r|ccrrrrr|}
\hline No. & $\begin{array}{c}\text { Ret.Time } \\
\text { min }\end{array}$ & Peak Name & $\begin{array}{c}\text { Height } \\
\text { mAU }\end{array}$ & $\begin{array}{c}\text { Area } \\
\text { mAU*min }\end{array}$ & $\begin{array}{r}\text { Rel.Area } \\
\%\end{array}$ & Amount & Type \\
\hline 1 & 14.65 & n.a. & 14.292 & 4.397 & 7.56 & n.a. & BM \\
2 & 15.75 & n.a. & 160.353 & 53.749 & 92.44 & n.a. & MB \\
\hline Total: & & & 174.645 & 58.147 & 100.00 & 0.000 & \\
\hline
\end{tabular}




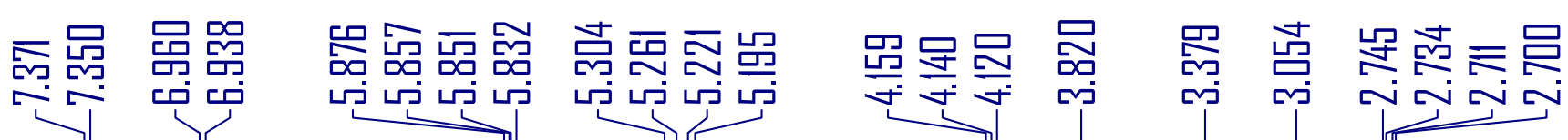
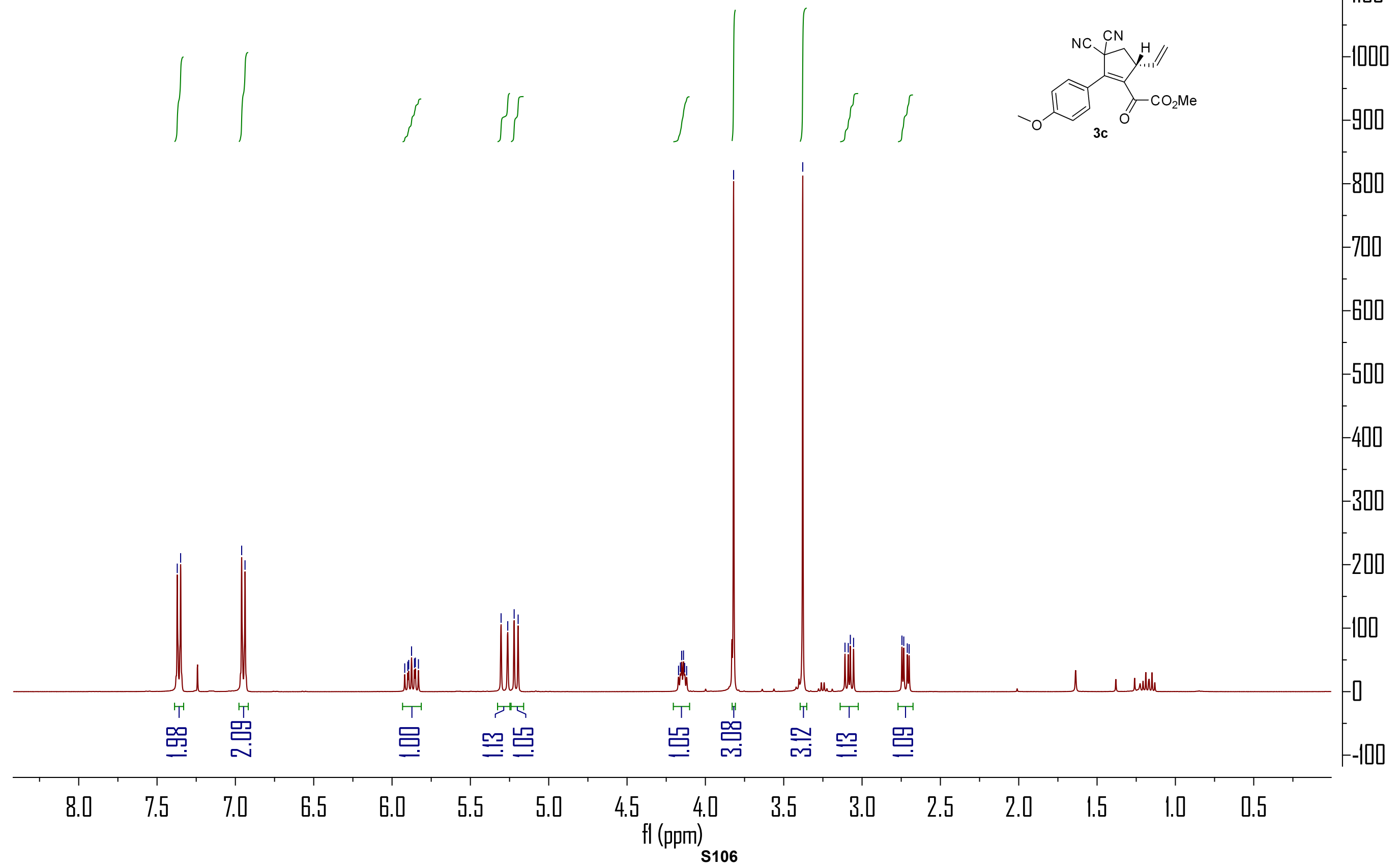


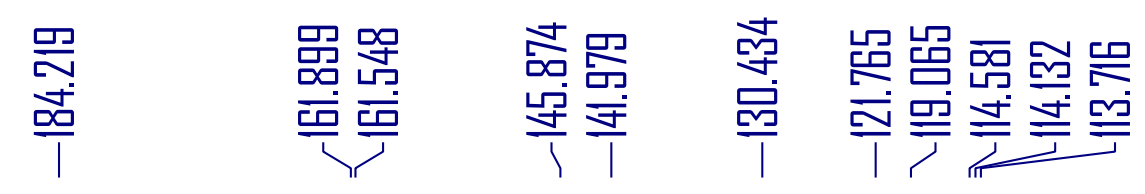

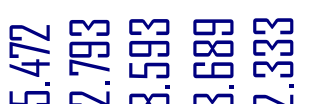

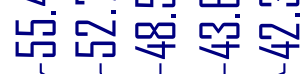

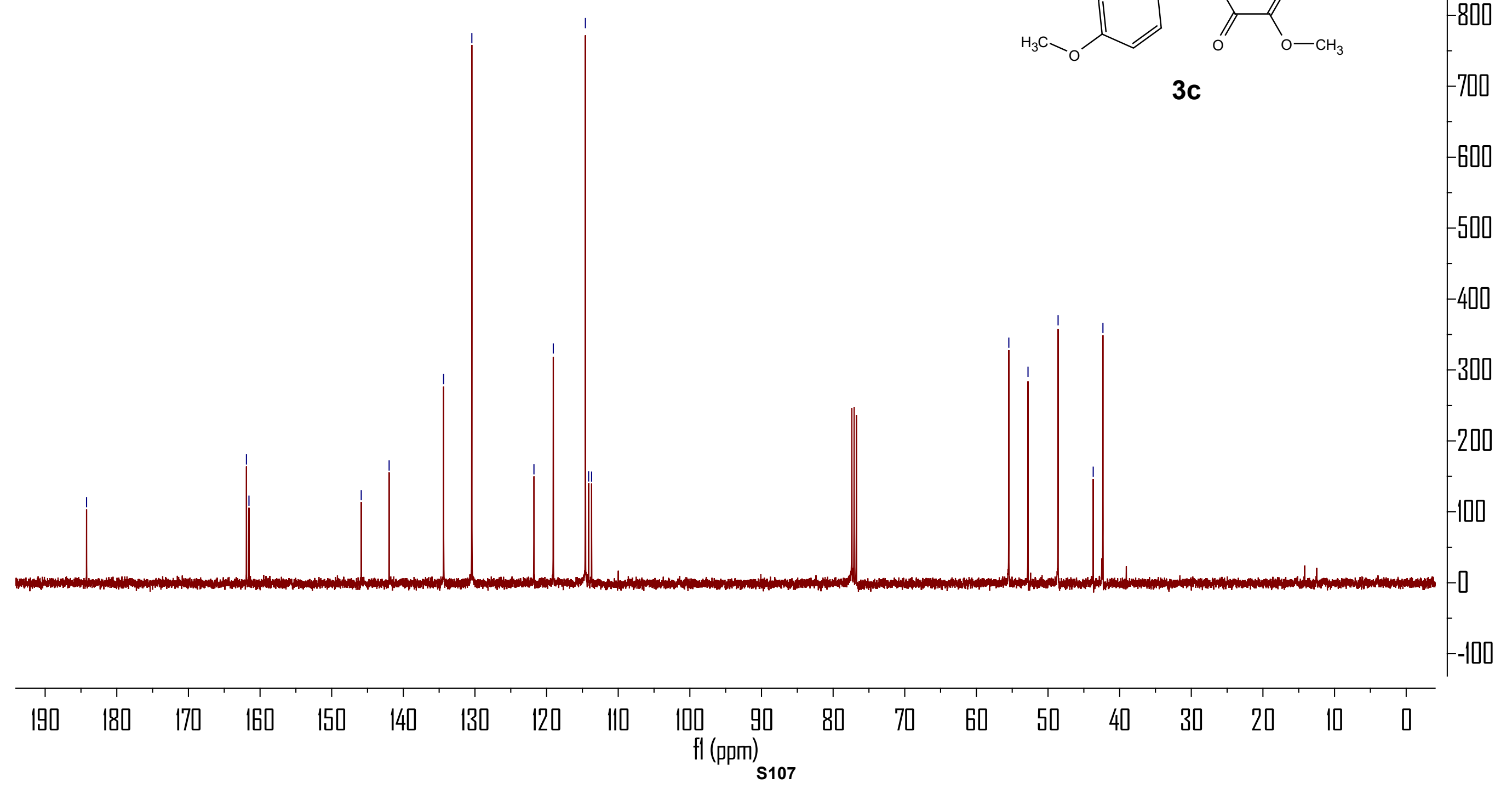




\begin{tabular}{|llll|}
\hline 4483 DWP-10-4+- IB 912540.7 & & \\
& & & \\
\hline Sample Name: & DWP-10-4+- IB 912540.7 & Injection Volume: & $\mathbf{5 . 0}$ \\
Vial Number: & GC4 & Channel: & EXT254NM \\
Sample Type: & unknown & Wavelength: & $\mathbf{2 5 4}$ \\
Control Program: & test-dad6 & Bandwidth: & $\mathbf{0}$ \\
Quantif. Method: & $\mathbf{2 0 1 7 0 6 0 8}$ & Dilution Factor: & $\mathbf{1 . 0 0 0 0}$ \\
Recording Time: & $\mathbf{2 0 1 9 - 1 - 1 1 ~ 2 : 3 7}$ & Sample Weight: & $\mathbf{1 . 0 0 0 0}$ \\
Run Time (min): & $\mathbf{3 0 . 0 0}$ & Sample Amount: & $\mathbf{1 . 0 0 0 0}$ \\
\hline
\end{tabular}

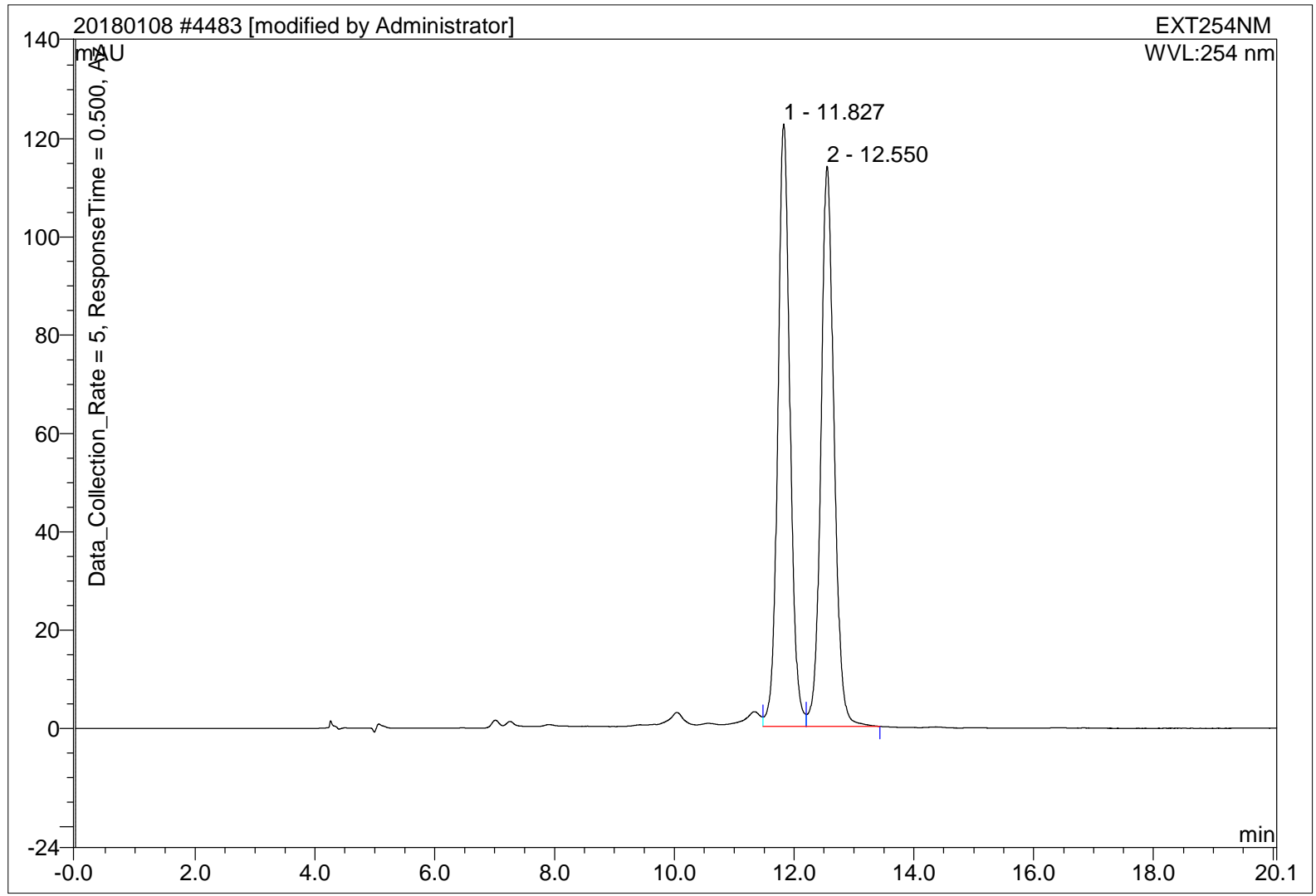

\begin{tabular}{|r|cccrrrr|}
\hline No. & $\begin{array}{c}\text { Ret.Time } \\
\text { min }\end{array}$ & Peak Name & $\begin{array}{c}\text { Height } \\
\text { mAU }\end{array}$ & $\begin{array}{c}\text { Area } \\
\text { mAU*min }\end{array}$ & $\begin{array}{r}\text { Rel.Area } \\
\%\end{array}$ & Amount & Type \\
\hline 1 & 11.83 & n.a. & 122.612 & 28.904 & 49.78 & n.a. & $\mathrm{M}^{*}$ \\
2 & 12.55 & n.a. & 114.024 & 29.154 & 50.22 & n.a. & MB $^{*}$ \\
\hline Total: & & & 236.637 & 58.059 & 100.00 & 0.000 & \\
\hline
\end{tabular}




\section{DWP-10-65 IB 912540.7}

\begin{tabular}{|llll|}
\hline Sample Name: & DWP-10-65 IB 912540.7 & Injection Volume: & $\mathbf{5 . 0}$ \\
Vial Number: & GA4 & Channel: & EXT254NM \\
Sample Type: & unknown & Wavelength: & $\mathbf{2 5 4}$ \\
Control Program: & test-dad6 & Bandwidth: & $\mathbf{0}$ \\
Quantif. Method: & $\mathbf{2 0 1 7 0 6 0 8}$ & Dilution Factor: & $\mathbf{1 . 0 0 0 0}$ \\
Recording Time: & $\mathbf{2 0 1 9 - 1 - 1 1 ~ 3 : 3 9}$ & Sample Weight: & $\mathbf{1 . 0 0 0 0}$ \\
Run Time (min): & $\mathbf{3 0 . 0 0}$ & Sample Amount: & $\mathbf{1 . 0 0 0 0}$ \\
\hline
\end{tabular}

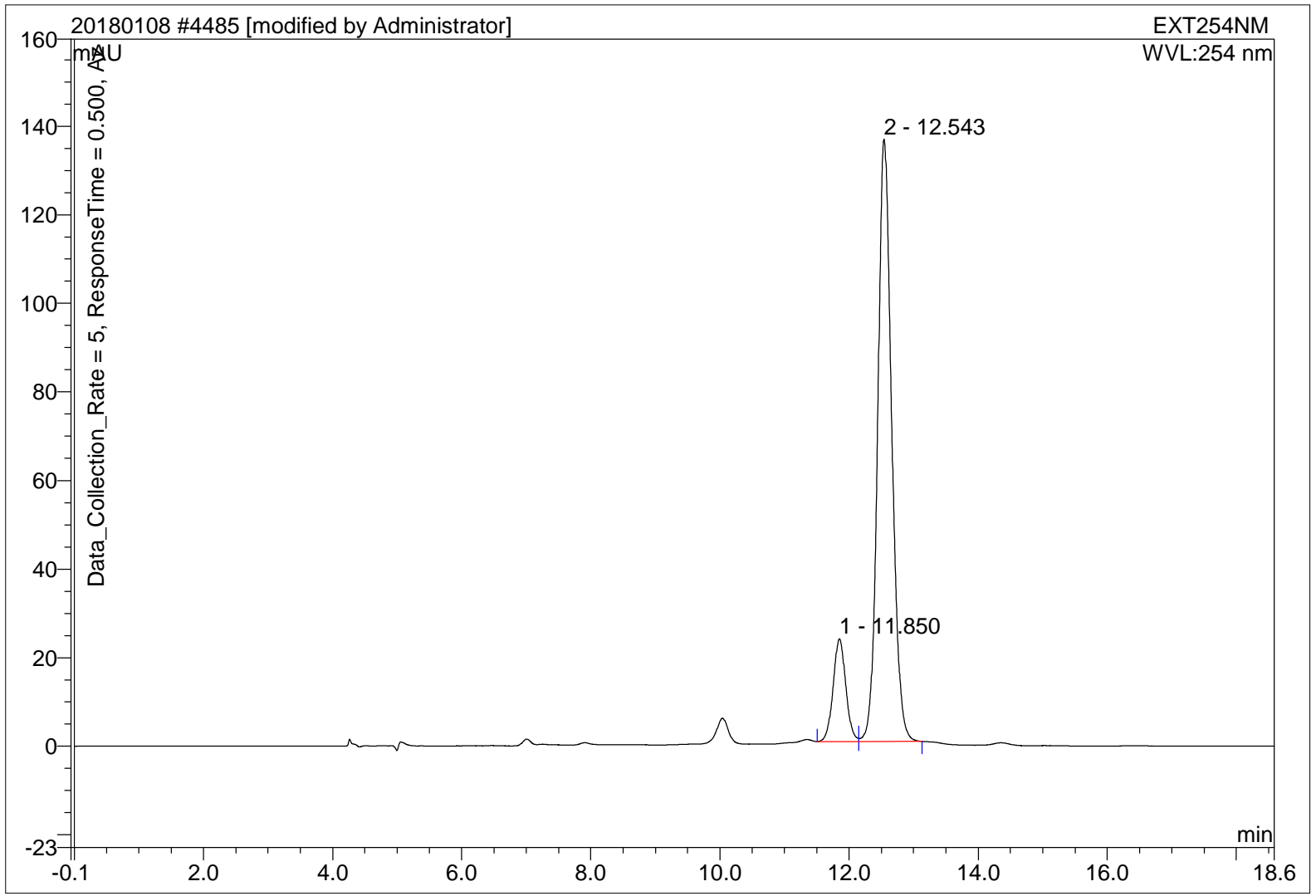

\begin{tabular}{|r|ccrrrrr|}
\hline No. & $\begin{array}{c}\text { Ret.Time } \\
\text { min }\end{array}$ & Peak Name & $\begin{array}{c}\text { Height } \\
\text { mAU }\end{array}$ & $\begin{array}{r}\text { Area } \\
\text { mAU*min }\end{array}$ & $\begin{array}{r}\text { Rel.Area } \\
\%\end{array}$ & Amount & Type \\
\hline 1 & 11.85 & n.a. & 23.244 & 5.297 & 13.41 & n.a. & BM $^{*}$ \\
2 & 12.54 & n.a. & 136.067 & 34.199 & 86.59 & n.a. & MB $^{*}$ \\
\hline Total: & & & 159.311 & 39.497 & 100.00 & 0.000 & \\
\hline
\end{tabular}




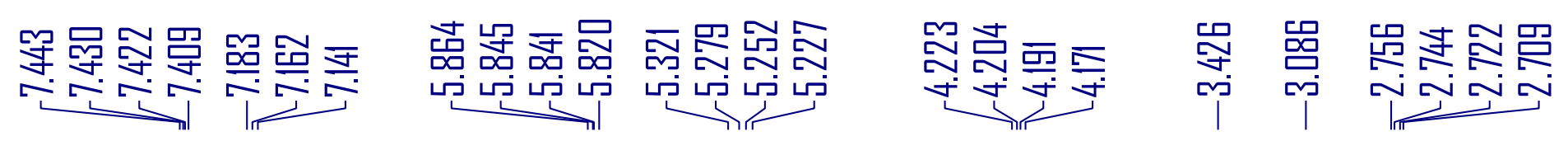
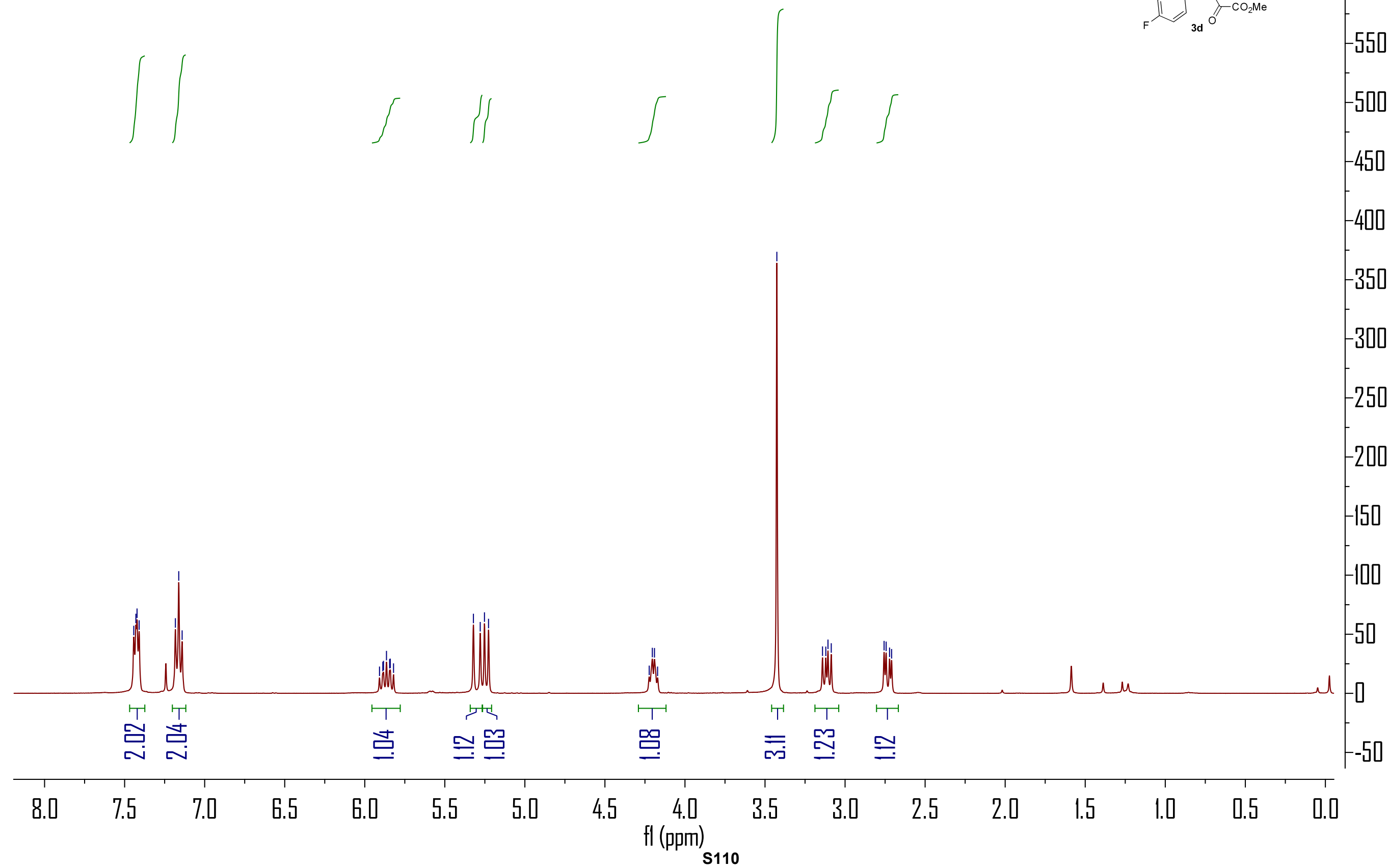


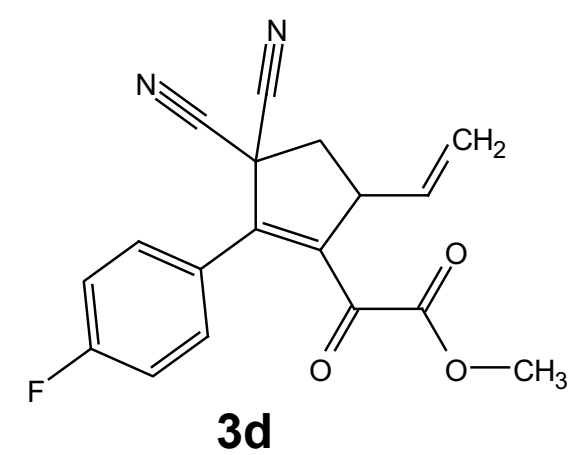

品

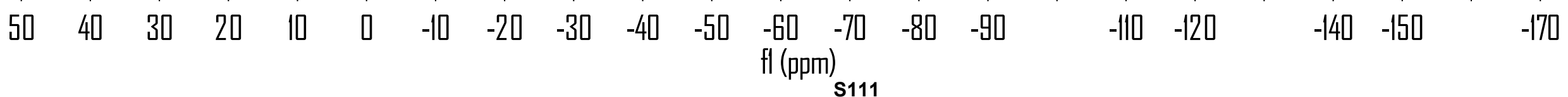




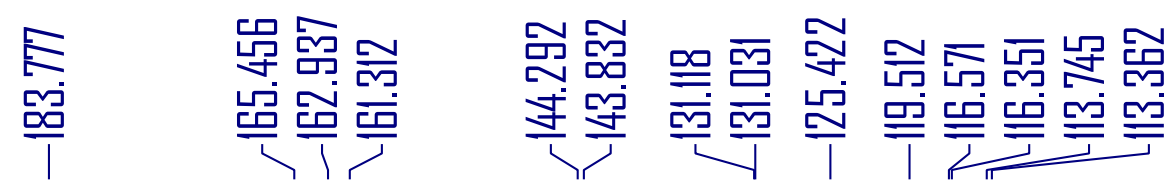

品器吕罢

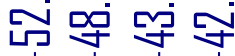
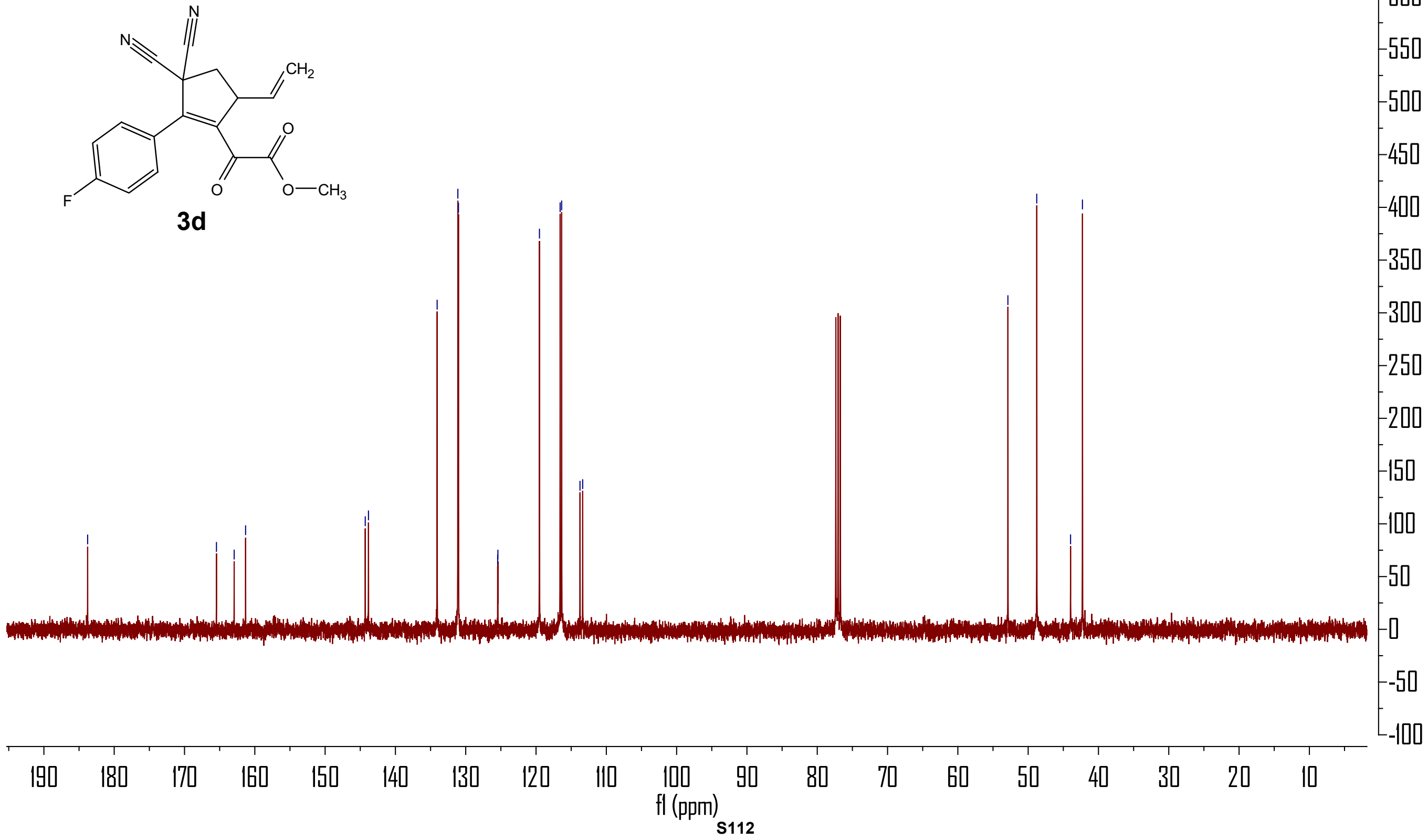


\section{DWP-10-73+- IC 912140.7}

\begin{tabular}{|llll|}
\hline Sample Name: & DWP-10-73+- IC 912140.7 & Injection Volume: & $\mathbf{5 . 0}$ \\
Vial Number: & GC3 & Channel: & UV_VIS_1 \\
Sample Type: & unknown & Wavelength: & $\mathbf{2 1 4}$ \\
Control Program: & $\mathbf{2 0 1 7 0 1 - 4}$ & Bandwidth: & n.a. \\
Quantif. Method: & $\mathbf{2 0 1 7 0 1}$ & Dilution Factor: & $\mathbf{1 . 0 0 0 0}$ \\
Recording Time: & $\mathbf{2 0 1 9 / 1 / 9 ~ 1 6 : 0 1}$ & Sample Weight: & $\mathbf{1 . 0 0 0 0}$ \\
Run Time (min): & $\mathbf{2 3 . 7 5}$ & Sample Amount: & $\mathbf{1 . 0 0 0 0}$ \\
\hline
\end{tabular}

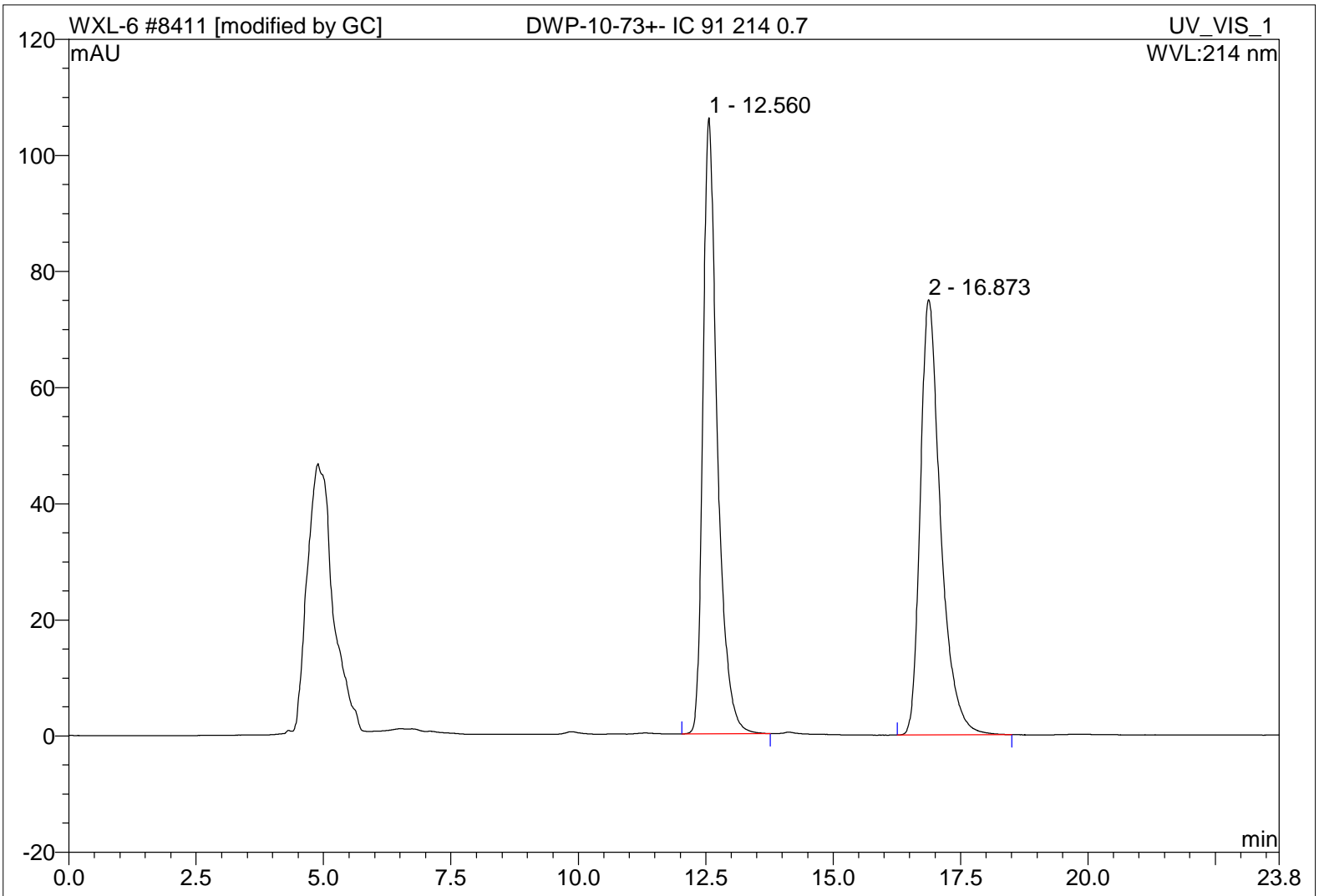

\begin{tabular}{|r|ccrrrrr|}
\hline No. & $\begin{array}{c}\text { Ret.Time } \\
\text { min }\end{array}$ & Peak Name & $\begin{array}{c}\text { Height } \\
\text { mAU }\end{array}$ & $\begin{array}{c}\text { Area } \\
\text { mAU*min }\end{array}$ & $\begin{array}{r}\text { Rel.Area } \\
\%\end{array}$ & Amount & Type \\
\hline 1 & 12.56 & n.a. & 106.141 & 34.873 & 50.11 & n.a. & BMB \\
2 & 16.87 & n.a. & 74.966 & 34.723 & 49.89 & n.a. & BMB \\
\hline Total: & & & 181.107 & 69.597 & 100.00 & 0.000 & \\
\hline
\end{tabular}




\section{DWP-10-64 IC 912140.7}

\begin{tabular}{|llll|}
\hline Sample Name: & DWP-10-64 IC 912140.7 & Injection Volume: & $\mathbf{5 . 0}$ \\
Vial Number: & GD3 & Channel: & UV_VIS_1 \\
Sample Type: & unknown & Wavelength: & $\mathbf{2 1 4}$ \\
Control Program: & $\mathbf{2 0 1 7 0 1 - 4}$ & Bandwidth: & n.a. \\
Quantif. Method: & $\mathbf{2 0 1 7 0 1}$ & Dilution Factor: & $\mathbf{1 . 0 0 0 0}$ \\
Recording Time: & $\mathbf{2 0 1 9 / 1 / 9 ~ 1 6 : 3 1}$ & Sample Weight: & $\mathbf{1 . 0 0 0 0}$ \\
Run Time (min): & $\mathbf{2 5 . 0 1}$ & Sample Amount: & $\mathbf{1 . 0 0 0 0}$ \\
\hline
\end{tabular}

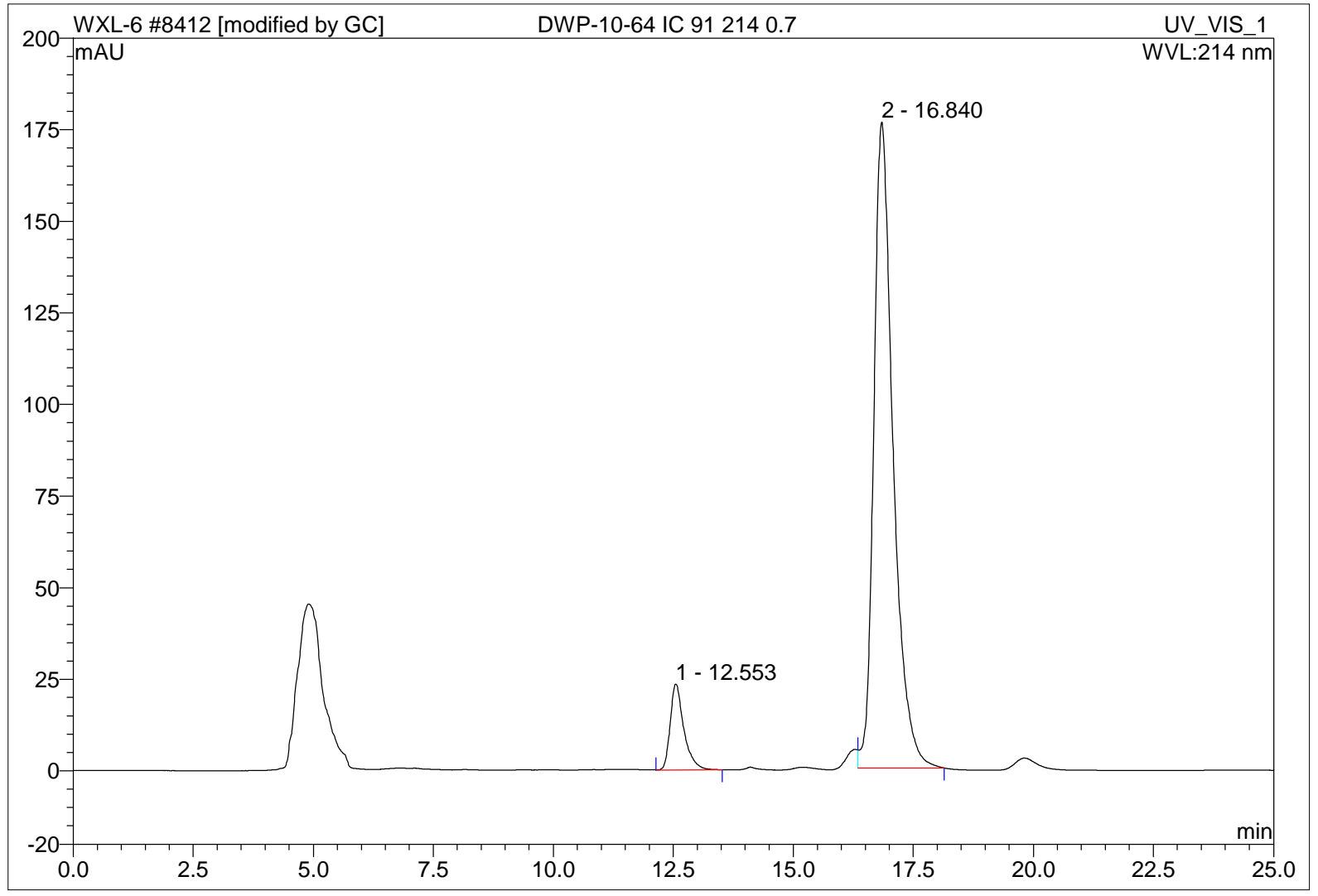

\begin{tabular}{|r|ccrrrrr|}
\hline No. & $\begin{array}{c}\text { Ret.Time } \\
\text { min }\end{array}$ & Peak Name & $\begin{array}{c}\text { Height } \\
\text { mAU }\end{array}$ & $\begin{array}{c}\text { Area } \\
\text { mAU*min }\end{array}$ & $\begin{array}{r}\text { Rel.Area } \\
\%\end{array}$ & Amount & Type \\
\hline 1 & 12.55 & n.a. & 23.378 & 7.694 & 8.55 & n.a. & BMB \\
2 & 16.84 & n.a. & 176.298 & 82.254 & 91.45 & n.a. & MB $^{*}$ \\
\hline Total: & & & 199.676 & 89.948 & 100.00 & 0.000 & \\
\hline
\end{tabular}



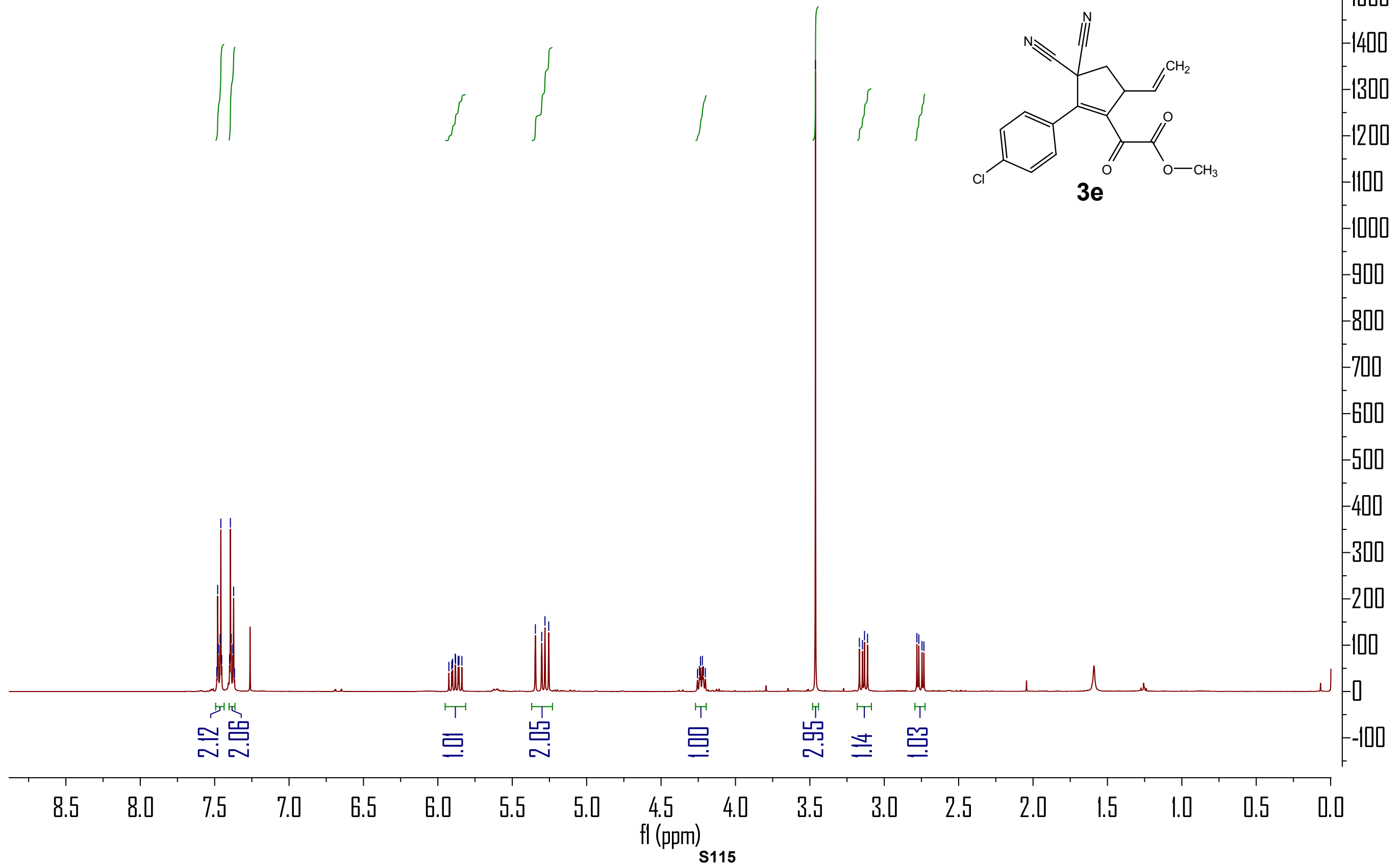


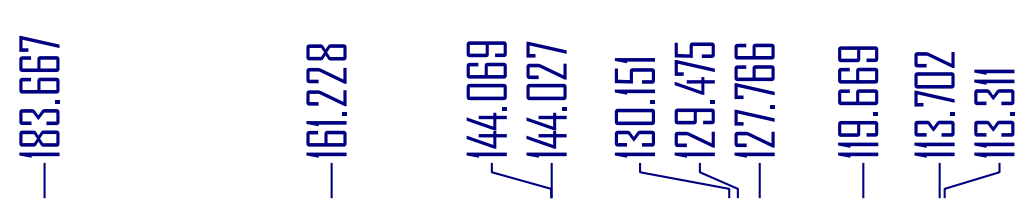

器品寻退

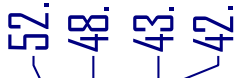
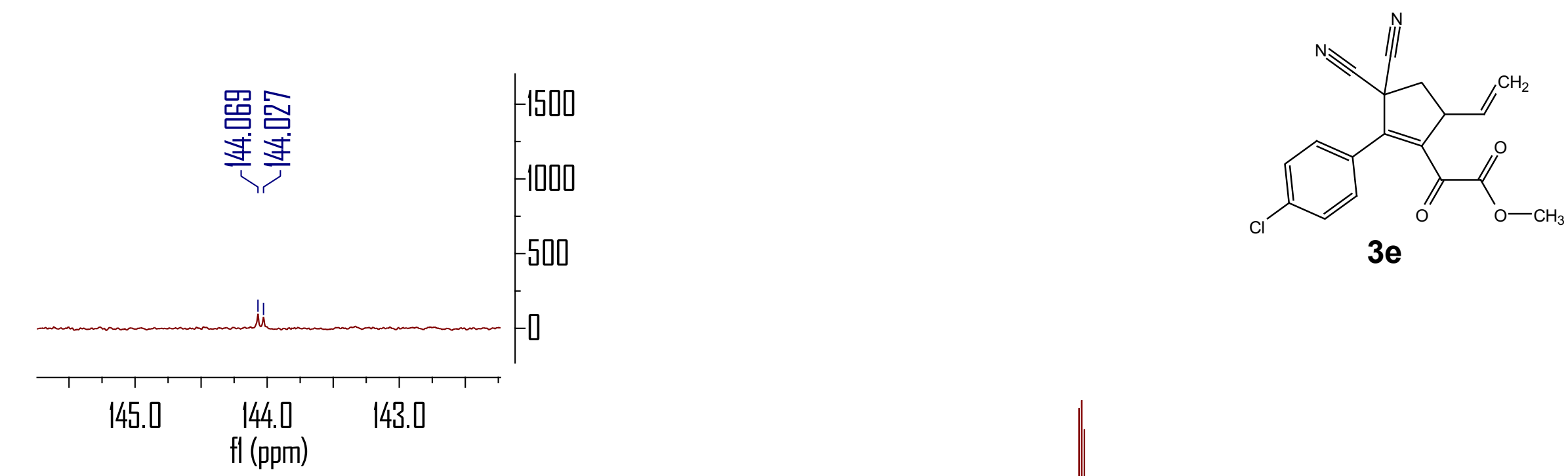

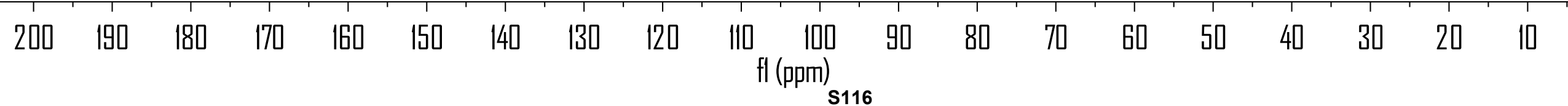




\section{DWP-10-50+- IB 912540.7}

\begin{tabular}{|llll|}
\hline Sample Name: & DWP-10-50+- IB 912540.7 & Injection Volume: & $\mathbf{5 . 0}$ \\
Vial Number: & GE6 & Channel: & EXT254NM \\
Sample Type: & unknown & Wavelength: & $\mathbf{2 5 4}$ \\
Control Program: & test-dad6 & Bandwidth: & $\mathbf{0}$ \\
Quantif. Method: & $\mathbf{2 0 1 7 0 6 0 8}$ & Dilution Factor: & $\mathbf{1 . 0 0 0 0}$ \\
Recording Time: & $\mathbf{2 0 1 9 - 1 - 1 0 ~ 2 0 : 2 4}$ & Sample Weight: & $\mathbf{1 . 0 0 0 0}$ \\
Run Time (min): & $\mathbf{3 0 . 0 0}$ & Sample Amount: & $\mathbf{1 . 0 0 0 0}$ \\
\hline
\end{tabular}

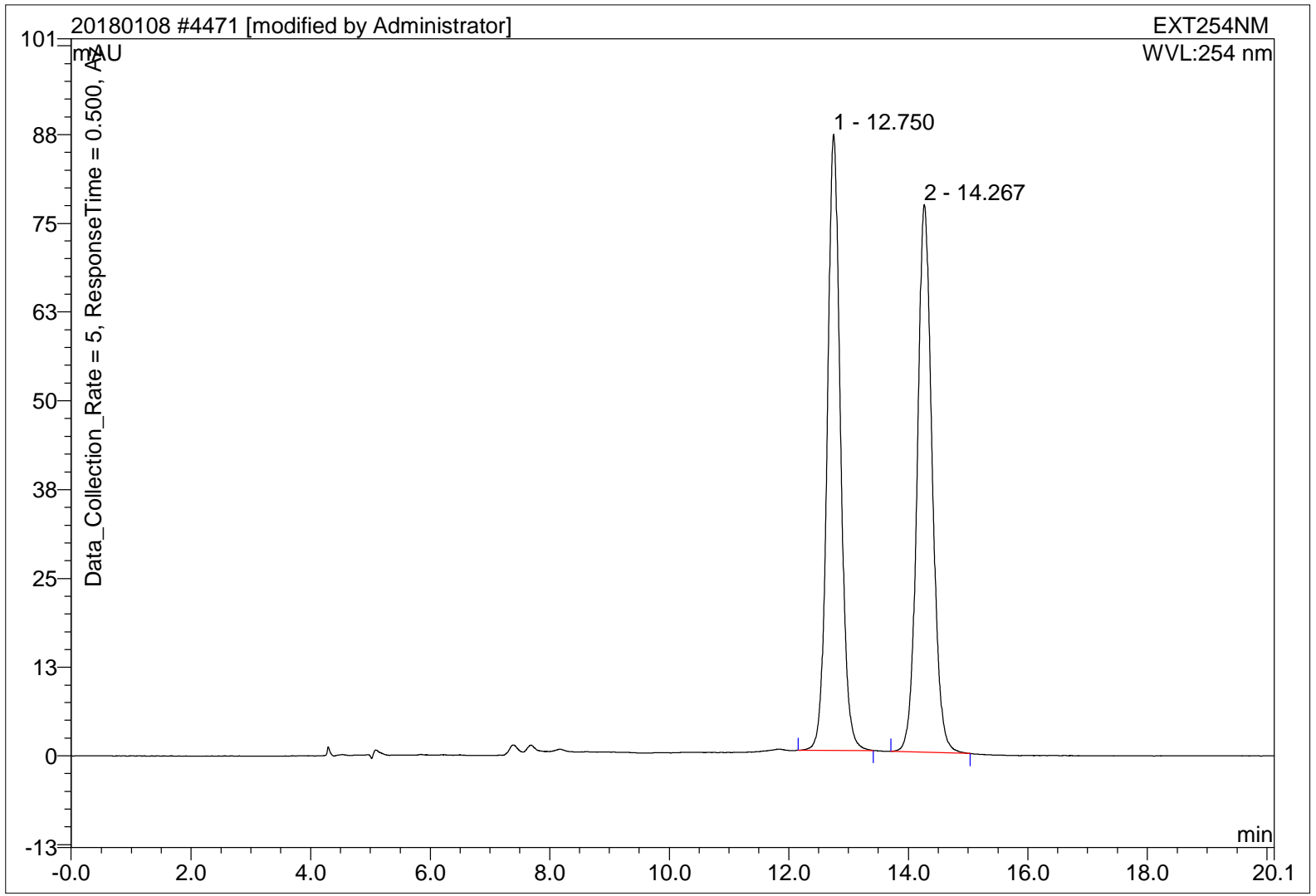

\begin{tabular}{|r|ccrrrrr|}
\hline No. & $\begin{array}{c}\text { Ret.Time } \\
\text { min }\end{array}$ & Peak Name & $\begin{array}{c}\text { Height } \\
\text { mAU }\end{array}$ & $\begin{array}{c}\text { Area } \\
\text { mAU*min }\end{array}$ & $\begin{array}{r}\text { Rel.Area } \\
\%\end{array}$ & Amount & Type \\
\hline 1 & 12.75 & n.a. & 86.797 & 22.265 & 49.90 & n.a. & BMB \\
2 & 14.27 & n.a. & 77.167 & 22.358 & 50.10 & n.a. & BMB $^{*}$ \\
\hline Total: & & & 163.964 & 44.624 & 100.00 & 0.000 & \\
\hline
\end{tabular}




\section{DWP-11-1 IB 912540.7}

\begin{tabular}{llll|}
\hline Sample Name: & DWP-11-1 IB 91 254 0.7 & Injection Volume: & $\mathbf{5 . 0}$ \\
Vial Number: & GC6 & Channel: & EXT254NM \\
Sample Type: & unknown & Wavelength: & $\mathbf{2 5 4}$ \\
Control Program: & test-dad6 & Bandwidth: & $\mathbf{0}$ \\
Quantif. Method: & $\mathbf{2 0 1 7 0 6 0 8}$ & Dilution Factor: & $\mathbf{1 . 0 0 0 0}$ \\
Recording Time: & $\mathbf{2 0 1 9 - 1 - 1 0 ~ 2 1 : 2 6}$ & Sample Weight: & $\mathbf{1 . 0 0 0 0}$ \\
Run Time (min): & $\mathbf{3 0 . 0 0}$ & Sample Amount: & $\mathbf{1 . 0 0 0 0}$ \\
\hline
\end{tabular}

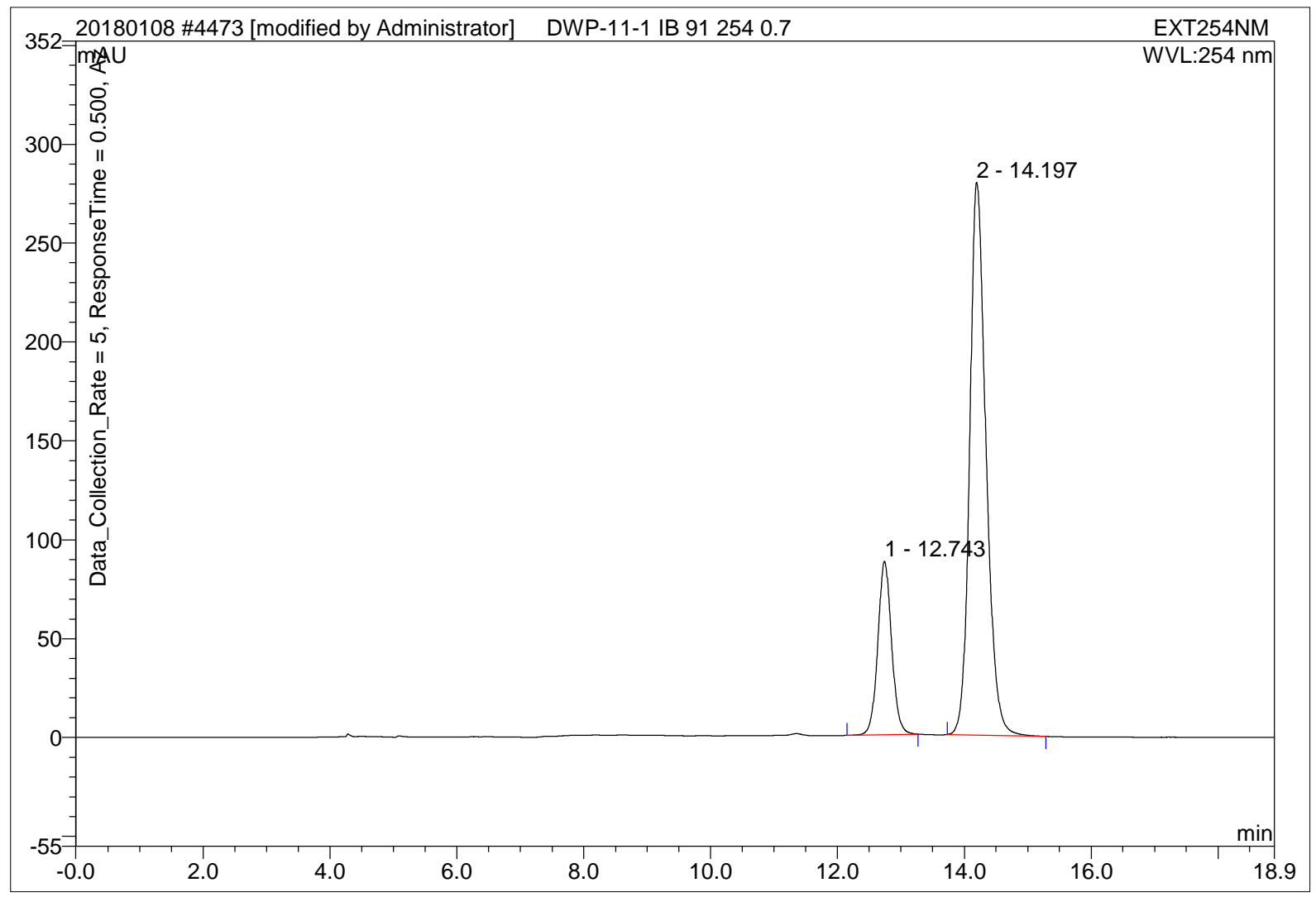

\begin{tabular}{|r|ccrrrrr|}
\hline No. & $\begin{array}{c}\text { Ret.Time } \\
\text { min }\end{array}$ & Peak Name & $\begin{array}{c}\text { Height } \\
\text { mAU }\end{array}$ & $\begin{array}{c}\text { Area } \\
\text { mAU*min }\end{array}$ & $\begin{array}{r}\text { Rel.Area } \\
\%\end{array}$ & Amount & Type \\
\hline 1 & 12.74 & n.a. & 87.808 & 22.152 & 21.00 & n.a. & BMB $^{*}$ \\
2 & 14.20 & n.a. & 279.685 & 83.335 & 79.00 & n.a. & BMB $^{*}$ \\
\hline Total: & & & 367.493 & 105.487 & 100.00 & 0.000 & \\
\hline
\end{tabular}




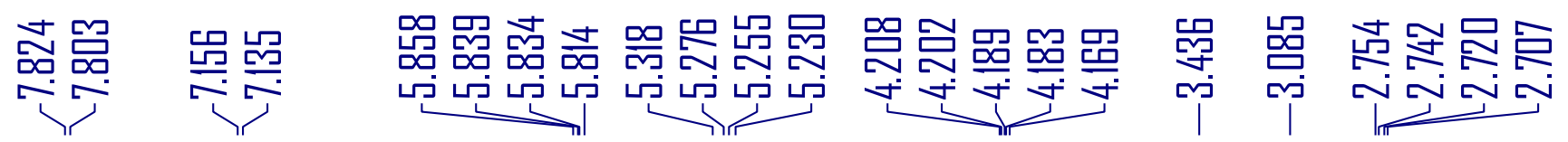
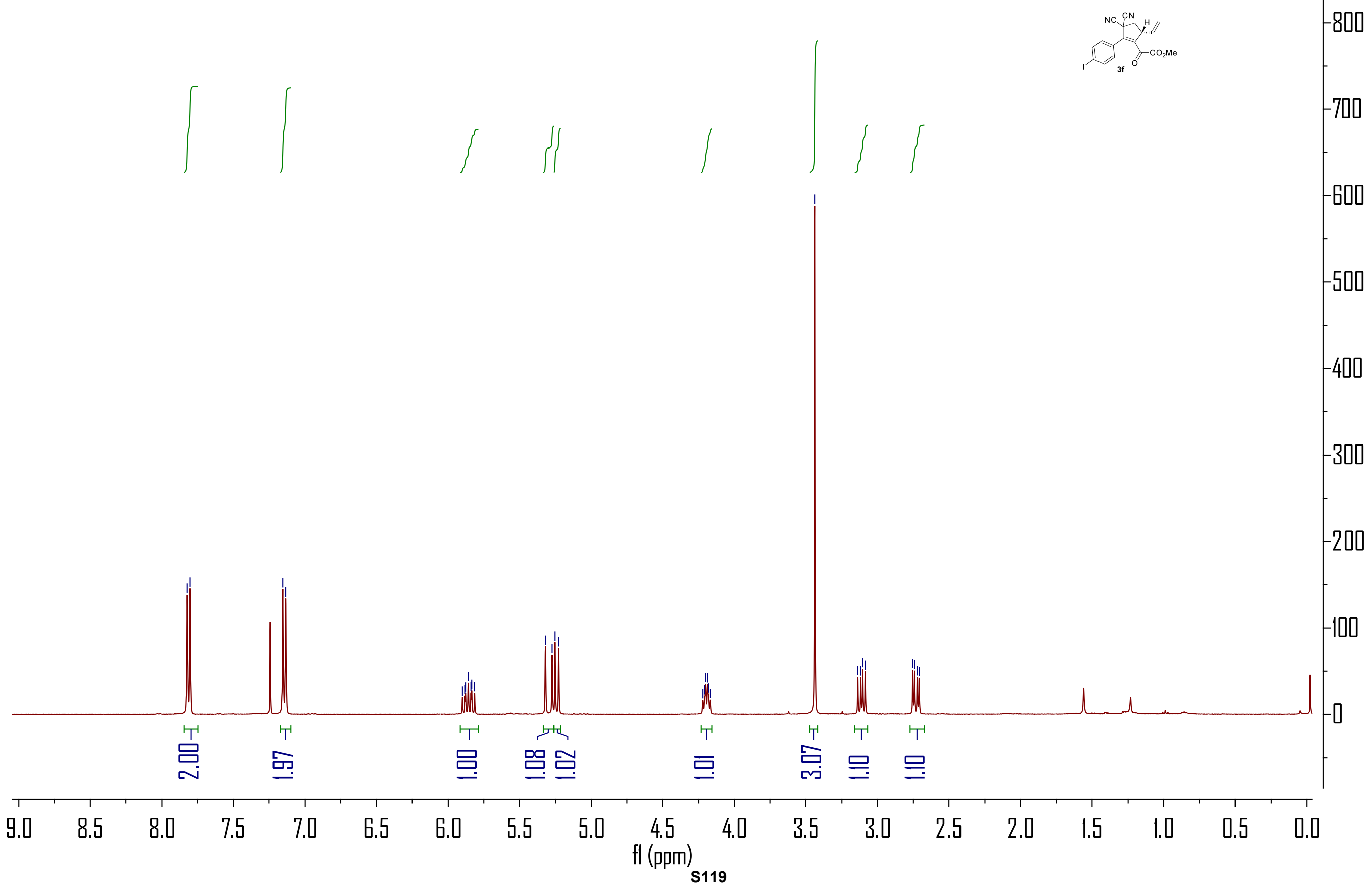


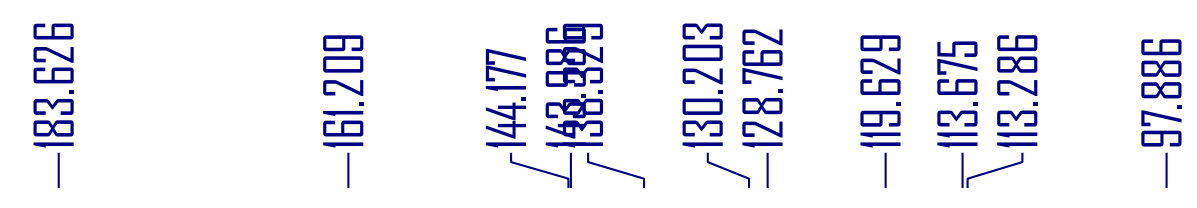

品氮品嵒

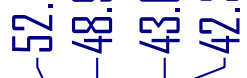

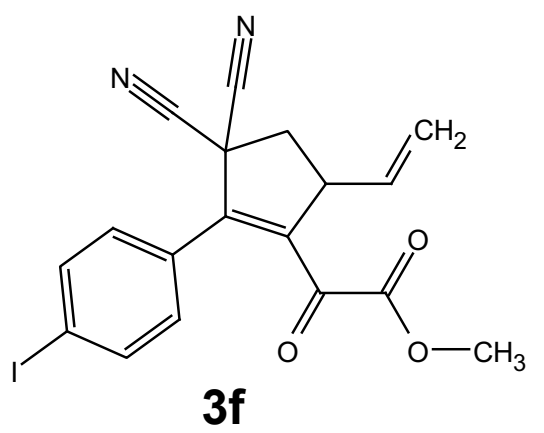

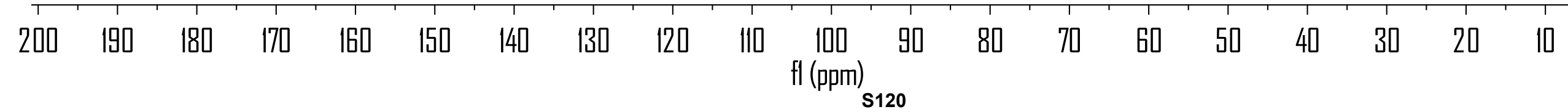




\section{DWP-10-7+- ID-3 912140.7}

\begin{tabular}{|llll|}
\hline Sample Name: & DWP-10-7+- ID-3 912140.7 & Injection Volume: & $\mathbf{3 . 0}$ \\
Vial Number: & GB2 & Channel: & UV_VIS_1 \\
Sample Type: & unknown & Wavelength: & $\mathbf{2 1 4}$ \\
Control Program: & $\mathbf{2 0 1 7 0 1 - 4}$ & Bandwidth: & n.a. \\
Quantif. Method: & $\mathbf{2 0 1 7 0 1}$ & Dilution Factor: & $\mathbf{1 . 0 0 0 0}$ \\
Recording Time: & $\mathbf{2 0 1 9 / 1 / 1 0 ~ 2 2 : 1 6}$ & Sample Weight: & $\mathbf{1 . 0 0 0 0}$ \\
Run Time (min): & $\mathbf{4 0 . 0 0}$ & Sample Amount: & $\mathbf{1 . 0 0 0 0}$ \\
\hline
\end{tabular}

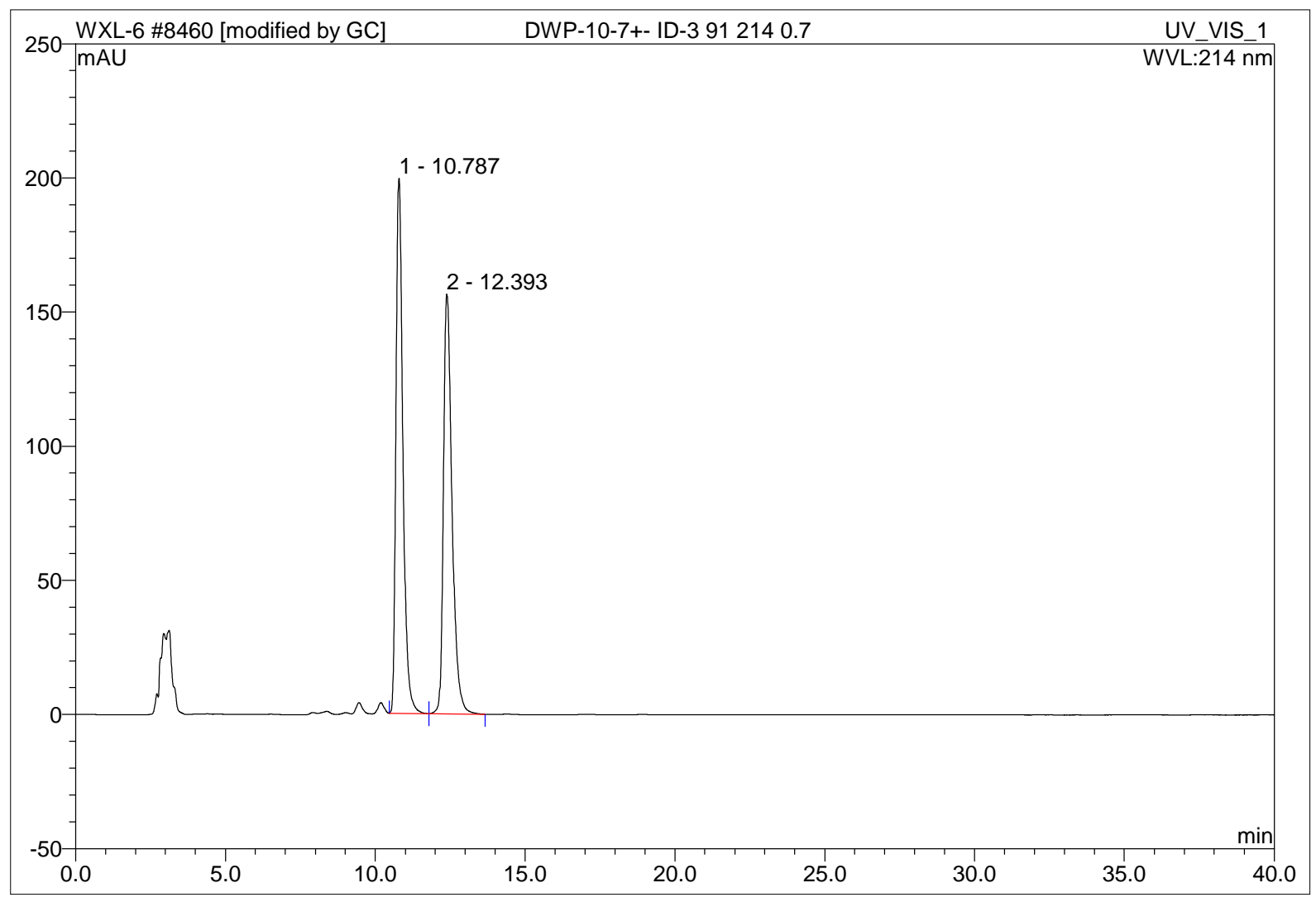

\begin{tabular}{|r|ccrrrrr|}
\hline No. & $\begin{array}{c}\text { Ret.Time } \\
\text { min }\end{array}$ & Peak Name & $\begin{array}{c}\text { Height } \\
\text { mAU }\end{array}$ & $\begin{array}{c}\text { Area } \\
\text { mAU*min }\end{array}$ & $\begin{array}{r}\text { Rel.Area } \\
\%\end{array}$ & Amount & Type \\
\hline 1 & 10.79 & n.a. & 199.494 & 53.561 & 50.73 & n.a. & BMb $^{*}$ \\
2 & 12.39 & n.a. & 156.511 & 52.013 & 49.27 & n.a. & bMB \\
\hline Total: & & & 356.005 & 105.574 & 100.00 & 0.000 & \\
\hline
\end{tabular}




\section{DWP-10-56 ID-3 912140.7}

\begin{tabular}{llll|}
\hline Sample Name: & DWP-10-56 ID-391 214 0.7 & Injection Volume: & $\mathbf{3 . 0}$ \\
Vial Number: & GB3 & Channel: & UV_VIS_1 \\
Sample Type: & unknown & Wavelength: & $\mathbf{2 1 4}$ \\
Control Program: & $\mathbf{2 0 1 7 0 1 - 4}$ & Bandwidth: & n.a. \\
Quantif. Method: & $\mathbf{2 0 1 7 0 1}$ & Dilution Factor: & $\mathbf{1 . 0 0 0 0}$ \\
Recording Time: & $\mathbf{2 0 1 9 / 1 / 1 0 ~ 2 2 : 5 8}$ & Sample Weight: & $\mathbf{1 . 0 0 0 0}$ \\
Run Time (min): & $\mathbf{4 0 . 0 0}$ & Sample Amount: & $\mathbf{1 . 0 0 0 0}$ \\
\hline
\end{tabular}

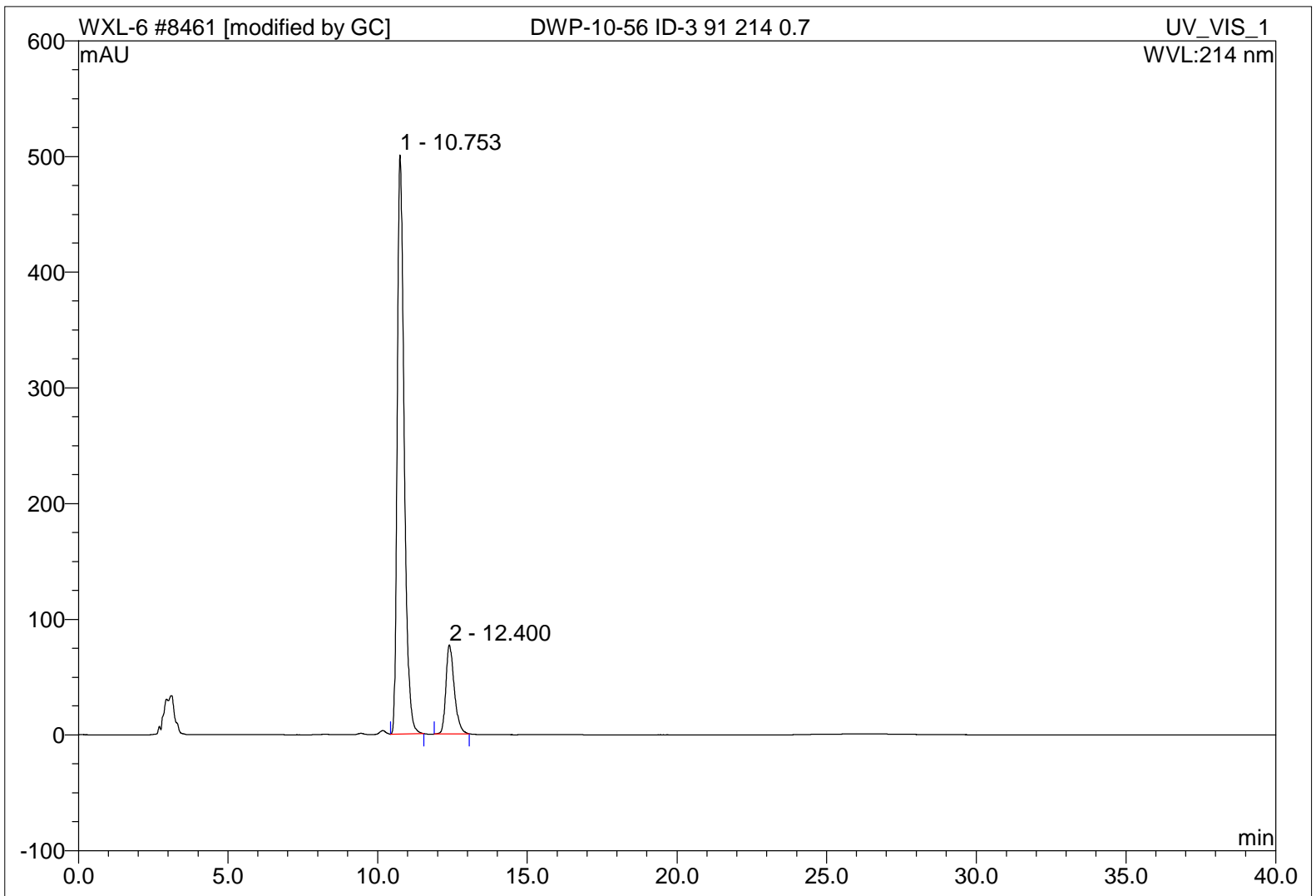

\begin{tabular}{|r|ccrrrrr|}
\hline No. & $\begin{array}{c}\text { Ret.Time } \\
\text { min }\end{array}$ & Peak Name & $\begin{array}{c}\text { Height } \\
\text { mAU }\end{array}$ & $\begin{array}{c}\text { Area } \\
\text { mAU*min }\end{array}$ & $\begin{array}{r}\text { Rel.Area } \\
\%\end{array}$ & Amount & Type \\
& 10.75 & n.a. & 500.728 & 134.600 & 84.29 & n.a. & BMB $^{*}$ \\
2 & 12.40 & n.a. & 77.141 & 25.093 & 15.71 & n.a. & BMB $^{*}$ \\
\hline Total: & & & 577.868 & 159.693 & 100.00 & 0.000 & \\
\hline
\end{tabular}




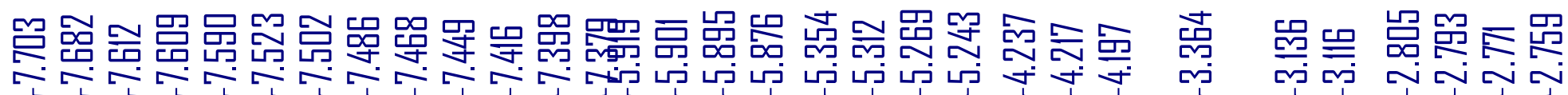

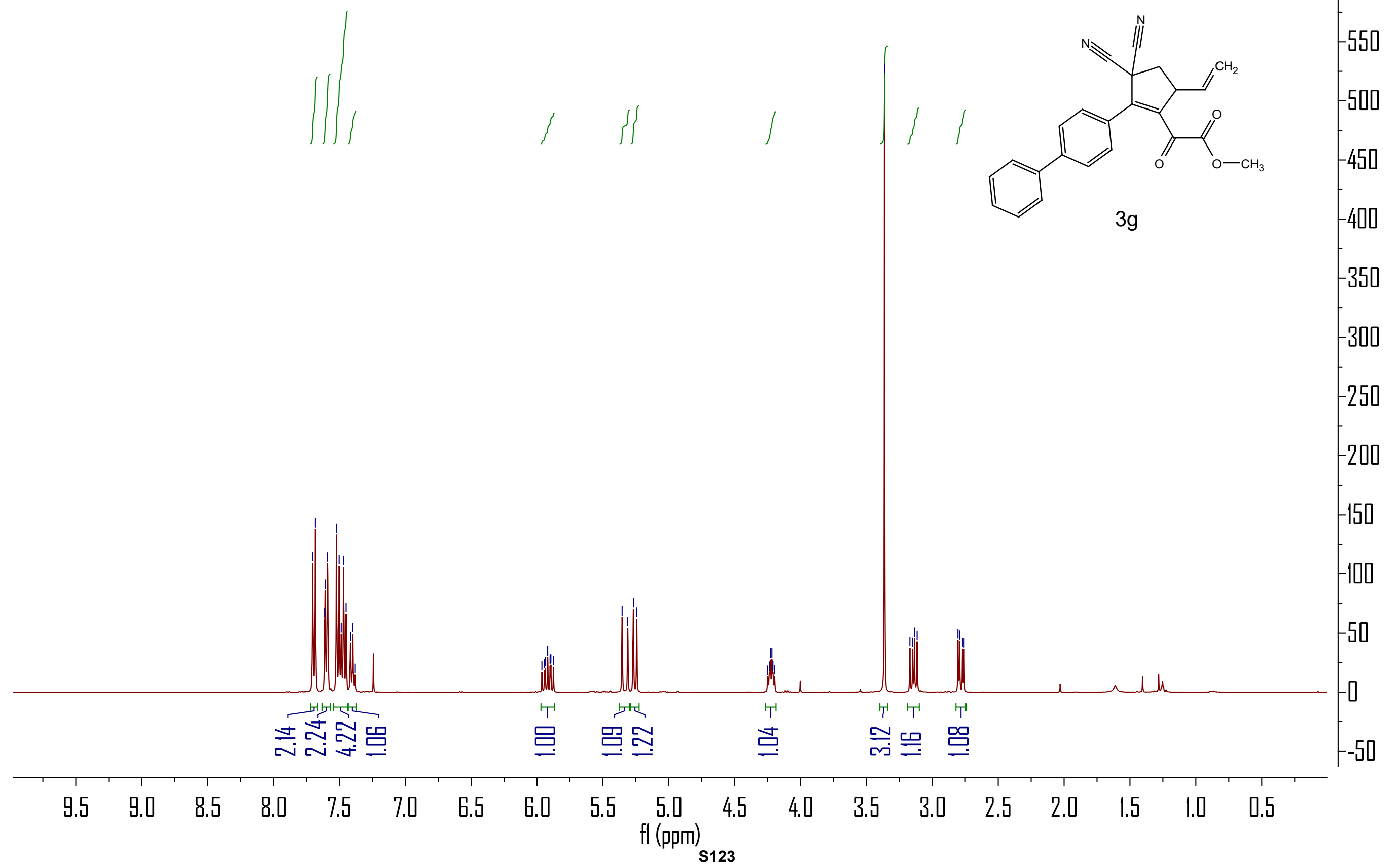




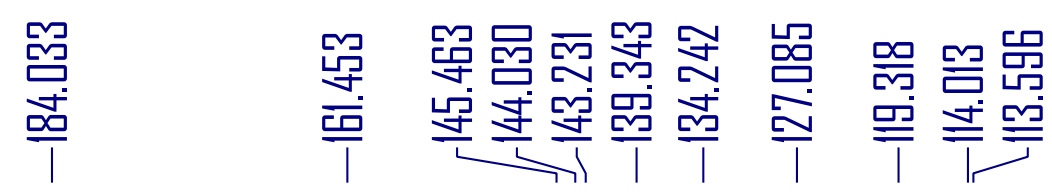

忿点学

垫骂 骂
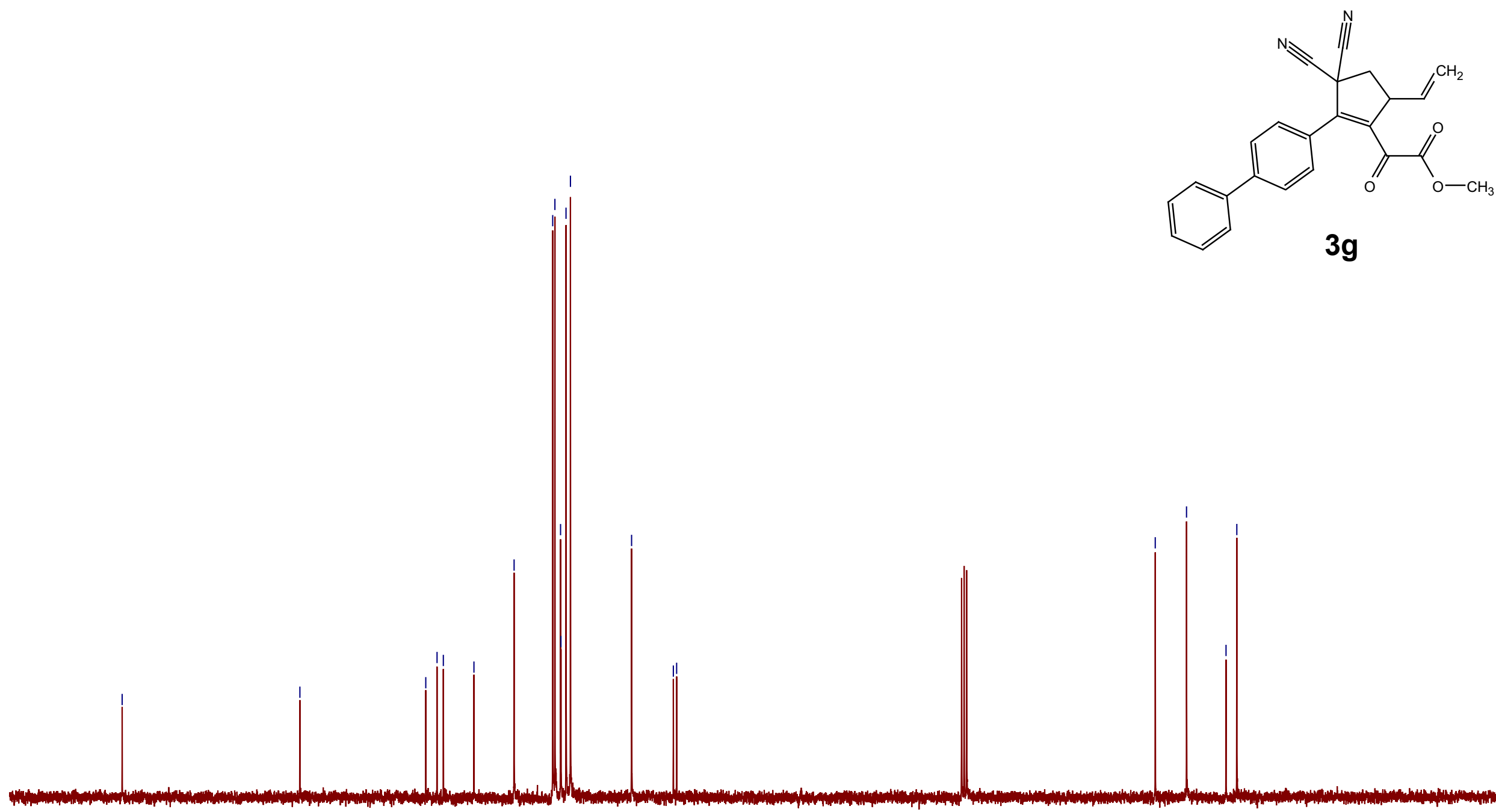

3g 


\section{DWP-9-105+- IB 9912140.7}

\begin{tabular}{|llll|}
\hline Sample Name: & DWP-9-105+- IB 9912140.7 & Injection Volume: & $\mathbf{3 . 0}$ \\
Vial Number: & GE2 & Channel: & UV_VIS_1 \\
Sample Type: & unknown & Wavelength: & $\mathbf{2 1 4}$ \\
Control Program: & $\mathbf{2 0 1 7 0 1 - 4}$ & Bandwidth: & n.a. \\
Quantif. Method: & $\mathbf{2 0 1 7 0 1}$ & Dilution Factor: & $\mathbf{1 . 0 0 0 0}$ \\
Recording Time: & $\mathbf{2 0 1 9 / 1 / 2 2 ~ 1 4 : 2 7}$ & Sample Weight: & $\mathbf{1 . 0 0 0 0}$ \\
Run Time (min): & $\mathbf{3 5 . 2 0}$ & Sample Amount: & $\mathbf{1 . 0 0 0 0}$ \\
\hline
\end{tabular}

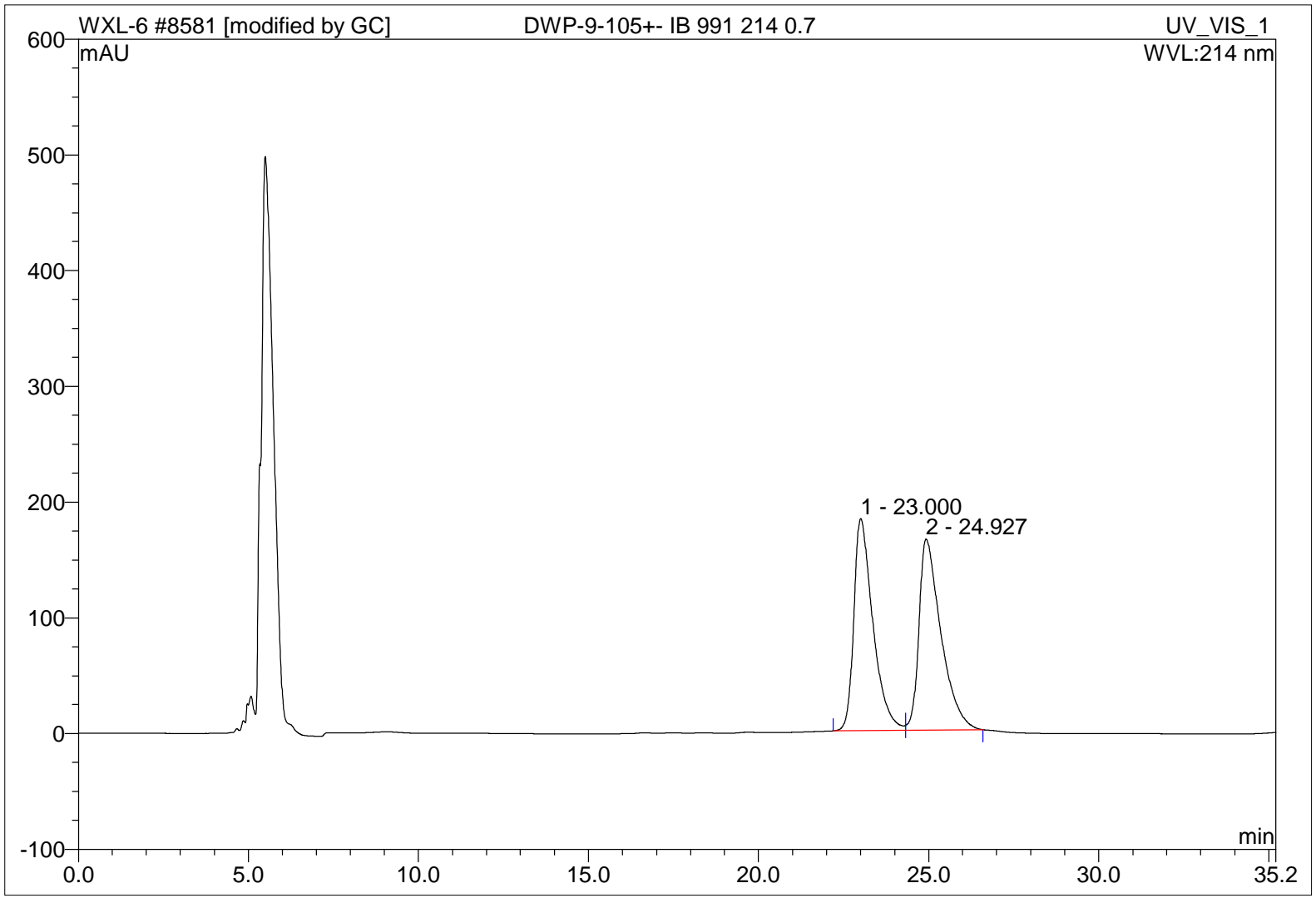

\begin{tabular}{|r|cccrrrr|}
\hline No. & $\begin{array}{c}\text { Ret.Time } \\
\text { min }\end{array}$ & Peak Name & $\begin{array}{c}\text { Height } \\
\text { mAU }\end{array}$ & $\begin{array}{c}\text { Area } \\
\text { mAU*min }\end{array}$ & $\begin{array}{r}\text { Rel.Area } \\
\%\end{array}$ & Amount & Type \\
\hline 1 & 23.00 & n.a. & 183.403 & 119.711 & 49.13 & n.a. & BM $^{*}$ \\
2 & 24.93 & n.a. & 165.334 & 123.970 & 50.87 & n.a. & MB $^{*}$ \\
\hline Total: & & & 348.738 & 243.680 & 100.00 & 0.000 & \\
\hline
\end{tabular}




\section{DWP-10-81 IB 9912140.7}

\begin{tabular}{|llll|}
\hline Sample Name: & DWP-10-81 IB 991 214 0.7 & Injection Volume: & $\mathbf{3 . 0}$ \\
Vial Number: & GE1 & Channel: & UV_VIS_1 \\
Sample Type: & unknown & Wavelength: & $\mathbf{2 1 4}$ \\
Control Program: & $\mathbf{2 0 1 7 0 1 - 4}$ & Bandwidth: & n.a. \\
Quantif. Method: & $\mathbf{2 0 1 7 0 1}$ & Dilution Factor: & $\mathbf{1 . 0 0 0 0}$ \\
Recording Time: & $\mathbf{2 0 1 9 / 1 / 2 2 ~ 1 5 : 0 4}$ & Sample Weight: & $\mathbf{1 . 0 0 0 0}$ \\
Run Time (min): & $\mathbf{4 3 . 5 3}$ & Sample Amount: & $\mathbf{1 . 0 0 0 0}$ \\
\hline
\end{tabular}

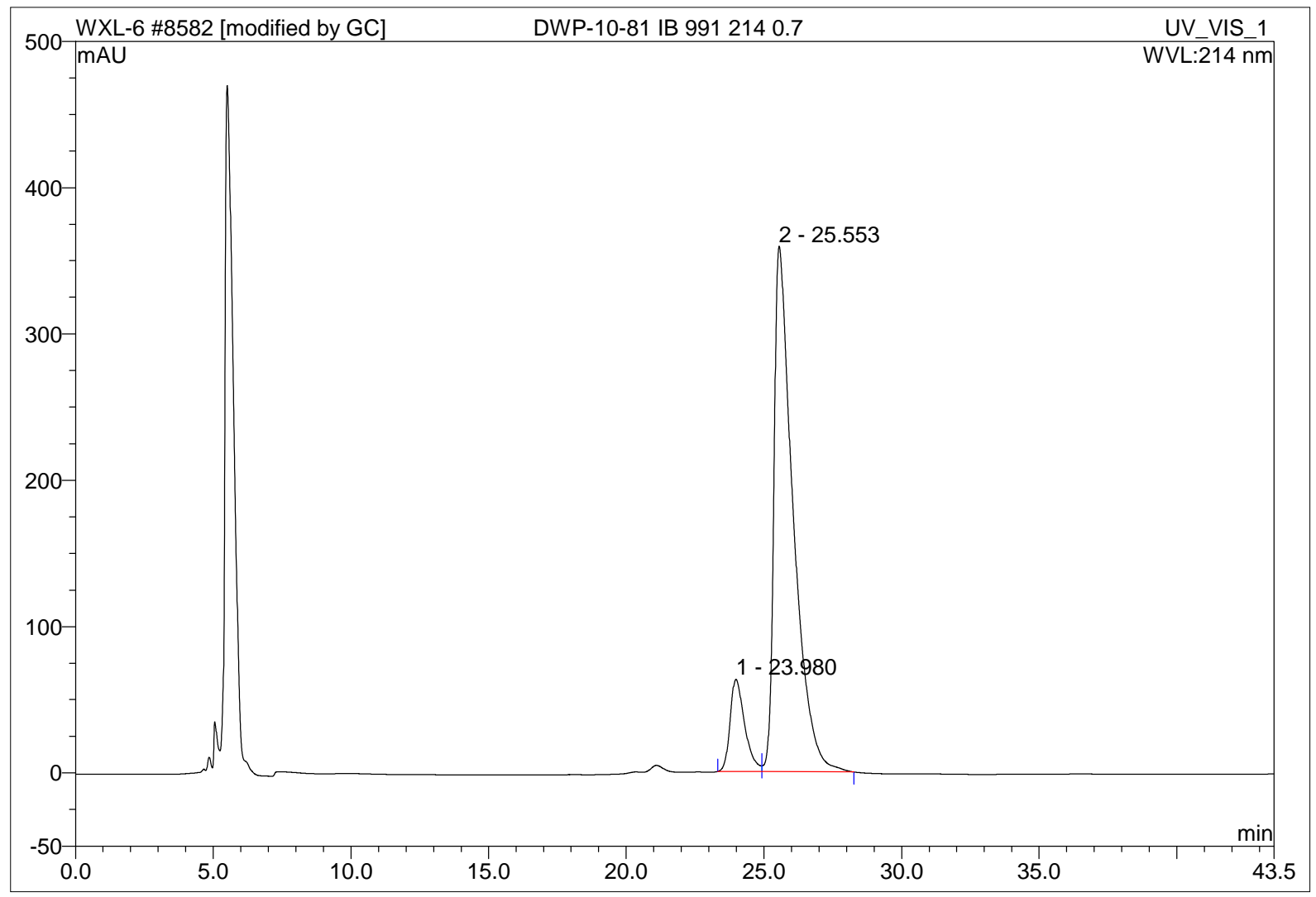

\begin{tabular}{|r|ccrrrrr|}
\hline No. & $\begin{array}{c}\text { Ret.Time } \\
\text { min }\end{array}$ & Peak Name & $\begin{array}{c}\text { Height } \\
\text { mAU }\end{array}$ & $\begin{array}{c}\text { Area } \\
\text { mAU*min }\end{array}$ & $\begin{array}{c}\text { Rel.Area } \\
\%\end{array}$ & Amount & Type \\
\hline 1 & 23.98 & n.a. & 63.138 & 38.805 & 11.80 & n.a. & BM $^{*}$ \\
2 & 25.55 & n.a. & 359.013 & 290.050 & 88.20 & n.a. & MB $^{*}$ \\
\hline Total: & & & 422.152 & 328.855 & 100.00 & 0.000 & \\
\hline
\end{tabular}




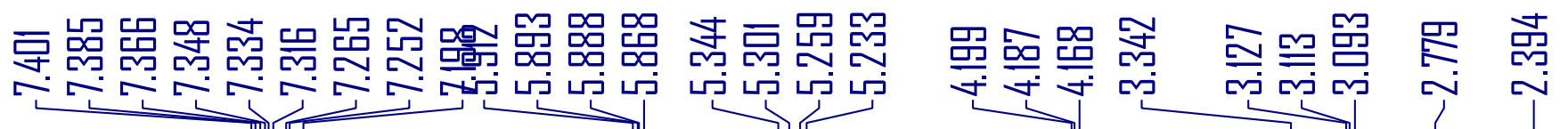
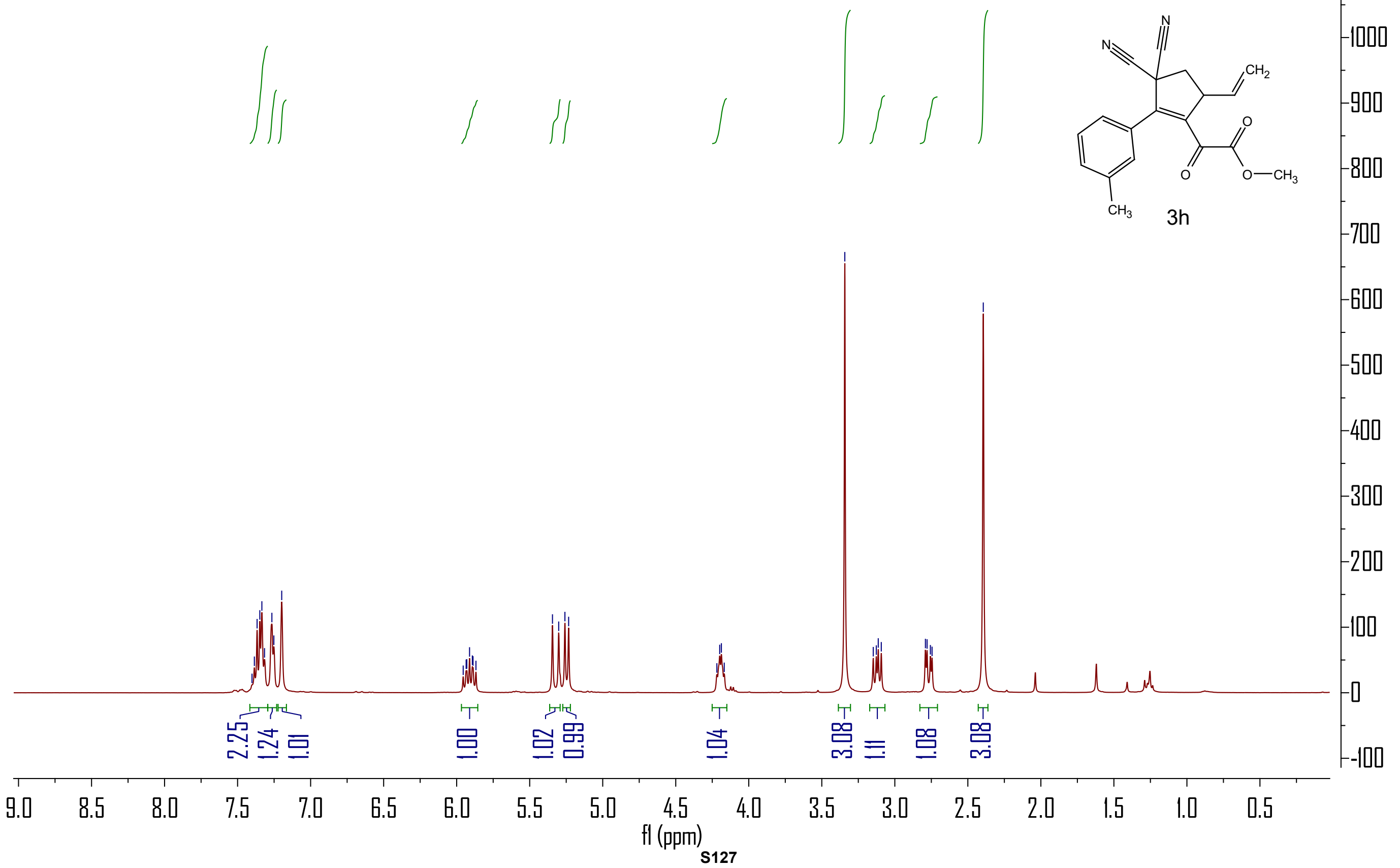


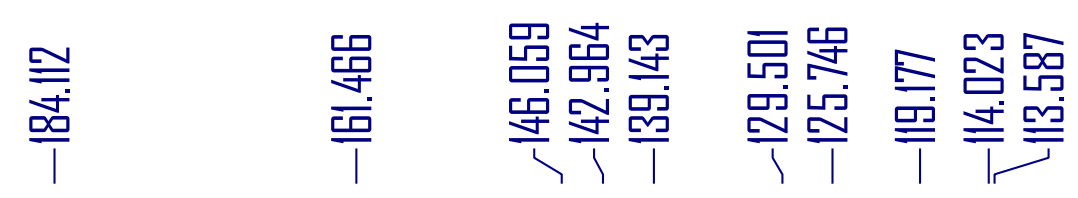

号品咒吕茄

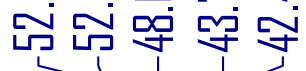

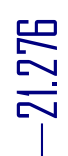
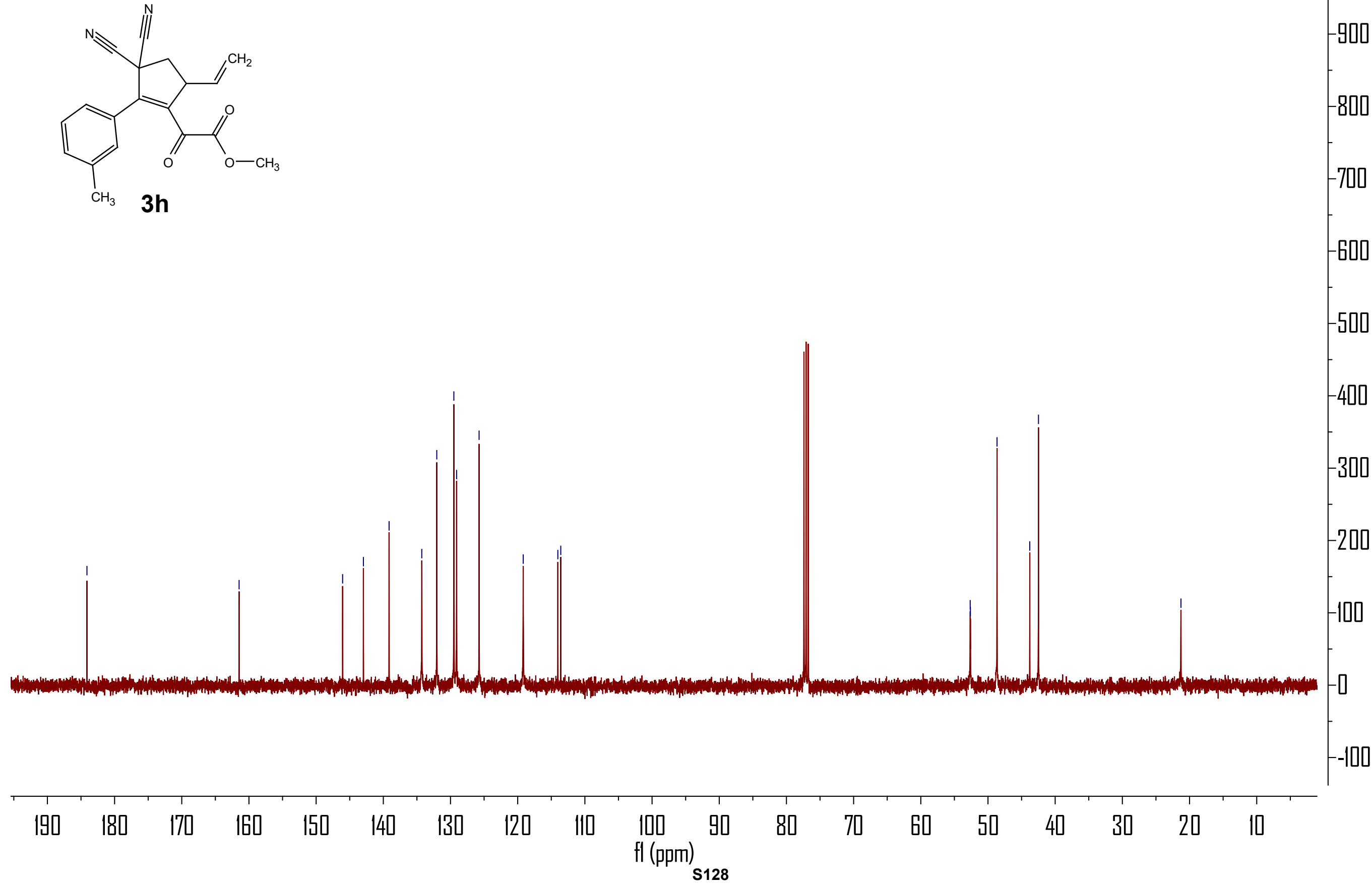


\begin{tabular}{|llll|}
\hline 4478 DWP-10-1+- IB 912140.7 & & \\
& & & \\
\hline Sample Name: & DWP-10-1+- IB 912140.7 & Injection Volume: & $\mathbf{5 . 0}$ \\
Vial Number: & GC5 & Channel: & UV_VIS_2 \\
Sample Type: & unknown & Wavelength: & $\mathbf{2 1 4 . 0}$ \\
Control Program: & test-dad6 & Bandwidth: & $\mathbf{4}$ \\
Quantif. Method: & $\mathbf{2 0 1 7 0 6 0 8}$ & Dilution Factor: & $\mathbf{1 . 0 0 0 0}$ \\
Recording Time: & $\mathbf{2 0 1 9 - 1 - 1 1 ~ 0 : 0 1}$ & Sample Weight: & $\mathbf{1 . 0 0 0 0}$ \\
Run Time (min): & $\mathbf{3 0 . 0 0}$ & Sample Amount: & $\mathbf{1 . 0 0 0 0}$ \\
\hline
\end{tabular}

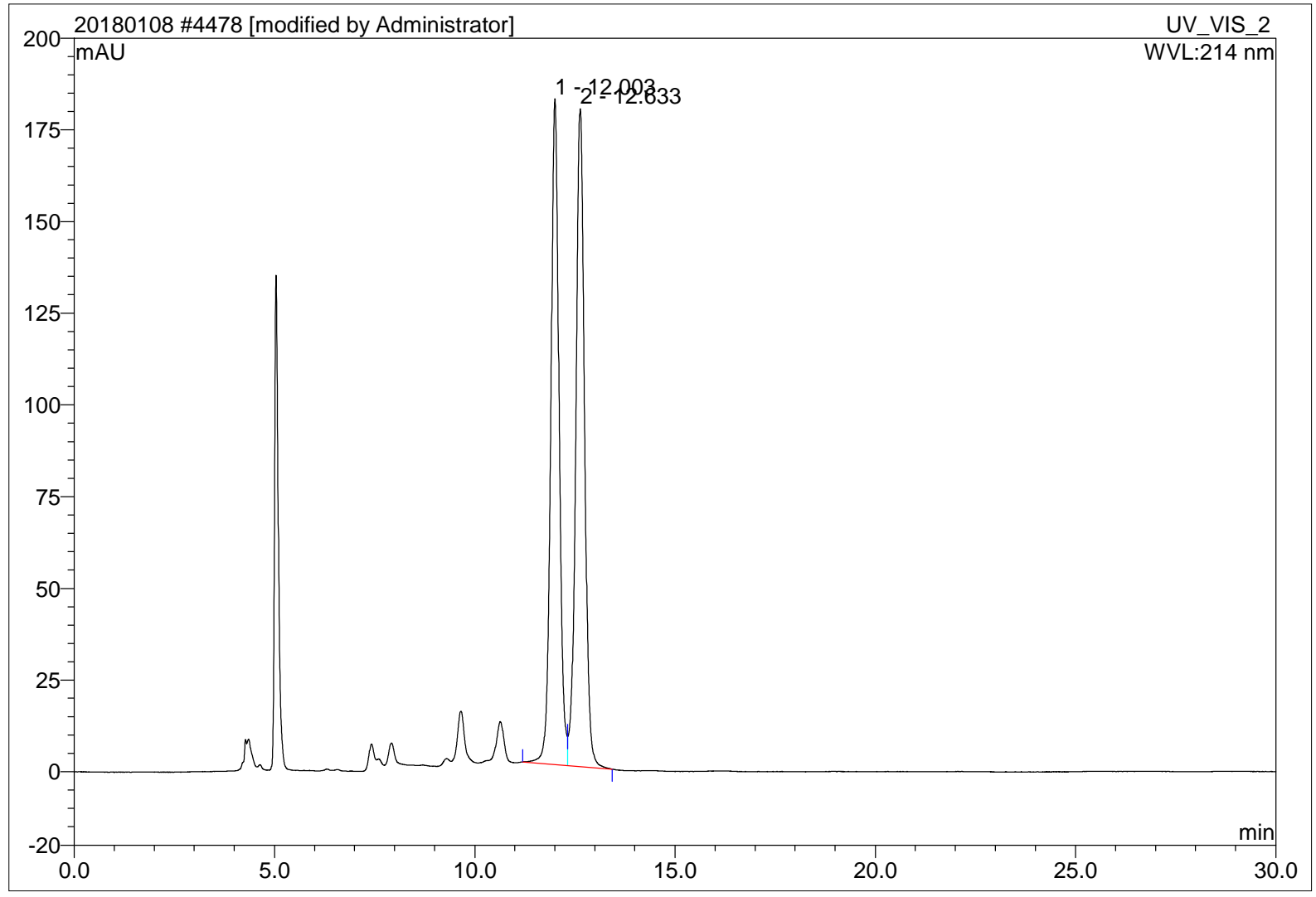

\begin{tabular}{|r|cccrrrr|}
\hline No. & $\begin{array}{c}\text { Ret.Time } \\
\text { min }\end{array}$ & Peak Name & $\begin{array}{c}\text { Height } \\
\text { mAU }\end{array}$ & $\begin{array}{c}\text { Area } \\
\text { mAU*min }\end{array}$ & $\begin{array}{r}\text { Rel.Area } \\
\%\end{array}$ & Amount & Type \\
\hline 1 & 12.00 & n.a. & 181.490 & 43.925 & 49.17 & n.a. & BM $^{*}$ \\
2 & 12.63 & n.a. & 179.374 & 45.417 & 50.83 & n.a. & MB $^{*}$ \\
\hline Total: & & & 360.864 & 89.342 & 100.00 & 0.000 & \\
\hline
\end{tabular}




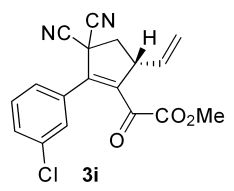

\begin{tabular}{|llll|}
\hline 4480 DWP-10-90 IB 912140.7 & & \\
& & & \\
\hline Sample Name: & DWP-10-90 IB 912140.7 & Injection Volume: & $\mathbf{5 . 0}$ \\
Vial Number: & GE5 & Channel: & UV_VIS_2 \\
Sample Type: & unknown & Wavelength: & $\mathbf{2 1 4 . 0}$ \\
Control Program: & test-dad6 & Bandwidth: & $\mathbf{4}$ \\
Quantif. Method: & $\mathbf{2 0 1 7 0 6 0 8}$ & Dilution Factor: & $\mathbf{1 . 0 0 0 0}$ \\
Recording Time: & $\mathbf{2 0 1 9 - 1 - 1 1 ~ 1 : 0 3}$ & Sample Weight: & $\mathbf{1 . 0 0 0 0}$ \\
Run Time (min): & $\mathbf{3 0 . 0 0}$ & Sample Amount: & $\mathbf{1 . 0 0 0 0}$ \\
\hline
\end{tabular}

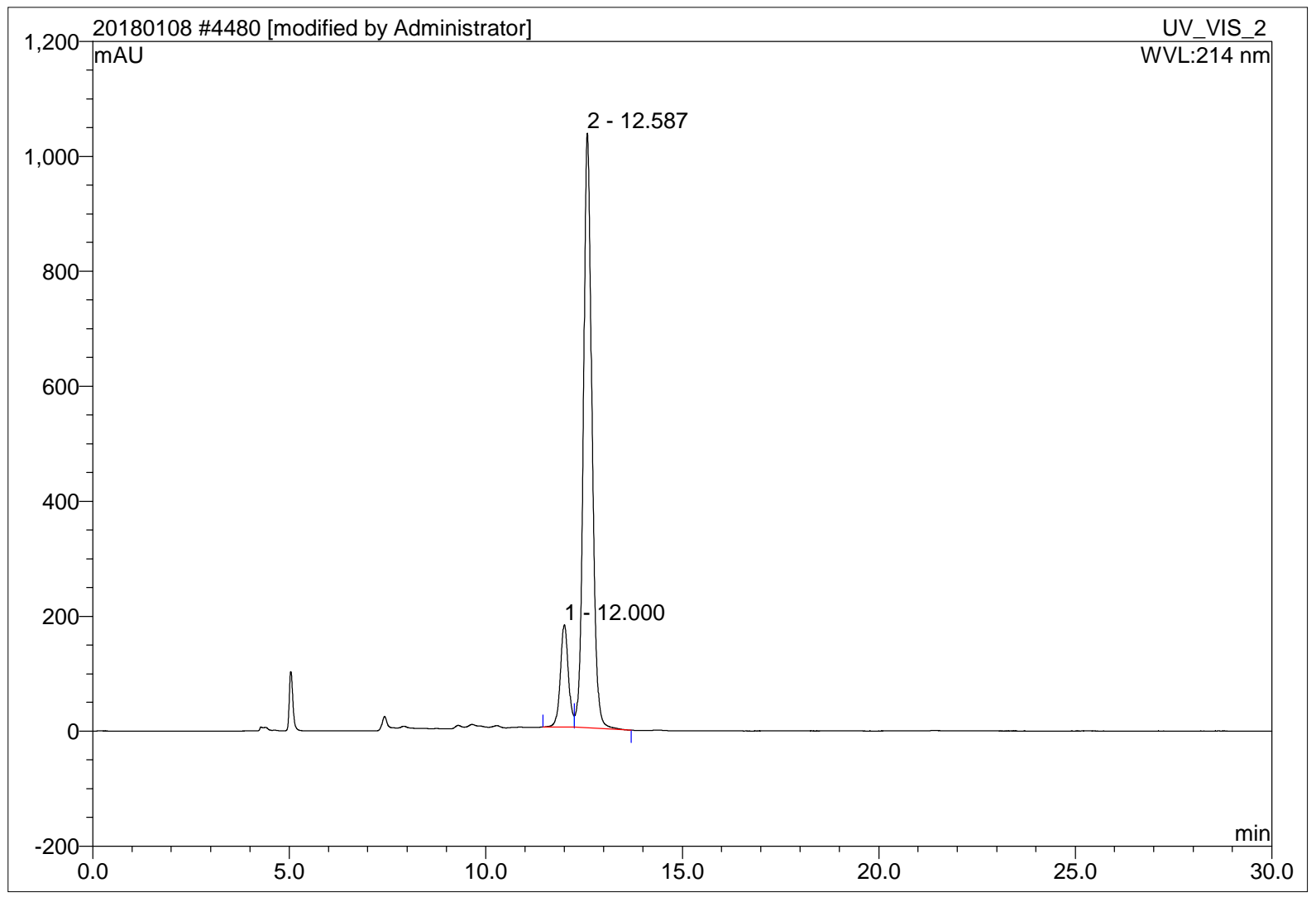

\begin{tabular}{|r|ccrrrrr|}
\hline No. & $\begin{array}{c}\text { Ret.Time } \\
\text { min }\end{array}$ & Peak Name & $\begin{array}{c}\text { Height } \\
\text { mAU }\end{array}$ & $\begin{array}{c}\text { Area } \\
\text { mAU*min }\end{array}$ & $\begin{array}{r}\text { Rel.Area } \\
\%\end{array}$ & Amount & Type \\
\hline 1 & 12.00 & n.a. & 178.719 & 42.430 & 13.92 & n.a. & BM $^{*}$ \\
2 & 12.59 & n.a. & 1034.866 & 262.333 & 86.08 & n.a. & MB $^{*}$ \\
\hline Total: & & & 1213.585 & 304.762 & 100.00 & 0.000 & \\
\hline
\end{tabular}




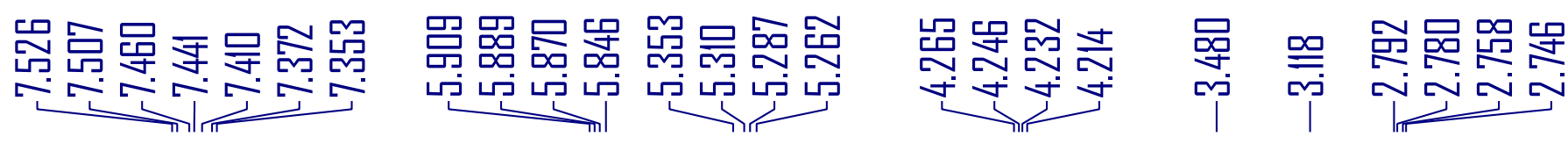
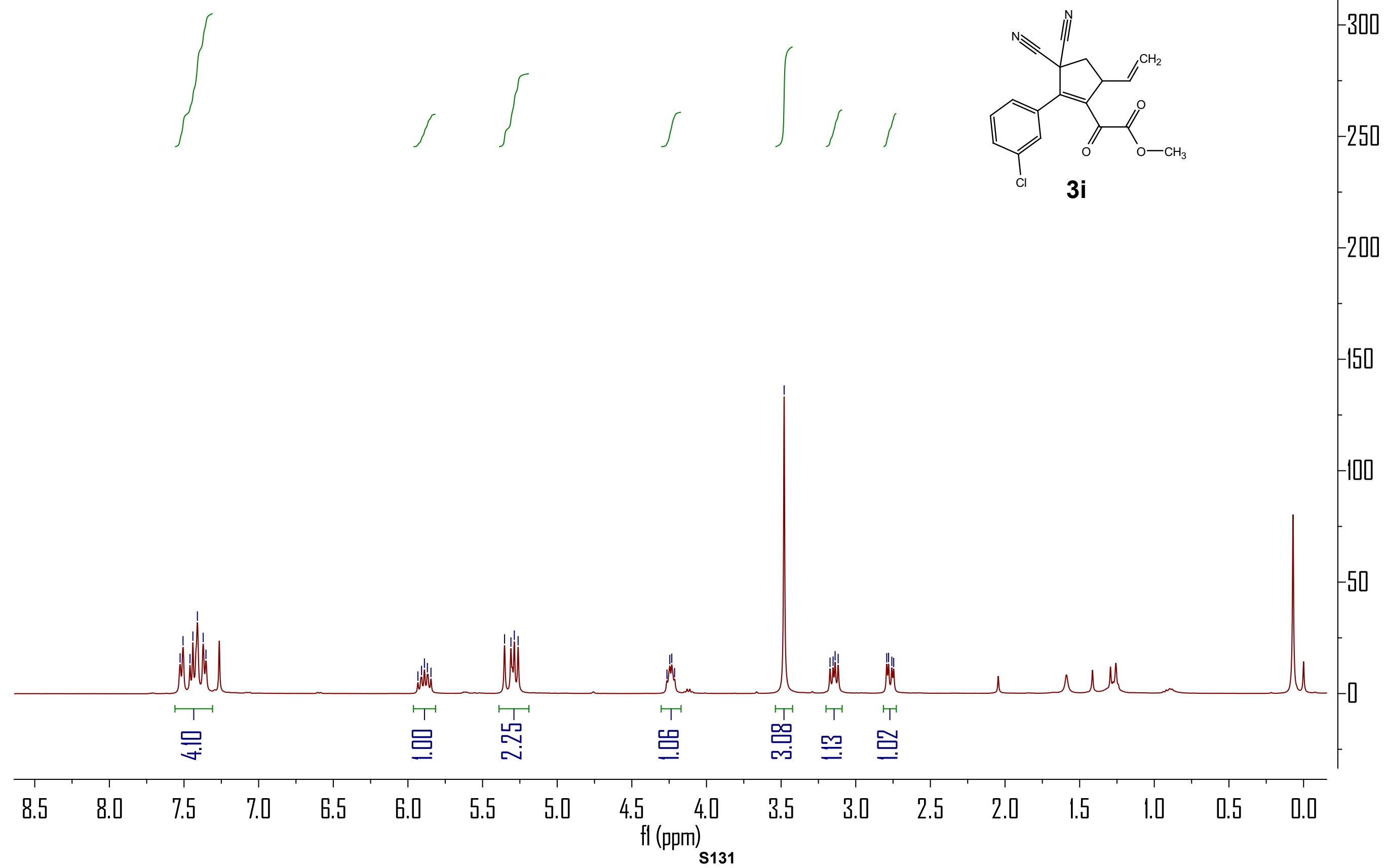


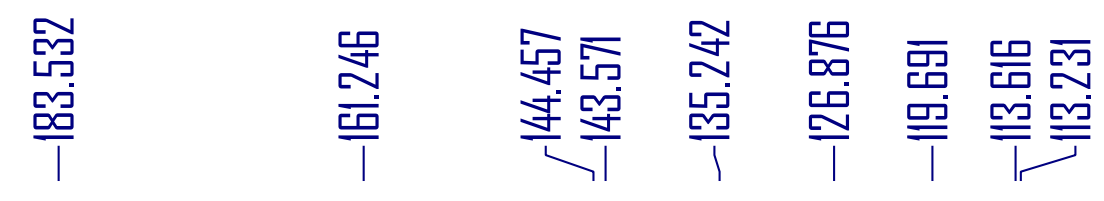

吕品器罢

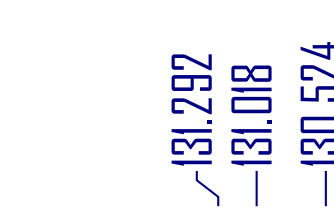

邑器芯

品
哭
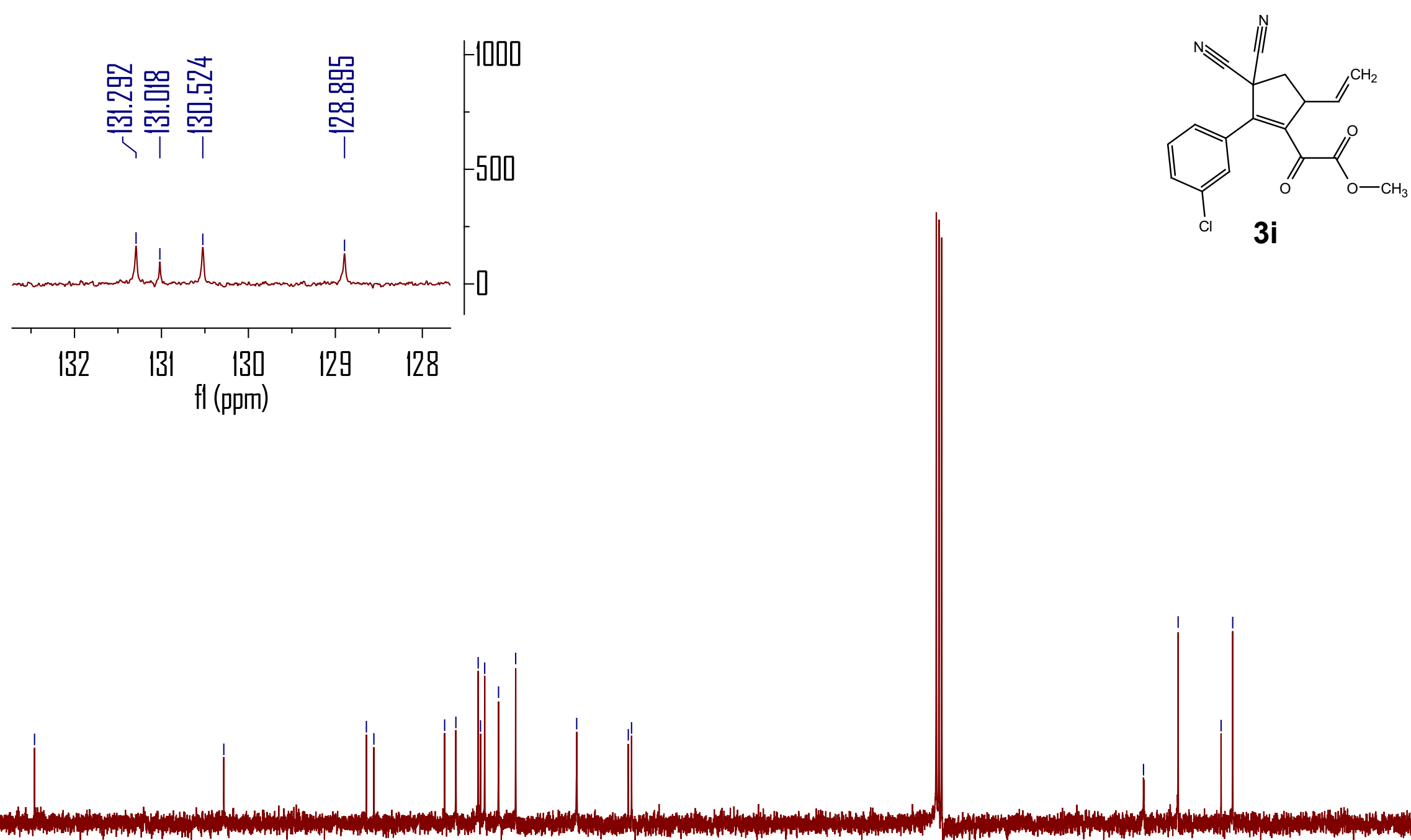

$\begin{array}{lllll}132 & 131 \\ \mathrm{fl}(\mathrm{ppm}) & 130 & 129 & 128\end{array}$

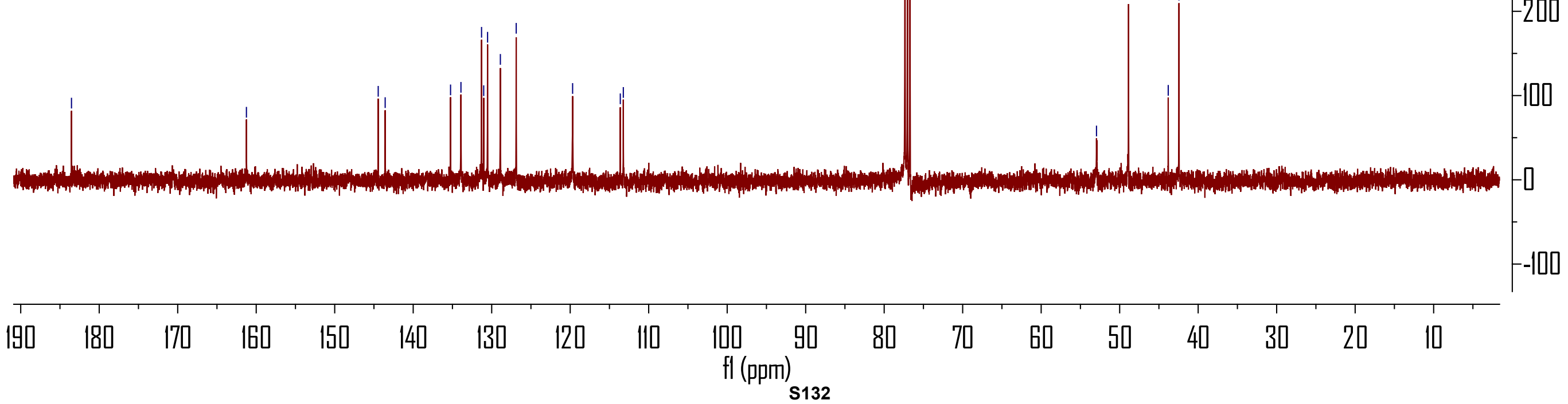




\begin{tabular}{|llll|}
\hline 4492 DWP-10-2+- IB 912540.7 & & \\
& & & \\
\hline Sample Name: & DWP-10-2+- IB 912540.7 & Injection Volume: & $\mathbf{5 . 0}$ \\
Vial Number: & GD2 & Channel: & EXT254NM \\
Sample Type: & unknown & Wavelength: & $\mathbf{2 5 4}$ \\
Control Program: & test-dad6 & Bandwidth: & $\mathbf{0}$ \\
Quantif. Method: & $\mathbf{2 0 1 7 0 6 0 8}$ & Dilution Factor: & $\mathbf{1 . 0 0 0 0}$ \\
Recording Time: & $\mathbf{2 0 1 9 - 1 - 1 1 ~ 7 : 1 6}$ & Sample Weight: & $\mathbf{1 . 0 0 0 0}$ \\
Run Time (min): & $\mathbf{3 0 . 0 0}$ & Sample Amount: & $\mathbf{1 . 0 0 0 0}$ \\
\hline
\end{tabular}

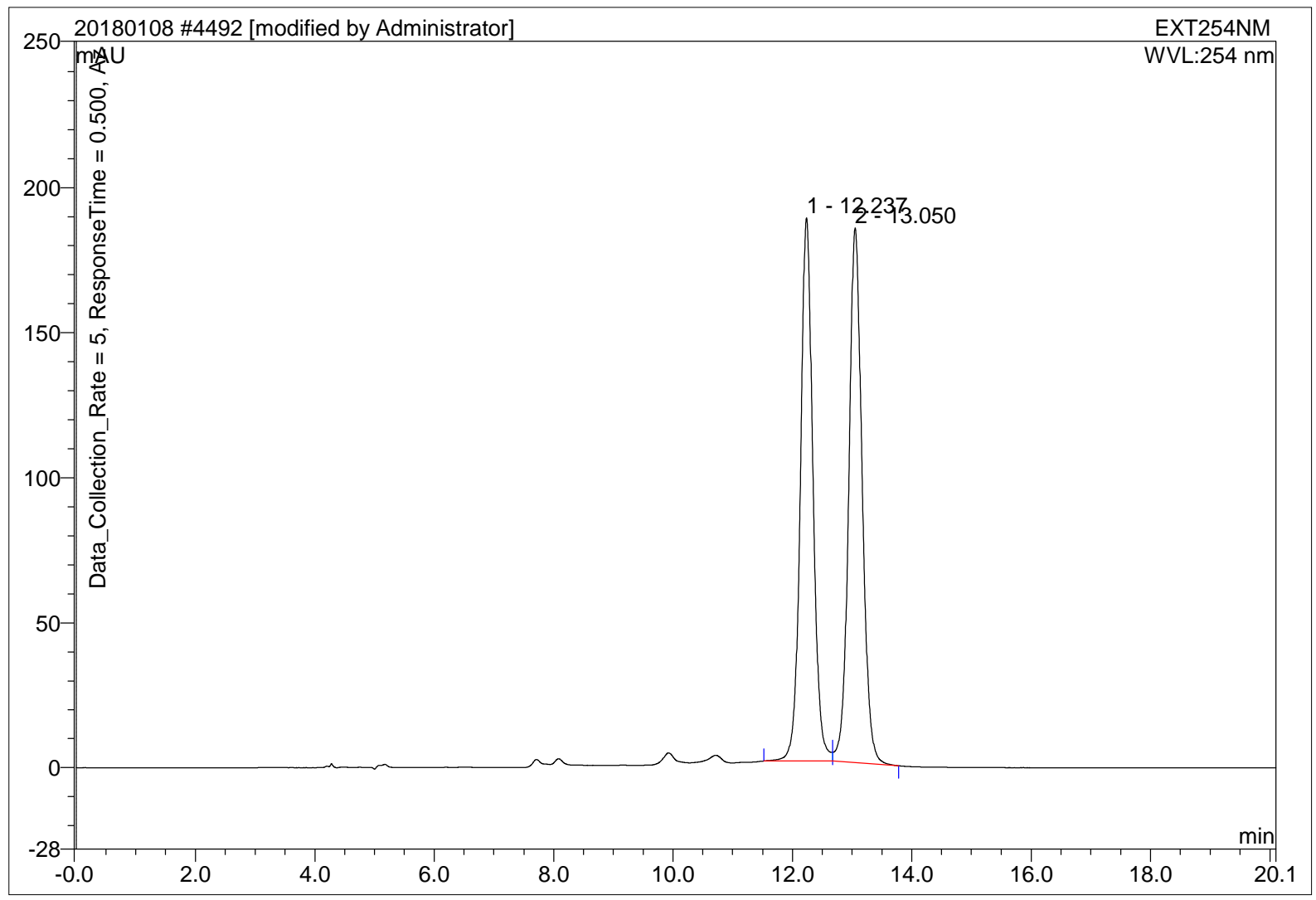

\begin{tabular}{|r|ccrrrrr|}
\hline No. & $\begin{array}{c}\text { Ret.Time } \\
\text { min }\end{array}$ & Peak Name & $\begin{array}{c}\text { Height } \\
\text { mAU }\end{array}$ & $\begin{array}{c}\text { Area } \\
\text { mAU*min }\end{array}$ & $\begin{array}{r}\text { Rel.Area } \\
\%\end{array}$ & Amount & Type \\
\hline 1 & 12.24 & n.a. & 187.375 & 45.793 & 48.82 & n.a. & BM $^{*}$ \\
2 & 13.05 & n.a. & 184.456 & 48.011 & 51.18 & n.a. & MB $^{*}$ \\
\hline Total: & & & 371.831 & 93.804 & 100.00 & 0.000 & \\
\hline
\end{tabular}




\begin{tabular}{|llll|}
\hline 4494 DWP-10-89 IB 912540.7 & & \\
& & & \\
\hline Sample Name: & DWP-10-89 IB 912540.7 & Injection Volume: & $\mathbf{5 . 0}$ \\
Vial Number: & GB2 & Channel: & EXT254NM \\
Sample Type: & unknown & Wavelength: & $\mathbf{2 5 4}$ \\
Control Program: & test-dad6 & Bandwidth: & $\mathbf{0}$ \\
Quantif. Method: & $\mathbf{2 0 1 7 0 6 0 8}$ & Dilution Factor: & $\mathbf{1 . 0 0 0 0}$ \\
Recording Time: & $\mathbf{2 0 1 9 - 1 - 1 1 ~ 8 : 1 8}$ & Sample Weight: & $\mathbf{1 . 0 0 0 0}$ \\
Run Time (min): & $\mathbf{1 8 . 0 0}$ & Sample Amount: & $\mathbf{1 . 0 0 0 0}$ \\
\hline
\end{tabular}

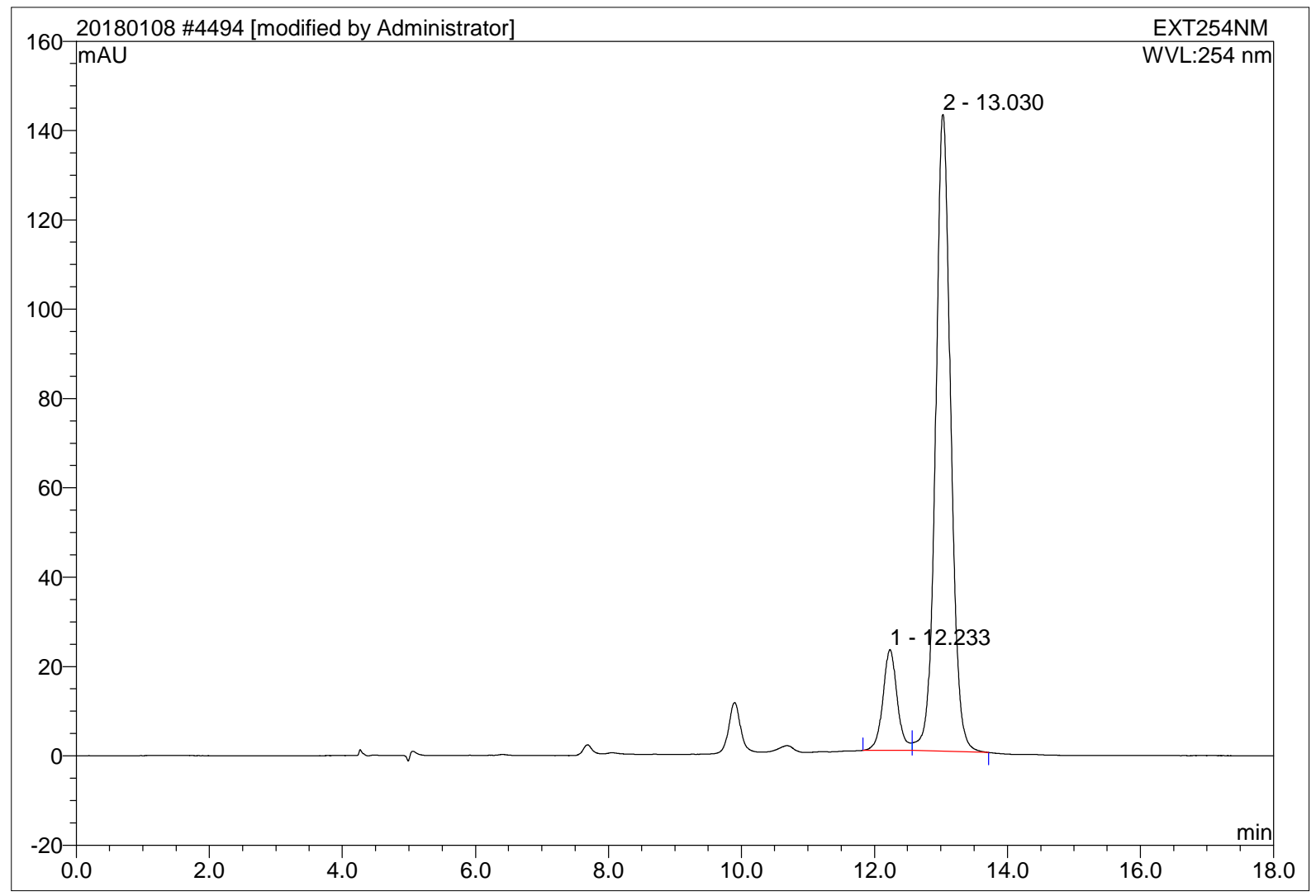

\begin{tabular}{|r|ccrrrrr|}
\hline No. & $\begin{array}{c}\text { Ret.Time } \\
\text { min }\end{array}$ & Peak Name & $\begin{array}{c}\text { Height } \\
\text { mAU }\end{array}$ & $\begin{array}{r}\text { Area } \\
\text { mAU*min }\end{array}$ & $\begin{array}{r}\text { Rel.Area } \\
\%\end{array}$ & Amount & Type \\
\hline 1 & 12.23 & n.a. & 22.616 & 5.641 & 13.02 & n.a. & BM $^{*}$ \\
2 & 13.03 & n.a. & 142.607 & 37.689 & 86.98 & n.a. & MB $^{*}$ \\
\hline Total: & & & 165.223 & 43.331 & 100.00 & 0.000 & \\
\hline
\end{tabular}




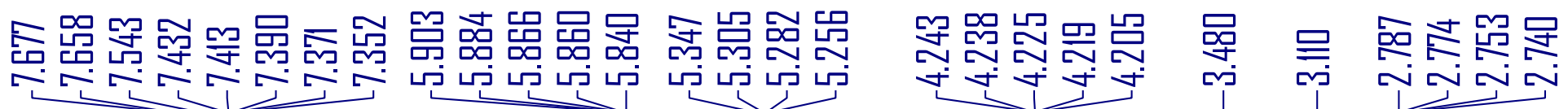
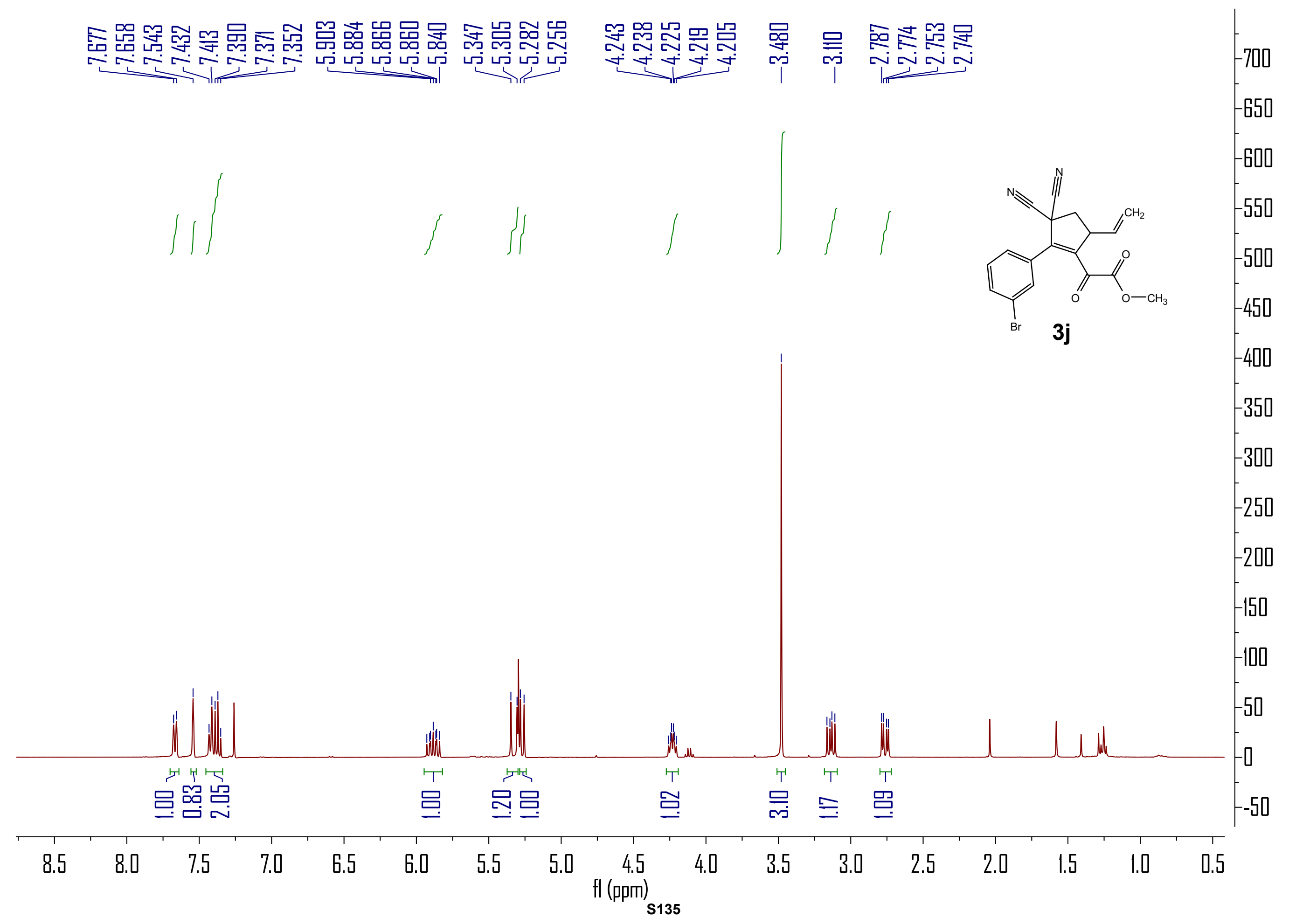


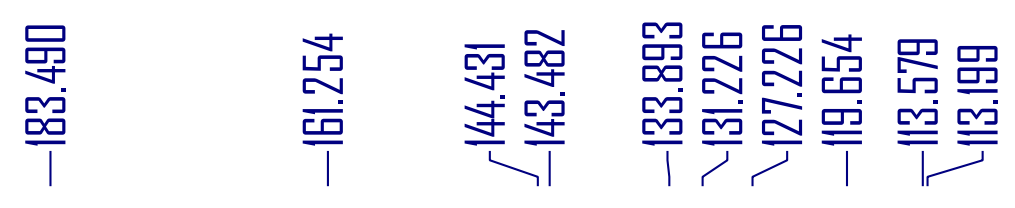

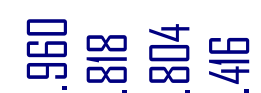

记 咛 羿

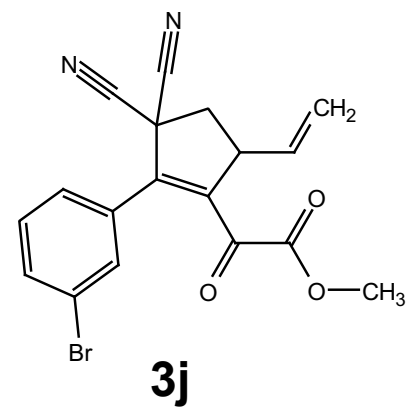




\begin{tabular}{|llll|}
\hline 8421 DWP-9-90+- IC 912140.7 & & \\
& & & \\
\hline Sample Name: & DWP-9-90+- IC 912140.7 & Injection Volume: & $\mathbf{5 . 0}$ \\
Vial Number: & GA6 & Channel: & UV_VIS_1 \\
Sample Type: & unknown & Wavelength: & $\mathbf{2 1 4}$ \\
Control Program: & $\mathbf{2 0 1 7 0 1 - 4}$ & Bandwidth: & n.a. \\
Quantif. Method: & $\mathbf{2 0 1 7 0 1}$ & Dilution Factor: & $\mathbf{1 . 0 0 0 0}$ \\
Recording Time: & $\mathbf{2 0 1 9 / 1 / 9 ~ 2 0 : 3 0}$ & Sample Weight: & $\mathbf{1 . 0 0 0 0}$ \\
Run Time (min): & $\mathbf{2 5 . 0 0}$ & Sample Amount: & $\mathbf{1 . 0 0 0 0}$ \\
\hline
\end{tabular}

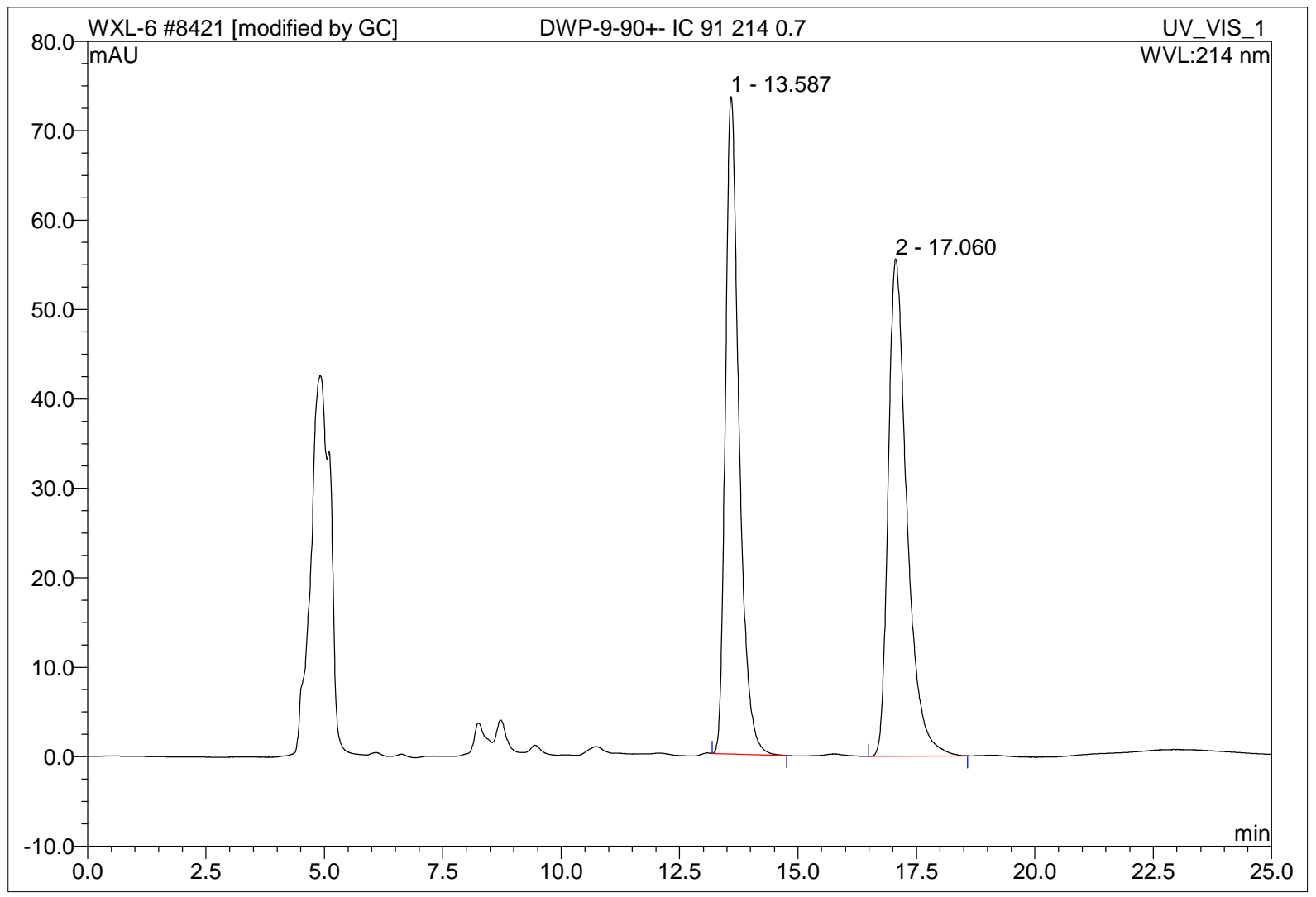

\begin{tabular}{|r|ccrrrrr|}
\hline No. & $\begin{array}{c}\text { Ret.Time } \\
\text { min }\end{array}$ & Peak Name & $\begin{array}{c}\text { Height } \\
\text { mAU }\end{array}$ & $\begin{array}{c}\text { Area } \\
\text { mAU*min }\end{array}$ & $\begin{array}{r}\text { Rel.Area } \\
\%\end{array}$ & Amount & Type \\
\hline 1 & 13.59 & n.a. & 73.545 & 24.579 & 49.07 & n.a. & BMB \\
2 & 17.06 & n.a. & 55.631 & 25.509 & 50.93 & n.a. & BMB \\
\hline Total: & & & 129.176 & 50.087 & 100.00 & 0.000 & \\
\hline
\end{tabular}




\section{DWP-10-63 IC 912140.7}

\begin{tabular}{|llll|}
\hline Sample Name: & DWP-10-63 IC 912140.7 & Injection Volume: & $\mathbf{5 . 0}$ \\
Vial Number: & GB5 & Channel: & UV_VIS_1 \\
Sample Type: & unknown & Wavelength: & $\mathbf{2 1 4}$ \\
Control Program: & $\mathbf{2 0 1 7 0 1 - 4}$ & Bandwidth: & n.a. \\
Quantif. Method: & $\mathbf{2 0 1 7 0 1}$ & Dilution Factor: & $\mathbf{1 . 0 0 0 0}$ \\
Recording Time: & $\mathbf{2 0 1 9 / 1 / 9 2 1 : 2 3}$ & Sample Weight: & $\mathbf{1 . 0 0 0 0}$ \\
Run Time (min): & $\mathbf{2 5 . 0 0}$ & Sample Amount: & $\mathbf{1 . 0 0 0 0}$ \\
\hline
\end{tabular}

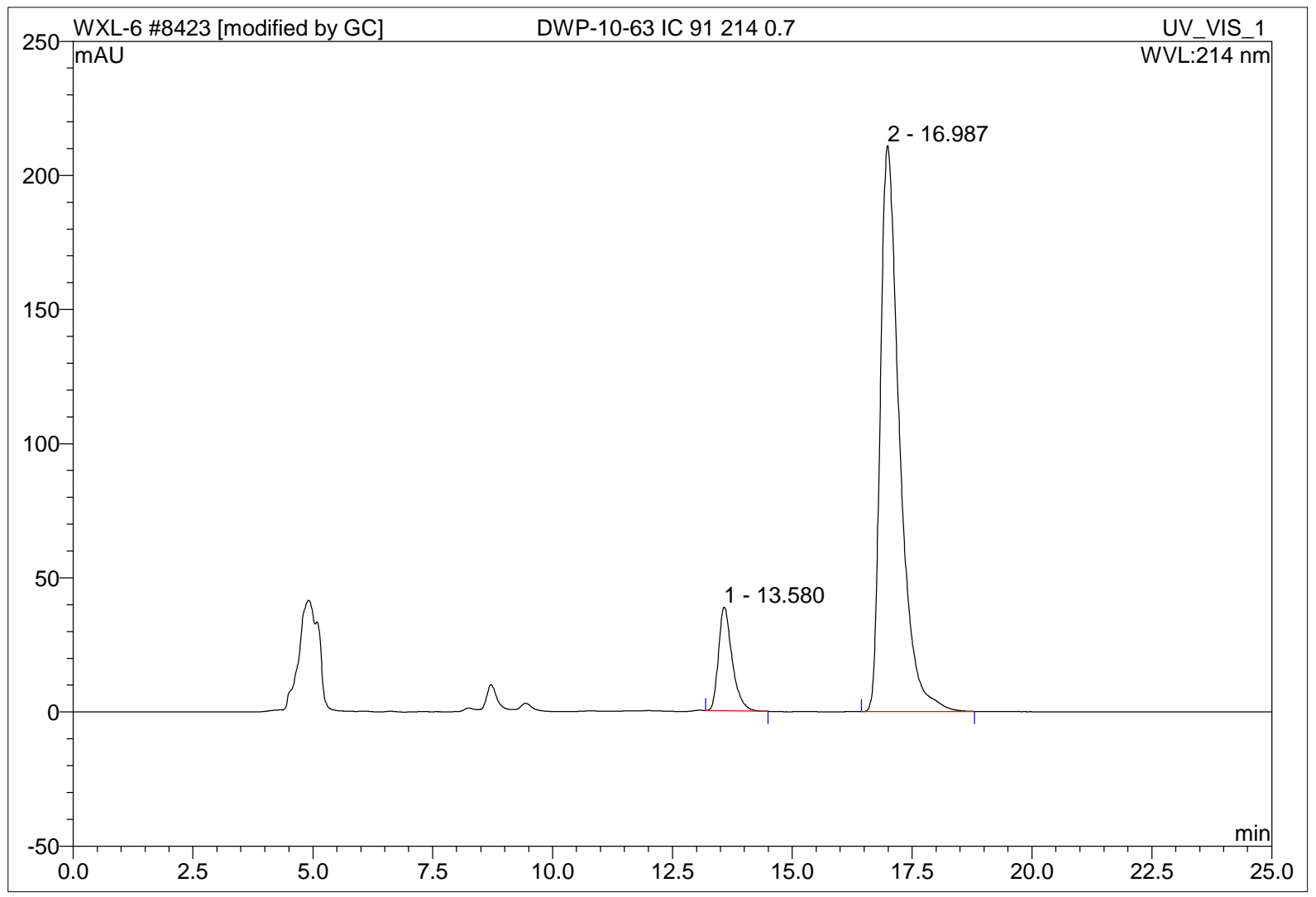

\begin{tabular}{|r|ccrrrrr|}
\hline No. & $\begin{array}{c}\text { Ret.Time } \\
\text { min }\end{array}$ & Peak Name & $\begin{array}{c}\text { Height } \\
\text { mAU }\end{array}$ & $\begin{array}{c}\text { Area } \\
\text { mAU*min }\end{array}$ & $\begin{array}{r}\text { Rel.Area } \\
\%\end{array}$ & Amount & Type \\
\hline 1 & 13.58 & n.a. & 38.698 & 12.822 & 11.57 & n.a. & BMB \\
2 & 16.99 & n.a. & 211.100 & 98.010 & 88.43 & n.a. & BMB \\
\hline Total: & & & 249.797 & 110.832 & 100.00 & 0.000 & \\
\hline
\end{tabular}




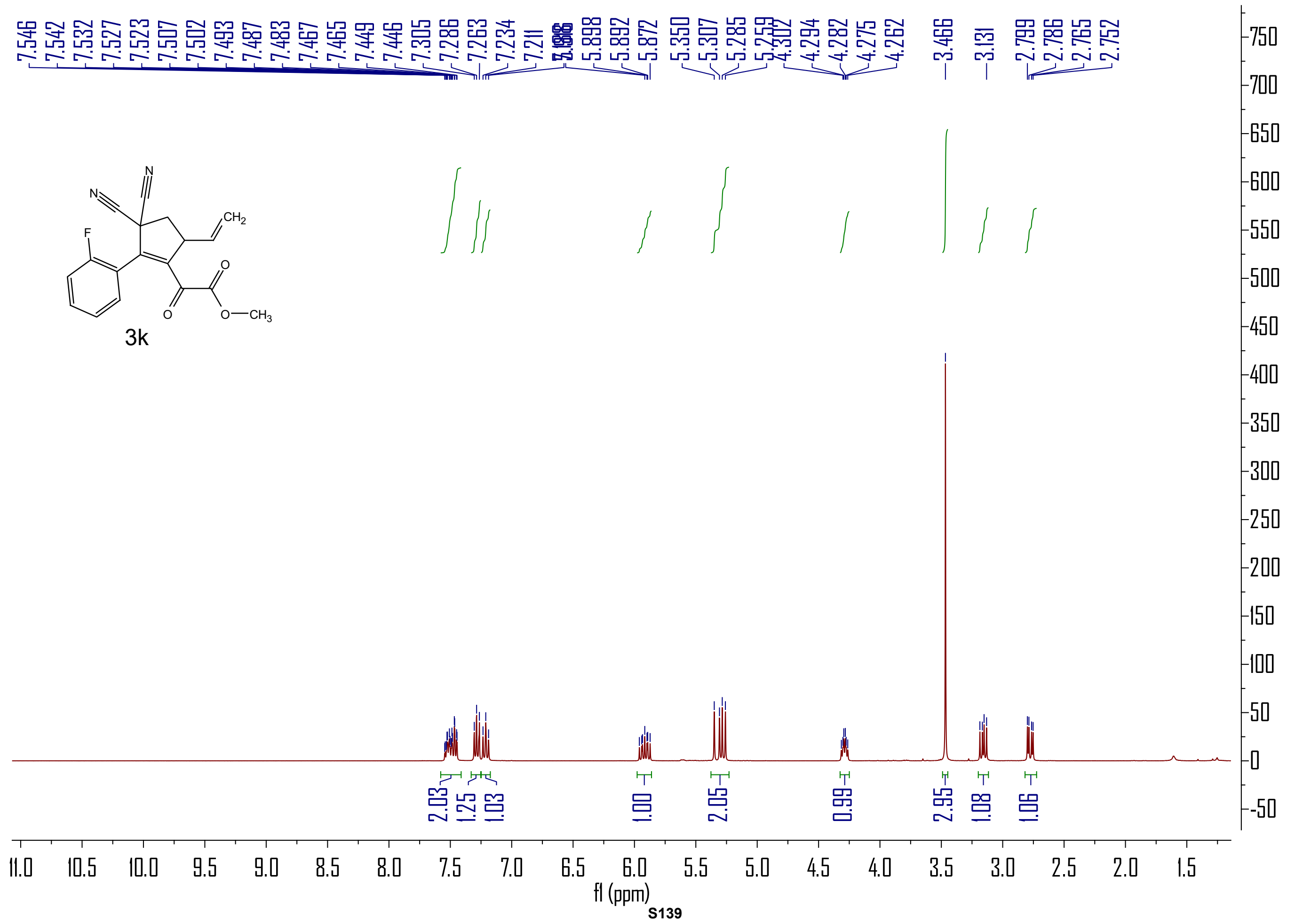


品

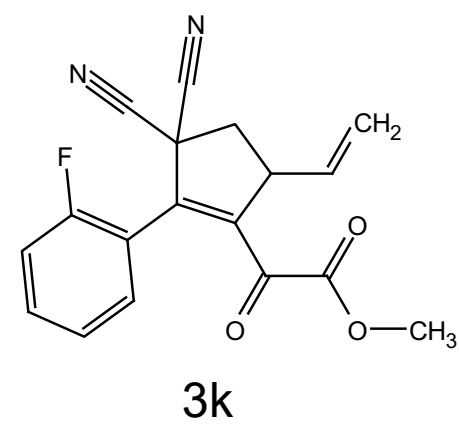

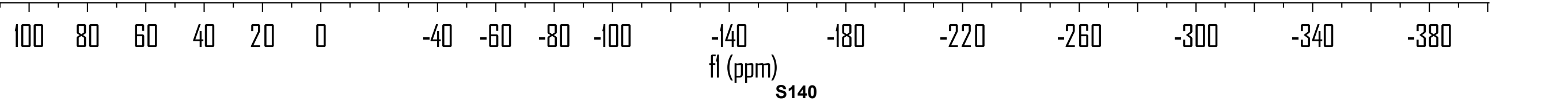




\section{DWP-10-20+- ID-3 912140.7}

\begin{tabular}{|llll|}
\hline Sample Name: & DWP-10-20+- ID-3 912140.7 & Injection Volume: & $\mathbf{3 . 0}$ \\
Vial Number: & GC3 & Channel: & UV_VIS_1 \\
Sample Type: & unknown & Wavelength: & $\mathbf{2 1 4}$ \\
Control Program: & $\mathbf{2 0 1 7 0 1 - 4}$ & Bandwidth: & n.a. \\
Quantif. Method: & $\mathbf{2 0 1 7 0 1}$ & Dilution Factor: & $\mathbf{1 . 0 0 0 0}$ \\
Recording Time: & $\mathbf{2 0 1 9 / 1 / 1 0 ~ 1 6 : 5 6}$ & Sample Weight: & $\mathbf{1 . 0 0 0 0}$ \\
Run Time (min): & $\mathbf{2 0 . 8 1}$ & Sample Amount: & $\mathbf{1 . 0 0 0 0}$ \\
\hline
\end{tabular}

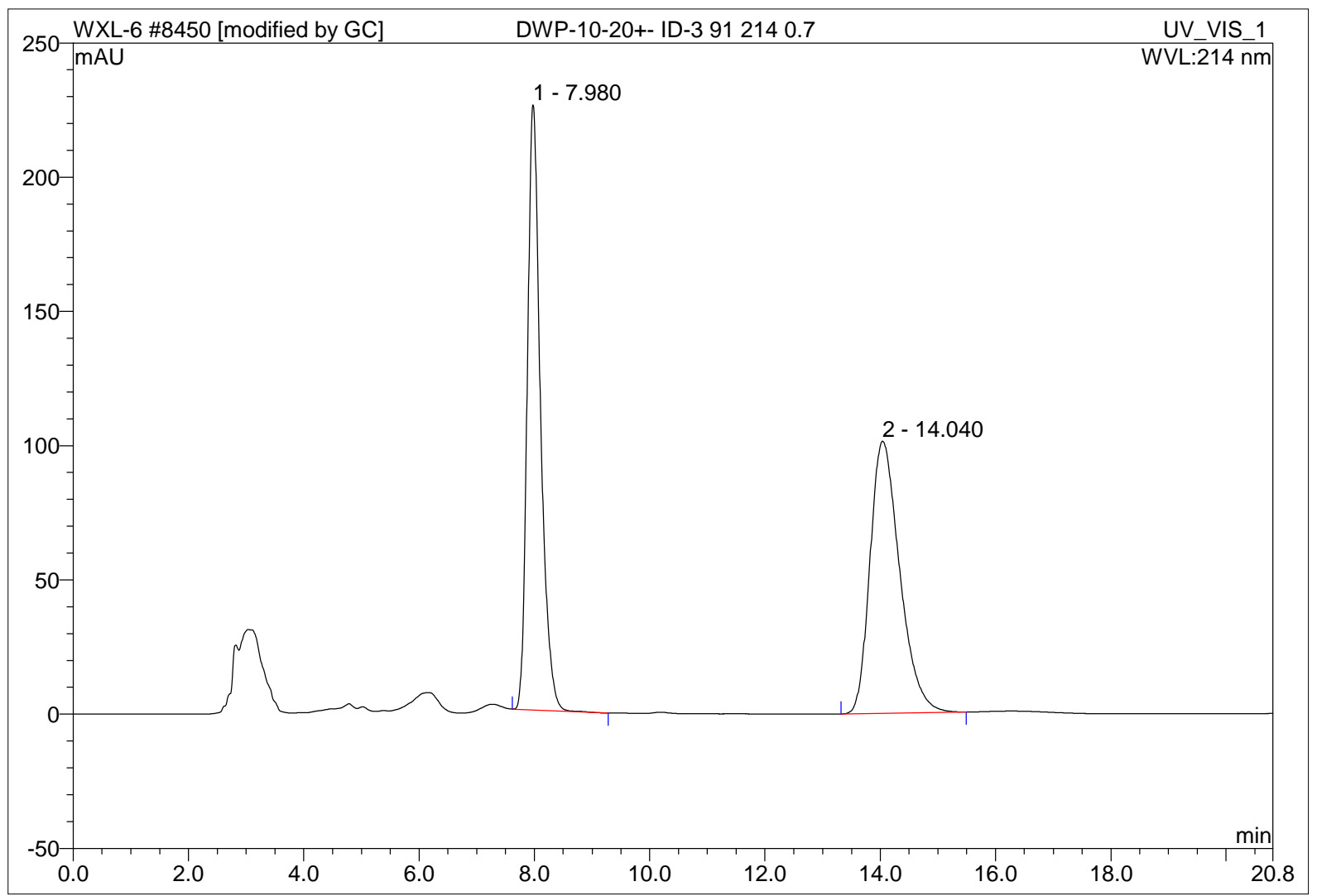

\begin{tabular}{|r|ccrrrrr|}
\hline No. & $\begin{array}{c}\text { Ret.Time } \\
\text { min }\end{array}$ & Peak Name & $\begin{array}{c}\text { Height } \\
\text { mAU }\end{array}$ & $\begin{array}{r}\text { Area } \\
\text { mAU*min }\end{array}$ & $\begin{array}{r}\text { Rel.Area } \\
\%\end{array}$ & Amount & Type \\
\hline 1 & 7.98 & n.a. & 225.496 & 59.796 & 49.71 & n.a. & BMB $^{*}$ \\
2 & 14.04 & n.a. & 101.429 & 60.502 & 50.29 & n.a. & BMB \\
\hline Total: & & & 326.925 & 120.298 & 100.00 & 0.000 & \\
\hline
\end{tabular}




\section{DWP-10-59 ID-3 912140.7}

\begin{tabular}{|llll|}
\hline Sample Name: & DWP-10-59 ID-3 912140.7 & Injection Volume: & $\mathbf{3 . 0}$ \\
Vial Number: & GC1 & Channel: & UV_VIS_1 \\
Sample Type: & unknown & Wavelength: & $\mathbf{2 1 4}$ \\
Control Program: & $\mathbf{2 0 1 7 0 1 - 4}$ & Bandwidth: & n.a. \\
Quantif. Method: & $\mathbf{2 0 1 7 0 1}$ & Dilution Factor: & $\mathbf{1 . 0 0 0 0}$ \\
Recording Time: & $\mathbf{2 0 1 9 / 1 / 1 0 ~ 1 7 : 4 9}$ & Sample Weight: & $\mathbf{1 . 0 0 0 0}$ \\
Run Time (min): & $\mathbf{1 7 . 6 4}$ & Sample Amount: & $\mathbf{1 . 0 0 0 0}$ \\
\hline
\end{tabular}

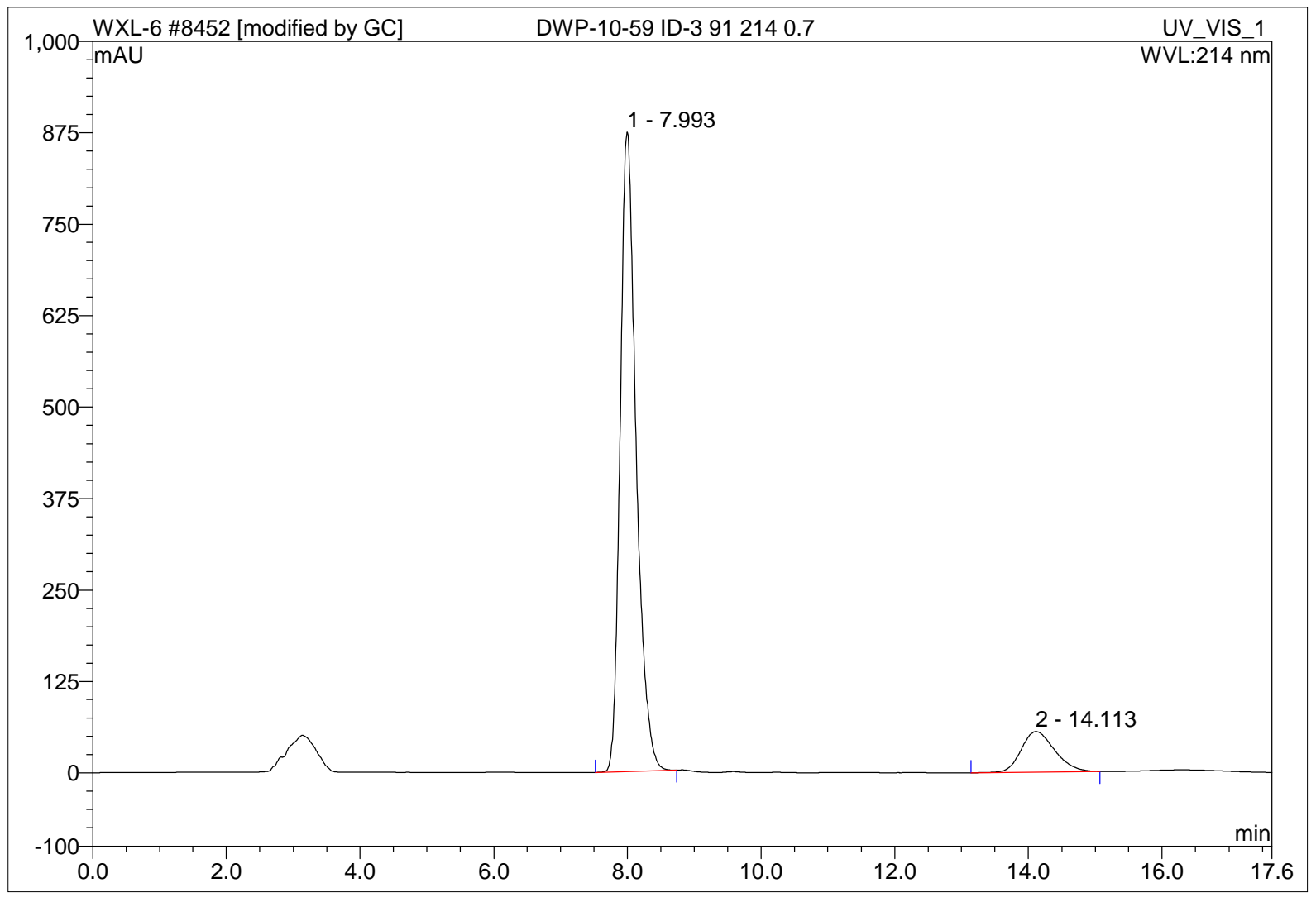

\begin{tabular}{|r|ccrrrrr|}
\hline No. & $\begin{array}{c}\text { Ret.Time } \\
\text { min }\end{array}$ & Peak Name & $\begin{array}{c}\text { Height } \\
\text { mAU }\end{array}$ & $\begin{array}{c}\text { Area } \\
\text { mAU*min }\end{array}$ & $\begin{array}{r}\text { Rel.Area } \\
\%\end{array}$ & Amount & Type \\
& 7.99 & n.a. & 874.093 & 242.160 & 88.34 & n.a. & BMB \\
2 & 14.11 & n.a. & 55.303 & 31.958 & 11.66 & n.a. & BMB $^{*}$ \\
\hline Total: & & & 929.396 & 274.118 & 100.00 & 0.000 & \\
\hline
\end{tabular}




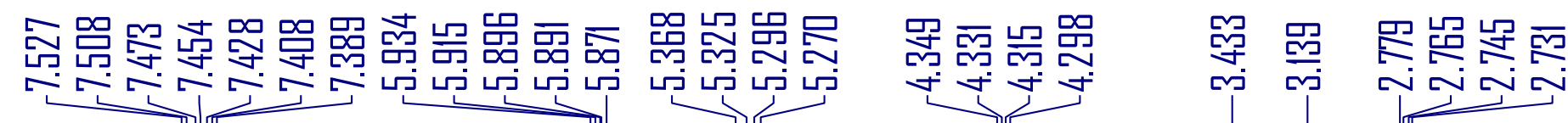

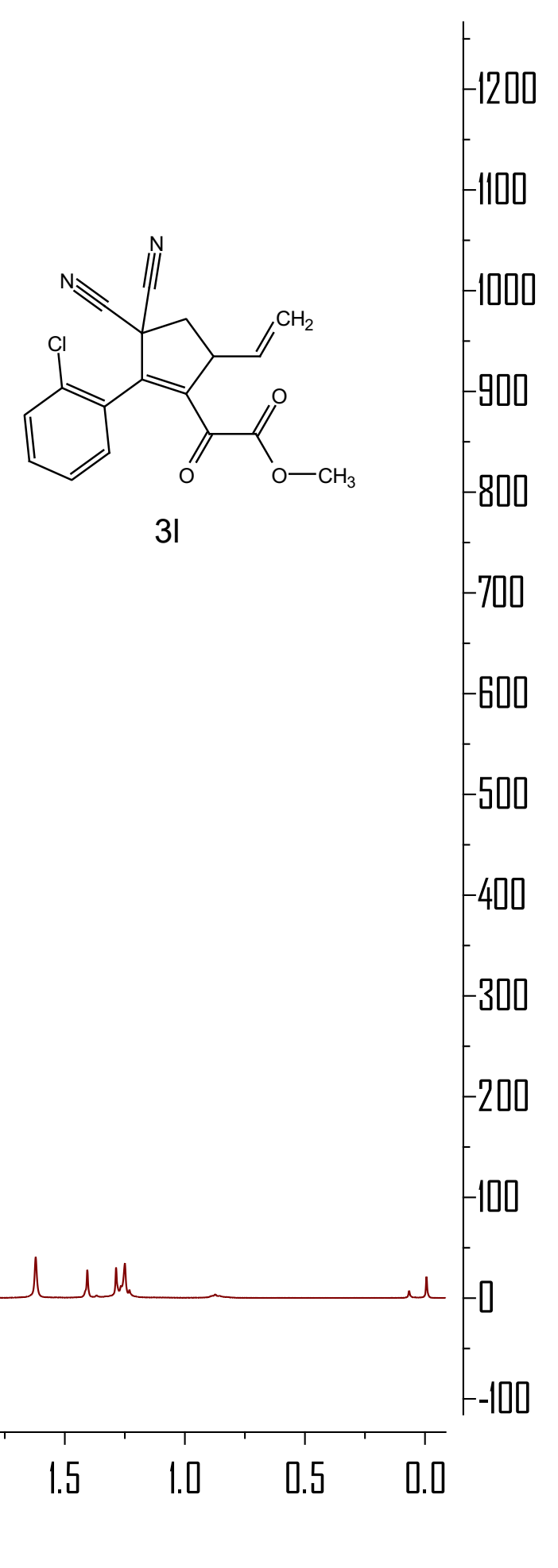




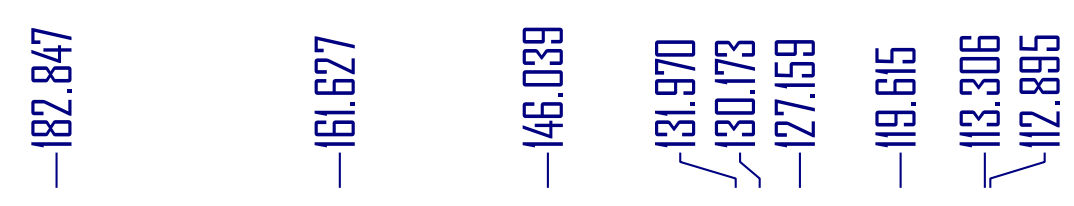

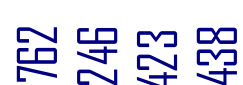

동 过
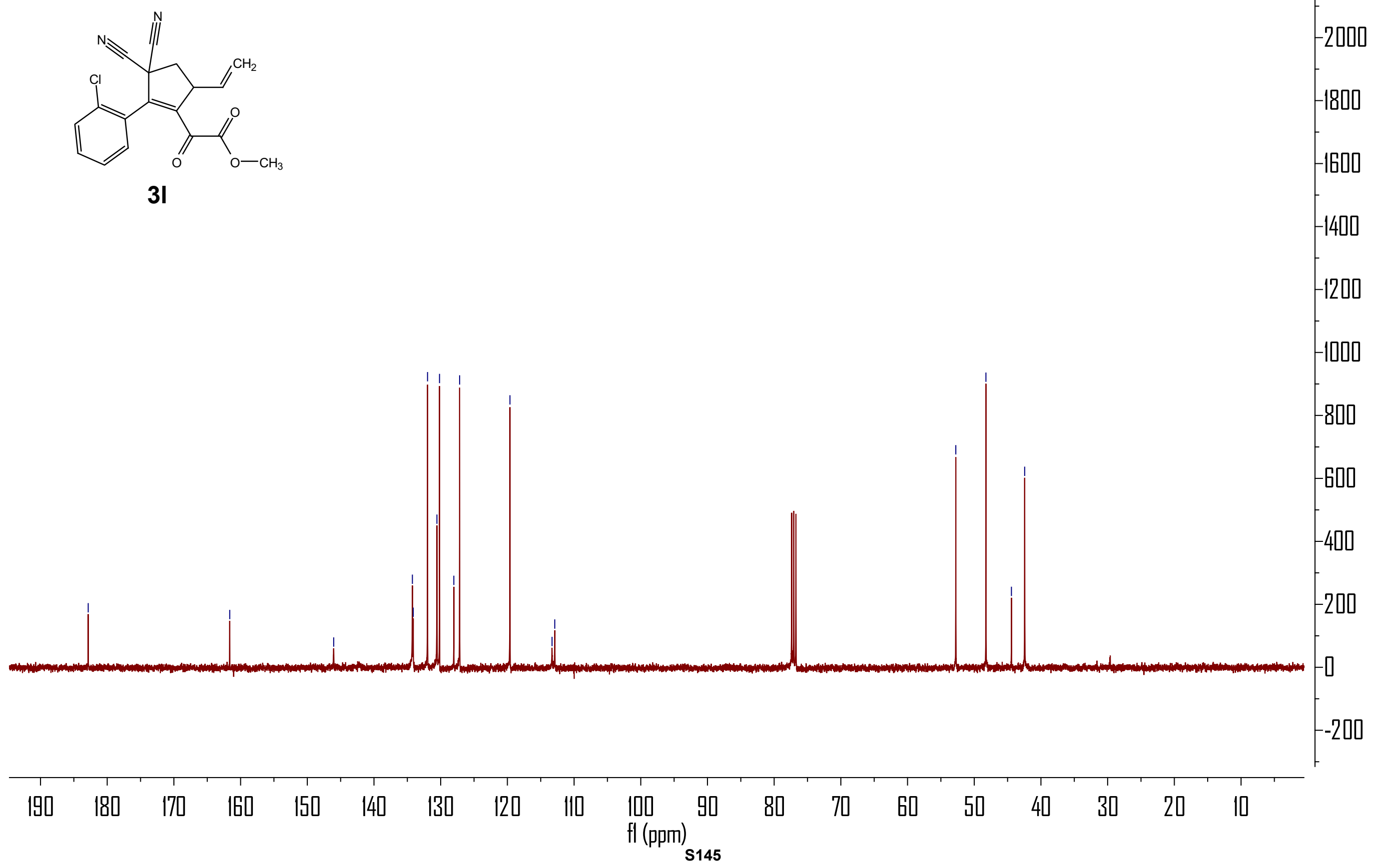


\section{DWP-10-21+- ID-3 9552140.7}

\begin{tabular}{|llll|}
\hline Sample Name: & DWP-10-21+- ID-3 9552140.7 & Injection Volume: & $\mathbf{1 . 0}$ \\
Vial Number: & GC7 & Channel: & UV_VIS_1 \\
Sample Type: & unknown & Wavelength: & $\mathbf{2 1 4}$ \\
Control Program: & $\mathbf{2 0 1 7 0 1 - 6}$ & Bandwidth: & n.a. \\
Quantif. Method: & $\mathbf{2 0 1 7 0 1}$ & Dilution Factor: & $\mathbf{1 . 0 0 0 0}$ \\
Recording Time: & $\mathbf{2 0 1 9 / 1 / 8 ~ 1 2 : 2 0}$ & Sample Weight: & $\mathbf{1 . 0 0 0 0}$ \\
Run Time (min): & $\mathbf{3 0 . 0 0}$ & Sample Amount: & $\mathbf{1 . 0 0 0 0}$ \\
\hline
\end{tabular}

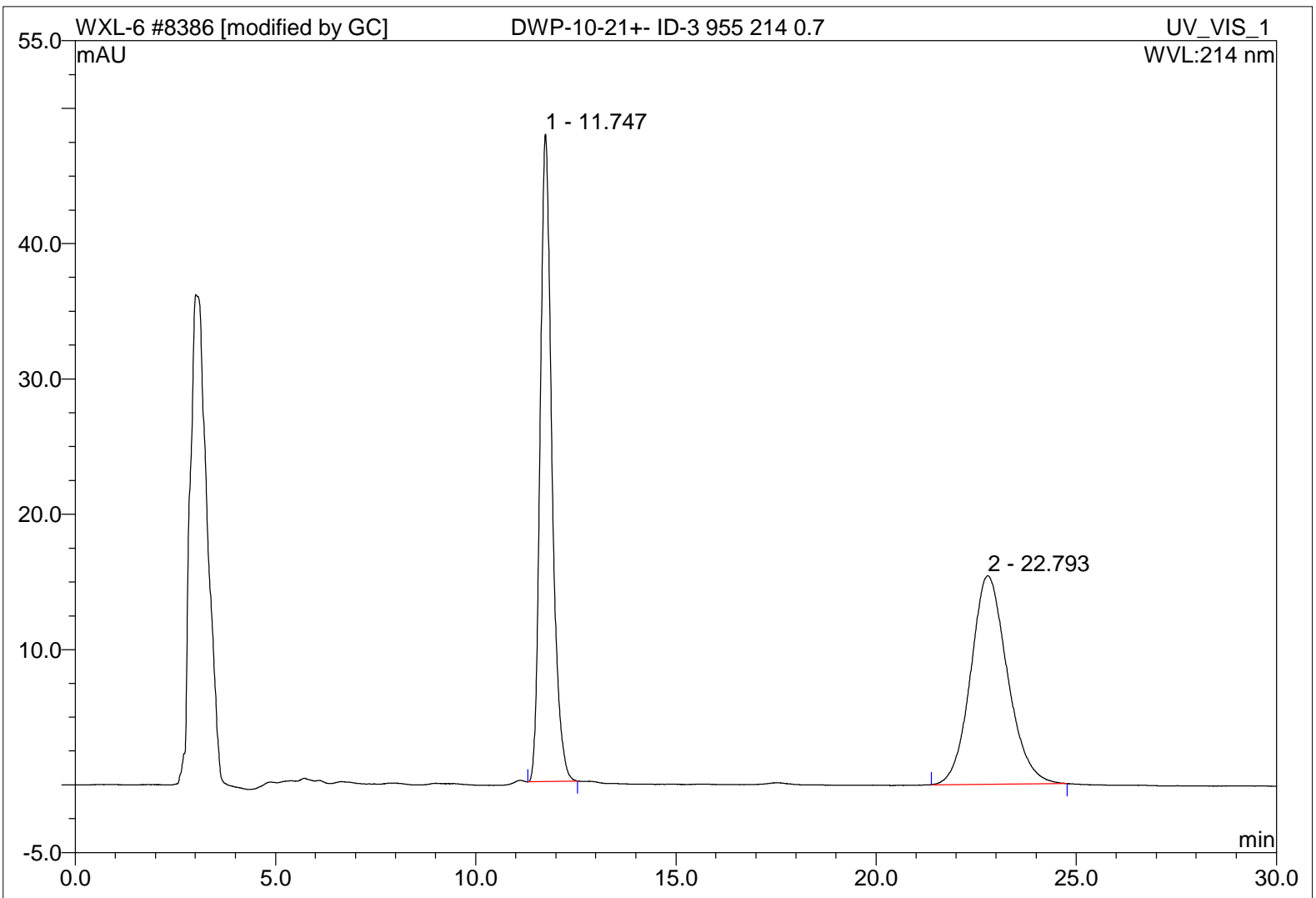

\begin{tabular}{|r|ccrrrrr|}
\hline No. & $\begin{array}{c}\text { Ret.Time } \\
\text { min }\end{array}$ & Peak Name & $\begin{array}{c}\text { Height } \\
\text { mAU }\end{array}$ & $\begin{array}{c}\text { Area } \\
\text { mAU*min }\end{array}$ & $\begin{array}{r}\text { Rel.Area } \\
\%\end{array}$ & Amount & Type \\
\hline 1 & 11.75 & n.a. & 47.875 & 16.306 & 49.87 & n.a. & BMB \\
2 & 22.79 & n.a. & 15.432 & 16.388 & 50.13 & n.a. & BMB \\
\hline Total: & & & 63.307 & 32.695 & 100.00 & 0.000 & \\
\hline
\end{tabular}




\section{DWP-10-62 ID-3 9552140.7}

\begin{tabular}{llll|}
\hline Sample Name: & DWP-10-62 ID-3 955 214 0.7 & Injection Volume: & $\mathbf{1 . 0}$ \\
Vial Number: & GC6 & Channel: & UV_VIS_1 \\
Sample Type: & unknown & Wavelength: & $\mathbf{2 1 4}$ \\
Control Program: & $\mathbf{2 0 1 7 0 1 - 6}$ & Bandwidth: & n.a. \\
Quantif. Method: & $\mathbf{2 0 1 7 0 1}$ & Dilution Factor: & $\mathbf{1 . 0 0 0 0}$ \\
Recording Time: & $\mathbf{2 0 1 9 / 1 / 8 1 1 : 3 7}$ & Sample Weight: & $\mathbf{1 . 0 0 0 0}$ \\
Run Time (min): & $\mathbf{4 1 . 0 7}$ & Sample Amount: & $\mathbf{1 . 0 0 0 0}$ \\
\hline
\end{tabular}

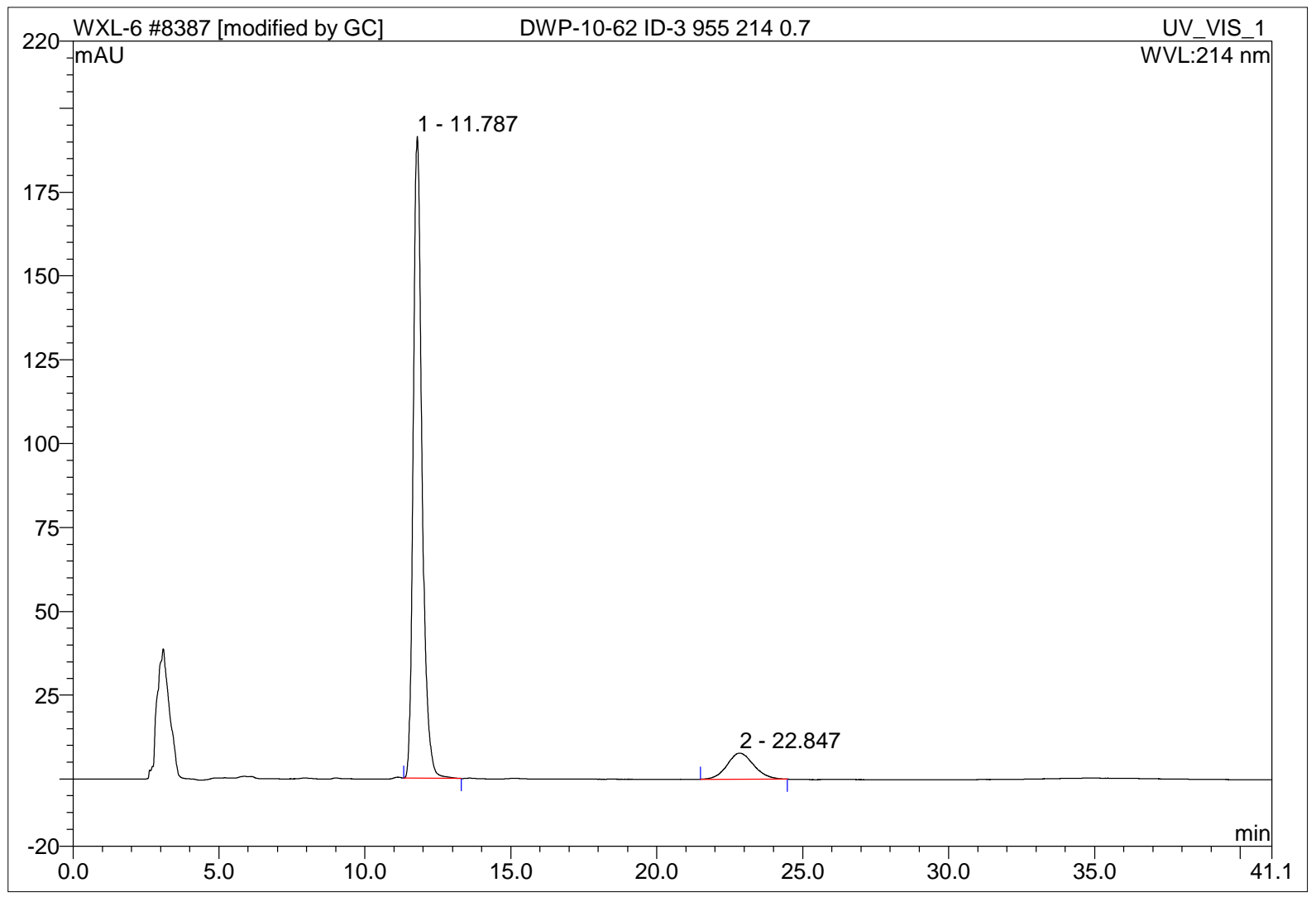

\begin{tabular}{|r|ccrrrrr|}
\hline No. & $\begin{array}{c}\text { Ret.Time } \\
\text { min }\end{array}$ & Peak Name & $\begin{array}{c}\text { Height } \\
\text { mAU }\end{array}$ & $\begin{array}{c}\text { Area } \\
\text { mAU*min }\end{array}$ & $\begin{array}{r}\text { Rel.Area } \\
\%\end{array}$ & Amount & Type \\
& 11.79 & n.a. & 191.423 & 66.706 & 88.98 & n.a. & BMB \\
2 & 22.85 & n.a. & 7.778 & 8.262 & 11.02 & n.a. & BMB \\
\hline Total: & & & 199.201 & 74.968 & 100.00 & 0.000 & \\
\hline
\end{tabular}




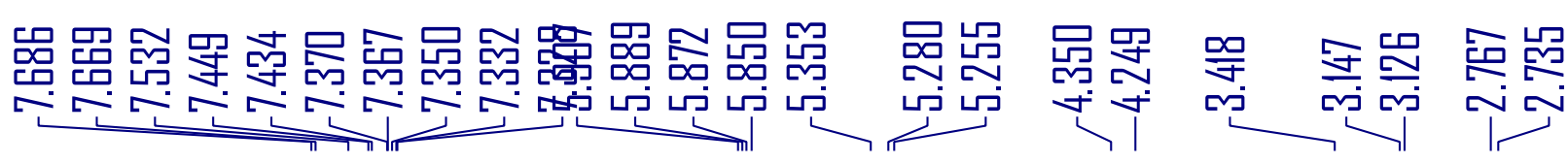
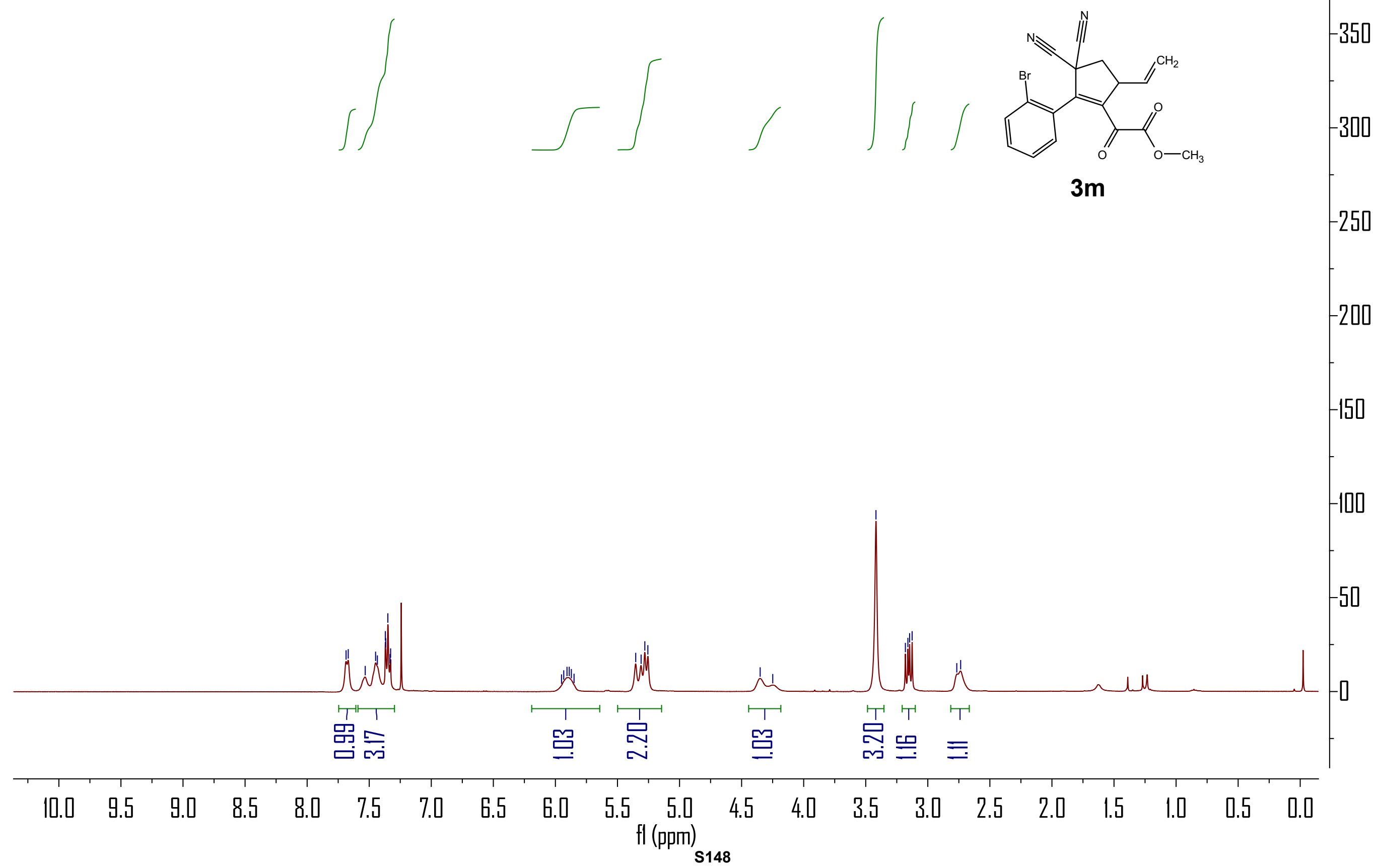


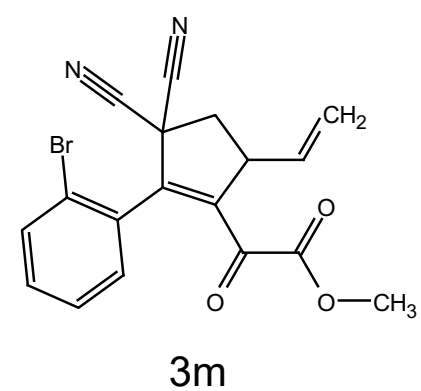




\section{DWP-10-23+- IB 912140.7}

\begin{tabular}{|llll|}
\hline Sample Name: & DWP-10-23+- IB 912140.7 & Injection Volume: & $\mathbf{5 . 0}$ \\
Vial Number: & GA3 & Channel: & UV_VIS_2 \\
Sample Type: & unknown & Wavelength: & $\mathbf{2 1 4 . 0}$ \\
Control Program: & test-dad3 & Bandwidth: & $\mathbf{4}$ \\
Quantif. Method: & $\mathbf{2 0 1 7 0 6 0 8}$ & Dilution Factor: & $\mathbf{1 . 0 0 0 0}$ \\
Recording Time: & $\mathbf{2 0 1 9 - 1 - 1 1 ~ 8 : 5 6}$ & Sample Weight: & $\mathbf{1 . 0 0 0 0}$ \\
Run Time (min): & $\mathbf{4 5 . 0 0}$ & Sample Amount: & $\mathbf{1 . 0 0 0 0}$ \\
\hline
\end{tabular}

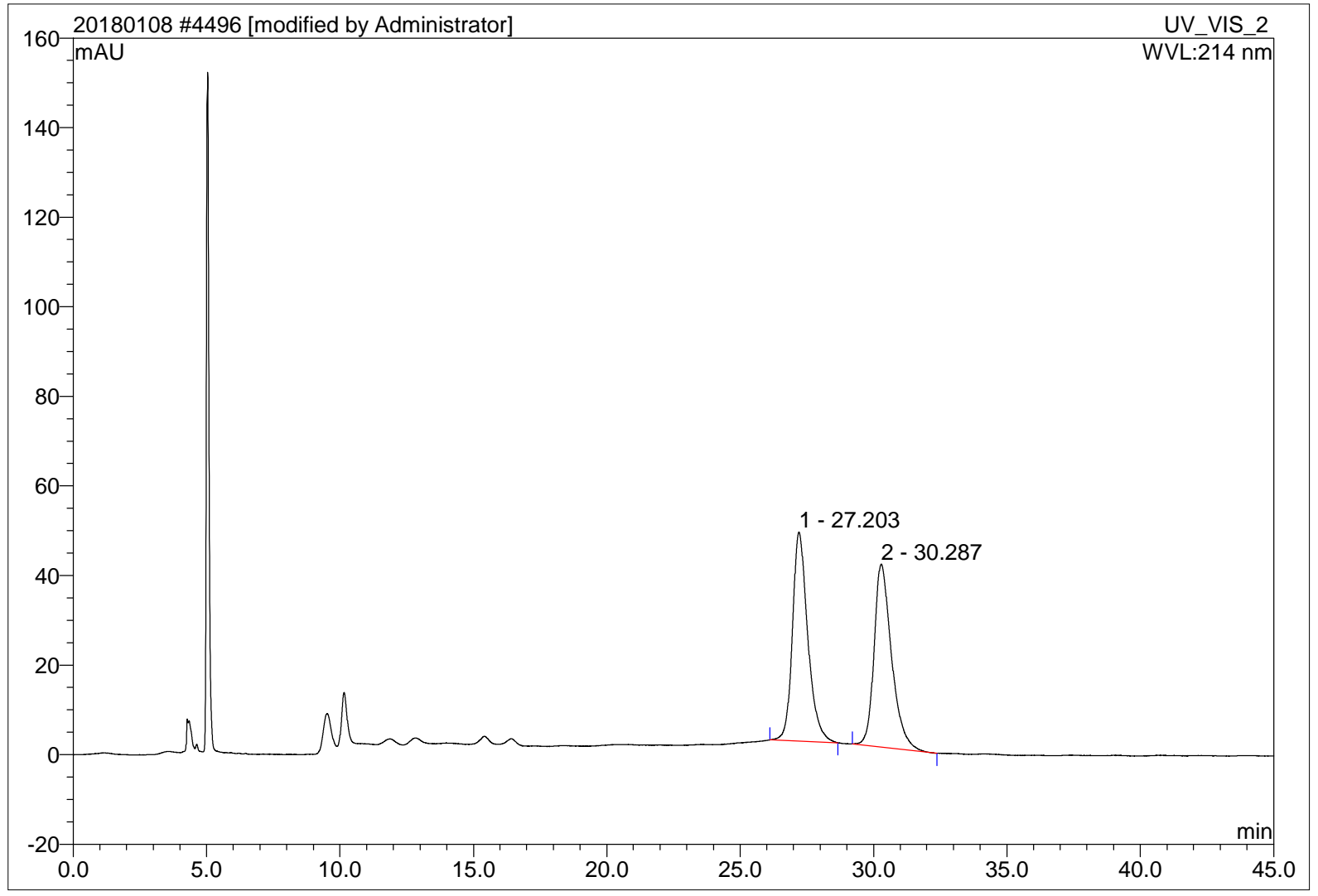

\begin{tabular}{|r|ccrrrrr|}
\hline No. & $\begin{array}{c}\text { Ret.Time } \\
\text { min }\end{array}$ & Peak Name & $\begin{array}{c}\text { Height } \\
\text { mAU }\end{array}$ & $\begin{array}{c}\text { Area } \\
\text { mAU*min }\end{array}$ & $\begin{array}{r}\text { Rel.Area } \\
\%\end{array}$ & Amount & Type \\
\hline 1 & 27.20 & n.a. & 46.691 & 31.094 & 50.02 & n.a. & BMB $^{*}$ \\
2 & 30.29 & n.a. & 40.890 & 31.071 & 49.98 & n.a. & BMB $^{*}$ \\
\hline Total: & & & 87.581 & 62.165 & 100.00 & 0.000 & \\
\hline
\end{tabular}




\section{DWP-10-100 IB 912140.7}

\begin{tabular}{llll|}
\hline Sample Name: & DWP-10-100 IB 91214 0.7 & Injection Volume: & $\mathbf{5 . 0}$ \\
Vial Number: & GB3 & Channel: & UV_VIS_2 \\
Sample Type: & unknown & Wavelength: & $\mathbf{2 1 4 . 0}$ \\
Control Program: & test-dad3 & Bandwidth: & $\mathbf{4}$ \\
Quantif. Method: & $\mathbf{2 0 1 7 0 6 0 8}$ & Dilution Factor: & $\mathbf{1 . 0 0 0 0}$ \\
Recording Time: & $\mathbf{2 0 1 9 - 1 - 1 1 ~ 9 : 4 2}$ & Sample Weight: & $\mathbf{1 . 0 0 0 0}$ \\
Run Time (min): & $\mathbf{3 8 . 2 6}$ & Sample Amount: & $\mathbf{1 . 0 0 0 0}$ \\
\hline
\end{tabular}

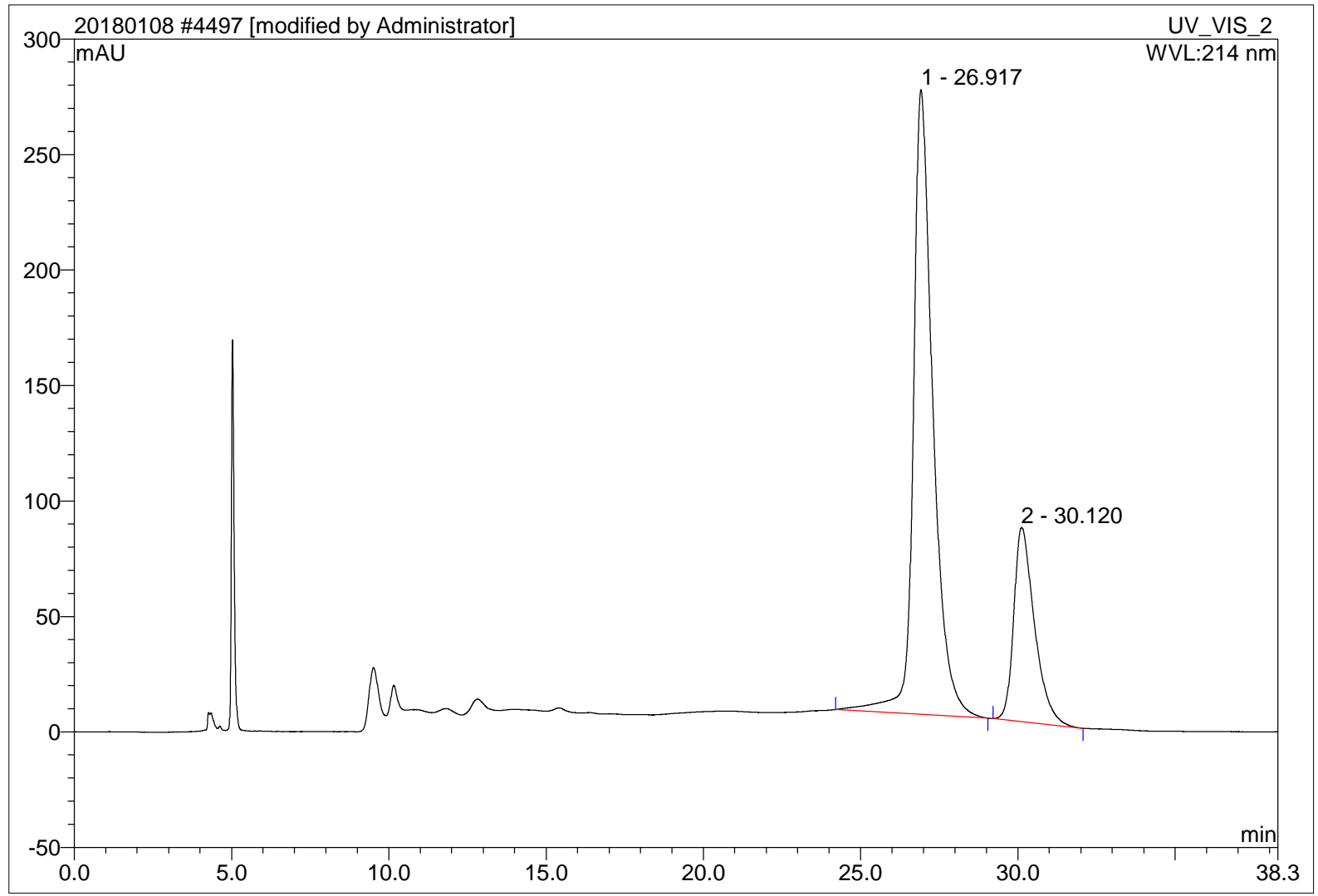

\begin{tabular}{|r|ccrrrrr|}
\hline No. & $\begin{array}{c}\text { Ret.Time } \\
\text { min }\end{array}$ & Peak Name & $\begin{array}{c}\text { Height } \\
\text { mAU }\end{array}$ & $\begin{array}{r}\text { Area } \\
\text { mAU*min }\end{array}$ & $\begin{array}{r}\text { Rel.Area } \\
\%\end{array}$ & Amount & Type \\
\hline 1 & 26.92 & n.a. & 270.584 & 196.016 & 75.23 & n.a. & BMB $^{*}$ \\
2 & 30.12 & n.a. & 84.143 & 64.523 & 24.77 & n.a. & BMB $^{*}$ \\
\hline Total: & & & 354.727 & 260.538 & 100.00 & 0.000 & \\
\hline
\end{tabular}




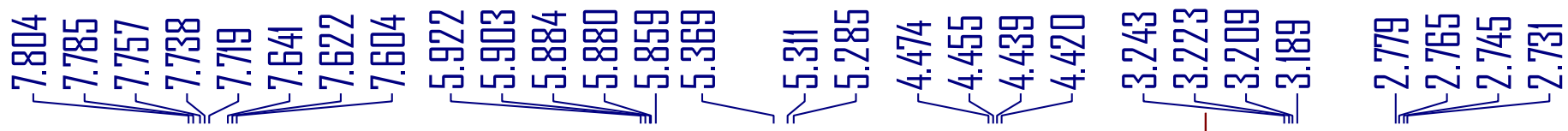

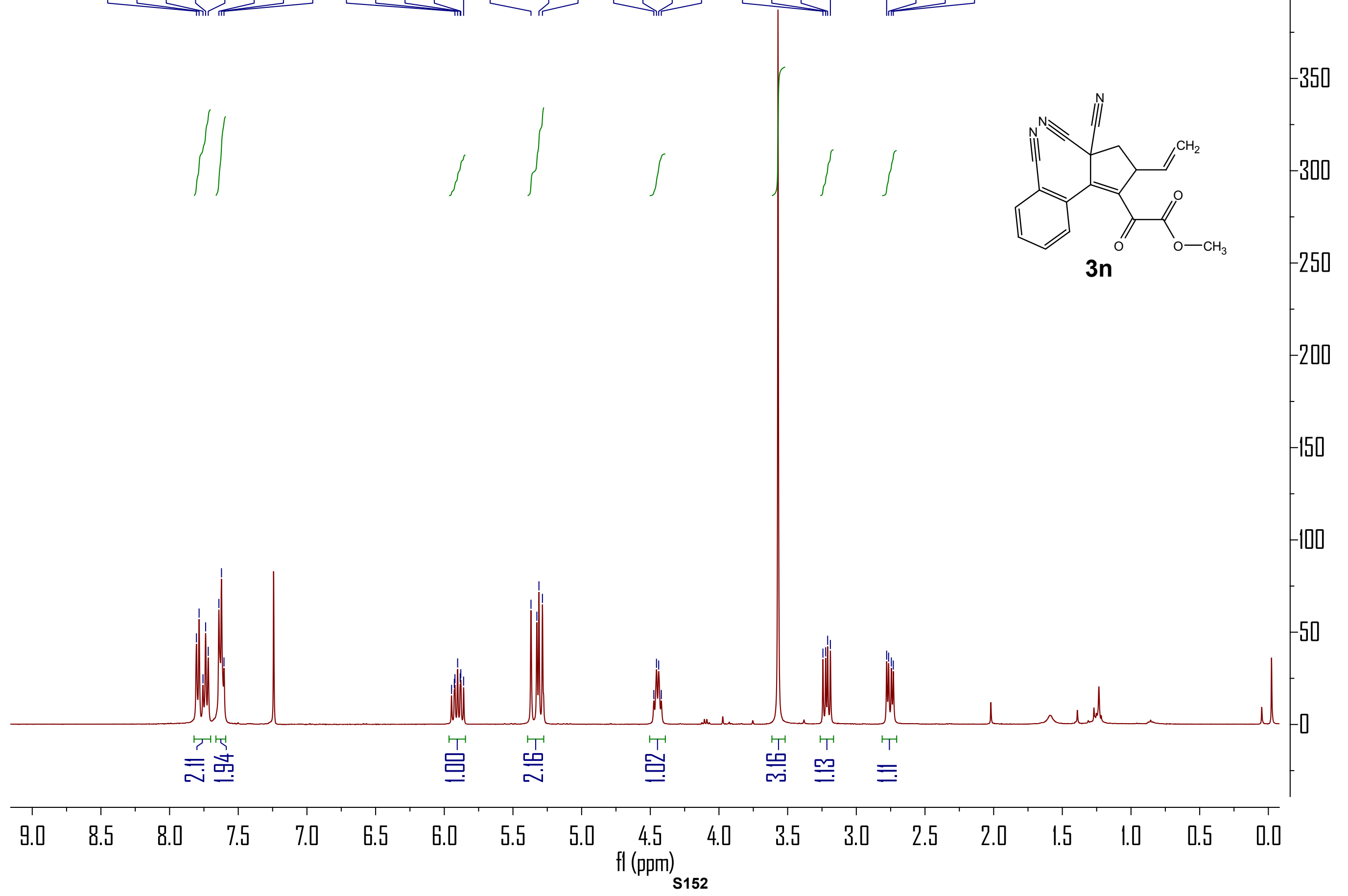




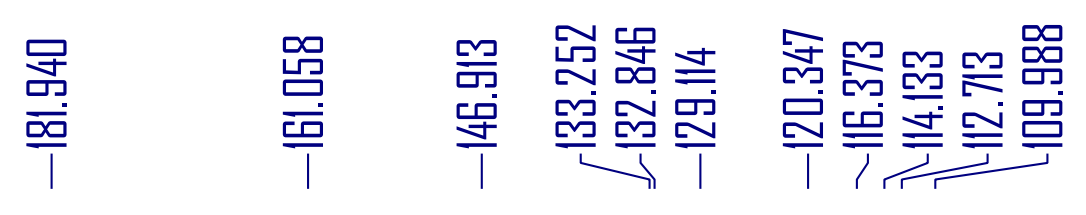

可吴需

啗品戈
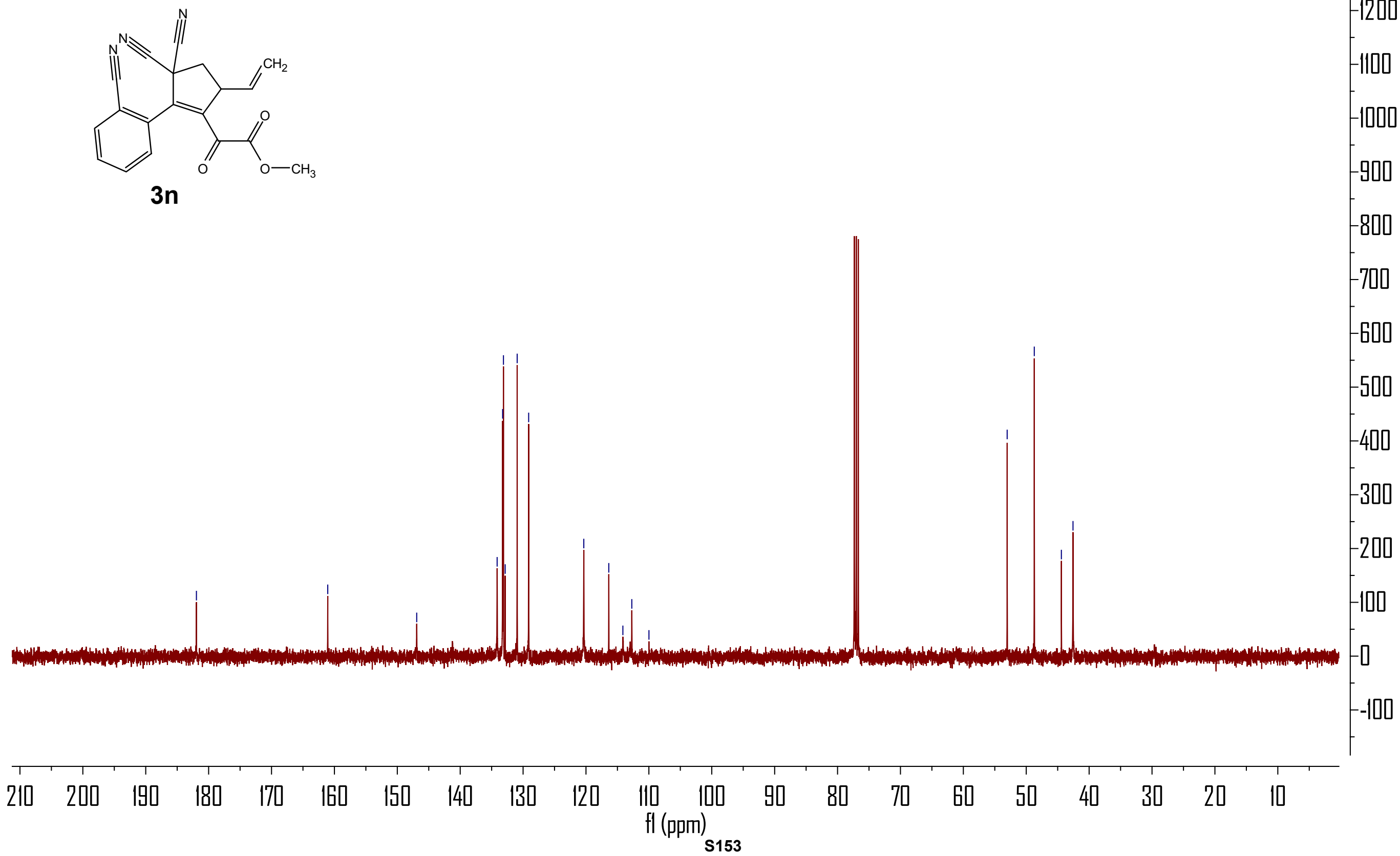


\section{DWP-10-11+- ID-3 912140.7}

\begin{tabular}{|llll|}
\hline Sample Name: & DWP-10-11+- ID-3 912140.7 & Injection Volume: & $\mathbf{3 . 0}$ \\
Vial Number: & GA1 & Channel: & UV_VIS_1 \\
Sample Type: & unknown & Wavelength: & $\mathbf{2 1 4}$ \\
Control Program: & $\mathbf{2 0 1 7 0 1 - 4}$ & Bandwidth: & n.a. \\
Quantif. Method: & $\mathbf{2 0 1 7 0 1}$ & Dilution Factor: & $\mathbf{1 . 0 0 0 0}$ \\
Recording Time: & $\mathbf{2 0 1 9 / 1 / 1 0 ~ 2 0 : 5 3}$ & Sample Weight: & $\mathbf{1 . 0 0 0 0}$ \\
Run Time (min): & $\mathbf{4 0 . 0 1}$ & Sample Amount: & $\mathbf{1 . 0 0 0 0}$ \\
\hline
\end{tabular}

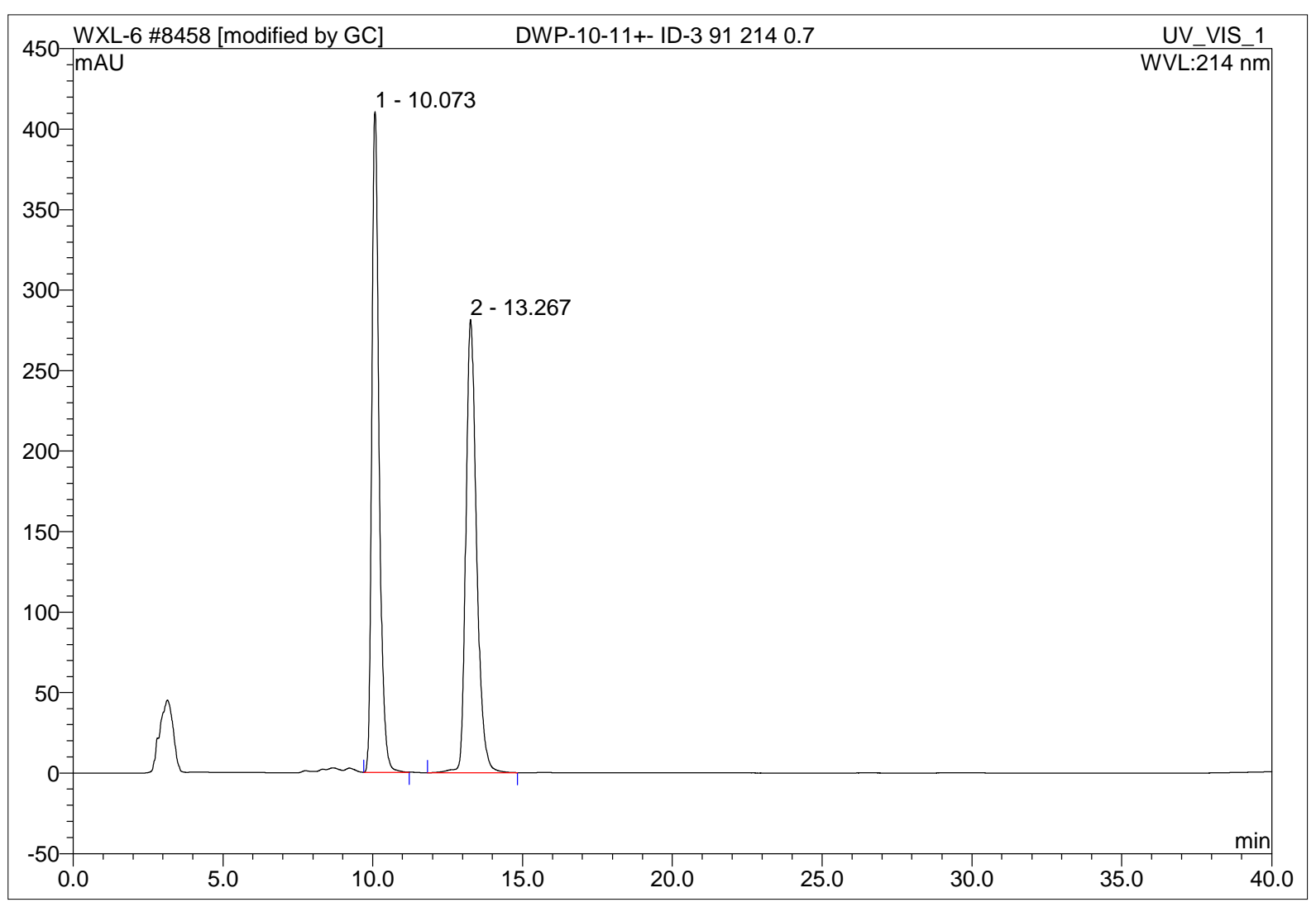

\begin{tabular}{|r|cccrrrr|}
\hline No. & $\begin{array}{c}\text { Ret.Time } \\
\text { min }\end{array}$ & Peak Name & $\begin{array}{c}\text { Height } \\
\text { mAU }\end{array}$ & $\begin{array}{c}\text { Area } \\
\text { mAU*min }\end{array}$ & $\begin{array}{c}\text { Rel.Area } \\
\%\end{array}$ & Amount & Type \\
\hline 1 & 10.07 & n.a. & 410.446 & 115.652 & 49.73 & n.a. & BMB \\
2 & 13.27 & n.a. & 281.487 & 116.919 & 50.27 & n.a. & BMB \\
\hline Total: & & & 691.934 & 232.571 & 100.00 & 0.000 & \\
\hline
\end{tabular}




\section{DWP-10-86 ID-3 912140.7}

\begin{tabular}{|llll|}
\hline Sample Name: & DWP-10-86 ID-3 91214 0.7 & Injection Volume: & $\mathbf{3 . 0}$ \\
Vial Number: & GB1 & Channel: & UV_VIS_1 \\
Sample Type: & unknown & Wavelength: & $\mathbf{2 1 4}$ \\
Control Program: & $\mathbf{2 0 1 7 0 1 - 4}$ & Bandwidth: & n.a. \\
Quantif. Method: & $\mathbf{2 0 1 7 0 1}$ & Dilution Factor: & $\mathbf{1 . 0 0 0 0}$ \\
Recording Time: & $\mathbf{2 0 1 9 / 1 / 1 0 ~ 2 1 : 3 5}$ & Sample Weight: & $\mathbf{1 . 0 0 0 0}$ \\
Run Time (min): & $\mathbf{4 0 . 0 0}$ & Sample Amount: & $\mathbf{1 . 0 0 0 0}$ \\
\hline
\end{tabular}

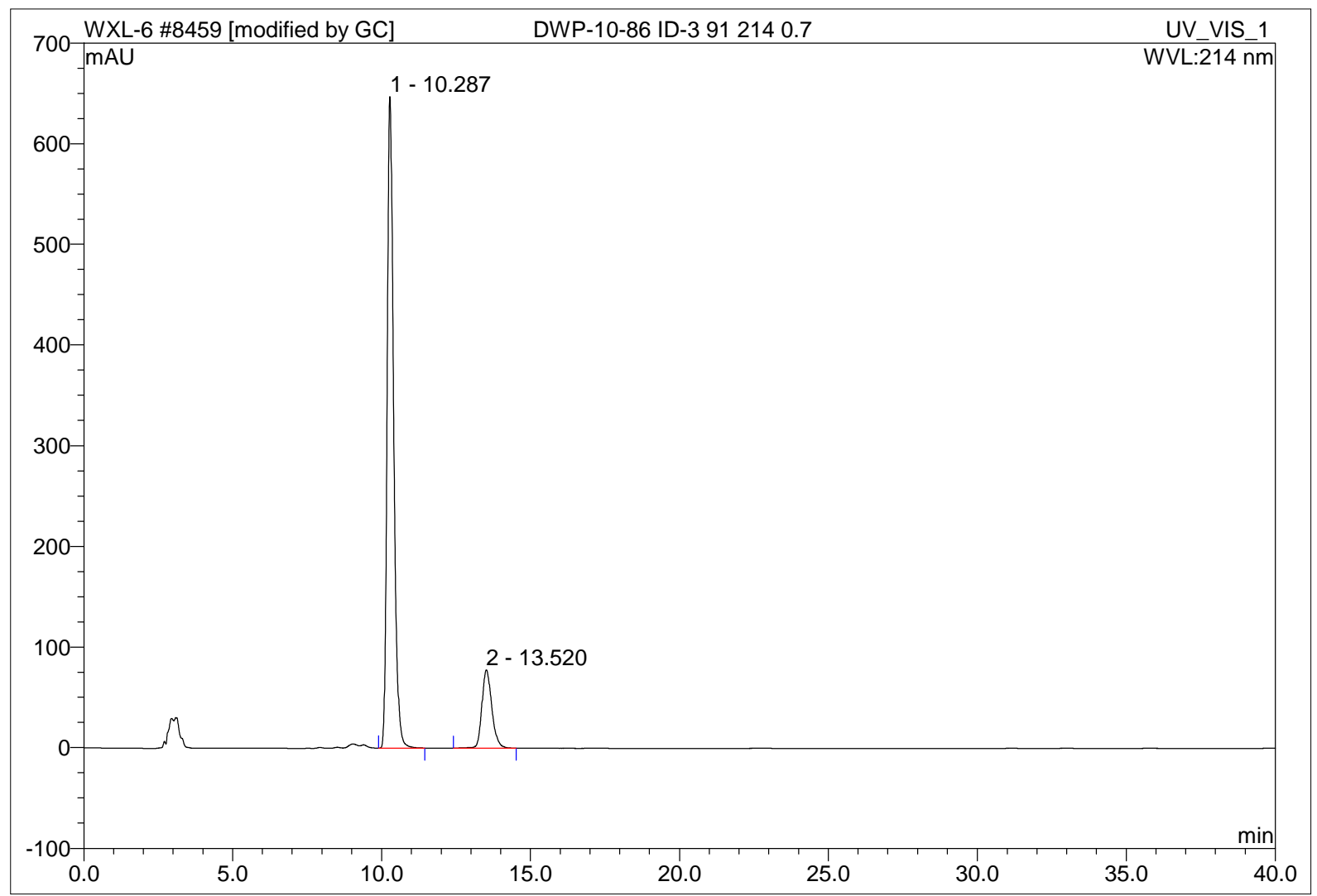

\begin{tabular}{|r|ccrrrrr|}
\hline No. & $\begin{array}{c}\text { Ret.Time } \\
\text { min }\end{array}$ & Peak Name & $\begin{array}{c}\text { Height } \\
\text { mAU }\end{array}$ & $\begin{array}{c}\text { Area } \\
\text { mAU*min }\end{array}$ & $\begin{array}{r}\text { Rel.Area } \\
\%\end{array}$ & Amount & Type \\
\hline 1 & 10.29 & n.a. & 647.146 & 162.982 & 84.39 & n.a. & BMB \\
2 & 13.52 & n.a. & 78.150 & 30.156 & 15.61 & n.a. & BMB \\
\hline Total: & & & 725.296 & 193.137 & 100.00 & 0.000 & \\
\hline
\end{tabular}




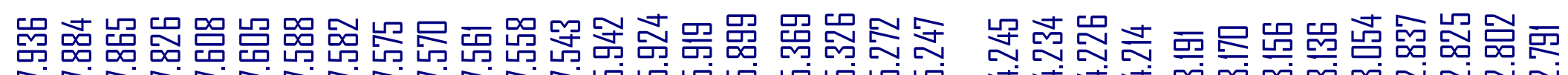
ح

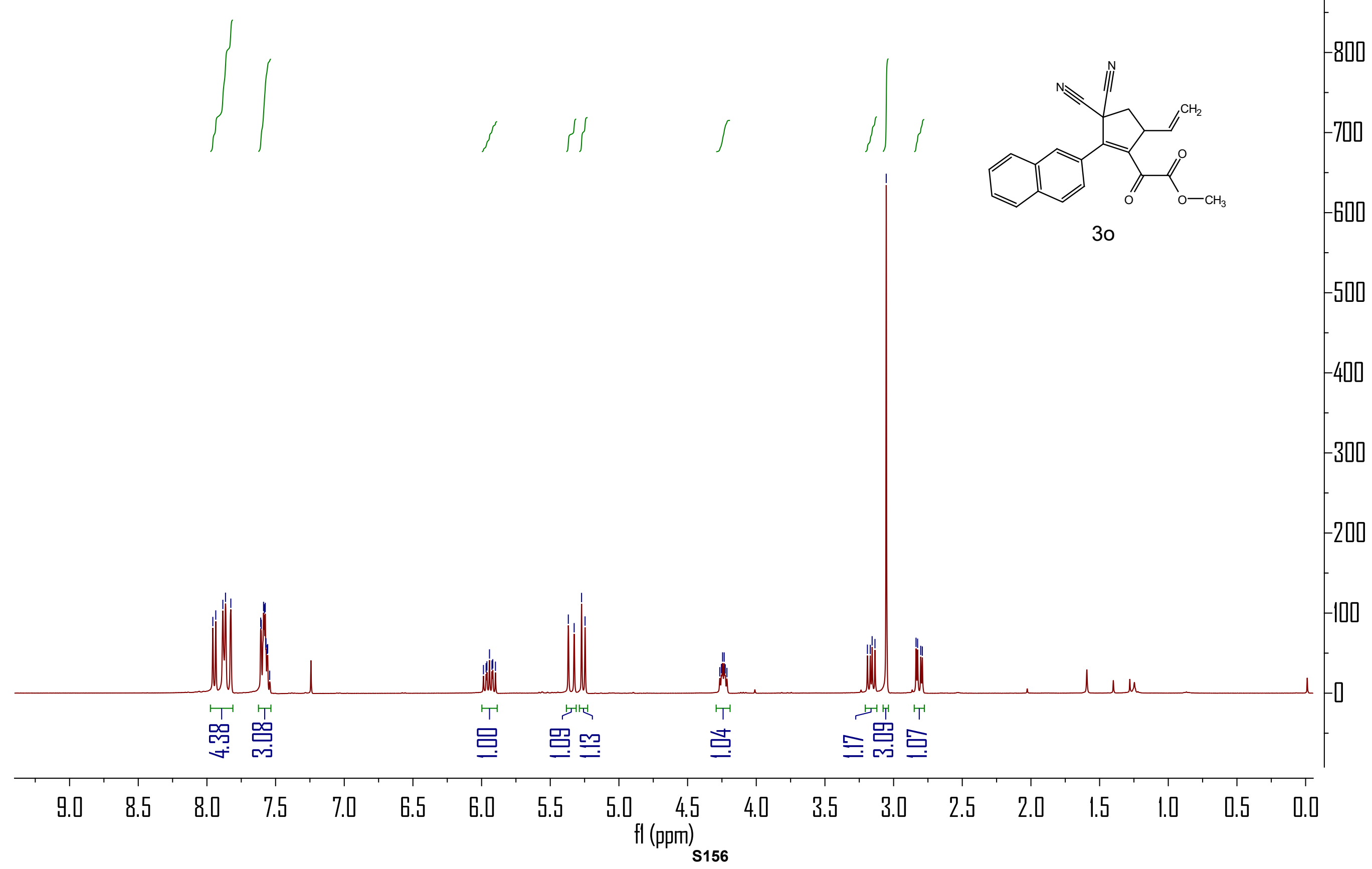




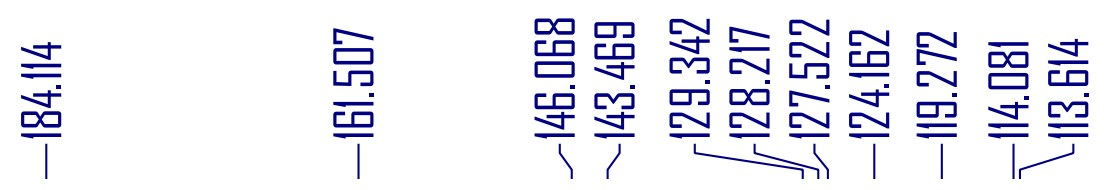

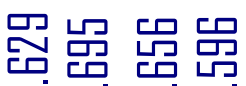

出骂 羿

$-1700$

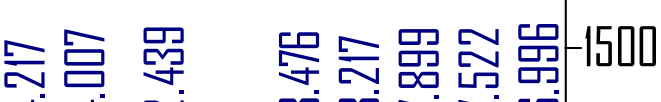

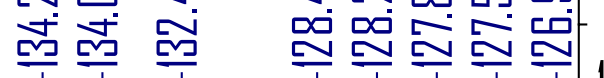

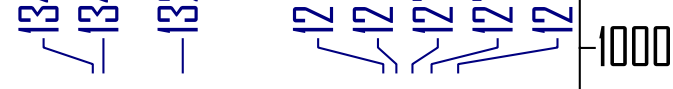
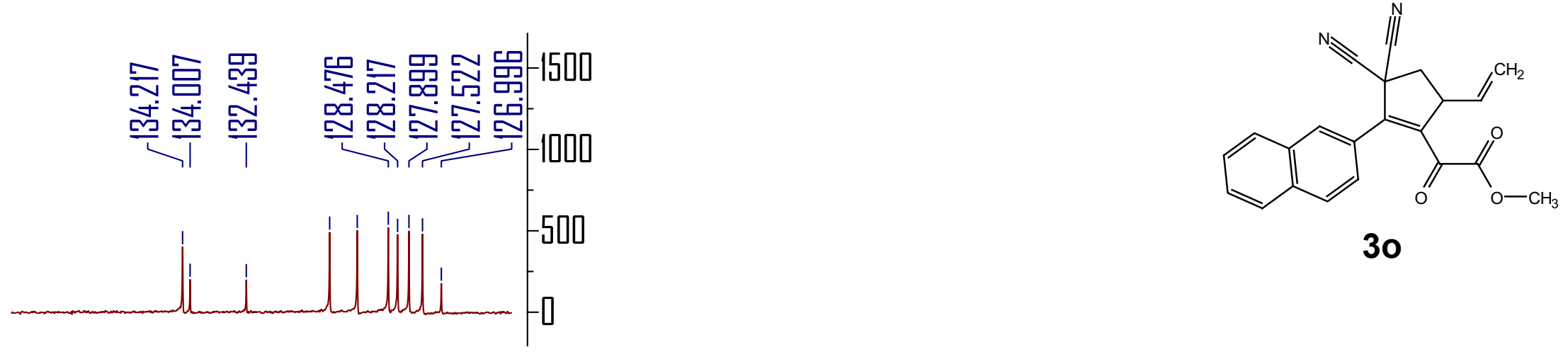

30

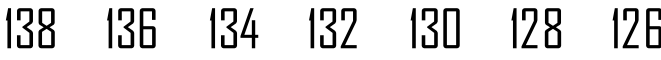
$\mathrm{fl}(\mathrm{ppm})$ 


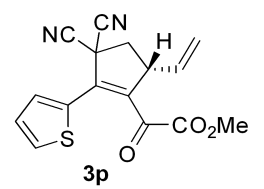

\section{DWP-9-104+- IB 912140.7}

\begin{tabular}{|llll|}
\hline Sample Name: & DWP-9-104+- IB 912140.7 & Injection Volume: & $\mathbf{5 . 0}$ \\
Vial Number: & GA1 & Channel: & UV_VIS_2 \\
Sample Type: & unknown & Wavelength: & $\mathbf{2 1 4 . 0}$ \\
Control Program: & test-dad6 & Bandwidth: & $\mathbf{4}$ \\
Quantif. Method: & $\mathbf{2 0 1 7 0 6 0 8}$ & Dilution Factor: & $\mathbf{1 . 0 0 0 0}$ \\
Recording Time: & $\mathbf{2 0 1 9 - 1 - 1 1 ~ 1 1 : 4 3}$ & Sample Weight: & $\mathbf{1 . 0 0 0 0}$ \\
Run Time (min): & $\mathbf{2 1 . 9 6}$ & Sample Amount: & $\mathbf{1 . 0 0 0 0}$ \\
\hline
\end{tabular}

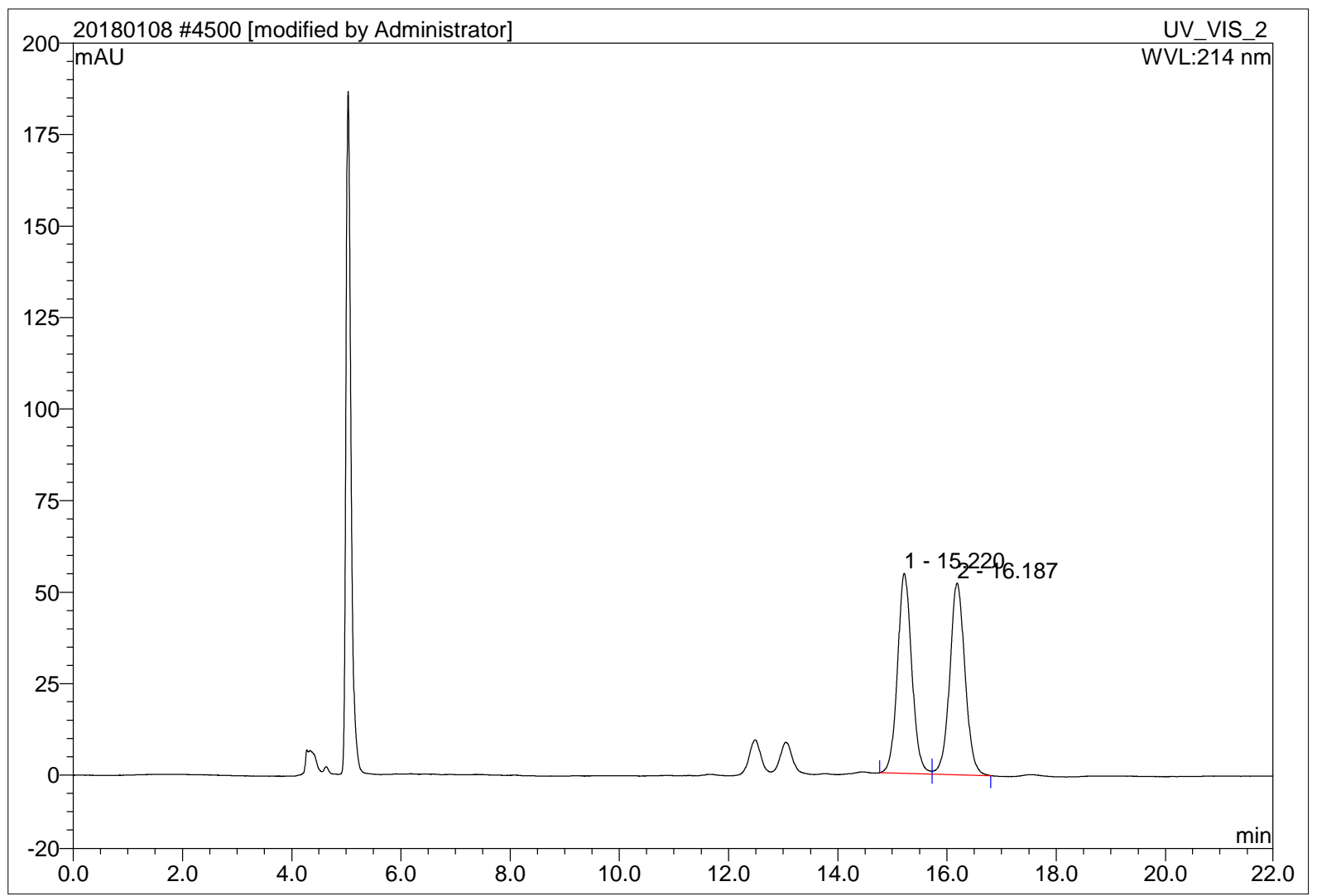

\begin{tabular}{|r|ccrrrrr|}
\hline No. & $\begin{array}{c}\text { Ret.Time } \\
\text { min }\end{array}$ & Peak Name & $\begin{array}{c}\text { Height } \\
\text { mAU }\end{array}$ & $\begin{array}{c}\text { Area } \\
\text { mAU*min }\end{array}$ & $\begin{array}{r}\text { Rel.Area } \\
\%\end{array}$ & Amount & Type \\
\hline 1 & 15.22 & n.a. & 54.701 & 16.502 & 49.38 & n.a. & BM \\
2 & 16.19 & n.a. & 52.379 & 16.918 & 50.62 & n.a. & MB \\
\hline Total: & & & 107.081 & 33.420 & 100.00 & 0.000 & \\
\hline
\end{tabular}




\section{DWP-10-103 IB 912140.7}

\begin{tabular}{|llll|}
\hline Sample Name: & DWP-10-103 IB 912140.7 & Injection Volume: & $\mathbf{5 . 0}$ \\
Vial Number: & GC1 & Channel: & UV_VIS_2 \\
Sample Type: & unknown & Wavelength: & $\mathbf{2 1 4 . 0}$ \\
Control Program: & test-dad6 & Bandwidth: & $\mathbf{4}$ \\
Quantif. Method: & $\mathbf{2 0 1 7 0 6 0 8}$ & Dilution Factor: & $\mathbf{1 . 0 0 0 0}$ \\
Recording Time: & $\mathbf{2 0 1 9 - 1 - 1 1 ~ 1 2 : 3 7}$ & Sample Weight: & $\mathbf{1 . 0 0 0 0}$ \\
Run Time (min): & $\mathbf{2 5 . 2 6}$ & Sample Amount: & $\mathbf{1 . 0 0 0 0}$ \\
\hline
\end{tabular}

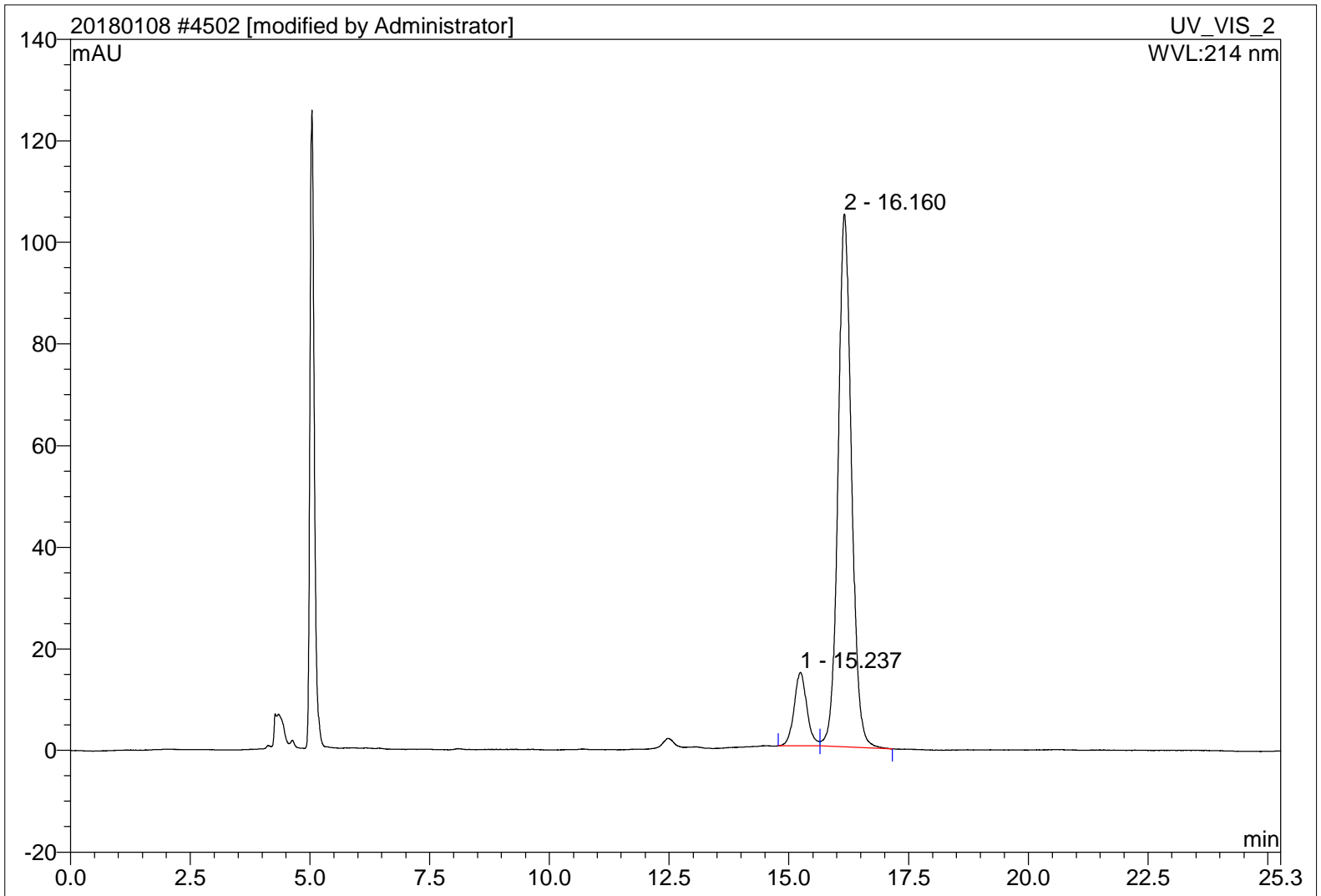

\begin{tabular}{|r|ccrrrrr|}
\hline No. & $\begin{array}{c}\text { Ret.Time } \\
\text { min }\end{array}$ & Peak Name & $\begin{array}{c}\text { Height } \\
\text { mAU }\end{array}$ & $\begin{array}{r}\text { Area } \\
\text { mAU*min }\end{array}$ & $\begin{array}{r}\text { Rel.Area } \\
\%\end{array}$ & Amount & Type \\
\hline 1 & 15.24 & n.a. & 14.534 & 4.486 & 11.59 & n.a. & BM $^{*}$ \\
2 & 16.16 & n.a. & 104.965 & 34.218 & 88.41 & n.a. & MB $^{*}$ \\
\hline Total: & & & 119.498 & 38.704 & 100.00 & 0.000 & \\
\hline
\end{tabular}




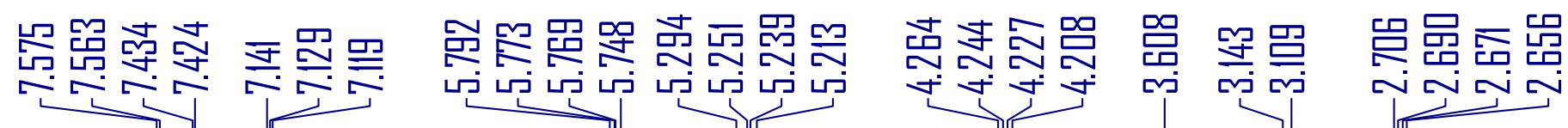

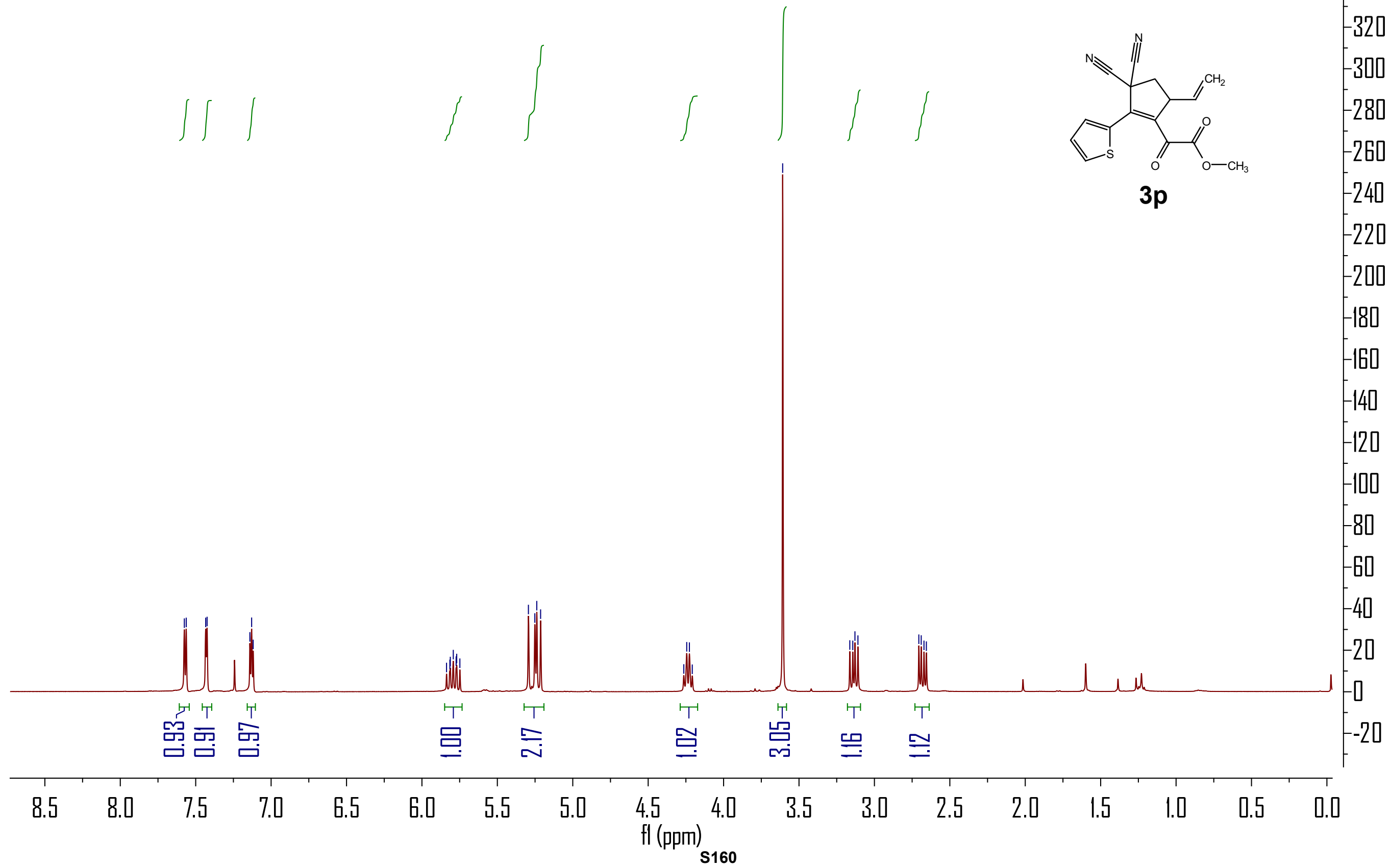




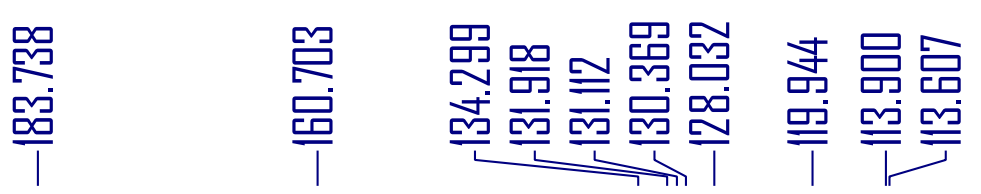

号嵒品

$-1800$

$3 p$

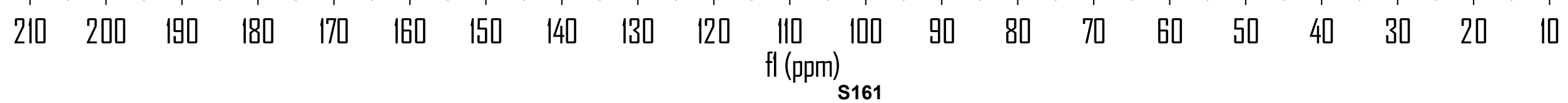




\section{DWP-10-12+- IC 912140.7}

\begin{tabular}{|llll|}
\hline Sample Name: & DWP-10-12+- IC $\mathbf{9 1 2 1 4} \mathbf{0 . 7}$ & Injection Volume: & $\mathbf{5 . 0}$ \\
Vial Number: & GB2 & Channel: & UV_VIS_1 \\
Sample Type: & unknown & Wavelength: & $\mathbf{2 1 4}$ \\
Control Program: & $\mathbf{2 0 1 7 0 1 - 4}$ & Bandwidth: & n.a. \\
Quantif. Method: & $\mathbf{2 0 1 7 0 1}$ & Dilution Factor: & $\mathbf{1 . 0 0 0 0}$ \\
Recording Time: & $\mathbf{2 0 1 9 / 1 / 9 2 2 : 4 3}$ & Sample Weight: & $\mathbf{1 . 0 0 0 0}$ \\
Run Time (min): & $\mathbf{2 5 . 0 1}$ & Sample Amount: & $\mathbf{1 . 0 0 0 0}$ \\
\hline
\end{tabular}

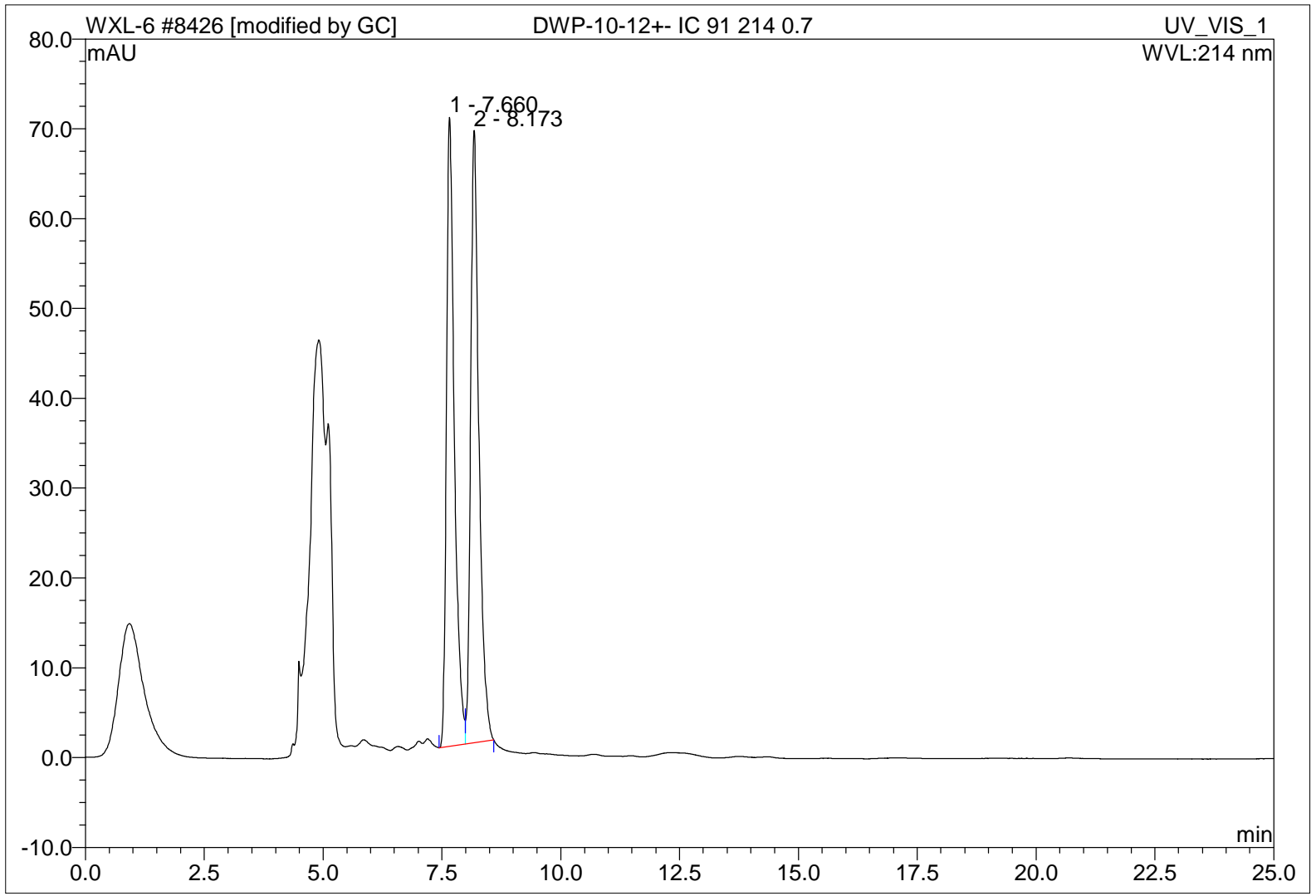

\begin{tabular}{|r|ccrrrrr|}
\hline No. & $\begin{array}{c}\text { Ret.Time } \\
\text { min }\end{array}$ & Peak Name & $\begin{array}{c}\text { Height } \\
\text { mAU }\end{array}$ & $\begin{array}{c}\text { Area } \\
\text { mAU*min }\end{array}$ & $\begin{array}{r}\text { Rel.Area } \\
\%\end{array}$ & Amount & Type \\
\hline 1 & 7.66 & n.a. & 70.049 & 13.473 & 49.42 & n.a. & BM $^{*}$ \\
2 & 8.17 & n.a. & 68.173 & 13.788 & 50.58 & n.a. & MB $^{*}$ \\
\hline Total: & & & 138.222 & 27.261 & 100.00 & 0.000 & \\
\hline
\end{tabular}




\section{DWP-10-76 IC 912140.7}

\begin{tabular}{llll|}
\hline Sample Name: & DWP-10-76 IC 912140.7 & Injection Volume: & $\mathbf{5 . 0}$ \\
Vial Number: & GB1 & Channel: & UV_VIS_1 \\
Sample Type: & unknown & Wavelength: & $\mathbf{2 1 4}$ \\
Control Program: & $\mathbf{2 0 1 7 0 1 - 4}$ & Bandwidth: & n.a. \\
Quantif. Method: & $\mathbf{2 0 1 7 0 1}$ & Dilution Factor: & $\mathbf{1 . 0 0 0 0}$ \\
Recording Time: & $\mathbf{2 0 1 9 / 1 / 9 2 3 : 0 9}$ & Sample Weight: & $\mathbf{1 . 0 0 0 0}$ \\
Run Time (min): & $\mathbf{2 5 . 0 0}$ & Sample Amount: & $\mathbf{1 . 0 0 0 0}$ \\
\hline
\end{tabular}

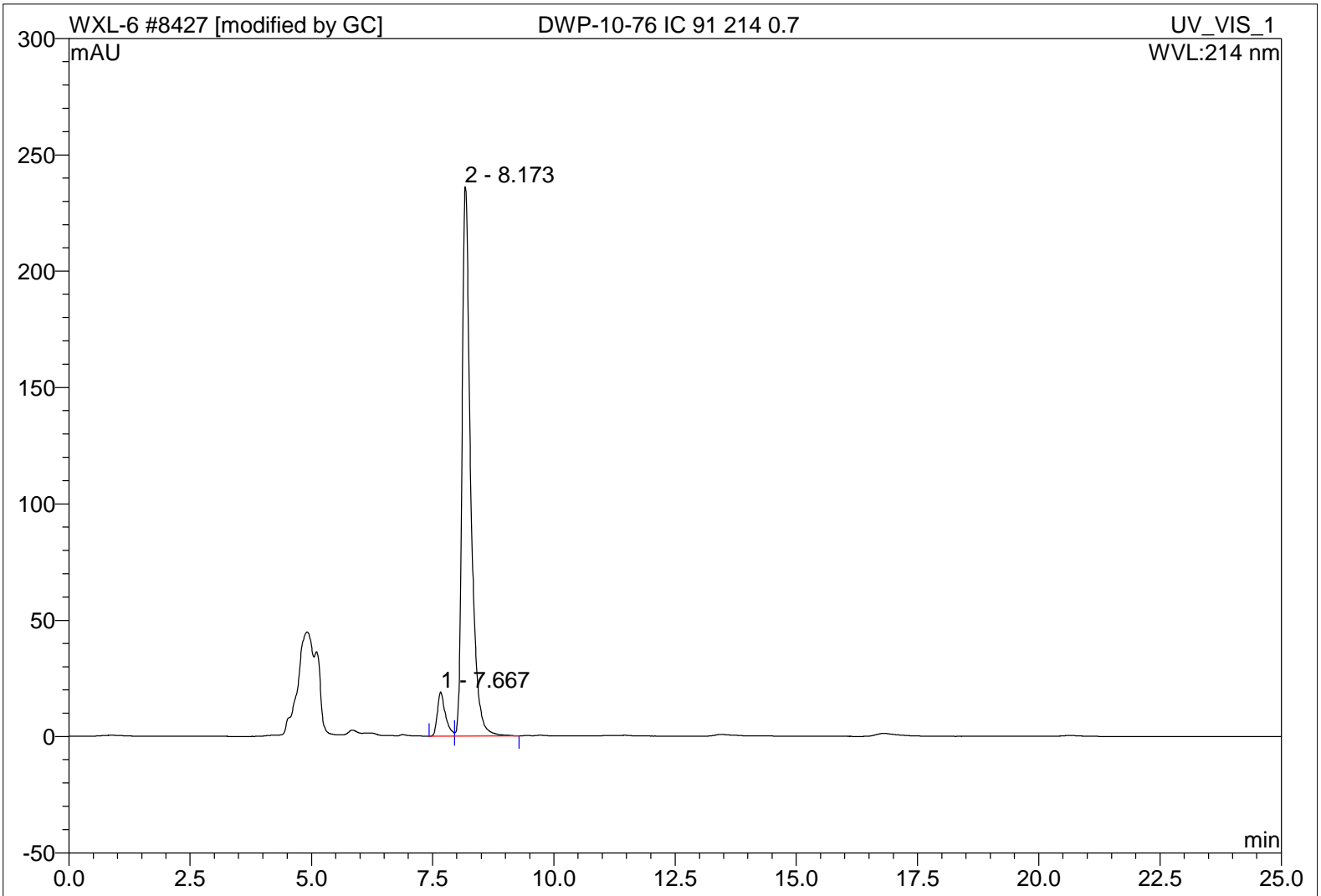

\begin{tabular}{|r|ccrrrrr|}
\hline No. & $\begin{array}{c}\text { Ret.Time } \\
\text { min }\end{array}$ & Peak Name & $\begin{array}{c}\text { Height } \\
\text { mAU }\end{array}$ & $\begin{array}{c}\text { Area } \\
\text { mAU*min }\end{array}$ & $\begin{array}{r}\text { Rel.Area } \\
\%\end{array}$ & Amount & Type \\
\hline 1 & 7.67 & n.a. & 18.927 & 3.634 & 6.92 & n.a. & BM \\
2 & 8.17 & n.a. & 236.118 & 48.857 & 93.08 & n.a. & MB \\
\hline Total: & & & 255.045 & 52.491 & 100.00 & 0.000 & \\
\hline
\end{tabular}




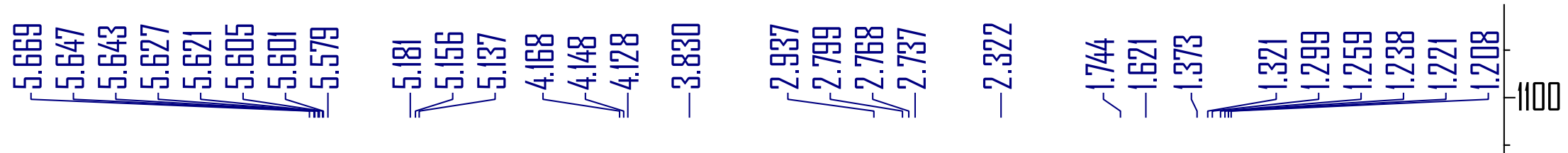

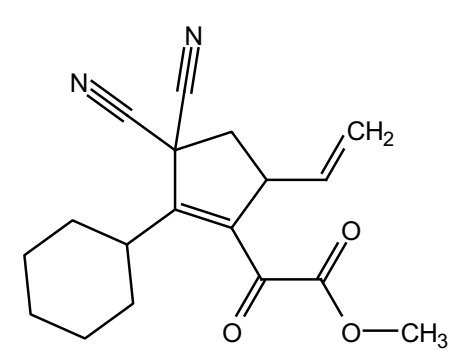

$3 q$
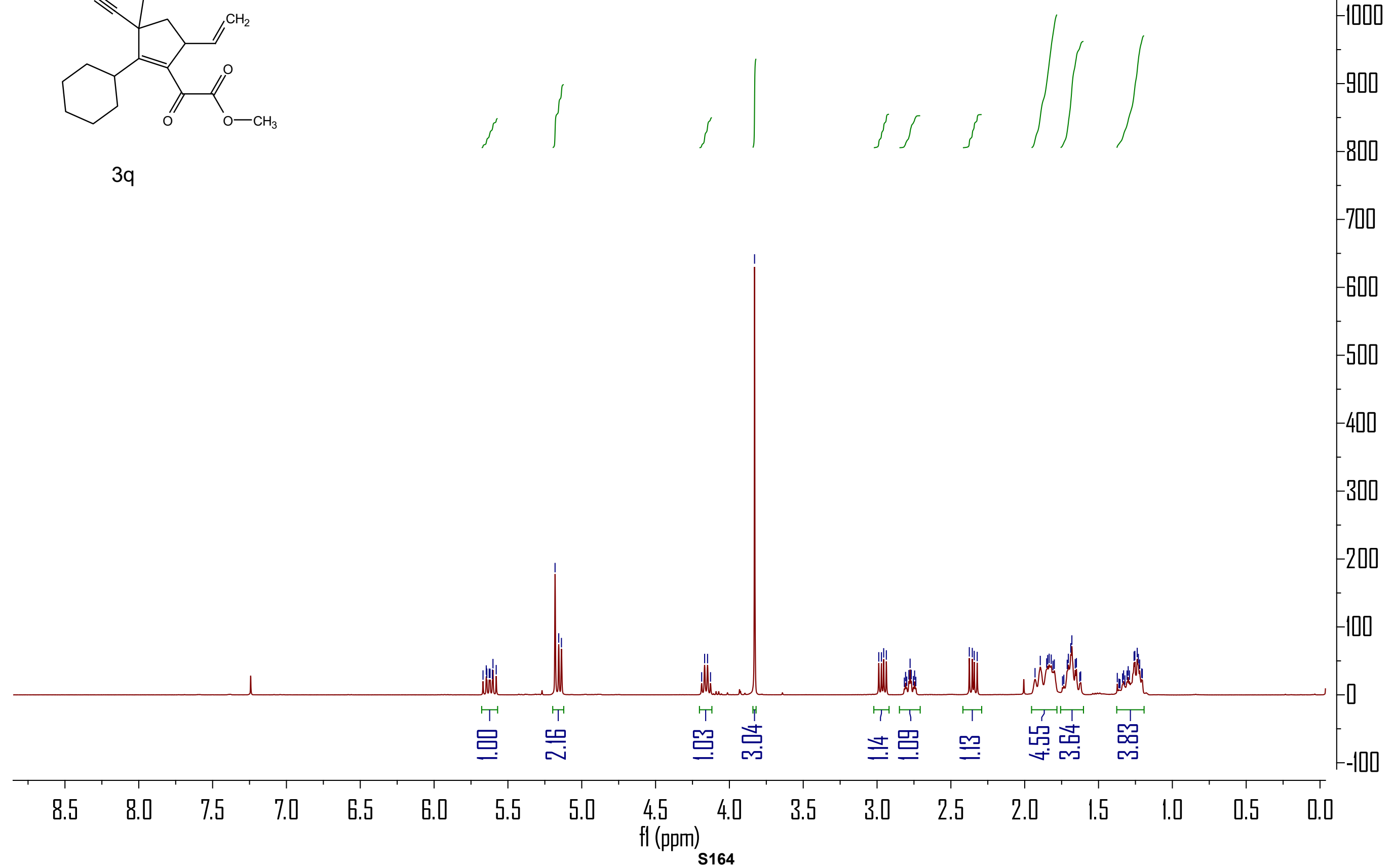


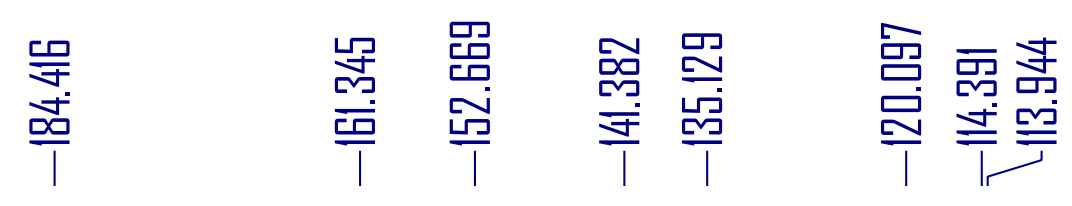

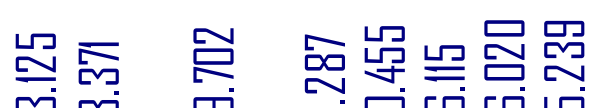

$-750$

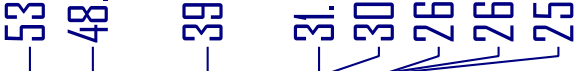

$-700$

$-650$

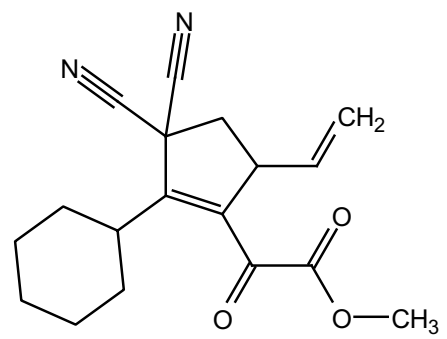

$-600$

$-550$

500

$3 q$

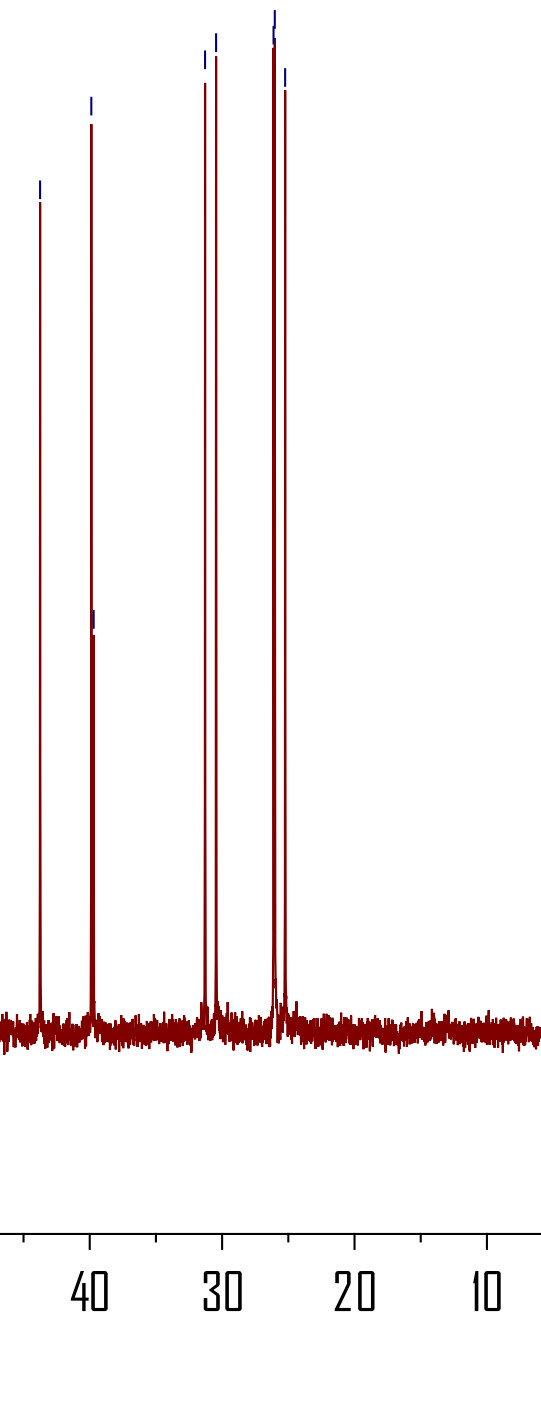

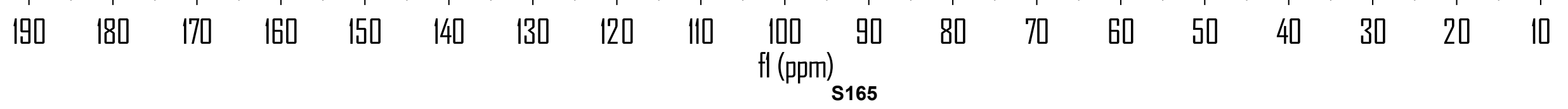




\begin{tabular}{|llll|}
\hline 4453 DWP-10-71+- IC 9822140.7 & & \\
& & & \\
\hline Sample Name: & DWP-10-71+- IC 9822140.7 & Injection Volume: & $\mathbf{3 . 0}$ \\
Vial Number: & RA5 & Channel: & UV_VIS_2 \\
Sample Type: & unknown & Wavelength: & $\mathbf{2 1 4 . 0}$ \\
Control Program: & test-dad6 & Bandwidth: & $\mathbf{4}$ \\
Quantif. Method: & $\mathbf{2 0 1 7 0 6 0 8}$ & Dilution Factor: & $\mathbf{1 . 0 0 0 0}$ \\
Recording Time: & $\mathbf{2 0 1 9 - 1 - 1 0 ~ 1 1 : 4 5}$ & Sample Weight: & $\mathbf{1 . 0 0 0 0}$ \\
Run Time (min): & $\mathbf{2 2 . 6 6}$ & Sample Amount: & $\mathbf{1 . 0 0 0 0}$ \\
\hline
\end{tabular}

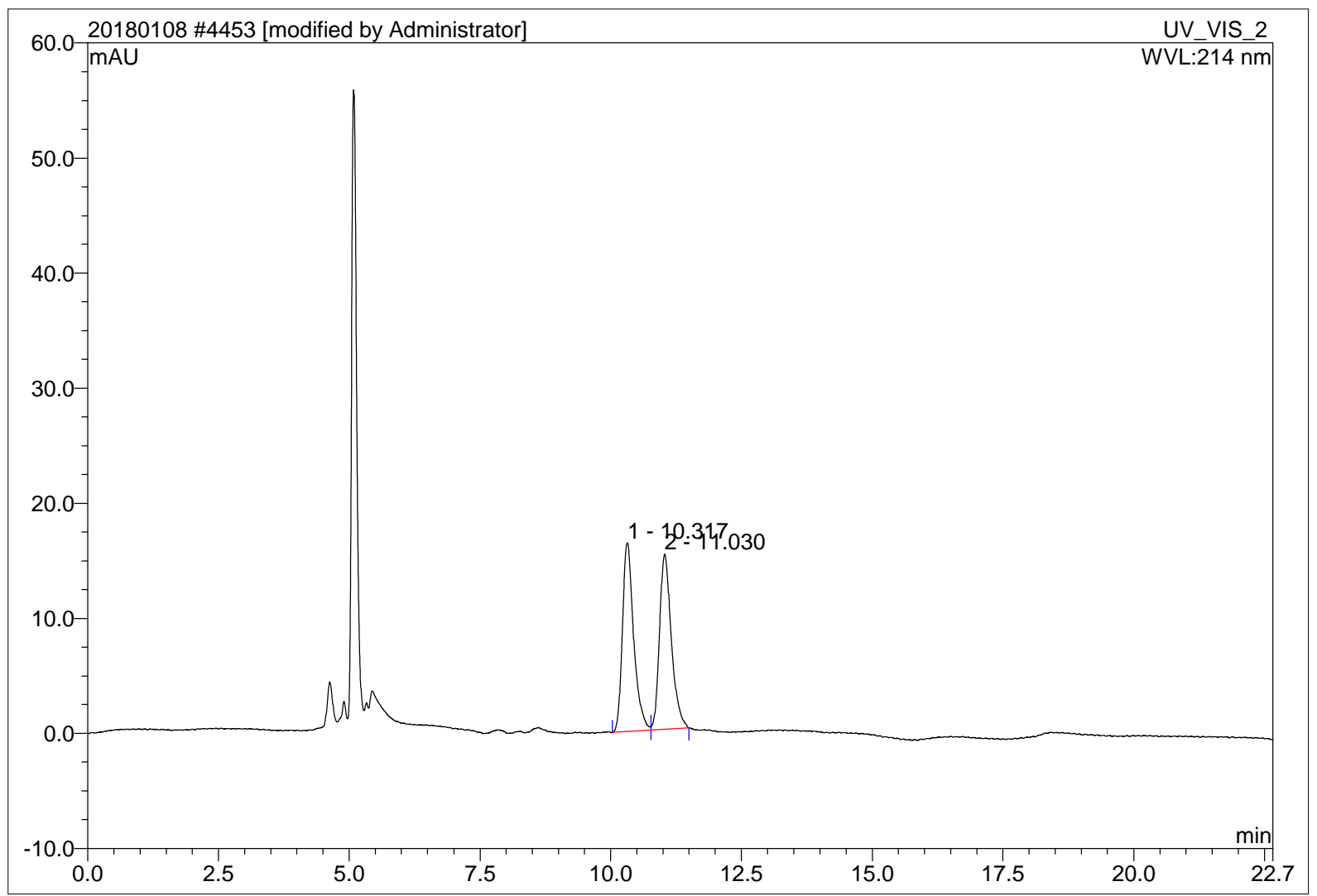

\begin{tabular}{|r|ccrrrrr|}
\hline No. & $\begin{array}{c}\text { Ret.Time } \\
\text { min }\end{array}$ & Peak Name & $\begin{array}{c}\text { Height } \\
\text { mAU }\end{array}$ & $\begin{array}{c}\text { Area } \\
\text { mAU*min }^{*}\end{array}$ & $\begin{array}{r}\text { Rel.Area } \\
\%\end{array}$ & Amount & Type \\
\hline 1 & 10.32 & n.a. & 16.424 & 4.108 & 50.54 & n.a. & BM \\
2 & 11.03 & n.a. & 15.239 & 4.020 & 49.46 & n.a. & MB \\
\hline Total: & & & 31.663 & 8.128 & 100.00 & 0.000 & \\
\hline
\end{tabular}




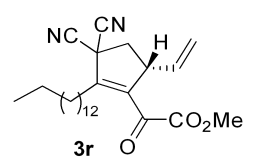

\begin{tabular}{|llll|}
\hline 4454 DWP-10-72 IC 9822140.7 & & \\
& & & \\
\hline Sample Name: & DWP-10-72 IC 9822140.7 & Injection Volume: & $\mathbf{3 . 0}$ \\
Vial Number: & RA4 & Channel: & UV_VIS_2 \\
Sample Type: & unknown & Wavelength: & $\mathbf{2 1 4 . 0}$ \\
Control Program: & test-dad6 & Bandwidth: & $\mathbf{4}$ \\
Quantif. Method: & $\mathbf{2 0 1 7 0 6 0 8}$ & Dilution Factor: & $\mathbf{1 . 0 0 0 0}$ \\
Recording Time: & $\mathbf{2 0 1 9 - 1 - 1 0 ~ 1 2 : 0 9}$ & Sample Weight: & $\mathbf{1 . 0 0 0 0}$ \\
Run Time (min): & $\mathbf{4 3 . 6 4}$ & Sample Amount: & $\mathbf{1 . 0 0 0 0}$ \\
\hline
\end{tabular}

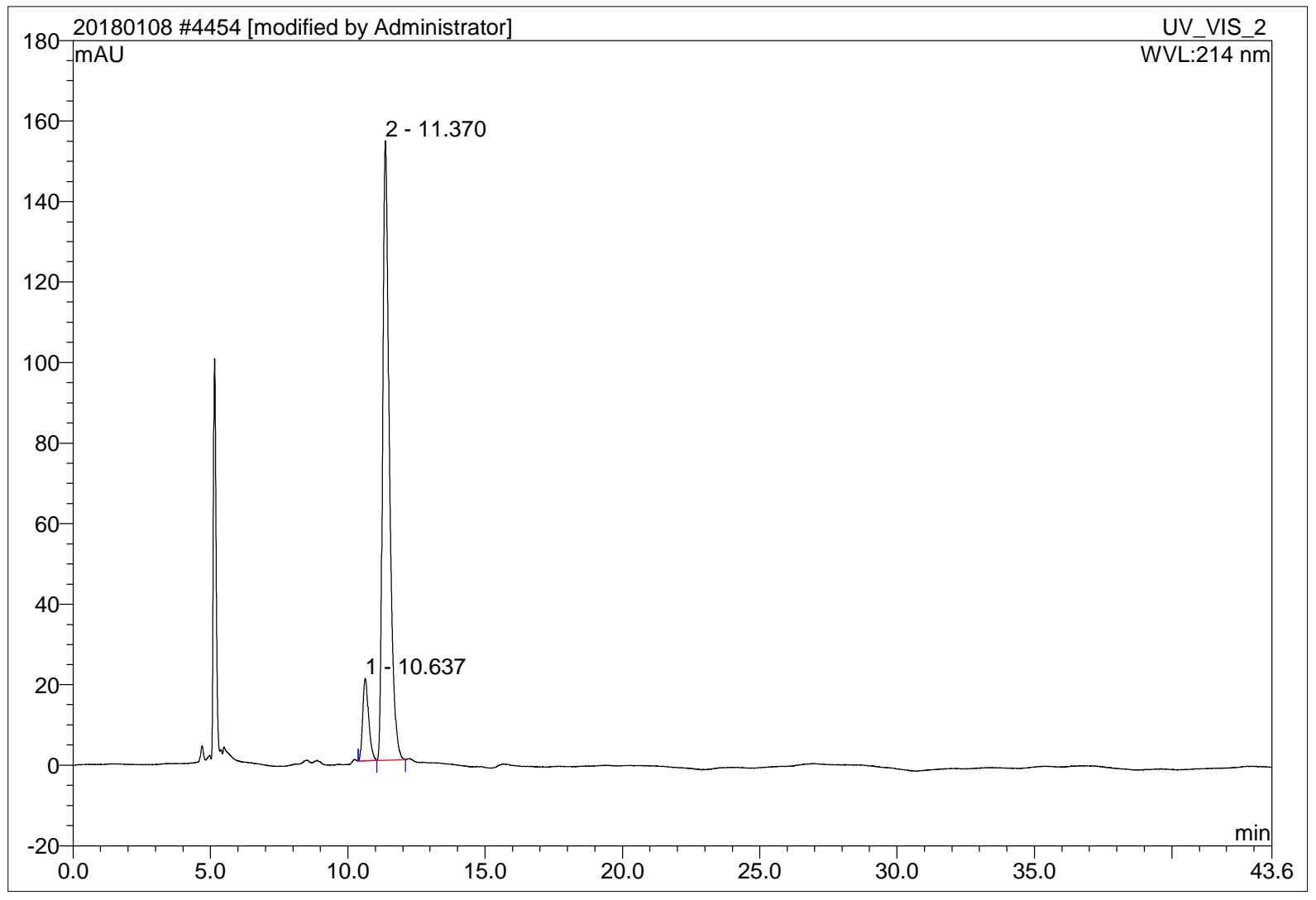

\begin{tabular}{|r|ccrrrrr|}
\hline No. & $\begin{array}{c}\text { Ret.Time } \\
\text { min }\end{array}$ & Peak Name & $\begin{array}{c}\text { Height } \\
\text { mAU }\end{array}$ & $\begin{array}{c}\text { Area } \\
\text { mAU*min }\end{array}$ & $\begin{array}{r}\text { Rel.Area } \\
\%\end{array}$ & Amount & Type \\
\hline 1 & 10.64 & n.a. & 20.496 & 5.140 & 10.45 & n.a. & Ru \\
2 & 11.37 & n.a. & 153.870 & 44.039 & 89.55 & n.a. & BMB $^{*}$ \\
\hline Total: & & & 174.366 & 49.179 & 100.00 & 0.000 & \\
\hline
\end{tabular}




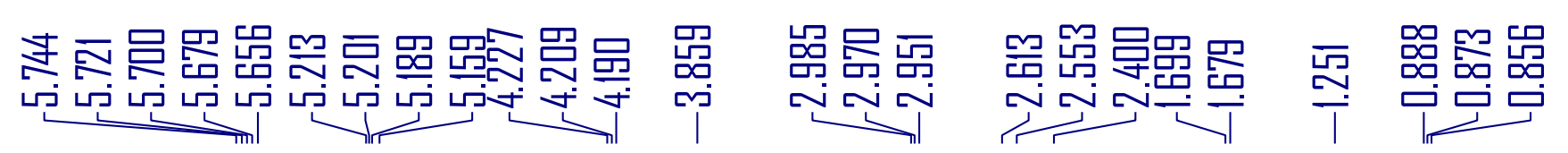

450

400
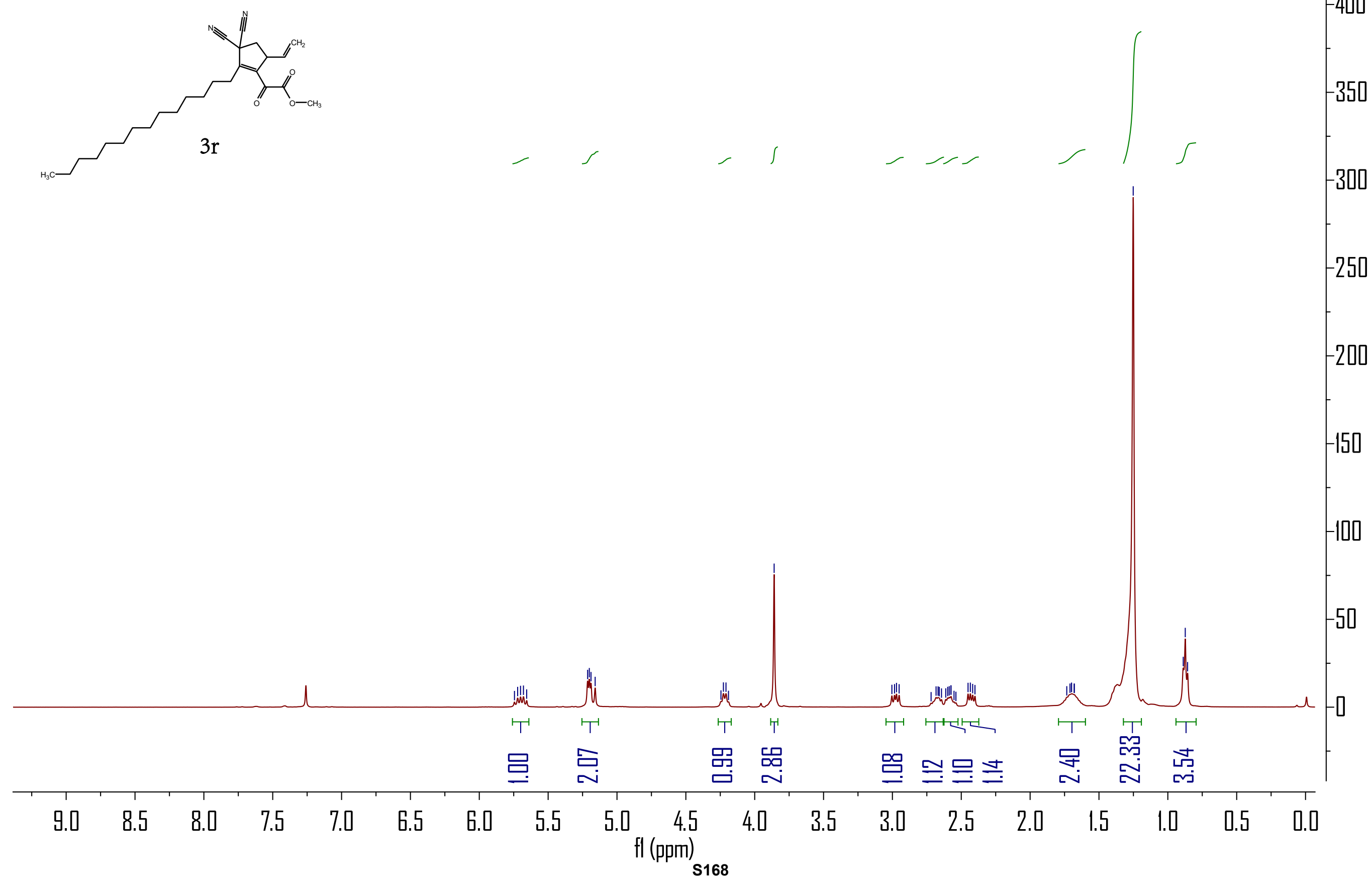


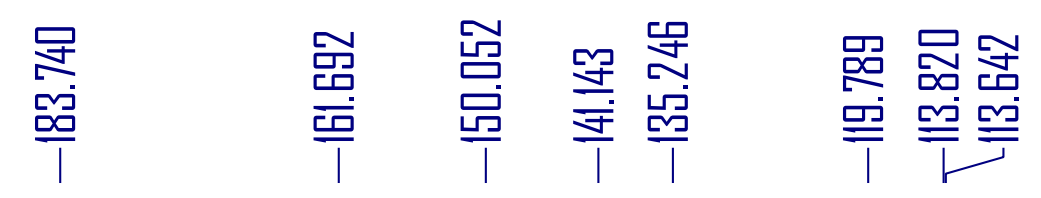

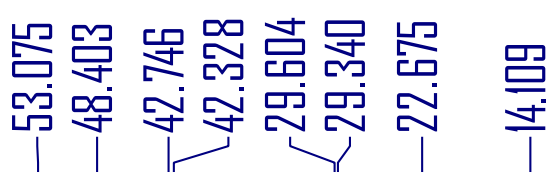
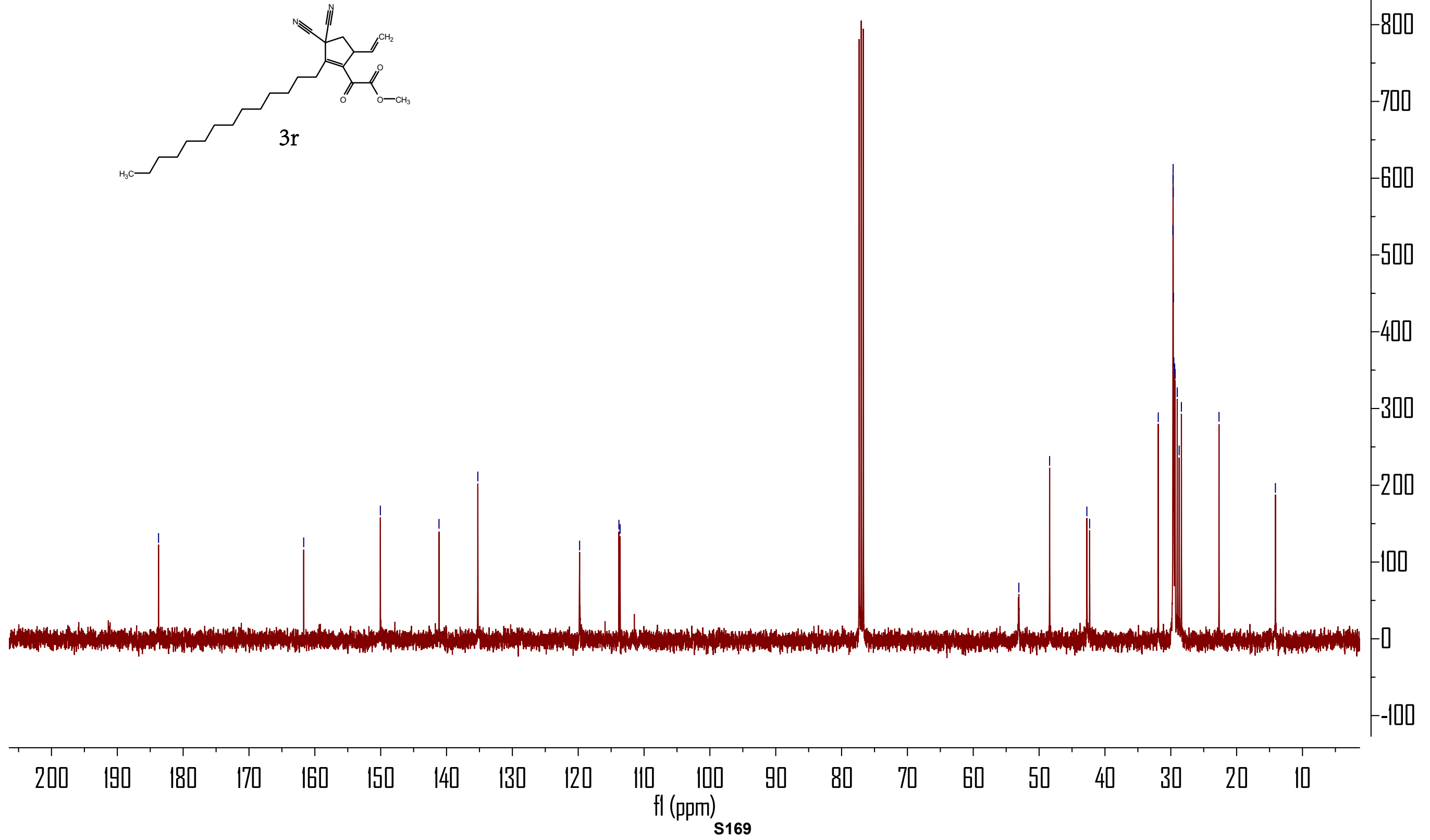


\section{DWP-10-13+- IC 912140.7}

\begin{tabular}{llll|}
\hline Sample Name: & DWP-10-13+- IC 912140.7 & Injection Volume: & $\mathbf{5 . 0}$ \\
Vial Number: & GC1 & Channel: & UV_VIS_1 \\
Sample Type: & unknown & Wavelength: & $\mathbf{2 1 4}$ \\
Control Program: & $\mathbf{2 0 1 7 0 1 - 4}$ & Bandwidth: & n.a. \\
Quantif. Method: & $\mathbf{2 0 1 7 0 1}$ & Dilution Factor: & $\mathbf{1 . 0 0 0 0}$ \\
Recording Time: & $\mathbf{2 0 1 9 / 1 / 9 ~ 2 3 : 3 6}$ & Sample Weight: & $\mathbf{1 . 0 0 0 0}$ \\
Run Time (min): & $\mathbf{2 5 . 0 0}$ & Sample Amount: & $\mathbf{1 . 0 0 0 0}$ \\
\hline
\end{tabular}

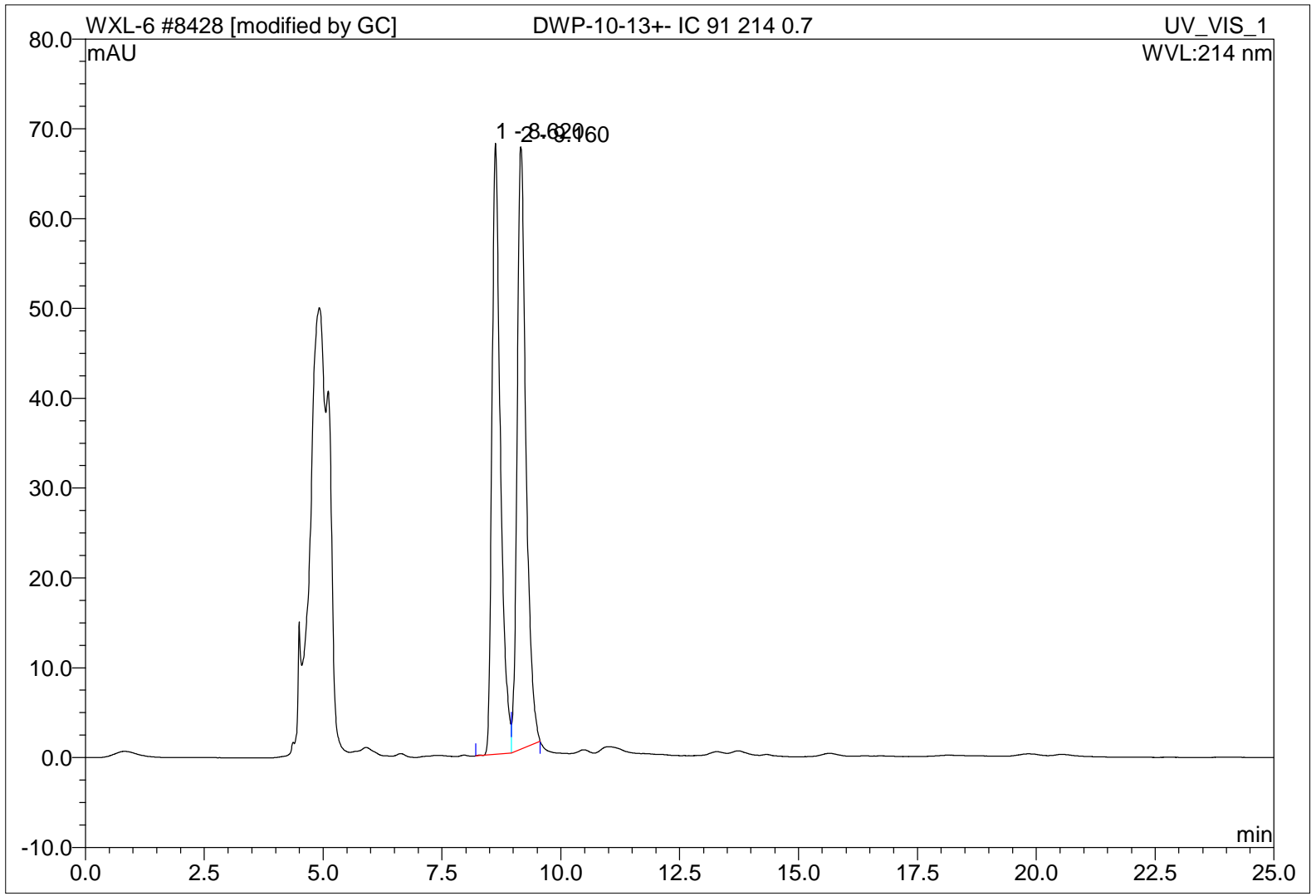

\begin{tabular}{|r|ccrrrrr|}
\hline No. & $\begin{array}{c}\text { Ret.Time } \\
\text { min }\end{array}$ & Peak Name & $\begin{array}{c}\text { Height } \\
\text { mAU }\end{array}$ & $\begin{array}{c}\text { Area } \\
\text { mAU*min }\end{array}$ & $\begin{array}{r}\text { Rel.Area } \\
\%\end{array}$ & Amount & Type \\
\hline 1 & 8.62 & n.a. & 68.061 & 14.076 & 49.11 & n.a. & BM $^{*}$ \\
2 & 9.16 & n.a. & 67.070 & 14.589 & 50.89 & n.a. & MB $^{*}$ \\
\hline Total: & & & 135.131 & 28.665 & 100.00 & 0.000 & \\
\hline
\end{tabular}




\section{DWP-10-78 IC 912140.7}

\begin{tabular}{|llll|}
\hline Sample Name: & DWP-10-78 IC 912140.7 & Injection Volume: & $\mathbf{5 . 0}$ \\
Vial Number: & GC2 & Channel: & UV_VIS_1 \\
Sample Type: & unknown & Wavelength: & $\mathbf{2 1 4}$ \\
Control Program: & $\mathbf{2 0 1 7 0 1 - 4}$ & Bandwidth: & n.a. \\
Quantif. Method: & $\mathbf{2 0 1 7 0 1}$ & Dilution Factor: & $\mathbf{1 . 0 0 0 0}$ \\
Recording Time: & $\mathbf{2 0 1 9 / 1 / 1 0 ~ 0 : 0 3}$ & Sample Weight: & $\mathbf{1 . 0 0 0 0}$ \\
Run Time (min): & $\mathbf{2 5 . 0 0}$ & Sample Amount: & $\mathbf{1 . 0 0 0 0}$ \\
\hline
\end{tabular}

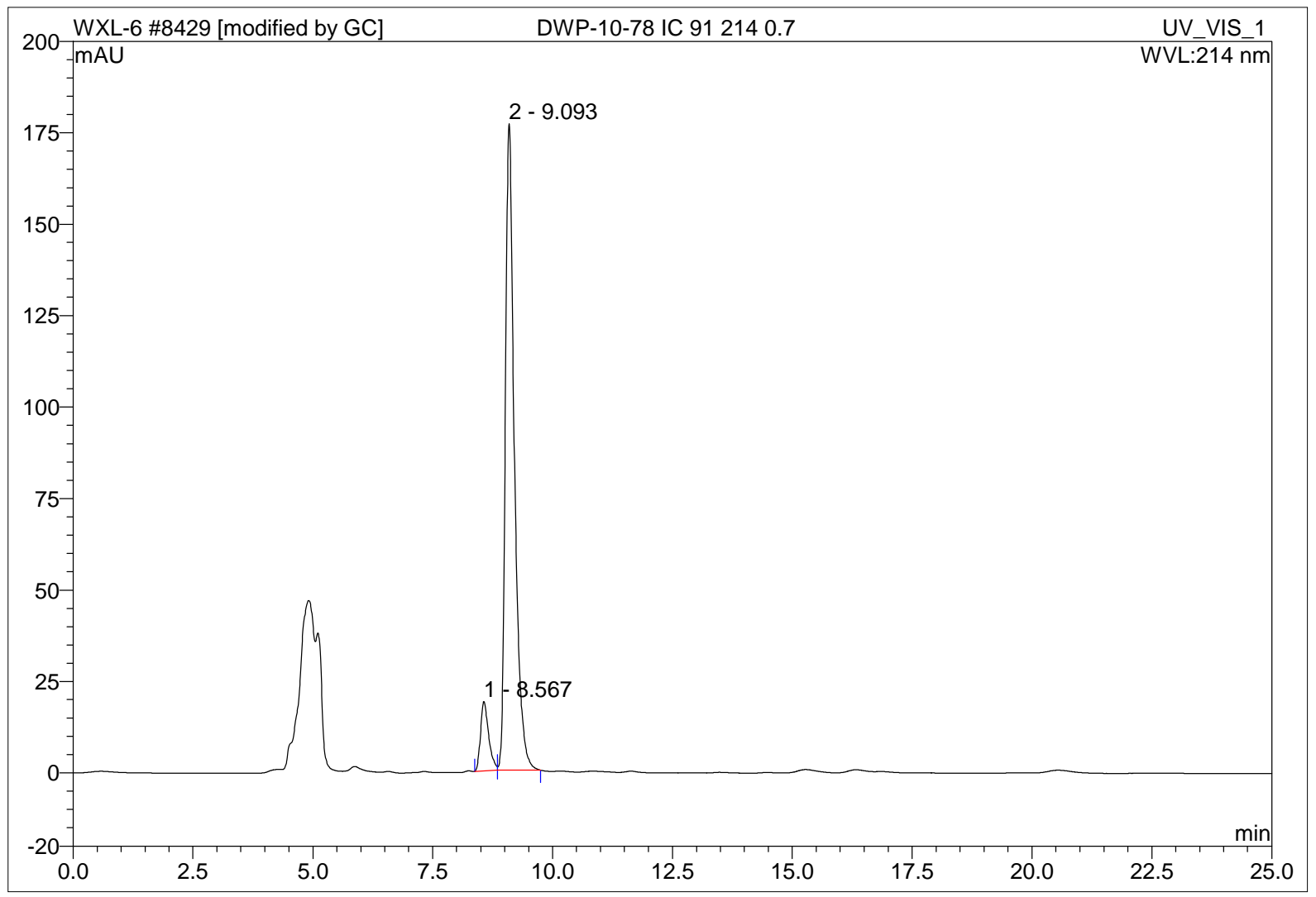

\begin{tabular}{|r|ccrrrrr|}
\hline No. & $\begin{array}{c}\text { Ret.Time } \\
\text { min }\end{array}$ & Peak Name & $\begin{array}{c}\text { Height } \\
\text { mAU }\end{array}$ & $\begin{array}{c}\text { Area } \\
\text { mAU*min }\end{array}$ & $\begin{array}{c}\text { Rel.Area } \\
\%\end{array}$ & Amount & Type \\
\hline 1 & 8.57 & n.a. & 18.981 & 3.721 & 8.76 & n.a. & BM $^{*}$ \\
2 & 9.09 & n.a. & 176.710 & 38.759 & 91.24 & n.a. & MB $^{*}$ \\
\hline Total: & & & 195.690 & 42.480 & 100.00 & 0.000 & \\
\hline
\end{tabular}




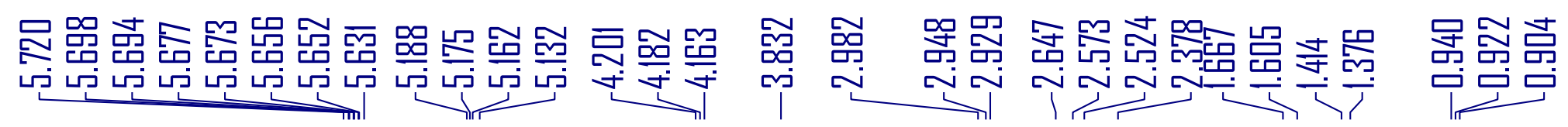
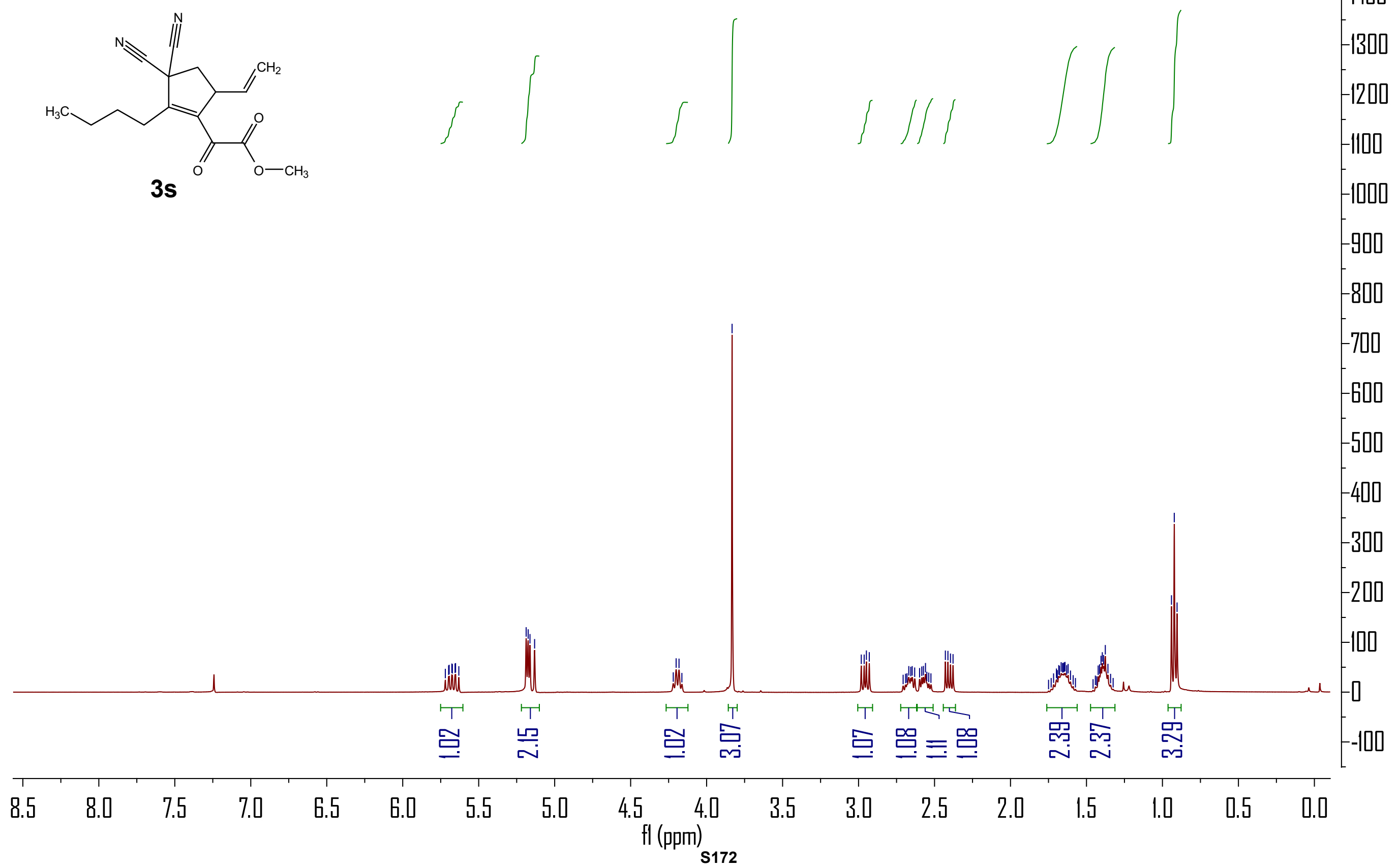


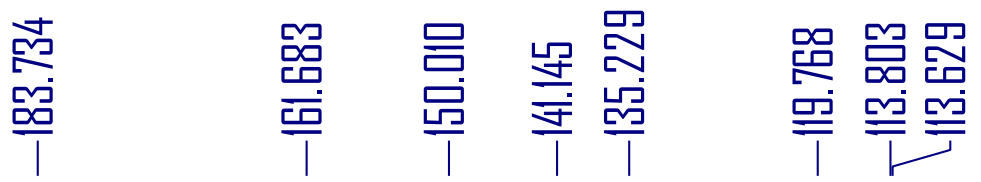

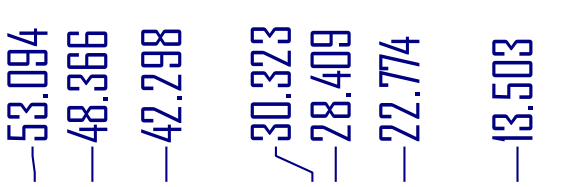

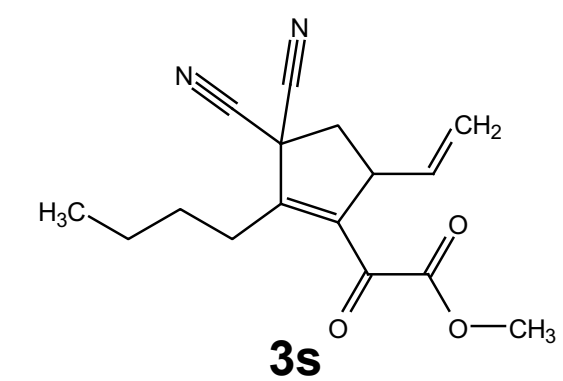

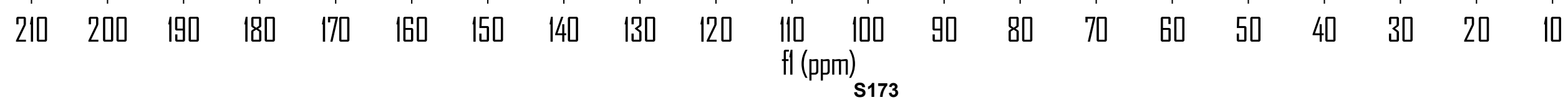




\section{DWP-9-38+- ID3 9822540.7}

\begin{tabular}{|llll|}
\hline Sample Name: & DWP-9-38+- ID3 982 254 0.7 & Injection Volume: & $\mathbf{3 . 0}$ \\
Vial Number: & RD1 & Channel: & EXT254NM \\
Sample Type: & unknown & Wavelength: & $\mathbf{2 5 4}$ \\
Control Program: & test-dad4 & Bandwidth: & $\mathbf{0}$ \\
Quantif. Method: & $\mathbf{2 0 1 7 0 6 0 8}$ & Dilution Factor: & $\mathbf{1 . 0 0 0 0}$ \\
Recording Time: & $\mathbf{2 0 1 9 - 1 - 1 9 ~ 2 0 : 4 8}$ & Sample Weight: & $\mathbf{1 . 0 0 0 0}$ \\
Run Time (min): & $\mathbf{1 4 . 4 3}$ & Sample Amount: & $\mathbf{1 . 0 0 0 0}$ \\
\hline
\end{tabular}

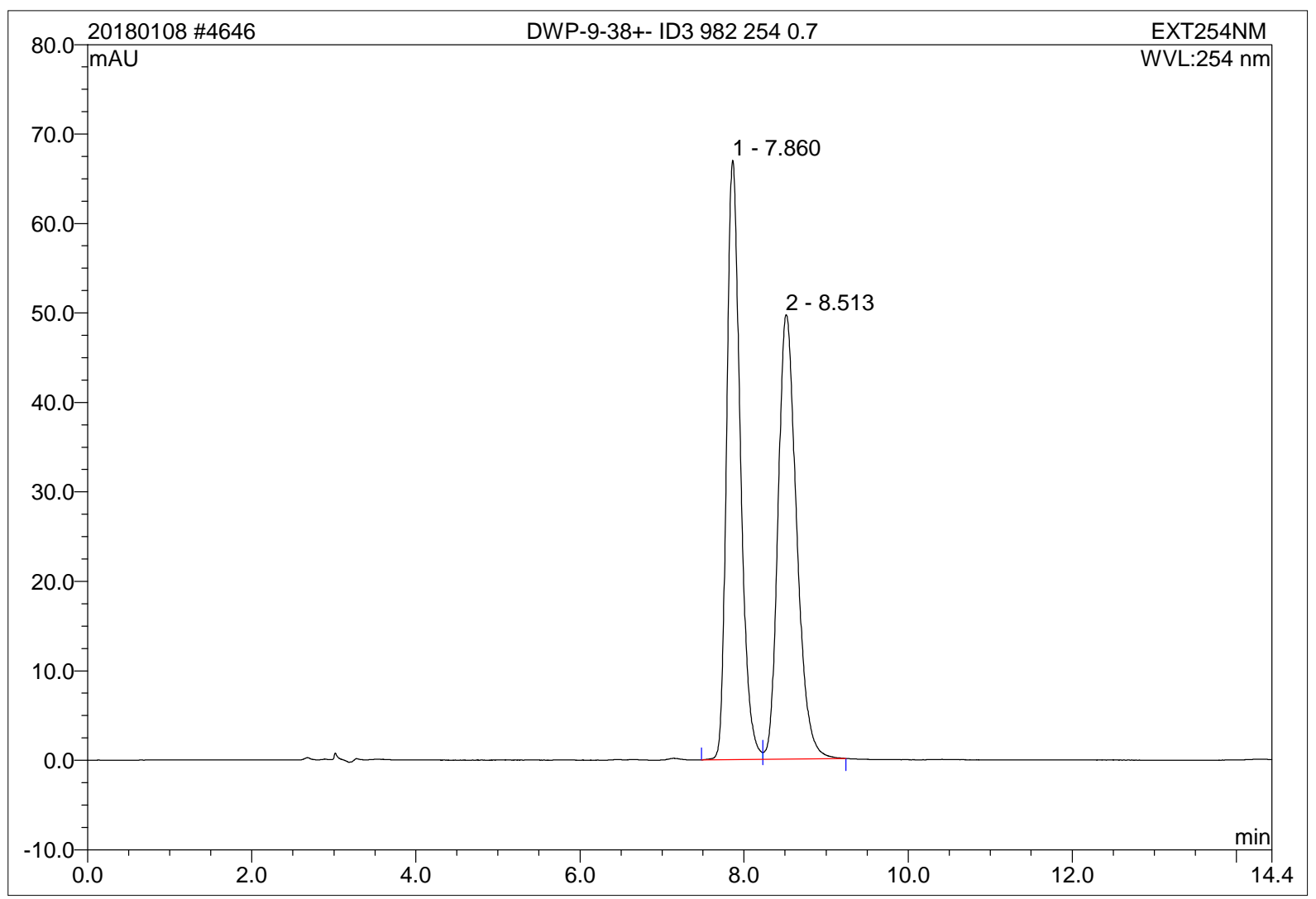

\begin{tabular}{|r|ccrrrrr|}
\hline No. & $\begin{array}{c}\text { Ret.Time } \\
\text { min }\end{array}$ & Peak Name & $\begin{array}{c}\text { Height } \\
\text { mAU }\end{array}$ & $\begin{array}{c}\text { Area } \\
\text { mAU*min }^{*}\end{array}$ & $\begin{array}{r}\text { Rel.Area } \\
\%\end{array}$ & Amount & Type \\
\hline 1 & 7.86 & n.a. & 67.032 & 13.000 & 49.88 & n.a. & BM \\
2 & 8.51 & n.a. & 49.690 & 13.062 & 50.12 & n.a. & MB \\
\hline Total: & & & 116.722 & 26.062 & 100.00 & 0.000 & \\
\hline
\end{tabular}




\section{DWP-10-77 ID3 9822540.7}

\begin{tabular}{|llll|}
\hline Sample Name: & DWP-10-77 ID3 982 254 0.7 & Injection Volume: & $\mathbf{3 . 0}$ \\
Vial Number: & RE1 & Channel: & EXT254NM \\
Sample Type: & unknown & Wavelength: & $\mathbf{2 5 4}$ \\
Control Program: & test-dad4 & Bandwidth: & $\mathbf{0}$ \\
Quantif. Method: & $\mathbf{2 0 1 7 0 6 0 8}$ & Dilution Factor: & $\mathbf{1 . 0 0 0 0}$ \\
Recording Time: & $\mathbf{2 0 1 9 - 1 - 1 9 ~ 2 1 : 0 4}$ & Sample Weight: & $\mathbf{1 . 0 0 0 0}$ \\
Run Time (min): & $\mathbf{1 1 . 3 5}$ & Sample Amount: & $\mathbf{1 . 0 0 0 0}$ \\
\hline
\end{tabular}

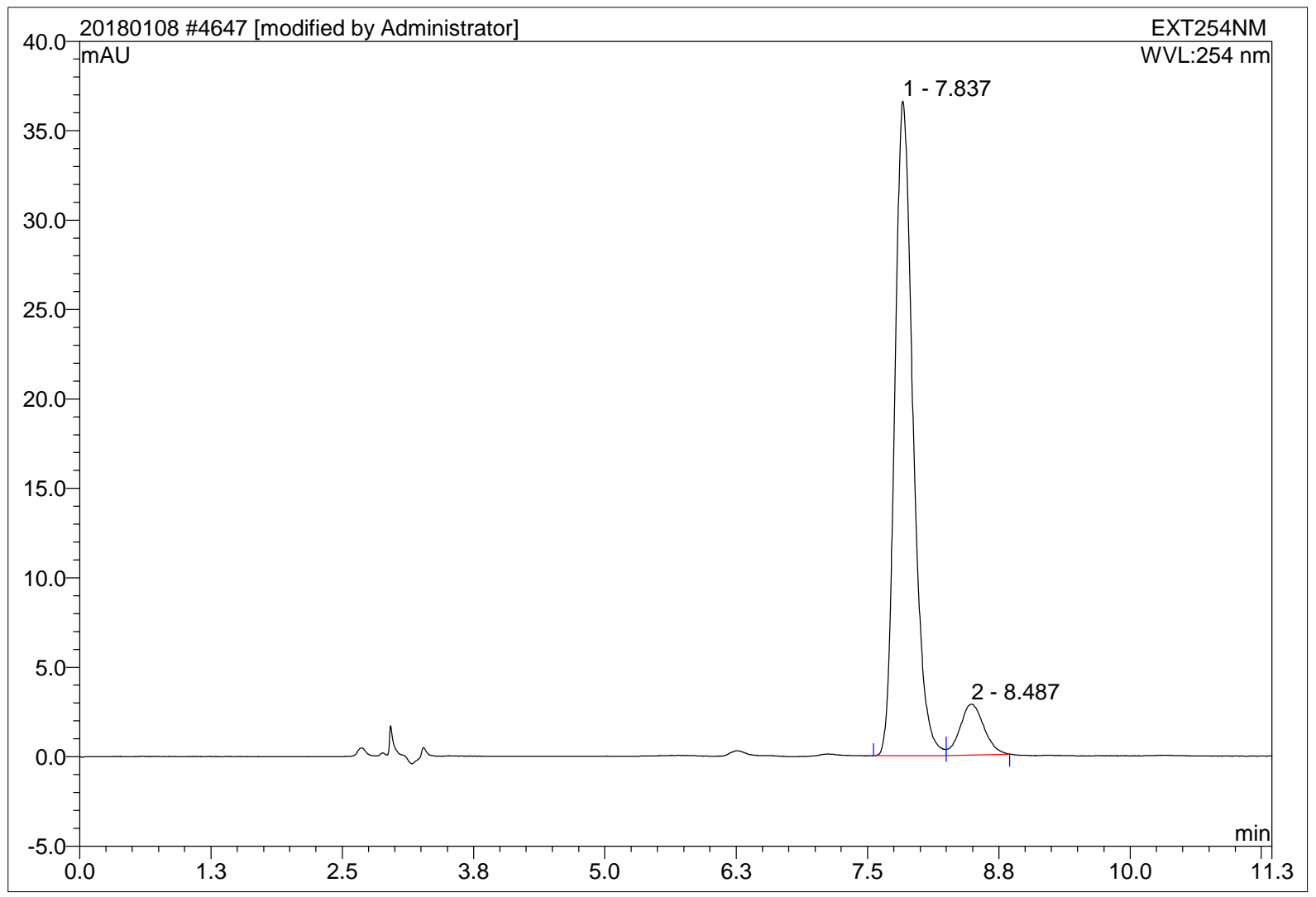

\begin{tabular}{|r|ccrrrrr|}
\hline No. & $\begin{array}{c}\text { Ret.Time } \\
\text { min }\end{array}$ & Peak Name & $\begin{array}{c}\text { Height } \\
\text { mAU }\end{array}$ & $\begin{array}{r}\text { Area } \\
\text { mAU*min }^{*}\end{array}$ & $\begin{array}{r}\text { Rel.Area } \\
\%\end{array}$ & Amount & Type \\
\hline 1 & 7.84 & n.a. & 36.614 & 7.373 & 90.74 & n.a. & BM $^{*}$ \\
2 & 8.49 & n.a. & 2.846 & 0.753 & 9.26 & n.a. & MB $^{*}$ \\
\hline Total: & & & 39.461 & 8.126 & 100.00 & 0.000 & \\
\hline
\end{tabular}




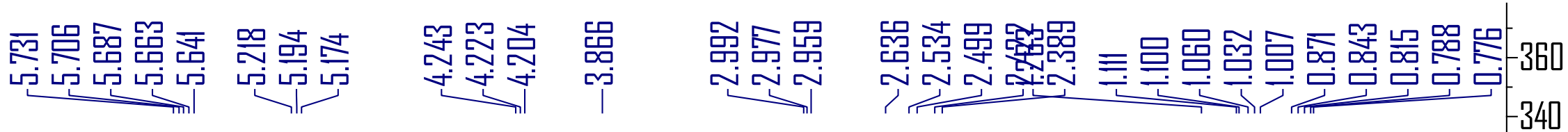
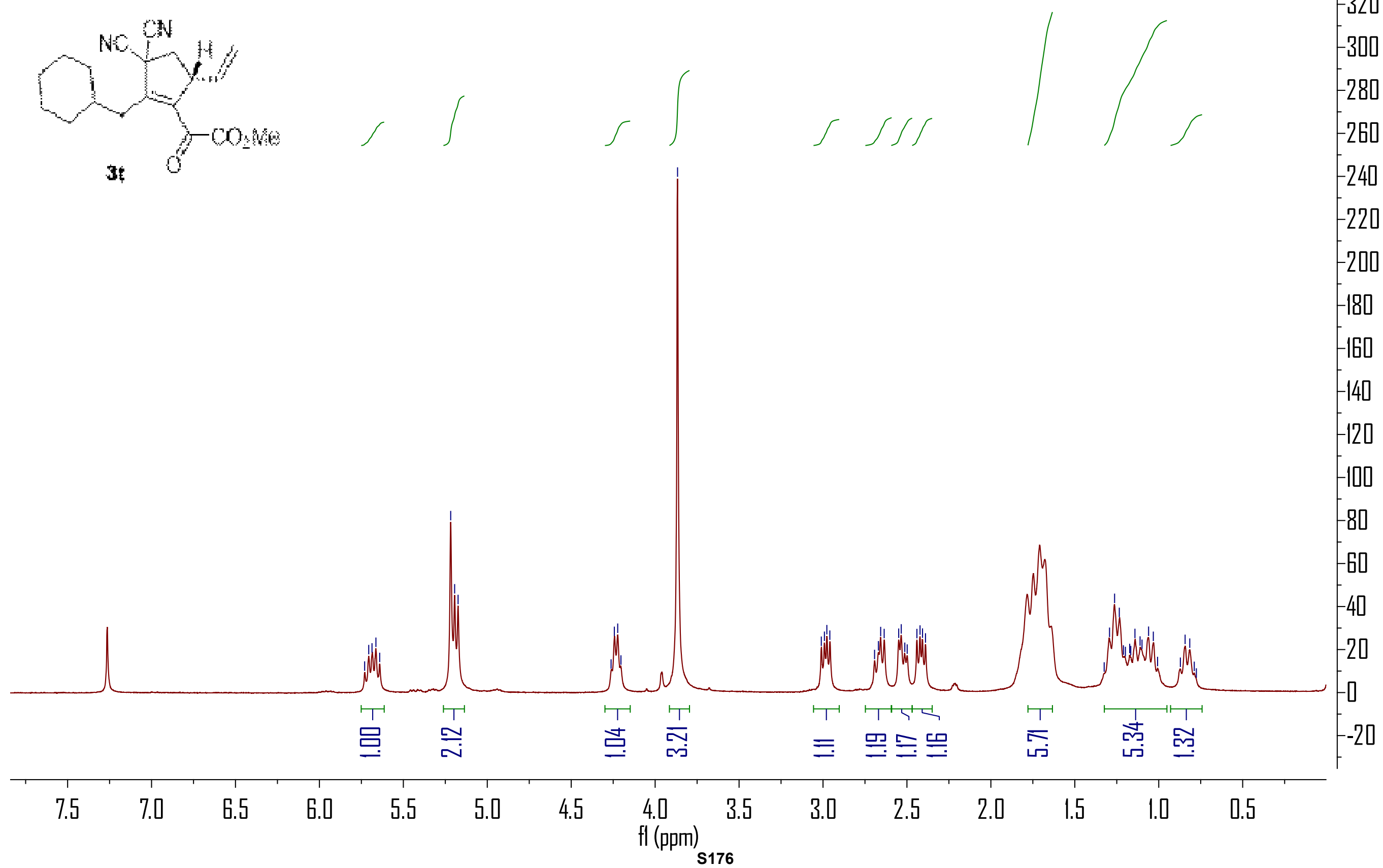


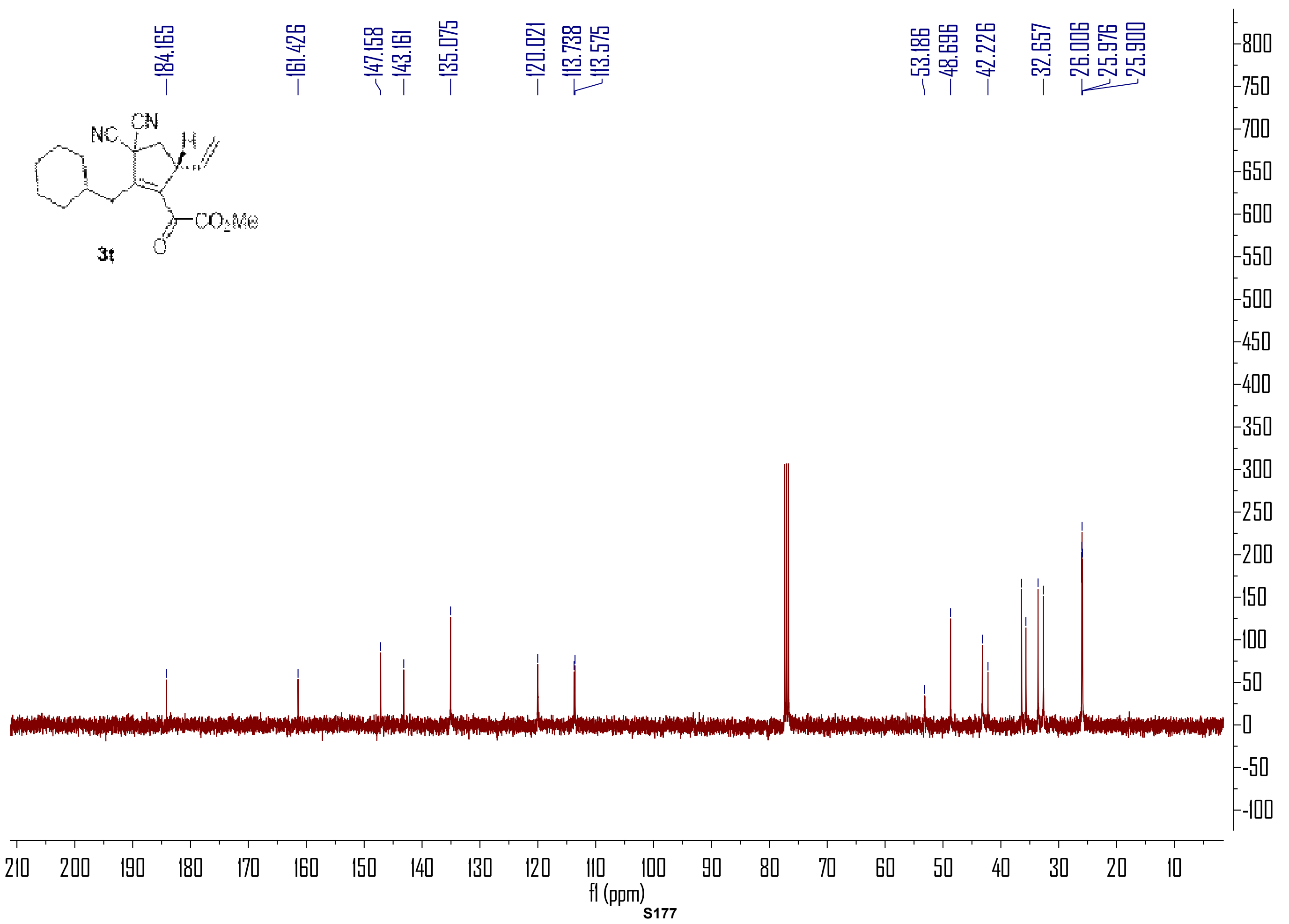


Operator:Administrator Timebase:HPLC Sequence:20180108

\section{DWP-10-16+- IB 9822540.7}

\begin{tabular}{|llll|}
\hline Sample Name: & DWP-10-16+- IB 982 254 0.7 & Injection Volume: & $\mathbf{5 . 0}$ \\
Vial Number: & GA7 & Channel: & EXT254NM \\
Sample Type: & unknown & Wavelength: & $\mathbf{2 5 4}$ \\
Control Program: & test-dad4 & Bandwidth: & $\mathbf{0}$ \\
Quantif. Method: & $\mathbf{2 0 1 7 0 6 0 8}$ & Dilution Factor: & $\mathbf{1 . 0 0 0 0}$ \\
Recording Time: & $\mathbf{2 0 1 9 - 1 - 1 1 ~ 1 6 : 5 2}$ & Sample Weight: & $\mathbf{1 . 0 0 0 0}$ \\
Run Time (min): & $\mathbf{4 0 . 6 1}$ & Sample Amount: & $\mathbf{1 . 0 0 0 0}$ \\
\hline
\end{tabular}

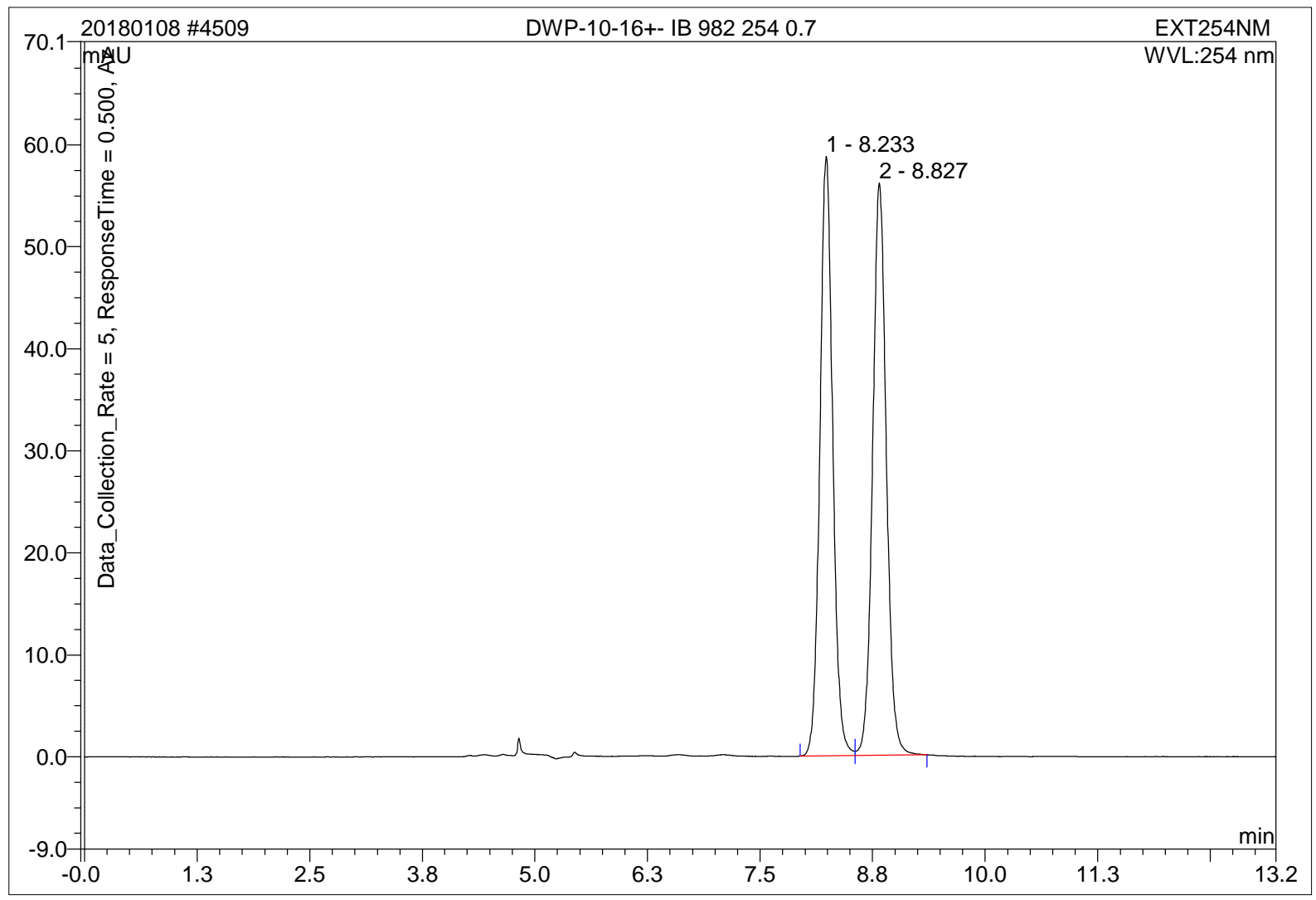

\begin{tabular}{|r|ccrrrrr|}
\hline No. & $\begin{array}{c}\text { Ret.Time } \\
\text { min }\end{array}$ & Peak Name & $\begin{array}{c}\text { Height } \\
\text { mAU }\end{array}$ & $\begin{array}{c}\text { Area } \\
\text { mAU*min }\end{array}$ & $\begin{array}{r}\text { Rel.Area } \\
\%\end{array}$ & Amount & Type \\
\hline 1 & 8.23 & n.a. & 58.801 & 9.729 & 49.56 & n.a. & BM \\
2 & 8.83 & n.a. & 56.131 & 9.901 & 50.44 & n.a. & MB \\
\hline Total: & & & 114.933 & 19.630 & 100.00 & 0.000 & \\
\hline
\end{tabular}


Operator:Administrator Timebase:HPLC Sequence:20180108

\section{DWP-10-105 IB 9822540.7}

\begin{tabular}{|llll|}
\hline Sample Name: & DWP-10-105 IB 982 254 0.7 & Injection Volume: & $\mathbf{5 . 0}$ \\
Vial Number: & GB7 & Channel: & EXT254NM \\
Sample Type: & unknown & Wavelength: & $\mathbf{2 5 4}$ \\
Control Program: & test-dad6 & Bandwidth: & $\mathbf{0}$ \\
Quantif. Method: & $\mathbf{2 0 1 7 0 6 0 8}$ & Dilution Factor: & $\mathbf{1 . 0 0 0 0}$ \\
Recording Time: & $\mathbf{2 0 1 9 - 1 - 1 4 ~ 1 0 : 0 5}$ & Sample Weight: & $\mathbf{1 . 0 0 0 0}$ \\
Run Time (min): & $\mathbf{2 6 . 2 3}$ & Sample Amount: & $\mathbf{1 . 0 0 0 0}$ \\
\hline
\end{tabular}

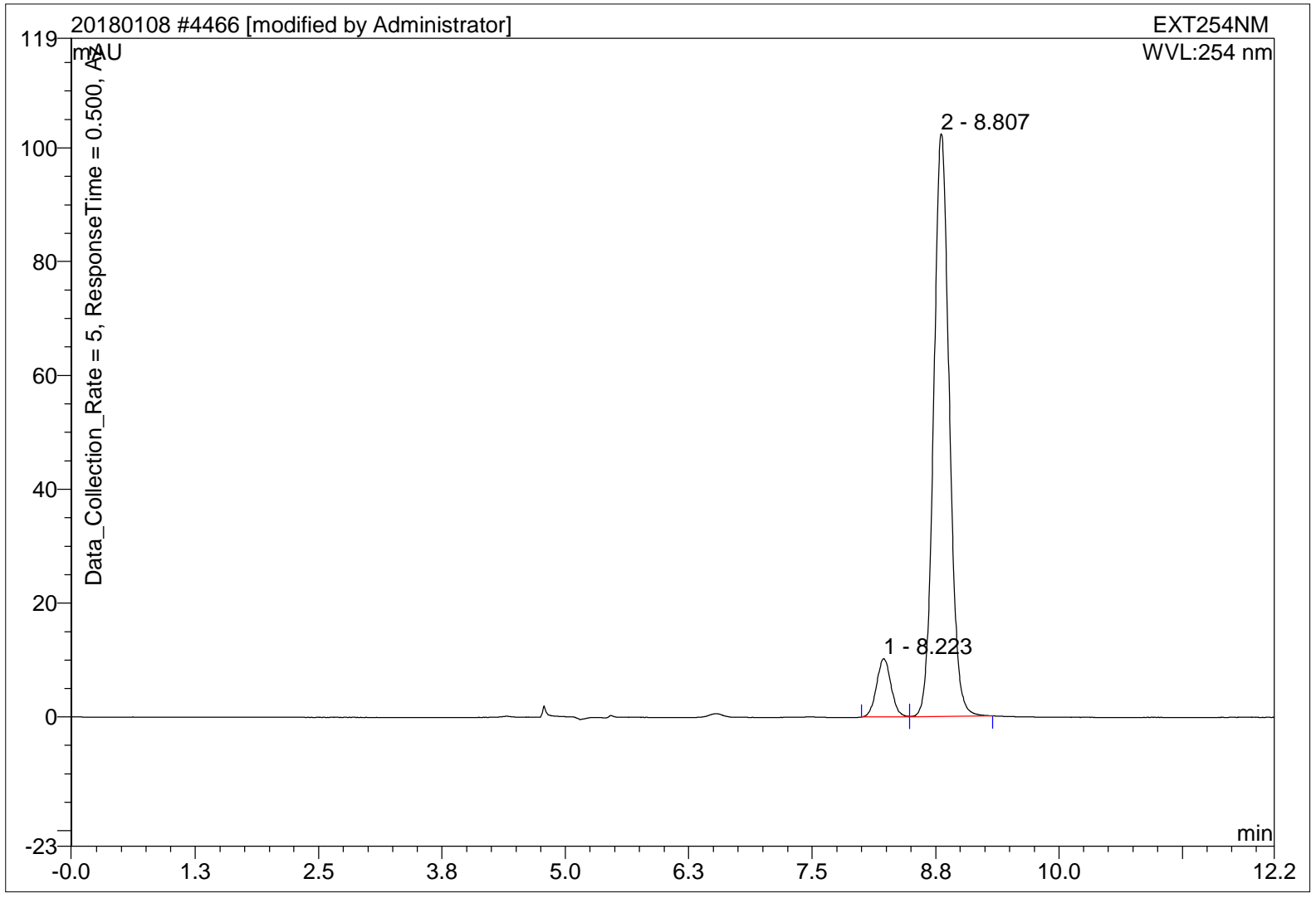

\begin{tabular}{|r|ccrrrrr|}
\hline No. & $\begin{array}{c}\text { Ret.Time } \\
\text { min }\end{array}$ & Peak Name & $\begin{array}{c}\text { Height } \\
\text { mAU }\end{array}$ & $\begin{array}{c}\text { Area } \\
\text { mAU*min }\end{array}$ & $\begin{array}{r}\text { Rel.Area } \\
\%\end{array}$ & Amount & Type \\
\hline 1 & 8.22 & n.a. & 10.312 & 1.753 & 8.51 & n.a. & BM $^{*}$ \\
2 & 8.81 & n.a. & 102.425 & 18.846 & 91.49 & n.a. & MB $^{*}$ \\
\hline Total: & & & 112.737 & 20.600 & 100.00 & 0.000 & \\
\hline
\end{tabular}




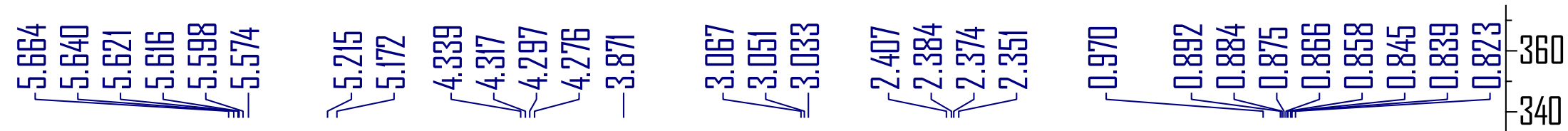

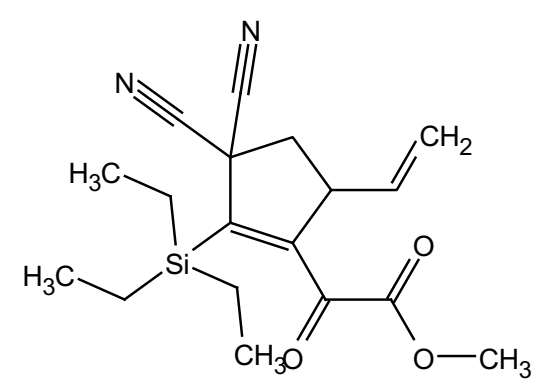

$-320$

3u

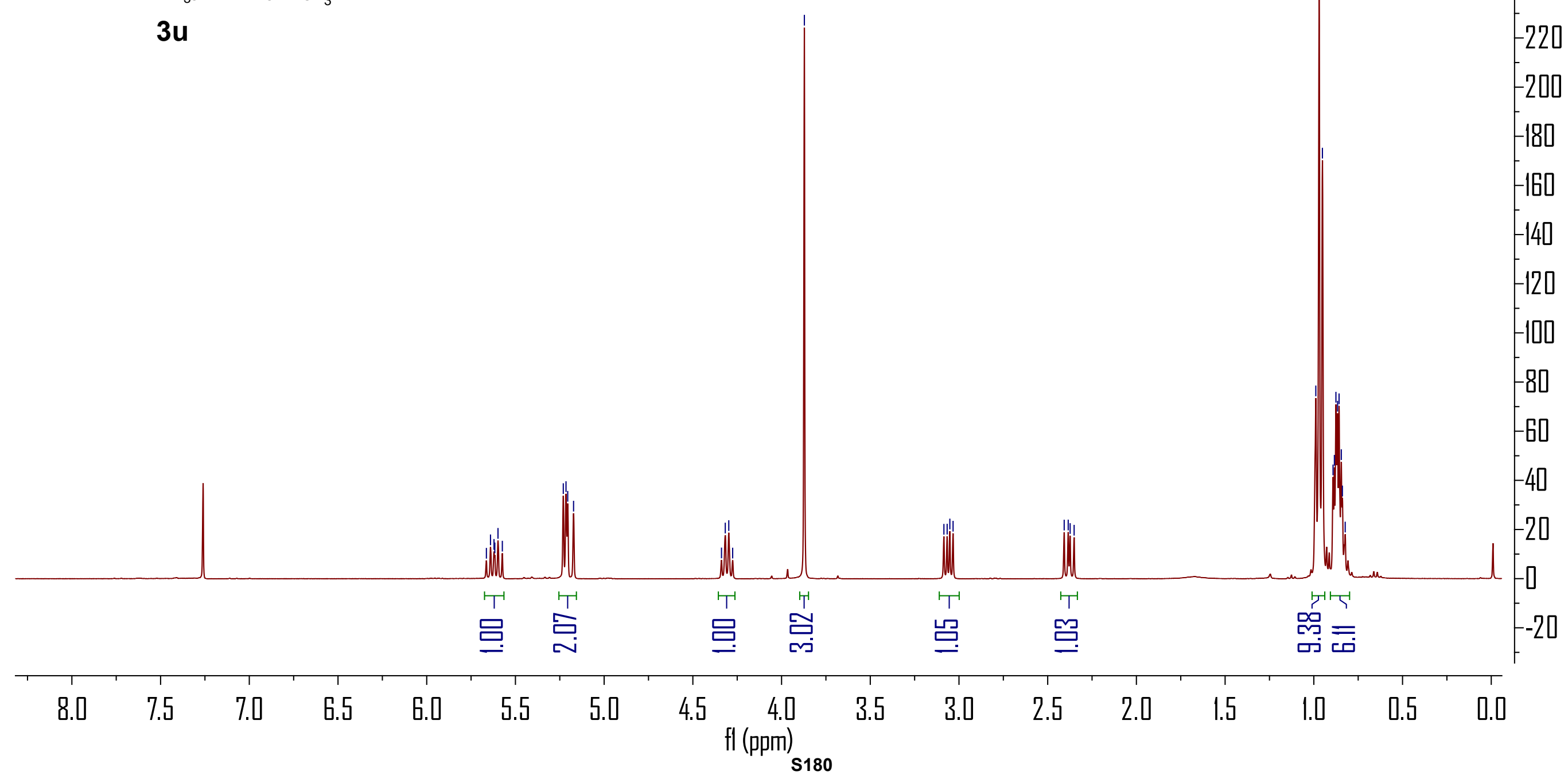




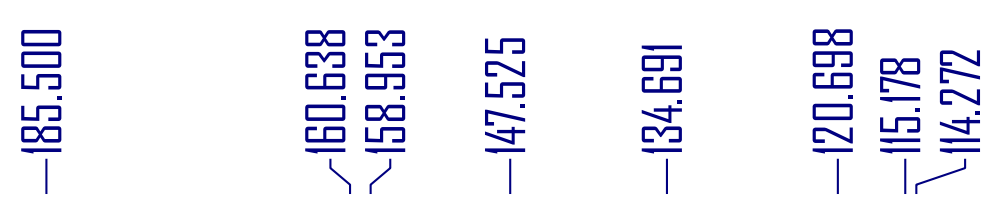

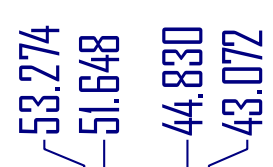

告哭

-2100
-2000
-1900

1800

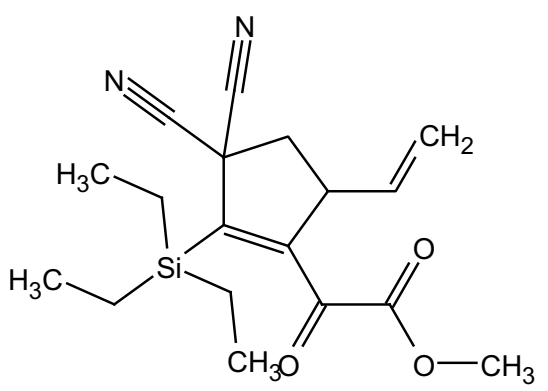

$-1700$

$-1600$

$-1500$

$-1400$

$-1300$

$3 \mathrm{u}$ 


\section{DWP-8-73+- IC 912140.7}

\begin{tabular}{|llll|}
\hline Sample Name: & DWP-8-73+- IC 912140.7 & Injection Volume: & $\mathbf{5 . 0}$ \\
Vial Number: & GA1 & Channel: & UV_VIS_1 \\
Sample Type: & unknown & Wavelength: & $\mathbf{2 1 4}$ \\
Control Program: & $\mathbf{2 0 1 7 0 1 - 4}$ & Bandwidth: & n.a. \\
Quantif. Method: & $\mathbf{2 0 1 7 0 1}$ & Dilution Factor: & $\mathbf{1 . 0 0 0 0}$ \\
Recording Time: & $\mathbf{2 0 1 9 / 1 / 9 ~ 1 8 : 1 7}$ & Sample Weight: & $\mathbf{1 . 0 0 0 0}$ \\
Run Time (min): & $\mathbf{2 5 . 0 0}$ & Sample Amount: & $\mathbf{1 . 0 0 0 0}$ \\
\hline
\end{tabular}

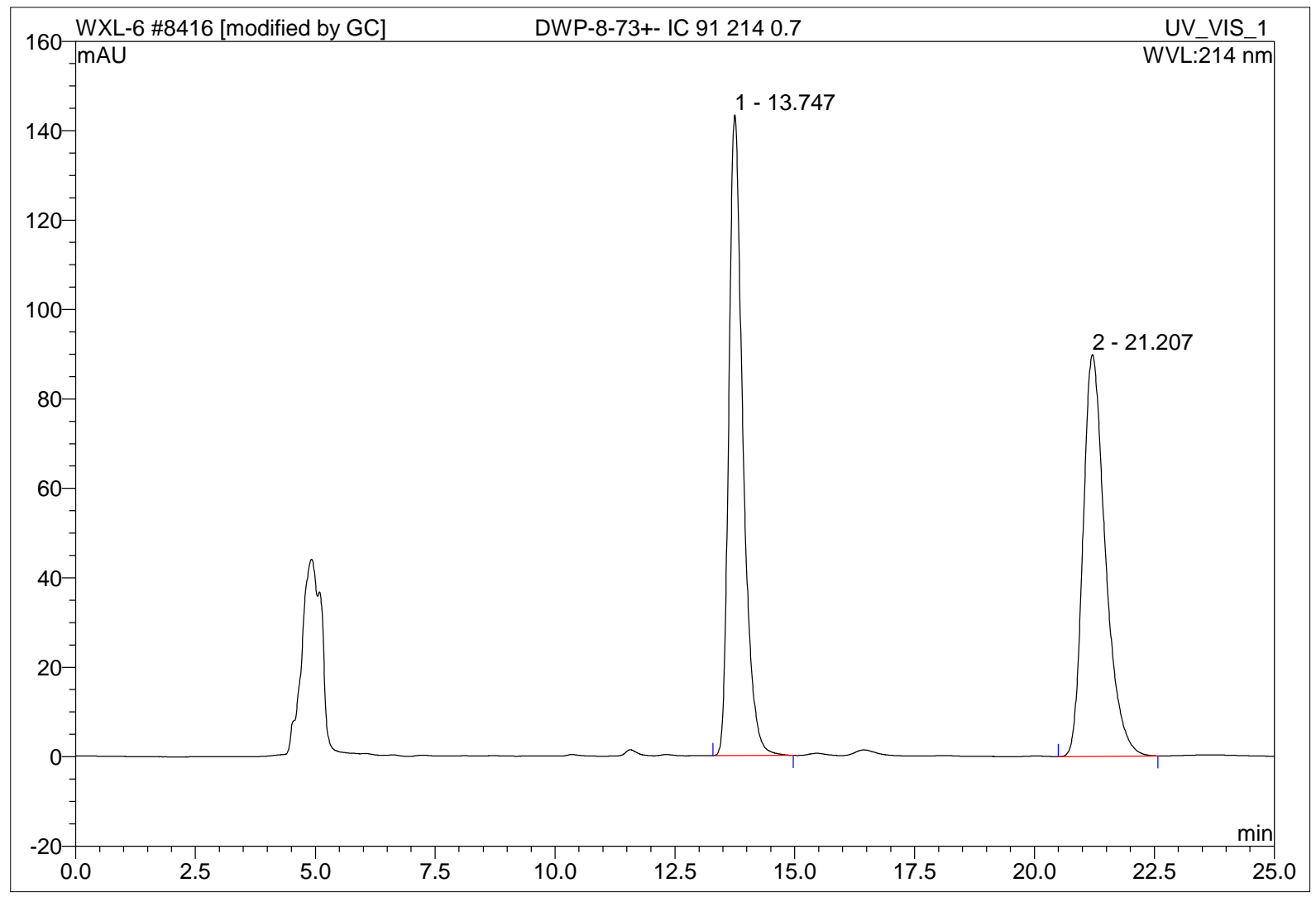

\begin{tabular}{|r|ccrrrrr|}
\hline No. & $\begin{array}{c}\text { Ret.Time } \\
\text { min }\end{array}$ & Peak Name & $\begin{array}{c}\text { Height } \\
\text { mAU }\end{array}$ & $\begin{array}{c}\text { Area } \\
\text { mAU*min }\end{array}$ & $\begin{array}{c}\text { Rel.Area } \\
\%\end{array}$ & Amount & Type \\
\hline 1 & 13.75 & n.a. & 143.346 & 48.906 & 50.19 & n.a. & BMB \\
2 & 21.21 & n.a. & 89.886 & 48.530 & 49.81 & n.a. & BMB \\
\hline Total: & & & 233.232 & 97.436 & 100.00 & 0.000 & \\
\hline
\end{tabular}




\section{DWP-10-84 IC 912140.7}

\begin{tabular}{llll|}
\hline Sample Name: & DWP-10-84 IC 91214 0.7 & Injection Volume: & $\mathbf{5 . 0}$ \\
Vial Number: & GA2 & Channel: & UV_VIS_1 \\
Sample Type: & unknown & Wavelength: & $\mathbf{2 1 4}$ \\
Control Program: & $\mathbf{2 0 1 7 0 1 - 4}$ & Bandwidth: & n.a. \\
Quantif. Method: & $\mathbf{2 0 1 7 0 1}$ & Dilution Factor: & $\mathbf{1 . 0 0 0 0}$ \\
Recording Time: & $\mathbf{2 0 1 9 / 1 / 9 ~ 1 8 : 4 4}$ & Sample Weight: & $\mathbf{1 . 0 0 0 0}$ \\
Run Time (min): & $\mathbf{2 5 . 0 1}$ & Sample Amount: & $\mathbf{1 . 0 0 0 0}$ \\
\hline
\end{tabular}

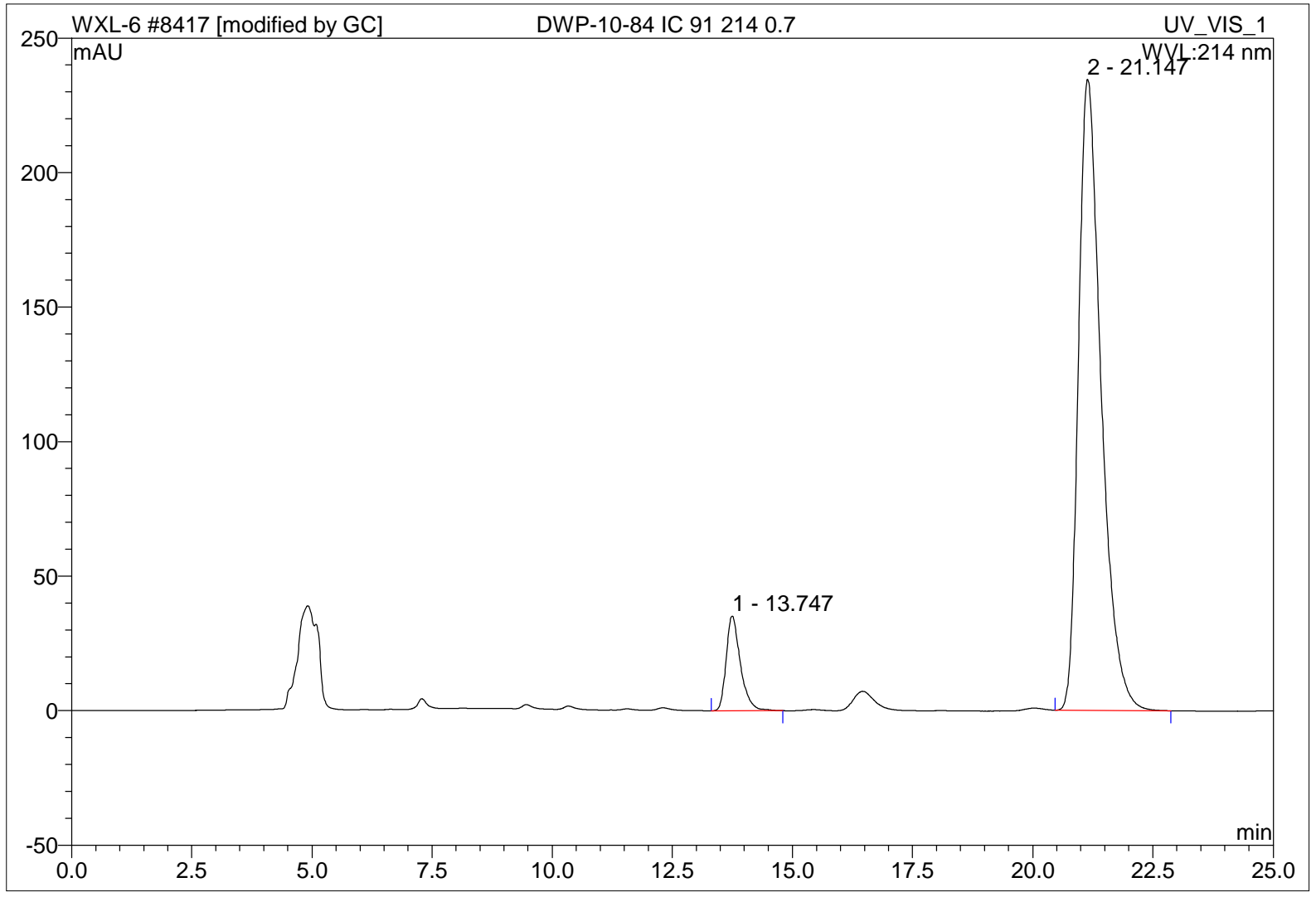

\begin{tabular}{|r|ccrrrrr|}
\hline No. & $\begin{array}{c}\text { Ret.Time } \\
\text { min }\end{array}$ & Peak Name & $\begin{array}{c}\text { Height } \\
\text { mAU }\end{array}$ & $\begin{array}{c}\text { Area } \\
\text { mAU*min }\end{array}$ & $\begin{array}{r}\text { Rel.Area } \\
\%\end{array}$ & Amount & Type \\
\hline 1 & 13.75 & n.a. & 35.271 & 12.141 & 8.59 & n.a. & BMB \\
2 & 21.15 & n.a. & 234.677 & 129.276 & 91.41 & n.a. & BMB \\
\hline Total: & & & 269.948 & 141.417 & 100.00 & 0.000 & \\
\hline
\end{tabular}




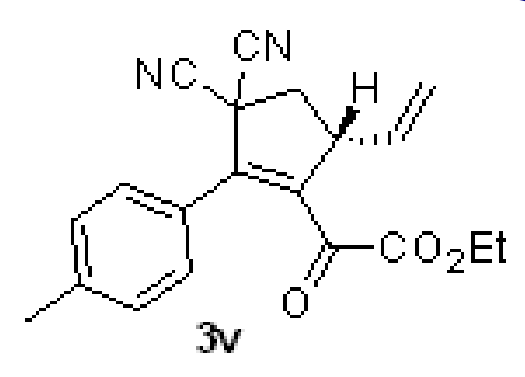

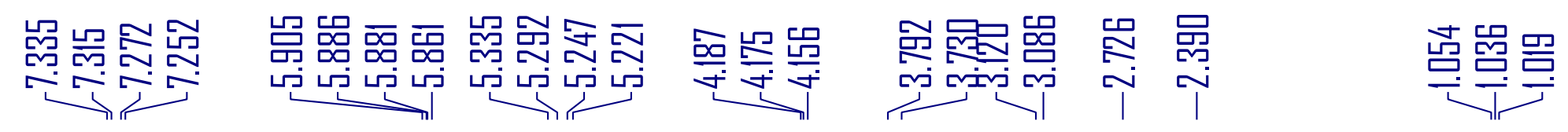

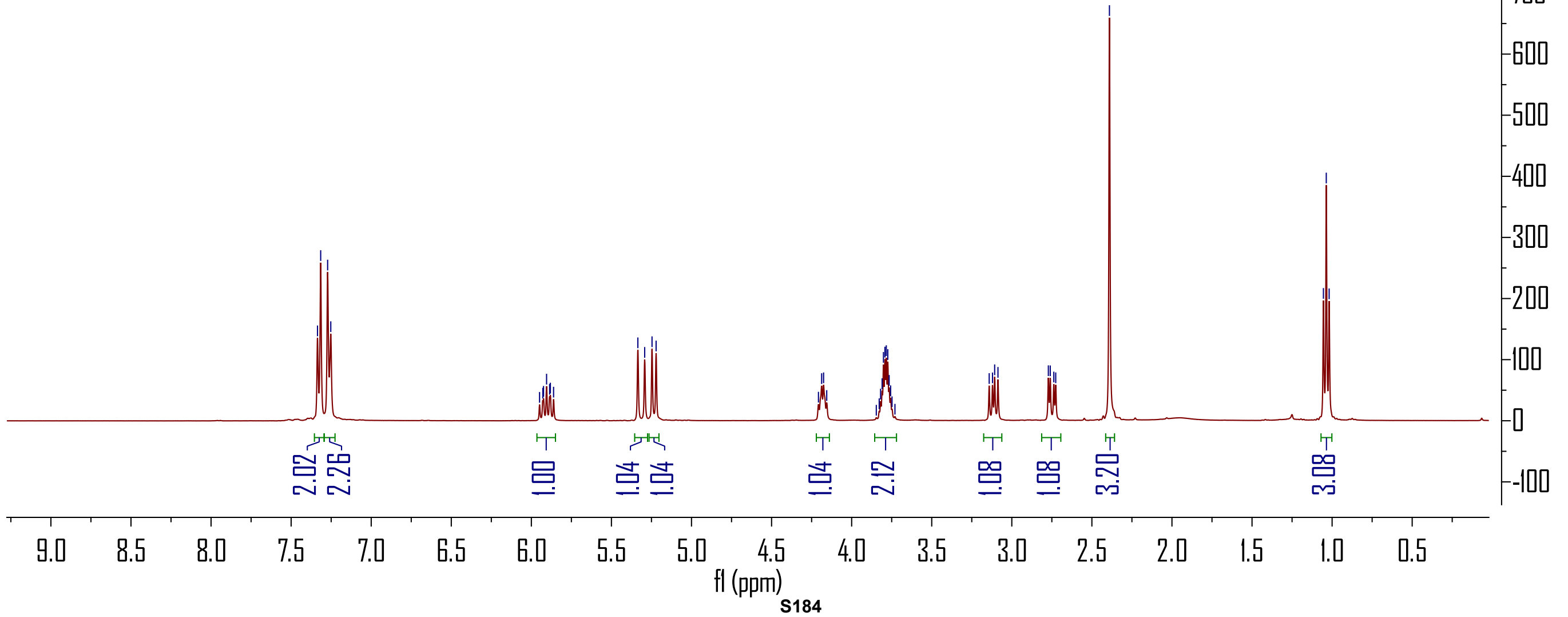




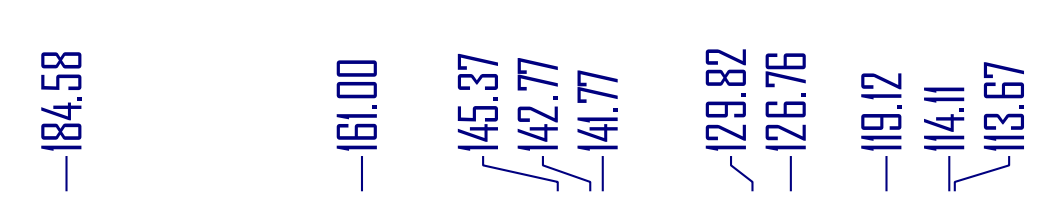

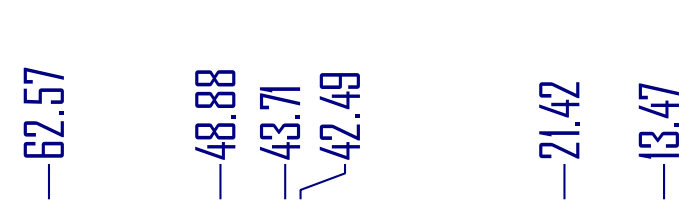
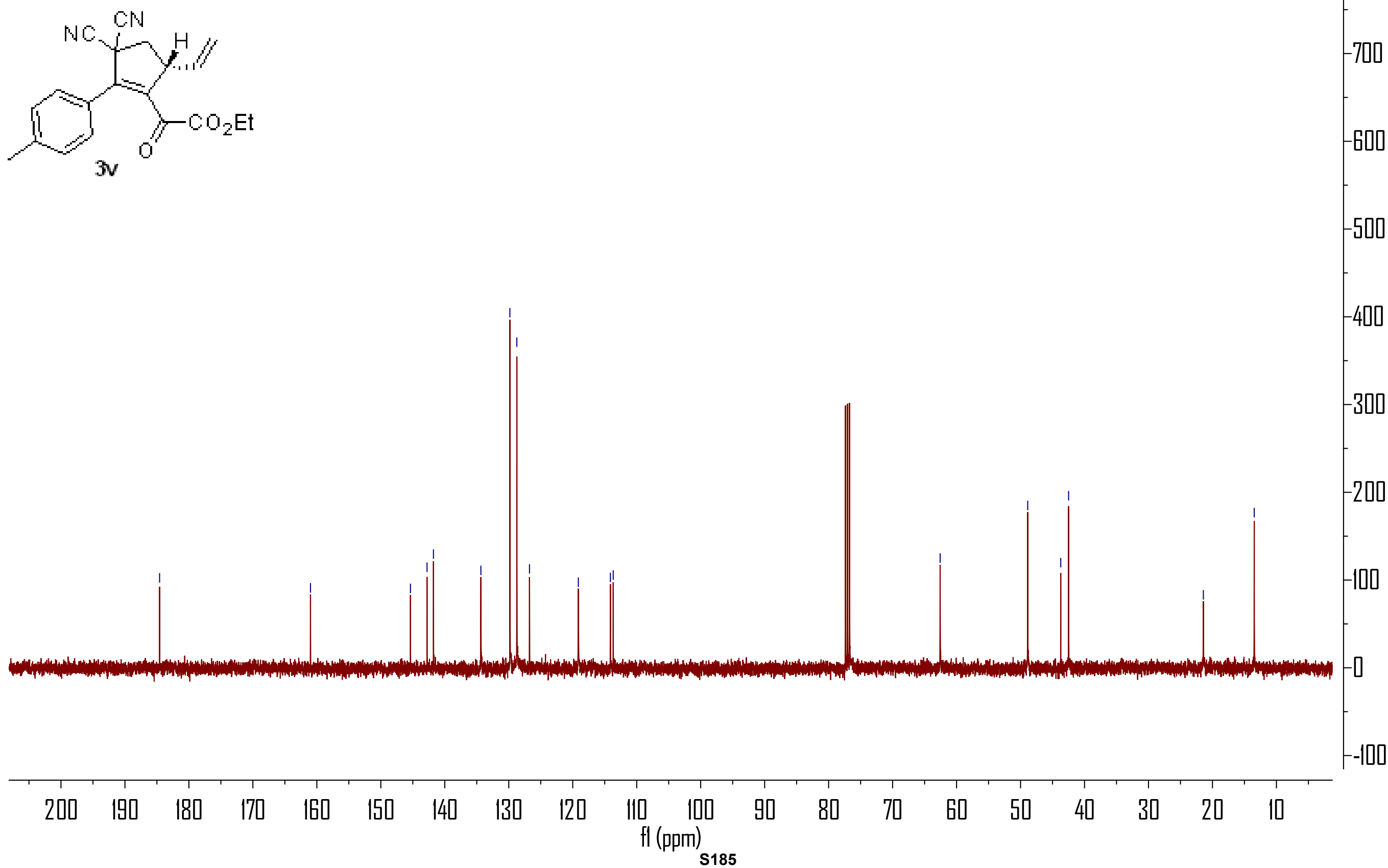


\section{DWP-8-80+- IC 912140.7}

\begin{tabular}{|llll|}
\hline Sample Name: & DWP-8-80+- IC 912140.7 & Injection Volume: & $\mathbf{5 . 0}$ \\
Vial Number: & GA3 & Channel: & UV_VIS_1 \\
Sample Type: & unknown & Wavelength: & $\mathbf{2 1 4}$ \\
Control Program: & $\mathbf{2 0 1 7 0 1 - 4}$ & Bandwidth: & n.a. \\
Quantif. Method: & $\mathbf{2 0 1 7 0 1}$ & Dilution Factor: & $\mathbf{1 . 0 0 0 0}$ \\
Recording Time: & $\mathbf{2 0 1 9 / 1 / 9 ~ 1 9 : 1 0}$ & Sample Weight: & $\mathbf{1 . 0 0 0 0}$ \\
Run Time (min): & $\mathbf{2 5 . 0 1}$ & Sample Amount: & $\mathbf{1 . 0 0 0 0}$ \\
\hline
\end{tabular}

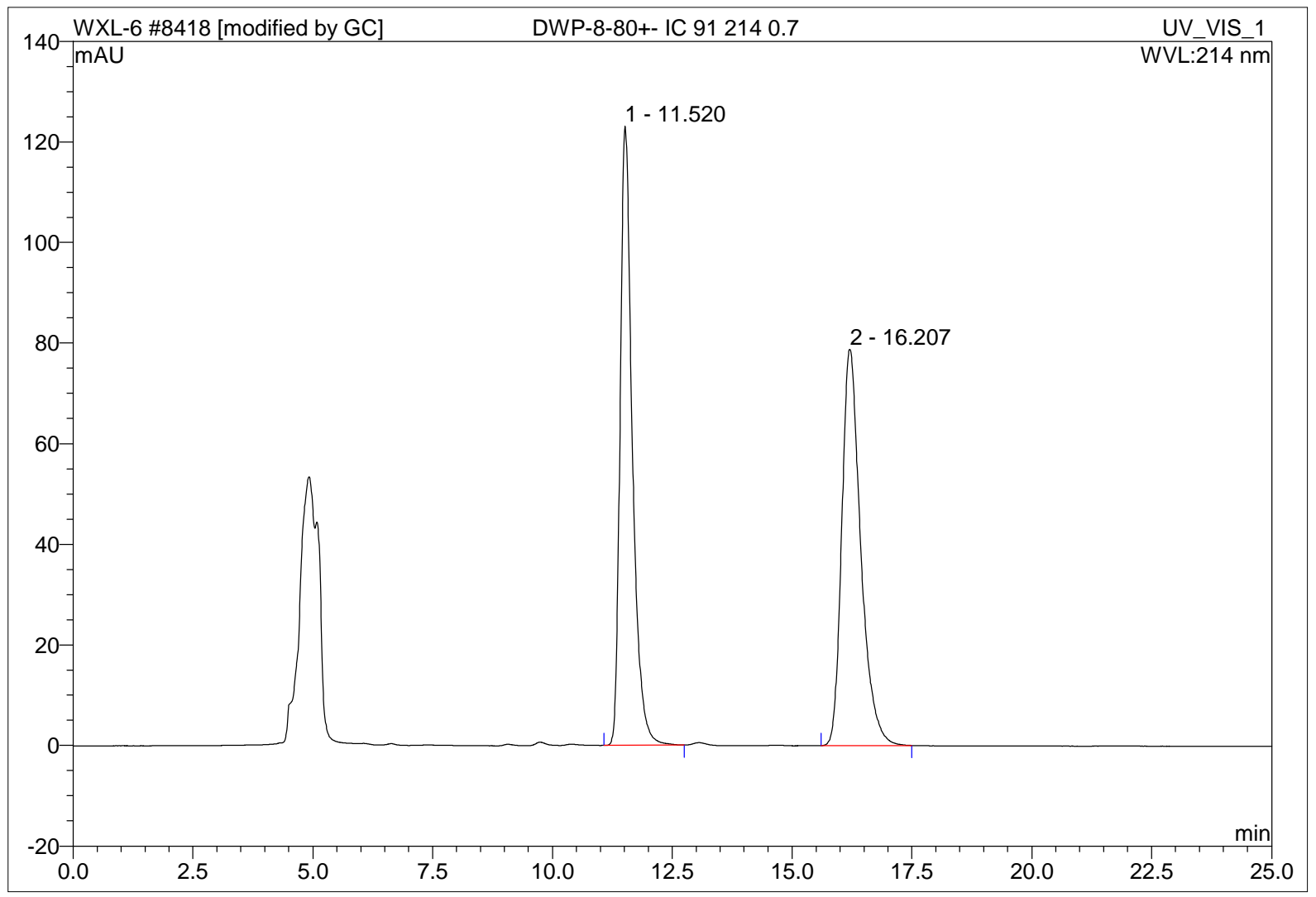

\begin{tabular}{|r|ccrrrrr|}
\hline No. & $\begin{array}{c}\text { Ret.Time } \\
\text { min }\end{array}$ & Peak Name & $\begin{array}{c}\text { Height } \\
\text { mAU }\end{array}$ & $\begin{array}{c}\text { Area } \\
\text { mAU*min }\end{array}$ & $\begin{array}{c}\text { Rel.Area } \\
\%\end{array}$ & Amount & Type \\
\hline 1 & 11.52 & n.a. & 123.117 & 36.276 & 50.08 & n.a. & BMB \\
2 & 16.21 & n.a. & 78.756 & 36.163 & 49.92 & n.a. & BMB \\
\hline Total: & & & 201.873 & 72.440 & 100.00 & 0.000 & \\
\hline
\end{tabular}




\section{DWP-10-79 IC 912140.7}

\begin{tabular}{llll|}
\hline Sample Name: & DWP-10-79 IC 91214 0.7 & Injection Volume: & $\mathbf{5 . 0}$ \\
Vial Number: & GA4 & Channel: & UV_VIS_1 \\
Sample Type: & unknown & Wavelength: & $\mathbf{2 1 4}$ \\
Control Program: & $\mathbf{2 0 1 7 0 1 - 4}$ & Bandwidth: & n.a. \\
Quantif. Method: & $\mathbf{2 0 1 7 0 1}$ & Dilution Factor: & $\mathbf{1 . 0 0 0 0}$ \\
Recording Time: & $\mathbf{2 0 1 9 / 1 / 9 ~ 1 9 : 3 7}$ & Sample Weight: & $\mathbf{1 . 0 0 0 0}$ \\
Run Time (min): & $\mathbf{2 5 . 0 0}$ & Sample Amount: & $\mathbf{1 . 0 0 0 0}$ \\
\hline
\end{tabular}

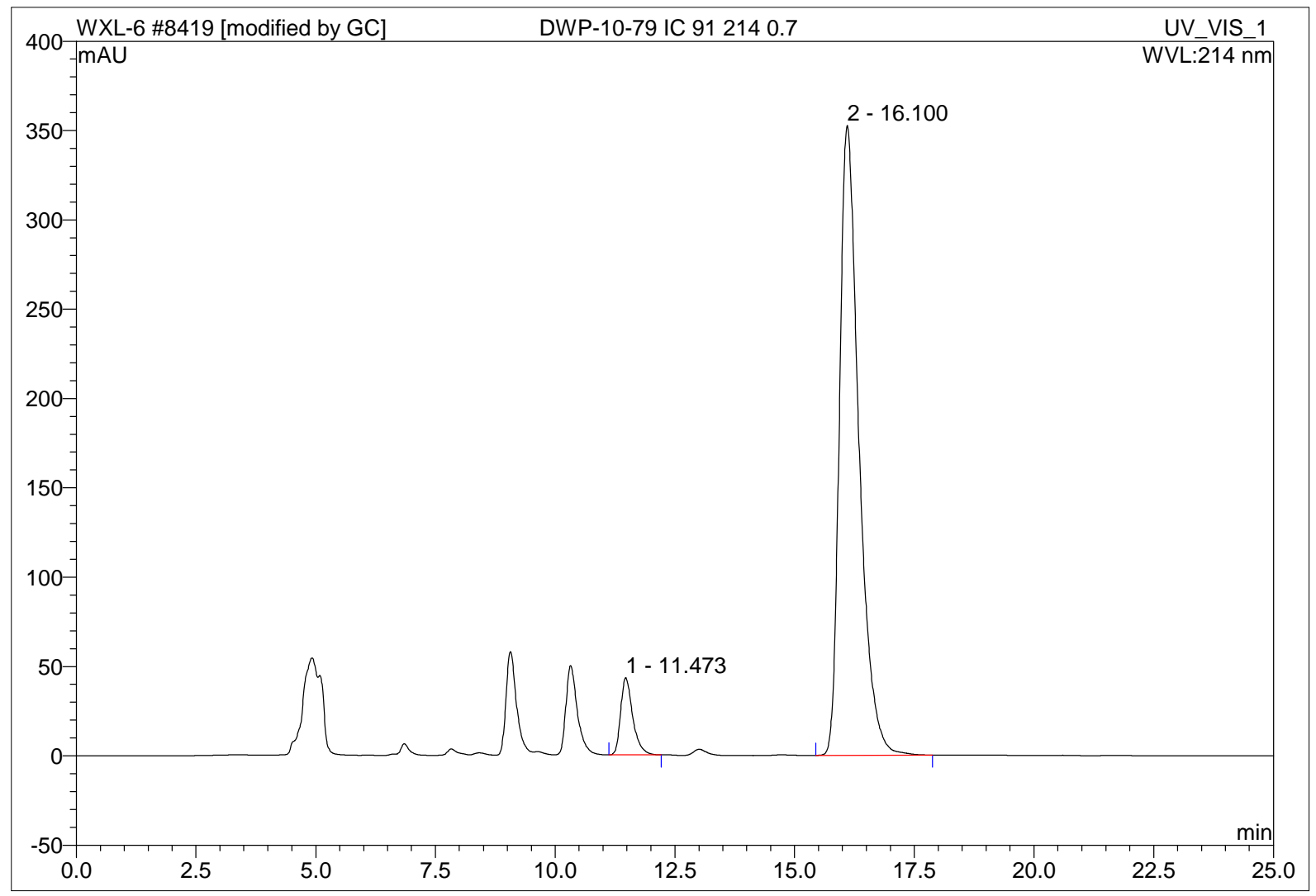

\begin{tabular}{|r|ccrrrrr|}
\hline No. & $\begin{array}{c}\text { Ret.Time } \\
\text { min }\end{array}$ & Peak Name & $\begin{array}{c}\text { Height } \\
\text { mAU }\end{array}$ & $\begin{array}{c}\text { Area } \\
\text { mAU*min }\end{array}$ & $\begin{array}{r}\text { Rel.Area } \\
\%\end{array}$ & Amount & Type \\
\hline 1 & 11.47 & n.a. & 43.433 & 12.693 & 7.11 & n.a. & BMB \\
2 & 16.10 & n.a. & 352.723 & 165.745 & 92.89 & n.a. & BMB \\
\hline Total: & & & 396.156 & 178.437 & 100.00 & 0.000 & \\
\hline
\end{tabular}




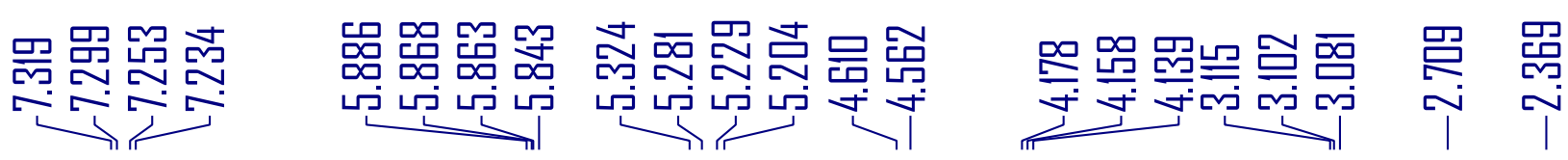
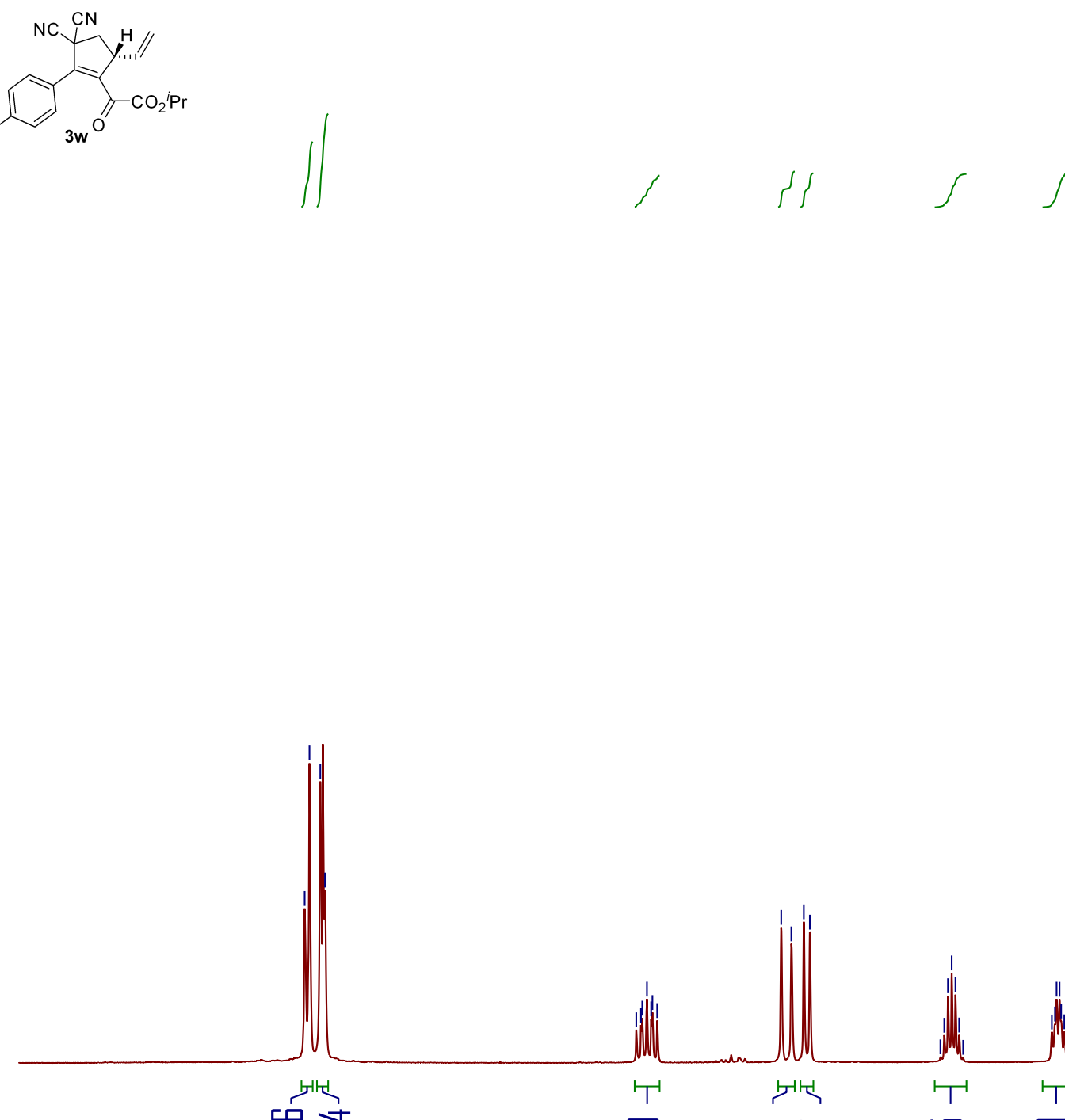

吕 兴㒵 员 吕

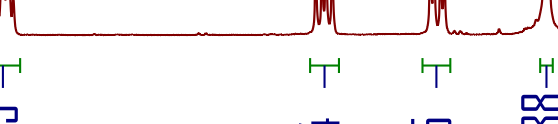

몸홍

\pm 点㗊

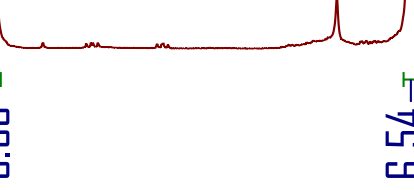

$6.5 \quad 6$.

$5.5 \quad 5$.

4.5 $\mathrm{fl}(\mathrm{ppm})$

$\begin{array}{lllll}3.5 & 3.0 & 2.5 & 2.0 & 1.5\end{array}$

$\begin{array}{llll}.5 & 1.0 & 0.5 & 0.0\end{array}$ 


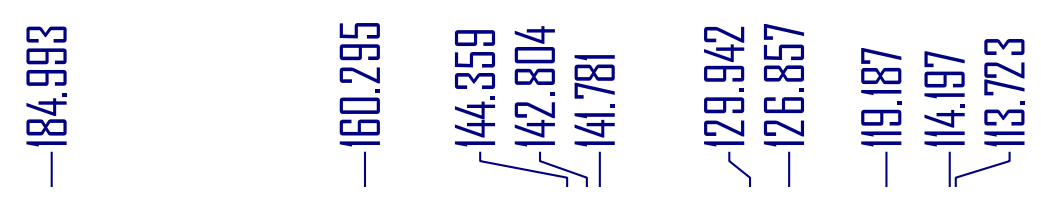

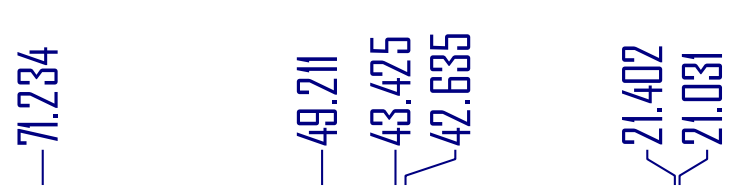

$-700$

650

$-600$

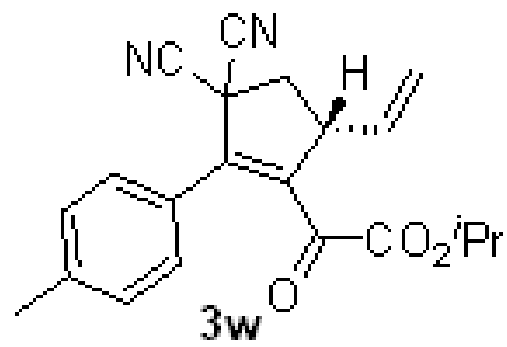

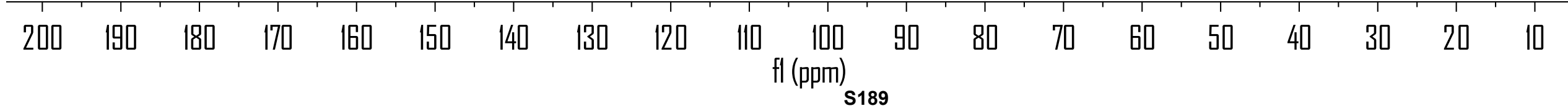


<smiles>CCCC/C=C/C1CN(C#N)C(C#N)(C#N)C1c1ccc(C)cc1</smiles>

\section{==== Shimadzu LCsolution Analysis Report ====}

$\begin{array}{ll}\text { Acquired by } & : \text { Admin } \\ \text { Sample Name } & : \text { dwp-11-47-2 } \\ \text { Sample ID } & : \text { IC,90/10,0.7,214 } \\ \text { Vail \# } & : \text { 1 uL } \\ \text { Injection Volume } & : \text { dwp-11-47-2.Icd } \\ \text { Data File Name } & : 1 . \text { Icm } \\ \text { Method File Name } & : \\ \text { Batch File Name } & : \text { Default.lcr } \\ \text { Report File Name } & : \text { 2019-5-16 19:15:33 } \\ \text { Data Acquired } & : 2019-5-16 \text { 19:36:26 } \\ \text { Data Processed } & \end{array}$

G:Idwp-11-47-2.Icd

$<$ Chromatogram>

$\mathrm{mV}$

G:Idwp-11-47-2.Icd

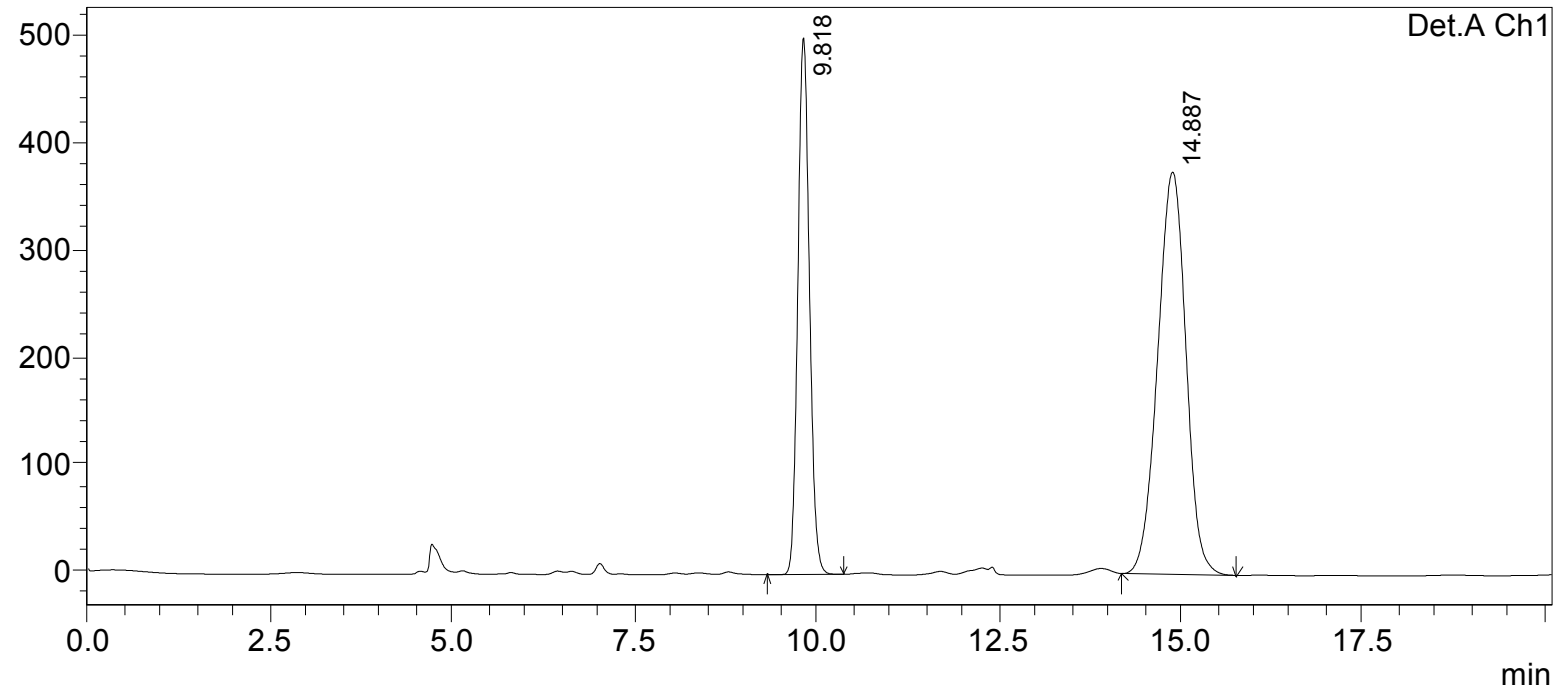

1 Det.A Ch1/214nm

PeakTable

Detector A Ch1 214nm

\begin{tabular}{|r|r|r|r|r|r|}
\hline \multicolumn{1}{|c|}{ Peak\# } & Ret. Time & \multicolumn{1}{c|}{ Area } & \multicolumn{1}{|c|}{ Height } & \multicolumn{1}{c|}{ Area \% } & \multicolumn{1}{c|}{ Height \% } \\
\hline 1 & 9.818 & 5535894 & 500126 & 35.362 & 57.141 \\
\hline 2 & 14.887 & 10119238 & 375117 & 64.638 & 42.859 \\
\hline Total & & 15655133 & 875242 & 100.000 & 100.000 \\
\hline
\end{tabular}




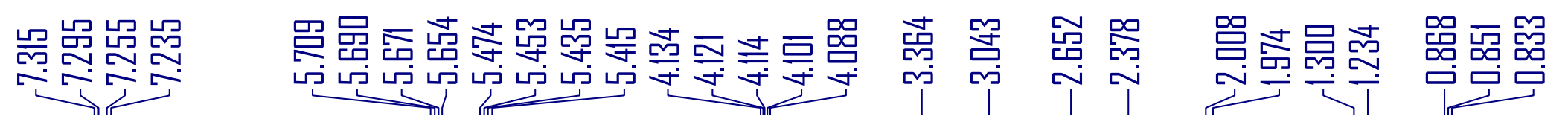

$-320$

300

280
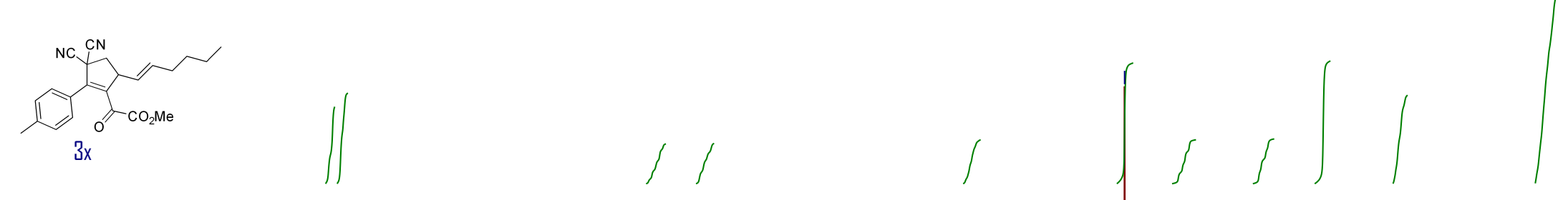

260

240

220

200

180

$-160$

140

$-120$

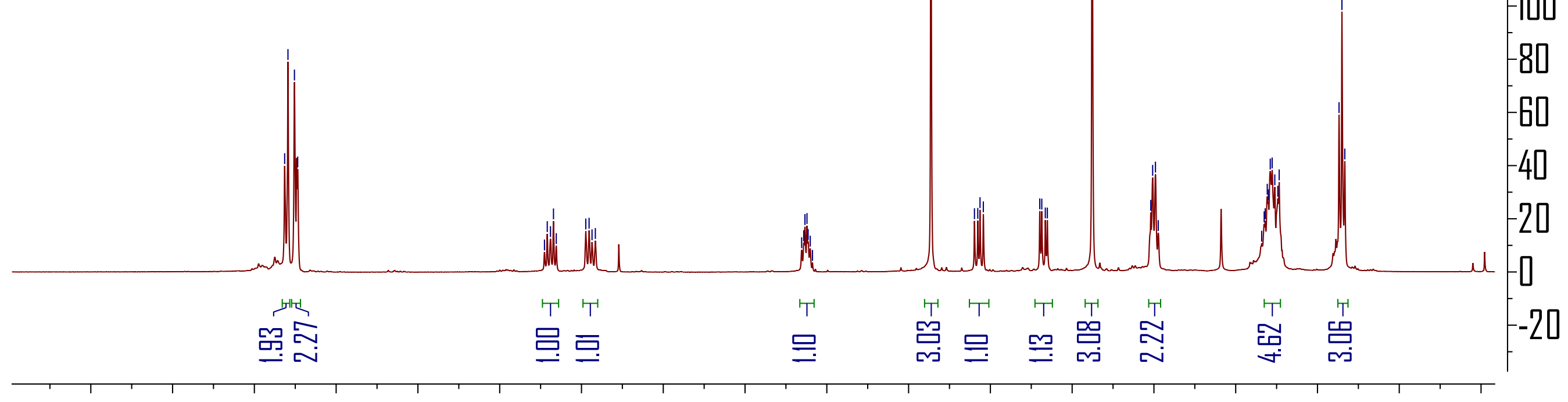

$\begin{array}{llllllllllllllllll}8.5 & 8.0 & 7.5 & 7.0 & 6.5 & 6.0 & 5.5 & 5.0 & 4.5 & 4.0 & 3.5 & 3.0 & 2.5 & 2.0 & 1.5 & 1.0 & 0.5 & 0.0\end{array}$ fl (ppm) 


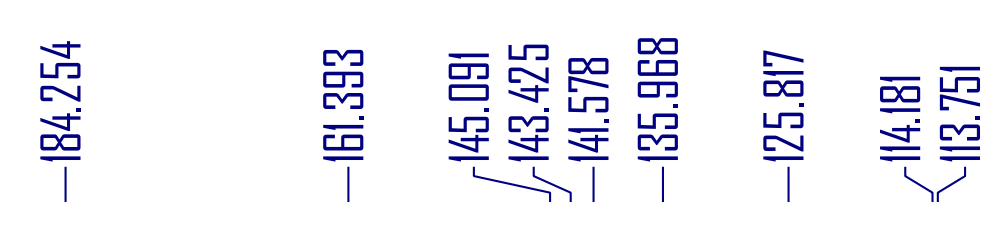
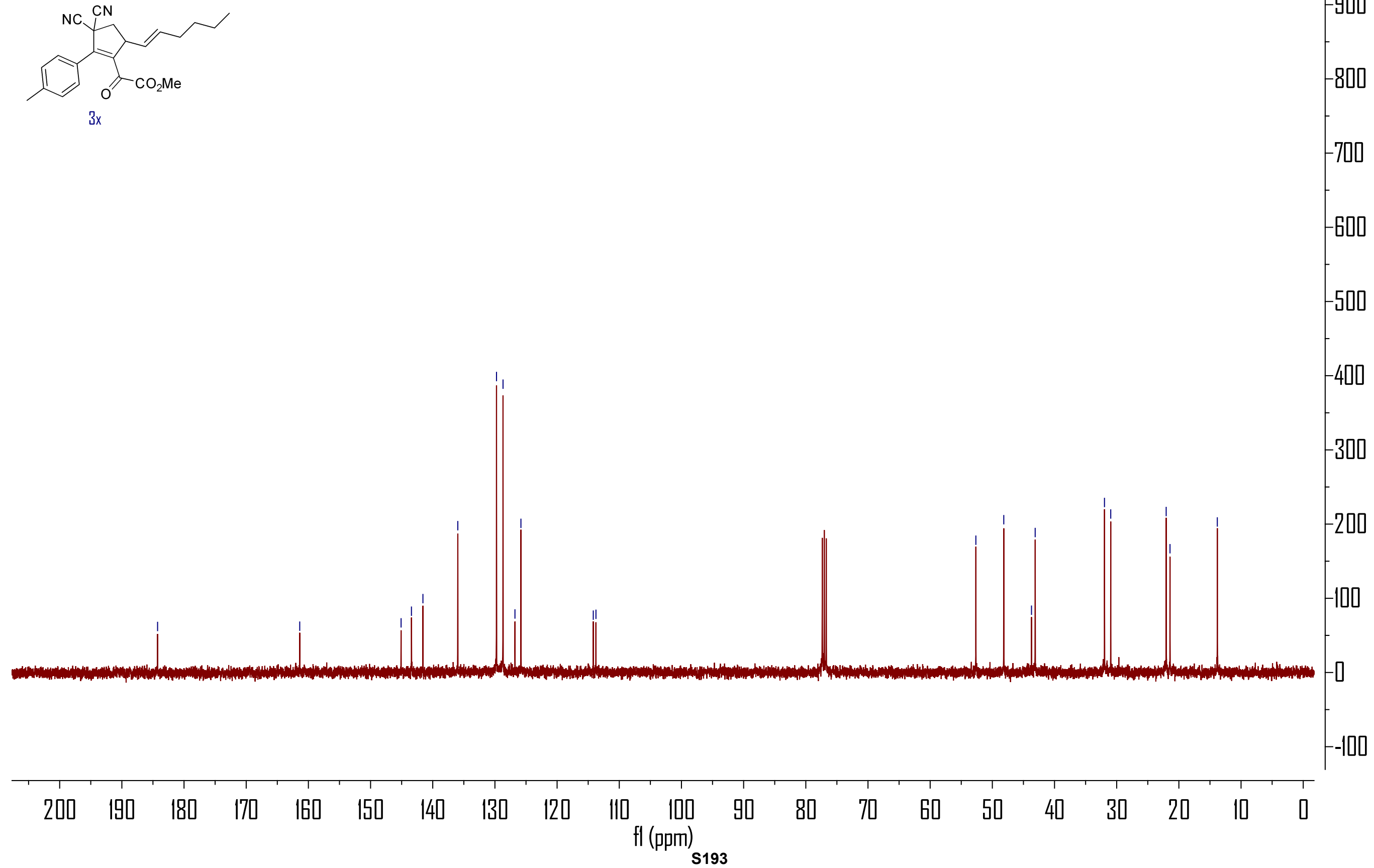


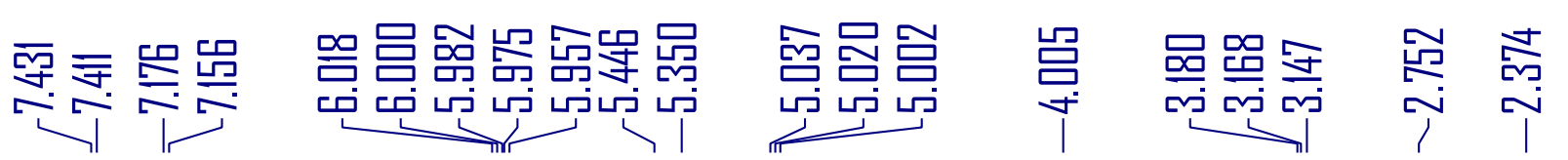
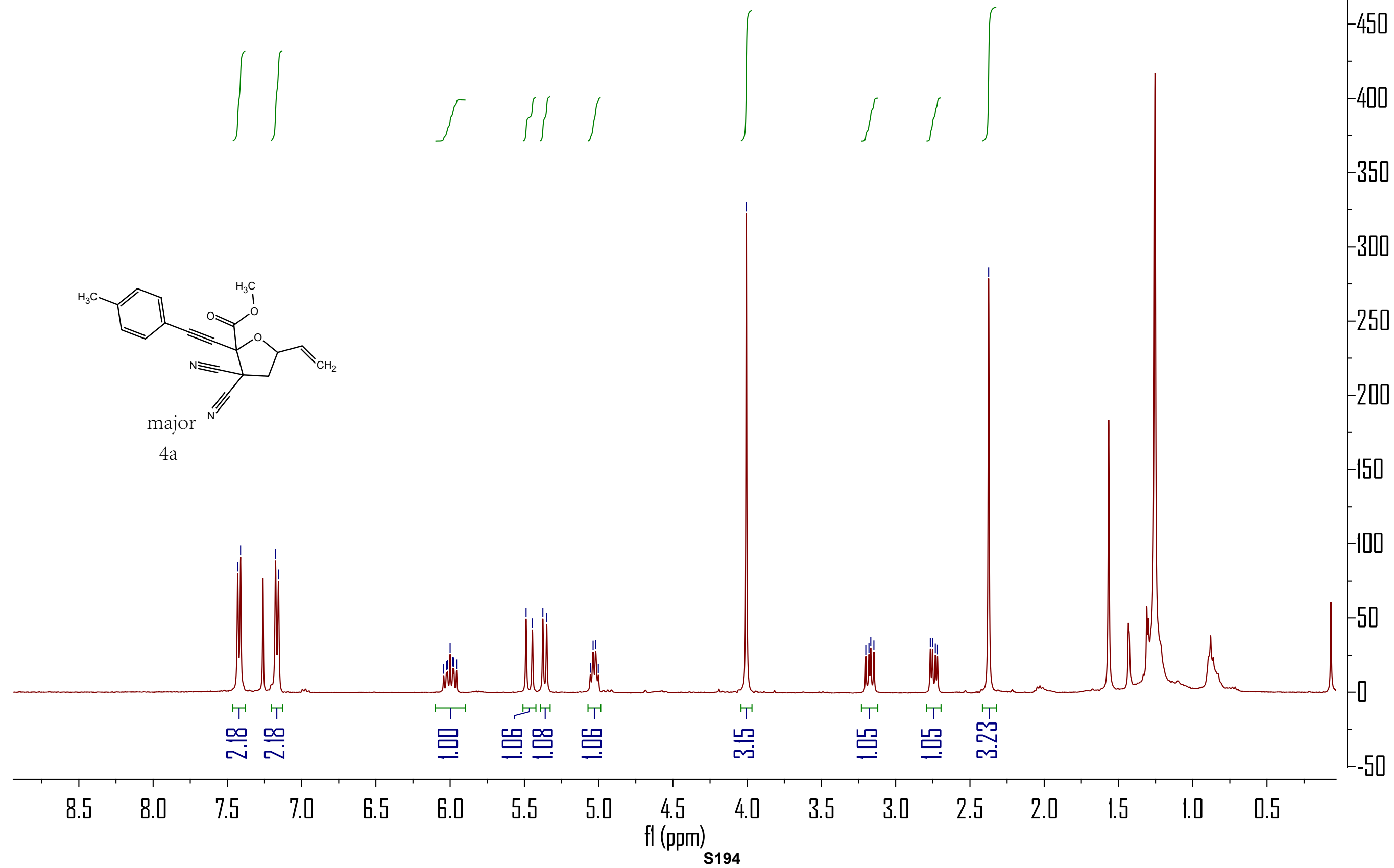


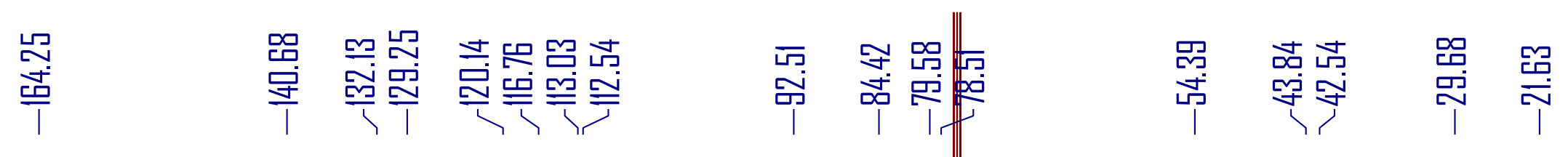

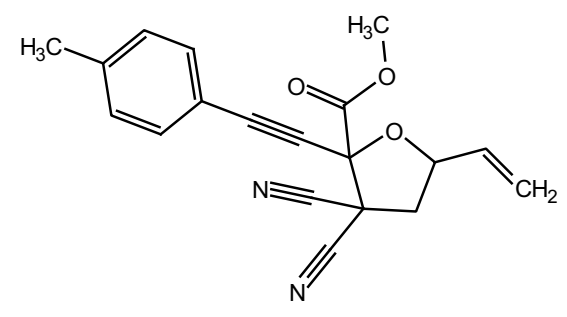

major

$4 a$

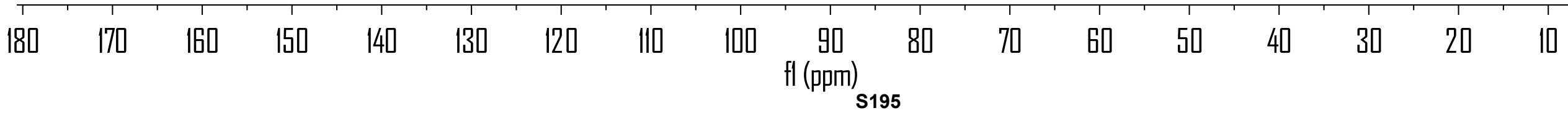




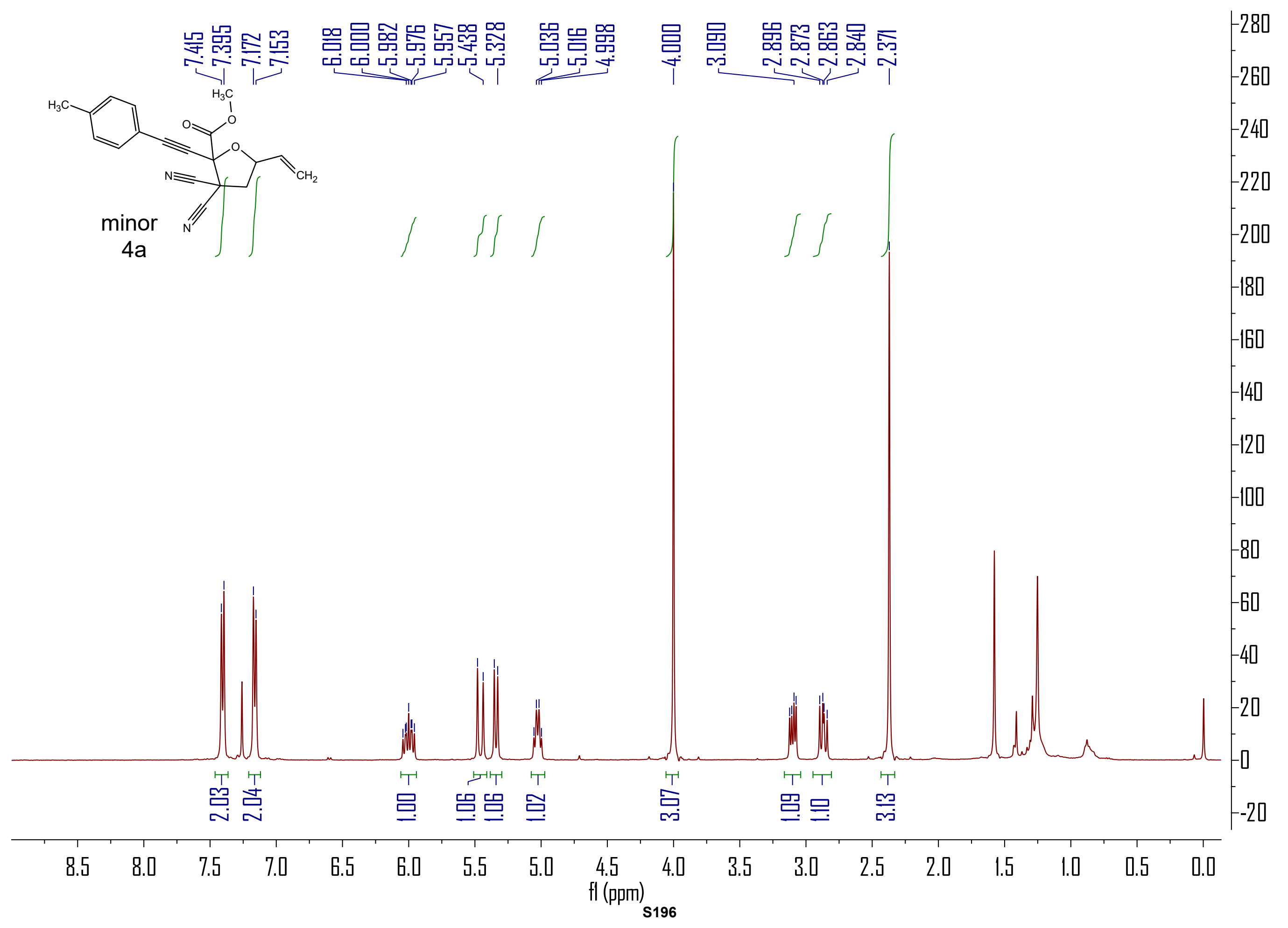




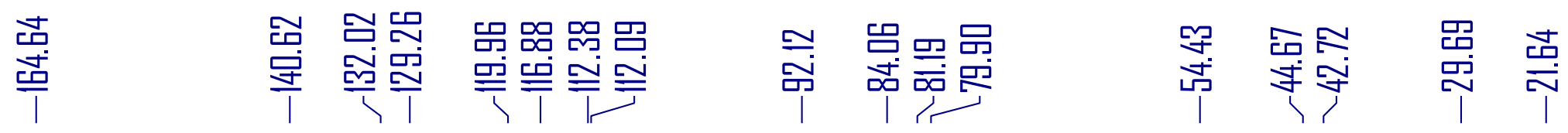
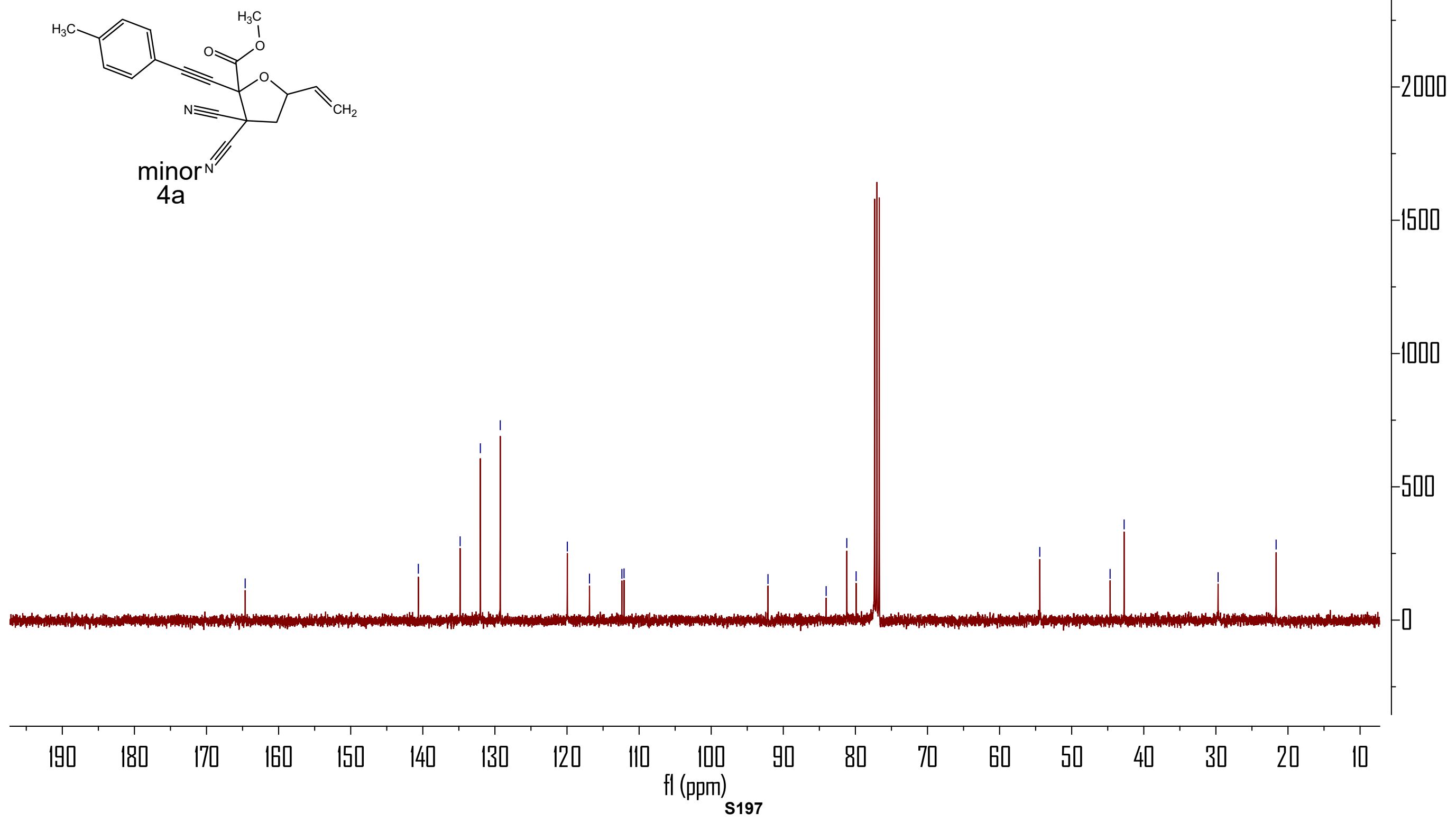


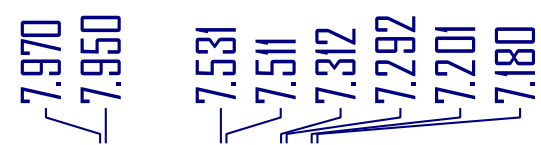
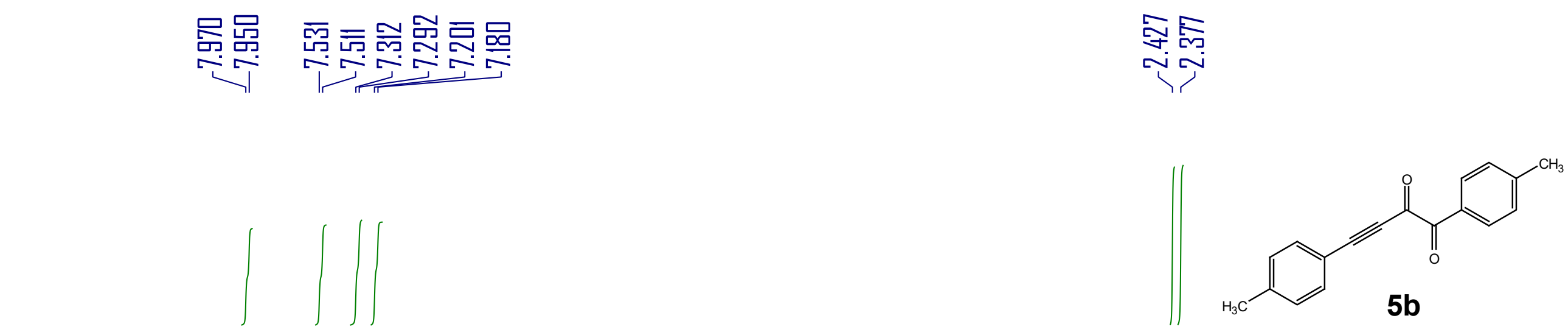

$-550$

$-500$

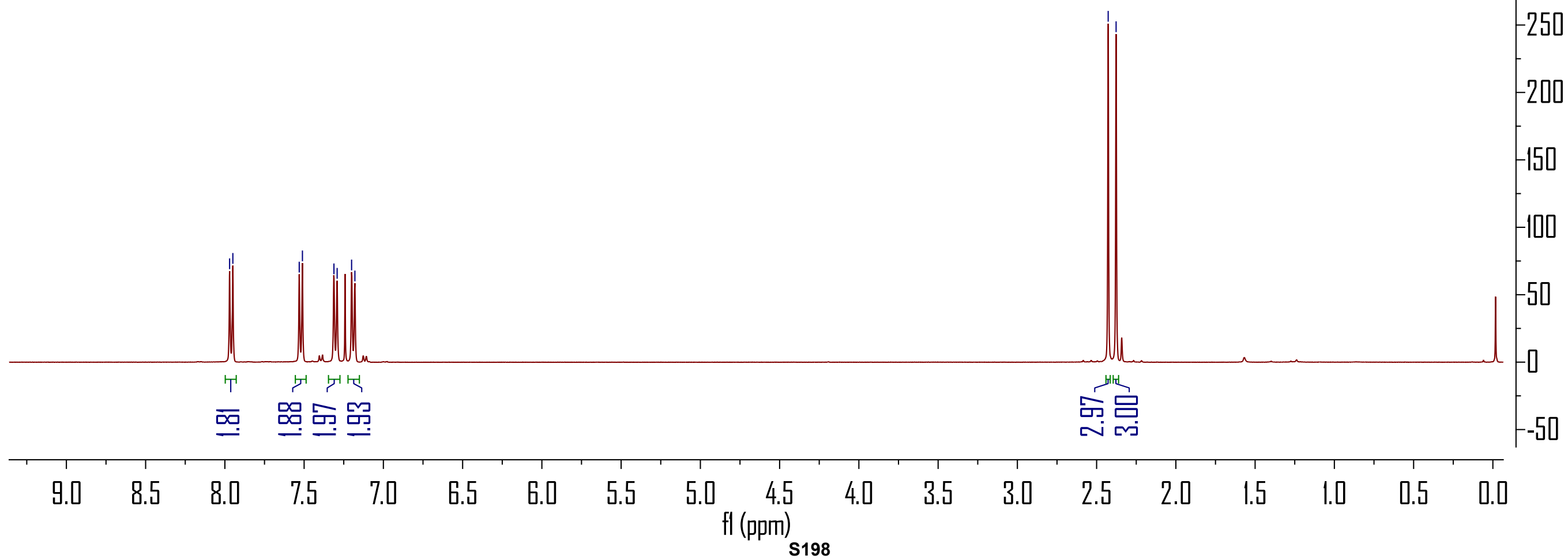




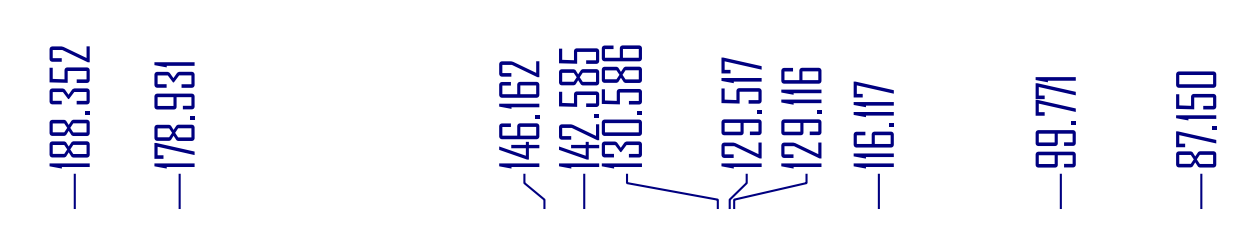$$
\text { 罂 }
$$

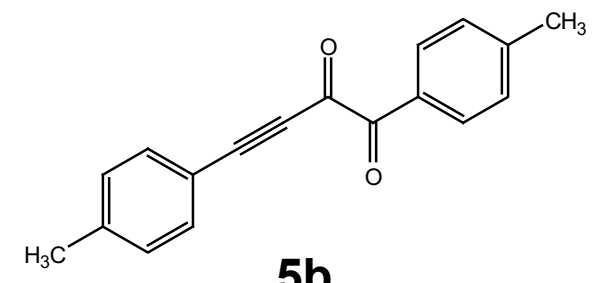

\section{$5 b$}




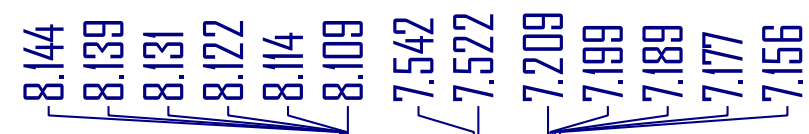

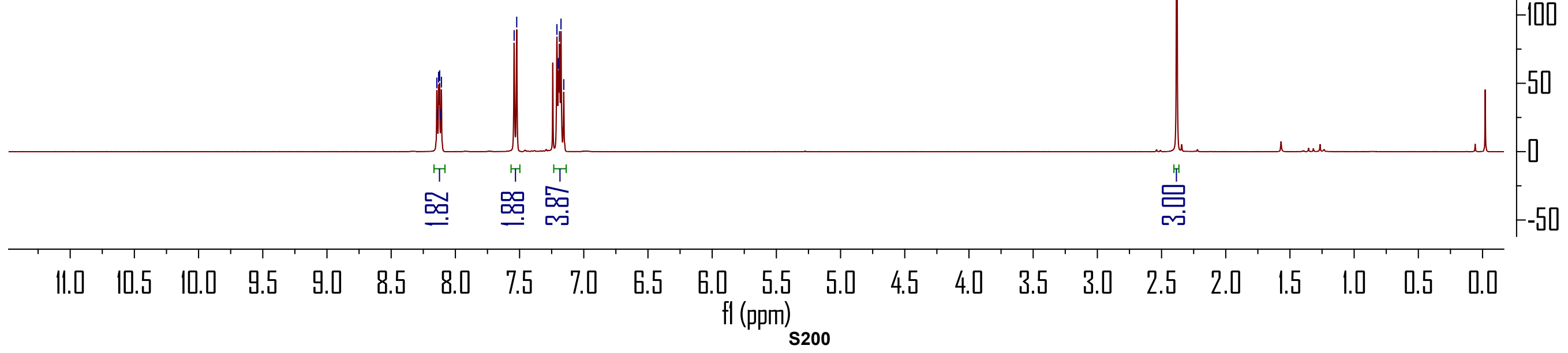




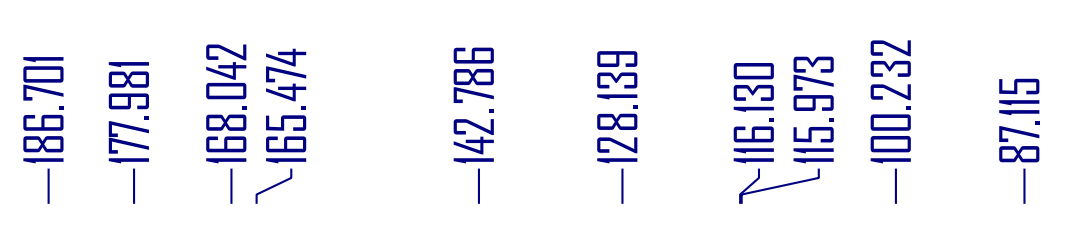

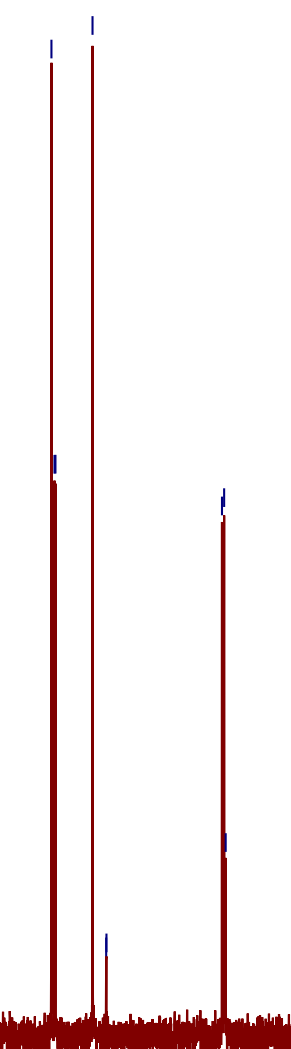




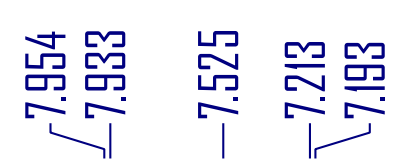

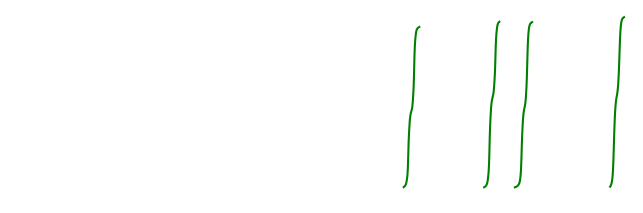

$$
\stackrel{\text { 鹗 }}{\uparrow}
$$

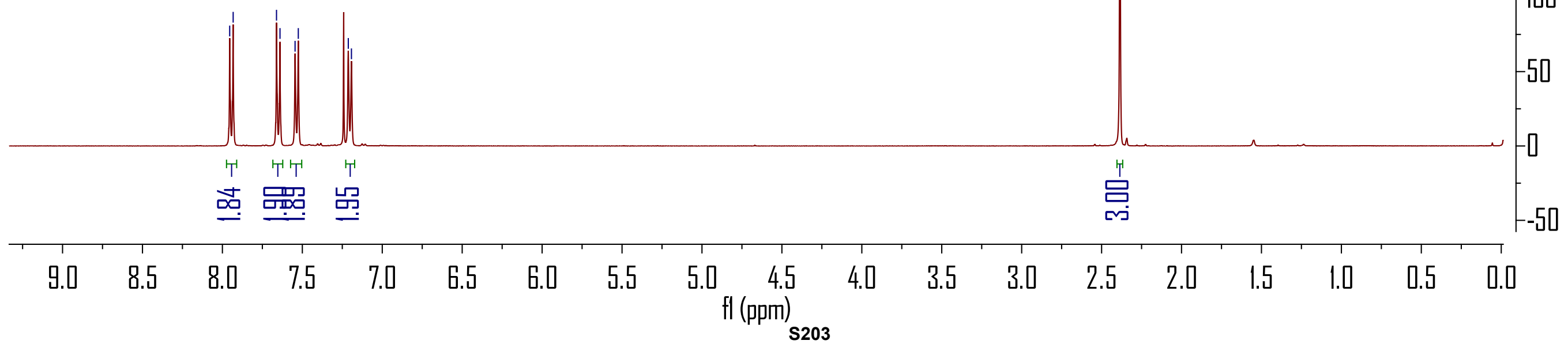




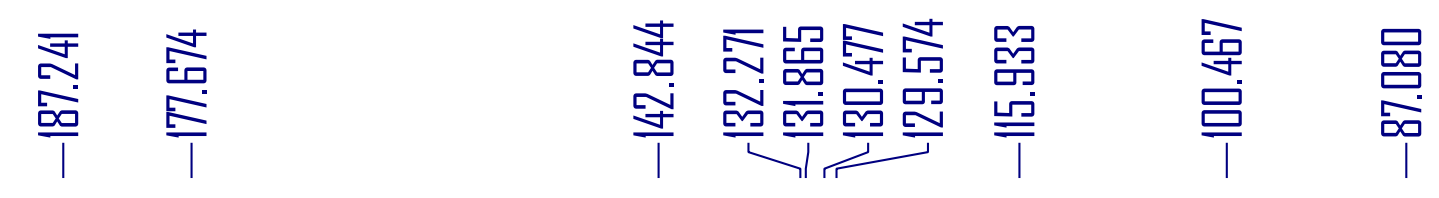

$\underset{1}{\bar{x}}$

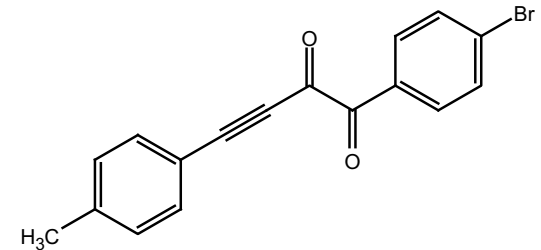

$5 d$

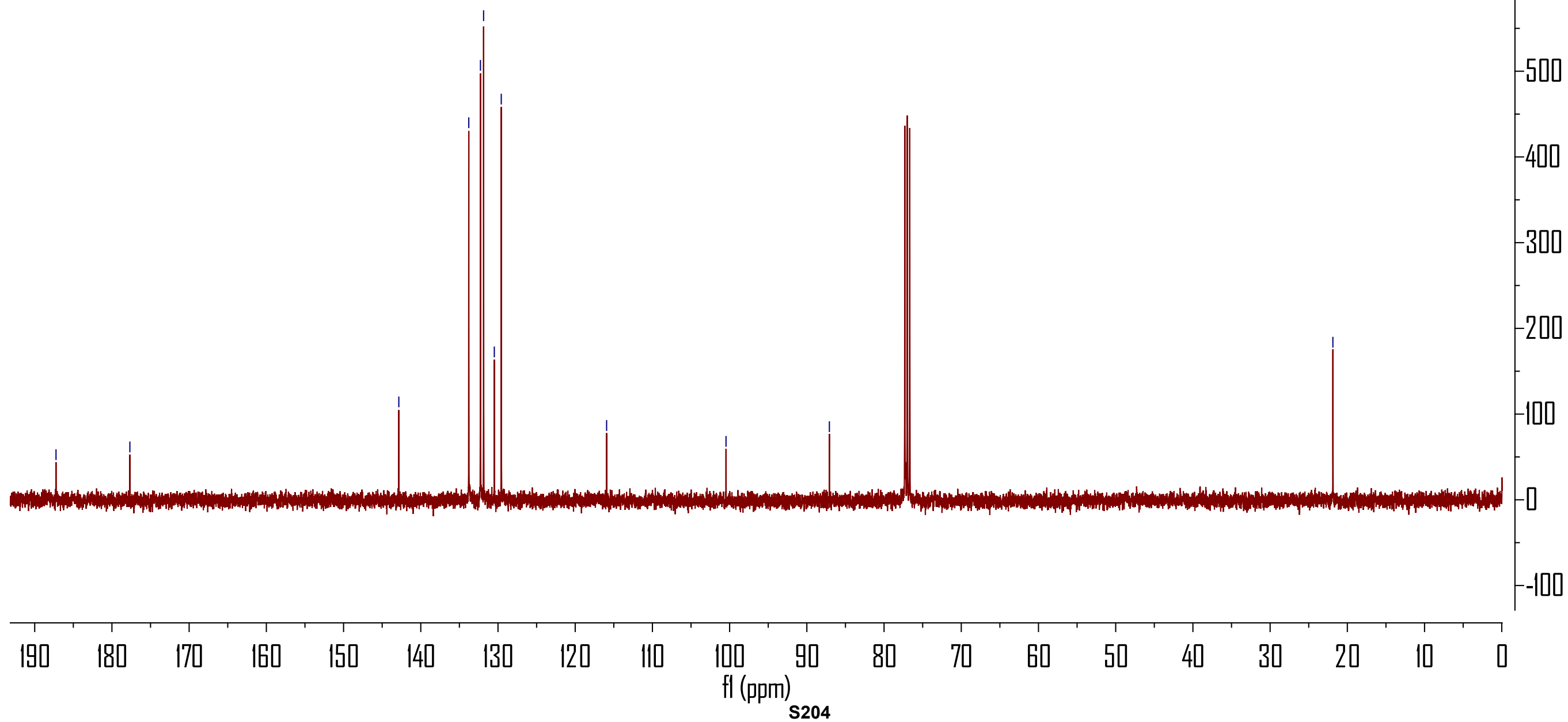




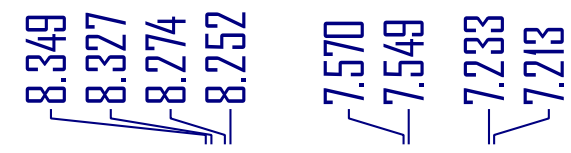

哿
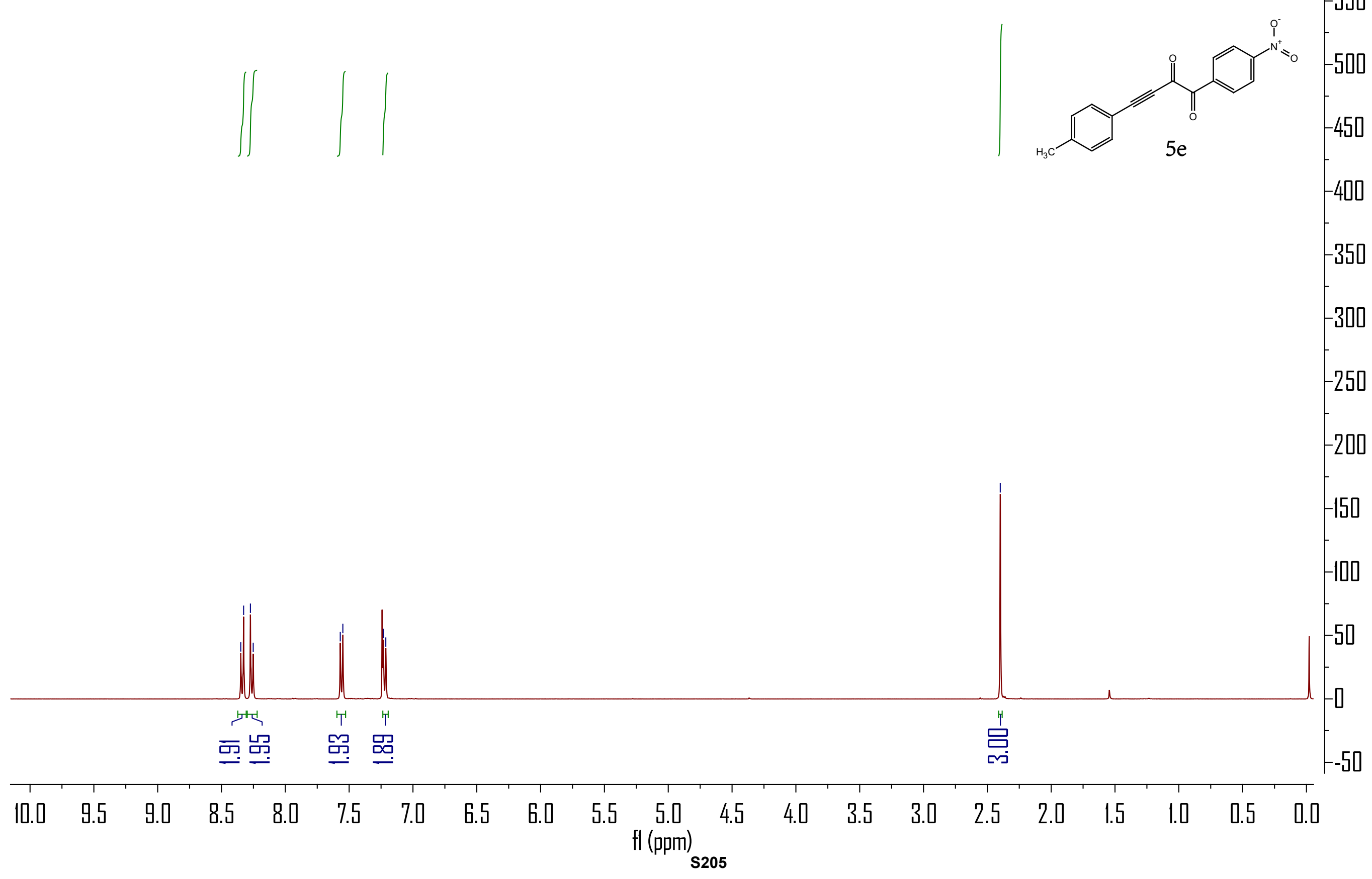


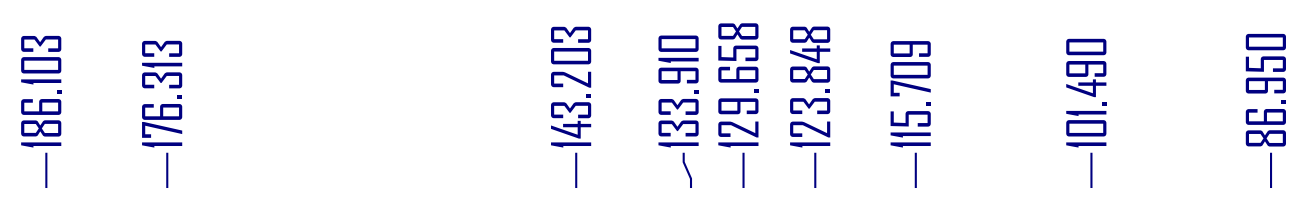

骂
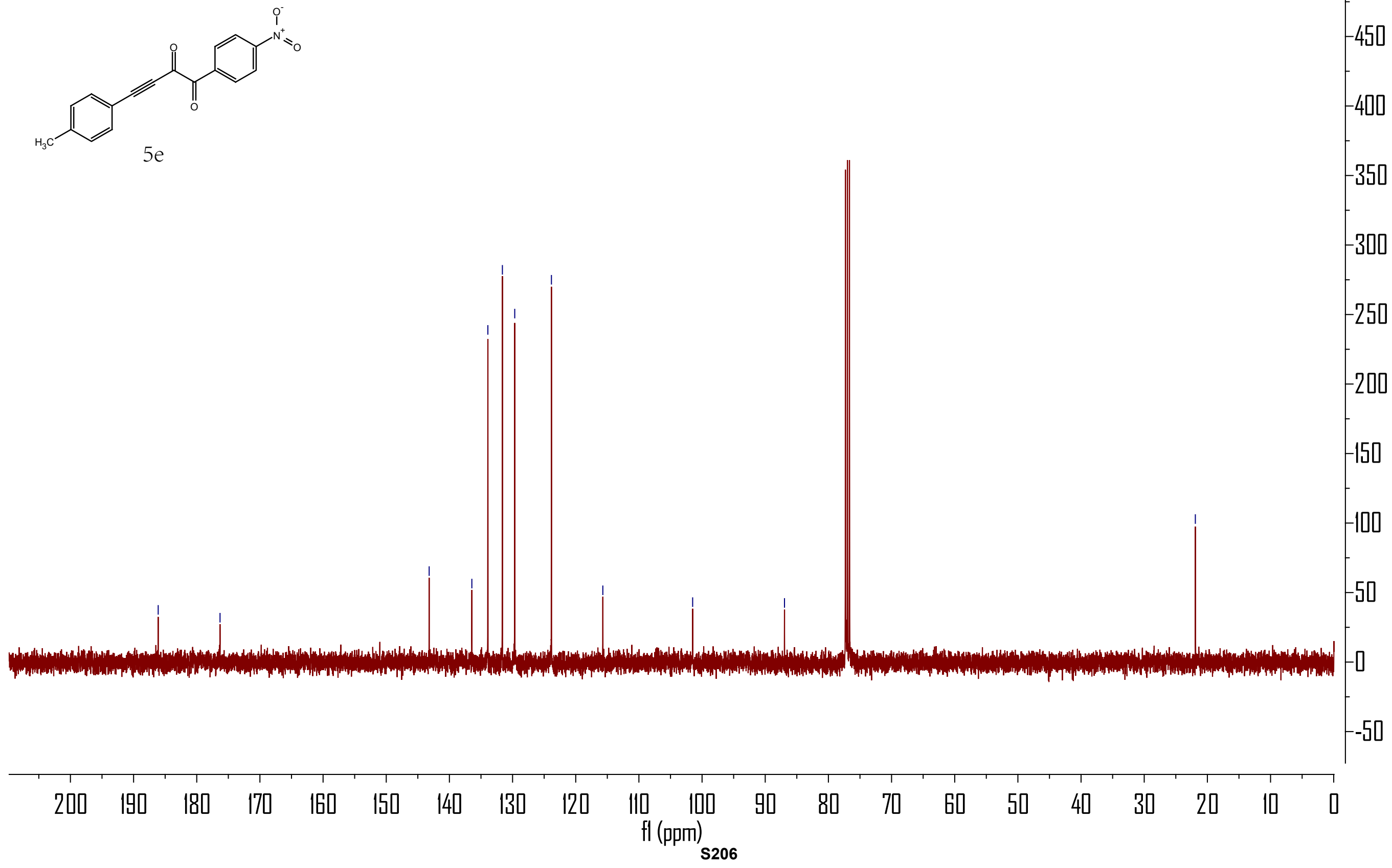


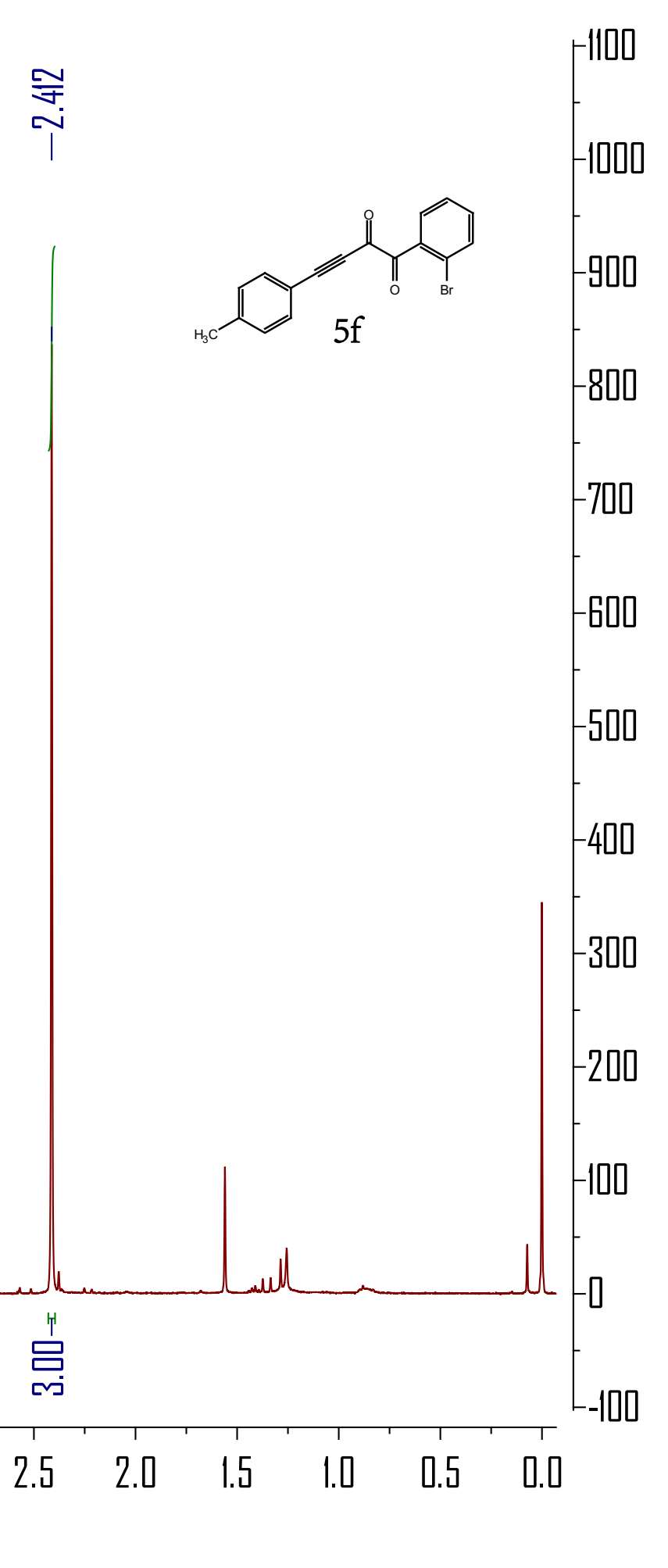




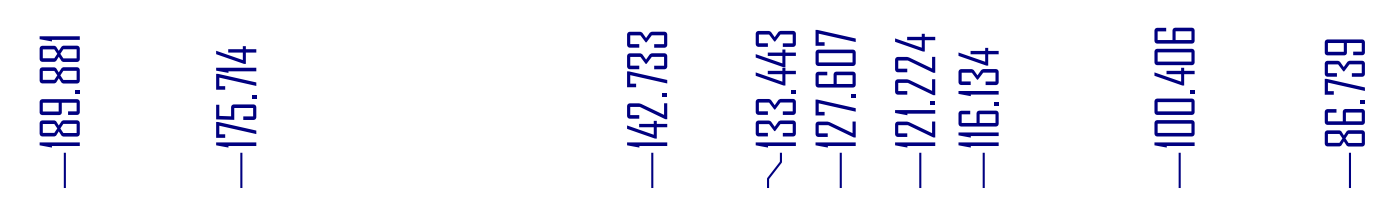

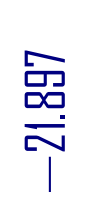

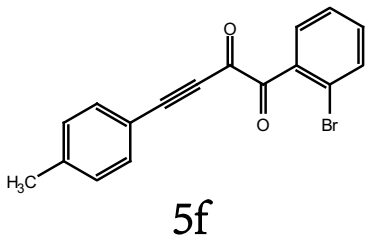


6a

\section{DWP-8-8+- IC 912140.7}

\begin{tabular}{|llll|}
\hline Sample Name: & DWP-8-8+- IC $\mathbf{9 1 2 1 4} \mathbf{0 . 7}$ & Injection Volume: & $\mathbf{5 . 0}$ \\
Vial Number: & GC3 & Channel: & UV_VIS_1 \\
Sample Type: & unknown & Wavelength: & $\mathbf{2 1 4}$ \\
Control Program: & $\mathbf{2 0 1 7 0 1 - 4}$ & Bandwidth: & n.a. \\
Quantif. Method: & $\mathbf{2 0 1 7 0 1}$ & Dilution Factor: & $\mathbf{1 . 0 0 0 0}$ \\
Recording Time: & $\mathbf{2 0 1 9 / 1 / 9 ~ 1 7 : 2 4}$ & Sample Weight: & $\mathbf{1 . 0 0 0 0}$ \\
Run Time (min): & $\mathbf{2 5 . 0 1}$ & Sample Amount: & $\mathbf{1 . 0 0 0 0}$ \\
\hline
\end{tabular}

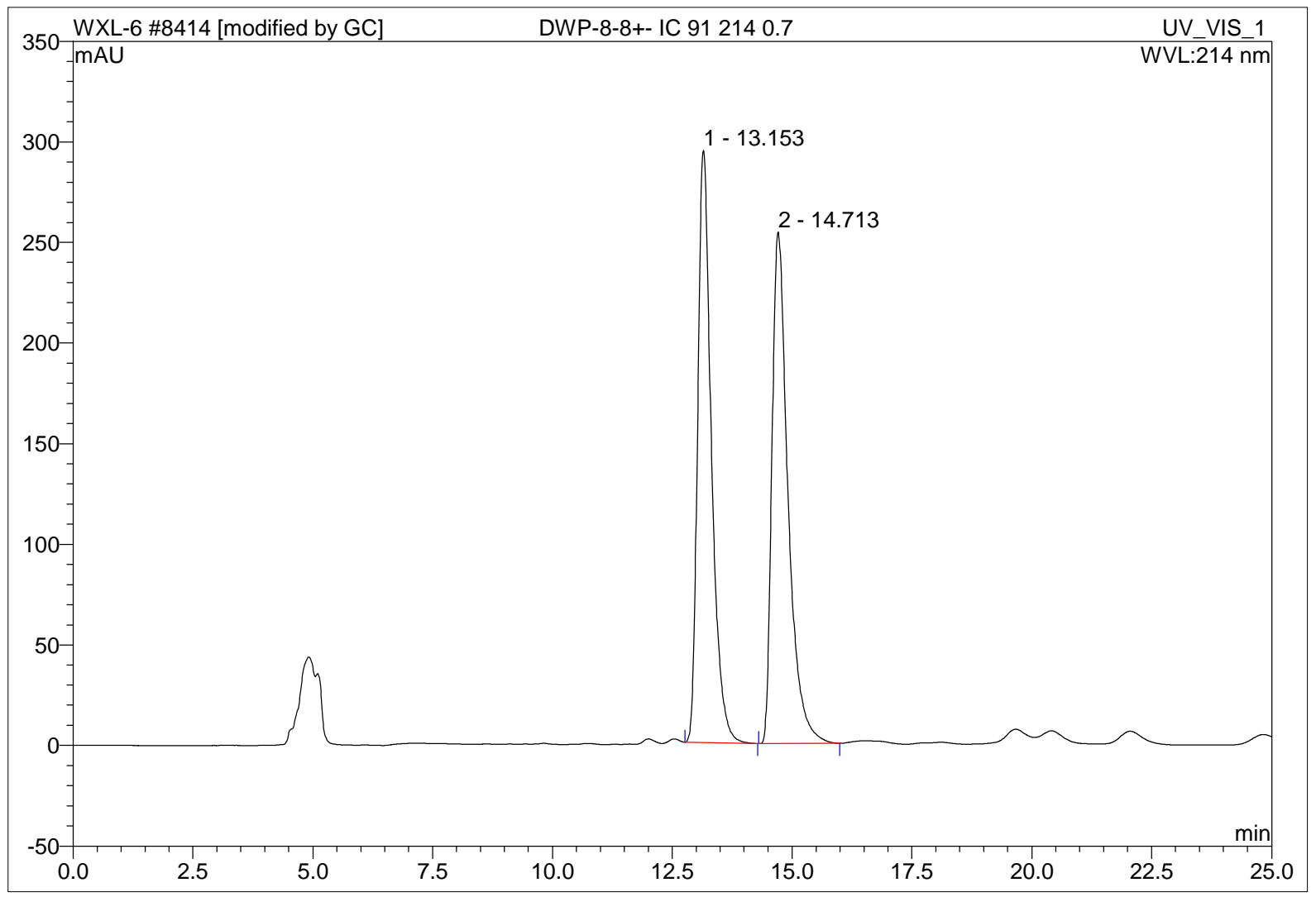

\begin{tabular}{|r|ccrrrrr|}
\hline No. & $\begin{array}{c}\text { Ret.Time } \\
\text { min }\end{array}$ & Peak Name & $\begin{array}{c}\text { Height } \\
\text { mAU }\end{array}$ & $\begin{array}{r}\text { Area } \\
\text { mAU*min }\end{array}$ & $\begin{array}{r}\text { Rel.Area } \\
\%\end{array}$ & Amount & Type \\
\hline 1 & 13.15 & n.a. & 294.467 & 97.807 & 49.71 & n.a. & BMB \\
2 & 14.71 & n.a. & 254.307 & 98.961 & 50.29 & n.a. & BMB $^{*}$ \\
\hline Total: & & & 548.774 & 196.767 & 100.00 & 0.000 & \\
\hline
\end{tabular}




\section{DWP-10-60 IC 912140.7}

\begin{tabular}{|llll|}
\hline Sample Name: & DWP-10-60 IC 912140.7 & Injection Volume: & $\mathbf{5 . 0}$ \\
Vial Number: & GD3 & Channel: & UV_VIS_1 \\
Sample Type: & unknown & Wavelength: & $\mathbf{2 1 4}$ \\
Control Program: & $\mathbf{2 0 1 7 0 1 - 4}$ & Bandwidth: & n.a. \\
Quantif. Method: & $\mathbf{2 0 1 7 0 1}$ & Dilution Factor: & $\mathbf{1 . 0 0 0 0}$ \\
Recording Time: & $\mathbf{2 0 1 9 / 1 / 9 1 7 : 5 1}$ & Sample Weight: & $\mathbf{1 . 0 0 0 0}$ \\
Run Time (min): & $\mathbf{2 5 . 0 0}$ & Sample Amount: & $\mathbf{1 . 0 0 0 0}$ \\
\hline
\end{tabular}

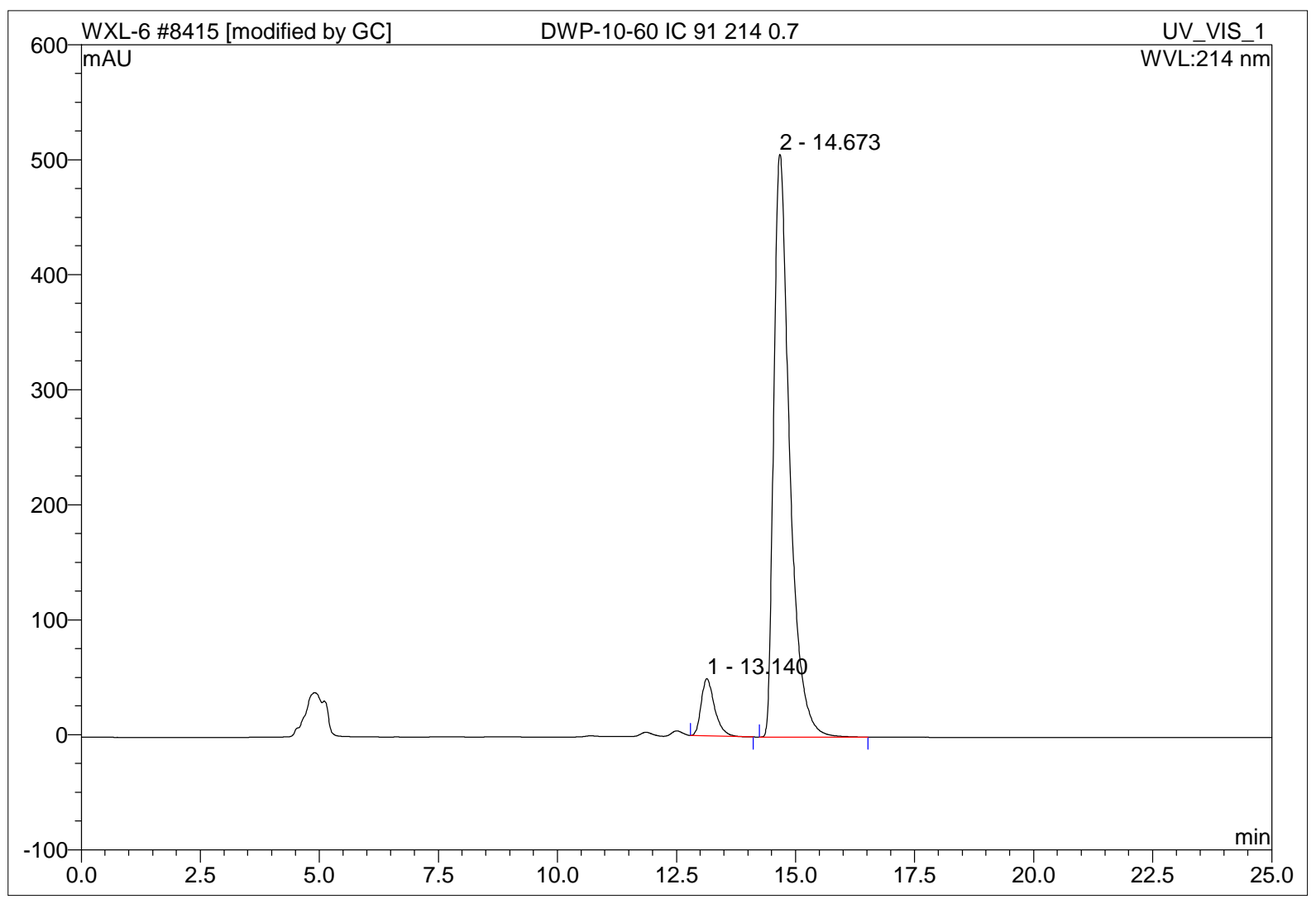

\begin{tabular}{|r|ccrrrrr|}
\hline No. & $\begin{array}{c}\text { Ret.Time } \\
\text { min }\end{array}$ & Peak Name & $\begin{array}{c}\text { Height } \\
\text { mAU }\end{array}$ & $\begin{array}{r}\text { Area } \\
\text { mAU*min }\end{array}$ & $\begin{array}{r}\text { Rel.Area } \\
\%\end{array}$ & Amount & Type \\
\hline 1 & 13.14 & n.a. & 50.103 & 16.308 & 7.67 & n.a. & BMB $^{*}$ \\
2 & 14.67 & n.a. & 506.659 & 196.439 & 92.33 & n.a. & BMB \\
\hline Total: & & & 556.762 & 212.746 & 100.00 & 0.000 & \\
\hline
\end{tabular}




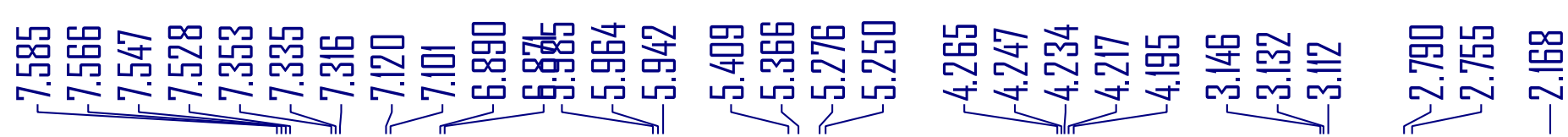
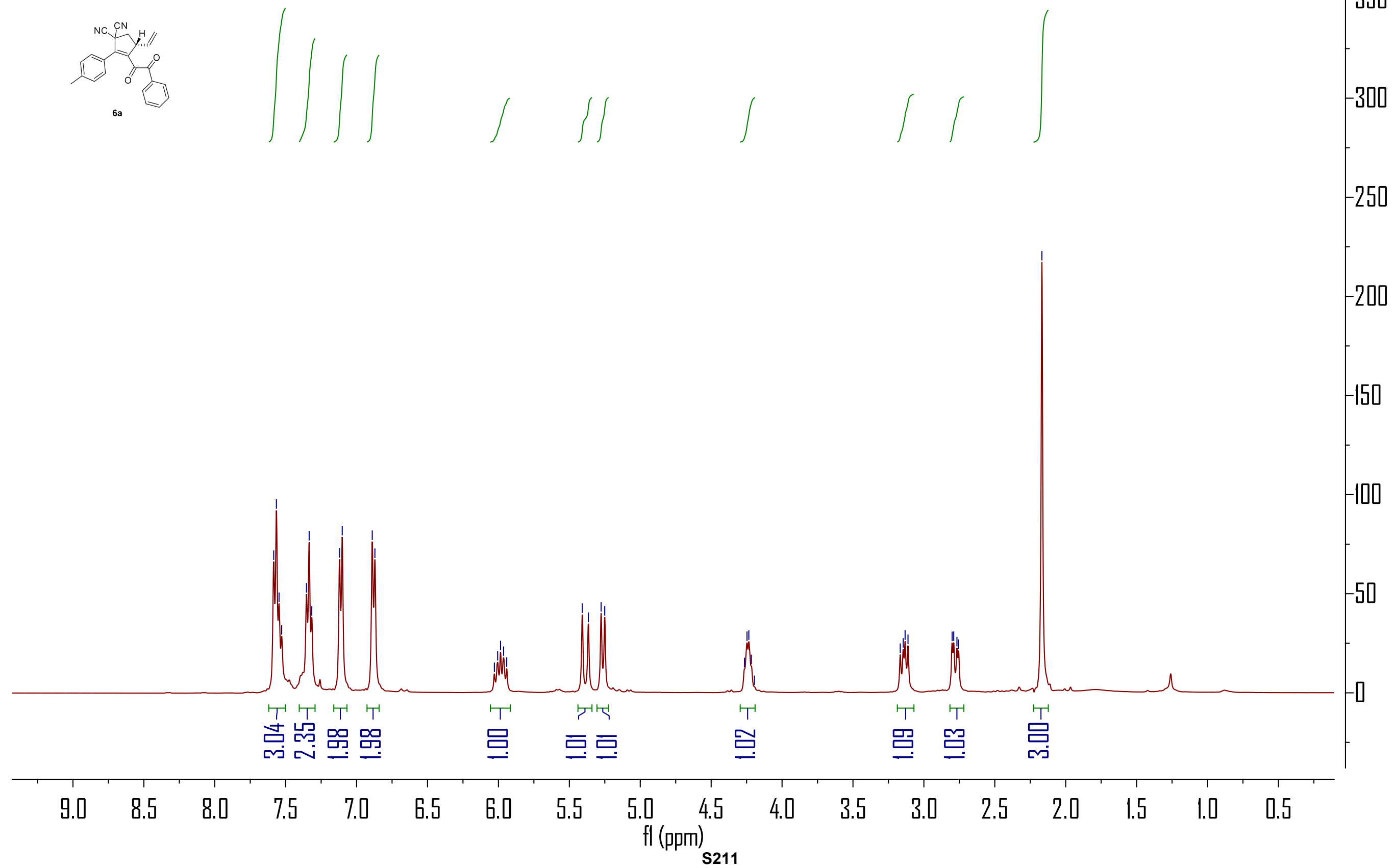


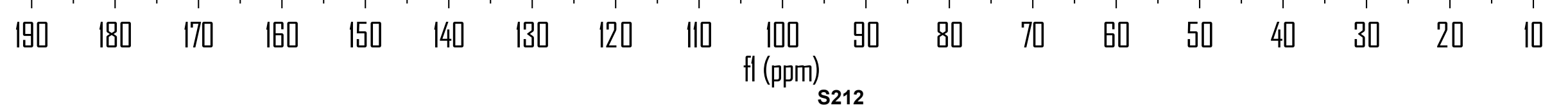


$6 b$

\section{DWP-9-99+- IB 9912140.7}

\begin{tabular}{|llll|}
\hline Sample Name: & DWP-9-99+- IB 9912140.7 & Injection Volume: & $\mathbf{5 . 0}$ \\
Vial Number: & RA2 & Channel: & UV_VIS_2 \\
Sample Type: & unknown & Wavelength: & $\mathbf{2 1 4 . 0}$ \\
Control Program: & test-dad4 & Bandwidth: & $\mathbf{4}$ \\
Quantif. Method: & $\mathbf{2 0 1 7 0 6 0 8}$ & Dilution Factor: & $\mathbf{1 . 0 0 0 0}$ \\
Recording Time: & $\mathbf{2 0 1 9 - 1 - 1 0 ~ 1 7 : 0 7}$ & Sample Weight: & $\mathbf{1 . 0 0 0 0}$ \\
Run Time (min): & $\mathbf{4 6 . 3 5}$ & Sample Amount: & $\mathbf{1 . 0 0 0 0}$ \\
\hline
\end{tabular}

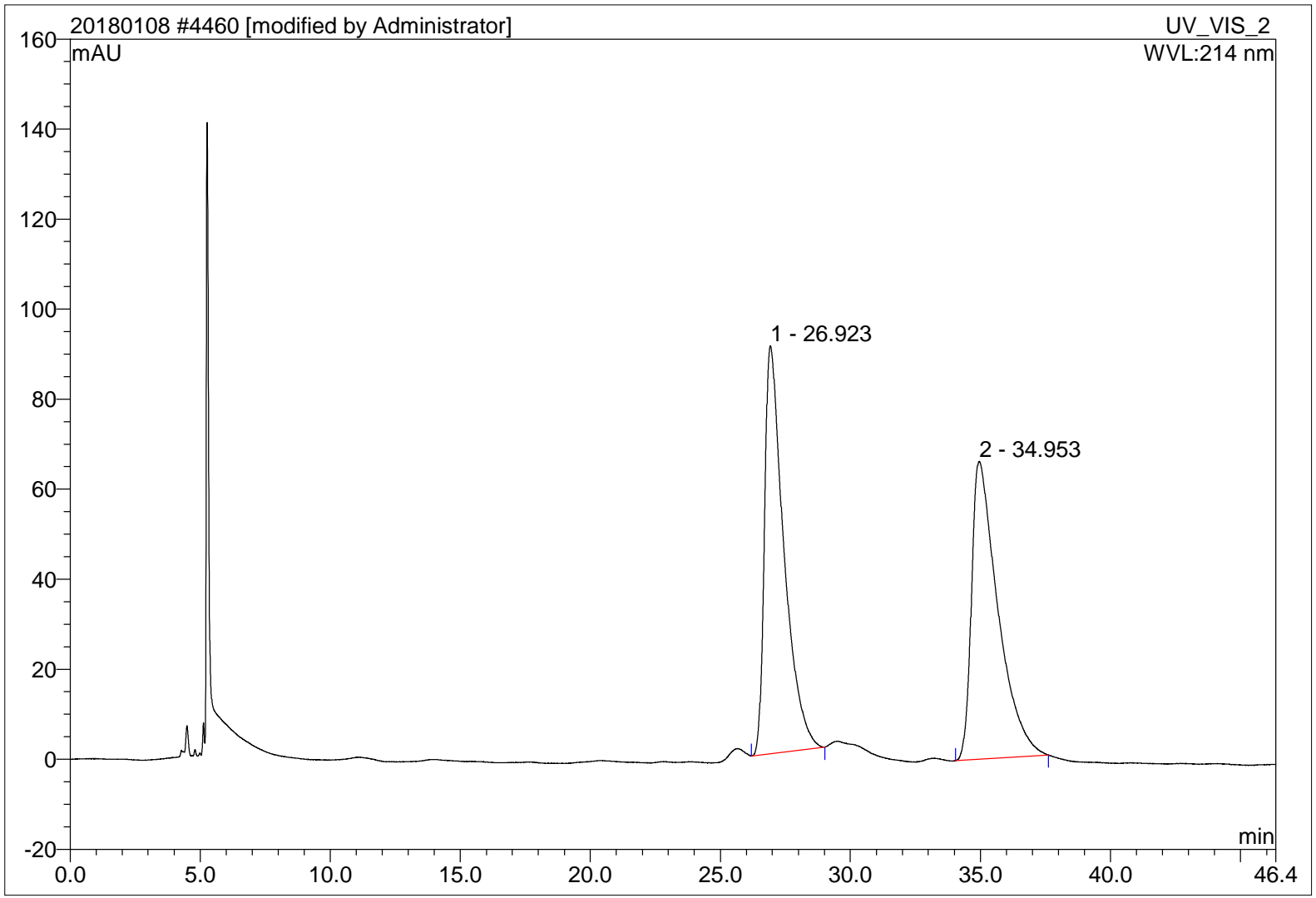

\begin{tabular}{|r|ccrrrrr|}
\hline No. & $\begin{array}{c}\text { Ret.Time } \\
\text { min }\end{array}$ & Peak Name & $\begin{array}{c}\text { Height } \\
\text { mAU }\end{array}$ & $\begin{array}{c}\text { Area } \\
\text { mAU*min }\end{array}$ & $\begin{array}{r}\text { Rel.Area } \\
\%\end{array}$ & Amount & Type \\
\hline 1 & 26.92 & n.a. & 90.669 & 78.492 & 50.24 & n.a. & BMB $^{*}$ \\
2 & 34.95 & n.a. & 66.197 & 77.756 & 49.76 & n.a. & BMB \\
\hline Total: & & & 156.866 & 156.248 & 100.00 & 0.000 & \\
\hline
\end{tabular}




$\begin{array}{ll} & \\ \text { Injection Volume: } & \mathbf{5 . 0} \\ \text { Channel: } & \mathbf{U V} \mathbf{V I S \_ 2} \\ \text { Wavelength: } & \mathbf{2 1 4 . 0} \\ \text { Bandwidth: } & \mathbf{4} \\ \text { Dilution Factor: } & \mathbf{1 . 0 0 0 0} \\ \text { Sample Weight: } & \mathbf{1 . 0 0 0 0} \\ \text { Sample Amount: } & \mathbf{1 . 0 0 0 0}\end{array}$

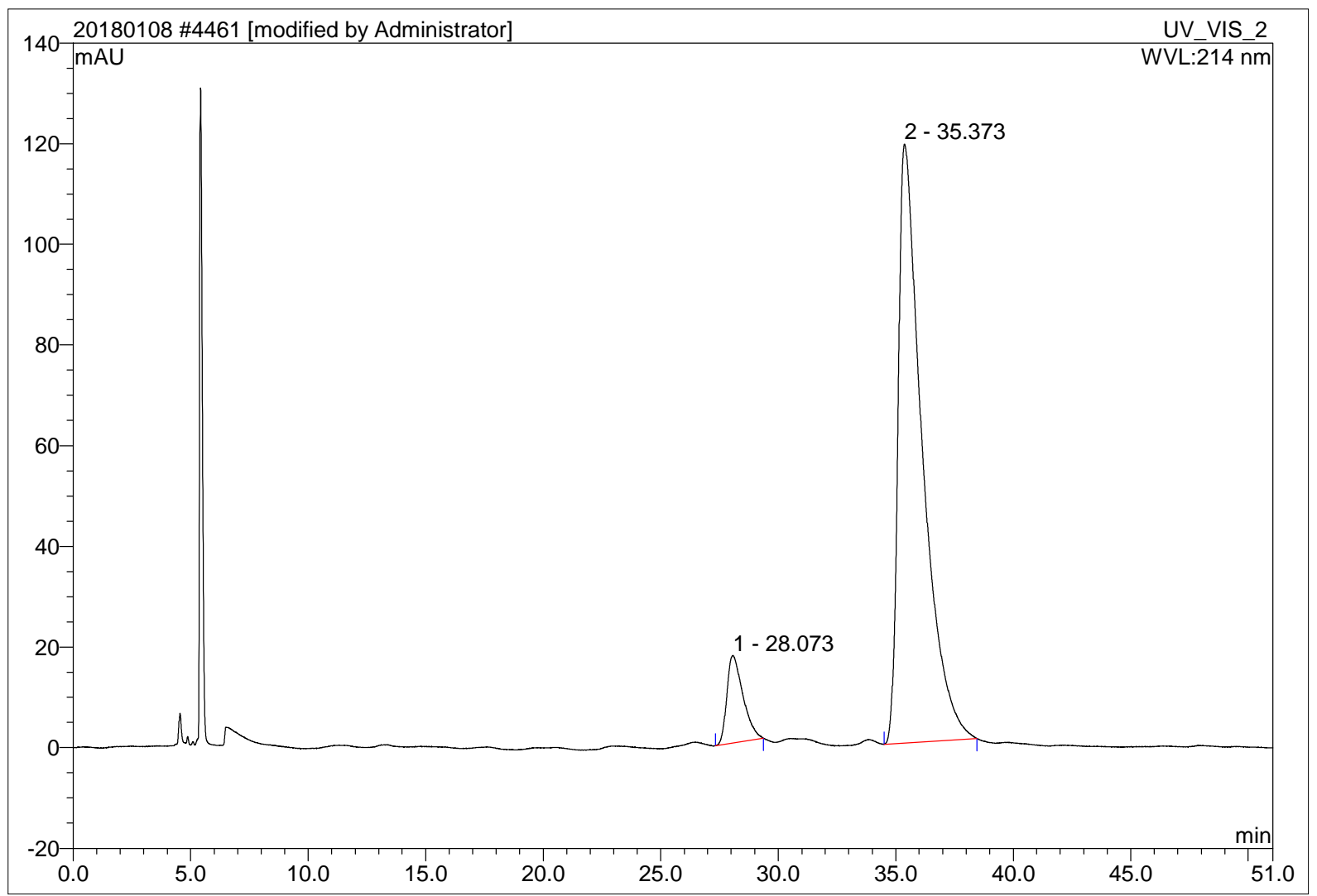

\begin{tabular}{|r|ccrrrrr|}
\hline No. & $\begin{array}{c}\text { Ret.Time } \\
\text { min }\end{array}$ & Peak Name & $\begin{array}{c}\text { Height } \\
\text { mAU }\end{array}$ & $\begin{array}{r}\text { Area } \\
\text { mAU*min }^{*}\end{array}$ & $\begin{array}{r}\text { Rel.Area } \\
\%\end{array}$ & Amount & Type \\
\hline 1 & 28.07 & n.a. & 17.466 & 13.844 & 8.89 & n.a. & BMB \\
2 & 35.37 & n.a. & 119.036 & 141.842 & 91.11 & n.a. & BMB \\
\hline Total: & & & 136.503 & 155.687 & 100.00 & 0.000 & \\
\hline
\end{tabular}




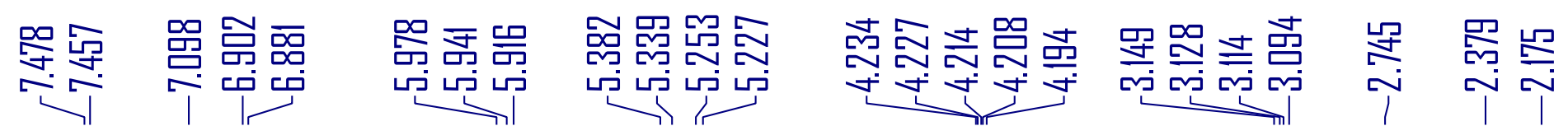
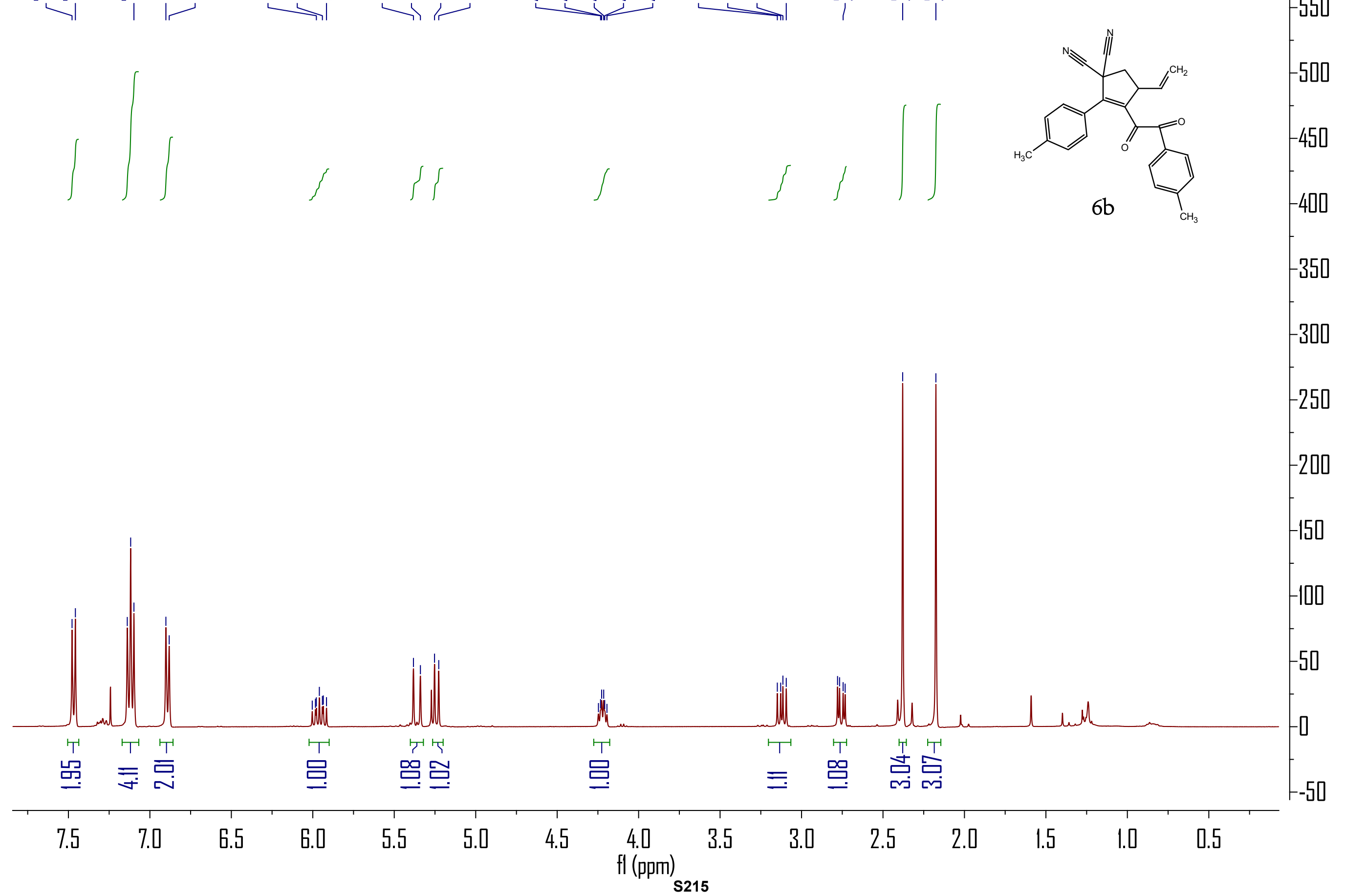


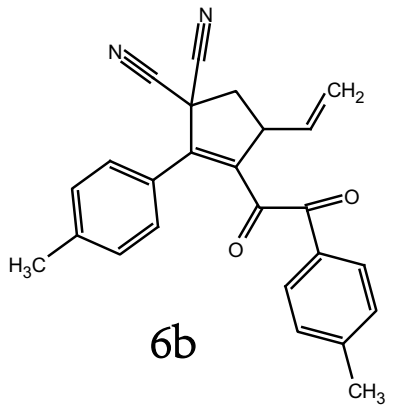




\begin{tabular}{|llll|}
\hline 4469 DWP-9-87+- IB 912140.7 & & \\
& & & \\
\hline Sample Name: & DWP-9-87+- IB 912140.7 & Injection Volume: & $\mathbf{5 . 0}$ \\
Vial Number: & GD7 & Channel: & UV_VIS_2 \\
Sample Type: & unknown & Wavelength: & $\mathbf{2 1 4 . 0}$ \\
Control Program: & test-dad6 & Bandwidth: & $\mathbf{4}$ \\
Quantif. Method: & $\mathbf{2 0 1 7 0 6 0 8}$ & Dilution Factor: & $\mathbf{1 . 0 0 0 0}$ \\
Recording Time: & $\mathbf{2 0 1 9 - 1 - 1 0 ~ 1 9 : 3 6}$ & Sample Weight: & $\mathbf{1 . 0 0 0 0}$ \\
Run Time (min): & $\mathbf{1 5 . 1 1}$ & Sample Amount: & $\mathbf{1 . 0 0 0 0}$ \\
\hline
\end{tabular}

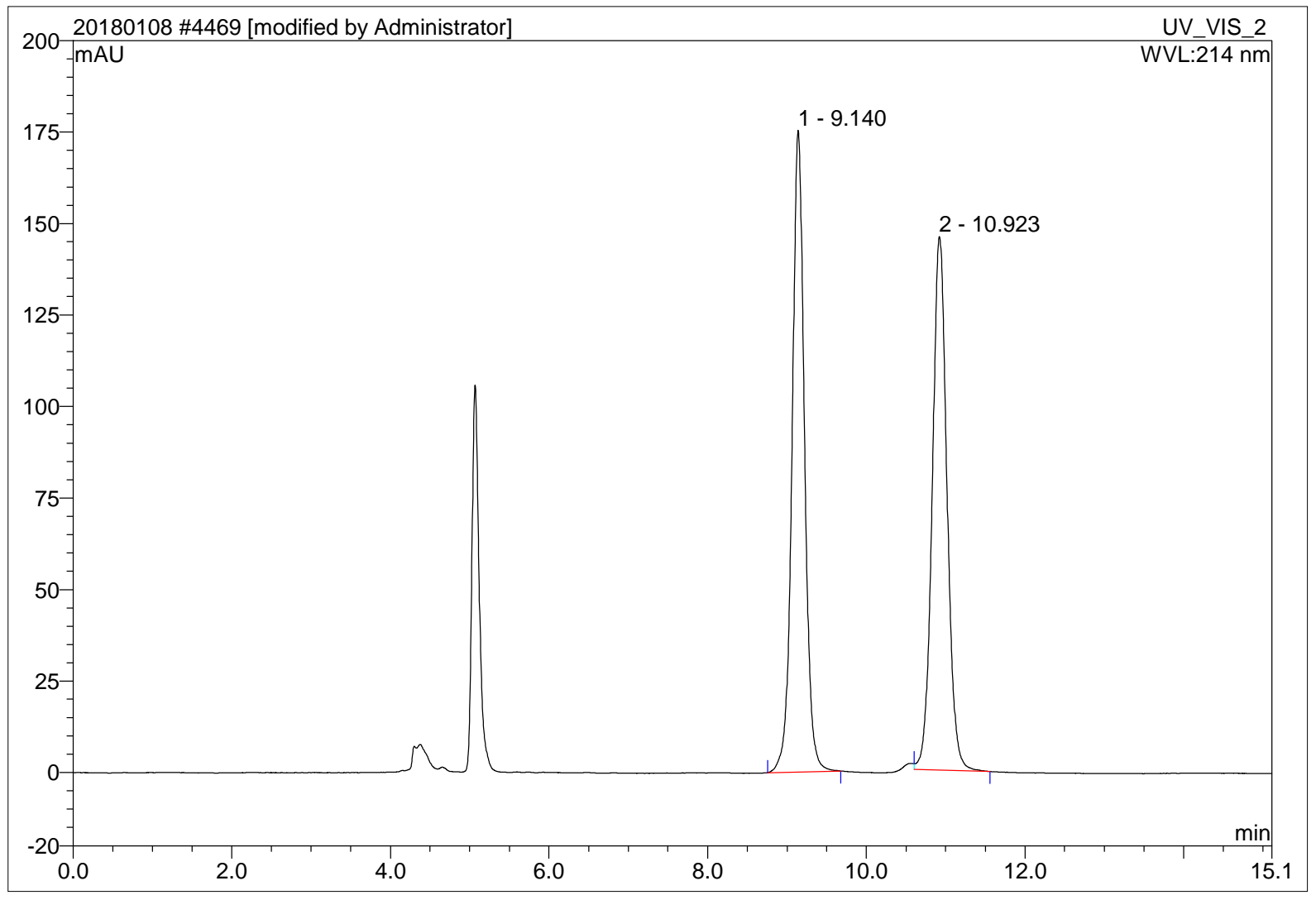

\begin{tabular}{|r|rrrrrrr|}
\hline No. & $\begin{array}{c}\text { Ret.Time } \\
\text { min }\end{array}$ & Peak Name & $\begin{array}{c}\text { Height } \\
\text { AAU }\end{array}$ & $\begin{array}{c}\text { Area } \\
\text { mAU*min }\end{array}$ & $\begin{array}{c}\text { Rel.Area } \\
\%\end{array}$ & Amount & Type \\
\hline 1 & 9.14 & n.a. & 175.350 & 31.925 & 50.43 & n.a. & BMB \\
2 & 10.92 & n.a. & 145.842 & 31.387 & 49.57 & n.a. & MB $^{*}$ \\
\hline Total: & & & 321.192 & 63.312 & 100.00 & 0.000 & \\
\hline
\end{tabular}




\section{DWP-10-61 IB 912140.7}

\begin{tabular}{|llll|}
\hline Sample Name: & DWP-10-61 IB 91 214 0.7 & Injection Volume: & $\mathbf{5 . 0}$ \\
Vial Number: & GE7 & Channel: & UV_VIS_2 \\
Sample Type: & unknown & Wavelength: & $\mathbf{2 1 4 . 0}$ \\
Control Program: & test-dad6 & Bandwidth: & $\mathbf{4}$ \\
Quantif. Method: & $\mathbf{2 0 1 7 0 6 0 8}$ & Dilution Factor: & $\mathbf{1 . 0 0 0 0}$ \\
Recording Time: & $\mathbf{2 0 1 9 - 1 - 1 0 ~ 1 9 : 5 3}$ & Sample Weight: & $\mathbf{1 . 0 0 0 0}$ \\
Run Time (min): & $\mathbf{2 9 . 7 7}$ & Sample Amount: & $\mathbf{1 . 0 0 0 0}$ \\
\hline
\end{tabular}

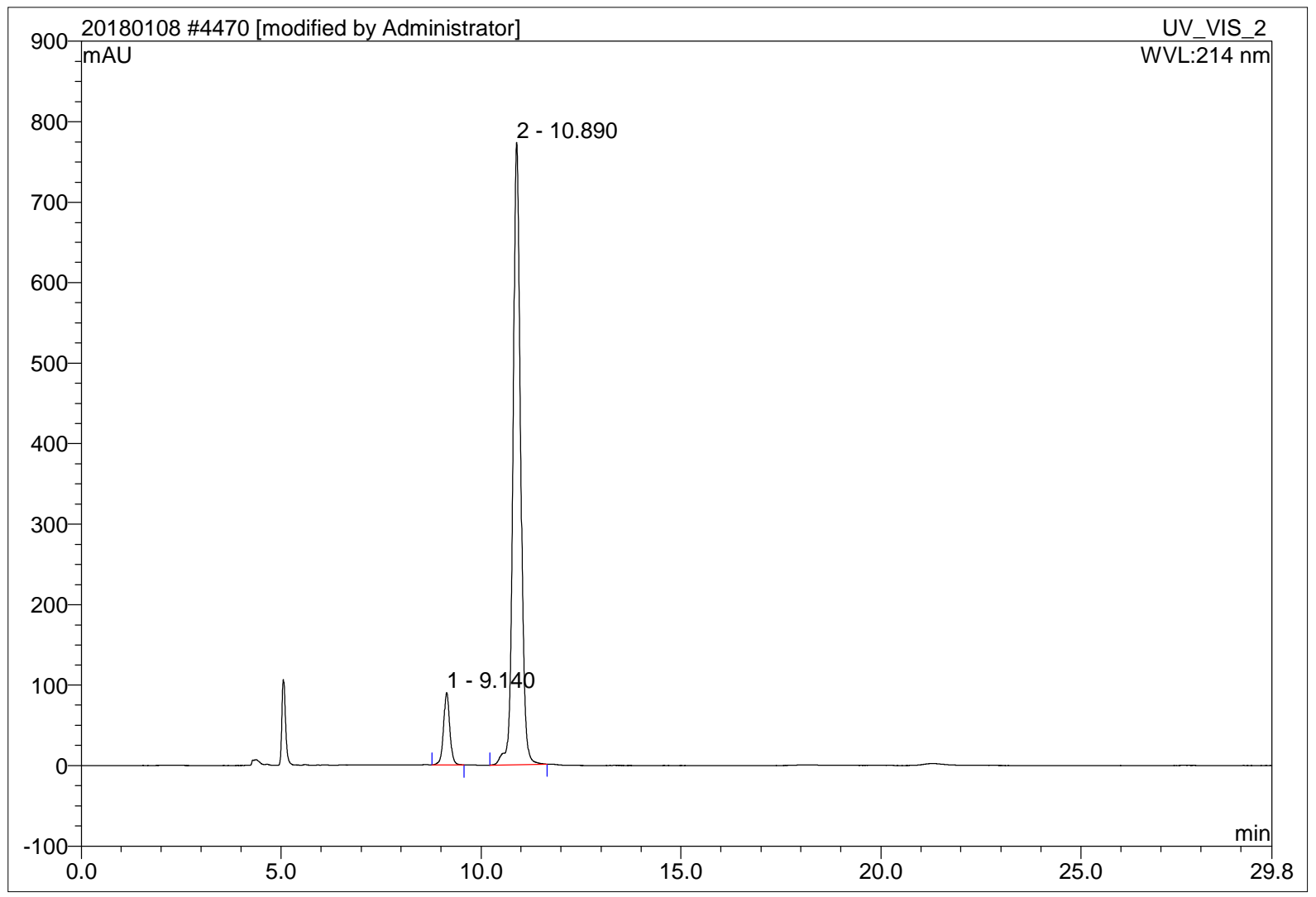

\begin{tabular}{|r|ccrrrrr|}
\hline No. & $\begin{array}{c}\text { Ret.Time } \\
\text { min }\end{array}$ & Peak Name & $\begin{array}{c}\text { Height } \\
\text { mAU }\end{array}$ & $\begin{array}{c}\text { Area } \\
\text { mAU*min }\end{array}$ & $\begin{array}{r}\text { Rel.Area } \\
\%\end{array}$ & Amount & Type \\
\hline 1 & 9.14 & n.a. & 89.946 & 16.353 & 8.68 & n.a. & BMB \\
2 & 10.89 & n.a. & 773.374 & 171.989 & 91.32 & n.a. & BMB \\
\hline Total: & & & 863.320 & 188.342 & 100.00 & 0.000 & \\
\hline
\end{tabular}




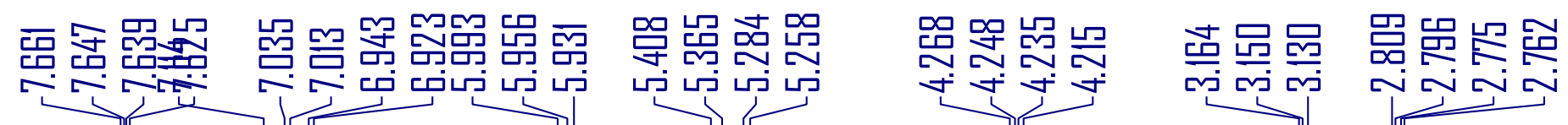
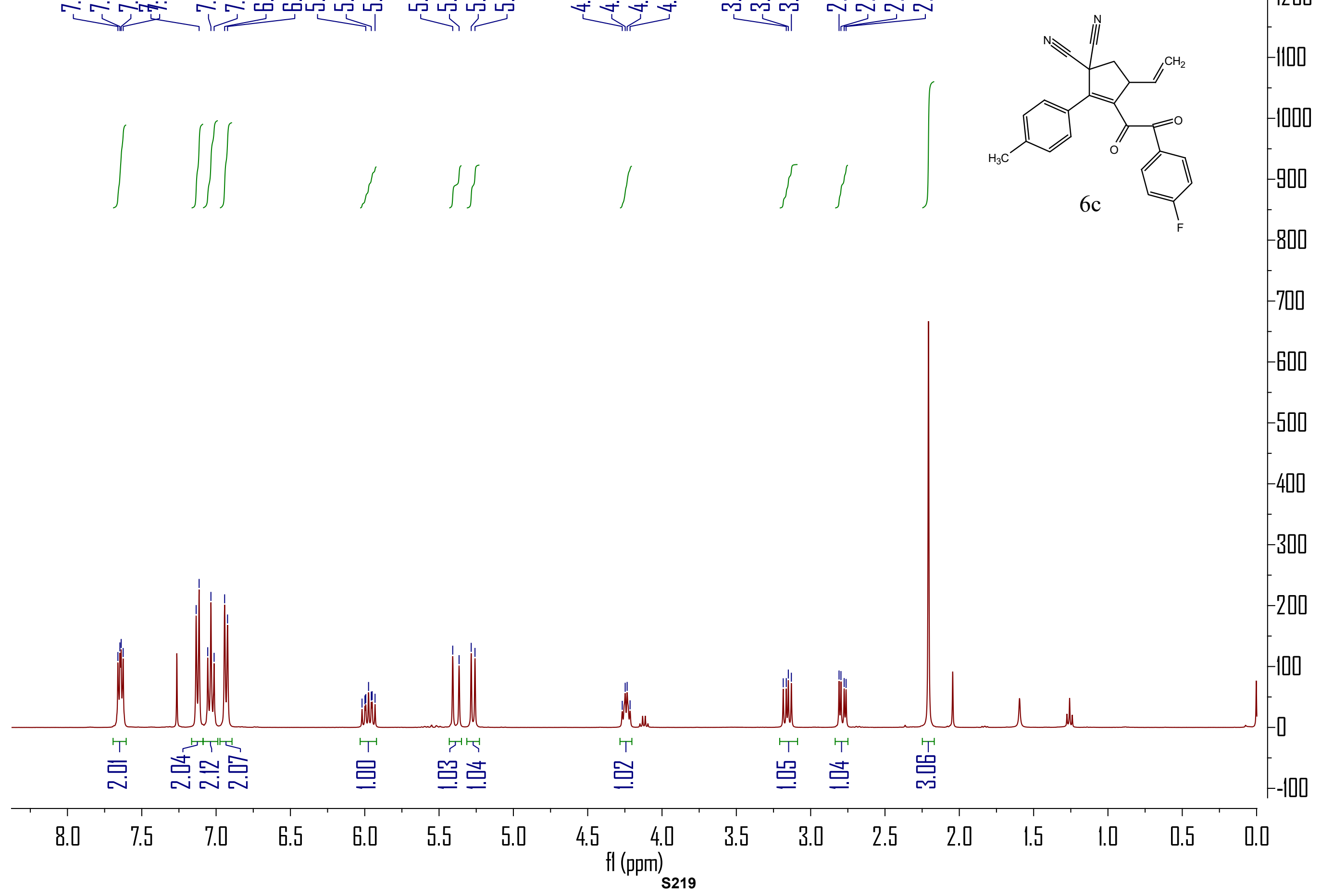
算

$-6500$

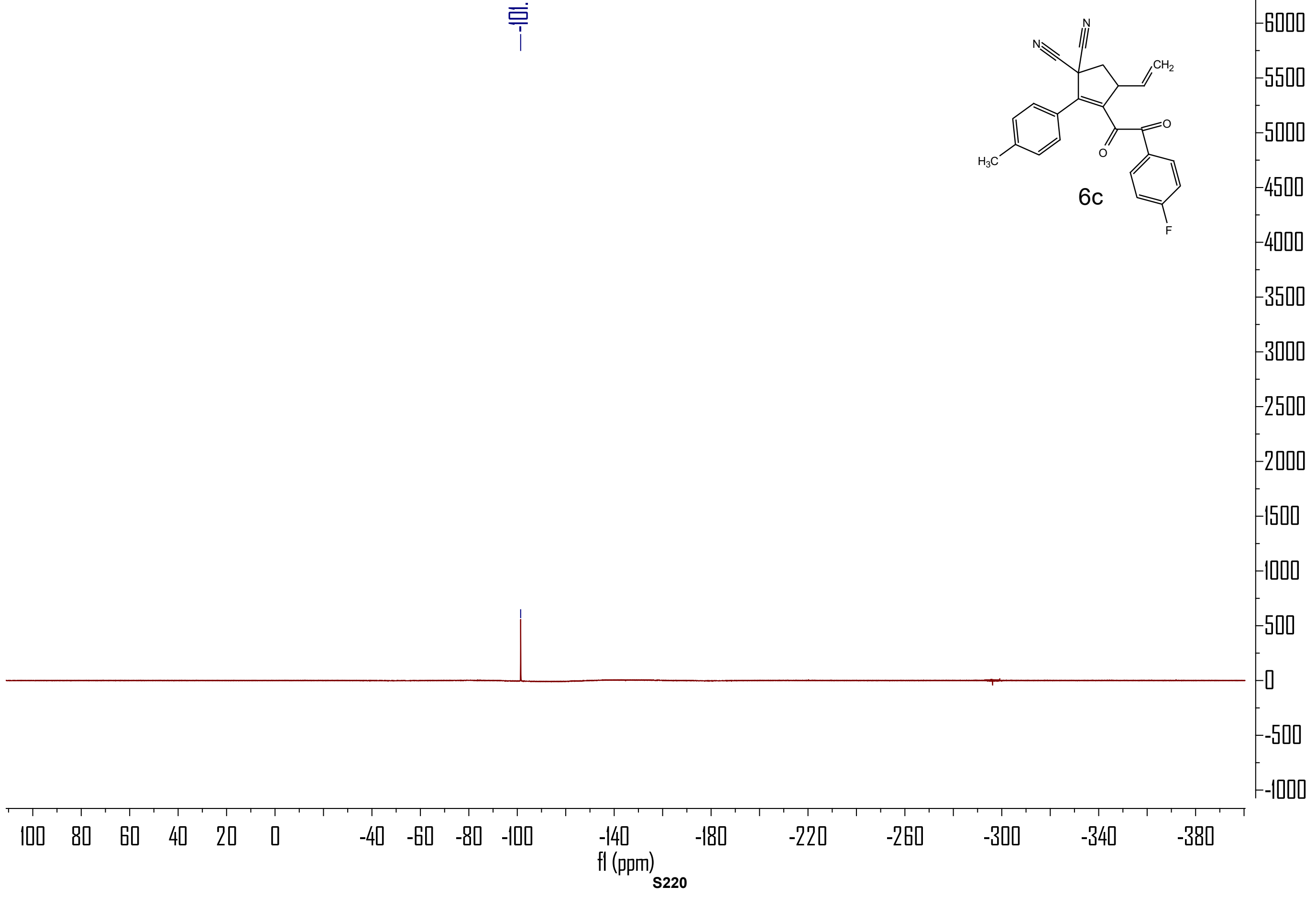




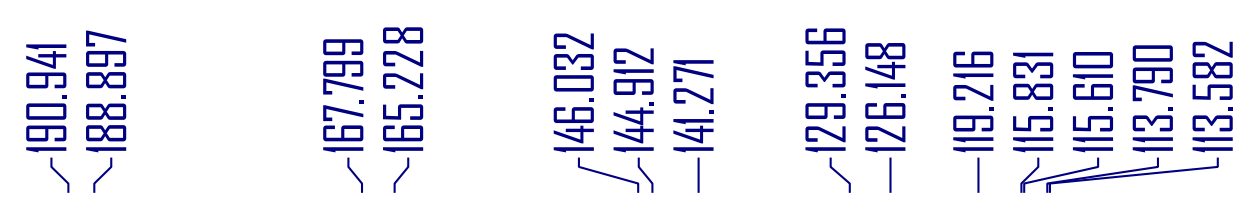

喿点志

总

$-2200$

$-2100$

$-2000$

$-1900$

$-1800$

$-1700$

$-1600$

$-1500$

$-1400$

$-1300$

$-1200$

- 1100

- 1000

900

$-800$

$-700$

$-600$

500

$-400$

$-300$

ل|

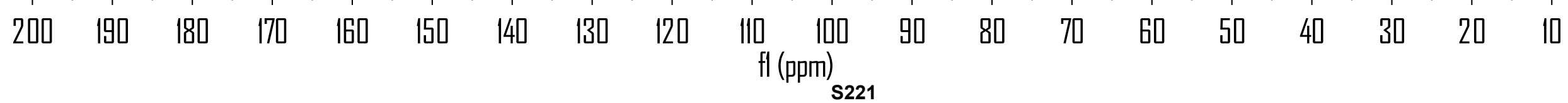


$6 d$

\section{DWP-9-100+- IB 912140.7}

\begin{tabular}{|llll|}
\hline Sample Name: & DWP-9-100+- IB 912140.7 & Injection Volume: & $\mathbf{5 . 0}$ \\
Vial Number: & GE1 & Channel: & UV_VIS_2 \\
Sample Type: & unknown & Wavelength: & $\mathbf{2 1 4 . 0}$ \\
Control Program: & test-dad6 & Bandwidth: & $\mathbf{4}$ \\
Quantif. Method: & $\mathbf{2 0 1 7 0 6 0 8}$ & Dilution Factor: & $\mathbf{1 . 0 0 0 0}$ \\
Recording Time: & $\mathbf{2 0 1 9 - 1 - 1 1 ~ 1 3 : 3 4}$ & Sample Weight: & $\mathbf{1 . 0 0 0 0}$ \\
Run Time (min): & $\mathbf{1 6 . 4 9}$ & Sample Amount: & $\mathbf{1 . 0 0 0 0}$ \\
\hline
\end{tabular}

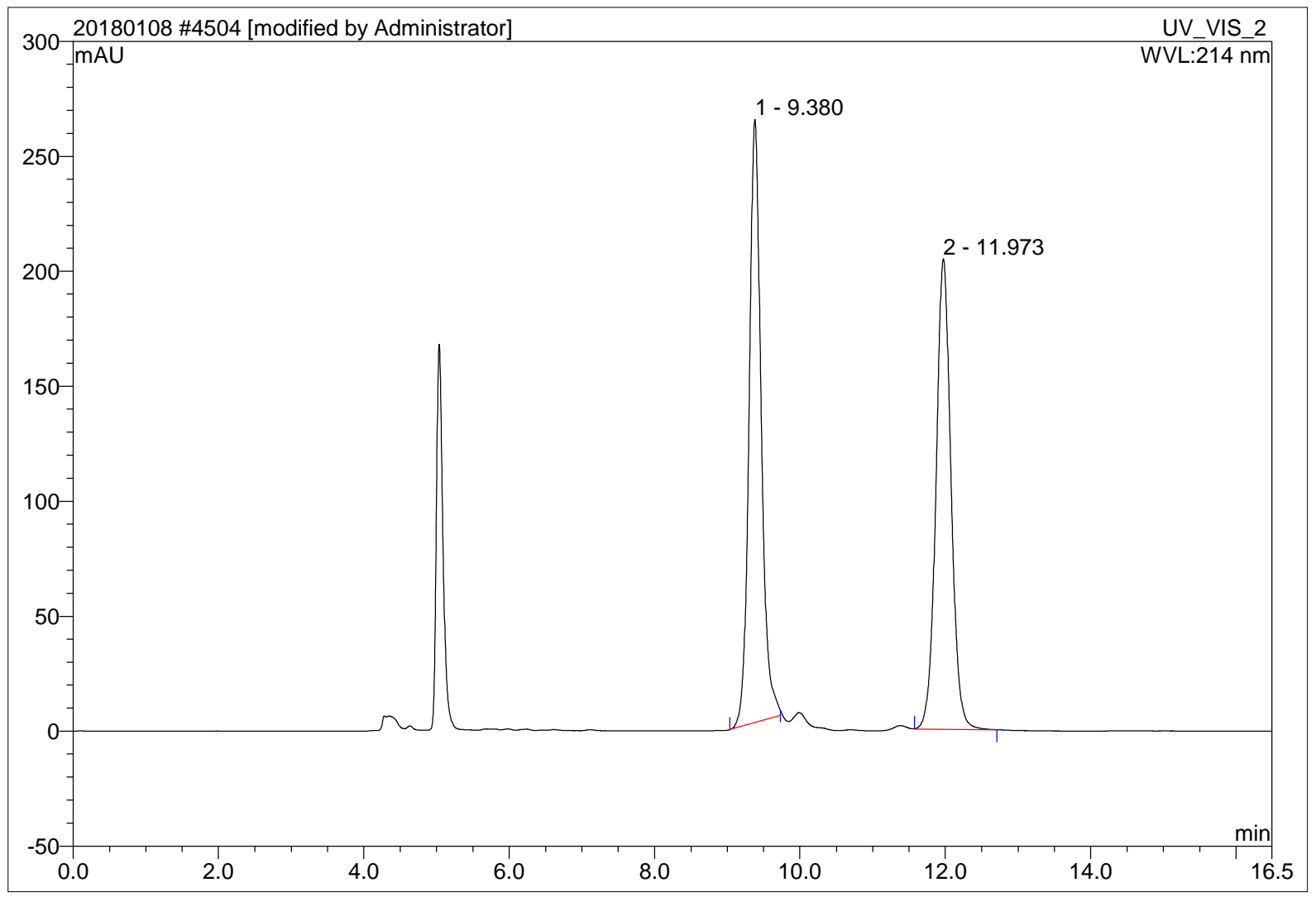

\begin{tabular}{|r|ccrrrrr|}
\hline No. & $\begin{array}{c}\text { Ret.Time } \\
\text { min }\end{array}$ & Peak Name & $\begin{array}{c}\text { Height } \\
\text { mAU }\end{array}$ & $\begin{array}{c}\text { Area } \\
\text { mAU*min }\end{array}$ & $\begin{array}{c}\text { Rel.Area } \\
\%\end{array}$ & Amount & Type \\
\hline 1 & 9.38 & n.a. & 262.365 & 50.551 & 50.70 & n.a. & BM $^{*}$ \\
2 & 11.97 & n.a. & 204.607 & 49.152 & 49.30 & n.a. & MB $^{*}$ \\
\hline Total: & & & 466.972 & 99.703 & 100.00 & 0.000 & \\
\hline
\end{tabular}


$6 d$

\section{DWP-10-93 IB 912140.7}

\begin{tabular}{llll|}
\hline Sample Name: & DWP-10-93 IB 91214 0.7 & Injection Volume: & $\mathbf{5 . 0}$ \\
Vial Number: & GE8 & Channel: & UV_VIS_2 \\
Sample Type: & unknown & Wavelength: & $\mathbf{2 1 4 . 0}$ \\
Control Program: & test-dad6 & Bandwidth: & $\mathbf{4}$ \\
Quantif. Method: & $\mathbf{2 0 1 7 0 6 0 8}$ & Dilution Factor: & $\mathbf{1 . 0 0 0 0}$ \\
Recording Time: & $\mathbf{2 0 1 9 - 1 - 1 1 ~ 1 3 : 5 2}$ & Sample Weight: & $\mathbf{1 . 0 0 0 0}$ \\
Run Time (min): & $\mathbf{1 5 . 0 0}$ & Sample Amount: & $\mathbf{1 . 0 0 0 0}$ \\
\hline
\end{tabular}

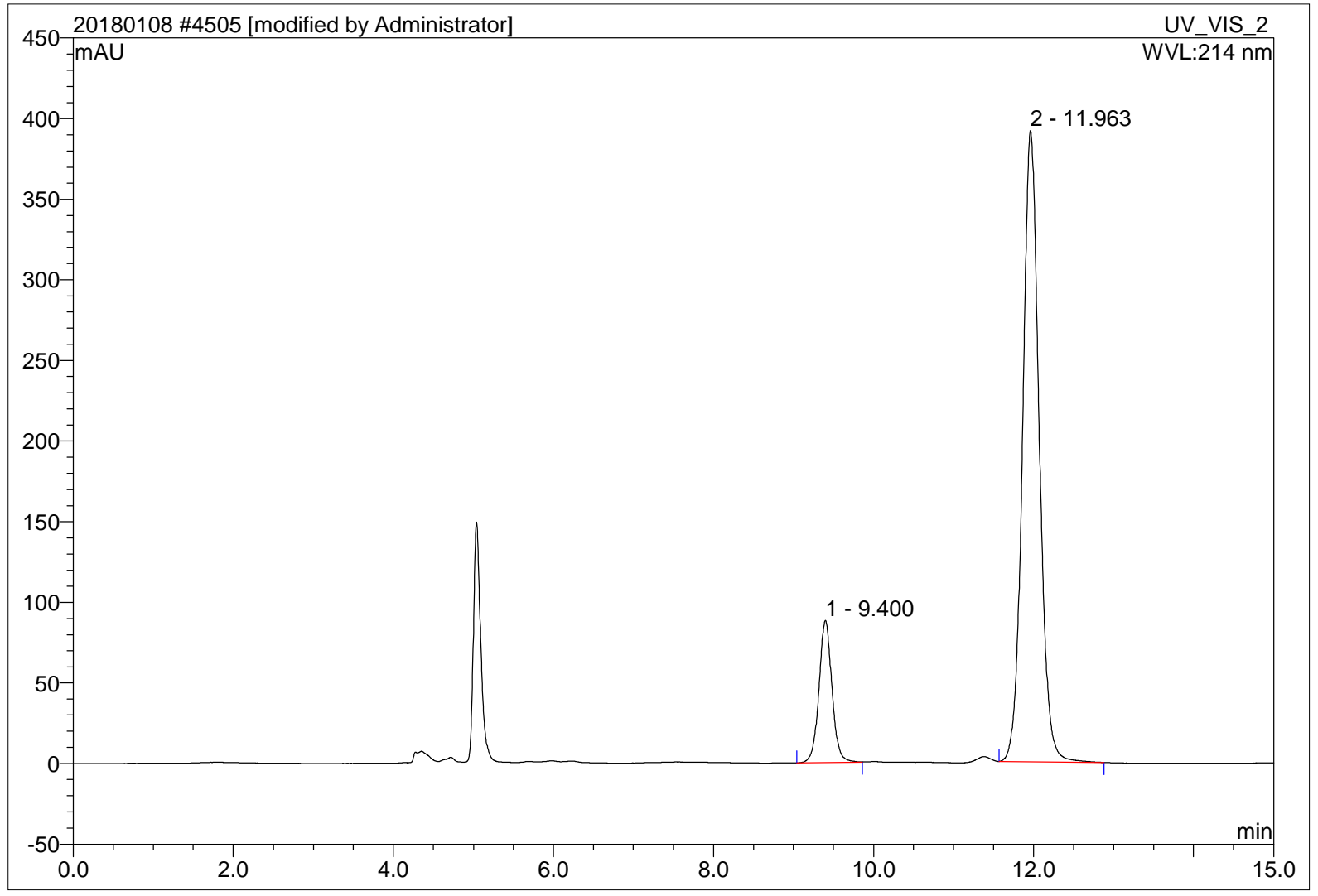

\begin{tabular}{|r|ccrrrrr|}
\hline No. & $\begin{array}{c}\text { Ret.Time } \\
\text { min }\end{array}$ & Peak Name & $\begin{array}{c}\text { Height } \\
\text { mAU }\end{array}$ & $\begin{array}{r}\text { Area } \\
\text { mAU*min }\end{array}$ & $\begin{array}{r}\text { Rel.Area } \\
\%\end{array}$ & Amount & Type \\
\hline 1 & 9.40 & n.a. & 88.260 & 16.627 & 15.05 & n.a. & BMB \\
2 & 11.96 & n.a. & 391.542 & 93.859 & 84.95 & n.a. & BMB $^{*}$ \\
\hline Total: & & & 479.802 & 110.486 & 100.00 & 0.000 & \\
\hline
\end{tabular}




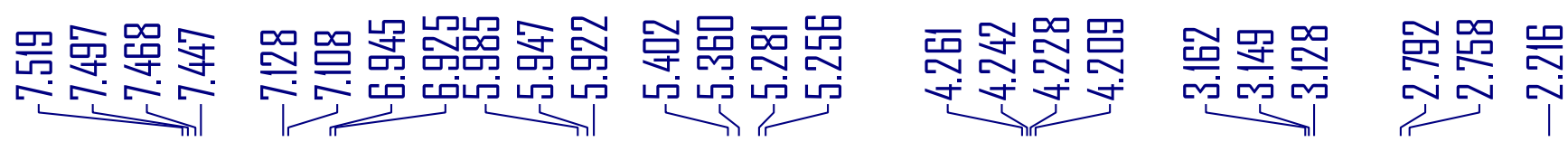

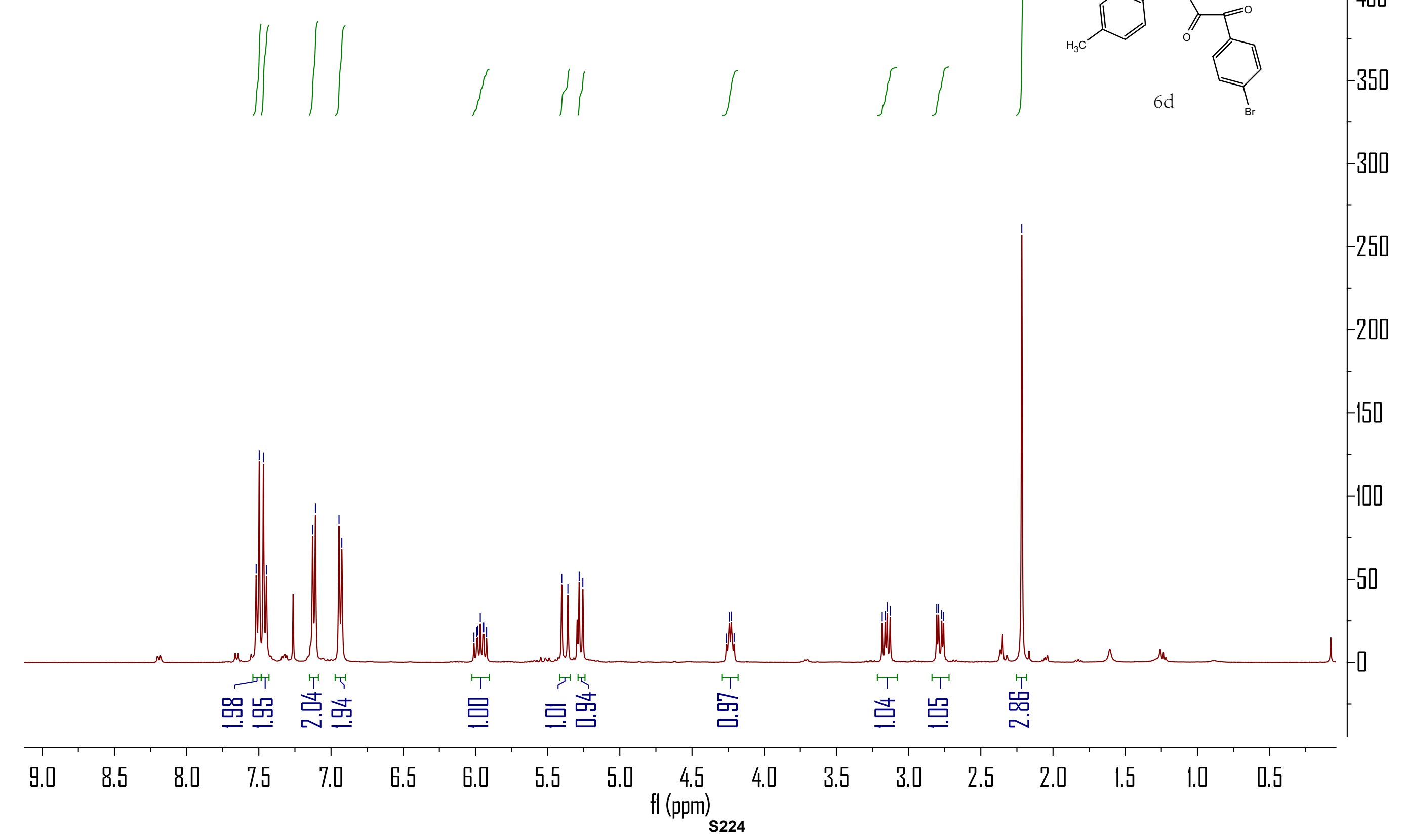



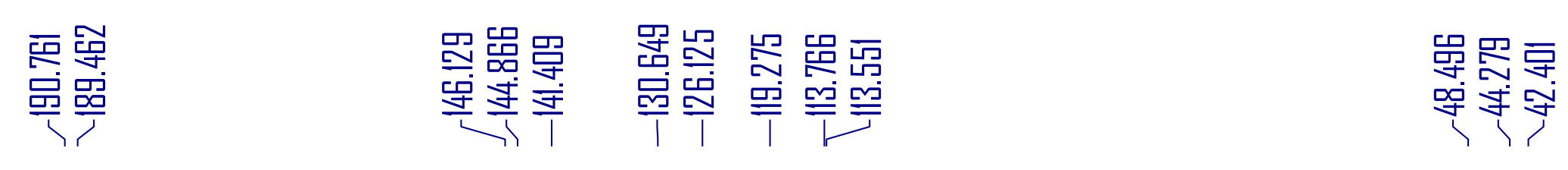

孚

$-1500$

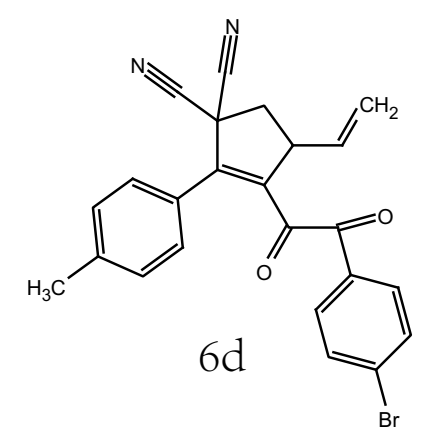

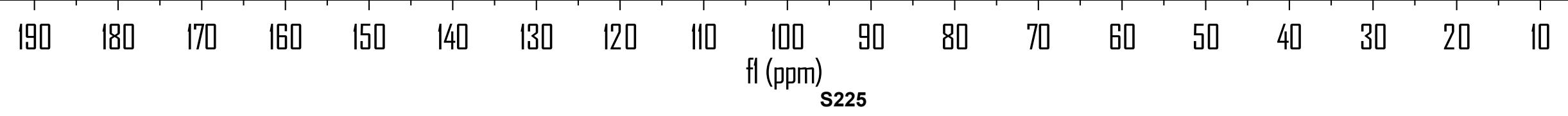




\begin{tabular}{|llll|}
\hline 4498 DWP-9-101+- IB 912140.7 & & \\
& & & \\
\hline Sample Name: & DWP-9-101+- IB 912140.7 & Injection Volume: & $\mathbf{5 . 0}$ \\
Vial Number: & GC3 & Channel: & UV_VIS_2 \\
Sample Type: & unknown & Wavelength: & $\mathbf{2 1 4 . 0}$ \\
Control Program: & test-dad3 & Bandwidth: & $\mathbf{4}$ \\
Quantif. Method: & $\mathbf{2 0 1 7 0 6 0 8}$ & Dilution Factor: & $\mathbf{1 . 0 0 0 0}$ \\
Recording Time: & $\mathbf{2 0 1 9 - 1 - 1 1 ~ 1 0 : 2 1}$ & Sample Weight: & $\mathbf{1 . 0 0 0 0}$ \\
Run Time (min): & $\mathbf{4 0 . 2 8}$ & Sample Amount: & $\mathbf{1 . 0 0 0 0}$ \\
\hline
\end{tabular}

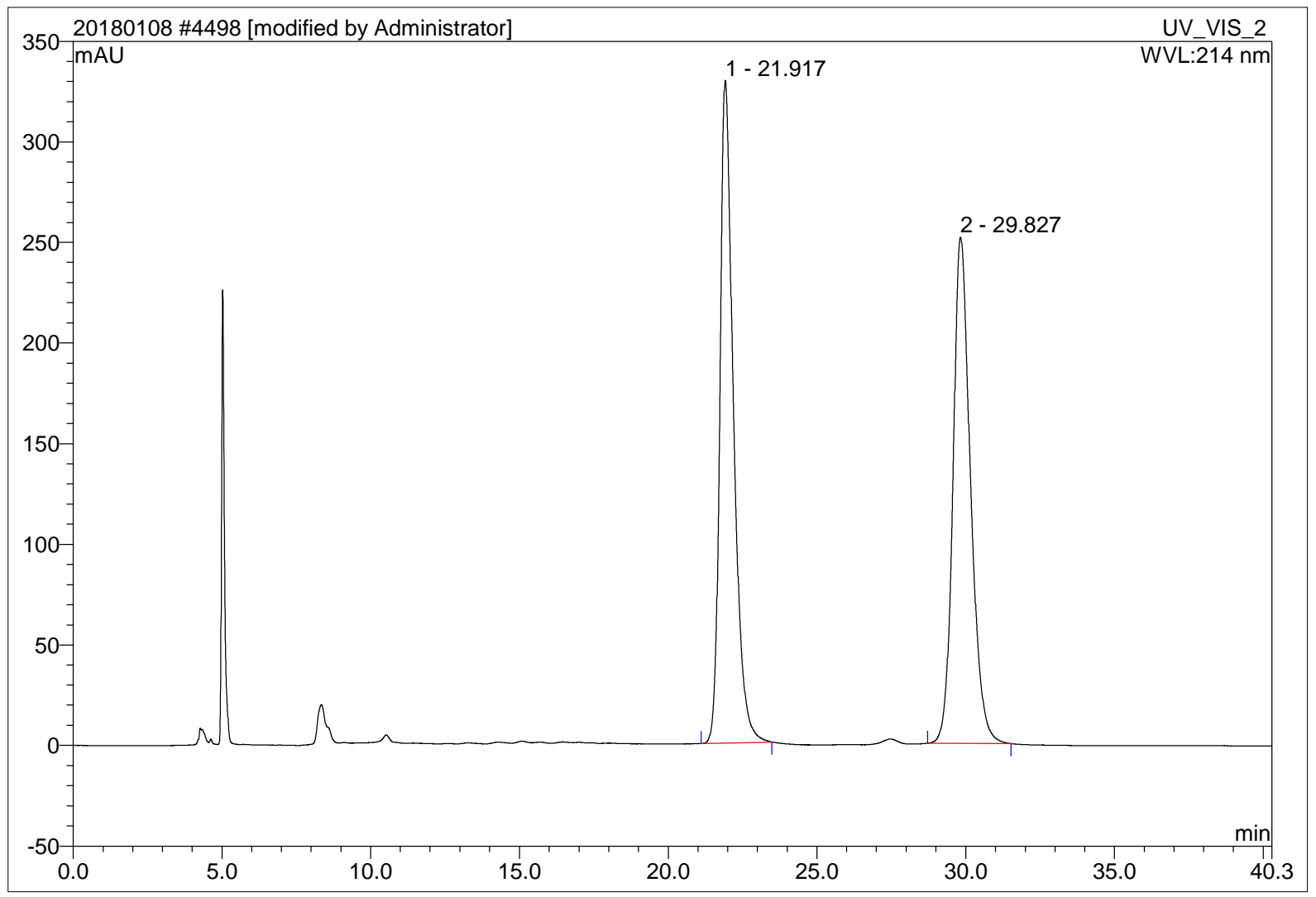

\begin{tabular}{|r|ccccccc|}
\hline No. & $\begin{array}{c}\text { Ret.Time } \\
\text { min }\end{array}$ & Peak Name & $\begin{array}{c}\text { Height } \\
\text { mAU }\end{array}$ & $\begin{array}{c}\text { Area } \\
\text { mAU*min }\end{array}$ & $\begin{array}{r}\text { Rel.Area } \\
\%\end{array}$ & Amount & Type \\
\hline 1 & 21.92 & n.a. & 329.364 & 173.635 & 50.27 & n.a. & BMB \\
2 & 29.83 & n.a. & 251.842 & 171.757 & 49.73 & n.a. & BMB \\
\hline Total: & & & 581.206 & 345.391 & 100.00 & 0.000 & \\
\hline
\end{tabular}




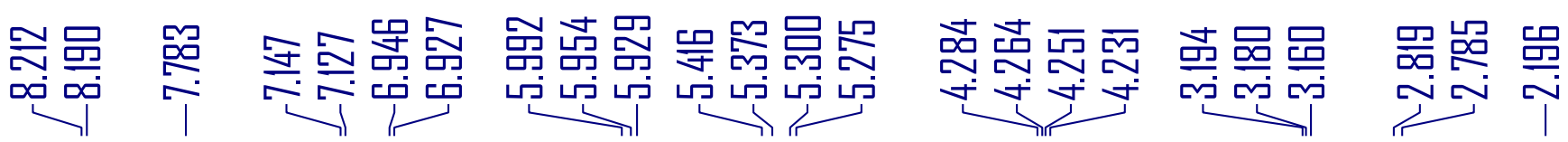
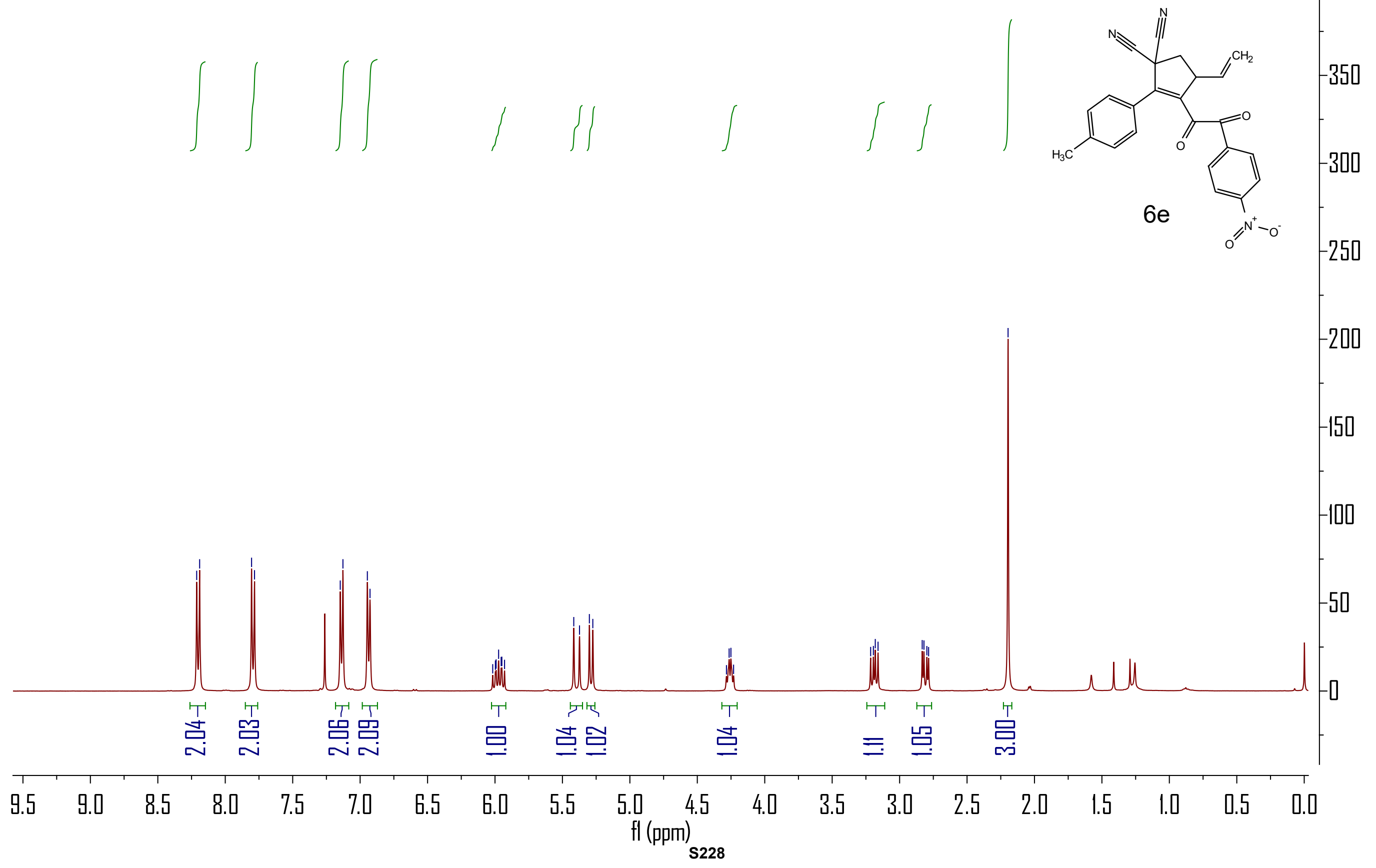


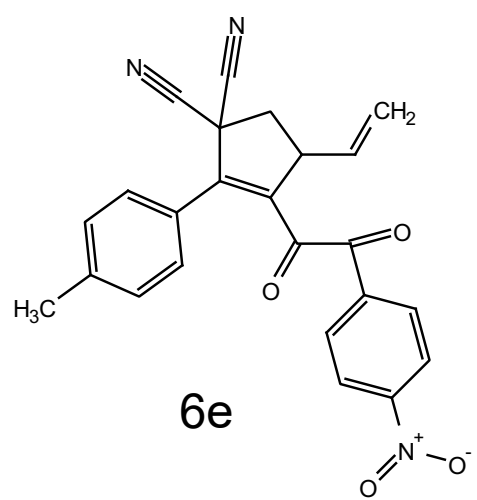

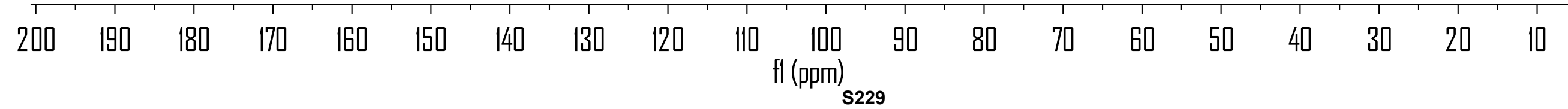




\section{$===$ Shimadzu LCsolution Analysis Report ====}

$\begin{array}{ll}\text { Acquired by } & \text { : Admin } \\ \text { Sample Name } & : \text { DWP-11-12-RAC } \\ \text { Sample ID } & : \text { IB,95/5,1.5,215 } \\ \text { Vail \# } & : \text { 1 uL } \\ \text { Injection Volume } & : \text { DWP-11-12-RAC.Icd } \\ \text { Data File Name } & : 1 . \text { Icm } \\ \text { Method File Name } & : \\ \text { Batch File Name } & : \text { Default.lcr } \\ \text { Report File Name } & : 2019-1-1613: 52: 00 \\ \text { Data Acquired } & : 2019-1-1614: 03: 23 \\ \text { Data Processed } & \end{array}$

G:IDWP-11-12-RAC.Icd

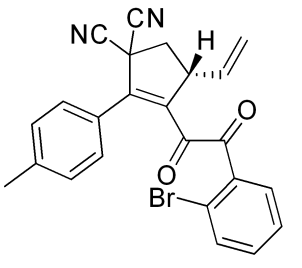

$6 f$

\section{<Chromatogram>}

$\mathrm{mV}$

G:IDWP-11-12-RAC.Icd

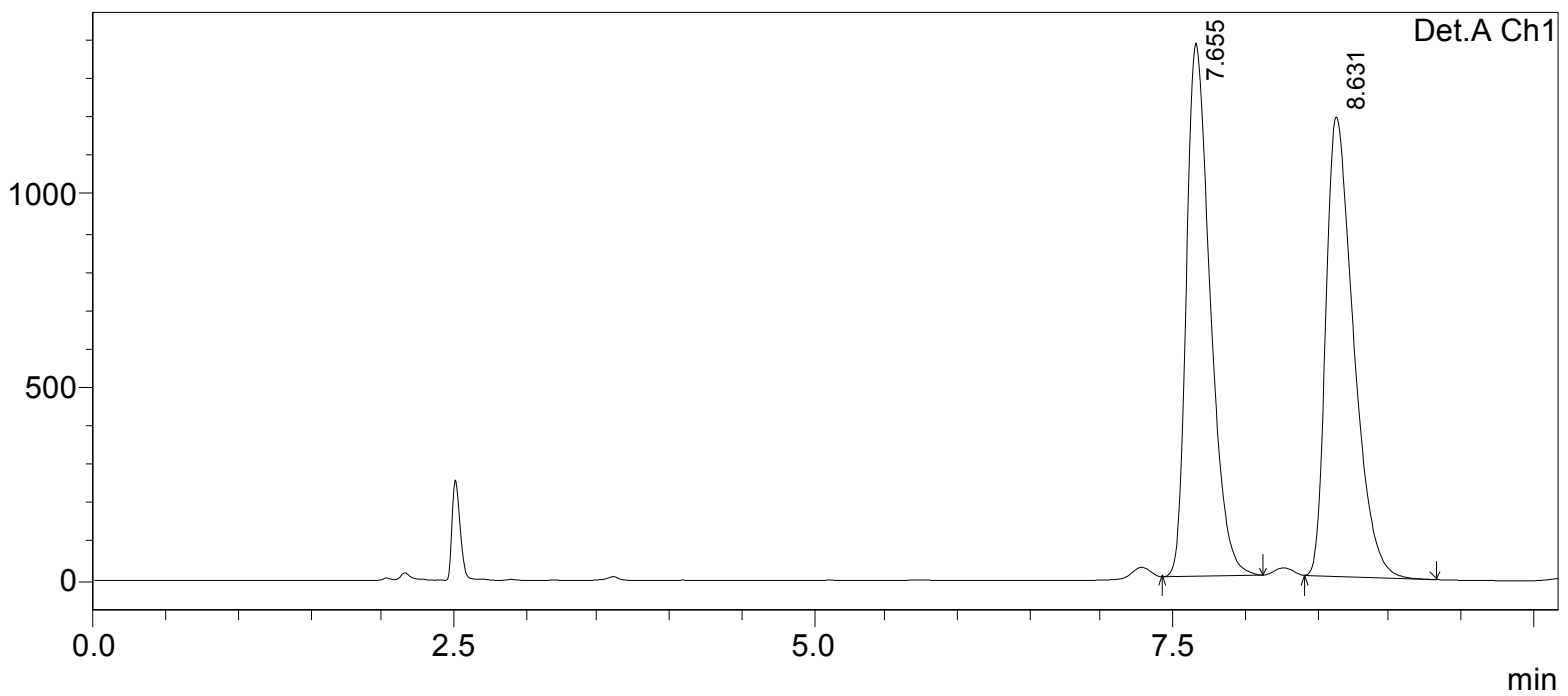

1 Det.A Ch1/215nm

PeakTable

Detector A Ch1 215nm

\begin{tabular}{|r|r|r|r|r|r|}
\hline \multicolumn{1}{|c|}{ Peak\# } & Ret. Time & \multicolumn{1}{|c|}{ Area } & Height & \multicolumn{1}{|c|}{ Area \% } & \multicolumn{1}{|c|}{ Height \% } \\
\hline 1 & 7.655 & 15853098 & 1381786 & 49.919 & 53.718 \\
\hline 2 & 8.631 & 15904721 & 1190492 & 50.081 & 46.282 \\
\hline Total & & 31757819 & 2572278 & 100.000 & 100.000 \\
\hline
\end{tabular}




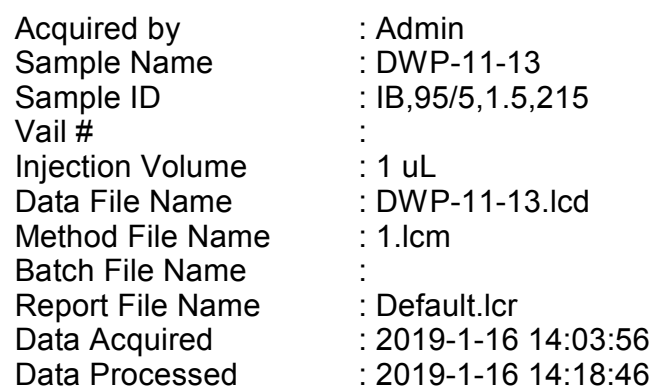

\section{$<$ Chromatogram $>$}

$\mathrm{mV}$

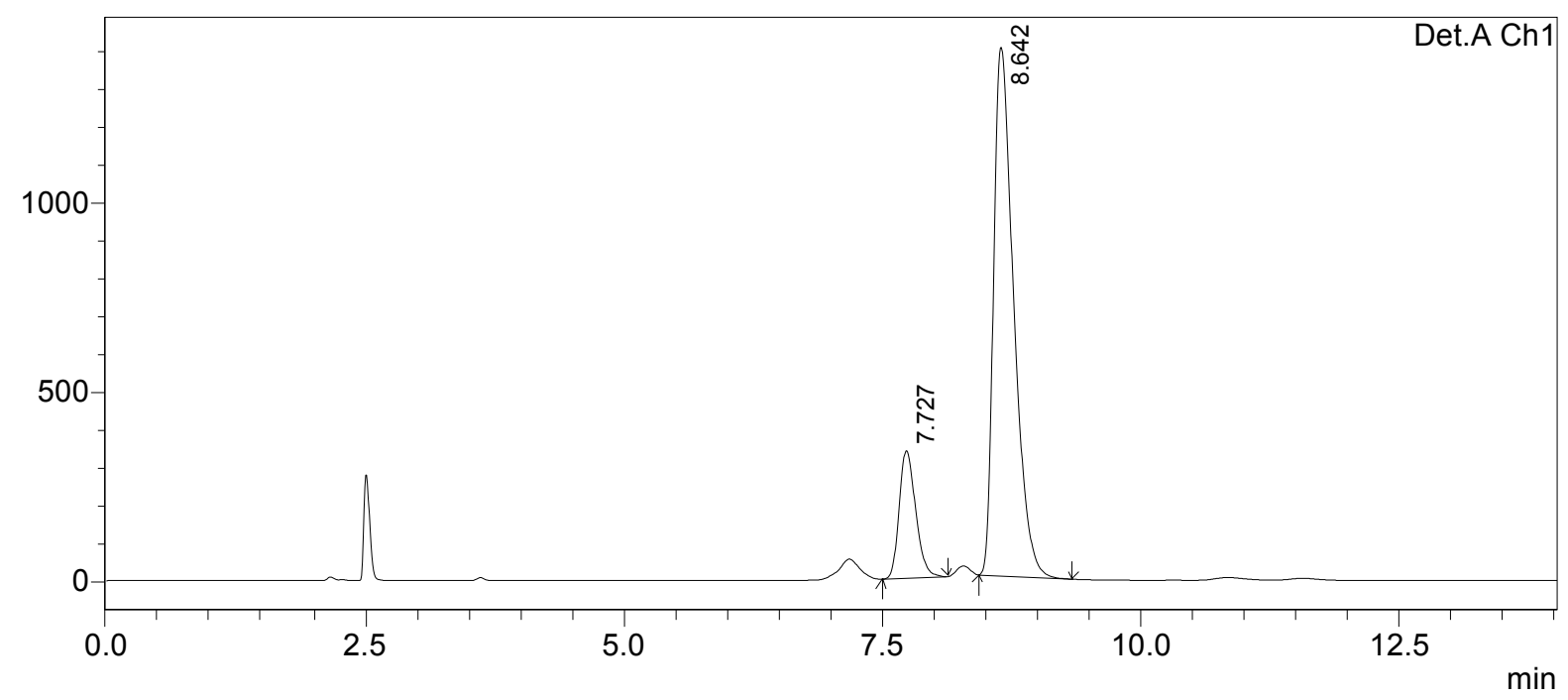

1 Det.A Ch1/215nm

Detector A Ch1 215nm

PeakTable

\begin{tabular}{|r|r|r|r|r|r|}
\hline \multicolumn{1}{|c|}{ Peak\# } & Ret. Time & \multicolumn{1}{c|}{ Area } & \multicolumn{1}{c|}{ Height } & Area \% & \multicolumn{1}{c|}{ Height \% } \\
\hline 1 & 7.727 & 3754376 & 337598 & 16.645 & 19.459 \\
\hline 2 & 8.642 & 18800538 & 1397317 & 83.355 & 80.541 \\
\hline Total & & 22554914 & 1734915 & 100.000 & 100.000 \\
\hline
\end{tabular}



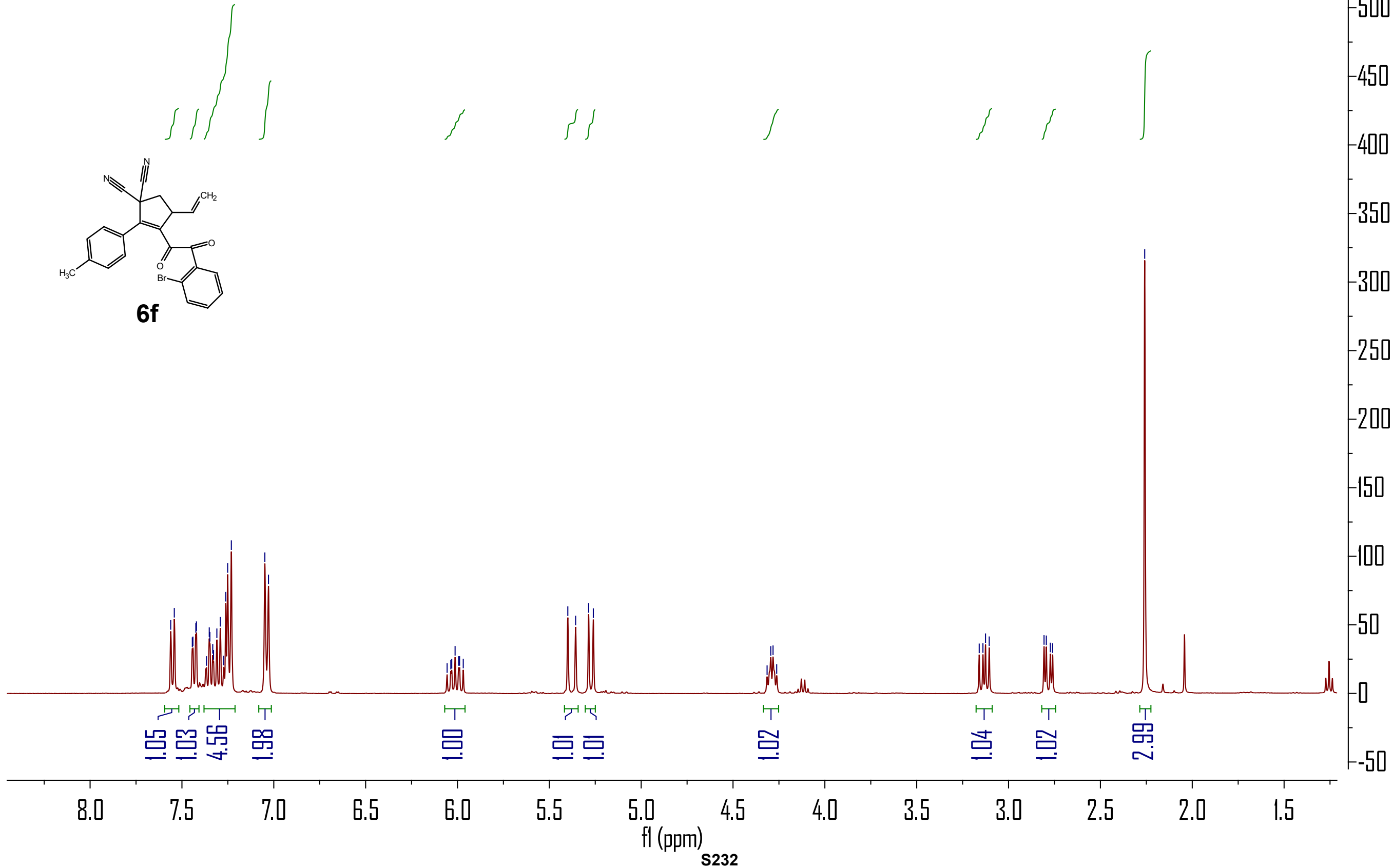


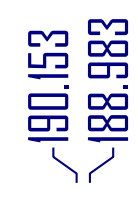

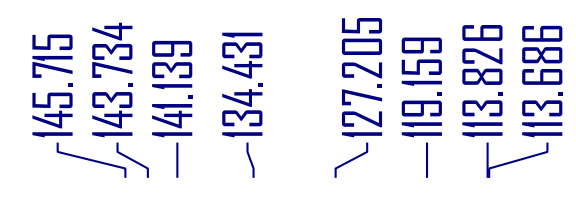

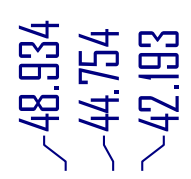

$\stackrel{\overline{9}}{\stackrel{\overline{9}}{i}}$

$-1700$
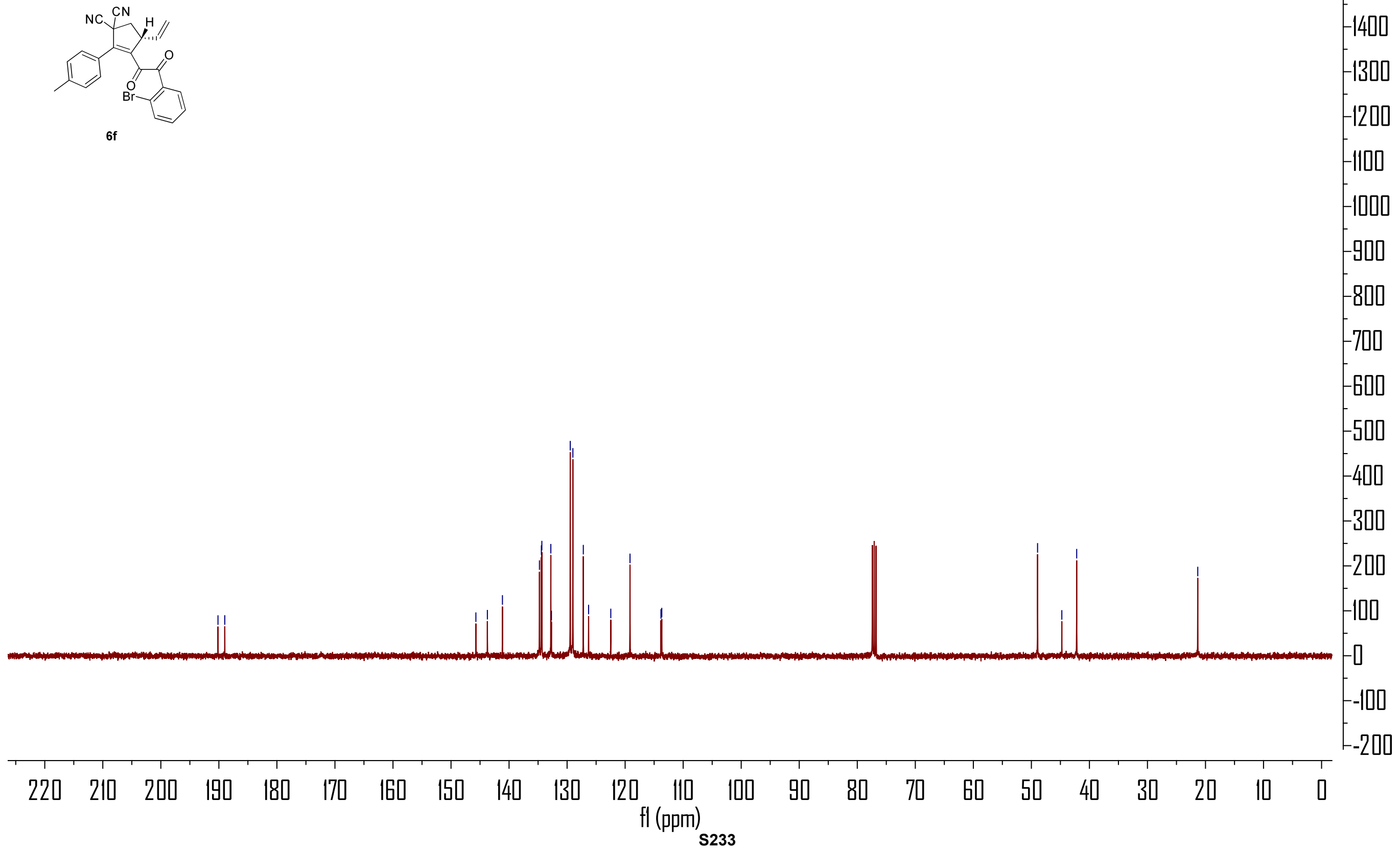


\section{==== Shimadzu LCsolution Analysis Report ====}

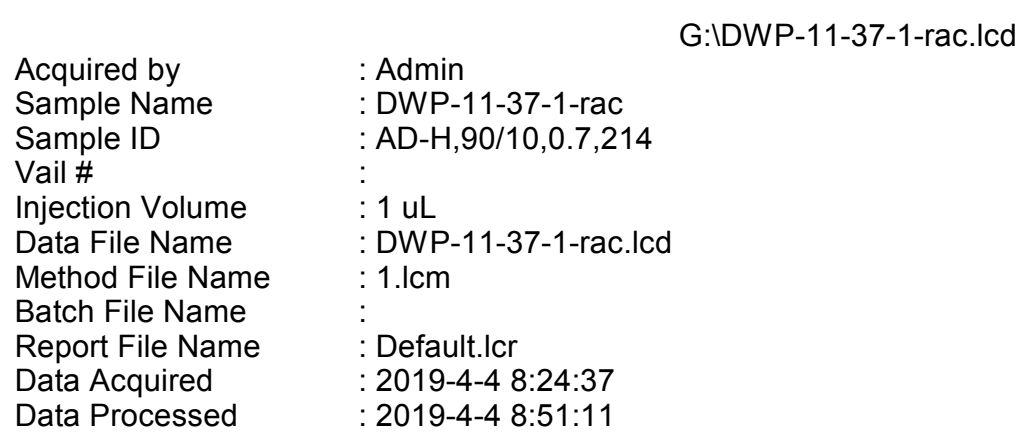

\section{G:IDWP-11-37-1-rac.Icd}

Data Processed

\section{$<$ Chromatogram $>$}

G:IDWP-11-37-1-rac.Icd

$\mathrm{mV}$

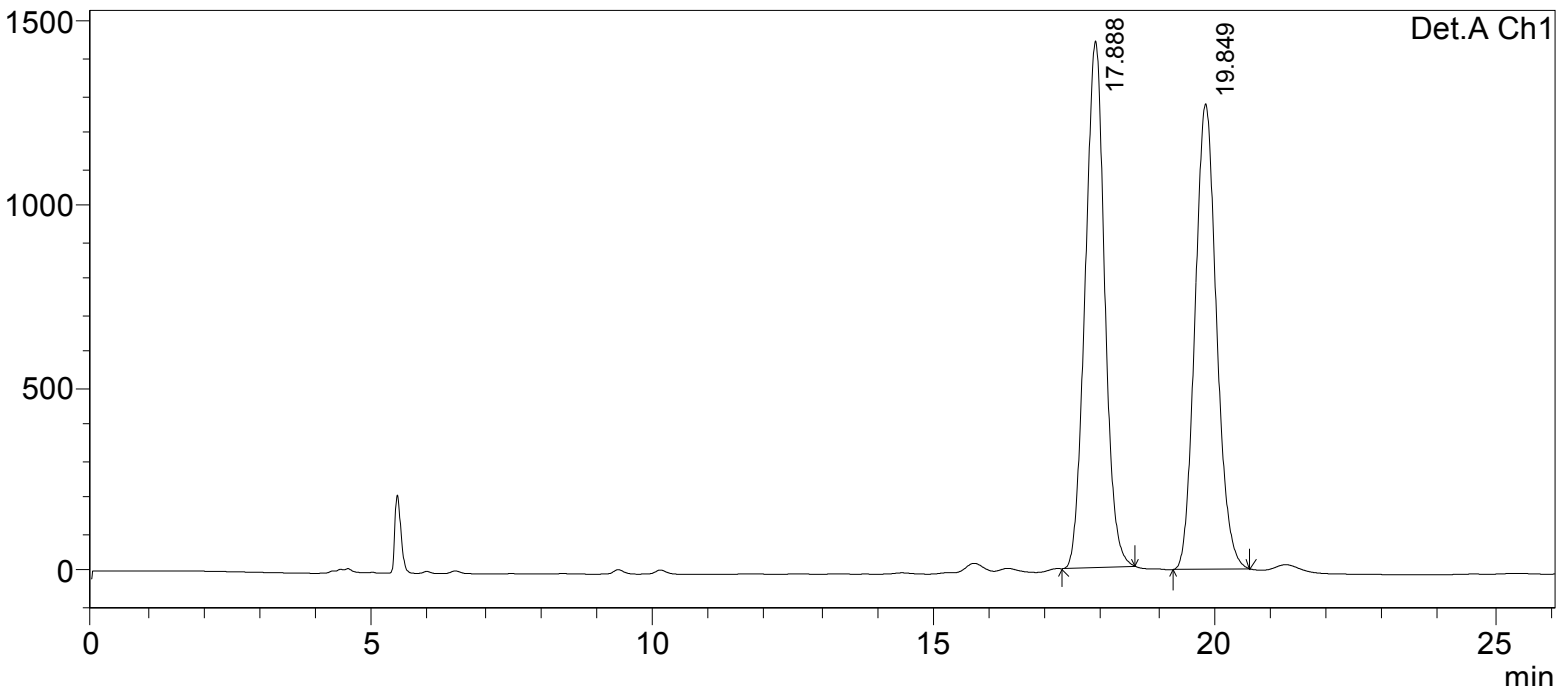

1 Det.A Ch1/214nm

PeakTable

Detector A Ch1 214nm

\begin{tabular}{|r|r|r|r|r|r|}
\hline Peak\# & Ret. Time & Area & Height & \multicolumn{1}{|c|}{ Area \% } & \multicolumn{1}{|c|}{ Height \% } \\
\hline 1 & 17.888 & 34778416 & 1440748 & 50.651 & 53.081 \\
\hline 2 & 19.849 & 33884977 & 1273504 & 49.349 & 46.919 \\
\hline Total & & 68663393 & 2714253 & 100.000 & 100.000 \\
\hline
\end{tabular}




\section{==== Shimadzu LCsolution Analysis Report ====}

$\begin{array}{ll}\text { Acquired by } & \text { : Admin } \\ \text { Sample Name } & \text { : DWP-11-37-1 } \\ \text { Sample ID } & : \text { AD-H,90/10,0.7,214 } \\ \text { Vail \# } & : \text { uL } \\ \text { Injection Volume } & : \text { DWP-11-37-1.Icd } \\ \text { Data File Name } & : \text { 1.lcm } \\ \text { Method File Name } & : \\ \text { Batch File Name } & : \text { Default.lcr } \\ \text { Report File Name } & : \text { 2019-4-4 8:52:58 } \\ \text { Data Acquired } & : 2019-4-49: 31: 26 \\ \text { Data Processed } & \end{array}$

G:IDWP-11-37-1.Icd<smiles>COC(=O)C1=C(c2ccc(C)cc2)[C@@](C#N)(C2CO2)C[C@H]1C#N</smiles>

major

\section{$<$ Chromatogram $>$}

$\mathrm{mV}$

G:IDWP-11-37-1.Icd

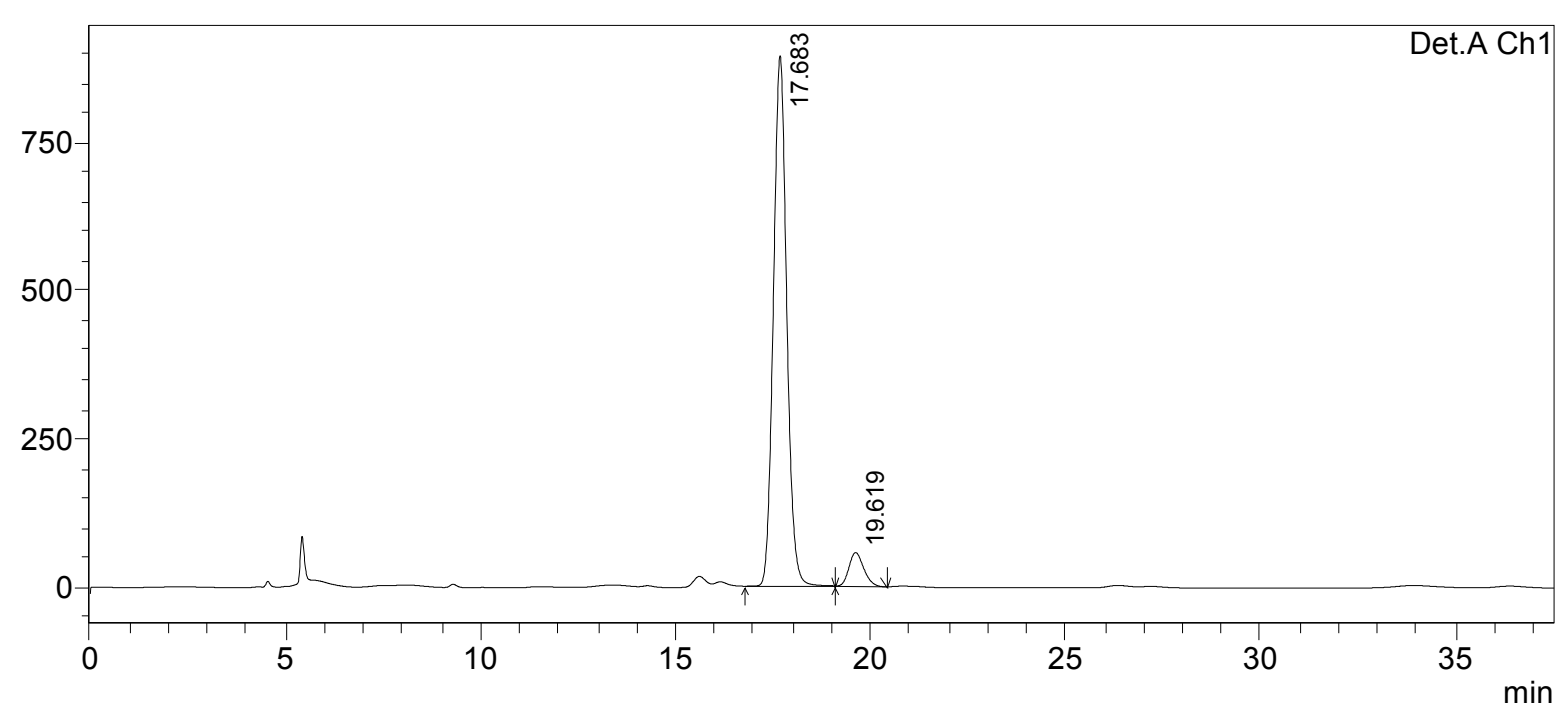

1 Det.A Ch1/214nm

PeakTable

Detector A Ch1 $214 \mathrm{~nm}$
\begin{tabular}{|r|r|r|r|r|r|}
\hline Peak\# & Ret. Time & \multicolumn{1}{|c|}{ Area } & Height & Area $\%$ & \multicolumn{1}{|c|}{ Height $\%$} \\
\hline 1 & 17.683 & 21264904 & 893905 & 93.241 & 93.952 \\
\hline 2 & 19.619 & 1541383 & 57544 & 6.759 & 6.048 \\
\hline Total & & 22806288 & 951449 & 100.000 & 100.000 \\
\hline
\end{tabular}



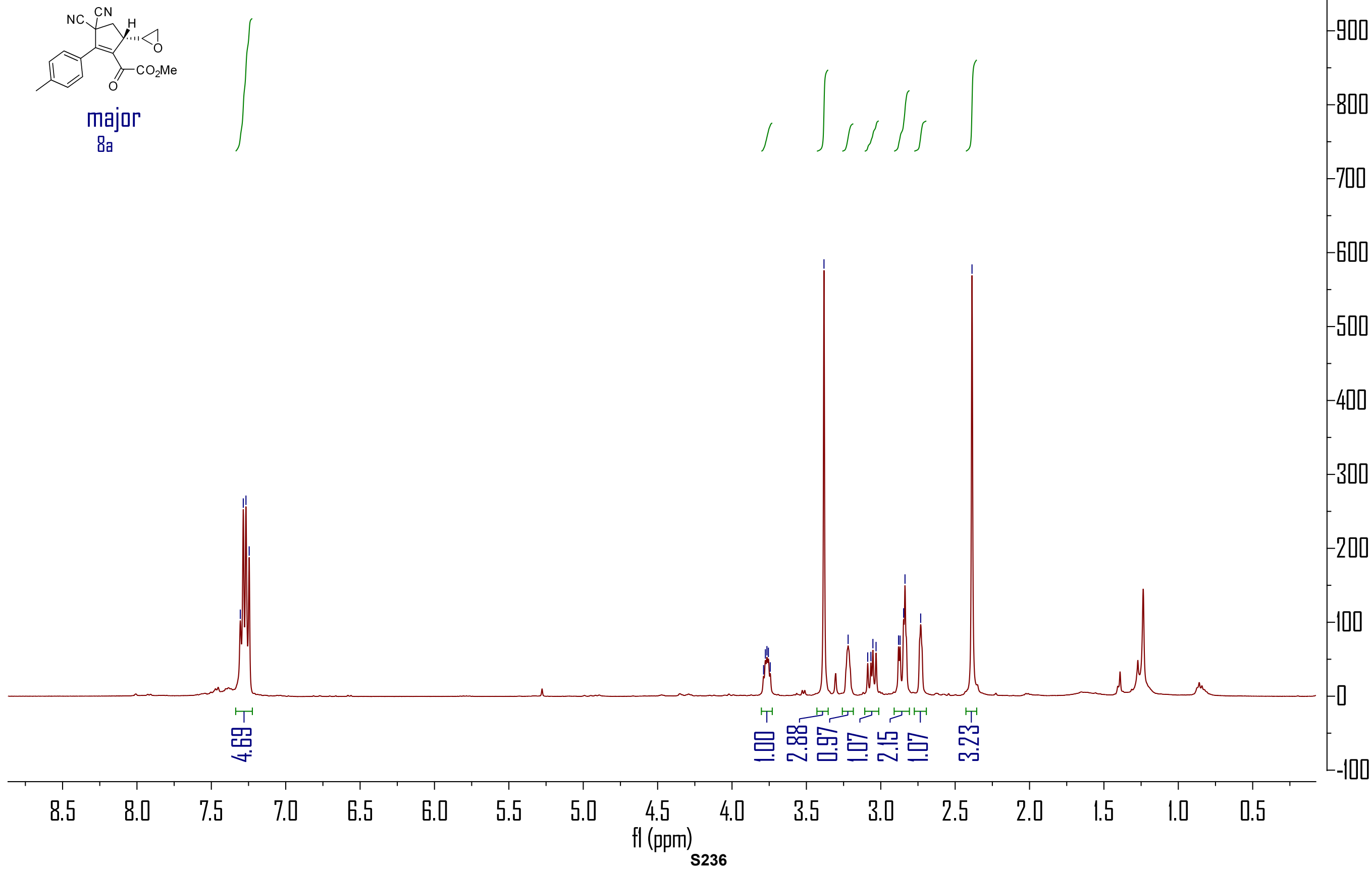


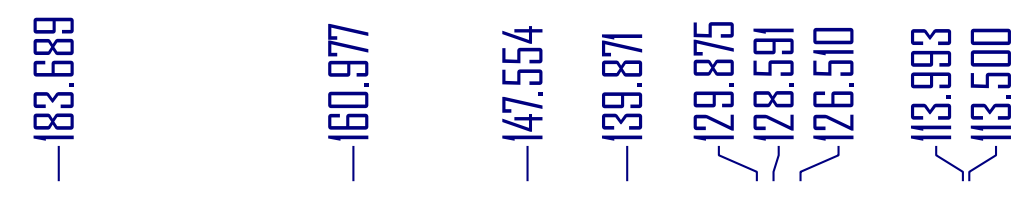

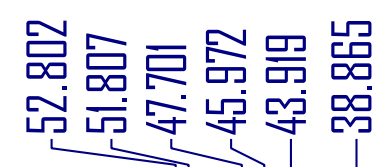

品

$-1200$

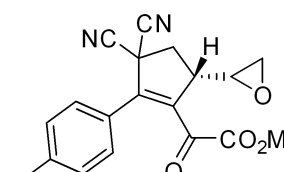

major

8 a

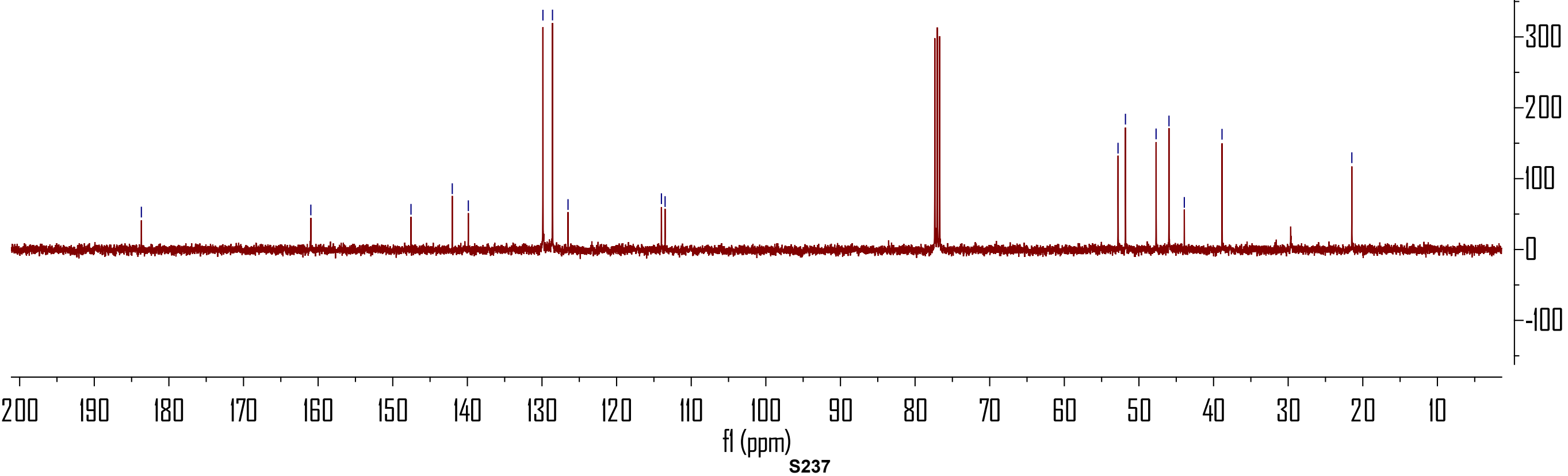




\section{==== Shimadzu LCsolution Analysis Report ====}

$\begin{array}{ll}\text { Acquired by } & \text { : Admin } \\ \text { Sample Name } & : \text { DWP-11-37-2-RAC-2 } \\ \text { Sample ID } & : \text { ID,90/10,0.7,254 } \\ \text { Vail \# } & : \\ \text { Injection Volume } & : \text { uL } \\ \text { Data File Name } & : \text { DWP-11-37-2-RAC-2.Icd } \\ \text { Method File Name } & : \text { 1.Icm } \\ \text { Batch File Name } & : \\ \text { Report File Name } & : \text { Default.Icr } \\ \text { Data Acquired } & : \text { 2019-4-8 13:44:41 } \\ \text { Data Processed } & : \text { 2019-4-8 14:41:07 }\end{array}$

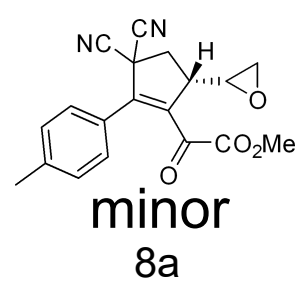

\section{$<$ Chromatogram $>$}

$\mathrm{mV}$

G:IDWP-11-37-2-RAC-2.Icd

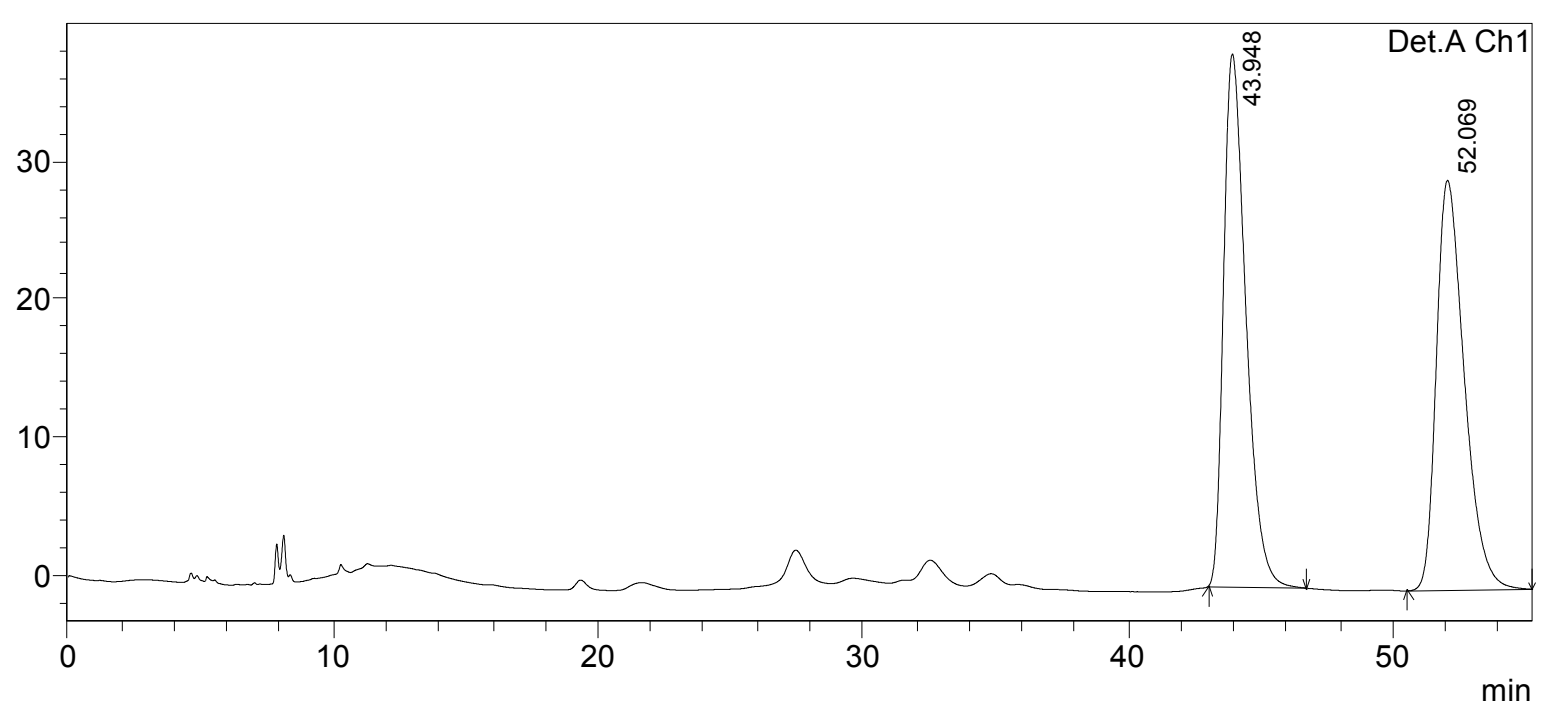

1 Det.A Ch1/254nm

PeakTable

Detector A Ch1 254nm

\begin{tabular}{|r|r|r|r|r|r|}
\hline Peak\# & Ret. Time & \multicolumn{1}{|c|}{ Area } & Height & Area $\%$ & Height \% \\
\hline 1 & 43.948 & 2261988 & 38611 & 50.934 & 56.526 \\
\hline 2 & 52.069 & 2179041 & 29696 & 49.066 & 43.474 \\
\hline Total & & 4441030 & 68306 & 100.000 & 100.000 \\
\hline
\end{tabular}




\section{==== Shimadzu LCsolution Analysis Report ====}

$\begin{array}{ll}\text { Acquired by } & : \text { Admin } \\ \text { Sample Name } & : \text { DWP-11-37-2 } \\ \text { Sample ID } & : \text { ID,90/10,0.7,254 } \\ \text { Vail \# } & : \text { 1 uL } \\ \text { Injection Volume } & : \text { DWP-11-37-2.Icd } \\ \text { Data File Name } & : \text { 1.lcm } \\ \text { Method File Name } & : \\ \text { Batch File Name } & : \text { Default.lcr } \\ \text { Report File Name } & : 2019-4-814: 44: 45 \\ \text { Data Acquired } & : 2019-4-815: 41: 51 \\ \text { Data Processed } & \end{array}$

G:IDWP-11-37-2.Icd

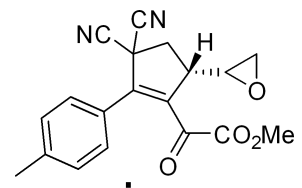

minor

$8 a$

\section{$<$ Chromatogram $>$}

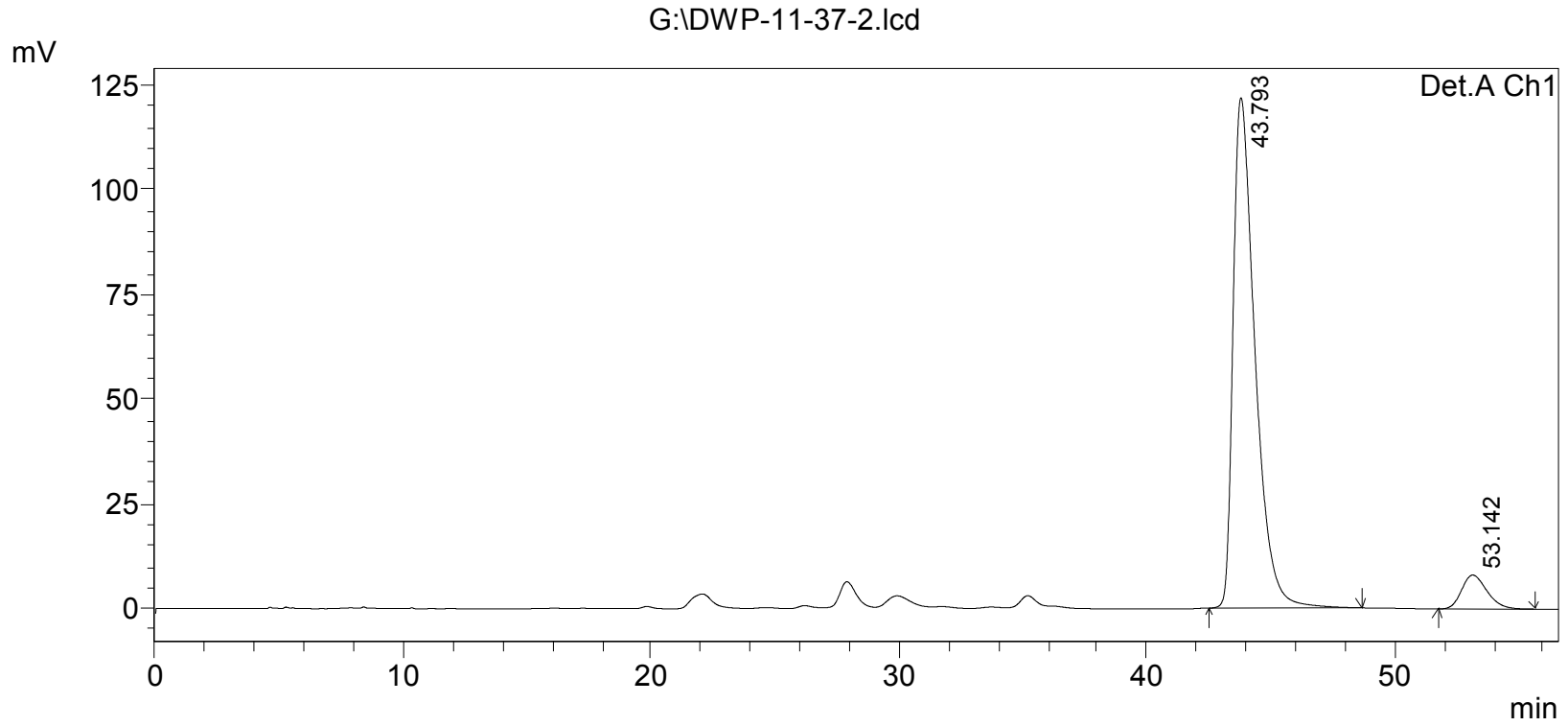

1 Det.A Ch1/254nm

Detector A Ch1 $254 \mathrm{~nm}$
\begin{tabular}{|r|r|r|r|r|r|}
\hline \multicolumn{1}{|c|}{ Peak\# } & Ret. Time & \multicolumn{1}{c|}{ Area } & Height & Area $\%$ & \multicolumn{1}{c|}{ Height $\%$} \\
\hline 1 & 43.793 & 7471554 & 121962 & 92.808 & 93.755 \\
\hline 2 & 53.142 & 579017 & 8124 & 7.192 & 6.245 \\
\hline Total & & 8050571 & 130086 & 100.000 & 100.000 \\
\hline
\end{tabular}




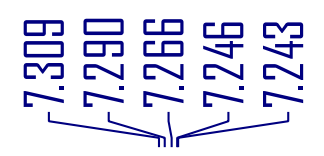

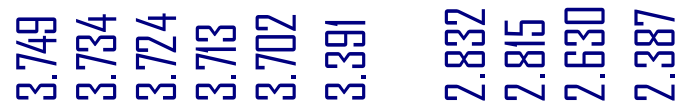

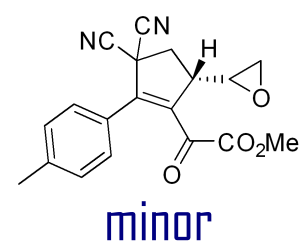

8 a

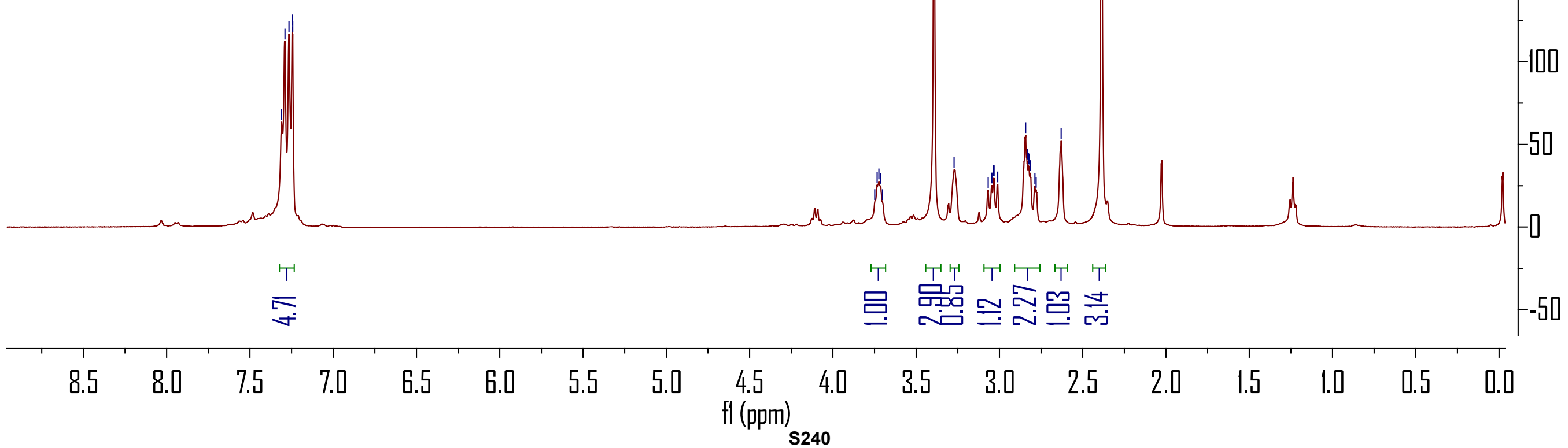



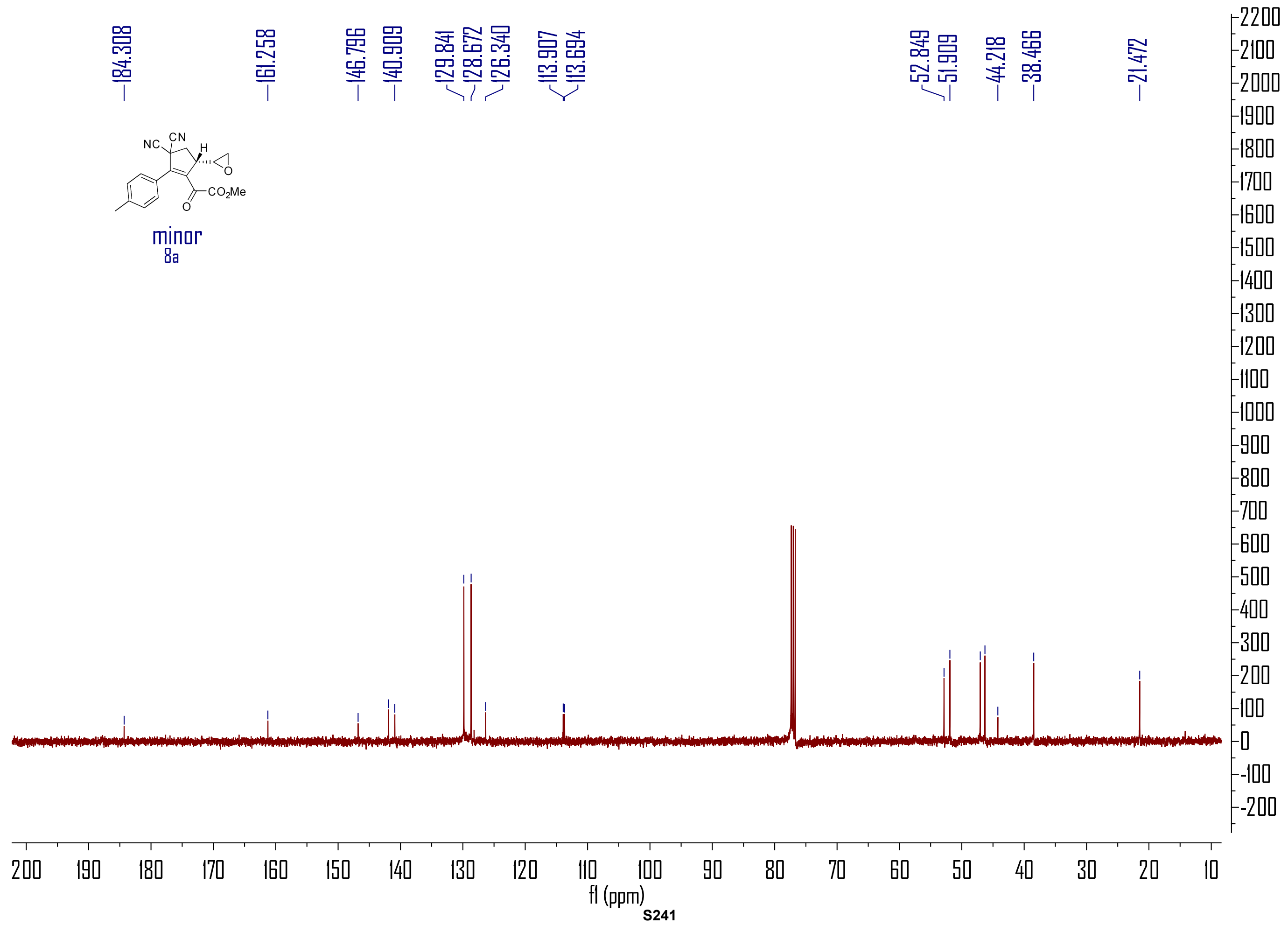


\section{==== Shimadzu LCsolution Analysis Report ====}

Acquired by

Sample Name

Sample ID

Vail \#

Injection Volume

Data File Name

Method File Name

Batch File Name

Report File Name

Data Acquired

Data Processed
G:IHPLC.5.29IDWP-11-51-RAC.Icd

: Admin

DWP-11-51-RAC

: ID,90/10,0.7,214

$: 1 \mathrm{uL}$

DWP-11-51-RAC.Icd

$1 . \mathrm{lcm}$

Default.Icr

2019-5-28 11:13:41

: 2019-5-29 8:15:29

\section{$<$ Chromatogram $>$}

$\mathrm{mV}$

G:IHPLC.5.29IDWP-11-51-RAC.Icd

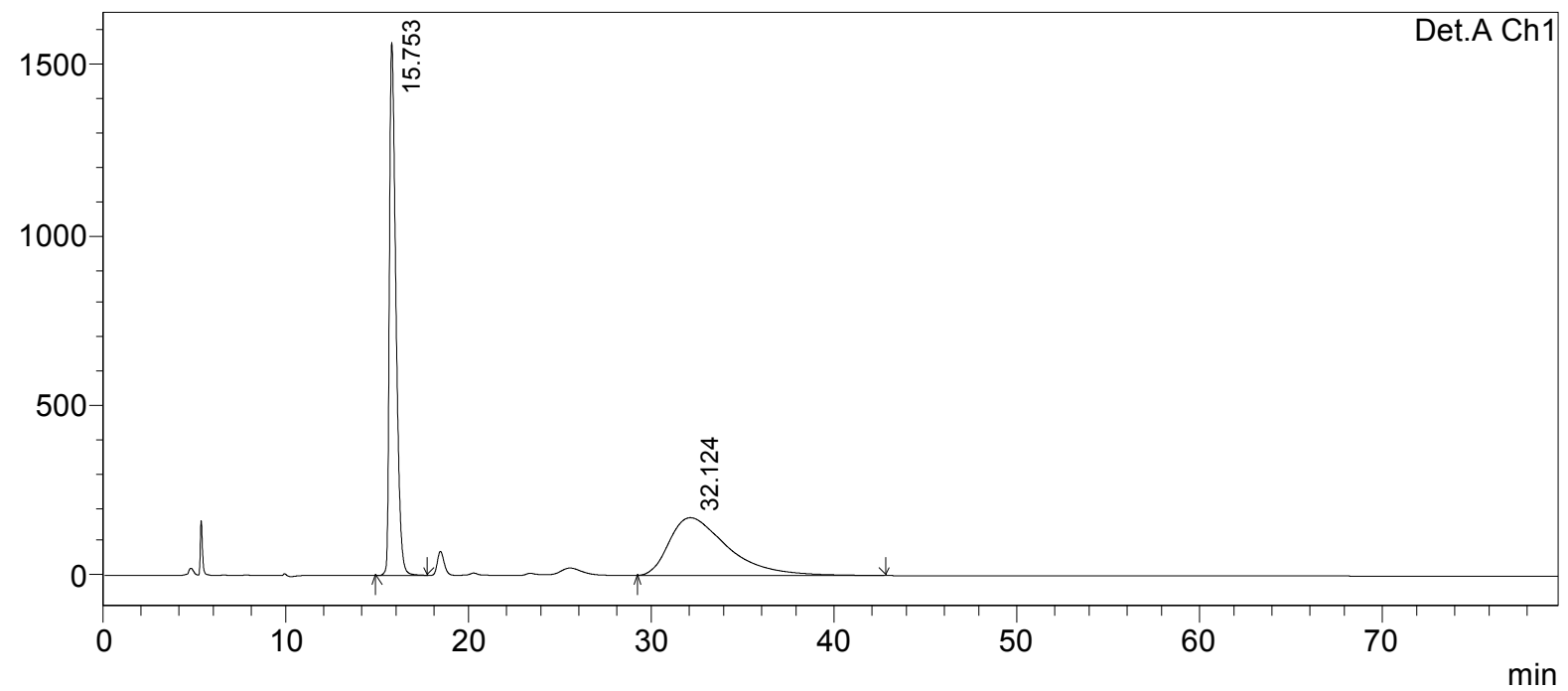

1 Det.A Ch1/214nm

PeakTable

Detector A Ch1 214nm
\begin{tabular}{|r|r|r|r|r|r|}
\hline Peak\# & Ret. Time & Area & Height & Area \% & \multicolumn{1}{|c|}{ Height \% } \\
\hline 1 & 15.753 & 38922810 & 1565357 & 50.734 & 90.225 \\
\hline 2 & 32.124 & 37797098 & 169589 & 49.266 & 9.775 \\
\hline Total & & 76719908 & 1734947 & 100.000 & 100.000 \\
\hline
\end{tabular}


==== Shimadzu LCsolution Analysis Report ====

G:IHPLC.5.29IDWP-11-50.Icd<smiles>C=C[C@H]1CC(C#N)(C#N)C(c2ccc(C)cc2)=C1[C@H](O)C(C)=O</smiles>

9a
Acquired by

Sample Name

Sample ID

Vail \#

Injection Volume

Data File Name

Method File Name

Batch File Name

Report File Name

Data Acquired

Data Processed
: Admin

: DWP-11-50

ID,90/10,0.7,214

: $1 \mathrm{uL}$

DWP-11-50.Icd

: $1 . \mathrm{lcm}$

: Default.Icr

2019-5-29 8:23:29

: 2019-5-29 9:14:27

\section{<Chromatogram>}

$\mathrm{mV}$

G:IHPLC.5.29IDWP-11-50.Icd

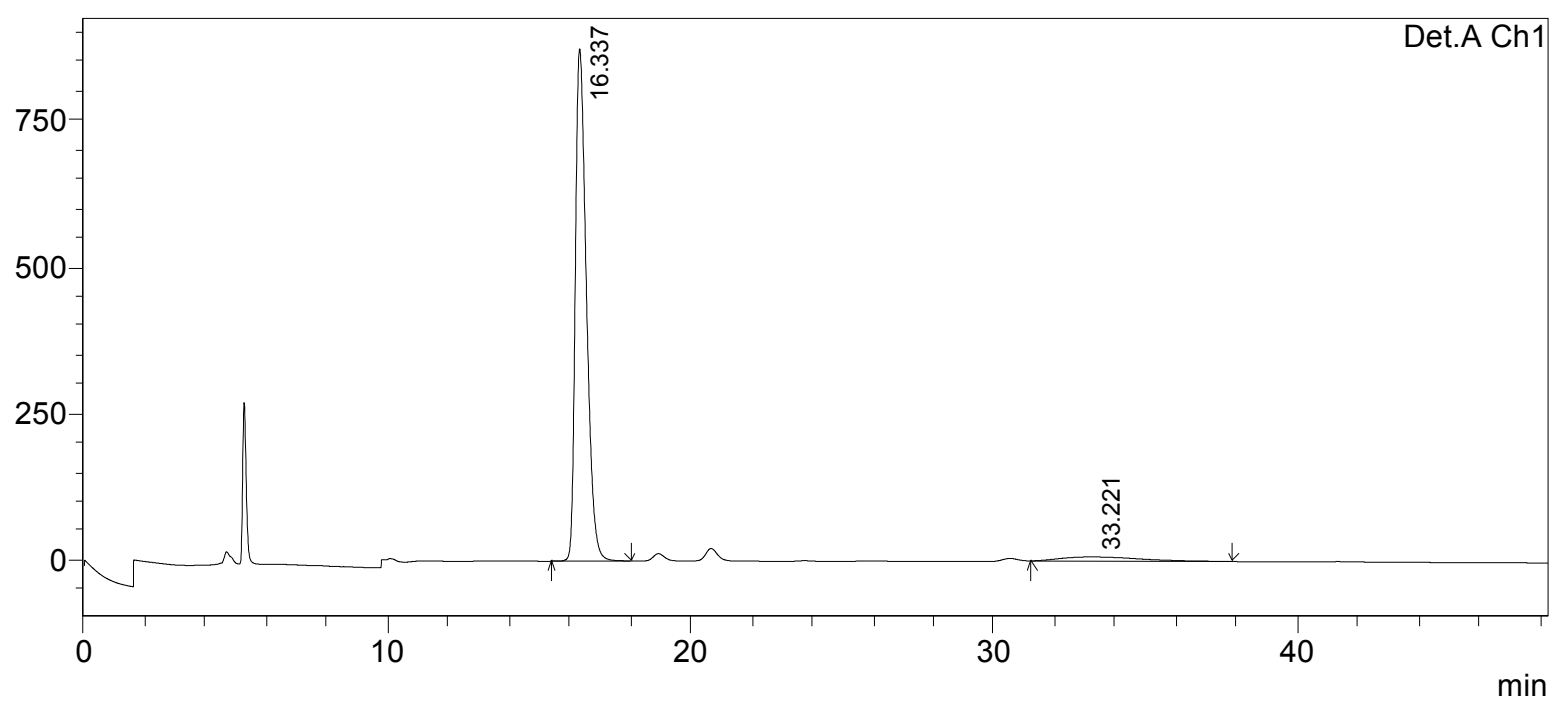

1 Det.A Ch1/214nm

Detector A Ch1 214nm

PeakTable

\begin{tabular}{|r|r|r|r|r|r|}
\hline \multicolumn{1}{|c|}{ Peak\# } & Ret. Time & \multicolumn{1}{c|}{ Area } & \multicolumn{1}{c|}{ Height } & \multicolumn{1}{c|}{ Area \% } & \multicolumn{1}{c|}{ Height \% } \\
\hline 1 & 16.337 & 22180303 & 874039 & 94.478 & 99.194 \\
\hline 2 & 33.221 & 1296342 & 7098 & 5.522 & 0.806 \\
\hline Total & & 23476645 & 881137 & 100.000 & 100.000 \\
\hline
\end{tabular}




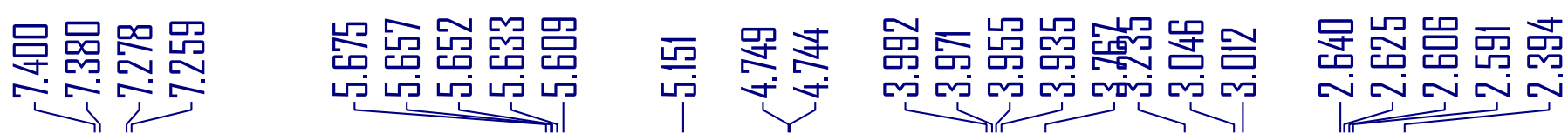
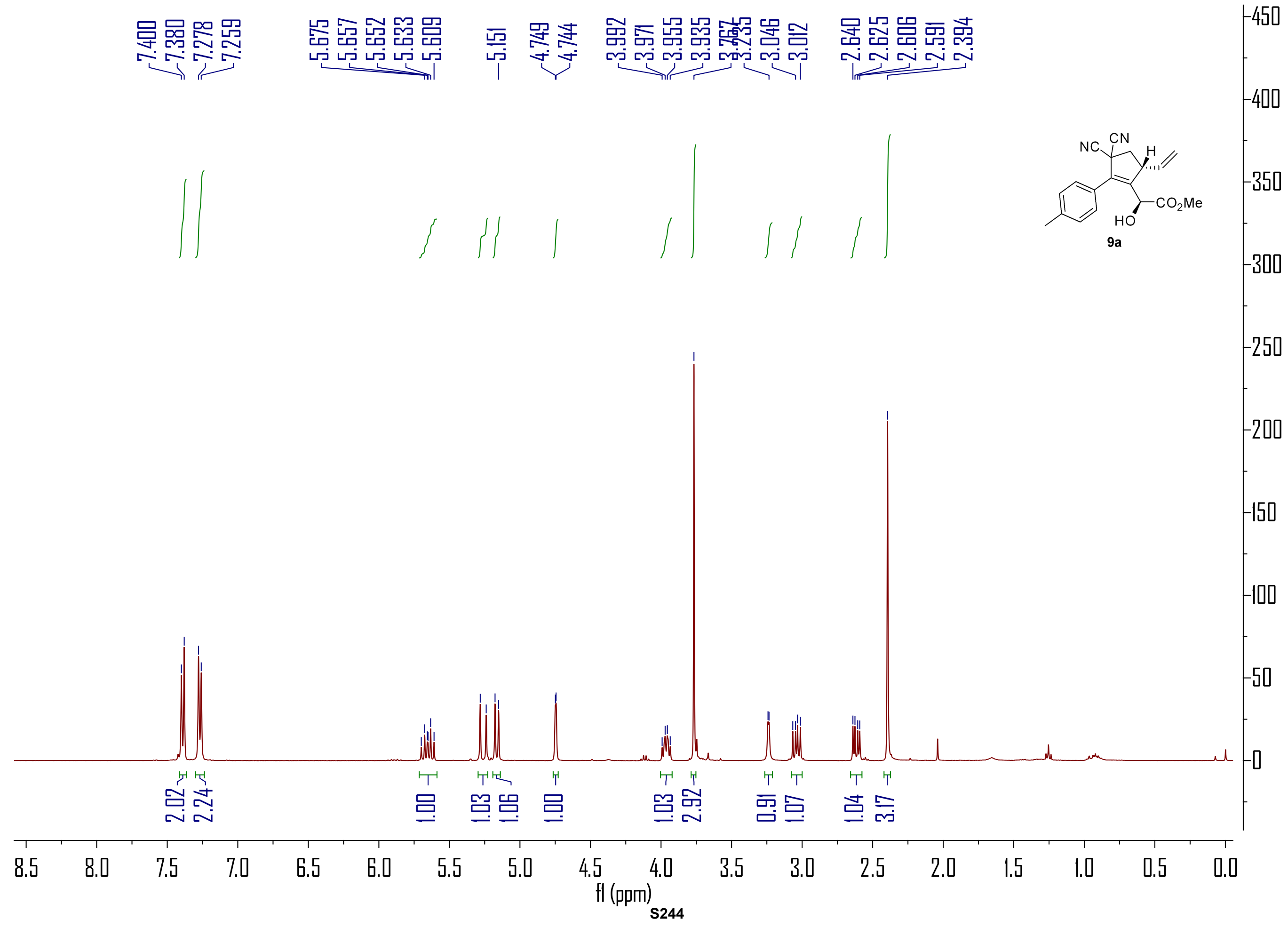

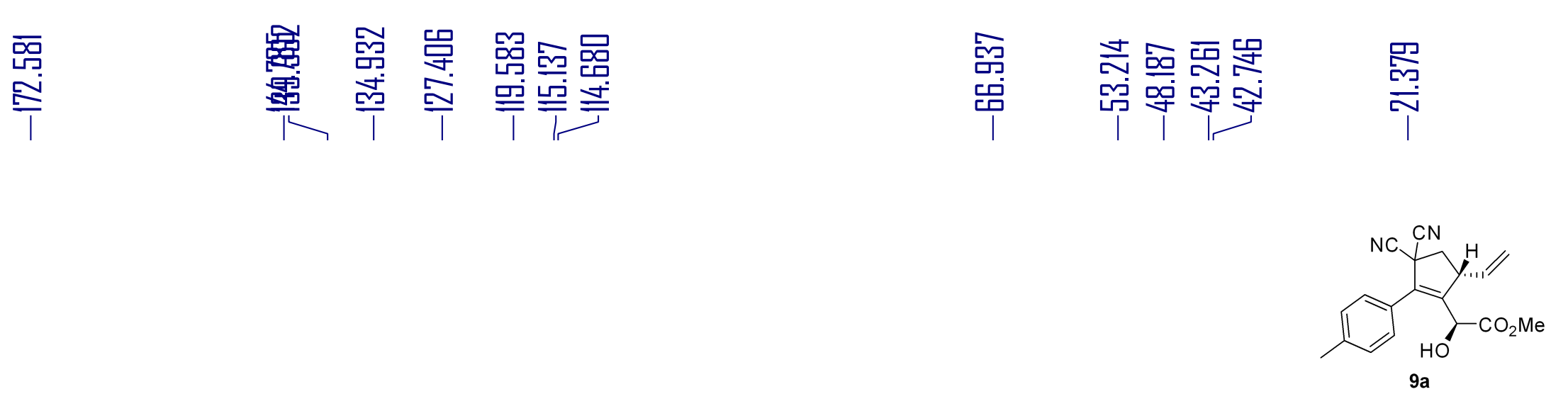

-1800
-1700
-1600
-1500
-1400
-1300
-1200
-1100
-1000
-900
-800
-700
-600
-500
-400
-300
-200
-100
-0
-100
--200
-




\section{$====$ Shimadzu LCsolution Analysis Report ====}

Acquired by

Sample Name

Sample ID

Vail \#

Injection Volume

Data File Name

Method File Name

Batch File Name

Report File Name

Data Acquired

Data Processed

G:IHPLC.5.29IDWP-11-56-2-RAC.Icd

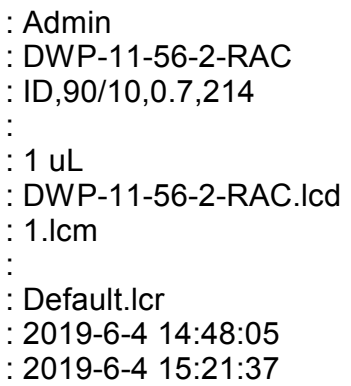

\section{$<$ Chromatogram>}

$\mathrm{mV}$

G:IHPLC.5.29IDWP-11-56-2-RAC.Icd

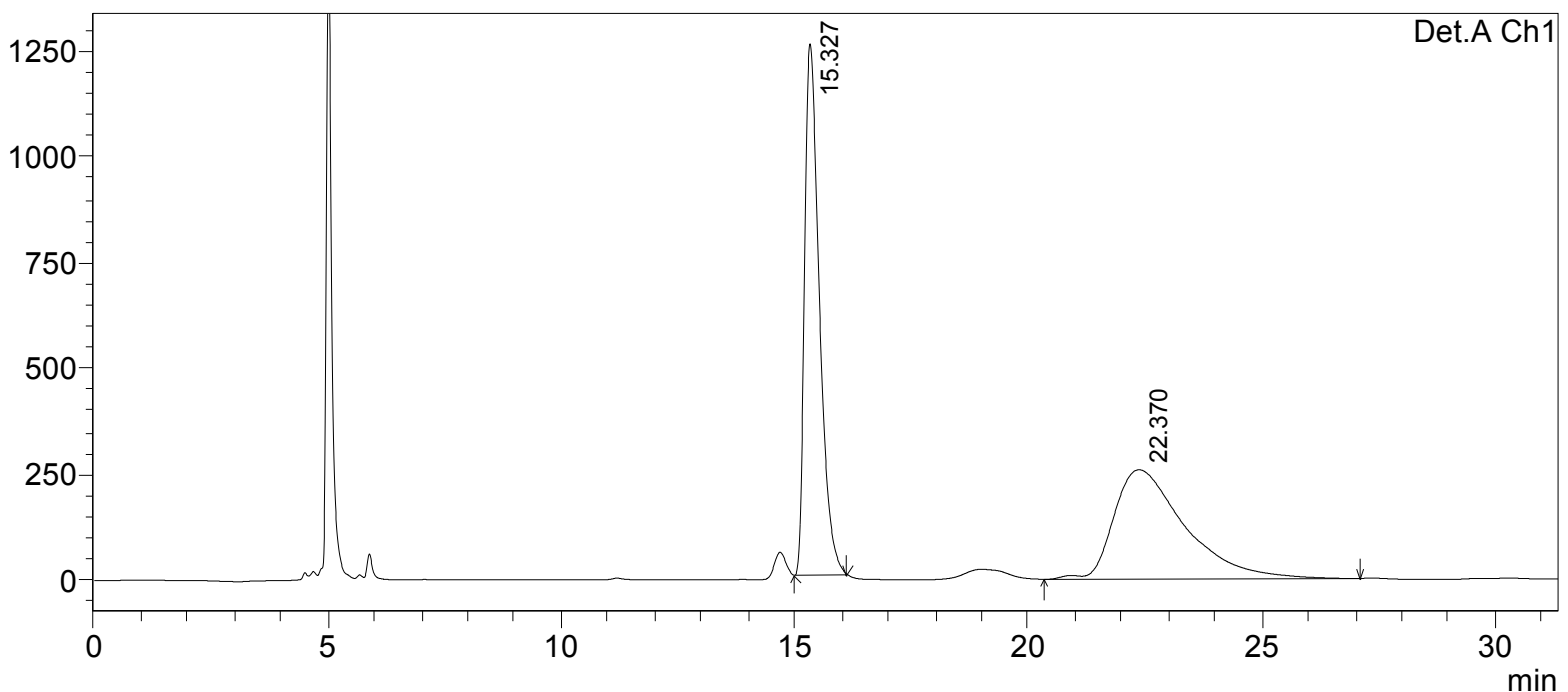

1 Det.A Ch1/214nm

PeakTable

Detector A Ch1 214nm

\begin{tabular}{|r|r|r|r|r|r|}
\hline \multicolumn{1}{|c|}{ Peak\# } & Ret. Time & \multicolumn{1}{|c|}{ Area } & \multicolumn{1}{|c|}{ Height } & Area \% & \multicolumn{1}{c|}{ Height \% } \\
\hline 1 & 15.327 & 27659646 & 1253583 & 50.392 & 82.934 \\
\hline 2 & 22.370 & 27228931 & 257968 & 49.608 & 17.066 \\
\hline Total & & 54888576 & 1511550 & 100.000 & 100.000 \\
\hline
\end{tabular}




\section{$====$ Shimadzu LCsolution Analysis Report ====}

Acquired by

Sample Name

Sample ID

Vail \#

Injection Volume

Data File Name

Method File Name

Batch File Name

Report File Name

Data Acquired

Data Processed
G:IHPLC.5.29IDWP-11-56-1.Icd

: Admin

: DWP-11-56-1

ID,90/10,0.7,214

$: 1 \mathrm{uL}$

DWP-11-56-1.Icd

$1 . \mathrm{lcm}$

Default.Icr

2019-6-4 15:55:16

: 2019-6-4 16:29:51

\section{$<$ Chromatogram>}

$\mathrm{mV}$

G:IHPLC.5.29IDWP-11-56-1.Icd

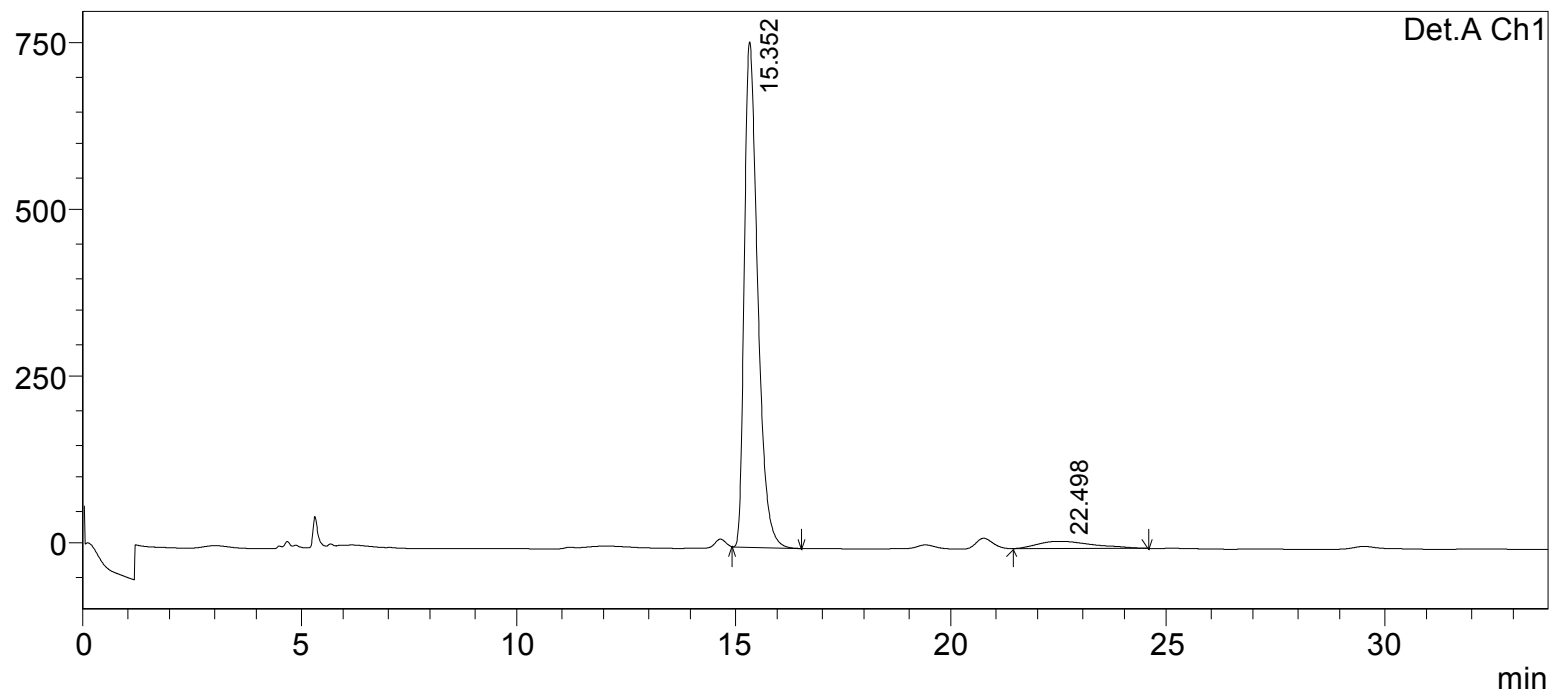

1 Det.A Ch1/214nm

Detector A Ch1 214nm

PeakTable

\begin{tabular}{|r|r|r|r|r|r|}
\hline Peak\# & Ret. Time & \multicolumn{1}{|c|}{ Area } & \multicolumn{1}{|c|}{ Height } & Area \% & \multicolumn{1}{|c|}{ Height \% } \\
\hline 1 & 15.352 & 16228369 & 757224 & 94.436 & 98.594 \\
\hline 2 & 22.498 & 956124 & 10798 & 5.564 & 1.406 \\
\hline Total & & 17184493 & 768022 & 100.000 & 100.000 \\
\hline
\end{tabular}




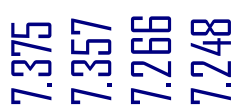
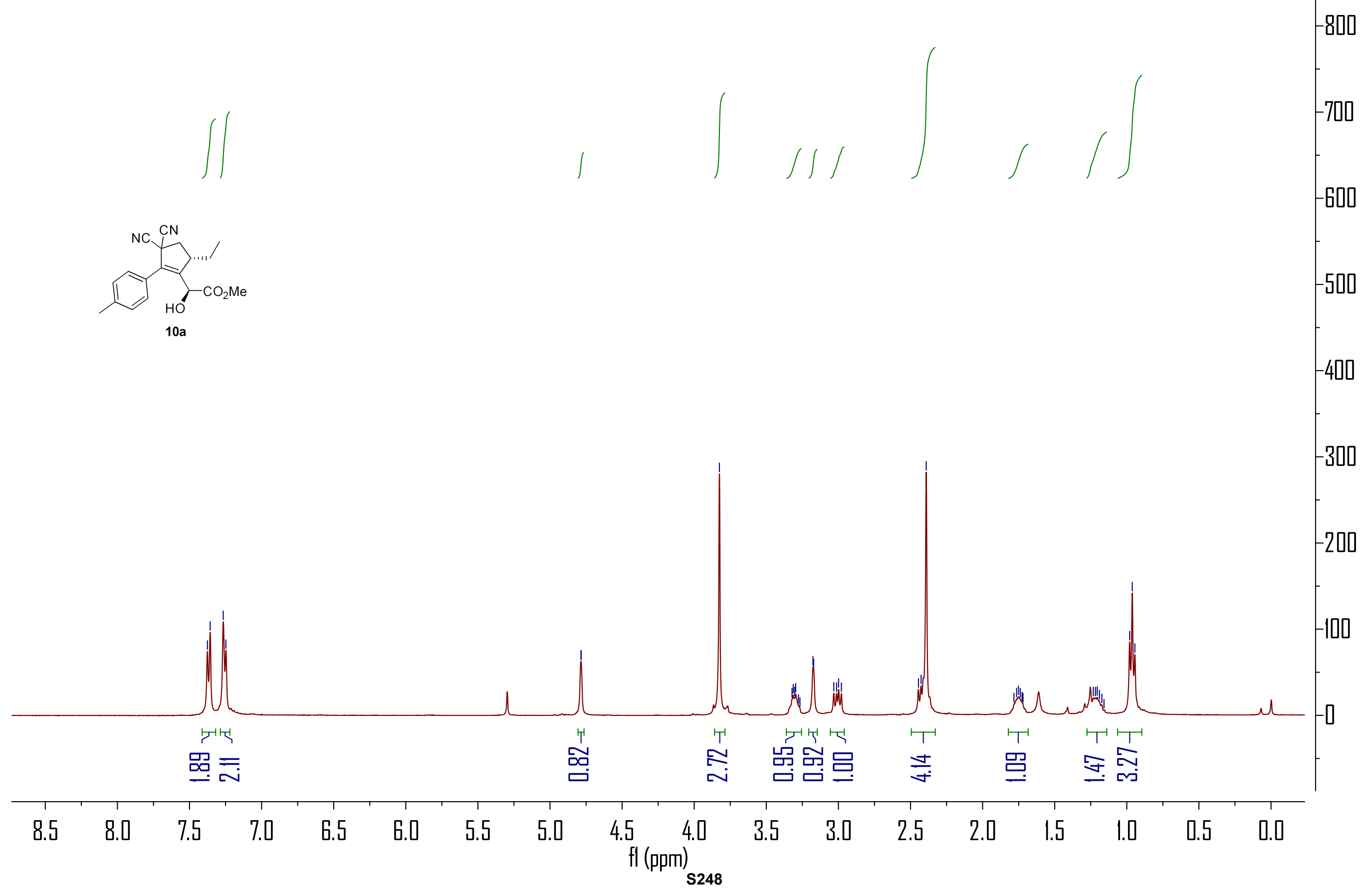


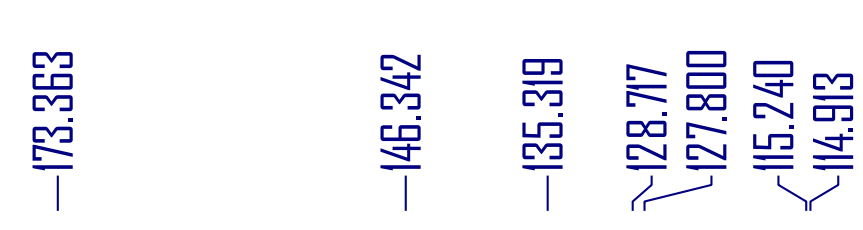

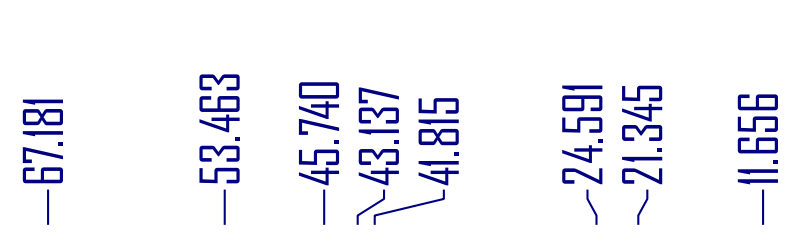

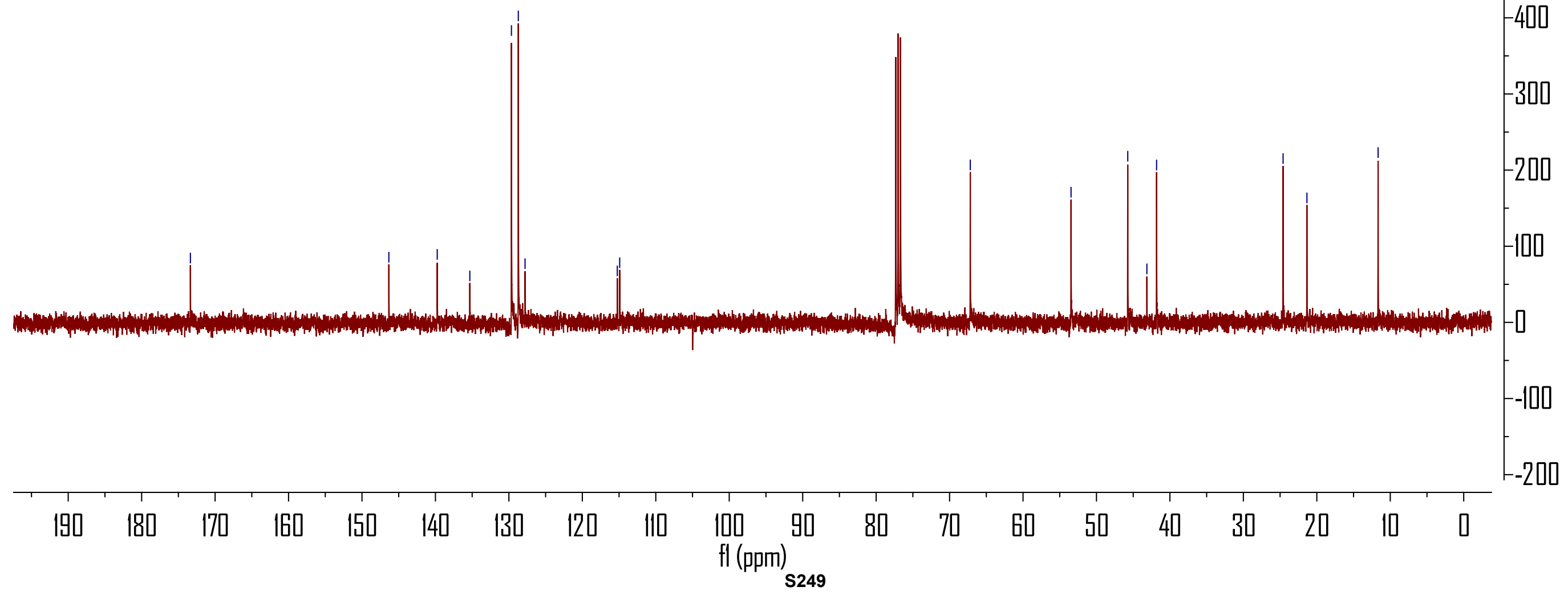


<smiles>COC(=O)C1=C(c2ccc(C)cc2)C2C(CBr)OC(CBr)C2N1</smiles>

\section{$====$ Shimadzu LCsolution Analysis Report ====}

Acquired by

Sample Name

Sample ID

Vail \#

Injection Volume

Data File Name

Method File Name

Batch File Name

Report File Name

Data Acquired

Data Processed
G:IDWP-11-60-rac-1b.Icd

: Admin

DWP-11-60-rac-1

ID,90/10,0.7,214

: $1 \mathrm{uL}$

DWP-11-60-rac-1b.lcd

: $1 . \mathrm{Icm}$

: Default.Icr

2019-6-8 13:35:22

2019-6-8 14:55:29

\section{$<$ Chromatogram>}

$\mathrm{mV}$

G:IDWP-11-60-rac-1b.Icd

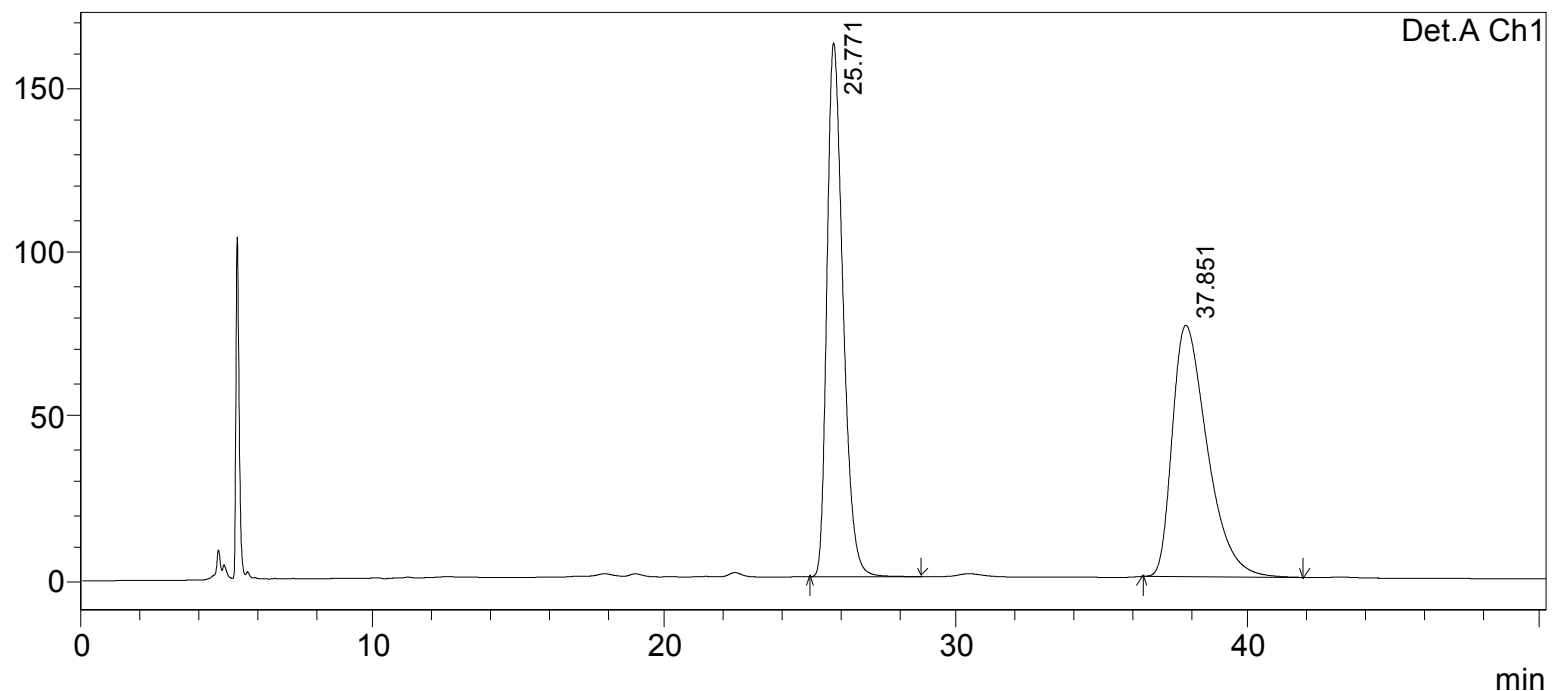

1 Det.A Ch1/214nm

PeakTable

Detector A Ch1 214nm

\begin{tabular}{|r|r|r|r|r|r|}
\hline Peak\# & Ret. Time & \multicolumn{1}{|c|}{ Area } & \multicolumn{1}{|c|}{ Height } & \multicolumn{1}{|c|}{ Area \% } & \multicolumn{1}{|c|}{ Height \% } \\
\hline 1 & 25.771 & 6224087 & 162607 & 49.352 & 67.989 \\
\hline 2 & 37.851 & 6387415 & 76560 & 50.648 & 32.011 \\
\hline Total & & 12611502 & 239167 & 100.000 & 100.000 \\
\hline
\end{tabular}


==== Shimadzu LCsolution Analysis Report ====

$\begin{array}{ll}\text { Acquired by } & \text { : Admin } \\ \text { Sample Name } & : \text { DWP-11-60-1b } \\ \text { Sample ID } & : \text { ID,90/10,0.7,214 } \\ \text { Vail \# } & : \text { 1 uL } \\ \text { Injection Volume } & : \text { DWP-11-60-1b.Icd } \\ \text { Data File Name } & : \text { 1.Icm } \\ \text { Method File Name } & : \\ \text { Batch File Name } & : \text { Default.lcr } \\ \text { Report File Name } & : 2019-6-814: 26: 15 \\ \text { Data Acquired } & : 2019-6-815: 17: 27 \\ \text { Data Processed } & \end{array}$

G:IDWP-11-60-1b.Icd

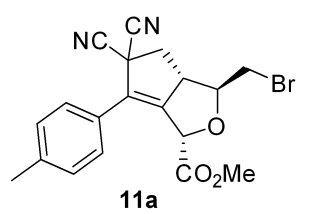

$<$ Chromatogram $>$

$\mathrm{mV}$

G:IDWP-11-60-1b.Icd

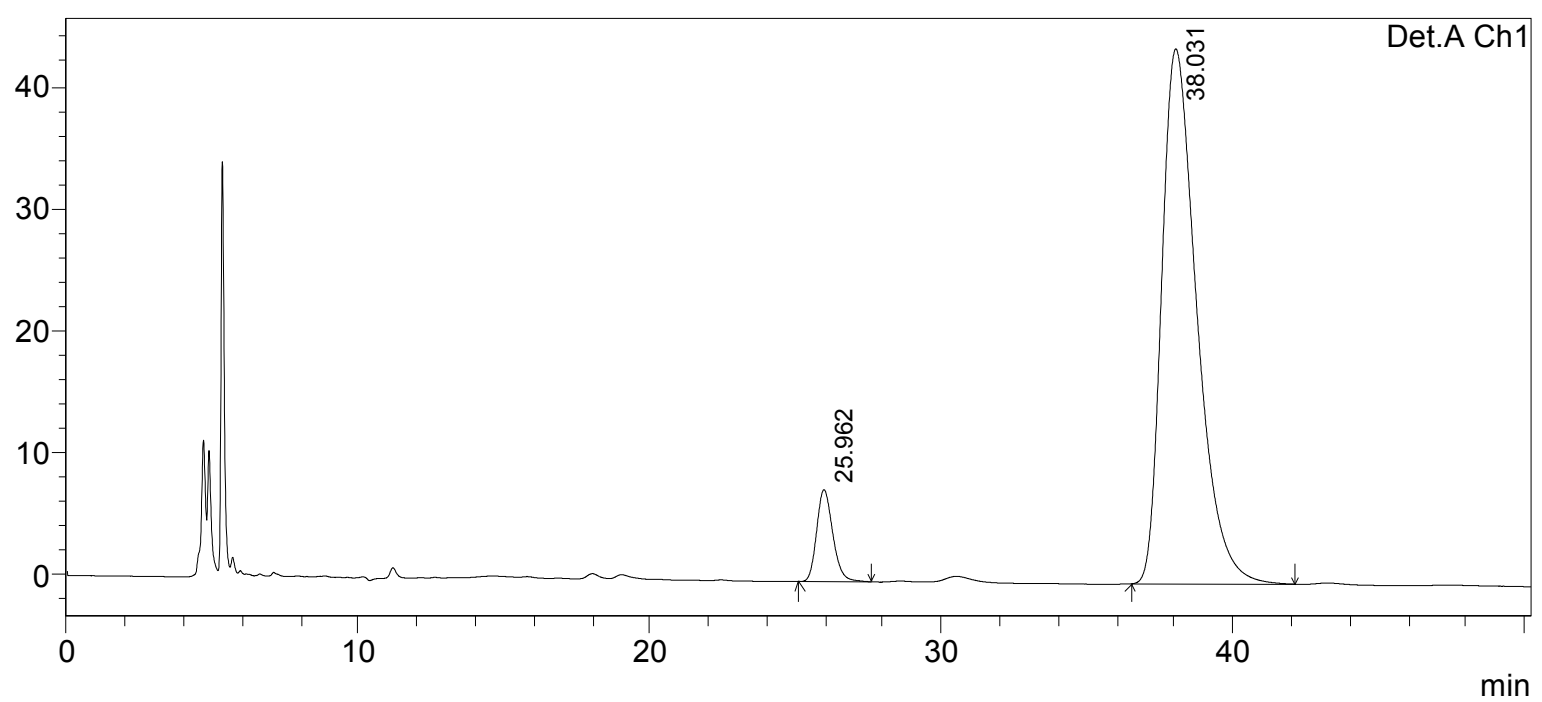

1 Det.A Ch1/214nm

PeakTable

Detector A Ch1 214nm

\begin{tabular}{|r|r|r|r|r|r|}
\hline Peak\# & Ret. Time & \multicolumn{1}{c|}{ Area } & Height & \multicolumn{1}{|c|}{ Area \% } & \multicolumn{1}{c|}{ Height \% } \\
\hline 1 & 25.962 & 290035 & 7516 & 7.348 & 14.662 \\
\hline 2 & 38.031 & 3656855 & 43745 & 92.652 & 85.338 \\
\hline Total & & 3946890 & 51261 & 100.000 & 100.000 \\
\hline
\end{tabular}



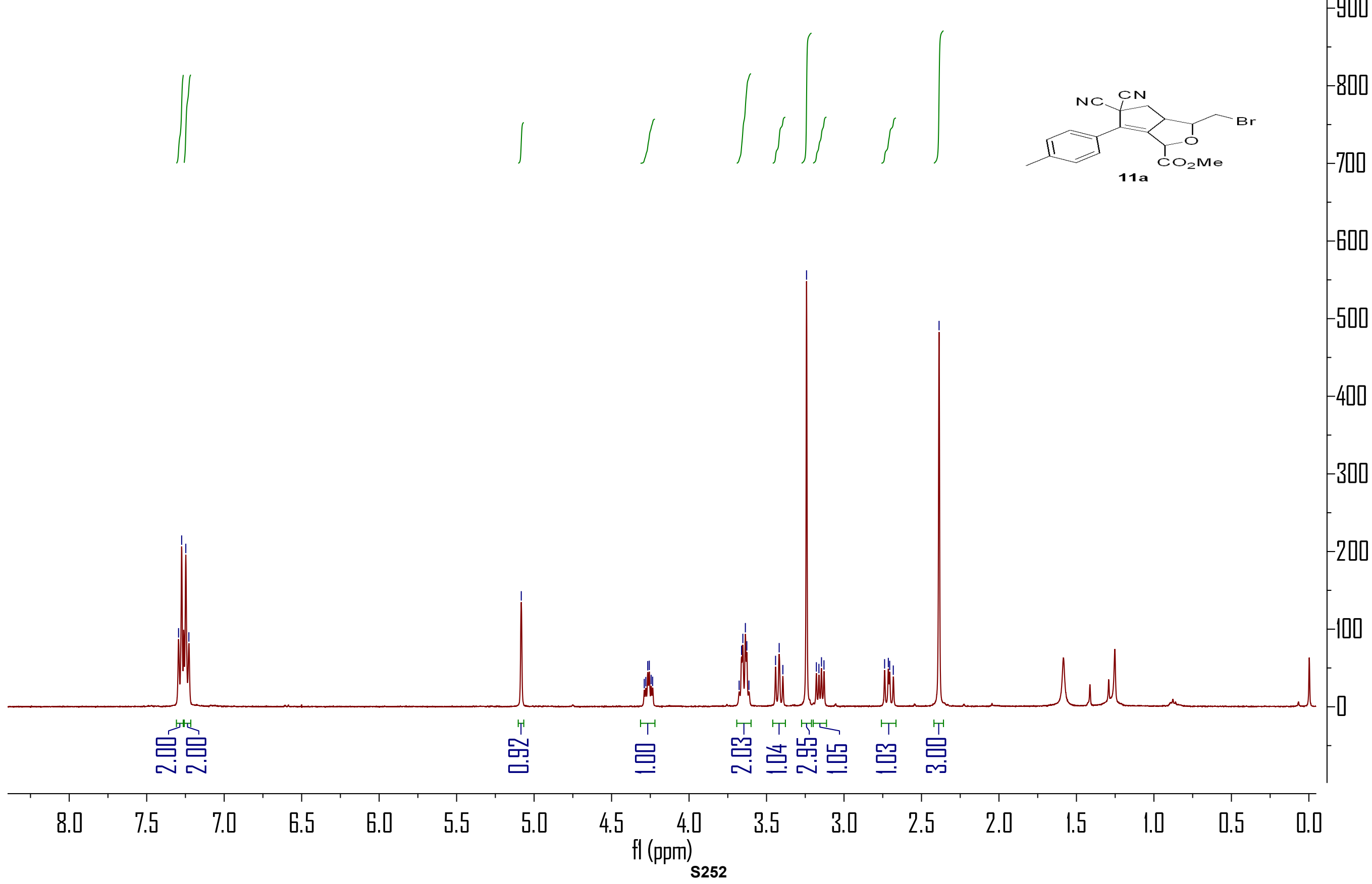


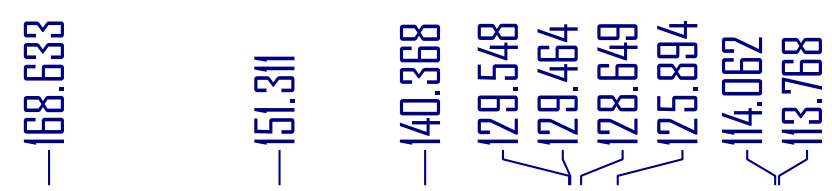

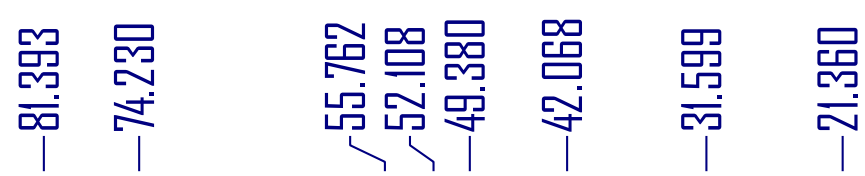
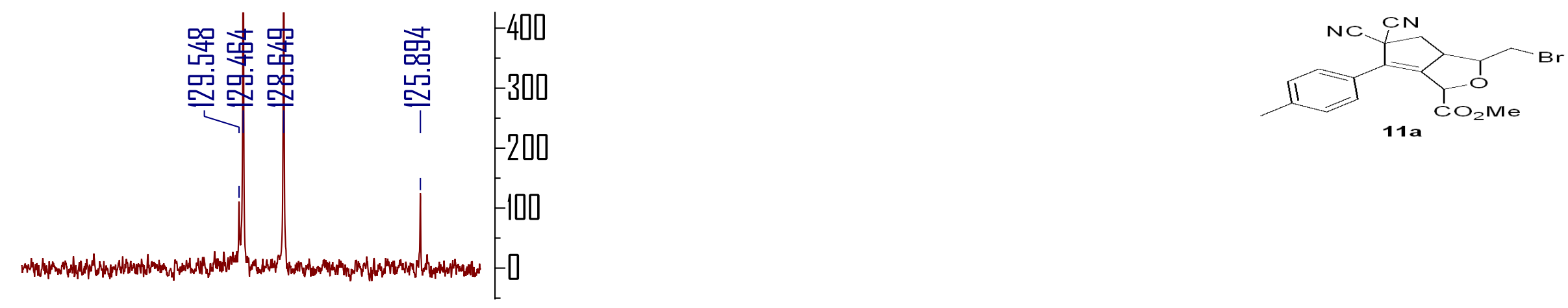

$\begin{array}{llll}332 & 130 \quad 128 & 126\end{array}$

$\mathrm{fl}$ (ppm)

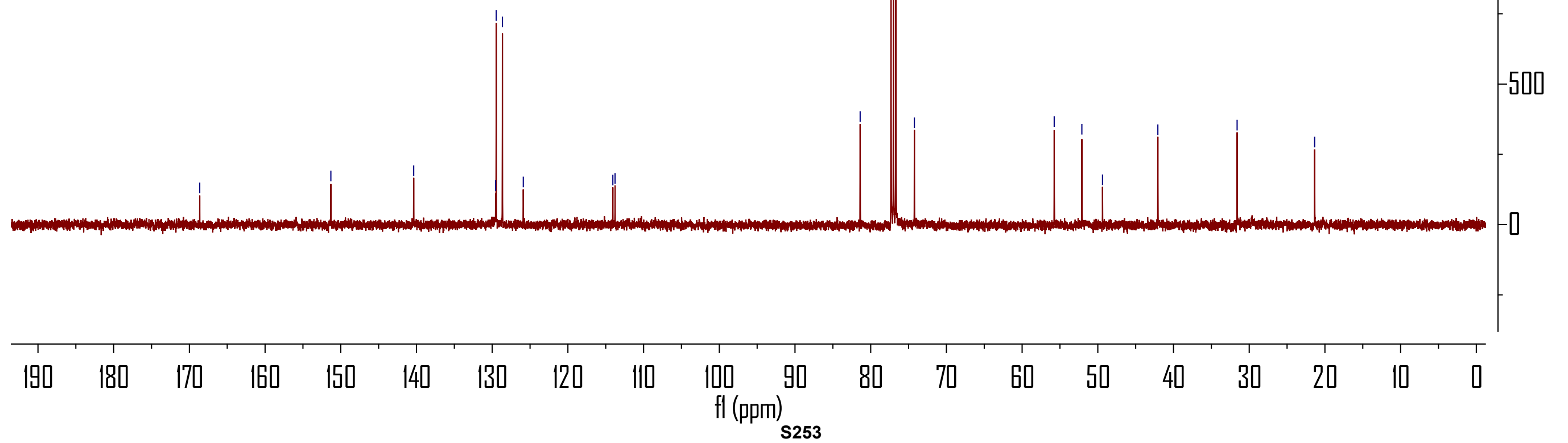




\section{HPLC pure DCM}

\section{DWP-D IC 912140.7}

\begin{tabular}{llll}
\hline Sample Name: & DWP-D IC 912140.7 & Injection Volume: & $\mathbf{1 . 0}$ \\
Vial Number: & RB3 & Channel: & UV_VIS_2 \\
Sample Type: & unknown & Wavelength: & $\mathbf{2 1 4 . 0}$ \\
Control Program: & test-dad6 & Bandwidth: & $\mathbf{4}$ \\
Quantif. Method: & $\mathbf{2 0 1 7 0 6 0 8}$ & Dilution Factor: & $\mathbf{1 . 0 0 0 0}$ \\
Recording Time: & $\mathbf{2 0 1 9 - 7 - 2 7 ~ 1 3 : 2 4}$ & Sample Weight: & $\mathbf{1 . 0 0 0 0}$ \\
Run Time (min): & $\mathbf{1 6 . 5 4}$ & Sample Amount: & $\mathbf{1 . 0 0 0 0}$ \\
\hline
\end{tabular}

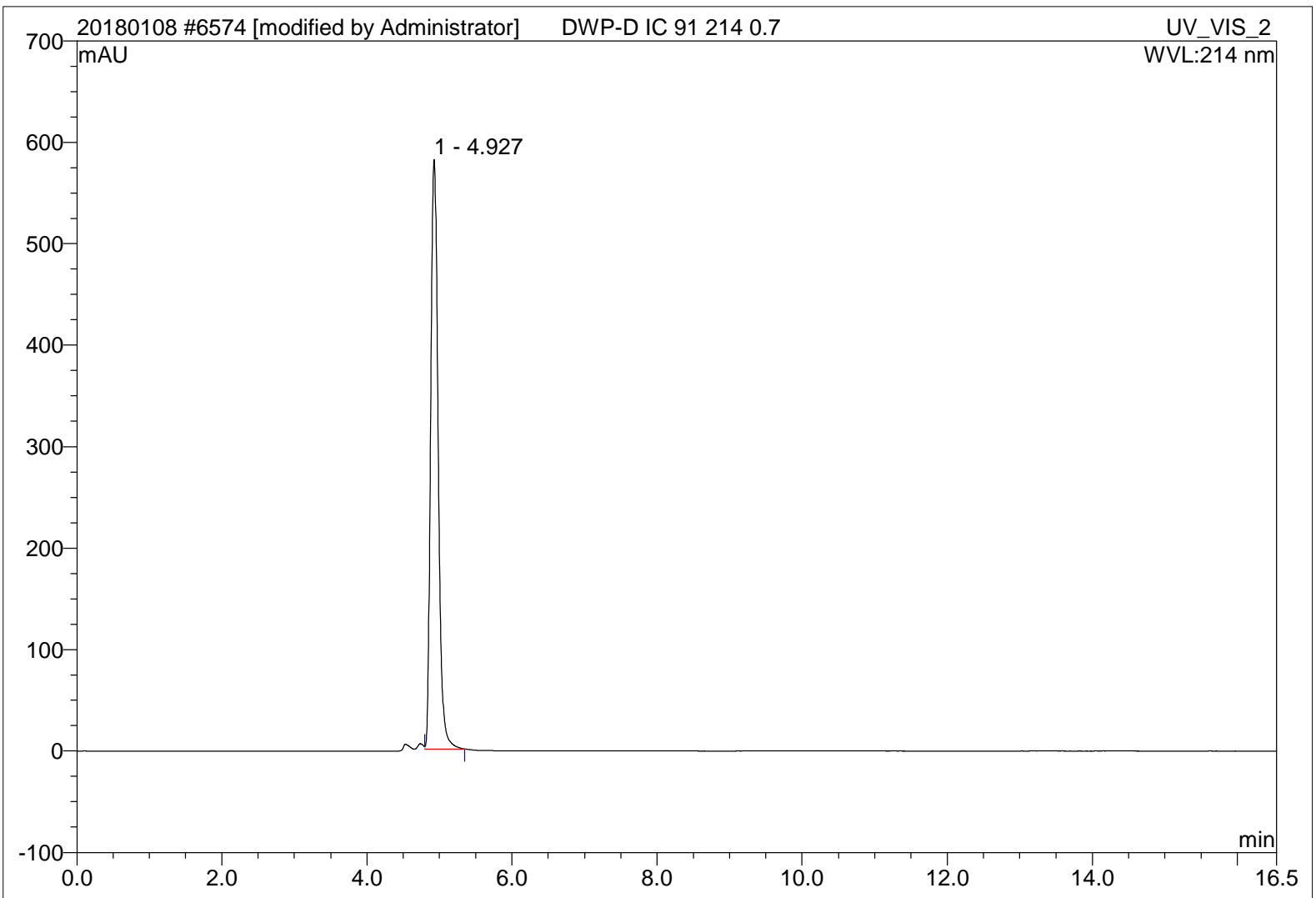

\begin{tabular}{|c|cccrccc|}
\hline No. & $\begin{array}{c}\text { Ret.Time } \\
\text { min }\end{array}$ & Peak Name & $\begin{array}{c}\text { Height } \\
\text { mAU }\end{array}$ & $\begin{array}{c}\text { Area } \\
\text { mAU*min }\end{array}$ & $\begin{array}{c}\text { Rel.Area } \\
\%\end{array}$ & Amount & Type \\
\hline 1 & 4.93 & n.a. & 581.301 & 69.961 & 100.00 & n.a. & MB $^{*}$ \\
\hline Total: & & & 581.301 & 69.961 & 100.00 & 0.000 & \\
\hline
\end{tabular}




\section{DWP-D ID3 9552140.7}

\begin{tabular}{llll|}
\hline Sample Name: & DWP-D ID3 9552140.7 & Injection Volume: & $\mathbf{1 . 0}$ \\
Vial Number: & RB3 & Channel: & UV_VIS_2 \\
Sample Type: & unknown & Wavelength: & $\mathbf{2 1 4 . 0}$ \\
Control Program: & test-dad4 & Bandwidth: & $\mathbf{4}$ \\
Quantif. Method: & $\mathbf{2 0 1 7 0 6 0 8}$ & Dilution Factor: & $\mathbf{1 . 0 0 0 0}$ \\
Recording Time: & $\mathbf{2 0 1 9 - 7 - 2 8 ~ 1 4 : 4 1}$ & Sample Weight: & $\mathbf{1 . 0 0 0 0}$ \\
Run Time (min): & $\mathbf{1 4 . 4 6}$ & Sample Amount: & $\mathbf{1 . 0 0 0 0}$ \\
\hline
\end{tabular}

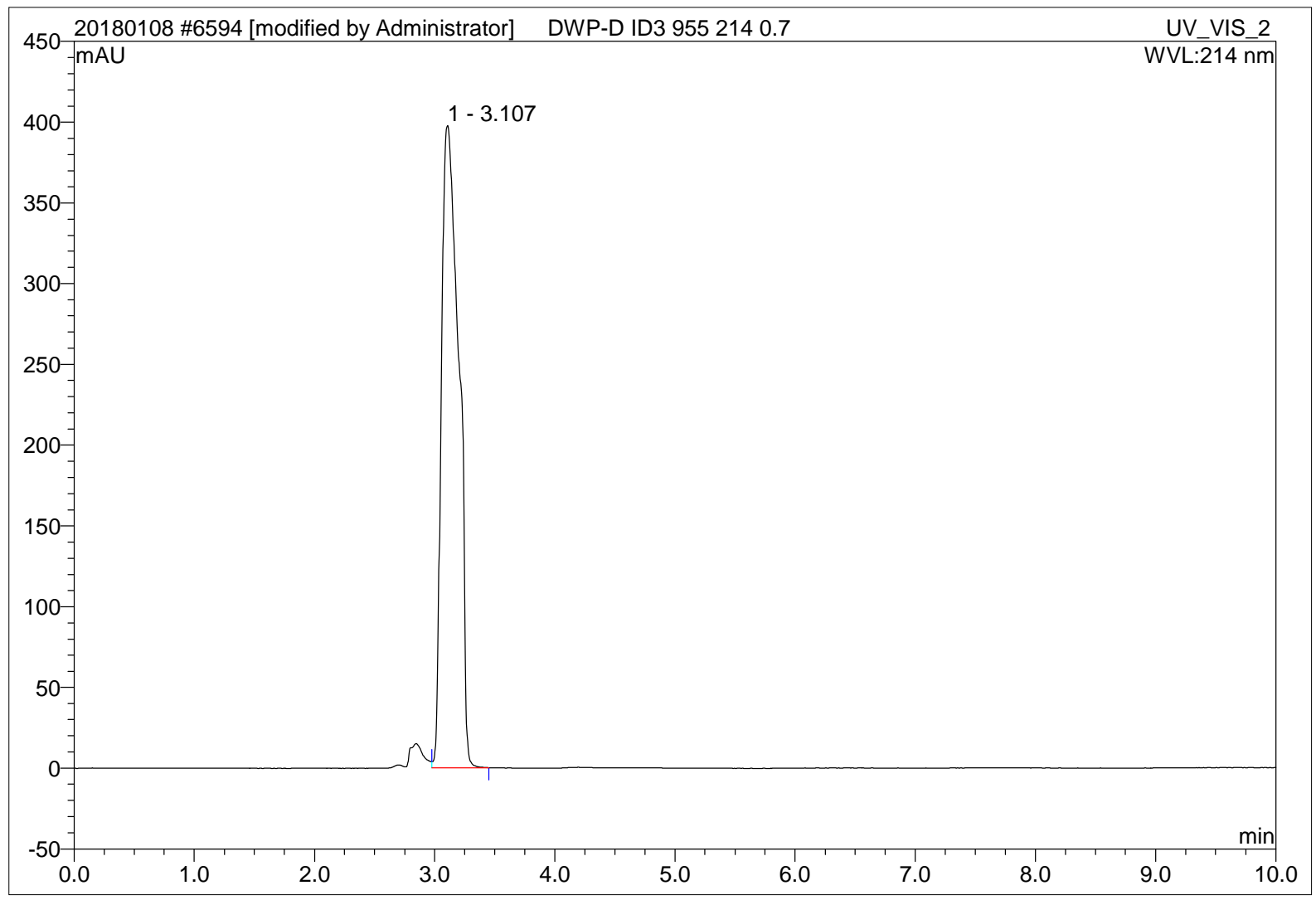

\begin{tabular}{|c|ccccccc|}
\hline No. & $\begin{array}{c}\text { Ret.Time } \\
\text { min }\end{array}$ & Peak Name & $\begin{array}{c}\text { Height } \\
\text { mAU }\end{array}$ & $\begin{array}{c}\text { Area } \\
\text { mAU*min }\end{array}$ & $\begin{array}{c}\text { Rel.Area } \\
\%\end{array}$ & Amount & Type \\
\hline 1 & 3.11 & n.a. & 397.608 & 66.578 & 100.00 & n.a. & MB $^{*}$ \\
\hline Total: & & & 397.608 & 66.578 & 100.00 & 0.000 & \\
\hline
\end{tabular}

